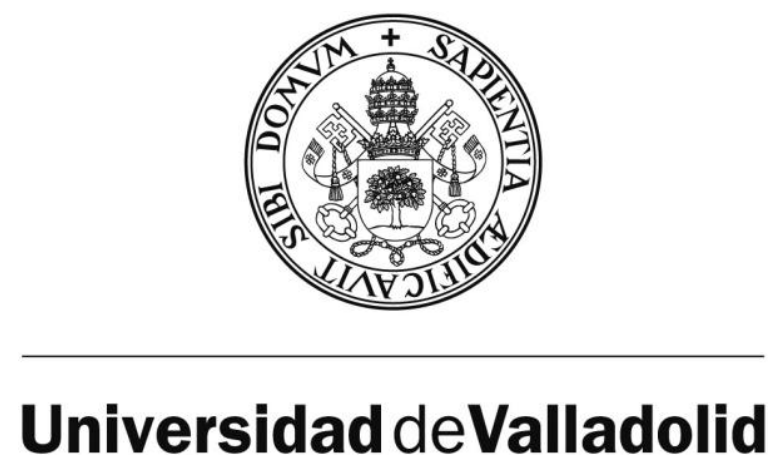

Tesis doctoral

\title{
Estudio de una década de intoxicaciones infantiles en un hospital terciario
}

\author{
Doctorando: \\ Ana Benito Justel \\ Médico de Emergencias Sanitarias Castilla y León.
}

Directores:

Antonio Dueñas Laita

Catedrático Área de Toxicología (ToxUVA)

Departamento de Medicina, Dermatología y Toxicología Universidad de Valladolid 
Miguel Ángel Castro Villamor

Médico de familia Centro de Salud Delicias I, La Cistérniga, Valladolid Profesor asociado del Departamento de Medicina, Dermatología y Toxicología.

Universidad de Valladolid. 
Antonio Dueñas Laita, Doctor en Medicina y Cirugía y Profesor Titular de la Facultad de Medicina de la Universidad de Valladolid,

\section{CERTIFICA:}

Que Doña Ana Benito Justel, Licenciada en Medicina y Cirugía por la Facultad de Medicina de la Universidad de Salamanca, ha realizado bajo mi dirección el trabajo titulado: "Estudio de una década de intoxicaciones infantiles en un hospital terciario".

Revisada la investigación realizada, que queda plasmada en la siguiente memoria, estimo que reúne los requisitos precisos para ser presentada y defendida para optar al grado de Doctor.

Para que conste a los efectos oportunos en Valladolid a 10 de Diciembre de 2014.

Fdo: Prof. Dr. Antonio Dueñas Laita 
Miguel Ángel Castro Villamor, Doctor en Medicina y Cirugía por la Facultad de Medicina de la Universidad de Valladolid,

\section{CERTIFICA:}

Que Doña Ana Benito Justel, Licenciada en Medicina y Cirugía por la Facultad de Medicina de la Universidad de Salamanca, ha realizado bajo mi dirección el trabajo titulado: "Estudio de una década de intoxicaciones infantiles en un hospital terciario".

Revisada la investigación realizada, que queda plasmada en la siguiente memoria, estimo que reúne los requisitos precisos para ser presentada y defendida para optar al grado de Doctor.

Para que conste a los efectos oportunos en Valladolid a 10 de Diciembre de 2014.

Fdo: Dr. Miguel Ángel Castro Villamor 


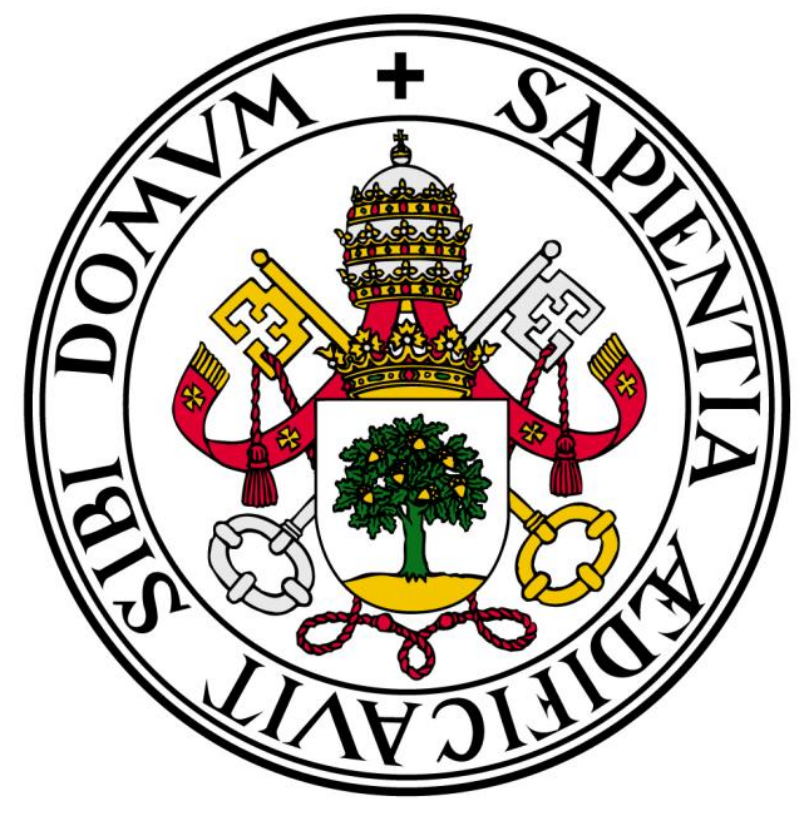

Agradecimientos 
Siempre he pensado que la palabra gracias es uno de los más importantes vocablos de nuestra lengua. $Y$ hoy quiero emplearlo varias veces.

En primer lugar, gracias al doctor Miguel Ángel Castro Villamor. La fortuna hizo que durante el tiempo que trabajé en el Servicio de Urgencias Hospitalario Río Hortega pudiera conocerle y aprender de él en todos los aspectos, profesionales y personales.

En segundo lugar, gracias al doctor Antonio Dueñas Laita, su figura supuso el estímulo y el empuje que necesitaba para realizar la labor de investigación que se me planteó.

Gracias a estos dos grandes profesionales que accedieron a ser tutores de mi tesis y que me han instruido, acompañado y animado desde el principio, sin ellos, nada de este trabajo hubiese sido posible.

Gracias a los compañeros del Servicio de Urgencias del Hospital Universitario Río Hortega por prestarme su ayuda en la recogida del material. A su coordinador, el Dr. M. Hernández Gajate por facilitarme el trabajo; a la Dra. Beatriz Martín Pérez, por ser un modelo con el que compararme, y al Dr. Raúl López Izquierdo, por su inestimable ayuda.

Gracias al personal de la administración tanto del Hospital Universitario Río Hortega, como de la Facultad de Medicina de Valladolid y de La Casa del Estudiante de Valladolid, por la facilidad con la que me han gestionado siempre lo que he necesitado.

Durante el tiempo de recogida de datos, elaboración y realización del estudio, muchas han sido las personas que me han ayudado, a todos ellos gracias.

La última parte de este agradecimiento, y no por ello la menos importante es a mi familia. En especial a mi padre, al que tanta ilusión le hacía ver a su hija convertida en doctora, y que estoy segura de que ahora está sonriendo y cuidándome desde el cielo junto a mis adorados abuelos. 
Y a las personas más importantes de mi vida:

A Rafa por ser a la vez mi marido, mi amigo, mi compañero.

A mi madre por ser la mejor madre del mundo y estar siempre a mi lado.

A mi hermano por cuidarme a su manera.

Y a la preciosa criatura que está a punto de nacer, por llenar mi vida de entusiasmo sin haberte visto aún la carita. 
A mi padre 


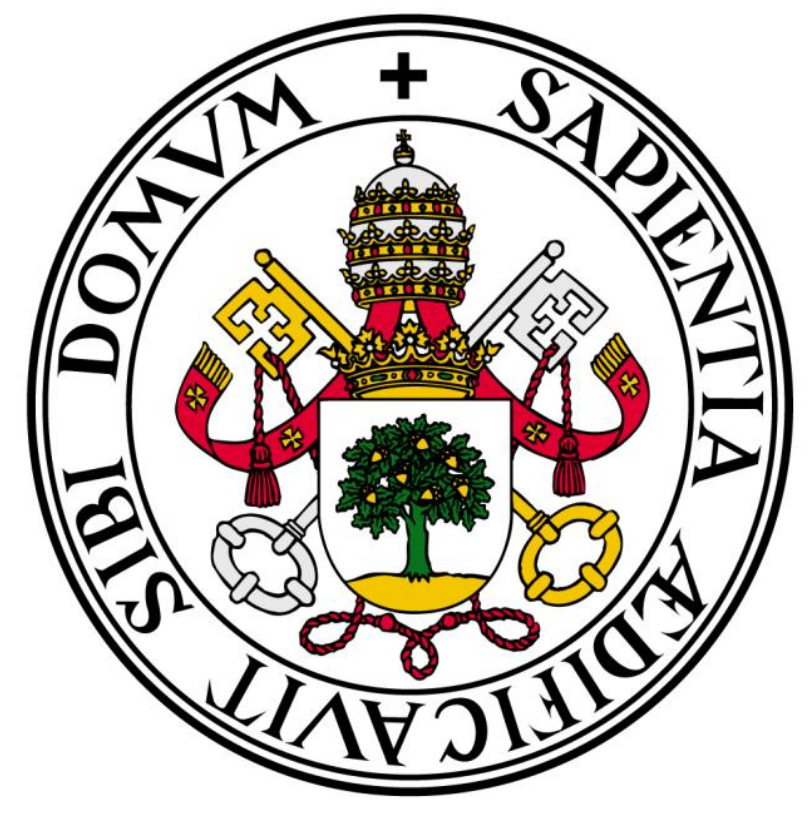

Índice 


\section{1. ÍNDICE}

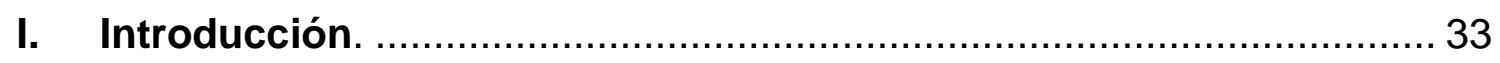

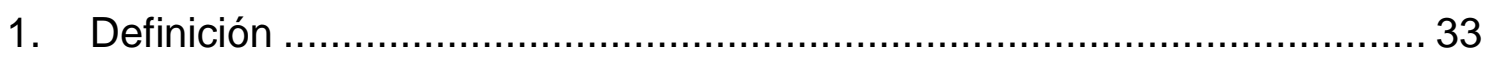

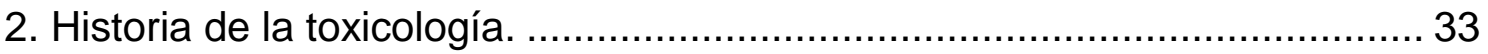

3. La toxicología en menores de edad a través de la historia........................... 39

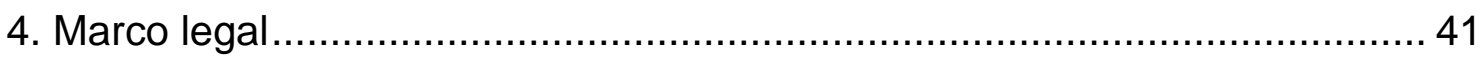

5. Estudios epidemiológicos en España..................................................... 42

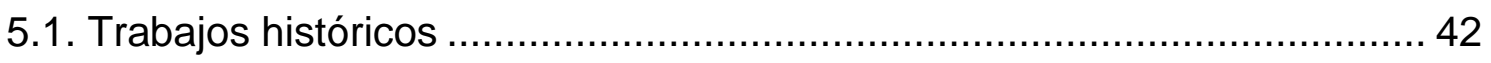

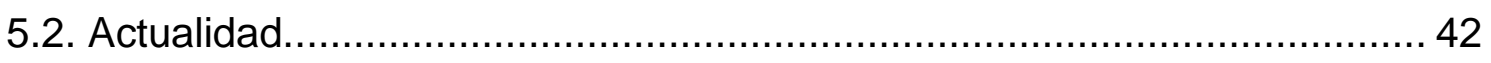

5.3. Análisis de los resultados de los estudios epidemiológicos....................... 45

5.4. Tesis españolas relacionadas con las intoxicaciones en nuestro país ...... 46

5.5. Estudios epidemiológicos de intoxicaciones en población infantil............. 46

5.6. Análisis de los estudios epidemiológicos sobre intoxicaciones en la

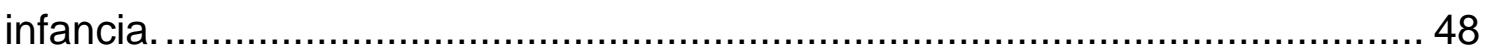

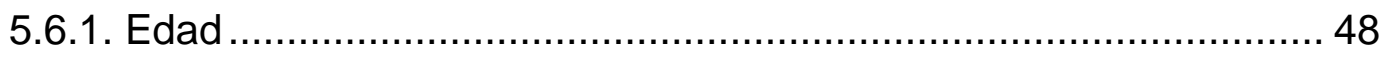

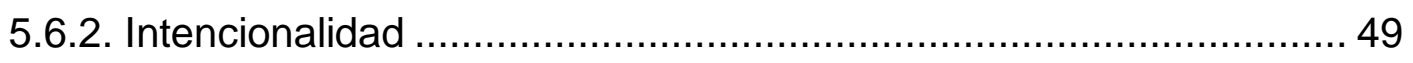

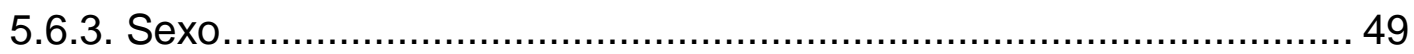

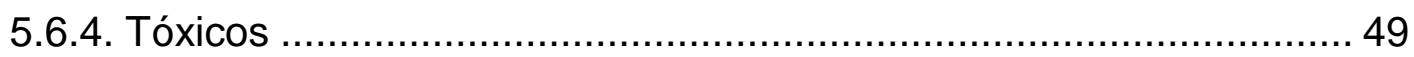

6. Incidencia de las intoxicaciones en el servicio de urgencias hospitalario en el

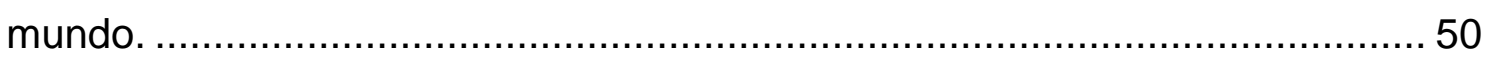

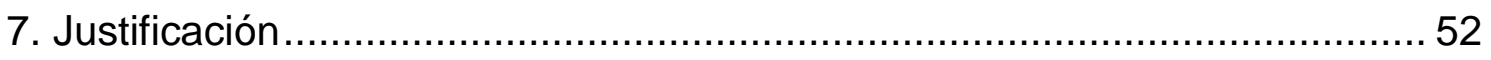

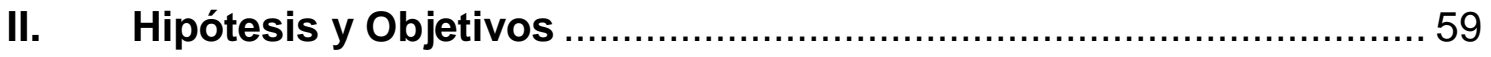

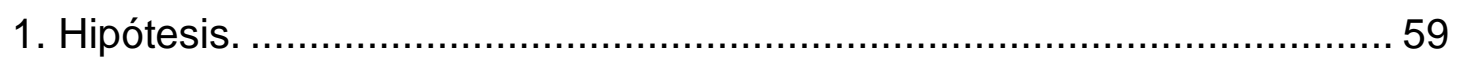




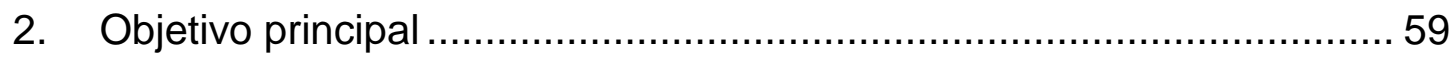

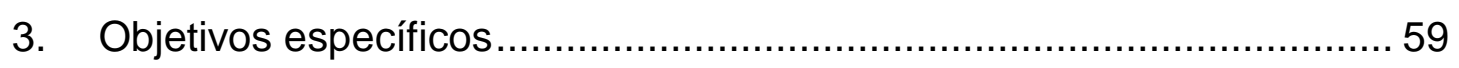

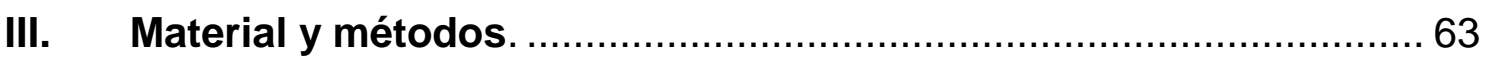

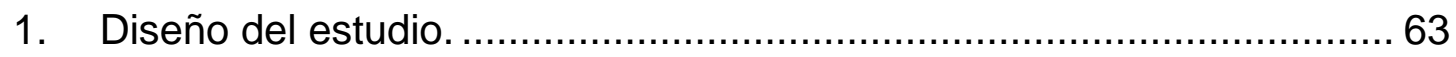

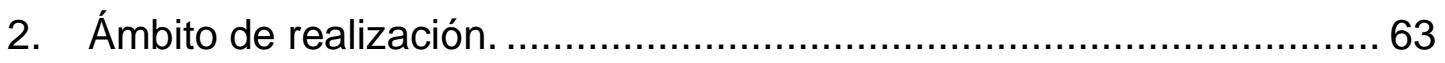

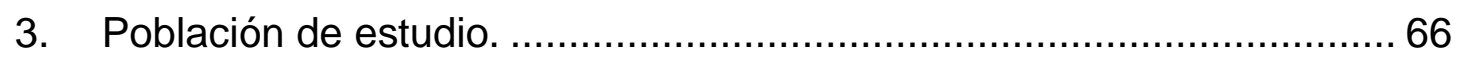

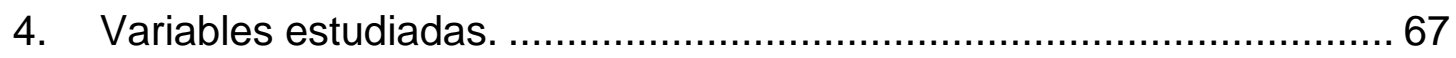

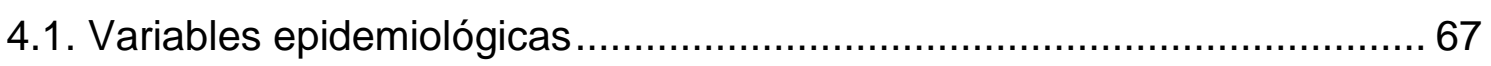

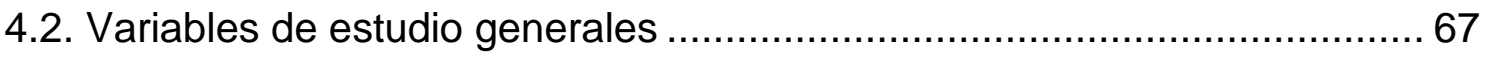

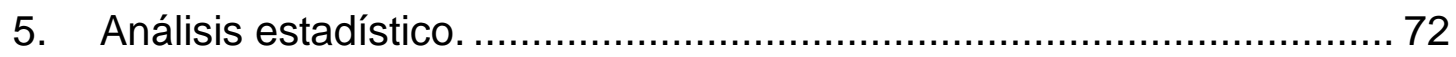

6. Tratamiento informático y control de calidad de los datos..................... 73

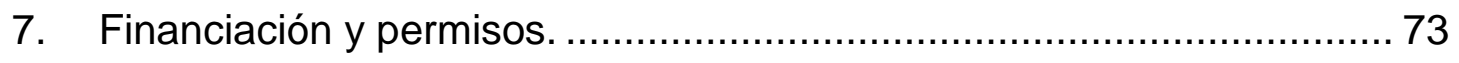

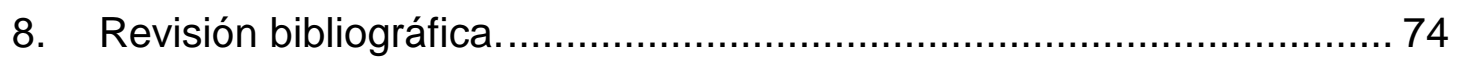

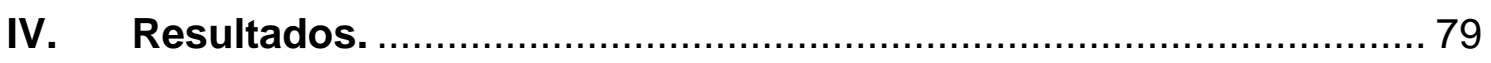

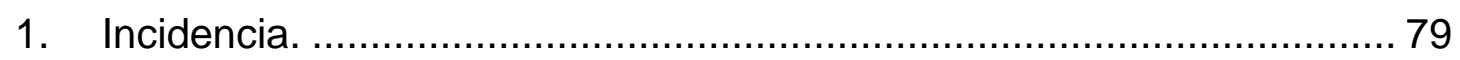

2. Diagnóstico principal establecido en el servicio de urgencias hospitalario.

2.1. Número de sustancias implicadas en la intoxicación. ............................... 83

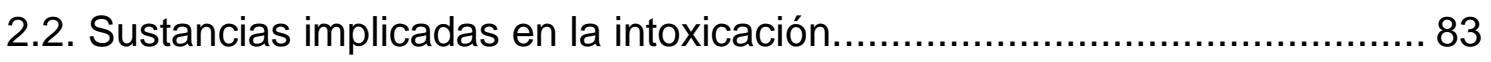

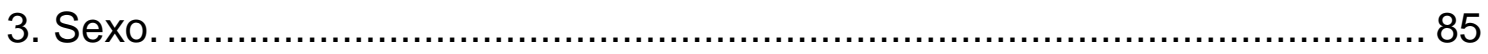

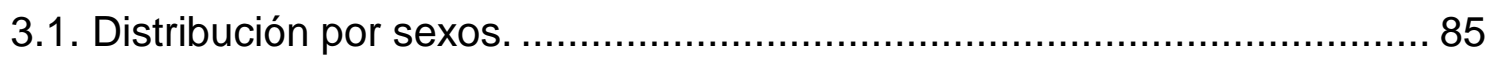

3.2. Distribución de los diferentes diagnósticos según el sexo. ........................ 86

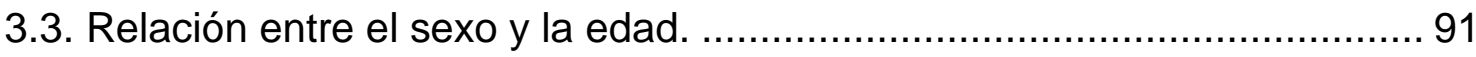


3.4. Relación entre el sexo y la distribución temporal de las intoxicaciones. ... 92 3.4.1. Relación entre el sexo y el año de atención en el servicio de urgencias hospitalario. 92

3.4.2. Relación entre el mes de atención en el servicio de urgencias hospitalario y el sexo del paciente. 93

3.5. Relación entre el sexo y otros parámetros. 95

3.6. Relación entre el sexo y la administración de Tiamina en su estancia en el servicio de urgencias hospitalario. 96

3.7. Relación entre el sexo del paciente y el destino tras la estancia en el servicio de urgencias hospitalario y el servicio hospitalario en el que va a ser ingresado. 96

3.8. Relación entre el sexo del paciente y el tipo de consulta por la que acude al servicio de urgencias hospitalario en el mismo año de la intoxicación, en años anteriores y en posteriores.

3.9. Relación entre el sexo y el número de consultas ambulatorias solicitadas desde su centro de salud. 98

3.10. Relación entre el sexo y la necesidad de realizar una interconsulta a la unidad de Psiquiatría durante su estancia en el servicio de urgencias hospitalario. 99

4 .Distribución por edades de las intoxicaciones en el servicio de urgencias hospitalario. 100

4.1. Relación entre la edad del paciente y los diferentes diagnósticos de intoxicación efectuados en el servicio de urgencias hospitalario 101

4.2. Relación entre la edad y el número de sustancias implicadas en la intoxicación. 103

4.3. Relación entre la edad y el tipo de sustancia implicada en la intoxicación. 
4.4. Relación entre la edad de los pacientes intoxicados y el año de su intoxicación.

4.5. Relación entre la edad y el mes de atención en el servicio de urgencias hospitalario. 110

4.6. Relación entre la edad y la hora de admisión al servicio de urgencias hospitalario.

4.7. Relación entre la edad y la presencia de antecedentes personales en los pacientes intoxicados. 114

4.8. Relación entre la edad del paciente y su localidad de origen 115

4.9. Relación entre la edad de los pacientes y la derivación previa de los pacientes al servicio de urgencias hospitalario por parte de otro servicio sanitario.

4.10. Acceso al servicio de urgencias hospitalario en vehículos de Emergencias Sanitarias teniendo en cuenta la edad del paciente. 118

4.11. Relación entre la edad y la realización de analítica y otras pruebas complementarias diagnósticas en el servicio de urgencias hospitalario. 120

4.12. Relación entre la edad y la administración de diferentes tratamientos en el servicio de urgencias hospitalario.

4.13. Relación entre la edad y el tiempo de permanencia en el servicio de urgencias hospitalario. 126

4.14. Relación entre la edad del paciente y el destino tras el paso por el servicio de urgencias hospitalario.

4.15. Relación entre la edad y otros parámetros. 129

4.16. Relación entre la edad y la necesidad de interconsulta a Psiquiatría durante su estancia en el servicio de urgencias hospitalario. 130

4.17. Relación entre la edad y la necesidad de consultar con Toxicología .... 131 
4.18. Relación entre la edad y la propiedad de la medicación implicada en las intoxicaciones medicamentosas.

4.19. Relación entre la edad, el mecanismo de origen y los síntomas iniciales de la intoxicación por monóxido de carbono. 133

4.20. Relación entre la edad y el número de veces que acuden al servicio de urgencias hospitalario los pacientes en el mismo año en el que se produce la intoxicación. 134

4.21. Relación entre la edad y el número de veces que acuden al servicio de urgencias hospitalario los pacientes en años previos al que se produce la intoxicación. 135

4.22. Relación entre la edad y el número de veces que acuden al servicio de urgencias hospitalario los pacientes en años posteriores al que se produce la intoxicación 135

4.23. Relación entre la edad y el número de consultas ambulatorias. 136

5. Incidencia temporal. 137

5.1. Pacientes atendidos cada año en el servicio de urgencias hospitalario. . 137 5.2 Pacientes atendidos cada mes en el servicio de urgencias hospitalario con diagnóstico de intoxicación. 138

5.3. Pacientes atendidos según la hora de admisión al servicio de urgencias hospitalario. 139

6. Agente causal de la intoxicación.

6..1. Intoxicación etílica.

6..1.1. Distribución de la intoxicación etílica según el sexo

6.1.2. Distribución por edades de la intoxicación etílica.

6.1.3. Distribución del diagnóstico de intoxicación etílica en el servicio de urgencias hospitalario según los años de atención. 
6.1.4. Distribución por meses en la intoxicación etílica. 146

6.1.5. Distribución por horas de la intoxicación etílica. 149

6.1.6. Distribución de la intoxicación etílica según la localidad de origen de los pacientes. 151

6.1.7. Antecedentes personales de los pacientes con intoxicación etílica . 153

6.1.8. Distribución de la intoxicación etílica según la derivación del paciente al servicio de urgencias hospitalario por parte de personal sanitario. 155

6.1.9. Acompañamiento en el servicio de urgencias hospitalario de los pacientes con intoxicación etílica 157

6.1.10. Distribución de la intoxicación etílica según la necesidad de transporte por vehículos de Emergencias Sanitarias. 159

6.1.11. Distribución de la intoxicación etílica según la realización de diferentes pruebas en el servicio de urgencias hospitalario. 161

6.1.12. Tratamiento recibido en los pacientes con intoxicación etílica ..... 172

6.1.13. Interconsulta con Psiquiatría durante la estancia en el servicio de urgencias hospitalarias en los pacientes con intoxicación etílica 184

6.1.14. Interconsulta con Toxicología durante la estancia en el servicio de urgencias hospitalario en los pacientes con intoxicación etílica 186

6.1.15. Distribución de la intoxicación etílica según el tiempo de estancia en el servicio de urgencias hospitalario. 187

6.1.16. Distribución de la intoxicación etílica según el destino del paciente tras su estancia en el servicio de urgencias hospitalario. 189

6.1.17. Distribución de la intoxicación etílica según la planta donde ingresa el paciente. 190

6.1.18. Tiempo de ingreso hospitalario en horas en los pacientes con intoxicación etílica 192 
6.1.19. Cifras de alcoholemia encontradas en los pacientes con intoxicación etílica 193

6.1.20. Consultas al servicio de urgencias hospitalario el mismo año y en años posteriores que acuden por la intoxicación etílica 195

6.1.21. Consultas al servicio de urgencias hospitalario en los años anteriores al que acuden por la intoxicación etílica 198

6.1.22. Número de consultas ambulatorias solicitadas en los pacientes con intoxicación etílica 201

6.2. Intoxicaciones medicamentosas. 202

6.2.1. Intoxicaciones medicamentosas y el sexo. 202

6.2.2. Edad de los pacientes en las intoxicaciones medicamentosas. 203

6.2.3. Año de atención en urgencias de los pacientes con diagnóstico de intoxicación medicamentosa. 204

6.2.4. Mes de atención en urgencias de los pacientes con diagnóstico de intoxicación medicamentosa. 205

6.2.5. Distribución por horas de llegada al servicio de Urgencias Hospitalario de los pacientes con intoxicación medicamentosa. 206

6.2.6. Localidad de origen de los pacientes con intoxicación medicamentosas. 207

6.2.7. Necesidad de vehículos de Emergencias Sanitarias en los pacientes con intoxicación medicamentosa 208

6.2.8. Necesidad de pruebas durante la estancia en el servicio de urgencias hospitalario de los intoxicados medicamentosos 208

6.2.9. Propietario de la medicación. 216

6.2.10. Necesidad de tratamiento durante la estancia en el servicio de urgencias hospitalario de los intoxicados medicamentosos. 216 
6.2.11. Interconsulta a Psiquiatría en los intoxicados medicamentosos.

6.2.12. Relación entre la necesidad de consulta con Toxicología y la intoxicación medicamentosa.

6.2.13. Estancia en el servicio de urgencias hospitalario de los pacientes con intoxicación medicamentosa. 229

6.2.14. Destino tras la estancia en el servicio de urgencias hospitalario de los pacientes con intoxicación medicamentosa. 230

6.2.15. Relación entre la intoxicación medicamentosa y diferentes parámetros.

6.2.16. Relación entre el número de consultas al servicio de Urgencias Hospitalario en el mismo año y en los posteriores al que lo hacen con el diagnóstico de la intoxicación y el diagnóstico de intoxicación medicamentosa.

6.3. Intoxicaciones por productos del hogar. 233

6.3.1. Sexo en las intoxicaciones por productos del hogar. 233

6.3.2. Edad en las intoxicaciones por productos del hogar. 233

6.3.3. Año en el que ocurren las intoxicaciones por productos del hogar. .... 234

6. 4. Intoxicaciones por monóxido de carbono. 235

6.4.1. Relación entre sexo e intoxicaciones por monóxido de carbono. 235

6.4.2. Relación entre la edad de los pacientes y la intoxicación por monóxido de carbono. 235

6.4.3. Relación entre el año de asistencia en el servicio de urgencias hospitalario de los pacientes y la intoxicación por monóxido de carbono. ..... 235

6.4.4. Mecanismo por el que se produce la intoxicación por monóxido de carbono 237 
6.4.5. Síntomas iniciales por los que demandan asistencia en los pacientes intoxicados por monóxido de carbono.

6.4.6. Relación entre el tratamiento con oxigenoterapia en el servicio de urgencias hospitalario en los pacientes con intoxicación por monóxido de carbono. 239

6.4.7. Relación entre la necesidad de interconsulta con Toxicología durante la estancia en el servicio de urgencias hospitalario en los pacientes con intoxicación por monóxido de carbono.

6.4.8. Relación entre el destino del paciente tras su paso por el servicio de urgencias hospitalario en los pacientes con intoxicación por monóxido de carbono. 240

6.5. Intoxicación por productos industriales. 241

6.5.1. Relación entre el sexo y la intoxicación por productos industriales 241

6.5.2. Relación entre la edad y la intoxicación por productos industriales. .... 241

6.6. Intoxicación por productos cosméticos. 242

6.6.1. Sexo en la intoxicación por productos cosméticos. 242

6.6.2. Edad en la intoxicación por productos cosméticos. 242

6.6.3. Relación entre la interconsulta a Toxicología durante la estancia en el servicio de urgencias hospitalario y la intoxicación por productos cosméticos. 243

6.6.4. Relación entre el destino tras la estancia en el servicio de urgencias hospitalario y la intoxicación por productos cosméticos. 243

6.7. Intoxicaciones por insecticidas. 244

6.7.1. Sexo en las intoxicaciones por insecticidas. 244

6.7.2. Edad en las intoxicaciones por insecticidas. 244 
6.7.3. Relación entre la necesidad de interconsulta con Toxicología en las intoxicaciones por insecticidas.

6.7.4. Relación entre el destino tras la estancia en el servicio de urgencias hospitalario en las intoxicaciones por insecticidas. 245

6.8. Intoxicación por cannabis 245

6.8.1. Sexo en las intoxicaciones por cannabis. 245

6.8.2. Edad en las intoxicaciones por cannabis. 246

6.8.3. Año de las intoxicaciones por cannabis. 246

6.8.4. Hora de admisión en el servicio de urgencias hospitalario de las intoxicaciones por cannabis. 247

6.8.5. Antecedentes personales de los pacientes con intoxicaciones por cannabis.

6.8.6. Necesidad de trasporte de los pacientes en vehículos de Emergencias Sanitarias en las intoxicaciones por cannabis.

6.8.7. Relación entre el número de sustancias implicadas en la intoxicación y el diagnóstico de intoxicación por cannabis.

6.8.8. Destino de los pacientes en las intoxicaciones por cannabis tras el paso por el servicio de urgencias hospitalario 250

6.9. Intoxicaciones por productos estimulantes. 251

6.9.1. Sexo de las intoxicaciones por productos estimulantes. 251

6.9.2. Edad de las intoxicaciones por productos estimulantes. 251

6.9.3. Relación entre el destino tras el paso por el servicio de urgencias hospitalario y las intoxicaciones por productos estimulantes. 252

6.10. Intoxicación por setas 252

6.10.1. Sexo en la intoxicación por setas. 252 
6.10.2. Edad de las intoxicaciones por setas.

6.10.3. Destino tras la estancia en el servicio de urgencias hospitalario de las intoxicaciones por setas.

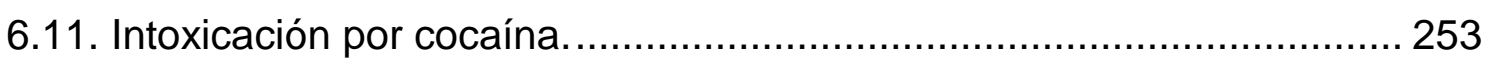

6.11.1. Sexo en la intoxicación por cocaína. ............................................... 253

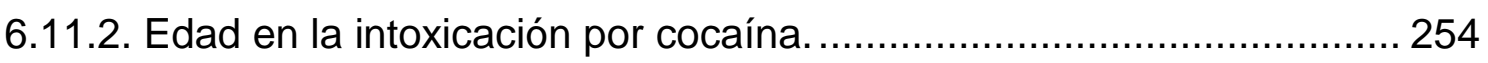

6.11.3. Número de sustancias implicadas en la intoxicación por cocaína......241

6.11.4. Destino tras la estancia en el servicio de urgencias hospitalario y la

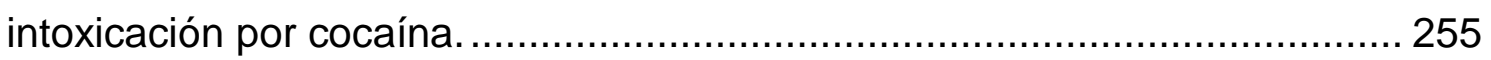

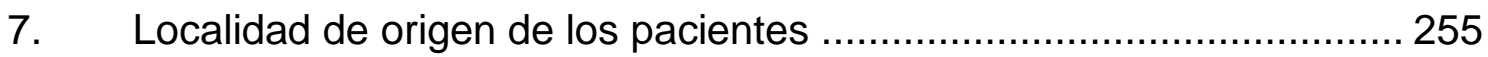

8. Antecedentes personales de los pacientes intoxicados....................... 257

9. Derivación de los pacientes intoxicados al servicio de urgencias hospitalario por personal sanitario......................................................... 258

10. Acompañamiento del paciente en su estancia en el servicio de urgencias hospitalario. 259

11. Acceso al servicio de urgencias hospitalario en vehículos de Emergencias Sanitarias 260

12. Realización de pruebas diagnósticas durante la estancia en el servicio de urgencias hospitalario. 261

12.1. Realización de analítica en el servicio de urgencias hospitalario. 261

12.2 Realización de diferentes tipos de analítica en el servicio de urgencias hospitalario. 262

12.3. Realización de electrocardiografía. 264

12.4. Realización de pruebas de imagen. 265

13. Tratamiento durante la estancia en el servicio de urgencias hospitalario. 266 
13.1. Administración de oxigenoterapia durante la estancia en el servicio de Urgencias hospitalario 266

13.2. Lavado gástrico. 267

13.3. Carbón activado. 268

13.4. Administración de antídoto específico en el servicio de urgencias hospitalario. 269

13.5. Administración de sueroterapia en el servicio de urgencias hospitalario. 269

13.6. Administración de antieméticos. 270

13.7. Administración de analgesia. 271

13.8. Administración de protectores gástricos 272

13.9. Administración de tiamina. 272

13.10. Administración de otro tipo de tratamiento. 273

14. Realización de interconsulta con el Servicio de Psiquiatría. 273

15. Realización de interconsulta con Toxicología 274

16. Tiempo de permanencia en el servicio de urgencias hospitalario. 275

17. Destino tras la estancia en el servicio de urgencias hospitalario. 276

18. Tiempo de ingreso hospitalario tras su estancia en el servicio de urgencias hospitalario. 277

19. Número de veces que acuden estos pacientes al servicio de urgencias hospitalario en el mismo año en el que se produce la asistencia por intoxicación. 279

20. Visitas al servicio de urgencias hospitalario en los años anteriores al registrado. 281 
21. Consultas en el servicio de urgencias hospitalario en los años posteriores a

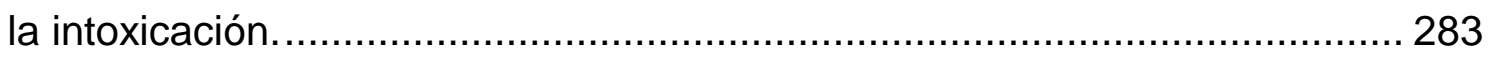

22. Consultas externas hospitalarias de los pacientes intoxicados............... 284

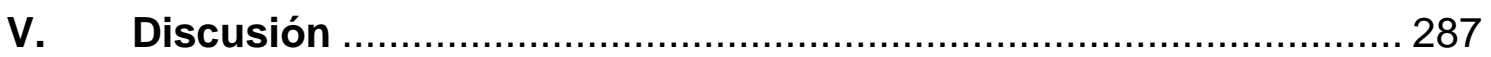

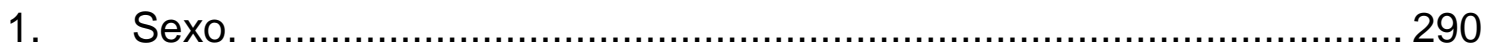

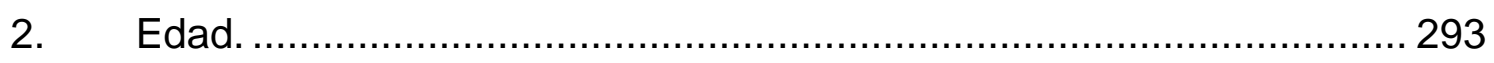

3. Año y mes de atención en el servicio de urgencias hospitalario.......... 294

4. Diagnóstico y tipo de sustancias implicadas en las intoxicaciones..... 295

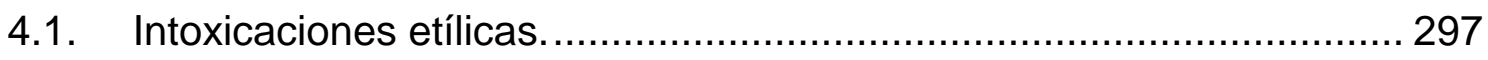

4.2. Intoxicaciones medicamentosas ................................................ 301

4.3. Intoxicaciones por monóxido de carbono. .................................... 302

4.4. Intoxicaciones por productos del hogar.................................. 303

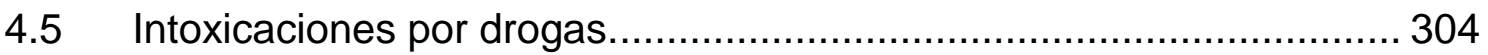

4.6. . Intoxicaciones por cosméticos.............................................. 308

4.7. Intoxicación por productos industriales.................................... 309

4.8. Intoxicaciones por pesticidas.............................................. 309

4.9. Intoxicaciones por setas. ........................................................ 310

5. Procedencia de los pacientes intoxicados............................... 310

6. Acceso al servicio de urgencias en vehículos de emergencias sanitarias. 310

7. Realización de pruebas durante su estancia en el servicio de urgencias. 312

8. Tratamiento de las intoxicaciones.

9. Interconsulta con la unidad de Salud Mental. 


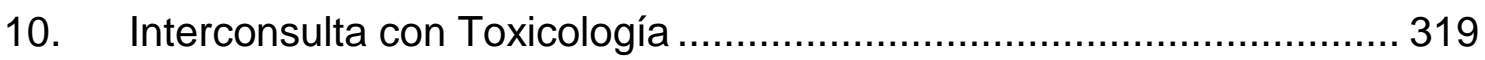

11. Tiempo de permanencia en el servicio de urgencias.......................... 320

12. Destino tras el paso por la urgencia hospitalaria................................ 321

13. Consultas en el servicio de urgencias hospitalario en el mismo año de la

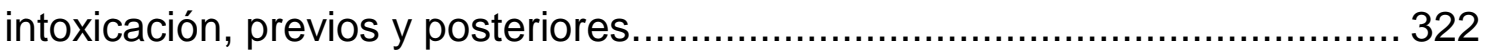

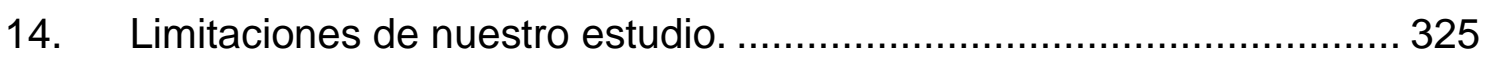

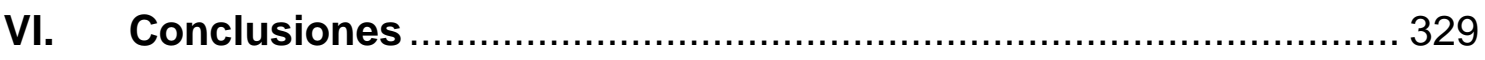

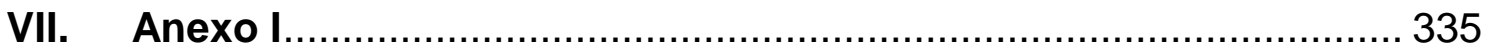

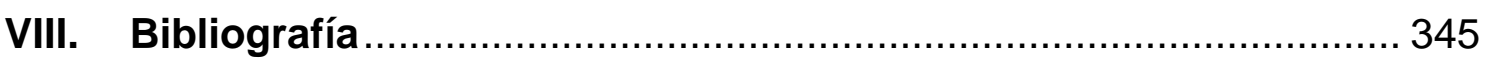




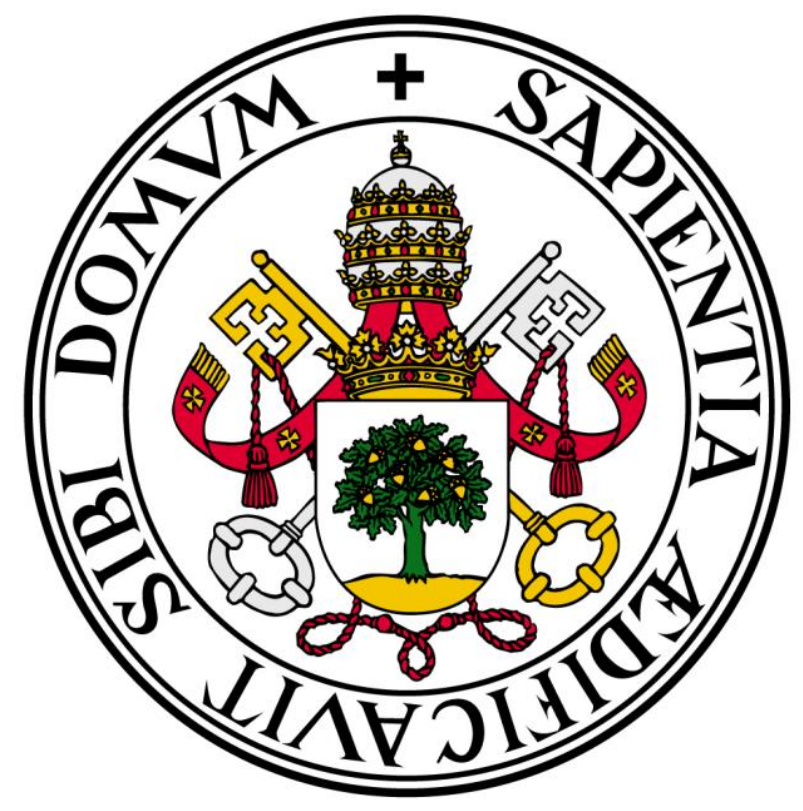

Introducción 



\section{Introducción.}

\section{Definición}

Según la RAE la toxicología se define como el estudio de las sustancias tóxicas y sus efectos, siendo fijada la significación de la palabra tóxico como perteneciente o relativo a un veneno o toxina.

Etimológicamente la toxicología se entiende como la "ciencia que estudia los venenos". La palabra toxikon procede del griego moderno y significa veneno de las flechas usadas en la caza en la antigüedad. Estas puntas de flechas se preparaban con material contaminado con bacterias con la intención de acelerar la muerte de los animales que cazaban.

El término "veneno", sin embargo, procede de la época romana, del latín venenum o poción del amor de Venus, refiriéndose a los tóxicos que se administraban en forma de pociones

Actualmente la toxicología es la ciencia que estudia las sustancias químicas y los agentes físicos en cuanto son capaces de producir alteraciones patológicas a los seres vivos, a la par que estudia los mecanismos de producción de tales alteraciones y los medios para contrarrestarlas, así como los procedimientos para detectar, identificar y determinar tales agentes y valorar su grado de toxicidad.

En la literatura actual, se encuentran definiciones clásicas de los términos tóxico e intoxicación como los descritos por Dueñas-Laita en $1999^{1}$, que define tóxico como cualquier sustancia química capaz de producir la muerte, heridas $u$ otros efectos perjudiciales en el organismo. Y el término intoxicación, como el conjunto de signos y síntomas resultantes de la acción de un tóxico sobre el organismo. 


\section{Historia de la toxicología.}

Puede decirse que la toxicología nace con el ser humano. Desde el Paleolítico el hombre se da cuenta de que algunas plantas producen la muerte de sus semejantes y de otros seres vivos.

Se tiene conocimiento de que los Masais de Kenia hace 18000 años ya utilizaban flechas impregnadas de estrofantina (sustancia similar a la digital) para capturar a sus presas

El Papiro de Ebers es el texto médico más antiguo conocido. Se encuentra fechado en el año 1500 ac. Escrito en hierático, consta de 110 páginas en las cuales se encuentran 700 fórmulas magistrales y remedios. En este texto se cita las primeras referencias escritas sobre venenos tales como el arsénico, el plomo, la cicuta, el acónito, el opio y los glucósidos cardiacos, así como sobre el uso terapéutico del carbón.

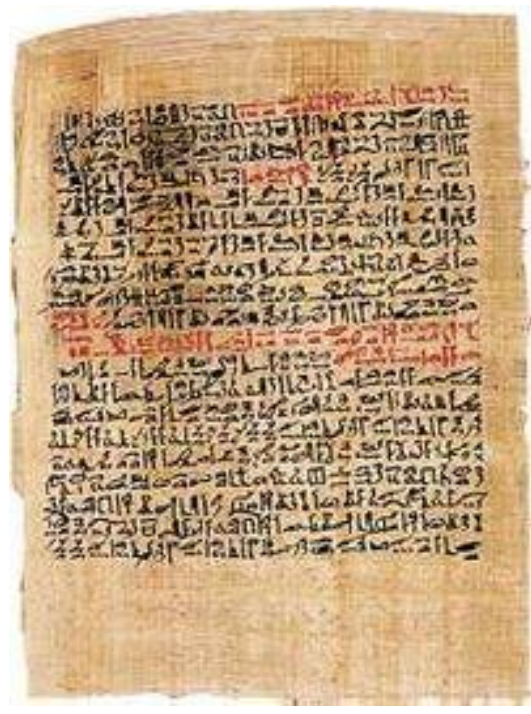

En la Grecia clásica es el estado quien controla los venenos como arma de ejecución. Cabe destacar el caso de la condena de Sócrates a morir bebiendo cicuta.

Por otro lado, en la Roma clásica algunos plebeyos trabajan como sicarios encargados de envenenar a otros por dinero. Por ello surge la Ley Cornelio en la que se regulan las penas a las que someter a este tipo de delitos.

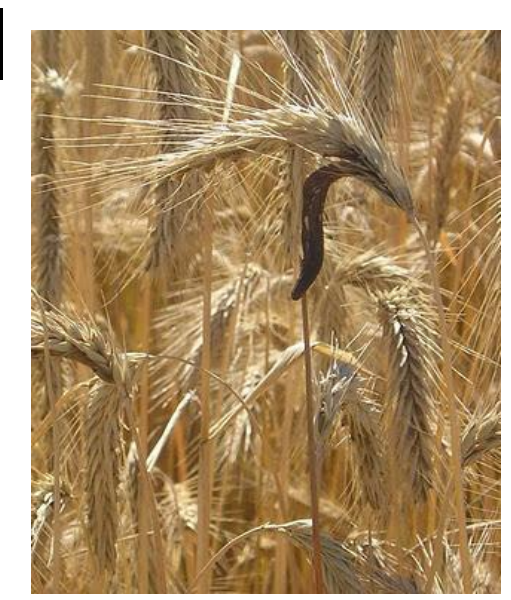

Durante el período de la Edad Media sucede uno de los grandes hitos toxicológicos: se abre el primer centro para atender exclusivamente a pacientes intoxicados por la epidemia de ergotismo que asoló a Francia, a cargo de la orden de San Antonio. Durante este período, 40000 personas murieron por comer trigo y centeno contaminados con 
micotoxinas.

Durante el mismo período de la Edad Media, Avicena, el conocido como Príncipe de los Médicos, dedica el libro $\mathrm{V}$ de su Canon de Medicinas a tratar las drogas $y$ sus prescripciones.

Por su parte, el filósofo y médico judío español Moisés ben Maimón o Maimónides, en su libro Los venenos y sus antídotos (1198), da consejos para evitar las intoxicaciones y prescribe su

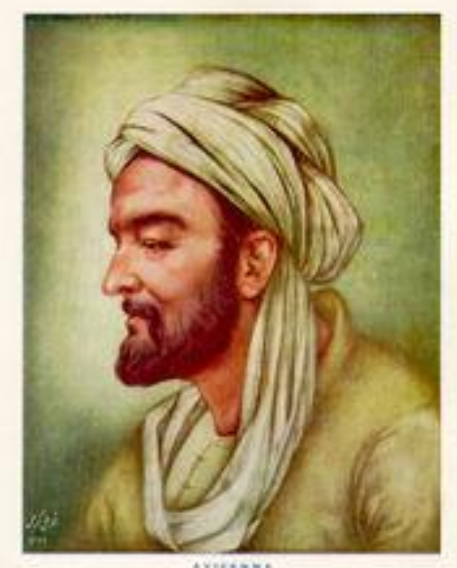
tratamiento.

Durante la época del Renacimiento (siglos XIV-XVI) destaca la figura de Paracelso. Considerado por algunos científicos como el "Padre de la toxicología". Célebre es su frase: "Dosis sola facit venenum", es decir "Todas las sustancias son venenos; no existe ninguna que no lo sea. La dosis diferencia a un veneno de una medicina..."

Enunció algunos del los principios básicos de la toxicología:

- la experimentación (animal) es fundamental para conocer cómo se desarrolla la respuesta del organismo frente a la sustancia tóxica.

- distingue la propiedad terapéutica de la propiedad tóxica de una sustancia.

- "dosis sola facit venenum", esto es, la dosis es determinante para la toxicidad de la sustancia.

En el siglo XVIII destaca por encima de todos, la figura del menorquín Mateo Orfila.

En 1813 publicó la obra Elementos de Química y Tratado de las exhumaciones Jurídicas, y en 1814, su Tratado de Toxicología en dos volúmenes, obra clásica y fundamental que aún hoy es reconocida

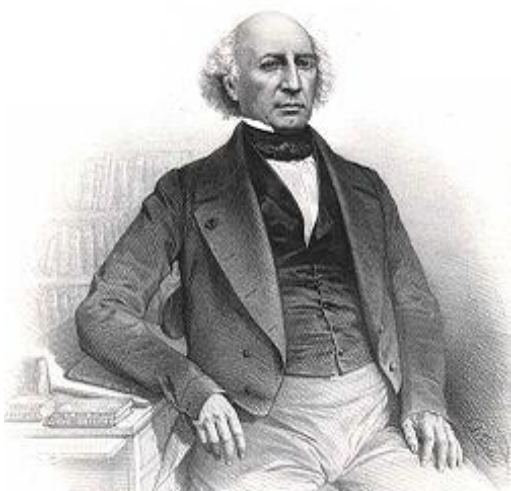


como la primera obra completa de importancia internacional

Desarrolló multitud de pruebas para identificar los tóxicos, que agrupa en seis clases. En su obra describe además las propiedades físicas, químicas, fisiológicas y tóxicas de las sustancias, deteniéndose en los métodos de tratamiento. Experimentó con animales a los que administraba cantidades conocidas de sustancias, observando la sintomatología de la intoxicación, y después de muertos examinaba los órganos y analizaba los tejidos.

Entre sus más importantes contribuciones destaca el descubrimiento de que los tóxicos se acumulan en diferentes tejidos.

Fue profesor de Medicina Legal de la Universidad de París, y en 1821 publicó su libro de texto titulado Lecciones de Medicina Legal.

Durante el siglo $X X$, el mundo sufre una nueva realidad, la guerra química. En 1915 Fritz Haber desarrolla ampollas de cloro y cianuro que se utilizan por primera vez en la Primera Guerra Mundial.

Esto ocasiona que en tengan que sentarse unas bases en el Protocolo de Ginebra de 1925 por las cuales se prohíbe el empleo de armas químicas.

La toxicología recibe un gran impulso tras la II Guerra mundial, cuando se dispara la producción de drogas, pesticidas y sustancias químicas de uso industrial.

Durante el siglo $\mathbf{X X}$ se suceden una serie de hitos en el mundo de la toxicología.

- A partir de 1945 podemos empezar a considerar a la toxicología como ciencia. En dicho año, aparece en Holanda, el primer centro de información a los farmacéuticos. En ese mismo año se desarrolla en Dinamarca el primer centro especializado en reanimación, volcado especialmente en los intentos de suicidio y a las sobredosis de medicamentos.

- En Inglaterra en 1950 se abre el primer centro de información y tratamiento toxicológico. 
- La aparición de los primeros "Centros de Lucha contra las Intoxicaciones», se inicia en 1952 en Chicago. Hoy en día se encuentran en numerosos países.

- En 1961 se configura la primera Sociedad de Toxicología, con 183 miembros.

La primera reunión anual de la Sociedad de Toxicología tuvo lugar en el Chevy Chase Sala del Hotel Marlborough-Blenheim en Atlantic City el 15 de abril de 1962.

- En 1962 surge la European Society for the Study of Drug Toxicity (ESSDT).

- De las observaciones estadísticas de los centros antitóxicos (CAT) surgió la necesidad de los Servicios de Farmacovigilancia y, más tarde, de los de Toxicovigilancia.

-Las nuevas áreas desarrolladas plenamente dentro el extenso campo que abarca la toxicología son:

- toxicología clínica

- toxicología ambiental

- toxicología farmacológica

- toxicología experimental ${ }^{2}$

Actualmente se calcula que un 1 por 100 de los ingresos generales en hospitales se debe a intoxicaciones, y el 8 por 100 de todas las autopsias que se realizan en el mundo son por muerte tóxica.

Durante el siglo XXI los terroristas han abierto una nueva forma de guerra tóxica. Cabe citar por ejemplo, el empleo de ántrax en sobres tras el atentado del 11 de Septiembre; o bien, el envenenamiento por Polonio 210 a Alexander LItvinenko, ex agente ruso. 
Por otra parte, la drogadicción es un hecho que afecta a todos los países del mundo en mayor o menor medida. La edad de inicio, lejos de ir aumentando, va disminuyendo, y las sustancias que se utilizan para dichos fines, van variando a lo largo de la historia, sintetizándose moléculas nuevas constantemente.

Todo ello, conduce a considerar la toxicología como una ciencia viva y en constante evolución. 


\section{La toxicología en menores de edad a través de la historia.}

Aparecen pocas referencias explícitas de historia de toxicología infantil como tal.

Los infanticidios y maltratos a menores han sido ejecutados durante toda la historia de la humanidad, muchos de ellos perpetrados con tóxicos.

La primera reseña que se evidencia de envenenamientos en menores de edad radica en la obra de Shakespeare, Romeo y Julieta.

"En el tierno cáliz de esta flor pequeña tiene su albergue el veneno y su poder la medicina: si se la huele, estimula el olfato y todos los sentidos; si se la prueba, con los sentidos acaba, matando el corazón. Así, del propio modo en que las plantas campean siempre en el pecho humano dos contrarios en lucha, la gracia y la voluntad rebelde, siendo pasto instantáneo del cáncer de la muerte la creación en que predomina el rival perverso" (Acto 2.2.1).

En el siglo XIX surge la pediatría como rama de la medicina, de la mano del considerado padre de la misma: Abraham Jacobi. Gracias a él se funda el primer hospital infantil, y se comienza a prestar atención a una correcta alimentación en la primera etapa de la vida.

En 1962 Kempen publica por primera vez el término de niño maltratado, pudiéndose leer en su trabajo la siguiente observación:

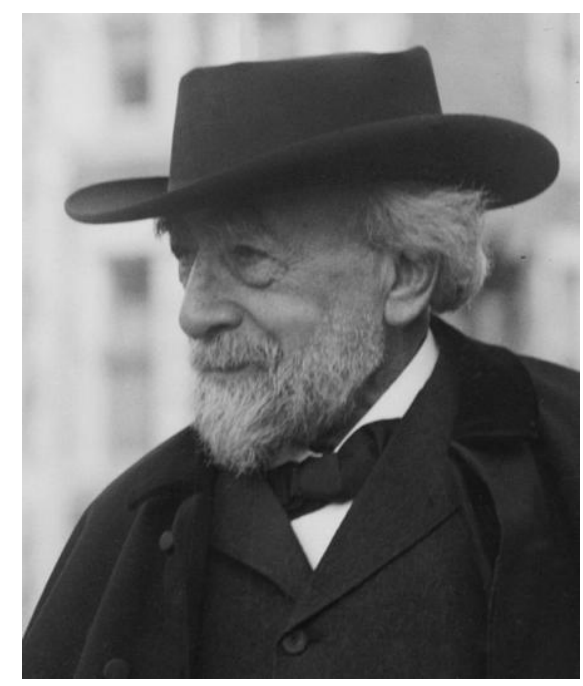

"Ocasionalmente los padres pueden agredir al niño administrándole una sobredosis de droga"

En 1971 se funda en Pittsburg el Pittsburgh Poison Center dentro del hospital infantil de la ciudad. Una de sus hazañas es la creación de Mr.Yuk, una mascota para que los niños entiendan los peligros de determinas sustancias y

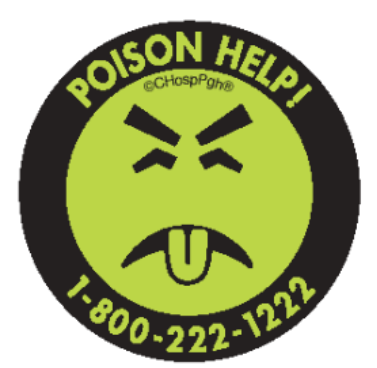
así evitar intoxicaciones fortuitas. 
Dicha mascota ha sido extendida ampliamente por el territorio americano a lo largo de los años.

En España se configura en octubre del

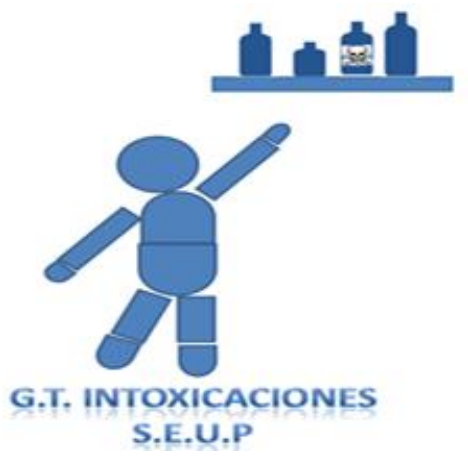
2008 un observatorio toxicológico infantil, al que pertenecen 50 servicios de urgencias pediátricos hospitalarios y epidemiólogos. Los objetivos de este observatorio son fundamentalmente dos: S.E.U.P detectar cambios en las intoxicaciones agudas pediátricas y, por otro, monitorizar el manejo del paciente pediátrico intoxicado en los servicios de urgencias pediátricas. Los hospitales integrados recogen de una manera prospectiva las intoxicaciones atendidas en sus servicios los días 13 de cada mes. Este observatorio publica cuatrimestralmente un boletín, al que también se puede acceder por la WEB de la Sociedad española de urgencias pediátricas. 


\section{Marco legal}

La Constitución Española fija en su artículo 12 la mayoría de edad en los 18 años.

Existen excepciones en cuanto a dicha edad en el ámbito sanitario. Así, La Ley 41/2002 de 14 de noviembre, reguladora de la autonomía del paciente y de derechos y obligaciones en materia de información y documentación clínica regula el consentimiento al tratamiento del llamado menor maduro en el ámbito sanitario, es decir, de los menores emancipados o con dieciséis años cumplidos, y señala que en estos casos no cabe prestar el consentimiento por representación. Sin embargo, en caso de actuación de grave riesgo, según el criterio del facultativo, los padres serán informados y su opinión será tenida en cuenta para la toma de la decisión correspondiente.

Las unidades de urgencias pediátricas hospitalarias españolas, atienden a las personas desde los primeros días de vida, hasta la edad de 14 años no incluidos. El corte en esta edad ha sido cuestionado en numerosas ocasiones, y el 5 de abril de 2013 fue aprobado por el consejo de ministros, el II Plan estratégico de infancia y adolescencia. En dicho documento se recoge un cambio respecto al previo: "7.13.1. Impulsar la atención de los menores hasta los 18 años en la hospitalización de las unidades pediátricas".

Esta nueva edad ha sembrado discrepancias entre las diferentes sociedades científicas que pueden tratar a este grupo poblacional 


\section{Estudios epidemiológicos en España.}

\subsection{Trabajos históricos}

El primer trabajo sobre epidemiología toxicológica registrado en España corresponde al año $1956^{3}$. . En él recogen los pacientes intoxicados que ingresan en los hospitales de Barcelona durante cinco años.

No se registra un segundo trabajo hasta 11 años después ${ }^{4}$ En este caso, Millá y cols. incluyen no sólo pacientes ingresados, sino todos los atendidos en urgencias hospitalarias, analizando por primera vez un tipo de intoxicación nueva, la etílica.

\subsection{Actualidad.}

Realizando una revisión de los estudios epidemiológicos sobre intoxicaciones realizados en España, encontramos un incremento de publicaciones en los últimos años.

Cabe destacar que la mayoría de ellos son llevados a cabo por servicios de urgencias hospitalarias, generales y pediátricas, y en algún caso por unidades de cuidados intensivos.

Dentro de estos estudios existe una gran heterogeneidad respecto a los datos de inclusión. La franja de edad que recogen es muy variable, y en pocos de ellos se valora exclusivamente los menores de edad desde el punto de vista legal, es decir, de 0 a 18 años no inclusive.

Se recoge en la siguiente tabla todos los artículos encontrados en Pubmed y en la Web of Science de la ISI Web of Knowledge, desde el 2000 al 2014, introduciendo como términos de búsqueda: intoxication, poison, poisoning y filtrando únicamente los datos epidemiológicos en España. Se procede a una comparativa entre los grupos de edad que comparan, los tóxicos, la localización y el periodo que abarcan 
Tabla 1: Estudios epidemiológicos españoles recogidos desde el año 2000 al 2013

\begin{tabular}{|c|c|c|c|c|c|c|c|c|c|}
\hline & Autor & $\begin{array}{c}\text { Añ } \\
0\end{array}$ & Revista & Centro & Tóxico & Lugar & Edad & Periodo & Casos \\
\hline $1^{5}$ & $\begin{array}{l}\text { Carpintero, } \\
\text { J.M. }\end{array}$ & $\begin{array}{l}20 \\
00\end{array}$ & Emergencias & H. La Rioja & Todos & Urgencias & Todos & 1998 & 618 \\
\hline $2^{6}$ & Riquelme, A. & $\begin{array}{l}20 \\
01\end{array}$ & At. Primaria & H. La Palma & Todos & Área de salud & Todos & 1998-1999 & 147 \\
\hline $3^{7}$ & Espinosa, G. & $\begin{array}{l}20 \\
01\end{array}$ & Med Clín & H. Clinic & Éxtasis líquido & Urgencias & Todos & 2000-2001 & 22 \\
\hline $4^{8}$ & Mintegui, S. & $\begin{array}{l}20 \\
02 \\
20\end{array}$ & An. Esp. Ped. & H. Cruces & Todos & Urgencias & $\begin{array}{l}\text { Pediátrica } \\
\text { s }\end{array}$ & 1988-1999 & 614 \\
\hline $5^{9}$ & Boscá, B. & $\begin{array}{l}20 \\
03\end{array}$ & At. Primaria & H. Requena & Todos & Urgencias & $\begin{array}{c}\text { Pediátrica } \\
\text { s }\end{array}$ & 1997-2002 & 55 \\
\hline $6_{0}^{1}$ & Burillo, G. & $\begin{array}{l}20 \\
03\end{array}$ & $\begin{array}{l}\text { Eur. J. Emerg. } \\
\text { Med. }\end{array}$ & 14 hospitales nacionales & Todos & Urgencias & Todos & $\begin{array}{c}14 \text { días febrero } \\
2000\end{array}$ & 419 \\
\hline $7^{1}$ & Sanjurjo, E. & $\begin{array}{l}20 \\
04\end{array}$ & Med Clín & H. Clinic & Éxtasis & Urgencias & Adultos & 2000-2002 & 135 \\
\hline $8^{1}$ & Sanjurjo, E. & $\begin{array}{l}20 \\
06\end{array}$ & Med Clín & H.Clinic & Cocaína & Urgencias & Adultos & 2002-2004 & 745 \\
\hline $9_{3}^{1}$ & $\begin{array}{c}\text { Pastó } \\
\text { Cardona, L }\end{array}$ & $\begin{array}{l}20 \\
07\end{array}$ & Rev. Toxicol & H. Bellvitge & $\begin{array}{l}\text { Todos salvo } \\
\text { alcohol }\end{array}$ & Urgencias & Todos & 1994-2004 & $\begin{array}{c}\text { No } \\
\text { aparec } \\
\text { e }\end{array}$ \\
\hline $\begin{array}{l}10 \\
14\end{array}$ & Burillo, G. & $\begin{array}{l}20 \\
08\end{array}$ & Emergencias & 24 hospitales nacionales & Todos & Urgencias & Adultos & $\begin{array}{c}\text { Septiembre 2005- } \\
\text { Agosto } 2006\end{array}$ & 2245 \\
\hline $\begin{array}{l}11 \\
15\end{array}$ & Galicia, M. & $\begin{array}{l}20 \\
08\end{array}$ & Emergencias & H. Clinic & Cocaína & Urgencias & Adultos & 2002-2007 & 1755 \\
\hline $\begin{array}{l}12 \\
16\end{array}$ & $\begin{array}{l}\text { Hernández, } \\
\text { SH. }\end{array}$ & $\begin{array}{l}20 \\
08\end{array}$ & Emergencias & & Cocaína & Urgencias & Adultos & & \\
\hline $\begin{array}{l}13 \\
17\end{array}$ & $\begin{array}{c}\text { Piñana López, } \\
\text { A. }\end{array}$ & $\begin{array}{l}20 \\
09\end{array}$ & $\begin{array}{l}\text { Siete Días } \\
\text { Médicos }\end{array}$ & & Alcohol & Urgencias & $\begin{array}{c}\text { No se } \\
\text { especifica }\end{array}$ & & \\
\hline $\begin{array}{l}14 \\
18\end{array}$ & $\begin{array}{c}\text { Macías Robles, } \\
\text { MD. }\end{array}$ & $\begin{array}{l}20 \\
09\end{array}$ & Emergencias & H. Avilés & $\begin{array}{l}\text { Productos } \\
\text { químicos }\end{array}$ & Urgencias & $\begin{array}{c}\text { No se } \\
\text { especifica }\end{array}$ & 2004-2007 & 204 \\
\hline
\end{tabular}




\begin{tabular}{|c|c|c|c|c|c|c|c|c|c|}
\hline $\begin{array}{l}15 \\
19\end{array}$ & $\begin{array}{c}\text { Clemente } \\
\text { Rodríguez, C. }\end{array}$ & $\begin{array}{l}20 \\
10\end{array}$ & Emergencias & H. Universitario del Mar & Todos & Urgencias & Adultos & 2003-2004 & 1531 \\
\hline $\begin{array}{l}16 \\
20\end{array}$ & $\begin{array}{l}\text { Sánchez } \\
\text { Sánchez, A. }\end{array}$ & $\begin{array}{l}20 \\
10\end{array}$ & $\begin{array}{l}\text { Rev. Esp. } \\
\text { Salud Pública }\end{array}$ & Castilla y León & Alcohol & Urgencias & $\begin{array}{l}10-30 \\
\text { años }\end{array}$ & 2003-2010 & 4429 \\
\hline $\begin{array}{l}17 \\
21\end{array}$ & $\begin{array}{c}\text { Arias } \\
\text { Constantini, V. }\end{array}$ & $\begin{array}{l}20 \\
10\end{array}$ & Med Clín & $\begin{array}{l}\text { H. San Joan de Deu } \\
\text { Barcelona }\end{array}$ & $\begin{array}{l}\text { Sustancias } \\
\text { psicoactivas }\end{array}$ & $\begin{array}{l}\text { Urgencias } \\
\text { pediátricas }\end{array}$ & $\begin{array}{c}\text { Adolescen } \\
\text { tes }\end{array}$ & 30 meses & 333 \\
\hline $\begin{array}{l}18 \\
22\end{array}$ & $\begin{array}{c}\text { Pitá- } \\
\text { Fernández, S. }\end{array}$ & $\begin{array}{l}20 \\
11\end{array}$ & $\begin{array}{l}\text { Arch. Gerontol. } \\
\text { Geriatr. }\end{array}$ & H. Coruña & Digoxina & Urgencias & Adultos & $\begin{array}{c}\text { Enero a } \\
\text { Septiembre } 2008\end{array}$ & \\
\hline $\begin{array}{l}19 \\
23\end{array}$ & $\begin{array}{c}\text { Croché } \\
\text { Santander, B. }\end{array}$ & $\begin{array}{l}20 \\
11\end{array}$ & $\begin{array}{l}\text { Arch. Argent } \\
\text { Pediatric }\end{array}$ & $\begin{array}{l}\text { H. Virgen del Rocío, } \\
\text { Sevilla }\end{array}$ & Cannabis & $\begin{array}{c}\text { UCl } \\
\text { pediátrica }\end{array}$ & Niños & 5 meses & 4 \\
\hline 20 & $\begin{array}{c}\text { Alonso Canal, } \\
\text { L. }\end{array}$ & $\begin{array}{l}20 \\
11\end{array}$ & $\begin{array}{l}\text { Anales } \\
\text { Pediatría }\end{array}$ & H. Niño Jesús (Madrid) & Vitamina D & $\begin{array}{l}\text { Urgencias } \\
\text { pediátricas }\end{array}$ & Niños & 2010 & 3 \\
\hline $\begin{array}{l}21 \\
25\end{array}$ & Galicia, M. & $\begin{array}{l}20 \\
11\end{array}$ & Emergencias & H. Clinic & Éxtasis & Urgencias & Adultos & 2000-2007 & 505 \\
\hline $\begin{array}{l}22 \\
26\end{array}$ & $\begin{array}{c}\text { Clemente } \\
\text { Rodríguez, C. }\end{array}$ & $\begin{array}{l}20 \\
11\end{array}$ & Emergencias & H. Del mar & Todos & Urgencias & Adultos & 2003-2004 & 1287 \\
\hline $\begin{array}{l}23 \\
27\end{array}$ & Badia, M. & $\begin{array}{l}20 \\
11\end{array}$ & Med. Intensiva & H. Lérida & Drogas abuso & $\mathrm{UCl}$ & Adultos & 2004-2008 & 146 \\
\hline $\begin{array}{l}24 \\
28\end{array}$ & López, JC. & $\begin{array}{l}20 \\
12\end{array}$ & Hemodial Int & H. Bellvitge & Litio & $\mathrm{UCl}$ & Adultos & 2004-2009 & 14 \\
\hline $\begin{array}{l}25 \\
29\end{array}$ & $\begin{array}{l}\text { De Miguel- } \\
\text { Bouzá, J.C. }\end{array}$ & $\begin{array}{l}20 \\
12\end{array}$ & Adicciones & Pontevedra & Todos & Urgencias & Adultos & 2005-2008 & 1893 \\
\hline $\begin{array}{l}26 \\
30\end{array}$ & Muñoz, R. & $\begin{array}{l}20 \\
13\end{array}$ & Emergencias & $\begin{array}{l}\text { H. Universitario La Paz } \\
\text { (Madrid) }\end{array}$ & Todos & Urgencias & Adultos & Abril-Julio 2011 & 1033 \\
\hline $\begin{array}{l}27 \\
31\end{array}$ & $\begin{array}{c}\text { Estévez } \\
\text { Rueda, M.J. }\end{array}$ & $\begin{array}{l}20 \\
13\end{array}$ & Emergencias & $\begin{array}{l}\text { H. Universitario Ramón y } \\
\text { Cajal (Madrid) }\end{array}$ & $\begin{array}{l}\text { Drogas ilegales } \\
\text { (body packers) }\end{array}$ & Urgencias & Adultos & 2007 & 826 \\
\hline
\end{tabular}




\subsection{Análisis de los resultados de los estudios epidemiológicos.}

Analizando los resultados obtenidos en estos estudios podemos definir una serie de tendencias en cuanto a las intoxicaciones en España.

En primer lugar, podemos destacar una incidencia de intoxicaciones agudas atendidas en los servicios de urgencias de nuestro país que oscila entre el $0,66 \%$ y el $0,77 \% .^{10}$

La edad media de los pacientes intoxicados se encuentra con pequeñas variaciones en la década de los treinta ${ }^{5,10}$

El sexo predominante en las intoxicaciones es el masculino, prácticamente en todas las franjas de edad ${ }^{10,32}$

Predominan las intoxicaciones agudas por sobredosis (más del $50 \%$.) Los gestos suicidas suponen alrededor del $30 \%$ de los casos.

Las intoxicaciones accidentales son actualmente menos frecuentes y se distinguen las domésticas (de predominio infantil) ${ }^{33}$

El tóxico que con mayor frecuencia está implicado en estas intoxicaciones es el alcohol, ya alcanza cifras de hasta $67 \%{ }^{10}$ y en segundo lugar los medicamentos (sobre todo, las benzodiacepinas, responsables de más de la mitad de este tipo de intoxicación.) 


\subsection{Tesis españolas relacionadas con las intoxicaciones en nuestro país}

Si introducimos dentro de la base de datos de tesis españolas Teseo, proporcionada por el Ministerio de Educación, Cultura y Deporte de España, los buscadores de intoxicaciones, intoxicación, envenenamiento o intoxicación en menores, encontramos 11 tesis relacionadas con el tema tratado.

De ellas, sólo 2 analizan la población menor de 14 años, una de forma única, y la otra porque engloba a pacientes desde 0 a 99 años.

Se puede leer un resumen de las mismas en el Anexo I, que se encuentra al final de la presente tesis.

\subsection{Estudios epidemiológicos de intoxicaciones en población infantil.}

Pese a que las intoxicaciones en niños suponen un número bajo de consultas en los servicios de urgencias, y que en los últimos años parece que se ha ido estabilizando dicha incidencia, ocasionan un fuerte sufrimiento para las familias que lo padecen y una situación de estrés para los profesionales que lo atienden.

Introduciendo en los buscadores de bases de datos las palabras intoxication, poisoning, children encontramos una mayor cantidad de trabajos al respecto, sobre todo en los últimos años.

Se presenta, al igual que se ha realizado en el caso de las intoxicaciones en general, un cuadro en el que se desgranan dichos trabajos 
Tabla 2: Estudios epidemiológicos intoxicaciones en población infantil en España desde año 1980 hasta el 2014

\begin{tabular}{|c|c|c|c|c|c|c|c|c|c|}
\hline & Autor & $\begin{array}{c}\text { Añ } \\
0\end{array}$ & Revista & Centro & Tóxico & Lugar & Edad & Periodo & $\begin{array}{c}\text { Cas } \\
\text { os }\end{array}$ \\
\hline $1^{34}$ & Salazar & $\begin{array}{c}201 \\
4\end{array}$ & An. Pediatría & 53 hospitales españoles & Todos & Urgencias pediatría & $<14$ & $\begin{array}{l}2008- \\
2013\end{array}$ & 556 \\
\hline $2^{35}$ & Azkunaga & $\begin{array}{c}201 \\
3\end{array}$ & An. Pediatría & 44 hospitales & Todos & Urgencias pediatría & $<7$ & $\begin{array}{l}2008- \\
2013\end{array}$ & 400 \\
\hline $3^{36}$ & Azkunaga & $\begin{array}{c}201 \\
2\end{array}$ & Emergencias & 38 hospitales & Todos & Urgencias pediatría & $\begin{array}{c}<14 \text {. Algún caso } \\
\text { hasta } 18\end{array}$ & $\begin{array}{l}2001- \\
2002, \\
2008- \\
2010\end{array}$ & 2432 \\
\hline $4^{37}$ & Azkunaga & 201 & Eur. J. Emerg. Med. & 40 hospitales & Todos & Urgencias pediatría & Masta to & 1 año & 130 \\
\hline $5^{24}$ & Alonso Canal & $\begin{array}{c}201 \\
1\end{array}$ & Anales Pediatría & Niño Jesús (Madrid) & Vitamina D & Urgencias pediatría & $<5$ & 5 meses & 3 \\
\hline $6^{23}$ & $\begin{array}{l}\text { Croche } \\
\text { Santander }\end{array}$ & $\begin{array}{c}201 \\
1\end{array}$ & $\begin{array}{l}\text { Arch. Argentinos } \\
\text { Pediatría }\end{array}$ & Virgen del Rocío Sevilla & Cannabis & Urgencias pediatría & $<3$ & 2009 & 4 \\
\hline $7^{38}$ & $\begin{array}{l}\text { Velasco } \\
\text { Arnaiz }\end{array}$ & $\begin{array}{c}201 \\
0\end{array}$ & Anales Pediatría & $\begin{array}{l}\text { Sant Joan de Deu } \\
\text { Barcelona }\end{array}$ & Cannabis & Urgencias pediatría & $<18$ & $\begin{array}{l}2007- \\
2008\end{array}$ & 533 \\
\hline $8^{33}$ & Mintegui & $\begin{array}{c}200 \\
6\end{array}$ & Ped. Emerg. Care & 17 hospitales & Todos & Urgencias pediatría & $\begin{array}{c}<15 \text { y en } 7 \\
\text { hospitales }<19\end{array}$ & $\begin{array}{l}2001- \\
2002\end{array}$ & 2157 \\
\hline $9^{9}$ & Boscá & $\begin{array}{c}200 \\
3\end{array}$ & Atención Primaria & Hospital Requena & Todos & Urgencias & No lo refleja & $\begin{array}{l}1997- \\
2002\end{array}$ & 55 \\
\hline $\begin{array}{l}10 \\
39\end{array}$ & $\begin{array}{l}\text { Conejo } \\
\text { Menor }\end{array}$ & $\begin{array}{c}200 \\
2\end{array}$ & An. Esp. Pediatría & Poison Control Center & Antipiréticos & Urgencias en general & $<14$ & $\begin{array}{l}1998- \\
2000\end{array}$ & $\begin{array}{c}1304 \\
4\end{array}$ \\
\hline $\begin{array}{l}11 \\
40\end{array}$ & Vázquez & $\begin{array}{c}199 \\
7\end{array}$ & Med. Clínica & $\begin{array}{l}\text { Hospital Gregorio } \\
\text { Marañón Madrid }\end{array}$ & $\mathrm{CO}$ & Urgencias pediatría & $<15$ & $\begin{array}{c}\text { No } \\
\text { aparece }\end{array}$ & 59 \\
\hline $\begin{array}{l}12 \\
41\end{array}$ & Repetto & $\begin{array}{c}199 \\
6\end{array}$ & $\begin{array}{l}\text { Vet and Human } \\
\text { toxicology }\end{array}$ & Sevilla & Detergentes & $\begin{array}{l}\text { Servicio intoxicación } \\
\text { toxicológica Sevilla }\end{array}$ & $<14$ & 1994 & 46 \\
\hline $\begin{array}{l}13 \\
42\end{array}$ & Mintegui & $\begin{array}{c}199 \\
0\end{array}$ & An. Pediatría & Hospital Cruces Bilbao & Todos & Urgencias pediatría & $<14$ & 1988 & 279 \\
\hline $\begin{array}{l}14 \\
43\end{array}$ & Armas & $\begin{array}{c}198 \\
0\end{array}$ & An. Esp. Pediatría & Hospitales Aragón & Todos & Urgencias & $<14$ & $\begin{array}{l}1977- \\
1978\end{array}$ & 1363 \\
\hline $\begin{array}{l}15 \\
44\end{array}$ & González & $\begin{array}{c}198 \\
0\end{array}$ & An. Esp. Pediatría & $\begin{array}{c}\text { Centros hospitalarios } \\
\text { Barcelona }\end{array}$ & $\begin{array}{l}\text { Mordedura } \\
\text { serpiente }\end{array}$ & Urgencias & $<16$ & $\begin{array}{l}1976- \\
1979\end{array}$ & 6 \\
\hline
\end{tabular}




\subsection{Análisis de los estudios epidemiológicos sobre intoxicaciones en}

\section{la infancia.}

Se puede observar que las características epidemiológicas de las intoxicaciones en menores han ido variando paulatinamente con el tiempo. Esto es debido a la introducción de nuevos tipos de tóxicos (tanto farmacológicos, industriales...); a la puesta en marcha de protocolos de seguridad por parte de los comerciantes, como tapones de seguridad en el caso de los fármacos utilizados por parte de la población infantil; y una mayor accesibilidad a algunos tipos de drogas, tales como el alcohol, cannabis.....

Al analizar los resultados de los estudios anteriormente descritos, podemos reflejar una serie de datos epidemiológicos.

Las intoxicaciones siguen suponiendo un tanto por ciento pequeño de las visitas a los servicios de urgencia pediátricos. Se calcula entorno al $0,6 \%$ de todas ellas.

\subsubsection{Edad}

En cuanto a la edad de atención de dichas intoxicaciones, existe un problema por la heterogeneidad de los estudios respecto a los criterios de inclusión en ellos. En la mayoría de centros hospitalarios la edad de atención en los servicios de pediatría es hasta los 14 años, no incluidos ${ }^{34,39,41}$; sin embargo, algunos artículos de los anteriormente mostrados, incluyen a jóvenes hasta los 17-18 años ${ }^{36,38}$

Normalmente, cuando se analizan las intoxicaciones pediátricas, se suelen distinguir dos grupos de edad bien diferenciados que engloban a la mayoría de las consultas en urgencias ${ }^{45}$

1. Los preescolares, por debajo de los 7- 5 años. En los estudios más recientes, se ha visto que la mayoría de las consultas a urgencias son en este grupo de edad. ${ }^{35}$

2. Los adolescentes. 
Ambos grupos muestran características diferentes en cuanto al tipo de intoxicaciones por las que son atendidos.

Los pequeños, suelen intoxicarse dentro del hogar, de forma involuntaria; acuden rápidamente a urgencias trasladados por sus padres junto con el tóxico responsable, por lo que en la mayoría de las ocasiones los facultativos los valoran prácticamente asintomáticos. El pronóstico de este tipo de intoxicaciones es muy favorable.

Los adolescentes por el contrario, suelen intoxicarse fuera del hogar, con un fin puramente recreacional, y no acuden a los servicios de urgencia inmediatamente, si no que esperan a tener síntomas. El tóxico muchas veces es desconocido, o mezcla de varios.

\subsubsection{Intencionalidad}

Respecto a la intencionalidad de la intoxicación se distinguen 3 supuestos:

1. Accidentales: sobre todo en menores de 7 años

2. Intencionales recreacionales: adolescentes con consumo de drogas

3. Intencionales con fines suicidas: este tipo de intoxicaciones son poco frecuentes en la edad pediátrica.

4. Intencionales por maltrato o acción criminal

\subsubsection{Sexo}

No se aprecian diferencias significativas en cuanto al género. Cabe destacar, que según va aumentando la edad de la intoxicación, se desvía ligeramente a favor del sexo femenino.

\subsubsection{Tóxicos}

Como ya se ha reflejado anteriormente, el tipo de tóxico depende sobre todo de la edad del paciente. Como se explica en Dueñas 2011 y $2014^{46,47}$ 
1. Los fármacos, continúan siendo a pesar de las medidas de seguridad que han tomado las empresas farmacéuticas, quienes ocasionan el mayor número de intoxicaciones. Son los responsables de la mayoría de las intoxicaciones graves atendidas en España, sobre todo, en menores de 6 años.

2. El segundo grupo causante lo engloban los productos del hogar, que afecta sobre todo, a niños menores de 3 años.

3. Etanol. En los últimos años, ha habido un aumento importante de la incidencia de este tipo de intoxicaciones, siendo la edad de consumo cada vez más baja.

4. Monóxido de carbono. Constituyen un $4-5 \%$ de las consultas en urgencias pediátricas. Siendo habitual la intoxicación de varios miembros de una misma familia $^{48}$

\section{Incidencia de las intoxicaciones en el servicio de urgencias hospitalario}

\section{en el mundo.}

Las consultas en urgencias hospitalarias por intoxicaciones suponen algo menos del $0,3 \%$ de todas las patologías atendidas en dichos servicios en nuestro medio. Mintegui 2012 ${ }^{45}$; Esta cifra ha supuesto un ligero aumento respecto a los datos previos $\left(<0,3 \%\right.$ ) (Mintegi 2008) ${ }^{53}$, a costa sobre todo de las intoxicaciones por alcohol.

Además, las consultas en urgencias, normalmente no se deben a una verdadera intoxicación, ya que la sustancia que lo ocasiona, o la dosis, no resultan tóxicas.(Nogúe; Dueñas-Laita) ${ }^{46,47,54}$

En España cada año desde 1999, se lleva a cabo un Sistema de Toxicovigilancia en Hospitales públicos españoles, promovido por la Subdirección General de Sanidad Ambiental y Salud Laboral de la Dirección General de Salud Pública del Ministerio de Sanidad. Gracias a un sistema de notificación voluntaria, coordinado por la Fundación Española de Toxicología Clínica, cada año se redacta un informe con los datos encontrados. ${ }^{55-64}$ 
Por otra parte, en España, el Servicio de Información Toxicológica (SIT), uno de los servicios del Instituto Nacional de Toxicología y Ciencias Forenses del Ministerio de Justicia; desde el año de su creación en 1971 desempeña funciones de Centro Antitóxico Español. Es el encargado de recibir por vía telefónica las consultas toxicológicas de todo aquel que lo necesite, administración de justicia, médicos, particulares.... Se ha comprobado mediante estudios como el de LoVecchio de $2008^{65}$, que este tipo de servicios ahorran mucho dinero a la Seguridad Social, ya que disminuyen el número de consultas en urgencias innecesarias.

Se recoge de forma anual sus estadísticas por medio de memorias. En el año $2011^{66}$ de las llamadas recibidas, el 22,5\% correspondían a menores de 2 años, el $21,8 \%$ de 3 a 14 años, y el 1\% de 15 a 19 años incluidos. Es decir, en total un $45,3 \%$ de todas las consultas atendidas corresponde a menores de edad.

La mejor base de datos sobre intoxicaciones de la que se dispone actualmente en el mundo, continúa siendo la norteamericana ${ }^{67-74}$ Los datos recogidos de intoxicaciones en menores de edad en Estados Unidos en el 2010 son los siguientes ${ }^{67}$ En este resumen anual dividen a la población según su edad, incluyendo en el grupo de jóvenes y adolescentes hasta los 19 años inclusive. Se recogen un total de 1512381 intoxicaciones, lo que corresponde al 63,42\% de todas las intoxicaciones recogidas por el Centro de Control de Enfermedades en dicho año.

En Europa no existe un documento único que refleje las intoxicaciones anuales existentes, sino que cada país tiene los suyos.

En el caso de Inglaterra, el National Poisons Information Service, emite de forma bianual las memorias con su actividad. ${ }^{75}$

En Irlanda, anualmente se lleva a cabo la redacción de una memoria con los datos de las consultas a su teléfono de atención toxicológica. ${ }^{76-84}$. En el caso del $2010^{84}$, de las 9330 consultas atendidas, 4664, es decir, el $50 \%$ correspondieron a menores de 10 años. 
En Francia, los encargados de recoger los datos toxicológicos son los Centros antienvenenamiento. En este caso, no disponen de memorias anuales 0 bianuales de acceso gratuito. La última a la que se puede acceder desde el años 2001 al 2010 , es la del $2006^{85}$, el $60 \%$ de las intoxicaciones accidentales registradas, pertenecen a menores o iguales a 19 años.

En el caso de Portugal, el CIAV (Centro de información antienvenenamiento), recoge las estadísticas de las intoxicaciones producidas en el país. En sus estadísticas aparecen todas las consultas recibidas desde el año 2001 al 2013, sin separar por años, un total de 375858 llamadas. Las intoxicaciones referidas a menores de 19 años corresponden a 9472.

\section{Justificación}

Las intoxicaciones en pediatría siguen constituyendo aunque con una baja incidencia, un problema para sus cuidadores y para los profesionales que las atienden.

Al realizar la revisión bibliográfica sobre los estudios españoles, el resultado es escaso. 15 estudios desde 1980 hasta el año 2014, es una baja producción.

Es cierto, que en los últimos años el interés en el tema ha ido en aumento; sobre todo, desde la creación del Observatorio toxicológico de la Sociedad Española de Urgencias Pediátricas en el 2008, al que se han ido agregando poco a poco los servicios de urgencias pediátricas de diferentes hospitales españoles, hasta ser en la actualidad 50 centros hospitalarios. Estos centros recogen de manera prospectiva los días 13 de cada mes, las intoxicaciones atendidas por su servicio durante ese periodo de tiempo.

Este observatorio se fundó con dos objetivos fundamentales: por una parte, detectar los cambios que van efectuándose en las intoxicaciones agudas infantiles (cada vez hay nuevos productos en el mercado, tanto farmacológicos, industriales, drogas...y mayor acceso a ellos) y en segundo lugar, monitorizar el manejo del paciente pediátrico. 
Cuatrimestralmente se publica además, en la página web de la Sociedad española de urgencias pediátricas, un boletín informativo de acceso libre.

Sin embargo, la información recogida por este observatorio no es completa, ya que no se especifica qué ha ocurrido en los años anteriores.

La población ha cambiado mucho en las últimas décadas. No son iguales los niños de los años 80, 90 que los de la generación del 2000. El acceso a nuevas sustancias, tanto farmacológicas, como drogas legales e ilegales no es el mismo. En la década del 2000 se observa en España un descenso en la edad de inicio del consumo de sustancia que con el paso de los años se ha hecho aún más manifiesto.

Desde el año 1994, se realiza en España de forma bianual una encuesta estatal sobre el consumo de drogas en España en enseñanza secundaria (ESTUDES) $^{49}$

En la siguiente tabla se observa la evolución de la edad media en el consumo de sustancias en España entre jóvenes de 14 a 18 años.

Tabla 3: Evolución de la edad media de consumo de sustancias en España según la encuesta ESTUDES

\begin{tabular}{|cccccccccc|}
\hline & 1994 & 1996 & 1998 & 2000 & 2002 & 2004 & 2006 & 2008 & 2010 \\
\hline Tabaco & 13,9 & 13,3 & 13,2 & 13,1 & 13,1 & 13,2 & 13,1 & 13,1 & 13,5 \\
Tabaco (consumo diario) & & 14,6 & 14,5 & 14,4 & 14,4 & 14,5 & 14,2 & 15,1 & 14,3 \\
Alcohol & 13,5 & 13,7 & 13,8 & 13,6 & 13,6 & 13,7 & 13,8 & 13,7 & 13,7 \\
Alcohol (consumo semanal) & & 15 & 15 & 14,9 & 15 & 15,1 & 15 & 15,6 & 14,8 \\
Hipnosedantes & 14,1 & 14,5 & 14,8 & 14,5 & 14,6 & 14,8 & 14,4 & 14,3 & 14,4 \\
Cannabis & 15,1 & 15,1 & 15 & 14,9 & 14,7 & 14,7 & 14,6 & 14,6 & 14,7 \\
Cocaína & 15,6 & 15,9 & 15,8 & 15,8 & 15,7 & 15,8 & 15,3 & 15,3 & 14,9 \\
Heroína & 14,4 & 14,7 & 14,4 & 15,4 & 14,9 & 14,4 & 14,7 & 14,3 & 14,4 \\
Anfetaminas & 15,5 & 15,7 & 15,6 & 15,6 & 15,6 & 15,7 & 15,6 & 15,4 & 15,5 \\
Alucinógenos & 15,4 & 15,6 & 15,4 & 15,5 & 15,5 & 15,8 & 15,5 & 15,4 & 15,4 \\
Inhalantes volátiles & 13,3 & 13,6 & 13,4 & 13,9 & 14,3 & 14 & 13,6 & 13,8 & 13,2 \\
Éxtasis & 15,6 & 15,7 & 15,5 & 15,7 & 15,4 & 15,6 & 15,5 & 15,2 & 15,3 \\
\hline
\end{tabular}


Por otra parte, no son exactamente iguales los niños y jóvenes de toda España. Cada comunidad autónoma tiene sus características y eso influye también en las intoxicaciones que sufren estos menores.

Véase por ejemplo, el caso de la encuesta anteriormente referida ESTUDES en el 2010; la proporción de bebedores es mayor a la estatal en el caso de las comunidades autónomas de Castilla y León, La Rioja, Aragón, Asturias, Navarra, Cantabria y Castilla La Mancha.

Por tanto, los datos obtenidos en los diferentes estudios sobre las intoxicaciones en pediatría, no serían totalmente a priori extrapolables a la población que se pretende incluir en el trabajo de investigación (población del área de Valladolid).

La edad de inclusión de los pacientes en los diferentes estudios mostrados es variable. En unos centros atienden únicamente a población menor de 14 años y en otros incluyen jóvenes hasta 18 o 19 años.

Dadas estas características, la población que se debería incluir en este trabajo de investigación, serían los jóvenes menores de 18 años, edad en la que en España comienza la mayoría de edad. Estos jóvenes son atendidos en Valladolid, no sólo en la urgencia pediátrica, sino también la urgencia general, con las peculiaridades que ello implica. No son los mismos profesionales los que trabajan en unas urgencias y otras; ni por tanto, su forma de actuar es exactamente la misma; por lo que parece interesante observar la diferencia establecida entre los dos grupos de edad.

Los tapones de seguridad en los fármacos y en los productos de limpieza han ayudado a que exista un menor número de intoxicaciones en la edad pediátrica. La legislación respecto a este tipo de tapones de seguridad no era obligatoria en España en los primeros años de recogida de datos que se plantea recoger en la muestra del trabajo de investigación, por lo que habría que evaluar si como es de esperar existía una mayor prevalencia de intoxicaciones por dichos tóxicos sobre todo, en los niños más pequeños.

Por otra parte, se recogen para la muestra que se plantea analizar, muchos más parámetros de las historias clínicas de los pacientes. 
Se registra el tratamiento ofrecido a los menores; haciendo especial hincapié en si está adaptado a lo largo de los años a las nuevas opciones terapéuticas, tales como el desuso del lavado gástrico (hace unos años era uno de los tratamientos que con mayor frecuencia se utilizaban).

Se evalúa el número de veces que acuden a urgencias en el mismo año y en los años previos y posteriores a la intoxicación, así como el número de consultas a las que acuden, para estudiar si la población que se intoxica es más o menos frecuentadora de las urgencias hospitalarias. 


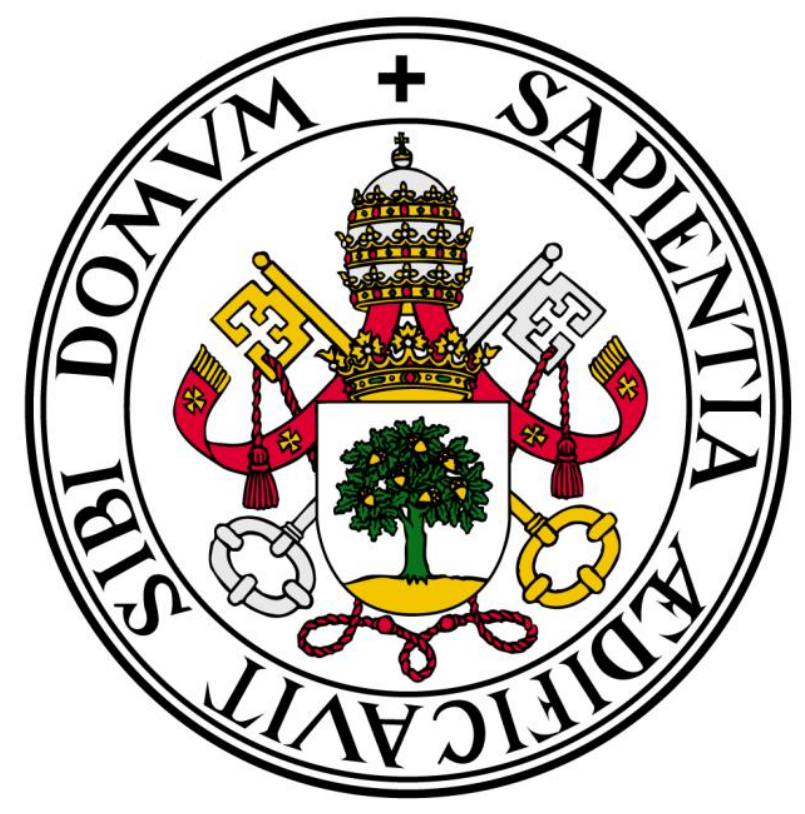

Hipótesis y objetivos 


\section{Hipótesis y Objetivos}

\section{Hipótesis.}

A pesar de que existen múltiples estudios sobre intoxicaciones agudas, son escasos los dedicados al estudio de las intoxicaciones en la edad pediátrica. Existen importantes diferencias organizativas entre los distintos modelos de asistencia hospitalaria urgente, a los que se asocia la falta de coordinación y establecimiento de guías clínicas del paciente intoxicado inter e intrahospitalarias, por lo que los escasos datos hasta ahora obtenidos en esta materia, podrían no ser representativos de la realidad asistencial en Castilla y León. Conocer la epidemiología de las intoxicaciones agudas en la edad infantil en nuestro entorno, es el punto de partida para establecer proyectos de mejora en este campo.

La hipótesis de este estudio es comprobar que la epidemiología de las intoxicaciones agudas en la población infantil en un hospital de tercer nivel de Castilla y León, no difiere de los resultados publicados a nivel nacional.

\section{Objetivo principal}

Conocer la asistencia que se muestra a los pacientes menores de edad que sufren una intoxicación aguda en un servicio de urgencias hospitalario de tercer nivel en la provincia de Valladolid.

\section{Objetivos específicos}

- Determinar las características epidemiológicas y toxicológicas de las intoxicaciones atendidas, en los pacientes menores de edad, en un servicio de urgencias hospitalario de tercer nivel en la provincia de Valladolid.

- Determinar las características clínicas y terapéuticas de las intoxicaciones atendidas en los pacientes menores de edad, en un servicio de urgencias hospitalario de tercer nivel en la provincia de Valladolid. 
- Conocer la evolución temporal, a lo largo de una década, de las características epidemiológicas, toxicológicas, clínicas y terapéuticas de las intoxicaciones atendidas, en los pacientes menores de edad, en un servicio de urgencias hospitalario de tercer nivel en la provincia de Valladolid. 


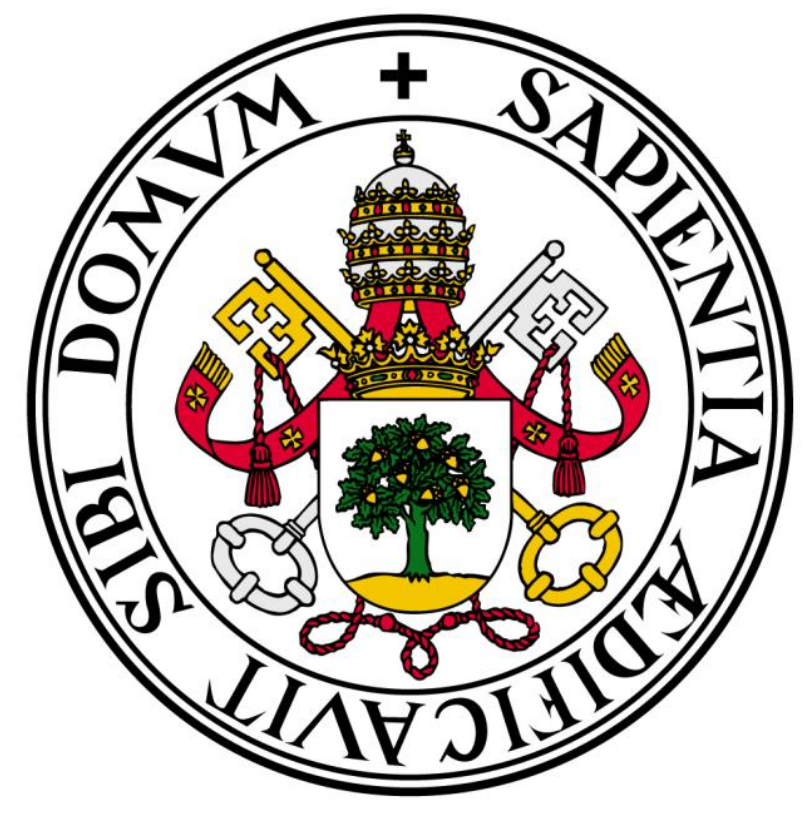

Material y métodos 


\section{Material y métodos.}

\section{Diseño del estudio.}

Considerando la hipótesis y los objetivos previamente marcados, se ha escogido un diseño de estudio retrospectivo, observacional y descriptivo de las historias atendidas en urgencias tanto pediátricas como generales de un hospital de tercer nivel durante un periodo de diez años.

\section{2. Ámbito de realización.}

Se recogen todas las historias de intoxicaciones salvo las alimentarias, atendidas durante el periodo englobado entre el 1 de Enero de 2001 y el 31 diciembre de 2010 en pacientes menores de 18 años, no inclusive; en las urgencias del Hospital Río Hortega de Valladolid.

Según los datos censales recogidos por el Instituto Nacional de Estadística en 2010, la provincia de Valladolid alberga a 533640 habitantes. Siendo censados en Valladolid capital, 315522 de los mismos.

Si se procede a filtrar habitantes de Valladolid capital de ambos sexos, menores de 19 años incluidos (en el Instituto Nacional de Estadística se muestran datos por franjas de edad, por lo que no es posible cribarlos en menores de 18 años), los datos serían los siguientes a fecha de 1 Enero de 2010.

Tabla 4: Habitantes de Valladolid capital menores de 19 años a fecha de 1 Enero 2010

\begin{tabular}{|ccccc|}
\hline & 0-4 años & 5-9 años & $10-14$ año & 15-19 años \\
\hline Habitantes & 13039 & 12965 & 12410 & 13416 \\
\hline
\end{tabular}

La provincia de Valladolid atiende a su población en dos Áreas de Salud diferentes, el Este y el Oeste, poseyendo cada una un hospital de referencia: el Hospital Clínico Universitario en Valladolid y el Hospital 
Comarcal de Medina del Campo, y la zona Oeste, cuyo hospital es Hospital Universitario Río Hortega.

Las zonas básicas de salud de Valladolid Oeste son:

- Arturo Eyries

- Campo Grande

- Cigales

- Delicias

- Esperanto

- Huerta del rey

- Laguna de Duero

- Mayorga

- Medina de Rioseco

- Mota del marqués

- Parquesol

- Pisuerga

- Tordesillas

- Valladolid rural II

- Parque Alameda - Covaresa

- Villafrechos

- Villalón de campos

Algunas de las poblaciones de dicho área de salud con mayor número de habitantes, sobre todo en edad pediátrica serían: Laguna de Duero, Cistérniga, Arroyo de la Encomienda y Tordesillas. 
En el año 2010 en el Instituto Nacional de Estadística, se encontraban censados los siguientes habitantes por franjas de edad.

Tabla 5: Habitantes Arroyo de la Encomienda y La Cistérniga según INE año 2010

\begin{tabular}{|c|cccc|}
\hline Edad & $0-4$ años & $5-9$ años & 10-14 años & $15-19$ años \\
\hline Habitantes Arroyo & 1247 & 832 & 547 & 411 \\
\hline \multicolumn{7}{|c|}{} & & & \\
\hline Habitantes La Cistérniga & 702 & 492 & 378 & 321 \\
\hline
\end{tabular}

Tabla 6: Habitantes de Laguna de Duero y Tordesillas según INE en 2010

\begin{tabular}{|c|c|c|c|c|}
\hline Edad & $0-4$ años & 5-9 años & 10-14 años & 15-19 años \\
\hline Habitantes Laguna de Duero & 1365 & 1431 & 1114 & 1075 \\
\hline Habitantes Tordesillas & 460 & 415 & 391 & 494 \\
\hline
\end{tabular}

En el año 2009 el hospital Río Hortega atendió 100840 urgencias. El número de consultas externas ascendió a 345732. Se ingresó a 22099 pacientes. En cuanto a las intervenciones quirúrgicas, se llevaron a cabo 19887. En la unidad de maternidad hubo 2196 partos y se realizaron 46 trasplantes.

Una característica especial del Hospital Universitario Río Hortega, es que posee la "Unidad Regional de Toxicología Clínica", centro toxicológico de referencia de Castilla y León.

Dicho hospital por tanto, se calificaría como "Hospitox", es decir, Hospital de tercer nivel que aglutina determinaciones analíticas que precisan técnicas complejas, que requieren personal especializado o que son de utilización muy esporádica, y que debería contemplarse al menos uno en cada Comunidad Autónoma. 


\section{Población de estudio.}

Para seleccionar la población de estudio, se realizó un cribado de las bases de datos de las urgencias atendidas en el Hospital Universitario Río Hortega, tanto en las urgencias generales como en las pediátricas durante el periodo comprendido entre 1 Enero de 2001 y 31 Diciembre de 2010.

Los criterios de inclusión para analizar los episodios de atención urgente fueron: pacientes menores de 18 años con diagnóstico médico de intoxicación, inhalación o ingesta de tóxicos u otras sustancias, así como los intentos autolíticos por productos tóxicos.

Los criterios de exclusión fueron los diagnósticos de:

- toxoinfecciones alimentarias

- reacciones adversas medicamentosas y efectos secundarios

- intoxicaciones crónicas

- ingesta de cuerpos extraños inertes

Se accedió a la historia clínica hospitalaria informatizada completa de los pacientes que cumplían los criterios de inclusión y no presentaban ninguno de los de exclusión.

El sistema de informatización de las historias clínicas hospitalarias se implantó progresivamente en el Hospital Universitario Río Hortega de Valladolid, por lo que éstas sólo se encuentran informatizadas completamente desde el año 2006 en adelante. Esto supone un claro problema a la hora del acceso a la información.

Entre los años 2001 y 2005, ambos inclusive, se accede a la información de la historia en papel. Por ello, determinados valores que aparecen escritos a mano, no pudieron ser registrados, salvo en casos especiales. 


\section{Variables estudiadas.}

Se diseñó una base de datos con diferentes variables a las que se accedió por medio de las historias clínicas tanto de urgencias como la general del paciente.

\subsection{Variables epidemiológicas}

Se registraron:

- Edad: edad de cada paciente atendido en el servicio de urgencias por intoxicación aguda, recogida en años.

- Sexo: mujer y hombre.

\subsection{Variables de estudio generales}

- Fecha de nacimiento: fecha de nacimiento del paciente intoxicado.

- Mes del año en el que el paciente acudió al servicio de urgencias: enero, febrero, marzo, abril, mayo, junio, julio, agosto, septiembre, octubre, noviembre y diciembre.

- Año en el que ocurrió la intoxicación.

- Hora de admisión: hora en la que el paciente solicita atención en el servicio de urgencias.

- Hora de salida de urgencias: hora en la que el paciente es dado de alta del servicio de urgencias a su domicilio, o es ingresado en planta, o trasladado a otro hospital.

- Tiempo de estancia en urgencias, recogido en horas: tiempo entre la hora de admisión en urgencias y la hora de salida de urgencias.

- Forma de acceder al servicio de urgencias. En este item se recoge si fue transportado por el servicio de emergencias sanitarias, no haciendo distinción entre las unidades de soporte vital avanzado (SVA) o las de soporte vital básico (SVB). 
- Derivación al servicio de urgencias. Se catalogan los pacientes entre los derivados por algún tipo de servicio sanitario, ya sea un servicio de atención primaria, u otro hospital; y los que acuden por propia iniciativa.

- Localidad de origen del paciente. Residentes en Valladolid capital y en los pueblos que corresponden al Área de Salud Oeste de Valladolid; recogiendo los municipios de la provincia correspondientes a esta área de salud con mayor población: Arroyo de la Encomienda, La Cistérniga, Laguna de Duero, Tordesillas, un grupo que engloba al resto de pueblos de la provincia de Valladolid, otro grupo que engloba a los pacientes residentes en otras localidades de Castilla y León y por último, el grupo que engloba a los pacientes residentes en otras localidades españolas.

- Destino del paciente: alta hospitalaria, ingreso hospitalario, alta y remisión a consulta externa o derivación interhospitalaria.

- Dentro de los pacientes ingresados se distingue el servicio en el que son atendidos: Unidad de cuidados intensivos (tanto pediátrica como general), Pediatría, Medicina Interna y otros.

- Sistemático de sangre: si se ha realizado sistemático de sangre o no.

- Bioquímica sanguínea: si se ha realizado bioquímica sanguínea o no.

- Hemostasia: si se ha realizado un estudio de hemostasia o no.

- Equilibrio venoso: si se ha realizado equilibrio venoso o no.

- Gasometría arterial: si se ha realizado gasometría arterial o no.

- Tóxicos en orina: si se ha realizado un estudio de tóxicos en orina o no.

- Alcoholemia: si se ha realizado una determinación de alcoholemia en sangre o no. 
- Realización por parte del servicio de urgencias de electrocardiograma.

- Solicitud de pruebas de imagen en su estancia en el Servicio de Urgencias. Tipo de prueba solicitada: radiografía de tórax, radiografía ósea, radiografía abdominal, ecografía o tomografía axial computerizada.

- Administración de oxigenoterapia suplementaria en el Servicio de Urgencias

- Diagnóstico clínico fundamental en la hoja de atención de Urgencias Hospitalarias.

- Número de sustancias con las que ha sufrido la intoxicación.

- Tipo de sustancia: tipo de sustancia que le ha ocasionado la intoxicación. Se establecen diferentes categorías:

- Alcohol

- Cocaína

- Anfetaminas

○ Hachís

○ Benzodiacepinas

- Antidepresivos

- Paracetamol

- AINEs

- Medicación habitual

- Setas

- Productos de cosmética 
- Productos de limpieza

- Productos de uso industrial

- Monóxido de carbono

- No especificados

- Insecticidas

- Propietario de la medicación: dentro de las producidas por medicación, se analiza quién es el propietario de la misma, si corresponde al mismo paciente o a su entorno.

- En las ocasionadas por monóxido de carbono, se distinguen dos epígrafes.

Mecanismo causante de la intoxicación: En primer lugar el mecanismo por el que se ha producido la intoxicación por CO: incendio, escape de gas, secundario a mala combustión de calderas o estufas o brasero, no recogido/no consta.

Síntoma inicial de la intoxicación por CO. En segundo lugar, el síntoma por el que han acudido a urgencias del hospital y se ha diagnosticado dicha inhalación: neurológicos, digestivos, respiratorios, síncope, resto de la familia intoxicada, no consta en la historia.

- Carboxihemoglobina: cifra de carboxihemoglobina cuando se realizó determinación analítica de la misma.

- Acompañamiento del paciente: se recoge si se registra en la historia si el paciente acude acompañado a urgencias y si en caso afirmativo se especifica quién es el acompañante. 
- Tiempo de observación en urgencias superior a 2 horas: se registra si los pacientes pasan en urgencias un tiempo de observación mayor o menor a dos horas.

- Alcoholemia: se registra la cifra de alcoholemia que presentan los menores de edad, cuando ésta se ha realizado y figura en la historia clínica.

- Consulta a Toxicología: necesidad o no de asistencia por parte de la Unidad de Toxicología, ya sea de manera telefónica o por consulta externa.

- Interconsulta a Psiquiatría: presencia o no de interconsulta a la Unidad de Psiquiatría.

- Horas de ingreso hospitalario: si el paciente ingresa, se registra número de horas que permanece hospitalizado y en la unidad en la que se lleva a cabo dicho ingreso.

- Tratamiento en urgencias: realización o no de tratamiento en el servicio de urgencias hospitalarias.

- Carbón activado: utilización o no de carbón activado.

- Lavado gástrico: realización de lavado gástrico o no.

- Empleo de antídotos: Se registra el empleo o no de antídoto específico.

- Sueroterapia: si el paciente recibió sueroterapia o no.

- Antieméticos: si el paciente recibió antieméticos o no.

- Analgésicos: si el paciente recibió analgésicos o no.

- Protector gástrico: si el paciente recibió gastroprotectores o no.

- Otra medicación: si el paciente recibió otros tratamientos o no. 
- Antecedentes personales: los antecedentes personales de los intoxicados, hasta un número de 3 como máximo, haciendo especial hincapié en los que más morbimortalidad pueden ocasionar.

- Visitas a urgencias el mismo año: número de veces que visitan la urgencia hospitalaria el mismo año y si es posible especificar el motivo general por el que acuden.

- Visitas a urgencias en los años previos: número de veces que visitan la urgencia hospitalaria en años previos y si es posible especificar el motivo general por el que acuden.

- Visitas a urgencias en posteriores: número de veces que visitan la urgencia hospitalaria en años posteriores y si es posible especificar el motivo general por el que acuden.

- Número de consultas externas: número de consultas externas citadas en su historia clínica a la que acuden a lo largo de su vida.

\section{Análisis estadístico.}

Se realizó una base de datos en el programa Microsoft Excel 2005, que tras ser depurada se exportó al programa SPSS 15.0 (SPSS Inc ${ }^{\circledR}$, Chicago III, EEUU), con el que se llevó a cabo el análisis estadístico posterior, con licencia de la Universidad de Valladolid.

Las variables continuas se describieron como media +/- DS (desviación estándar) cuando la distribución de la variable cumplía criterios de normalidad; o como mediana o rango intercuartílico, cuando la distribución de la variable no cumplía criterios de normalidad. Las variables cualitativas se describieron como frecuencias absolutas y relativas o porcentajes. Los datos se recogieron en tablas y se representaron en gráficos (diagramas de barras para las variables cualitativas, histograma de frecuencia, diagrama de cajas y barras de error para las variables cuantitativas.) 
El test de Kolmogorov-Smirnov se usó para determinar la normalidad de las distribuciones. Para estudiar las diferencias entre las medias se utilizaron los tests estadísticos paramétricos y no paramétricos exigidos por las condiciones de aplicación: $t$ de Student o $U$ de Mann-Whitney (para 2 grupos). Para el estudio de asociación entre variables cualitativas se necesitó el test de la Ji cuadrado, en caso necesario (porcentaje de casillas con valores esperados menor de cinco, mayor del $20 \%$ ) se utilizó la prueba exacta de Fisher. El nivel de significación se consideró con una $p \leq 0,05$.

\section{Tratamiento informático y control de calidad de los datos.}

El tratamiento informático de los datos, adecuadamente codificados, fue realizado en un ordenador personal portátil tipo Toshiba, sistema operativo Windows Vista y herramientas de Microsoft Office 2005. La búsqueda bibliográfica se efectuó a través de Internet en Microsoft Internet Explorer 6.0. Los datos recogidos fueron introducidos en una base de datos Excel 2007 diseñada ex profeso y exportada al programa SPSS 15.0. Se aseguró la calidad de los datos comprobándose al menos en dos ocasiones antes de su procesamiento definitivo para detectar datos perdidos, omisiones o errores.

\section{Financiación y permisos.}

La elaboración del estudio ha sido llevada a cabo según la ley 14/2007 de Investigación Biomédica, publicada en el Boletín Oficial del Estado número 159 del 4/7/2007, de acuerdo a la confidencialidad de los datos obtenidos. 


\section{Revisión bibliográfica.}

La revisión bibliográfica ha sido realizada a través de buscadores médicos: la página de Internet PubMed (http://www.ncbi.nlm.nih.gov/PubMed) un servicio de acceso libre a la base de datos Medline proporcionado por la National Library of Medicine (NLM), Doyma (http://www.elsevier.es) y Blackwell - Synergy (http://www.3.interscience.wiley.com). Para la búsqueda inicial se han empleado términos libres y vocabulario controlado (Servicio de Urgencias: Emergency Services; Intoxicación aguda: Acute poisoning; Tóxico: poisoned; Intoxicación medicamentosa aguda: Acute drug poisoning; Intoxicación en edad pediátrica: Pediatric poisoning; Niños: children; Descontaminación digestiva: gastrointestinal descontamination; Epidemiología: epidemiology; Intentos de suicidio: suicide attempts.) La búsqueda libre ha sido completada con la utilización de vocabulario controlado $(\mathrm{MeSH})$ restringiéndola a un término o ampliándola a varios si ha sido preciso. Los términos de búsqueda han sido relacionados mediante operadores lógicos e implementados mediante truncadores, limitándose a los años 1970-2014, todos los idiomas, investigación en humanos y artículos con abstract. De los artículos recuperados han sido seleccionados los que aportaban la información más válida e importante según criterio personal.

Han sido utilizados libros de texto, monografías y manuales actualizados de interés relevante. Así mismo, se usó el fondo bibliográfico de la biblioteca del Hospital Universitario Río Hortega y de las bibliotecas de Ciencias de la Salud de Valladolid. Una parte de la bibliografía ha sido obtenida a partir de los estudios de o revisiones procedentes de las fuentes anteriores. La revisión bibliográfica se hizo en dos tiempos: previa al diseño del estudio, para lograr una idea del estado actual de los estudios sobre intoxicaciones agudas, sobre todo en España; y una nueva revisión posterior al diseño, con el objetivo de priorizar los datos obtenidos y establecer modelos comparativos. 
Todas las referencias obtenidas fueron incluidas en una base del programa Reference Manager, versión 12, de archivo, edición y manejo de referencias bibliográficas.

En cuanto a la redacción de las referencias bibliográficas, se utilizó el estilo recomendado por el International Comittee of Medical Journals Editors conocido como Normas de Vancouver y disponibles en las direcciones electrónicas de www.icmje.org y www.nlm.nih.gov/bsd/uniform requirements.ht/m 


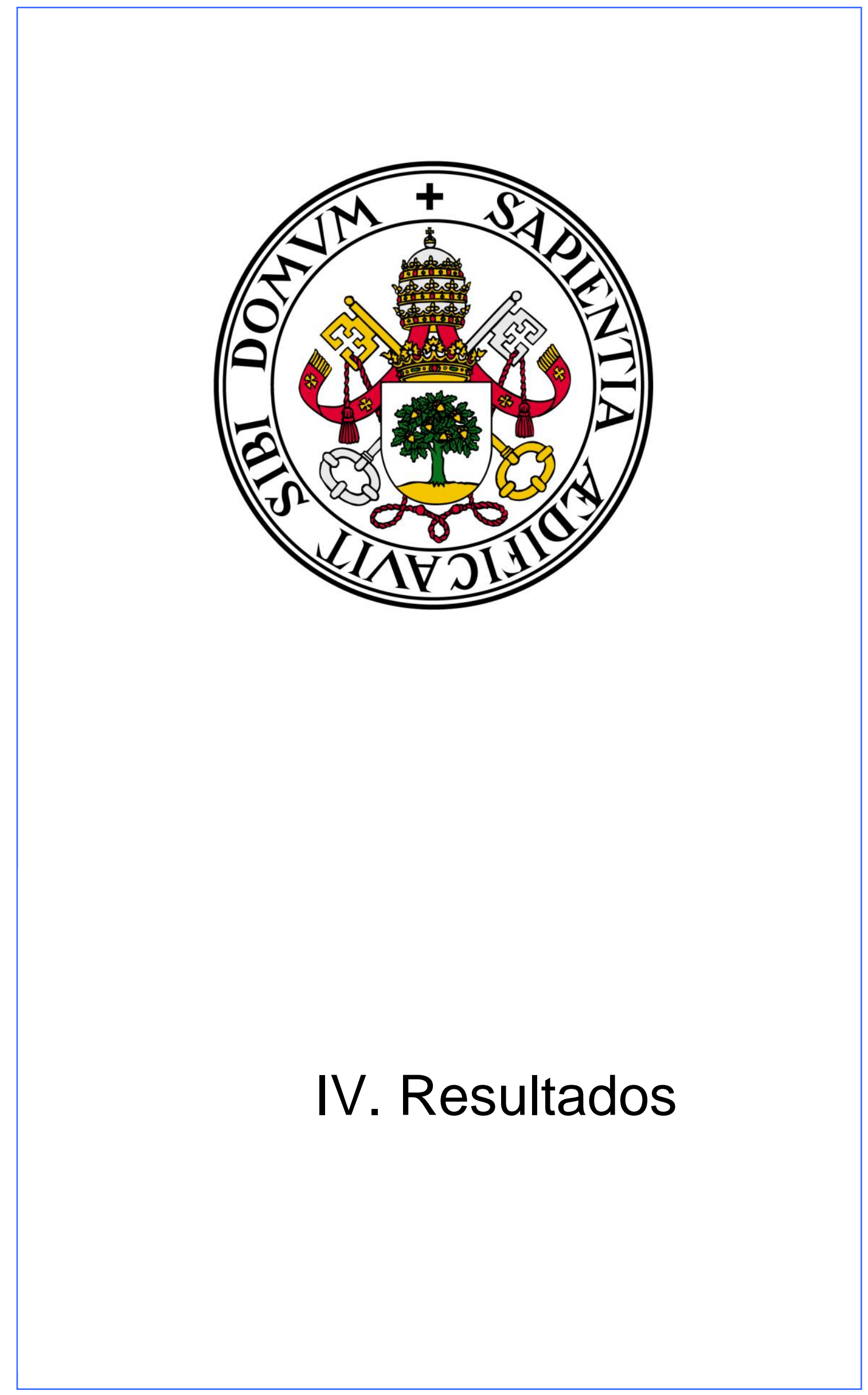




\section{Resultados.}

\section{Incidencia.}

Se recogieron en total 994 pacientes menores de 18 años, que acudieron a urgencias del Hospital Universitario Río Hortega de Valladolid con el diagnóstico de intoxicación, durante el periodo comprendido entre 1 Enero de año 2001 hasta el 31 de diciembre de 2010, ambos inclusive.

Como se ha hecho referencia en el apartado de material y métodos, entre los años 2001 y 2005, ambos incluidos, se accede a la información de la historia en papel, por lo que determinados valores escritos a mano no pudieron ser registrados, salvo en casos especiales.

Por tanto, de las 994 historias clínicas de las que se pueden obtener datos completos; sólo en 584 se pueden recogen todos los parámetros a estudiar.

Durante esos 10 años fueron atendidos por el Servicio de Urgencias del Hospital Universitario Río Hortega, un total de 239631 pacientes menores de 18 años, por lo que las consultas por intoxicaciones en menores de edad suponen el $0,41 \%$ de todas las consultas atendidas en dicho servicio en menores de 18 años. (Tabla 7.) 
Tabla 7: Incidencia de las intoxicaciones en menores de edad respecto los pacientes atendidos en el Servicio de Urgencias Hospitalario

\begin{tabular}{|c|c|c|c|c|c|c|}
\hline $\begin{array}{c}\text { Año } \\
\text { de } \\
\text { ate } \\
\text { nció } \\
\mathrm{n} \\
\end{array}$ & $\begin{array}{c}\text { Pacientes } \\
<18 \text { años } \\
\text { atendidos } \\
\text { en } \\
\text { urgencias }\end{array}$ & $\begin{array}{c}\text { Total de } \\
\text { pacientes } \\
\text { atendidos } \\
\text { en } \\
\text { urgencias }\end{array}$ & $\begin{array}{c}\text { Tasa de incidencia (\%) } \\
\text { de los menores de } 18 \\
\text { años respecto a las } \\
\text { urgencias generales }\end{array}$ & 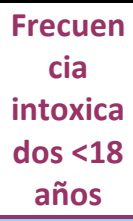 & $\begin{array}{c}\text { Tasa de } \\
\text { incidencia (\%) } \\
\text { respecto las } \\
\text { urgencias en } \\
<18 \text { años } \\
\end{array}$ & $\begin{array}{c}\text { Tasa de } \\
\text { incidencia (\%) } \\
\text { respecto las } \\
\text { urgencias en } \\
\text { general } \\
\end{array}$ \\
\hline $\begin{array}{c}200 \\
1\end{array}$ & 18201 & 69842 & 26,06 & 87 & 0,47 & 0,12 \\
\hline $\begin{array}{c}200 \\
2\end{array}$ & 23071 & 93273 & 24,73 & 92 & 0,39 & 0,09 \\
\hline $\begin{array}{c}200 \\
3\end{array}$ & 23711 & 96560 & 24,55 & 103 & 0,43 & 0,10 \\
\hline $\begin{array}{c}200 \\
4\end{array}$ & 20680 & 93876 & 22,02 & 55 & 0,26 & 0,05 \\
\hline $\begin{array}{c}200 \\
5\end{array}$ & 22932 & 102886 & 22,28 & 65 & 0,28 & 0,06 \\
\hline $\begin{array}{c}200 \\
6\end{array}$ & 24865 & 98940 & 25,13 & 92 & 0,37 & 0,09 \\
\hline $\begin{array}{c}200 \\
7\end{array}$ & 24552 & 99473 & 24,68 & 149 & 0,60 & 0,14 \\
\hline $\begin{array}{c}200 \\
8\end{array}$ & 22261 & 95410 & 23,33 & 119 & 0,53 & 0,12 \\
\hline $\begin{array}{c}200 \\
9\end{array}$ & 28144 & 100844 & 27,9 & 119 & 0,42 & 0,11 \\
\hline $\begin{array}{c}201 \\
0\end{array}$ & 31214 & 104939 & 29,74 & 113 & 0,36 & 0,10 \\
\hline $\begin{array}{c}\text { Tot } \\
\text { al }\end{array}$ & 239631 & 956043 & 25,06 & 994 & 0,41 & 0,10 \\
\hline
\end{tabular}

Si nos fijamos en los pacientes menores de 18 años pertenecientes al Área de Salud de Valladolid Oeste con tarjeta sanitaria en los años comprendidos entre 2002 y 2010 ( los datos referentes al año 2001 no están disponibles), la tasa de incidencia sería de $0,27 \%$ de todos los jóvenes registrados en dicha Área de Salud. (Tabla 8) 
Tabla 8: Incidencia de pacientes intoxicados atendidos en el servicio de urgencias hospitalarias, respecto a los menores de 18 años pertenecientes al Área de Salud de Valladolid Oeste

\begin{tabular}{|c|c|cc|c|}
\hline $\begin{array}{c}\text { Año } \\
\text { de } \\
\text { atenc } \\
\text { ión }\end{array}$ & $\begin{array}{c}\text { Inscritos menores } 18 \\
\text { años tarjeta sanitaria } \\
\text { Valladolid Oeste }\end{array}$ & $\begin{array}{c}\text { Pacientes }<18 \\
\text { años atendidos } \\
\text { en urgencias }\end{array}$ & $\begin{array}{c}\text { Frecuencia } \\
\text { intoxicados } \\
<18 \text { años }\end{array}$ & $\begin{array}{c}\text { Tasa de incidencia (\%) } \\
\text { respecto las tarjetas } \\
\text { sanitarias <18 años }\end{array}$ \\
\hline 2002 & 32811 & 23071 & 92 & 0,28 \\
2003 & 35938 & 23711 & 103 & 0,28 \\
2004 & 38382 & 20680 & 55 & 0,14 \\
2005 & 39353 & 22932 & 65 & 0,16 \\
2006 & 40607 & 24865 & 92 & 0,22 \\
2007 & 42091 & 24552 & 149 & 0,35 \\
2008 & 43167 & 22261 & 119 & 0,27 \\
2009 & 41893 & 28144 & 119 & 0,28 \\
2010 & 42537 & 31214 & 113 & 0,26 \\
\hline Total & 356779 & 239631 & 994 & 0,27 \\
\hline
\end{tabular}




\section{Diagnóstico principal establecido en el servicio de urgencias hospitalario.}

Se recogen los diferentes diagnósticos establecidos en el servicio de urgencias hospitalario para los pacientes intoxicados. Tabla 9 , figura 1.

Tabla 9: Diagnóstico

\begin{tabular}{|cc|c|}
\hline Diagnóstico & Frecuencia & Porcentaje \\
\hline Intoxicación etílica & 434 & 43,66 \\
Intoxicación medicamentosa & 267 & 26,86 \\
Intoxicación por productos de limpieza & 81 & 8,14 \\
\hline Intoxicación por CO & 80 & 8,04 \\
No especificado & 35 & 3,52 \\
Intoxicación por productos industriales & 25 & 2,51 \\
Intoxicación por productos cosméticos & 22 & 2,21 \\
Intoxicación por insecticidas y raticidas & 21 & 2,11 \\
\hline Intoxicación por cannabis & 13 & 1,30 \\
Intoxicación por estimulantes & 9 & 0,90 \\
Intoxicación por setas & 6 & 0,60 \\
Intoxicación por cocaína & 1 & 0,10 \\
\hline Total & 994 & 100 \\
\hline \multicolumn{2}{|c}{}
\end{tabular}

Figura 1: Diagnóstico principal

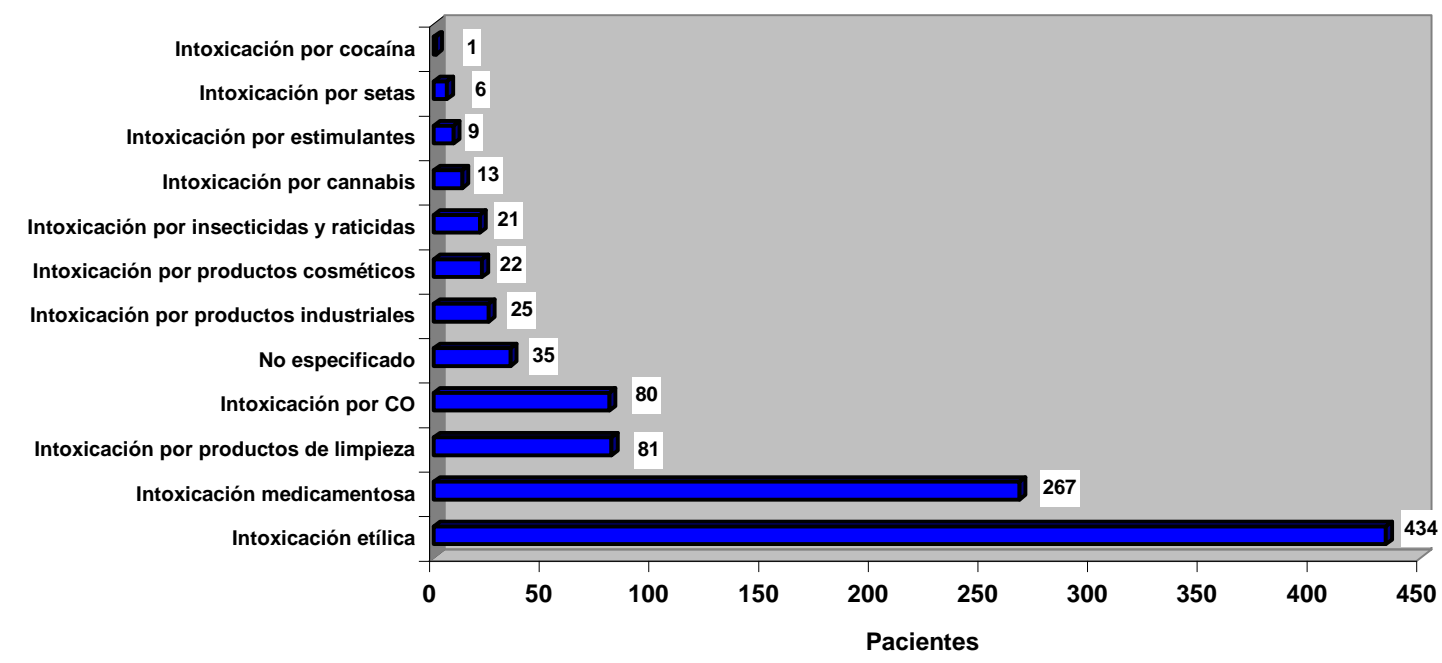




\subsection{Número de sustancias implicadas en la intoxicación.}

Ocasionalmente, las intoxicaciones agudas son acontecidas por más de un agente causal, lo que dificulta la labor diagnóstica y el pronóstico del enfermo. Se especifica el número de sustancias por las que son atendidos los pacientes intoxicados. El 97,5\% de los pacientes han sido víctimas de la intoxicación por una sustancia, y en ningún caso, por más de 3.Tabla 10, figura 2.

Tabla 10: Número de sustancias implicadas en la intoxicación

\begin{tabular}{|ccc|}
\hline Número de sustancias & Frecuencia & Porcentaje \\
\hline 1 & 969 & 97,48 \\
2 & 20 & 2,01 \\
3 & 5 & 0,50 \\
Total & 994 & 100 \\
\hline
\end{tabular}

Figura 2: Número de sustancias implicadas en la intoxicación.

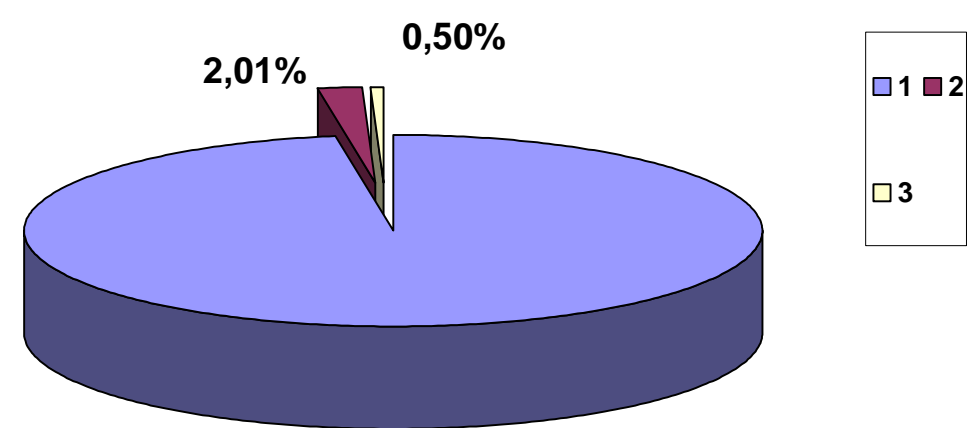

$97,48 \%$

\subsection{Sustancias implicadas en la intoxicación.}

Se recogen las dos primeras sustancias que ocasionan la asistencia en urgencias con el diagnóstico de intoxicación; estableciendo como primera sustancia aquella que ocasiona más clínica dentro de la necesidad de $\begin{array}{llllll}\text { cuidados. Tabla } & 11-12, & \text { figuras } & 3 & y & 4 .\end{array}$ 
Tabla 11: Sustancia principal implicada en la intoxicación

\begin{tabular}{|ccc|}
\hline Sustancia & Frecuencia & Porcentaje \\
\hline Alcohol & 434 & 43,70 \\
Medicación habitual & 150 & 15,10 \\
Co & 80 & 8,00 \\
Productos de limpieza & 78 & 7,80 \\
Paracetamol & 55 & 5,50 \\
No especificado & 43 & 4,30 \\
Benzodiacepinas & 27 & 2,70 \\
Productos industriales & 27 & 2,70 \\
Productos de cosmética & 26 & 2,60 \\
\hline AlNES & 21 & 2,10 \\
Insecticidas & 21 & 2,10 \\
Hachís & 13 & 1,30 \\
Anfetaminas & 6 & 0,60 \\
Antidepresivos & 6 & 0,60 \\
Setas & 6 & 0,60 \\
Cocaína & 1 & 0,10 \\
\hline Total & 994 & 100 \\
\hline
\end{tabular}

Figura 3: Sustancia principal implicada en la intoxicación

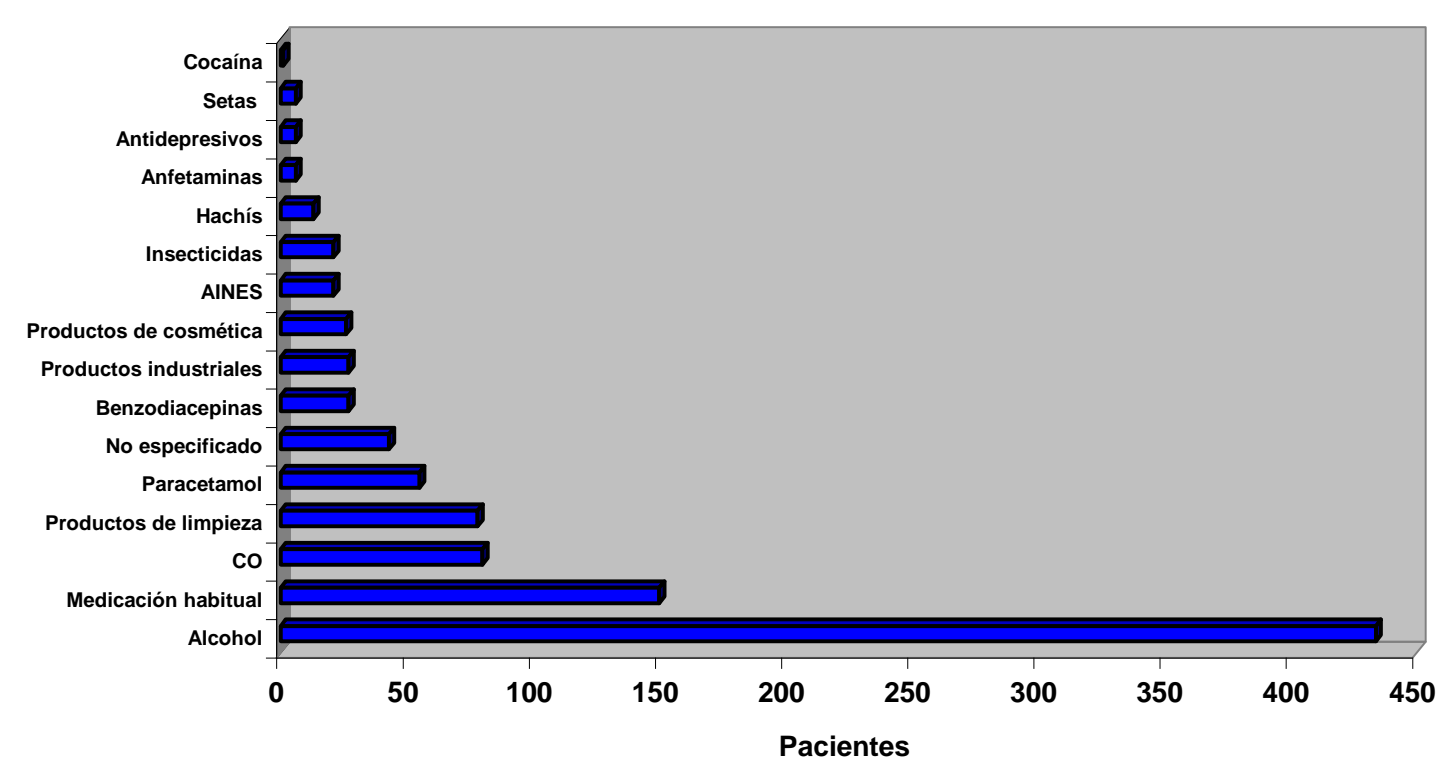


Tabla 12: Sustancia secundaria implicada en la intoxicación

\begin{tabular}{|ccc|}
\hline Sustancia & Frecuencia & Porcentaje \\
\hline Hachís & 12 & 46,15 \\
\hline Benzodiacepinas & 4 & 15,38 \\
Medicación habitual & 4 & 15,38 \\
\hline Anfetaminas & 2 & 7,69 \\
AINES & 2 & 7,69 \\
Antidepresivos & 1 & 3,85 \\
No especificado & 1 & 3,85 \\
\hline Total & 26 & 100 \\
\hline
\end{tabular}

Figura 4: Sustancia secundaria implicada en la intoxicación
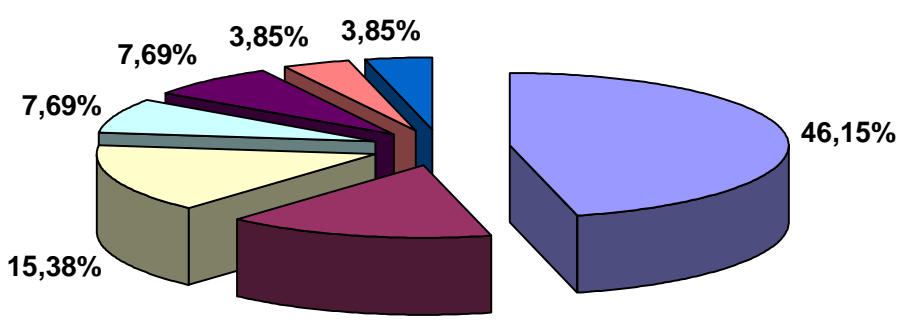

$\square$ Hachís

$\square$ Benzodiacepinas

$\square$ Medicación habitual

$\square$ Anfetaminas

$\square$ AINES

$\square$ Antidepresivos

$\square$ No especificado

$15,38 \%$

\section{Sexo.}

\subsection{Distribución por sexos.}

La distribución por sexos corresponde a 534 hombres y 460 mujeres, como se muestra en la tabla 13 y figura 5 .

Tabla 13: Distribución por sexos

\begin{tabular}{|ccc|}
\hline Sexo & Frecuencia & Porcentaje \\
\hline Hombre & 534 & 53,72 \\
Mujer & 460 & 46,28 \\
Total & 994 & 100 \\
\hline
\end{tabular}



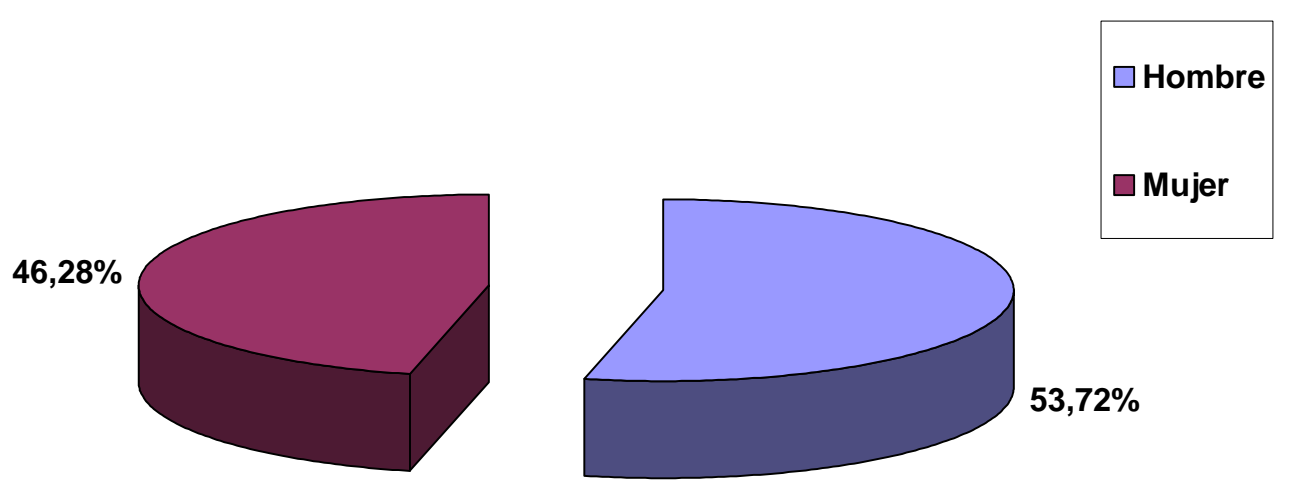

\subsection{Distribución de los diferentes diagnósticos según el sexo.}

No se evidenciaron diferencias estadísticamente significativas entre el diagnóstico de intoxicación etílica teniendo en cuenta su sexo ( $p$ valor= 0,38 ). Tabla 14.

Tabla 14: Relación entre el diagnóstico de intoxicación etílica y el sexo del paciente

\begin{tabular}{|c|cc|c|}
\hline & Resto diagnósticos & Diagnóstico etílico & Total \\
\hline Hombre & 294 & 240 & 534 \\
Mujer & 266 & 194 & 460 \\
\hline Total & 560 & 434 & 994 \\
\hline \multicolumn{3}{|c|}{$\mathrm{p}=0,38$} \\
\hline
\end{tabular}

Se evidenciaron diferencias estadísticamente significativas entre el diagnóstico de intoxicación medicamentosa teniendo en cuenta su sexo. $(p=0,008)$ Tabla 15. 
Tabla 15: Relación entre el diagnóstico de intoxicación medicamentosa y el sexo del enfermo

\begin{tabular}{|c|cc|c|}
\hline & Resto diagnósticos & Medicamentosas & Total \\
\hline Hombre & 409 & 125 & 534 \\
Mujer & 318 & 142 & 460 \\
\hline Total & 727 & 267 & 994 \\
\hline \multicolumn{2}{|r}{$\mathrm{p}=0,008$} \\
\hline
\end{tabular}

No se evidenciaron diferencias estadísticamente significativas entre el diagnóstico de intoxicación por productos de limpieza teniendo en cuenta su sexo. $(p=0,297)$ Tabla 16 .

Tabla 16: Relación entre el diagnóstico de intoxicación por productos de limpieza y el sexo del paciente

\begin{tabular}{|c|cc|c|}
\hline \multirow{2}{*}{ Hombre } & Resto diagnósticos & Intoxicación por productos de limpieza & Total \\
\cline { 2 - 4 } & 486 & 48 & 534 \\
Mujer & 427 & 33 & 460 \\
Total & 913 & 81 & 994 \\
\hline
\end{tabular}

No se evidenciaron diferencias estadísticamente significativas entre el diagnóstico de intoxicación por monóxido de carbono teniendo en cuenta su sexo. $(p=0,062)$ Tabla 17 .

Tabla 17: Relación entre el diagnóstico de intoxicación por monóxido de carbono y el sexo del paciente

\begin{tabular}{|c|ccc|}
\hline \multirow{2}{*}{ Hombre } & Resto diagnósticos & Intoxicación por CO & Total \\
\cline { 2 - 5 } Mujer & 499 & 35 & 534 \\
\hline Total & 415 & 45 & 460 \\
\hline \multicolumn{5}{r}{$\mathrm{p}=0,062$} \\
\hline
\end{tabular}


No se evidenciaron diferencias estadísticamente significativas entre el diagnóstico de intoxicación por productos no especificados teniendo en cuenta su sexo. $(p=0,448)$ Tabla 18

Tabla 18: Relación entre el diagnóstico de intoxicaciones por productos no especificados y el sexo del intoxicado

\begin{tabular}{|c|cc|c|}
\hline & Resto intoxicaciones & Intoxicaciones por productos no especificados & Total \\
\hline Hombre & 513 & 21 & 534 \\
Mujer & 446 & 14 & 460 \\
\hline Total & 959 & 35 & 994 \\
\hline \multicolumn{3}{|c}{} \\
\hline
\end{tabular}

No se evidenciaron diferencias estadísticamente significativas entre el diagnóstico de intoxicación por productos industriales teniendo en cuenta su sexo. $(p=0,297)$ Tabla 19.

Tabla 19: Relación entre el diagnóstico de intoxicación por productos industriales y el sexo del intoxicado

\begin{tabular}{|c|cc|c|}
\hline & Resto intoxicaciones & Intoxicación por productos industriales & Total \\
\hline Hombre & 518 & 16 & 534 \\
Mujer & 451 & 9 & 460 \\
Total & 969 & 25 & 994 \\
& & $\mathrm{p}=0,297$ & \\
\hline
\end{tabular}

No se evidenciaron diferencias estadísticamente significativas entre el diagnóstico de intoxicación por productos de cosmética teniendo en cuenta su sexo. $(p=0,610)$ Tabla 20

Tabla 20: Relación entre el diagnóstico de intoxicación por productos cosméticos y el sexo del intoxicado

\begin{tabular}{|c|cc|c|}
\hline & Resto diagnósticos & Intoxicación productos cosméticos & Total \\
\hline Hombre & 521 & 13 & 534 \\
Mujer & 451 & 9 & 460 \\
\hline Total & 972 & 22 & 994 \\
\hline \multicolumn{4}{|r}{} \\
\hline
\end{tabular}


Se evidenciaron diferencias estadísticamente significativas entre el diagnóstico de intoxicación por insecticidas teniendo en cuenta su sexo. ( $p=$ $0,008)$ Tabla 21

Tabla 21: Relación entre el diagnóstico de intoxicación por plaguicidas y el sexo del paciente

\begin{tabular}{|c|cccc|}
\hline & Resto diagnósticos & Intoxicación por insecticidas & Total \\
\cline { 2 - 5 } Hombre & 409 & 125 & 534 \\
Mujer & 318 & 142 & 460 \\
Total & 727 & 267 & 994 \\
& & $\mathrm{p}=0,008$ & & \\
\hline
\end{tabular}

No se evidenciaron diferencias estadísticamente significativas entre el diagnóstico de intoxicación por cannabis teniendo en cuenta su sexo. $(p=0$, 091)Tabla 22.

Tabla 22: Relación entre el diagnóstico de intoxicación por cannabis y el sexo del paciente

\begin{tabular}{|c|cc|c|}
\hline & Resto diagnósticos & Intoxicación por cannabis & Total \\
\hline Hombre & 524 & 10 & 534 \\
Mujer & 457 & 3 & 460 \\
\hline Total & 981 & 13 & 994 \\
\hline \multicolumn{2}{|r|}{$\mathrm{p}=0,091$} \\
\hline
\end{tabular}

No se evidenciaron diferencias estadísticamente significativas entre el diagnóstico de intoxicación por productos estimulantes teniendo en cuenta su sexo. $(p=0,146)$ Tabla 23

Tabla 23: Relación entre el diagnóstico por intoxicaciones por productos estimulantes y el sexo del intoxicado

\begin{tabular}{|c|cc|c|}
\hline & Resto intoxicaciones & Intoxicaciones por productos estimulantes & Total \\
\hline Hombre & 527 & 7 & 534 \\
Mujer & 458 & 2 & 460 \\
\hline Total & 985 & 9 & 994 \\
\hline \multicolumn{3}{|c}{} \\
\hline
\end{tabular}


No se evidenciaron diferencias estadísticamente significativas entre el diagnóstico de intoxicación por setas teniendo en cuenta su sexo. $(p=0$, 524)Tabla 24

Tabla 24: Relación entre las intoxicaciones por setas y el sexo del intoxicado

\begin{tabular}{|c|c|c|c|}
\hline & Resto intoxicaciones & Intoxicaciones por setas & Total \\
\hline Hombre & 530 & 4 & 534 \\
\hline Mujer & 458 & 2 & 460 \\
\hline Total & 988 & 6 & 994 \\
\hline
\end{tabular}

No se evidenciaron diferencias estadísticamente significativas entre el diagnóstico de intoxicación por cocaína teniendo en cuenta su sexo. $(p=0$, 353) Tabla 25.

Tabla 25: Relación entre el diagnóstico de intoxicación por cocaína y el sexo del paciente

\begin{tabular}{|c|cc|c|}
\hline & Resto diagnósticos & Intoxicación por cocaína & Total \\
\hline Hombre & 533 & 1 & 534 \\
Mujer & 460 & 0 & 460 \\
\hline Total & 993 & 1 & 994 \\
\hline \multicolumn{2}{|c|}{$\mathrm{p}=0,353$} \\
\hline
\end{tabular}

Tampoco se evidenciaron diferencias estadísticamente significativas entre el número de sustancias implicadas en las intoxicaciones y su tipo, teniendo en $\begin{array}{lllll}\text { cuenta su } & \text { sexo } & (\mathrm{p}=0,082) & \text { Tabla } & 26 .\end{array}$ 
Tabla 26: Relación entre el sexo y el número de sustancias implicadas en la intoxicación

\begin{tabular}{|c|cccccc|c|}
\hline & \multicolumn{7}{|c|}{ Número de Sustancias } \\
\hline & 1 & $\%$ & 2 & $\%$ & 3 & $\%$ & Total \\
\hline & 52 & & & & & & \\
Hombre & 1 & $53,76 \%$ & 11 & $55 \%$ & 2 & $40 \%$ & 534 \\
& 44 & & & & & & 460 \\
\hline Mujer & 8 & $46,23 \%$ & 9 & $45 \%$ & 3 & $60 \%$ & 994 \\
\hline & 96 & $100 \%$ & 20 & $100 \%$ & 5 & $100 \%$ & \\
\hline
\end{tabular}

\subsection{Relación entre el sexo y la edad.}

La media de edad de atención de los hombres es de 9,82 +/- 6,5 años; y la de las mujeres es de 10,53 +/- 6,3 años. Tabla 27, figura 6 .

No se evidenciaron diferencias estadísticamente significativas en la edad de los intoxicados teniendo en cuenta su sexo $(p=0,84)$

Tabla 27: Edad media de cada sexo

\begin{tabular}{|ccc|}
\hline Sexo & Frecuencia & Edad media \\
\hline Hombre & 534 & $9,82+/-6,5$ \\
Mujer & 460 & $10,53+/-6,3$ \\
Total & 994 & $10,15+/-6,43$ \\
\hline \multicolumn{3}{c}{$\mathrm{p}=0,84$} \\
\hline
\end{tabular}




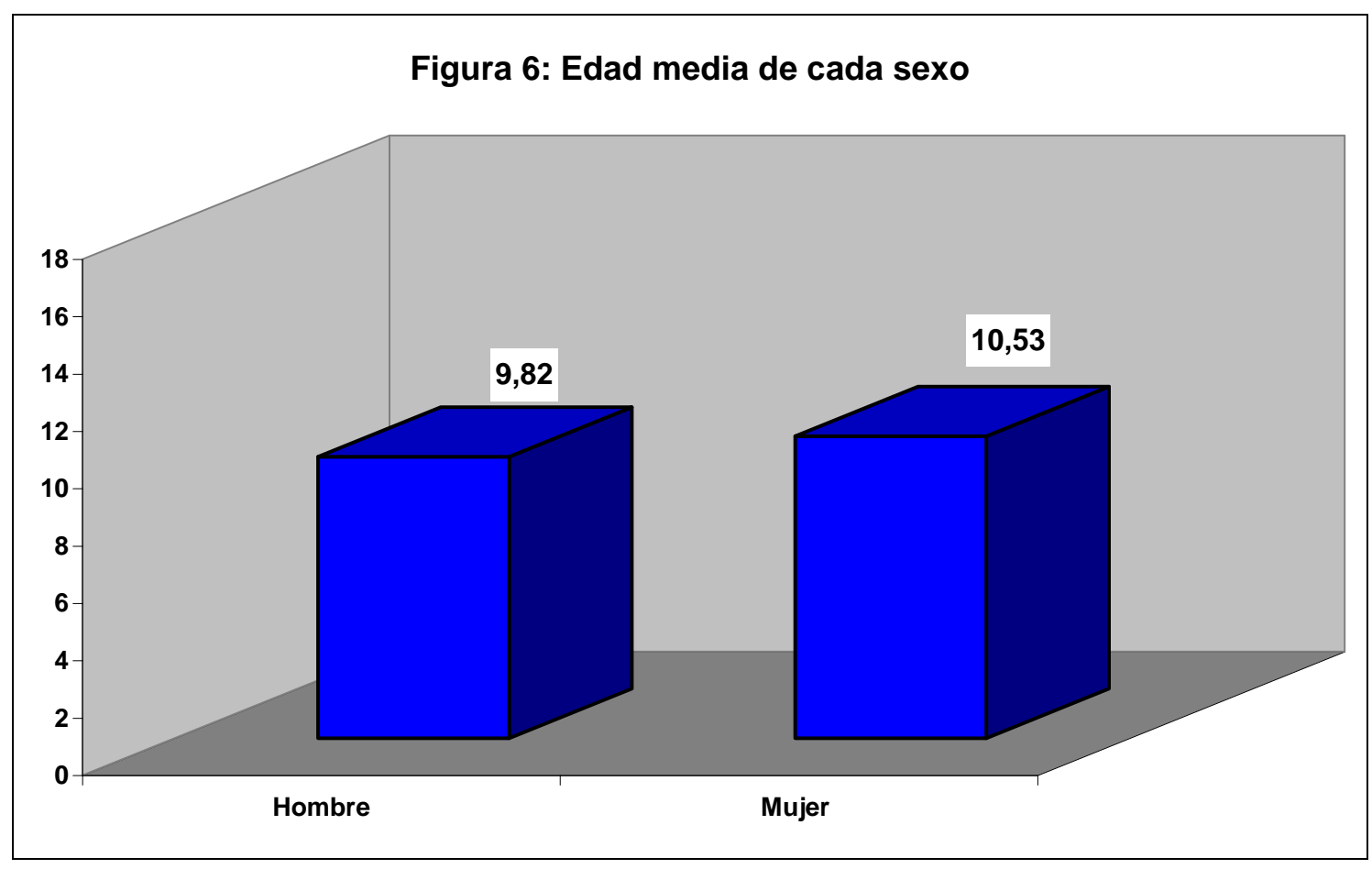

$p=0,84$

\subsection{Relación entre el sexo y la distribución temporal de las} intoxicaciones.

\subsubsection{Relación entre el sexo y el año de atención en el servicio de urgencias hospitalario.}

No se evidenciaron diferencias estadísticamente significativas en el año de atención de los pacientes intoxicados teniendo en cuenta su sexo $(p=0,20)$. Tablas 28, figuras 7 .

Tabla 28: Relación entre el sexo y el año de atención

\begin{tabular}{|c|cccccccccc|}
\hline & 2001 & 2002 & 2003 & 2004 & 2005 & 2006 & 2007 & 2008 & 2009 & 2010 \\
\hline Hombre & 45 & 53 & 58 & 23 & 29 & 46 & 84 & 71 & 60 & 65 \\
\hline Mujer & 42 & 39 & 45 & 32 & 36 & 46 & 65 & 48 & 59 & 48 \\
& 87 & 92 & 103 & 55 & 65 & 92 & 149 & 119 & 119 & 113 \\
& & & & & 0,20 & & & & & \\
\hline
\end{tabular}




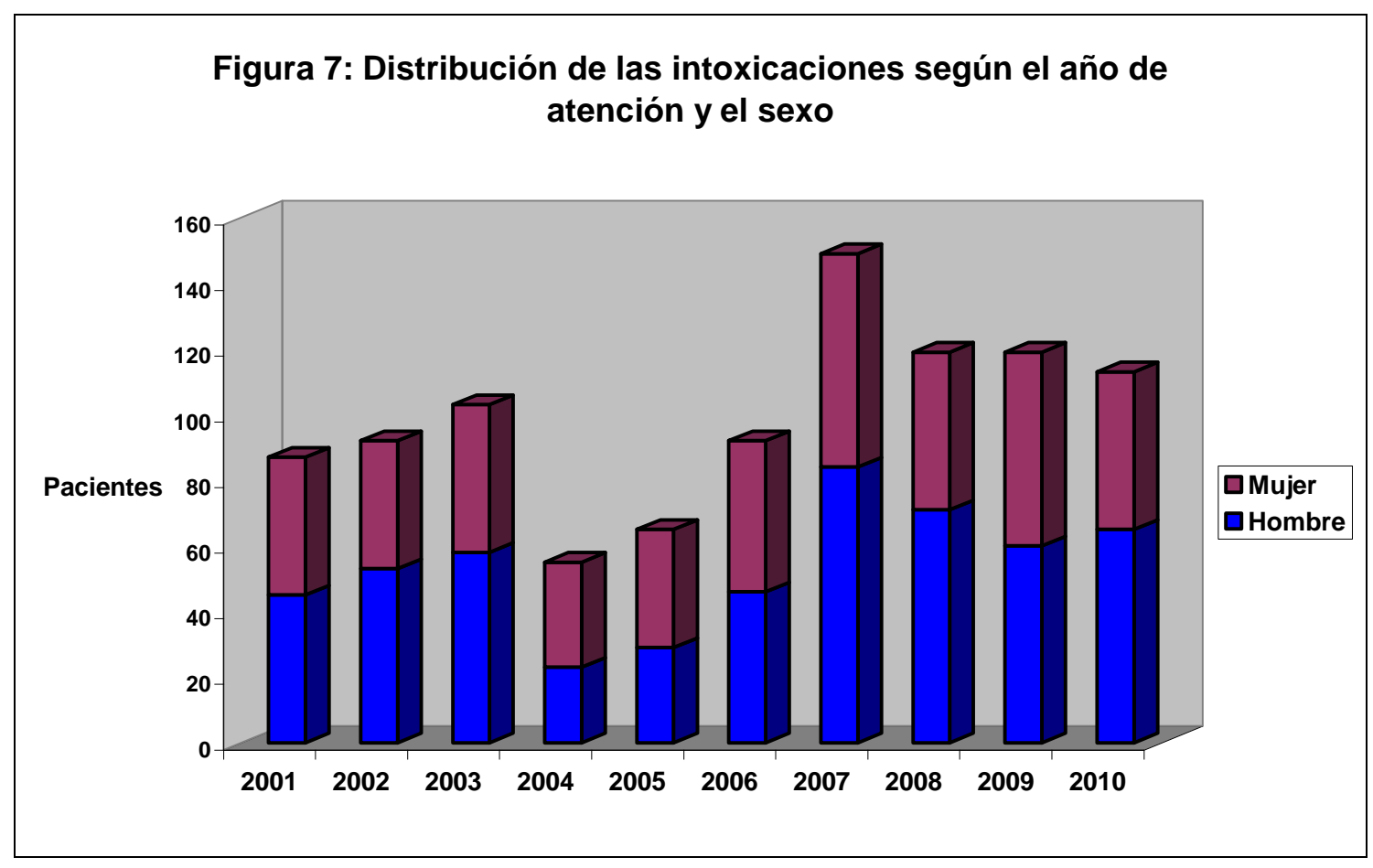

$p=0,20$

\subsubsection{Relación entre el mes de atención en el servicio de urgencias} hospitalario y el sexo del paciente.

No se evidenciaron diferencias estadísticamente significativas en el mes de atención de los pacientes intoxicados en el servicio de urgencias hospitalario teniendo en cuenta su sexo $(p=0,92)$. Tablas 29 , figura 8 .

Tabla 29: Relación entre el sexo y el mes de atención

\begin{tabular}{|c|c|c|c|c|c|c|c|c|c|c|c|c|}
\hline & Enero & Febrero & Marzo & Abril & Mayo & Junio & Julio & Agosto & Septiembre & Octubre & Noviembre & Diciembre \\
\hline Hombre & 36 & 46 & 54 & 35 & 47 & 49 & 54 & 35 & 45 & 41 & 46 & 46 \\
\hline Mujer & 30 & 36 & 44 & 48 & 40 & 47 & 22 & 34 & 37 & 40 & 35 & 47 \\
\hline \multicolumn{12}{|c|}{$p=0,92$} & 81 \\
\hline
\end{tabular}




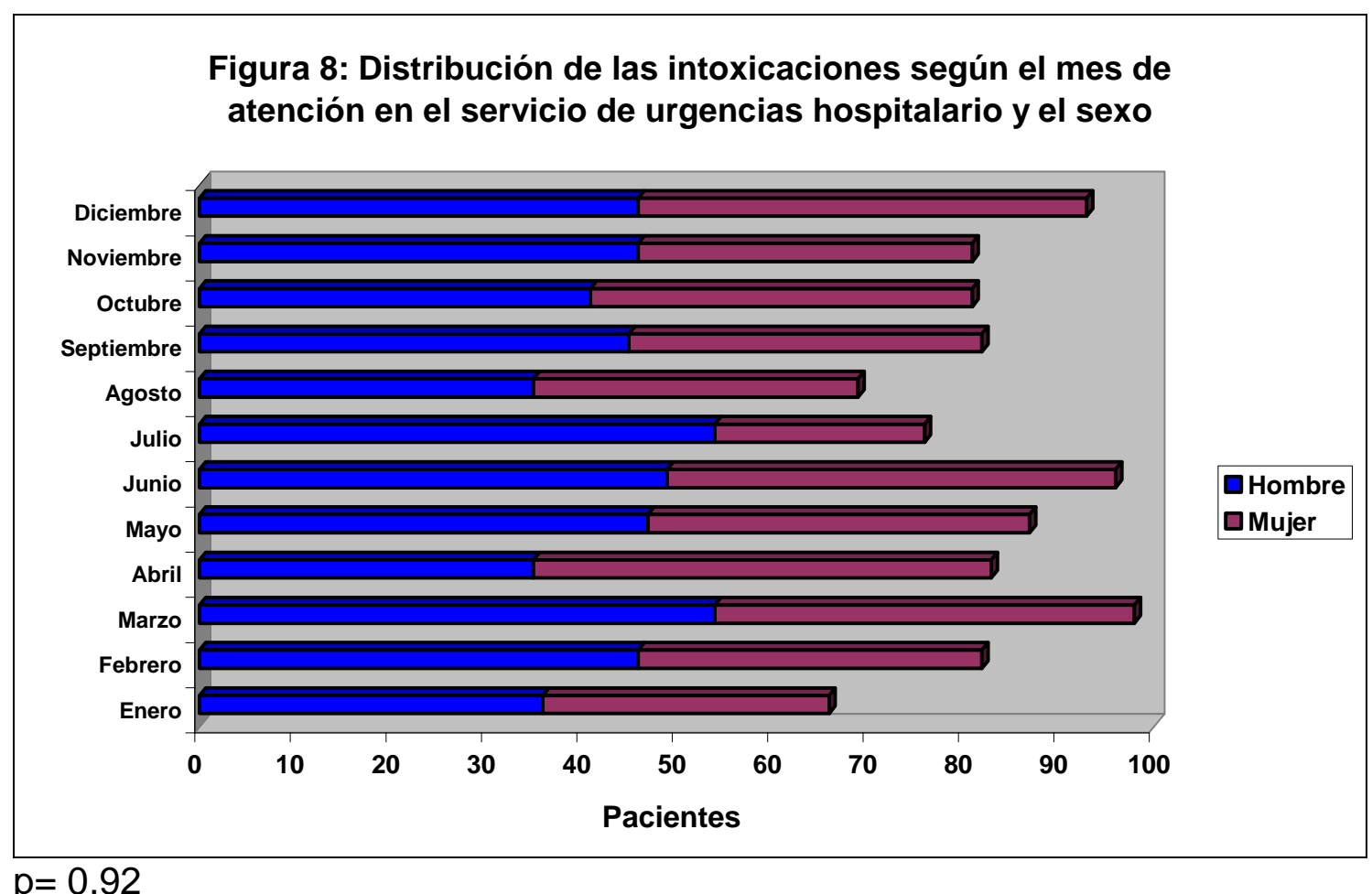




\subsection{Relación entre el sexo y otros parámetros.}

No se evidenciaron diferencias estadísticamente significativas entre los siguientes parámetros teniendo en cuenta su sexo (tabla 30):

Tabla 30: Relación entre diferentes parámetros y el sexo del paciente

\begin{tabular}{|c|c|}
\hline Relación de diferentes parámetros y sexo & p valor \\
\hline Hora de atención & 0,20 \\
\hline Tiempo permanencia en urgencias & 0,18 \\
\hline Transporte en vehículos de emergencia & 0,10 \\
\hline Derivación del paciente a urgencias & 0,20 \\
\hline Localidad de origen & 0,60 \\
\hline Acompañamiento en urgencias & 0,60 \\
\hline Síntomas iniciales intoxicación CO & 0,90 \\
\hline Mecanismo producción intoxicación CO & 0,30 \\
\hline Propietario medicación & 0,50 \\
\hline Urgencias el mismo año de la intoxicación & 0,19 \\
\hline Urgencias años anteriores a la intoxicación & 0,24 \\
\hline Urgencias años posteriores a la intoxicación & 0,20 \\
\hline Realización analítica en urgencias & 0,30 \\
\hline Realización ECG en urgencias & 0,70 \\
\hline Realización pruebas imagen en urgencias & 0,90 \\
\hline Oxigenoterapia en urgencias & 0,10 \\
\hline Tratamiento en urgencias & 0,37 \\
\hline Lavado gástrico en urgencias & 0,08 \\
\hline Carbón activado en urgencias & 0,30 \\
\hline Antídoto en urgencias & 0,49 \\
\hline Suero fisiológico en urgencias & 0,39 \\
\hline Suero glucosado en urgencias & 0,16 \\
\hline Antiemético en urgencias & 0,26 \\
\hline Analgésico en urgencias & 0,14 \\
\hline Protector gástrico en urgencias & 0,24 \\
\hline Consulta toxicología & 0,61 \\
\hline Tiempo estancia en servicio de urgencias & 0,18 \\
\hline Tiempo de ingreso hospitalario & 0,74 \\
\hline Alcoholemia & 0,18 \\
\hline Cifra de carboxihemoglobina & 0,29 \\
\hline
\end{tabular}


3.6. Relación entre el sexo y la administración de Tiamina en su estancia en el servicio de urgencias hospitalario.

Se evidenciaron diferencias estadísticamente significativas entre la administración de tiamina en urgencias como tratamiento teniendo en cuenta su sexo $(p=0,011)$ Tabla 31 .

Tabla 31: Relación entre el sexo del paciente y el tratamiento con Tiamina en el servicio de urgencias hospitalario

\begin{tabular}{|c|cccc|c|}
\hline & NO & $\%$ & Sí & $\%$ & Total \\
\hline Hombre & 315 & $54,87 \%$ & 8 & $100 \%$ & 323 \\
Mujer & 259 & $45,12 \%$ & 0 & $0 \%$ & 259 \\
\hline Total & 574 & $100 \%$ & 8 & $100 \%$ & 582 \\
\hline \multicolumn{7}{|c}{0,011} \\
\hline
\end{tabular}

3.7. Relación entre el sexo del paciente y el destino tras la estancia en el servicio de urgencias hospitalario y el servicio hospitalario en el que va a ser ingresado.

Se evidenciaron diferencias estadísticamente significativas entre el destino del paciente tras su paso por el servicio de urgencias hospitalario teniendo en cuenta su sexo $(\mathbf{p}=\mathbf{0 , 0 0 0 1 )}$. Tabla 32 .

Tabla 32: Relación entre el sexo del paciente y su destino tras el servicio de urgencias hospitalario

\begin{tabular}{|c|cccccccc|c|}
\hline Sexo & Alta & $\%$ & $\begin{array}{c}\text { Ingreso } \\
\text { hospitalario }\end{array}$ & $\%$ & $\begin{array}{c}\text { Consulta } \\
\text { externa }\end{array}$ & $\%$ & Derivación & $\%$ \\
\hline Hombre & 459 & $55,50 \%$ & 69 & $53,07 \%$ & 3 & $30 \%$ & 3 & $11,11 \%$ \\
Mujer & 368 & $44,49 \%$ & 61 & $46,92 \%$ & 7 & $70 \%$ & 24 & $88,88 \%$ \\
\hline Total & 827 & $100 \%$ & 130 & $100 \%$ & 10 & $100 \%$ & 27 & $100 \%$ \\
\hline \multicolumn{7}{|c}{$\mathrm{p}=0,0001$} \\
\hline
\end{tabular}

Se evidenciaron diferencias estadísticamente significativas entre el servicio hospitalario en el que ingresa el paciente tras su paso por el 
servicio de urgencias hospitalario teniendo en cuenta su sexo $(p=0,002)$. Tabla 33 y figura 9.

Tabla 33: Relación entre el sexo y el servicio de ingreso hospitalario del paciente intoxicado

\begin{tabular}{|c|cccccccc|}
\hline & Pediatría & $\%$ & Medicina Interna & $\%$ & UCI & $\%$ & Otros & $\%$ \\
\hline Hombre & 54 & $54,54 \%$ & 3 & $33,33 \%$ & 9 & $47,36 \%$ & 5 & $17,85 \%$ \\
Mujer & 45 & $45,45 \%$ & 6 & $66,66 \%$ & 10 & $52,63 \%$ & 23 & $82,14 \%$ \\
\hline Total & 99 & $100 \%$ & 9 & $100 \%$ & 19 & $100 \%$ & 28 & $100 \%$ \\
\hline \multicolumn{8}{|c|}{$\mathrm{p}=0,002$} \\
\hline
\end{tabular}

Figura 9: Relación entre el servicio de ingreso hospitalario del paciente intoxicado y el sexo

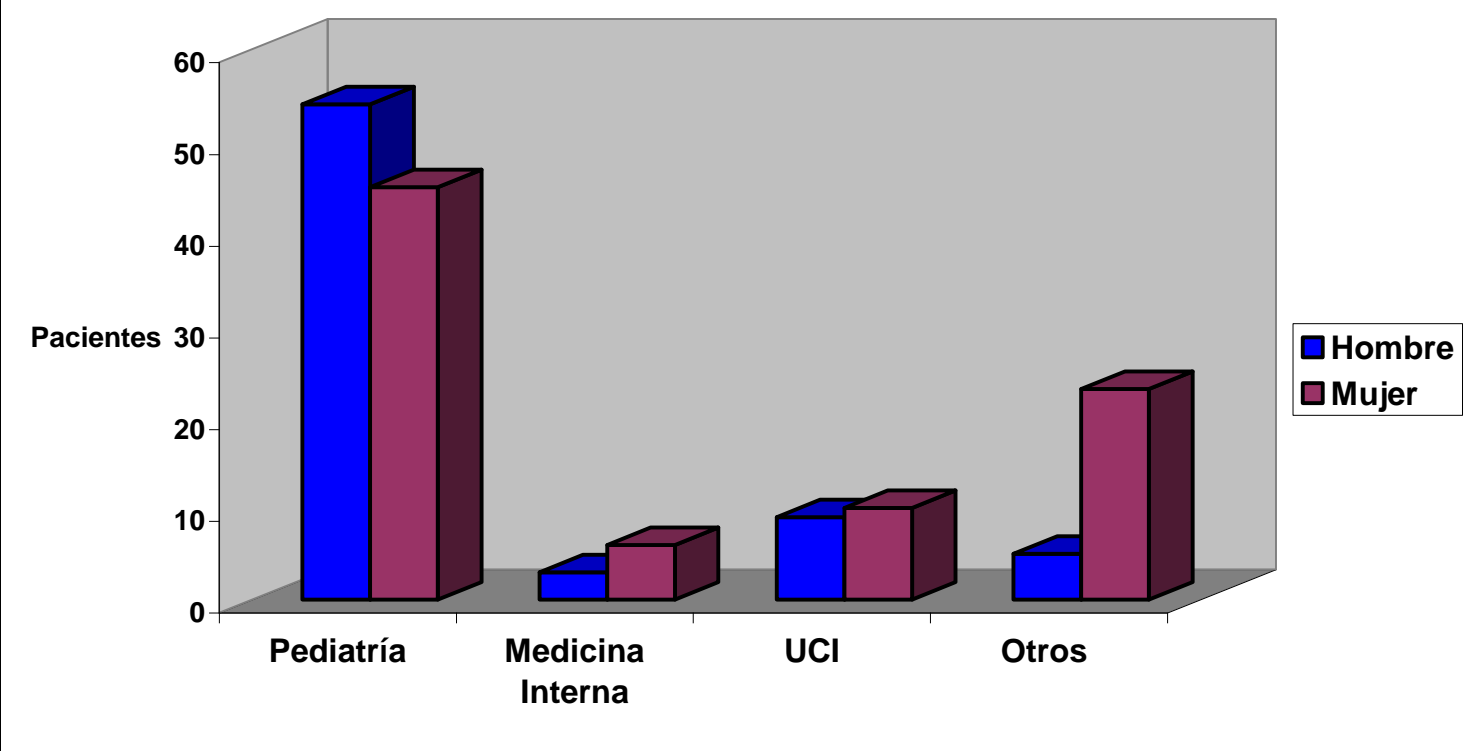

$p=0,002$

3.8. Relación entre el sexo del paciente y el tipo de consulta por la que acude al servicio de urgencias hospitalario en el mismo año de la intoxicación, en años anteriores y en posteriores.

Se evidenciaron diferencias estadísticamente significativas entre el tipo de consulta por la que acude al servicio de urgencias hospitalario nuevamente el año de su intoxicación, teniendo en cuenta su sexo ( $p=$ $0,017)$ Tabla 34. 
Tabla 34: Relación entre el sexo y el tipo de consulta por la que acude al servicio de urgencias hospitalario el mismo año de la intoxicación

\begin{tabular}{|c|c|c|c|c|c|c|c|c|c|c|c|c|c|c|c|c|c|c|c|c|}
\hline & $\begin{array}{c}\text { No } \\
\text { consult } \\
\text { a }\end{array}$ & $\%$ & $\begin{array}{c}\text { Gene } \\
\text { ral }\end{array}$ & $\%$ & $\begin{array}{l}T \\
r\end{array}$ & $\%$ & $\begin{array}{l}0 \\
f\end{array}$ & $\%$ & $\begin{array}{c}0 \\
\mathrm{RL}\end{array}$ & $\%$ & $\begin{array}{l}\text { Gi } \\
\text { ne }\end{array}$ & $\%$ & $\begin{array}{c}\text { Int } \\
x\end{array}$ & $\%$ & $\begin{array}{c}\text { Ps } \\
q\end{array}$ & $\%$ & $\begin{array}{l}\text { Otr } \\
\text { as }\end{array}$ & $\%$ & $\begin{array}{c}\text { Ingre } \\
\text { so }\end{array}$ & $\%$ \\
\hline Hom & & 51, & & 60 , & 3 & 66, & & & & 28 & & & & & & 10 & & 10 & & 10 \\
\hline bre & 383 & 61 & 92 & 52 & 4 & & 1 & 25 & 2 & 57 & 0 & 0 & 14 & 50 & 3 & 0 & 3 & 0 & 2 & 0 \\
\hline $\begin{array}{c}\text { Muje } \\
r\end{array}$ & 359 & $\begin{array}{l}48 \\
38\end{array}$ & 60 & $\begin{array}{l}39 \\
47\end{array}$ & $\begin{array}{l}1 \\
7\end{array}$ & $\begin{array}{c}33 \\
33\end{array}$ & 3 & 75 & 5 & $\begin{array}{l}71, \\
42\end{array}$ & 2 & $\begin{array}{c}10 \\
0\end{array}$ & 14 & 50 & 0 & 0 & 0 & 0 & 0 & 0 \\
\hline & & & & & 5 & & & 10 & & & & 10 & & 10 & & 10 & & 10 & & 10 \\
\hline & 742 & 100 & 152 & 100 & 1 & 100 & 4 & 0 & 7 & 100 & 2 & 0 & 28 & 0 & 3 & 0 & 3 & 0 & 2 & 0 \\
\hline \multicolumn{21}{|c|}{$p=0,017$} \\
\hline
\end{tabular}

Nota: Tr: Traumatología, Of: oftalmología, ORL: otorrinolaringología, Gine: ginecología; Intx: Intoxicación, Psq: Psiquiatría.

\subsection{Relación entre el sexo y el número de consultas ambulatorias} solicitadas desde su centro de salud.

Se evidenciaron diferencias estadísticamente significativas entre el número de consultas ambulatorias solicitadas desde el centro del salud teniendo en cuenta su sexo $(\mathbf{p}=\mathbf{0 , 0 0 7})$ Tabla 35 , figura 10 .

La media en hombres es de 1,49 +/- 1,32 consultas; $y$ en el caso de las mujeres es de $1,73+/-1,43$ consultas.

Tabla 35: Relación entre el sexo y el número de consultas ambulatorias solicitadas desde su centro de salud

\begin{tabular}{|c|c|c|c|c|c|c|}
\hline & 0 & 1 & 2 & 3 & 4 & Total \\
\hline Hombre & 160 & 125 & 116 & 70 & 54 & 525 \\
\hline Mujer & 128 & 87 & 96 & 70 & 75 & 456 \\
\hline Total & 288 & 212 & 212 & 140 & 129 & 981 \\
\hline \multicolumn{7}{|c|}{$p=0,007$} \\
\hline
\end{tabular}




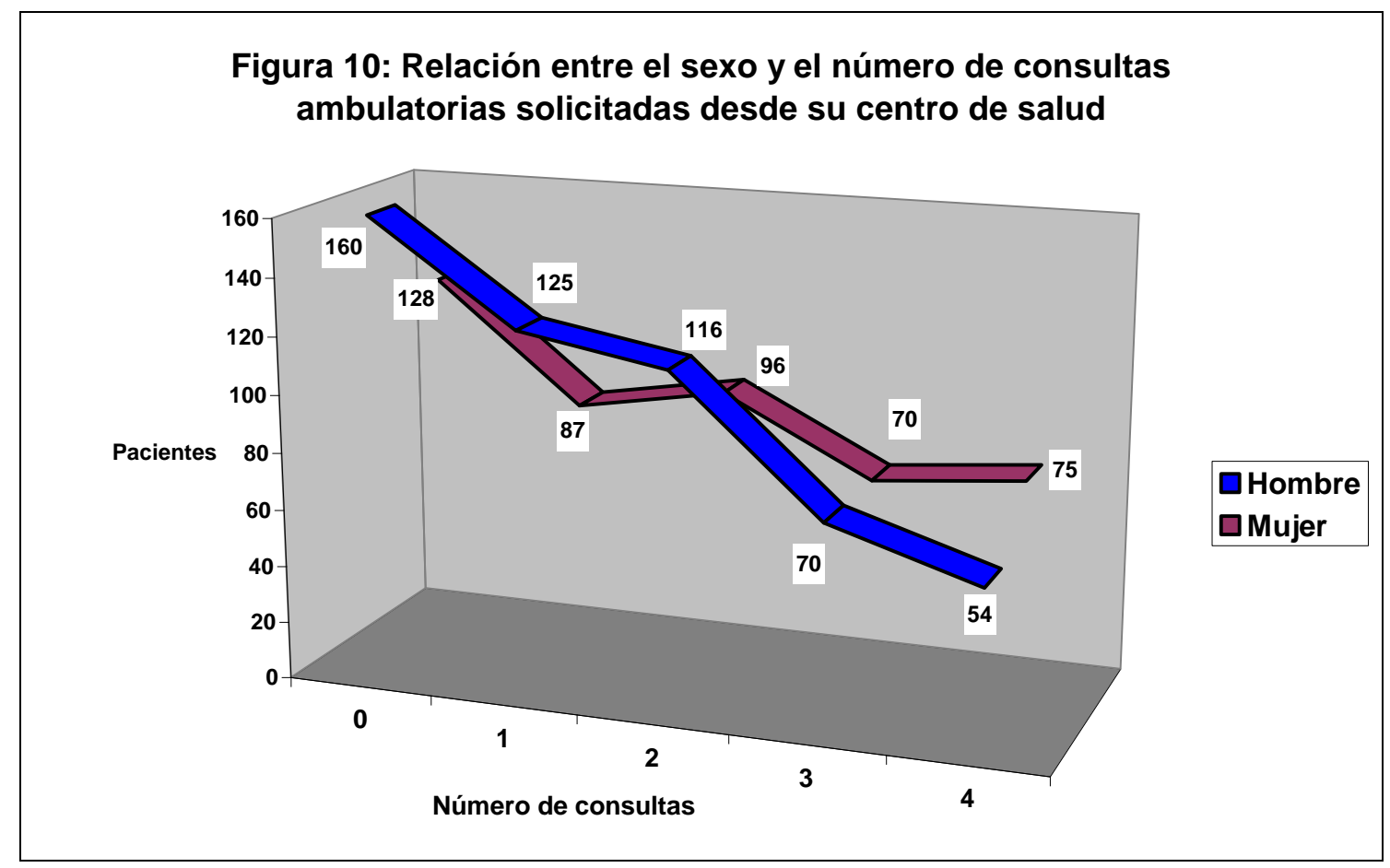

$p=0,007$

3.10. Relación entre el sexo y la necesidad de realizar una interconsulta a la unidad de Psiquiatría durante su estancia en el servicio de urgencias hospitalario.

Se evidenciaron diferencias estadísticamente significativas entre la necesidad de interconsulta con el servicio de Psiquiatria durante la estancia en el servicio de urgencias hospitalario del paciente intoxicado teniendo en cuenta su sexo $(\mathbf{p}=\mathbf{0 , 0 0 0 1 )}$ Tabla 36 .

Tabla 36: Relación entre el sexo del paciente intoxicado y la necesidad de interconsulta al servicio de Psiquiatría durante su estancia en el servicio de urgencias hospitalario

\begin{tabular}{|c|cccc|c|}
\hline & NO & $\%$ & SI & $\%$ & Total \\
\hline Hombre & 222 & $58,88 \%$ & 7 & $18,91 \%$ & 229 \\
Mujer & 155 & $41,11 \%$ & 30 & $81,08 \%$ & 185 \\
\hline Total & 377 & $100 \%$ & 37 & $100 \%$ & 414 \\
\hline \multicolumn{7}{|c|}{$\mathrm{p}=0,0001$} \\
\hline
\end{tabular}




\section{$\underline{4 . D i s t r i b u c i o ́ n ~ p o r ~ e d a d e s ~ d e ~ l a s ~ i n t o x i c a c i o n e s ~ e n ~ e l ~ s e r v i c i o ~ d e ~}$}

\section{urgencias hospitalario.}

Podemos encontrar pacientes de todas las edades entre 0 meses y 17,9 años.

La media de edad corresponde a 10,15 +/- 6,43 años.

La mediana de edad corresponde a 14 años, y la moda a 16 años.

Tabla 37, figura 11.

Tabla 37: Distribución por edades

\begin{tabular}{|ccc|}
\hline Edad en años & Frecuencia & Porcentaje \\
\hline$<1$ & 15 & 1,50 \\
1 & 112 & 11,30 \\
2 & 110 & 11,10 \\
3 & 73 & 7,30 \\
4 & 35 & 3,50 \\
5 & 22 & 2,20 \\
6 & 10 & 1,00 \\
7 & 12 & 1,20 \\
8 & 10 & 1,00 \\
9 & 7 & 0,70 \\
10 & 5 & 0,50 \\
11 & 9 & 0,90 \\
12 & 14 & 1,40 \\
13 & 33 & 3,30 \\
14 & 88 & 8,90 \\
15 & 139 & 14,00 \\
16 & 162 & 16,30 \\
17 & 138 & 13,90 \\
\hline
\end{tabular}




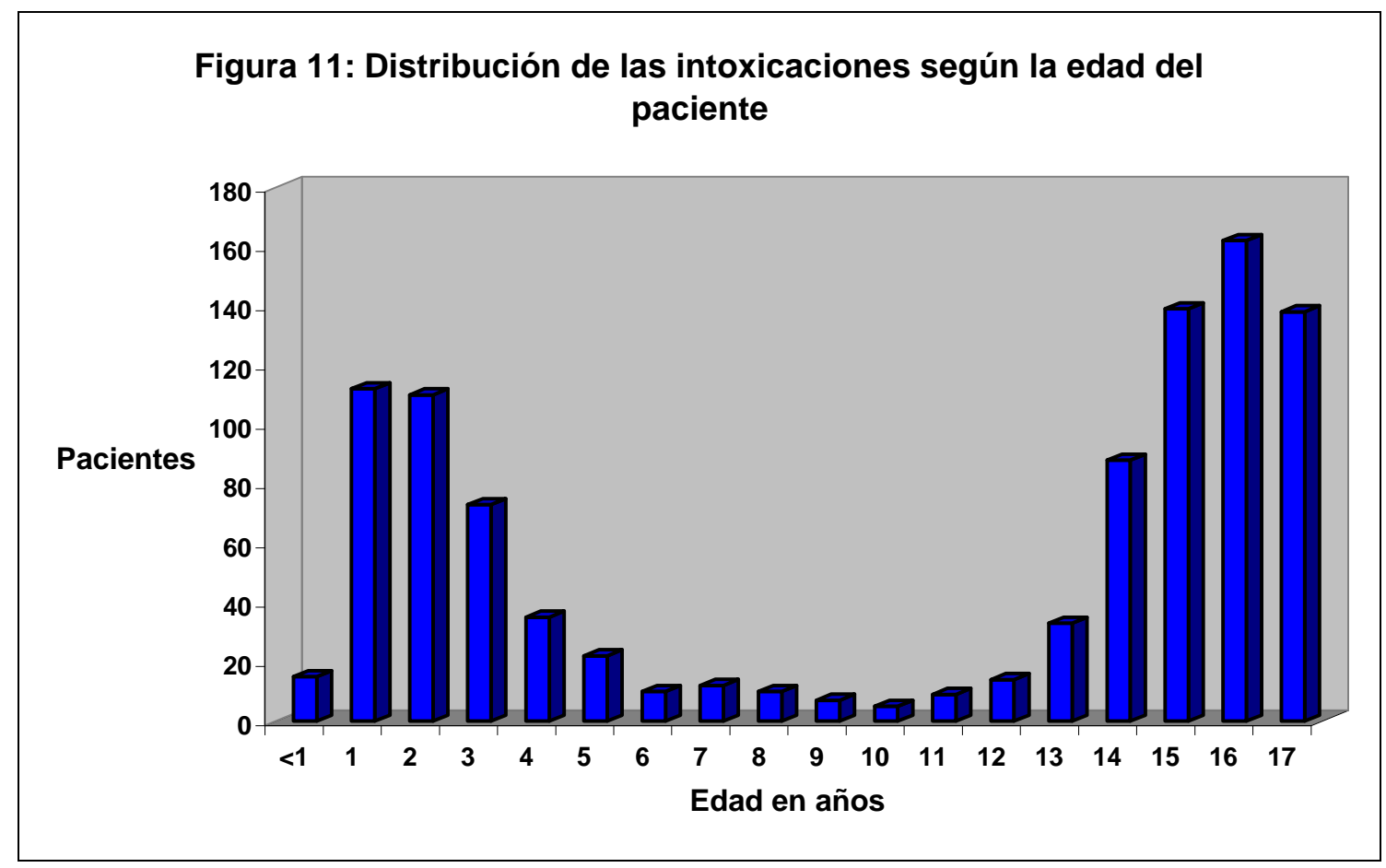

\subsection{Relación entre la edad del paciente y los diferentes diagnósticos de} intoxicación efectuados en el servicio de urgencias hospitalario.

Se recogen los diagnósticos de las diferentes intoxicaciones que acuden a urgencias y la edad media de cada uno de ellos. (Tabla 38, figura 12)

Tabla 38: Relación entre los diagnósticos y la edad media de cada uno

\begin{tabular}{|ccccc|}
\hline Diagnóstico & Edad media & Desviación típica & Mediana & $\mathbf{N}$ \\
\hline Cocaína & 17 & 0 & 17 & 1 \\
Cannabis & 15,46 & 1,12 & 16 & 13 \\
Etílica & 15,4 & 1,65 & 16 & 434 \\
Otras intoxicaciones & 15 & 2,44 & 16 & 9 \\
CO & 9,6 & 5,09 & 10,5 & 80 \\
No especificadas & 6,08 & 6,48 & 3 & 25 \\
Medicamentosa & 5,89 & 5,74 & 3 & 267 \\
Productos industriales & 5,5 & 5,39 & 3,5 & 6 \\
Bebidas estimulantes & 5,04 & 5,05 & 3 & 25 \\
Insecticidas & 3,95 & 3,73 & 3 & 21 \\
Setas & 3,8 & 4,82 & 1,5 & 10 \\
Productos de limpieza & 3,01 & 3,92 & 1 & 81 \\
Productos cosméticos & 1,73 & 1,42 & 1 & 22 \\
\hline
\end{tabular}




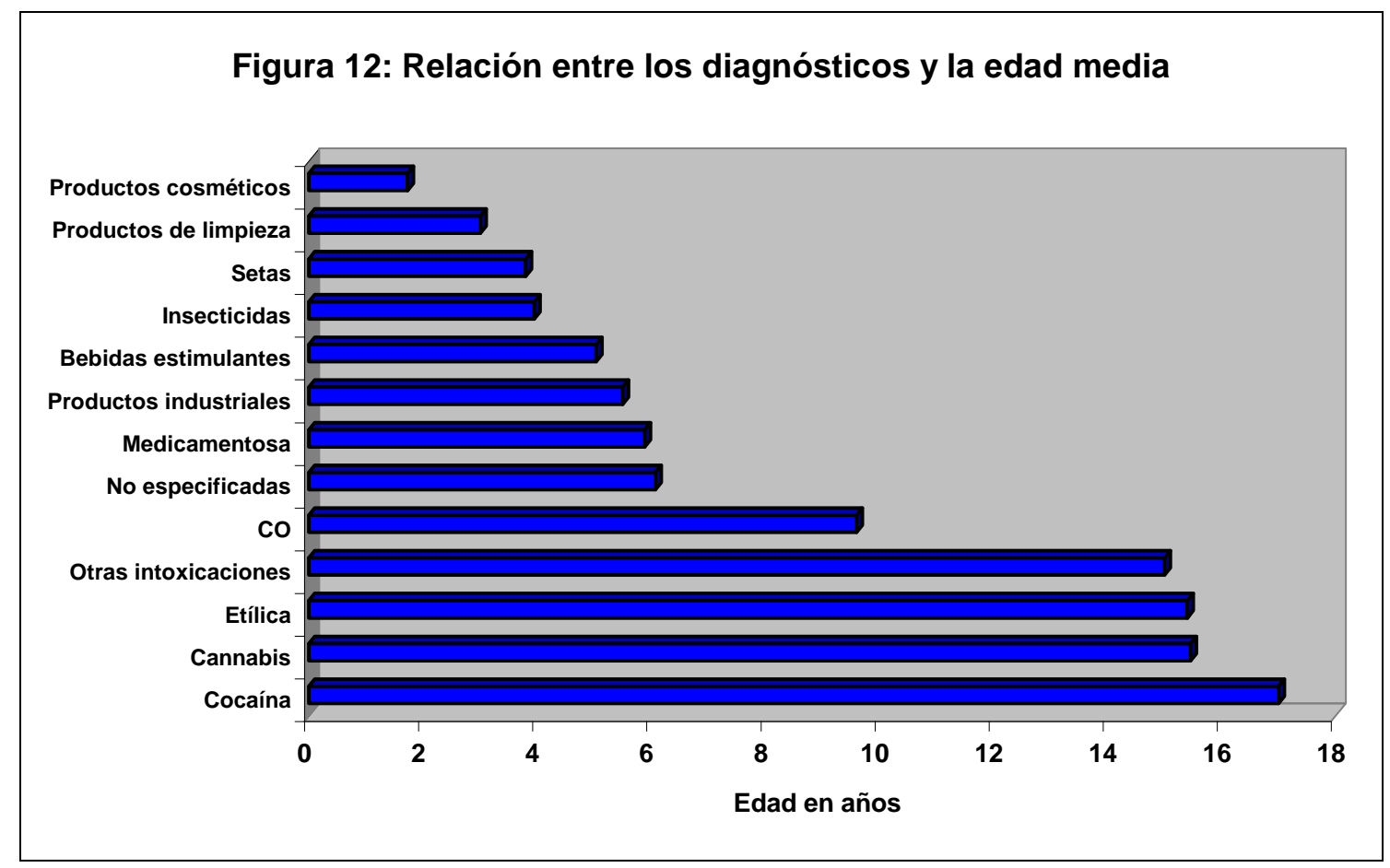

En la tabla 39 se muestran los diferentes diagnósticos de urgencias según los grupos de edad que los presentan.

Tabla 39: Distribución por grupos de edad de los diferentes diagnósticos de los pacientes que acuden al servicio de urgencias hospitalario por intoxicaciones

\begin{tabular}{|c|cccccc|}
\hline & \multicolumn{5}{c}{$\begin{array}{c}\text { Intoxicación } \\
\text { por }\end{array}$} \\
$\begin{array}{c}\text { Intoxicación } \\
\text { etílica }\end{array}$ & $\begin{array}{c}\text { Intoxicación } \\
\text { por cannabis }\end{array}$ & $\begin{array}{c}\text { Intoxic } \\
\text { ción por } \\
\text { cocaína }\end{array}$ & $\begin{array}{c}\text { Intoxicación } \\
\text { ación } \\
\text { por Co }\end{array}$ & $\begin{array}{c}\text { medicamento } \\
\text { sa }\end{array}$ & $\begin{array}{c}\text { Insecticidas y } \\
\text { raticidas }\end{array}$ \\
\hline $\begin{array}{c}\text { Menor o } \\
\text { igual 7 } \\
\text { años }\end{array}$ & 3 & 0 & 0 & 31 & 193 & 18 \\
$\begin{array}{c}8-13 \\
\text { años }\end{array}$ & 20 & 1 & 0 & 28 & 19 & 2 \\
$\begin{array}{c}\text { Mayor o } \\
\text { igual 14 } \\
\text { años }\end{array}$ & 411 & 12 & 1 & 21 & 55 & 1 \\
\hline
\end{tabular}

\begin{tabular}{|c|c|c|c|c|c|c|}
\hline $\begin{array}{c}\text { Intoxicación } \\
\text { por } \\
\text { productos } \\
\text { de limpieza }\end{array}$ & $\begin{array}{l}\text { Intoxicación } \\
\text { por productos } \\
\text { de cosmética }\end{array}$ & $\begin{array}{l}\text { Intoxica } \\
\text { ción por } \\
\text { otros } \\
\text { product } \\
\text { os }\end{array}$ & $\begin{array}{l}\text { Intoxic } \\
\text { ación } \\
\text { por } \\
\text { setas }\end{array}$ & $\begin{array}{c}\text { Intoxicación } \\
\text { por } \\
\text { productos } \\
\text { industriales }\end{array}$ & $\begin{array}{l}\text { Intoxicación } \\
\text { por productos } \\
\text { estimulantes }\end{array}$ & $\begin{array}{c}\text { No } \\
\text { especific } \\
\text { ado } \\
\end{array}$ \\
\hline 72 & 22 & 8 & 4 & 20 & 0 & 18 \\
\hline 4 & 0 & 1 & 1 & 1 & 1 & 0 \\
\hline 5 & 0 & 1 & 1 & 4 & 8 & 7 \\
\hline
\end{tabular}


Si se divide a la población en mayores o iguales de 14 años, que son los pacientes atendidos habitualmente en los servicios de urgencias generales, y los menores de 14 años atendidos en Urgencias pediátricas; se evidencian diferencias estadísticamente significativas en los diagnósticos de los pacientes, teniendo en cuenta esta división de edad $(p=0,0001)$

Si la división en grupos de edad se establece en los 7 años, como en muchos trabajos de epidemiológicos españoles, se evidencian diferencias estadísticamente significativas entre el diagnóstico en urgencias de los pacientes, teniendo en cuenta esta división de edad $(p=0,0001)$

\subsection{Relación entre la edad y el número de sustancias implicadas en la intoxicación.}

Se evidenciaron diferencias estadísticamente significativas entre el número de sustancias implicadas en la intoxicación en el servicio de urgencias hospitalario teniendo en cuenta su edad $(p=0,001)$. Tabla 40 , figura 13 .

Tabla 40: Relación entre la edad y el número de sustancias implicadas en la intoxicación

\begin{tabular}{|cccc|}
\hline Número de Sustancias & N & Media & Desviación típica \\
\hline 1 & 969 & 10,03 & 6,44 \\
Más de 1 & 25 & 14,52 & 4,08 \\
& & & \\
& & &
\end{tabular}




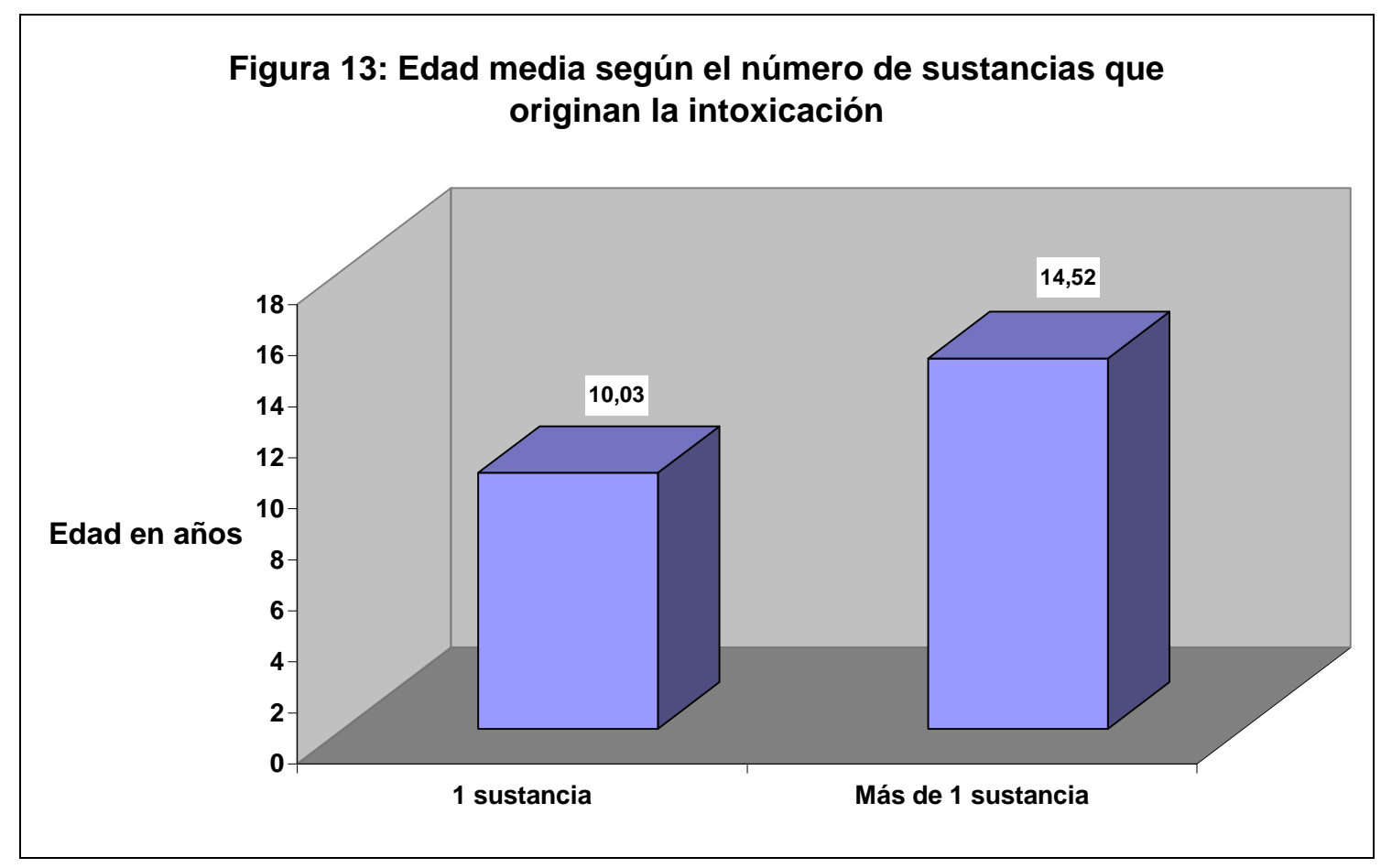

$$
p=0,001
$$

En la tabla 41 se muestra el número de sustancias implicadas en la intoxicación distribuidas por grupos de edad.

Tabla 41: Número de sustancias implicadas en la intoxicación distribuidas por grupo de edad

\begin{tabular}{|c|ccc|}
\hline & 1 & 2 & 3 \\
\hline Menor o igual 7 años & 387 & 1 & 1 \\
8-13 años & 76 & 2 & 0 \\
Mayor o igual 14 años & 506 & 17 & 4 \\
\hline
\end{tabular}

\subsection{Relación entre la edad y el tipo de sustancia implicada en la intoxicación.}

Se muestra la edad media de los pacientes intoxicados por las diferentes sustancias que se muestran a continuación. (Tabla 42, figura 14) 
Tabla 42: Relación entre la edad y el tipo de sustancia implicada en la intoxicación

\begin{tabular}{|cccc|}
\hline Sustancia & Edad media & Desviación típica & $\mathrm{N}$ \\
\hline Cocaína & 17 & 0 & 1 \\
Cannabis & 15,46 & 1,12 & 13 \\
Alcohol & 15,4 & 1,65 & 434 \\
Anfetaminas & 14,67 & 2,87 & 6 \\
CO & 9,6 & 5,09 & 80 \\
AlNEs & 8,48 & 6,9 & 21 \\
Benzodiacepinas & 8,11 & 6,84 & 27 \\
\hline Antidepresivos & 6,67 & 6,37 & 6 \\
Medicación habitual & 5,92 & 5,62 & 150 \\
Productos industriales & 5,5 & 5,39 & 6 \\
No especiificado & 3,95 & 3,73 & 21 \\
Insecticidas & 3,95 & 3,73 & 21 \\
\hline Setas & 3,8 & 4,82 & 10 \\
Paracetamol & 3,09 & 3,19 & 55 \\
Productos de limpieza & 3,01 & 3,92 & 81 \\
Productos cosméticos & 1,73 & 1,42 & 22 \\
\hline
\end{tabular}

\section{Figura 14: Relación entre la edad y la sustancia que causa la intoxicación}

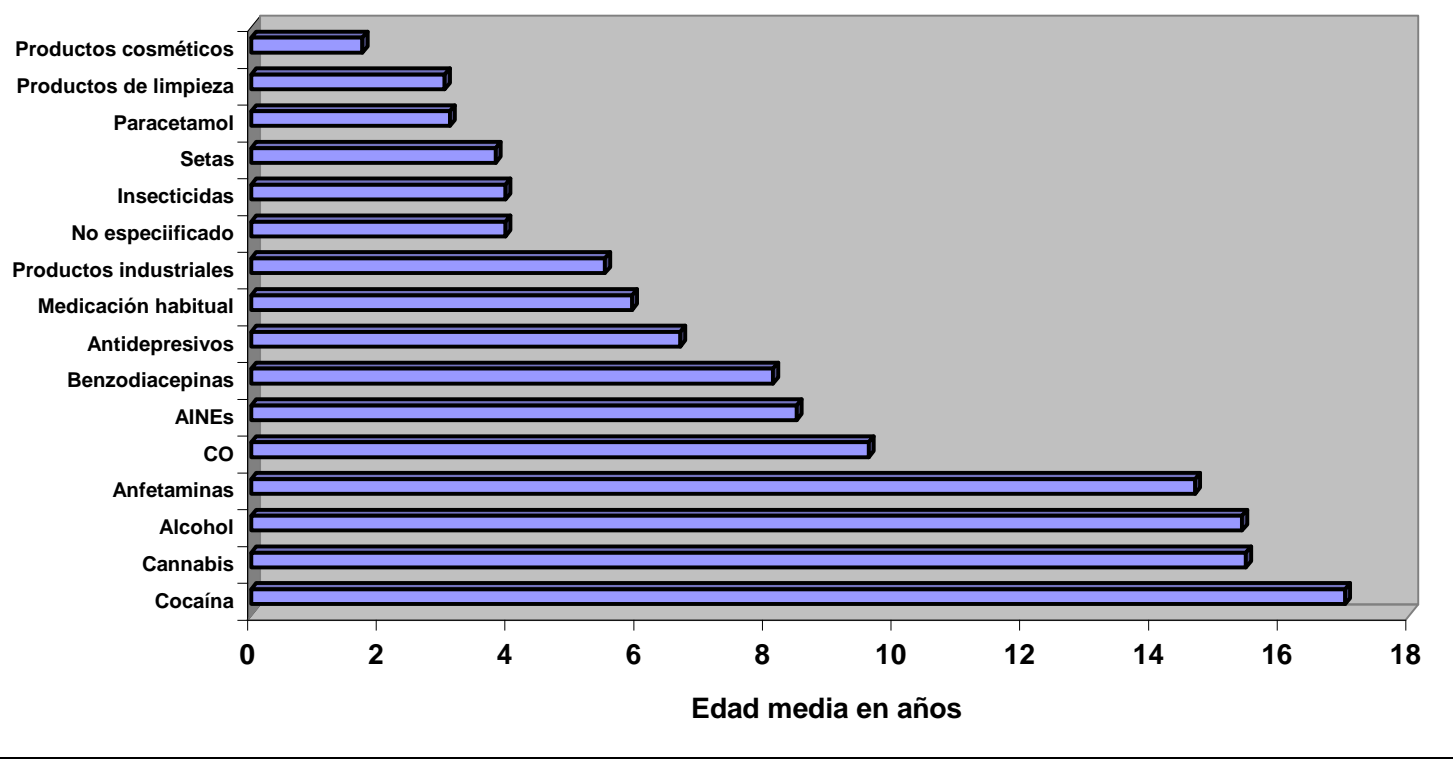


En la tabla 43, se muestra el tipo de sustancia implicada en la intoxicación distribuidas por grupos de edad.

Tabla 43: Tipo de sustancia implicada en la intoxicación distribuidos por grupos de edad

\begin{tabular}{|ccccccccc|}
\hline & Alcohol & Cocaína & $\begin{array}{c}\text { Anfetamin } \\
\text { as }\end{array}$ & Hachís & $\begin{array}{c}\text { Bezodiace } \\
\text { pinas }\end{array}$ & $\begin{array}{c}\text { Antidepre } \\
\text { sivos }\end{array}$ & $\begin{array}{c}\text { Paracet } \\
\text { amol }\end{array}$ & AINEs \\
\hline $\begin{array}{c}\text { Menor o } \\
\text { igual 7 } \\
\text { años }\end{array}$ & 3 & 0 & 0 & 0 & 15 & 4 & 52 & 12 \\
$\begin{array}{c}8-13 \text { años } \\
\begin{array}{c}\text { Mayor o } \\
\text { igual 14 } \\
\text { años }\end{array}\end{array}$ & 411 & 0 & 1 & 1 & 2 & 1 & 1 & 0 \\
\hline
\end{tabular}

\begin{tabular}{|cccccccc|}
\hline $\begin{array}{c}\text { Medicac } \\
\text { ión } \\
\text { habitual }\end{array}$ & Setas & $\begin{array}{c}\text { Productos } \\
\text { de } \\
\text { cosmética }\end{array}$ & $\begin{array}{c}\text { Producto } \\
\text { s de } \\
\text { limpieza }\end{array}$ & $\begin{array}{c}\text { Productos } \\
\text { industrial } \\
\text { es }\end{array}$ & $\begin{array}{c}\text { Monóxido } \\
\text { de } \\
\text { carbono }\end{array}$ & $\begin{array}{c}\text { No } \\
\text { especifi } \\
\text { cado }\end{array}$ & $\begin{array}{c}\text { Insectici } \\
\text { das }\end{array}$ \\
\hline 106 & 4 & 24 & 69 & 23 & 31 & 28 & 18 \\
15 & 1 & 0 & 4 & 1 & 28 & 1 & 2 \\
29 & 1 & 2 & 5 & 3 & 21 & 14 & 1 \\
\hline
\end{tabular}

Si se divide a la población en mayores o iguales de 14 años, que son los pacientes atendidos habitualmente en los servicios de urgencias generales, y los menores de 14 años atendidos en Urgencias pediátricas; se evidencian diferencias estadísticamente significativas entre las sustancias que ocasionan las intoxicaciones de los pacientes, teniendo en cuenta esta división de edad $(p=0,0001)$

Si la división en grupos de edad se establece en los 7 años, como en muchos trabajos de epidemiológicos españoles, se evidencian diferencias estadísticamente significativas entre las sustancias que ocasionan las intoxicaciones de los pacientes, teniendo en cuenta esta división de edad ( $\mathbf{p}=$ 0,0001)

\subsection{Relación entre la edad de los pacientes intoxicados y el año de su intoxicación.}

La edad media de los pacientes atendidos cada año en urgencias es la que se visualiza en la tabla 44, figura 15. 
Tabla 44: Edad media de los pacientes atendidos cada año

\begin{tabular}{|c|c|c|c|c|c|c|c|c|c|c|}
\hline Edad & 2001 & 2002 & 2003 & 2004 & 2005 & 2006 & 2007 & 2008 & 2009 & 2010 \\
\hline 0 & 4 & 2 & 1 & 0 & 4 & 1 & 0 & 1 & 0 & 2 \\
\hline 1 & 7 & 13 & 14 & 7 & 7 & 6 & 23 & 9 & 4 & 22 \\
\hline 2 & 13 & 15 & 17 & 8 & 4 & 11 & 15 & 8 & 15 & 4 \\
\hline 3 & 6 & 9 & 9 & 1 & 6 & 6 & 15 & 7 & 10 & 4 \\
\hline 4 & 1 & 1 & 5 & 2 & 5 & 5 & 1 & 6 & 5 & 4 \\
\hline 5 & 2 & 4 & 2 & 0 & 3 & 3 & 2 & 1 & 4 & 1 \\
\hline 6 & 0 & 3 & 1 & 0 & 1 & 0 & 1 & 1 & 2 & 1 \\
\hline 7 & 2 & 1 & 1 & 0 & 0 & 2 & 0 & 1 & 3 & 2 \\
\hline 8 & 1 & 3 & 0 & 0 & 0 & 1 & 3 & 1 & 1 & 0 \\
\hline 9 & 0 & 1 & 1 & 1 & 1 & 0 & 0 & 2 & 1 & 0 \\
\hline 10 & 0 & 0 & 0 & 0 & 1 & 3 & 1 & 0 & 0 & 0 \\
\hline 11 & 1 & 3 & 2 & 1 & 0 & 0 & 2 & 0 & 0 & 0 \\
\hline 12 & 1 & 3 & 1 & 1 & 0 & 3 & 0 & 1 & 2 & 2 \\
\hline 13 & 1 & 1 & 5 & 2 & 0 & 1 & 5 & 3 & 8 & 7 \\
\hline 14 & 5 & 3 & 6 & 6 & 8 & 7 & 11 & 17 & 11 & 14 \\
\hline 15 & 21 & 8 & 10 & 10 & 6 & 11 & 21 & 16 & 17 & 19 \\
\hline 16 & 16 & 10 & 13 & 7 & 7 & 21 & 30 & 25 & 16 & 17 \\
\hline \multirow[t]{2}{*}{17} & 6 & 12 & 15 & 9 & 12 & 11 & 19 & 20 & 20 & 14 \\
\hline & 87 & 92 & 103 & 55 & 65 & 92 & 149 & 119 & 119 & 113 \\
\hline & & & & & & & 10,1 & 11,6 & 10,8 & 10,5 \\
\hline Edad media & 9,95 & 8,26 & 8,96 & 10,75 & 9,4 & 10,52 & 4 & 2 & 2 & 2 \\
\hline $\begin{array}{l}\text { Desviación } \\
\text { estándar }\end{array}$ & 6,55 & 6,46 & 6,64 & 6,43 & 6,71 & 6,23 & 6,59 & 5,98 & 6,02 & 6,43 \\
\hline
\end{tabular}


Figura 15: Edad media de los pacientes atendidos en el servicio de urgencias hospitalario cada año

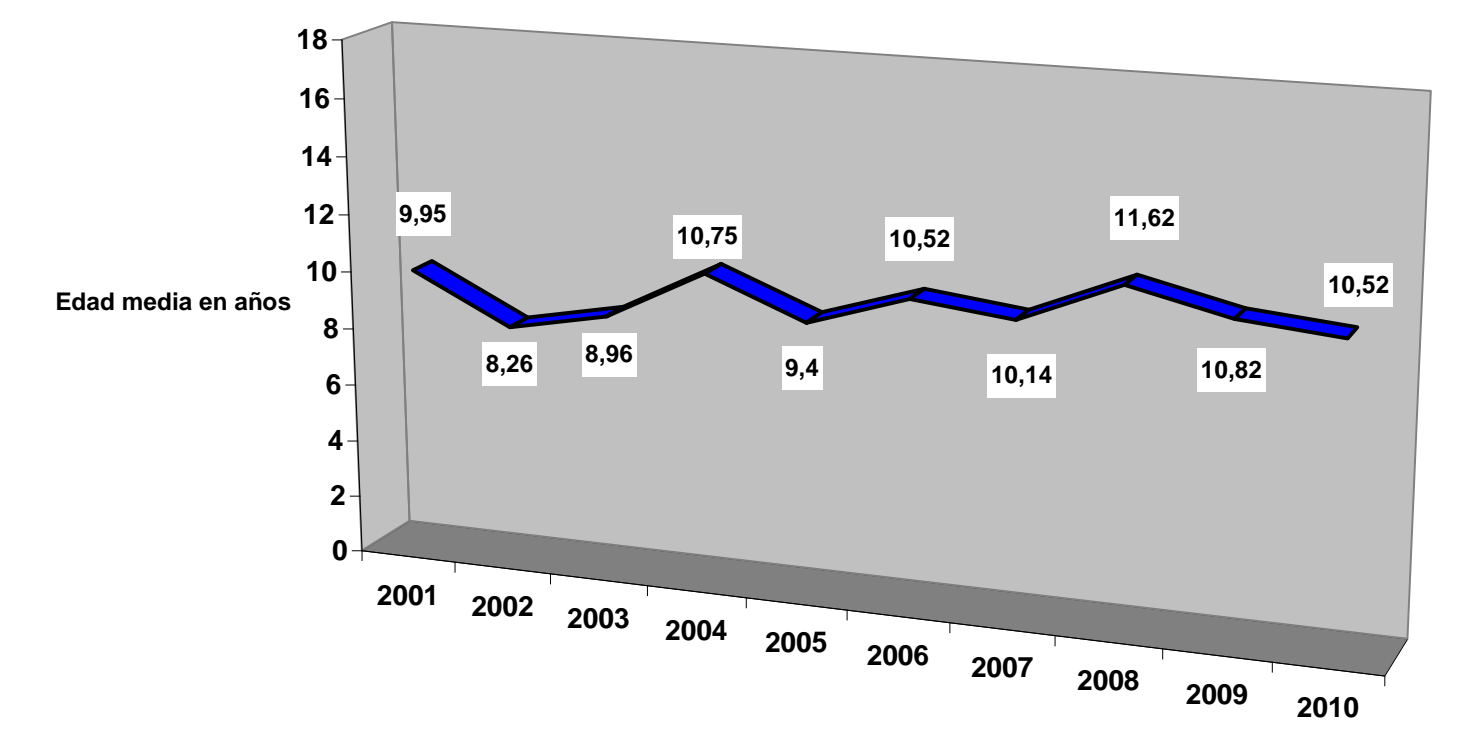

La edad media de atención de los pacientes intoxicados a través de los años es de $10,15+/-6,43$ años.

Se evidenciaron diferencias estadísticamente significativas entre el año de atención en urgencias teniendo en cuenta su edad $(p=0,001)$

Si se divide a la población en mayores o iguales de 14 años, que son los pacientes atendidos habitualmente en los servicios de urgencias generales, y los menores de 14 años atendidos en Urgencias pediátricas; se evidencian diferencias estadísticamente significativas entre el año de atención de los pacientes, teniendo en cuenta esta división de edad $(p=0,004)$

Si la división en grupos de edad se establece en los 7 años, como en muchos trabajos de epidemiológicos españoles, se evidencian diferencias estadísticamente significativas entre el año de atención de los pacientes, teniendo en cuenta esta división de edad $(p=0,002)$ 
Tabla 45: Distribución por años de atención en el servicio de urgencias hospitalario de los diferentes grupos de edad

\begin{tabular}{|c|c|c|c|c|c|c|c|c|c|c|}
\hline & 2001 & $\%$ & 2002 & $\%$ & 2003 & $\%$ & 2004 & $\%$ & 2005 & $\%$ \\
\hline \multirow{2}{*}{$\begin{array}{c}\text { Menor o } \\
\text { igual } 7 \text { años }\end{array}$} & & 40,22 & & & & 48,54 & & 32,72 & & 46,15 \\
\hline & 35 & $\%$ & 48 & $52,17 \%$ & 50 & $\%$ & 18 & $\%$ & 30 & $\%$ \\
\hline \multirow[b]{2}{*}{8 -13 años } & & 4,59 & & & & & & & & \\
\hline & 4 & $\%$ & 11 & $11,95 \%$ & 9 & $8,73 \%$ & 5 & $9,09 \%$ & 2 & $3,07 \%$ \\
\hline \multirow{2}{*}{$\begin{array}{c}\text { Mayor o } \\
\text { igual } 14 \text { años }\end{array}$} & & 55,17 & & & & 42,71 & & 58,18 & & 50,76 \\
\hline & 48 & $\%$ & 33 & $35,82 \%$ & 44 & $\%$ & 32 & $\%$ & 33 & $\%$ \\
\hline Total & 87 & $100 \%$ & 92 & $100 \%$ & 103 & $100 \%$ & 55 & $100 \%$ & 65 & $100 \%$ \\
\hline
\end{tabular}

\begin{tabular}{|c|c|c|c|c|c|c|c|c|c|}
\hline 2006 & $\%$ & 2007 & $\%$ & 2008 & $\%$ & 2009 & $\%$ & 2010 & $\%$ \\
\hline & 36,95 & & & & 28,57 & & 36,13 & & 35,39 \\
\hline 34 & $\%$ & 57 & $38,25 \%$ & 34 & $\%$ & 43 & $\%$ & 40 & $\%$ \\
\hline & 8,69 & & & & & & 10,08 & & \\
\hline 8 & $\%$ & 11 & $7,38 \%$ & 7 & $5,88 \%$ & 12 & $\%$ & 9 & $7,96 \%$ \\
\hline & 54,34 & & & & 65,54 & & 53,78 & & 56,63 \\
\hline 50 & $\%$ & 81 & $54,36 \%$ & 78 & $\%$ & 64 & $\%$ & 64 & $\%$ \\
\hline 92 & $100 \%$ & 149 & $100 \%$ & 119 & $100 \%$ & 119 & $100 \%$ & 113 & $100 \%$ \\
\hline
\end{tabular}

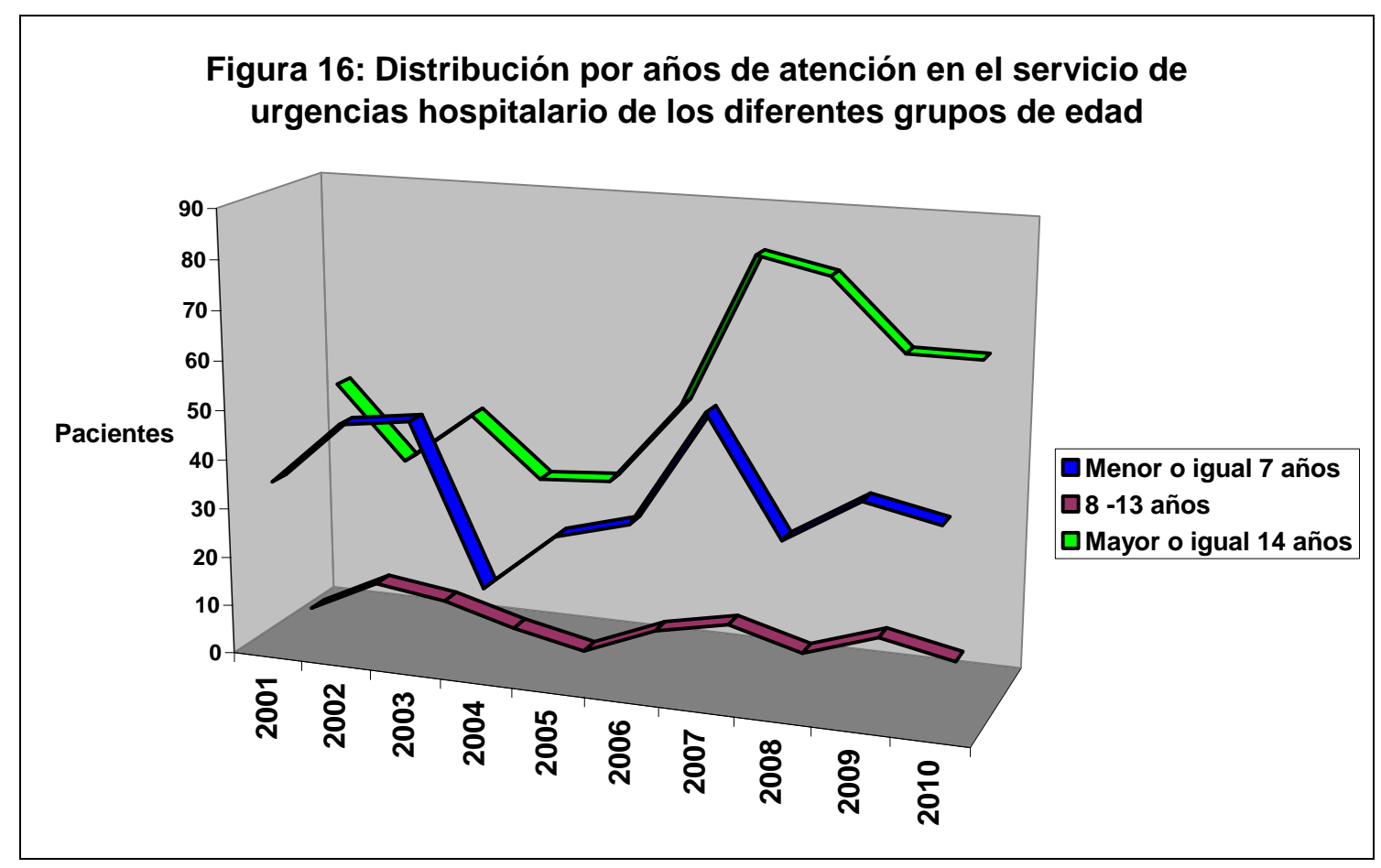




\subsection{Relación entre la edad y el mes de atención en el servicio de urgencias hospitalario.}

La edad media de los pacientes atendidos cada mes es la que se muestra en la tabla 46 y figura 17.

Tabla 46: Relación entre la edad y el mes de atención en el servicio de urgencias hospitalario.

\begin{tabular}{|c|cccccccccccc|}
\hline & Enero & $\begin{array}{c}\text { Febrer } \\
\text { o }\end{array}$ & $\begin{array}{c}\text { Mar } \\
\text { zo }\end{array}$ & $\begin{array}{c}\text { Abr } \\
\text { il }\end{array}$ & $\begin{array}{c}\text { Ma } \\
\text { yo }\end{array}$ & $\begin{array}{c}\text { Juni } \\
\text { o }\end{array}$ & $\begin{array}{c}\text { Juli } \\
\text { o }\end{array}$ & $\begin{array}{c}\text { Agos } \\
\text { to }\end{array}$ & $\begin{array}{c}\text { Septiem } \\
\text { bre }\end{array}$ & $\begin{array}{c}\text { Octub } \\
\text { re }\end{array}$ & $\begin{array}{c}\text { Noviem } \\
\text { bre }\end{array}$ & $\begin{array}{c}\text { Diciem } \\
\text { bre }\end{array}$ \\
\hline Total & 66 & 82 & 98 & 83 & 87 & 96 & 76 & 69 & 82 & 81 & 81 & 93 \\
\hline $\begin{array}{c}\text { Media } \\
\text { edad }\end{array}$ & 9,74 & 9,48 & 7 & 9 & 9,91 & 4 & 2 & 11,1 & 11,85 & 8,64 & 10,3 & 11,34 \\
\hline $\begin{array}{c}\text { Desviaci } \\
\text { ón típica }\end{array}$ & 6,029 & 6,59 & 6,31 & 8 & 6,77 & 7 & 6 & 6,49 & 6,07 & 6,72 & 6,35 & 5,71 \\
\hline
\end{tabular}

Figura 17: Media de edad de los pacientes atendidos cada mes con diagnóstico de intoxicación

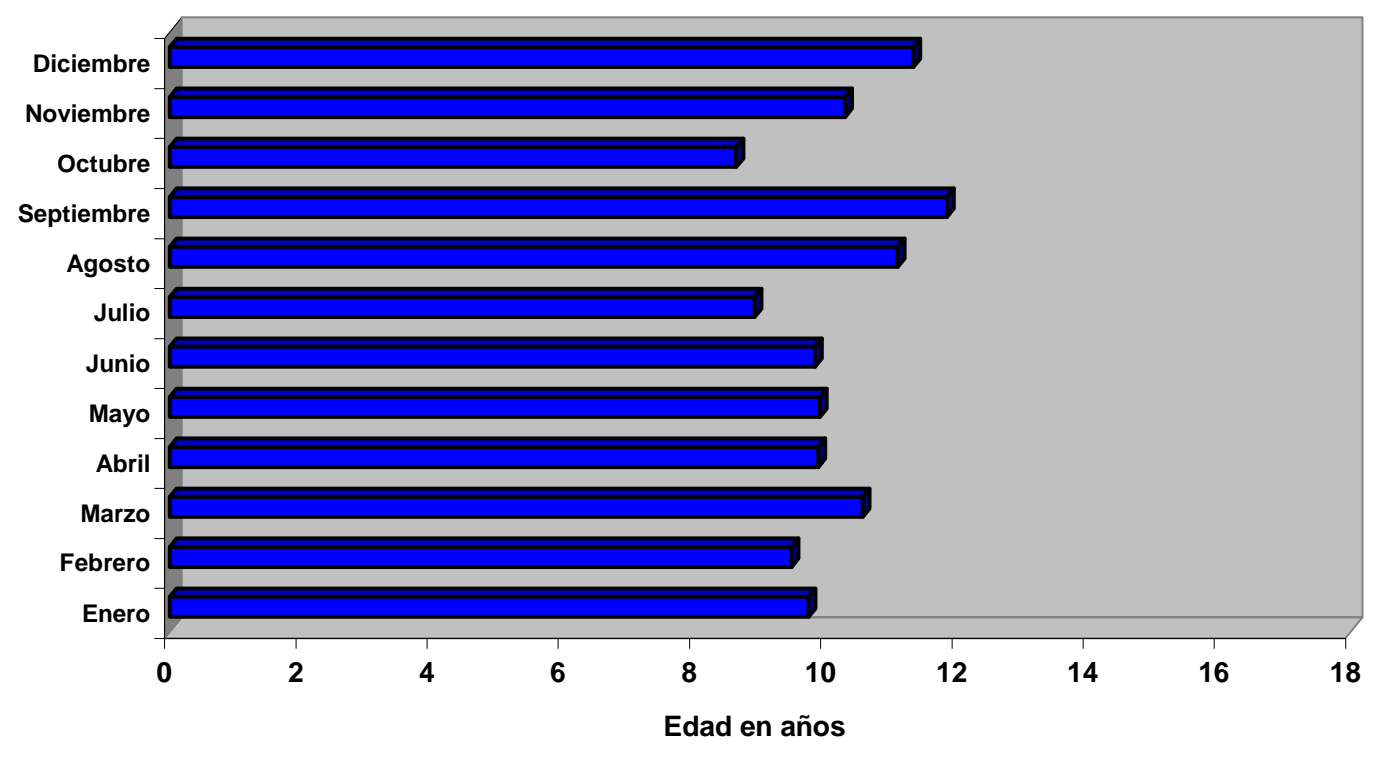

Si se divide a la población en mayores o iguales de 14 años, que son los pacientes atendidos habitualmente en los servicios de urgencias generales, y los menores de 14 años atendidos en Urgencias pediátricas; no se evidencian 
diferencias estadísticamente significativas entre el mes de atención de los pacientes, teniendo en cuenta esta división de edad $(p=0,44)$

Si la división en grupos de edad se establece en los 7 años, como en muchos trabajos de epidemiológicos españoles, no se evidencian diferencias estadísticamente significativas entre el mes de atención de los pacientes, teniendo en cuenta esta división de edad $(p=\mathbf{0 , 2 5 6 )}$

Tabla 47: Distribución por grupos de edades según la atención en el servicio de urgencias hospitalario por meses

\begin{tabular}{|c|c|c|c|c|c|c|c|c|c|c|c|c|}
\hline & $\begin{array}{c}\text { Ene } \\
\text { ro }\end{array}$ & $\%$ & $\begin{array}{c}\text { Febr } \\
\text { ero }\end{array}$ & $\%$ & Marzo & $\%$ & Abril & $\%$ & Mayo & $\%$ & Junio & $\%$ \\
\hline Menor o igual & & 43,9 & & 43,9 & & 34,6 & & 37,3 & & 43,6 & & 40,6 \\
\hline \multirow[t]{3}{*}{7 años } & 29 & $3 \%$ & 36 & $0 \%$ & 34 & $9 \%$ & 31 & $4 \%$ & 38 & $7 \%$ & 39 & $2 \%$ \\
\hline & & 9,09 & & 7,31 & & 12,2 & & 10,8 & & 1,14 & & 4,16 \\
\hline & 6 & $\%$ & 6 & $\%$ & 12 & $4 \%$ & 9 & $4 \%$ & 1 & $\%$ & 4 & $\%$ \\
\hline Mayor o igual & & 46,9 & & 48,7 & & 53,0 & & 51,8 & & 55,1 & & 55,2 \\
\hline 14 años & 31 & $6 \%$ & 40 & $8 \%$ & 52 & $6 \%$ & 43 & $0 \%$ & 48 & $7 \%$ & 53 & $0 \%$ \\
\hline \multirow{11}{*}{ Total } & & 100 & & 100 & & 100 & & 100 & & 100 & & 100 \\
\hline & 66 & $\%$ & 82 & $\%$ & 98 & $\%$ & 83 & $\%$ & 87 & $\%$ & 96 & $\%$ \\
\hline & $\begin{array}{c}\text { Juli } \\
\text { o } \\
\end{array}$ & $\%$ & $\begin{array}{c}\text { Agos } \\
\text { to }\end{array}$ & $\%$ & $\begin{array}{c}\text { Septie } \\
\text { mbre }\end{array}$ & $\%$ & $\begin{array}{c}\text { Octu } \\
\text { bre }\end{array}$ & $\%$ & $\begin{array}{c}\text { Novie } \\
\text { mbre }\end{array}$ & $\%$ & $\begin{array}{c}\text { Diciem } \\
\text { bre }\end{array}$ & $\%$ \\
\hline & & 48,6 & & 34,7 & & 29,2 & & 48,1 & & 37,0 & & 30,1 \\
\hline & 37 & $8 \%$ & 24 & $8 \%$ & 24 & $6 \%$ & 39 & $4 \%$ & 30 & $3 \%$ & 28 & $0 \%$ \\
\hline & & 3,94 & & 4,34 & & & & 12,3 & & 13,5 & & 13,9 \\
\hline & 3 & $\%$ & 3 & $\%$ & 0 & $0 \%$ & 10 & $4 \%$ & 11 & $8 \%$ & 13 & $7 \%$ \\
\hline & & 47,3 & & 60,8 & & 70,7 & & 39,5 & & 49,3 & & 55,9 \\
\hline & 36 & $6 \%$ & 42 & $6 \%$ & 58 & $3 \%$ & 32 & $0 \%$ & 40 & $8 \%$ & 52 & $1 \%$ \\
\hline & & 100 & & 100 & & 100 & & 100 & & 100 & & 100 \\
\hline & 76 & $\%$ & 69 & $\%$ & 82 & $\%$ & 81 & $\%$ & 81 & $\%$ & 93 & $\%$ \\
\hline
\end{tabular}

\subsection{Relación entre la edad y la hora de admisión al servicio de urgencias hospitalario.}

La edad media de admisión en cada hora del día en el servicio de urgencias hospitalario se muestra en la siguiente tabla 48, figura 18. 
Tabla 48: Media de edad admitida cada hora en el servicio de urgencias hospitalario

\begin{tabular}{|c|c|c|c|c|c|c|c|c|c|c|c|c|}
\hline & $\begin{array}{c}00: 00 \\
- \\
00: 59\end{array}$ & $\begin{array}{c}\text { 01:00 } \\
- \\
01: 59\end{array}$ & $\begin{array}{c}02: 00 \\
- \\
02: 59\end{array}$ & $\begin{array}{c}03: 00 \\
- \\
03: 59\end{array}$ & $\begin{array}{c}04: 00 \\
- \\
04: 59\end{array}$ & $\begin{array}{c}05: 00 \\
- \\
05: 59\end{array}$ & $\begin{array}{c}06: 00 \\
- \\
06: 59\end{array}$ & $\begin{array}{c}07: 00 \\
- \\
07: 59\end{array}$ & $\begin{array}{c}08: 00 \\
- \\
08: 59\end{array}$ & $\begin{array}{c}09: 00 \\
- \\
09: 59\end{array}$ & $\begin{array}{c}10: 00 \\
- \\
10: 59\end{array}$ & $\begin{array}{c}11: 00 \\
- \\
11: 59\end{array}$ \\
\hline $\begin{array}{c}\text { Media } \\
\text { edad en } \\
\text { años }\end{array}$ & 13 & 12,98 & 16,04 & 13,65 & 14,62 & 16,4 & 16,3 & 14,33 & 10,25 & 5,7 & 7,61 & 4,6 \\
\hline & $12: 00$ & $13: 00$ & $14: 00$ & $15: 00$ & $16: 00$ & $17: 00$ & $18: 00$ & $19: 00$ & $20: 00$ & $21: 00$ & $22: 00$ & $23: 00$ \\
\hline & - & - & - & - & - & - & - & - & - & - & - & - \\
\hline & $12: 59$ & $13: 59$ & $14: 59$ & $15: 59$ & $16: 59$ & $17: 59$ & $18: 59$ & $19: 59$ & $20: 59$ & $21: 59$ & $22: 59$ & $23: 59$ \\
\hline & 4,53 & 6,72 & 4,43 & 5,02 & 5,96 & 6,61 & 7,21 & 6,53 & 9,53 & 11,64 & 11,77 & 11,99 \\
\hline
\end{tabular}

Figura 18: Media de edad según la hora de atención en urgencias

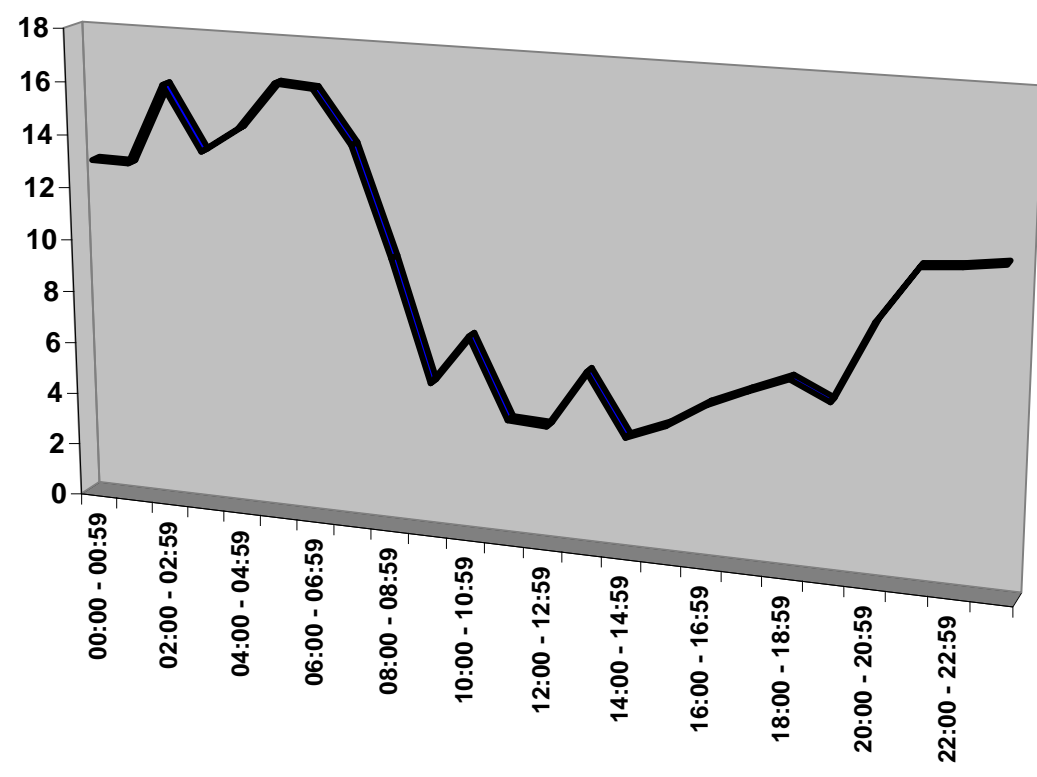

Si se divide a la población en mayores o iguales de 14 años, que son los pacientes atendidos habitualmente en los servicios de urgencias generales, y los menores de 14 años atendidos en Urgencias pediátricas; se evidencian 
diferencias estadísticamente significativas entre la hora de atención en urgencias de los pacientes, teniendo en cuenta esta división de edad $(p=\mathbf{0 , 0 0 8})$

Si la división en grupos de edad se establece en los 7 años, como en muchos trabajos de epidemiológicos españoles, se evidencian diferencias estadísticamente significativas entre la hora de atención en urgencias de los pacientes, teniendo en cuenta esta división de edad $(p=0,005)$

En la tabla 49 y figura 19, se muestra la distribución por grupos de edades de la asistencia en el servicio de urgencias hospitalario, según la hora de admisión.

Tabla 49: Distribución por horas de admisión en el servicio de urgencias hospitalario de los diferentes grupos de edad

\begin{tabular}{|c|c|c|c|c|c|c|c|c|c|c|c|c|}
\hline & $\begin{array}{c}00: 00 \\
- \\
00: 59\end{array}$ & $\begin{array}{c}01: 00 \\
- \\
01: 59\end{array}$ & $\begin{array}{c}02: 00 \\
- \\
02: 59\end{array}$ & $\begin{array}{c}03: 00 \\
- \\
03: 59\end{array}$ & $\begin{array}{c}04: 00 \\
- \\
04: 59\end{array}$ & $\begin{array}{c}05: 00 \\
- \\
05: 59\end{array}$ & $\begin{array}{c}06: 00 \\
- \\
06: 59\end{array}$ & $\begin{array}{c}07: 00 \\
- \\
07: 59\end{array}$ & $\begin{array}{c}08: 00 \\
- \\
08: 59\end{array}$ & $\begin{array}{c}09: 00 \\
- \\
09: 59\end{array}$ & $\begin{array}{c}10: 00 \\
- \\
10: 59\end{array}$ & $\begin{array}{c}11: 00 \\
- \\
11: 59\end{array}$ \\
\hline $\begin{array}{c}\text { menor o } \\
\text { igual } 7 \\
\text { años }\end{array}$ & 14 & 9 & 1 & 3 & 1 & 0 & 0 & 0 & 2 & 6 & 8 & 11 \\
\hline 8-13 años & 2 & 5 & 0 & 3 & 3 & 0 & 1 & 1 & 0 & 3 & 0 & 2 \\
\hline $\begin{array}{c}\text { Mayor o } \\
\text { igual } 14 \\
\text { años }\end{array}$ & 50 & 37 & 22 & 20 & 12 & 10 & 9 & 2 & 2 & 1 & 5 & 2 \\
\hline Total & 66 & 51 & 23 & 26 & 16 & 10 & 10 & 3 & 4 & 10 & 13 & 15 \\
\hline & $\begin{array}{c}12: 00 \\
- \\
12: 59\end{array}$ & $\begin{array}{c}13: 00 \\
- \\
13: 59\end{array}$ & $\begin{array}{c}14: 00 \\
- \\
14: 59 \\
\end{array}$ & $\begin{array}{c}15: 00 \\
- \\
15: 59\end{array}$ & $\begin{array}{c}16: 00 \\
- \\
16: 59 \\
\end{array}$ & $\begin{array}{c}17: 00 \\
- \\
17: 59 \\
\end{array}$ & $\begin{array}{c}18: 00 \\
- \\
18: 59\end{array}$ & $\begin{array}{c}19: 00 \\
- \\
19: 59 \\
\end{array}$ & $\begin{array}{c}20: 00 \\
- \\
20: 59\end{array}$ & $\begin{array}{c}21: 00 \\
- \\
21: 59 \\
\end{array}$ & $\begin{array}{c}22: 00 \\
- \\
22: 59 \\
\end{array}$ & $\begin{array}{c}23: 00 \\
- \\
23: 59\end{array}$ \\
\hline & 25 & 22 & 28 & 34 & 23 & 22 & 20 & 29 & 33 & 30 & 38 & 30 \\
\hline & 2 & 2 & 1 & 4 & 1 & 4 & 1 & 4 & 7 & 10 & 12 & 10 \\
\hline & 5 & 9 & 3 & 5 & 8 & 10 & 12 & 10 & 38 & 77 & 99 & 79 \\
\hline & 32 & 33 & 32 & 43 & 32 & 36 & 33 & 43 & 78 & 117 & 149 & 119 \\
\hline
\end{tabular}




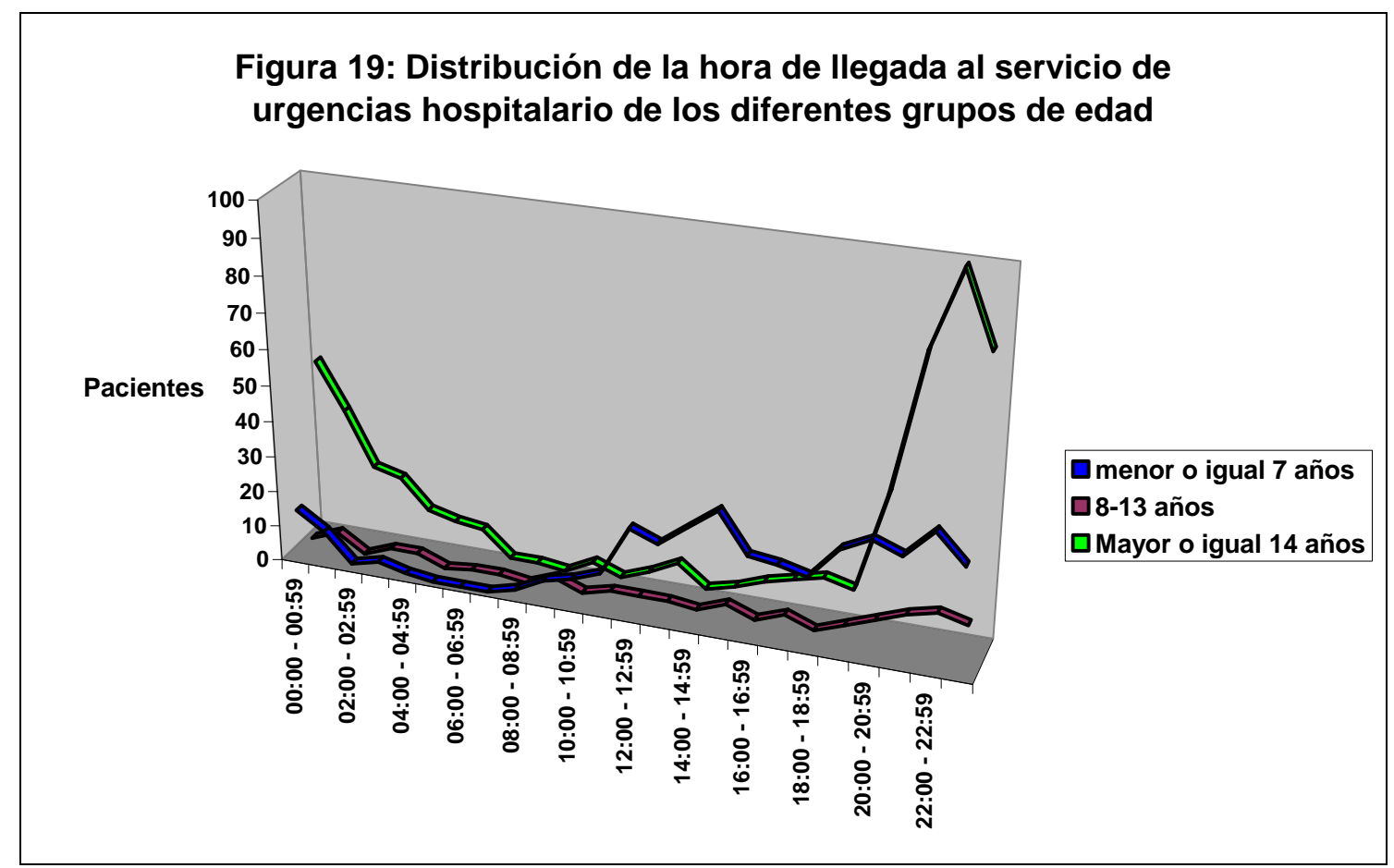

\subsection{Relación entre la edad y la presencia de antecedentes personales en} los pacientes intoxicados.

Se evidenciaron diferencias estadísticamente significativas entre la presencia de antecedentes personales de los pacientes intoxicados atendidos en el servicio de urgencias hospitalario teniendo en cuenta su edad $(\mathrm{p}=\mathbf{0 , 0 0 1 )}$. Tabla 50, figura 20 .

Tabla 50: Relación entre la edad de los pacientes y la presencia de antecedentes personales

\begin{tabular}{|c|cccc|}
\hline & Antecedentes Personales & N & Media & Desviación típ. \\
\hline Edad & NO & 628 & 9,65 & 6,58 \\
& SI & 366 & 10,99 & 6,08 \\
& \multicolumn{2}{c}{$\mathrm{p}=0,001$} & \\
\hline
\end{tabular}


Figura 20: Edad media en años de los pacientes con diagnóstico de intoxicación, según la presencia o no de antecedentes personales

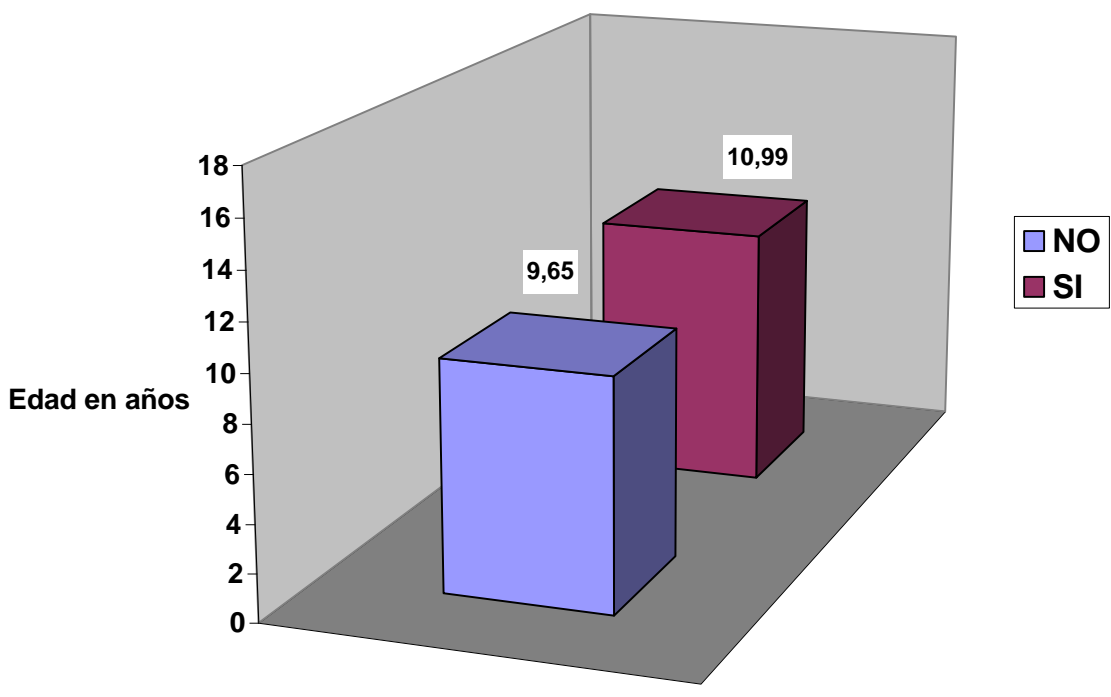

$p=0,001$

\subsection{Relación entre la edad del paciente y su localidad de origen.}

Se evidenciaron diferencias estadísticamente significativas entre la localidad de origen del intoxicado (Valladolid capital o resto de localidades) teniendo en cuenta su edad $(\mathbf{p}=0,0001)$. Tabla 51 , figura 21 .

Tabla 51 : Relación entre la edad y la procedencia del intoxicado

\begin{tabular}{|c|cccc|}
\hline \multirow{3}{*}{ Edad } & Localidad origen & $\mathrm{N}$ & Media & Desviación típ. \\
\cline { 2 - 5 } & Valladolid capital & 634 & 10,93 & 6,29 \\
& Otros lugares & 360 & 8,76 & 6,46 \\
& & $\mathrm{p}=0,0001$ & \\
\hline
\end{tabular}


Figura 21: Relación entre la edad y la procedencia del intoxicado

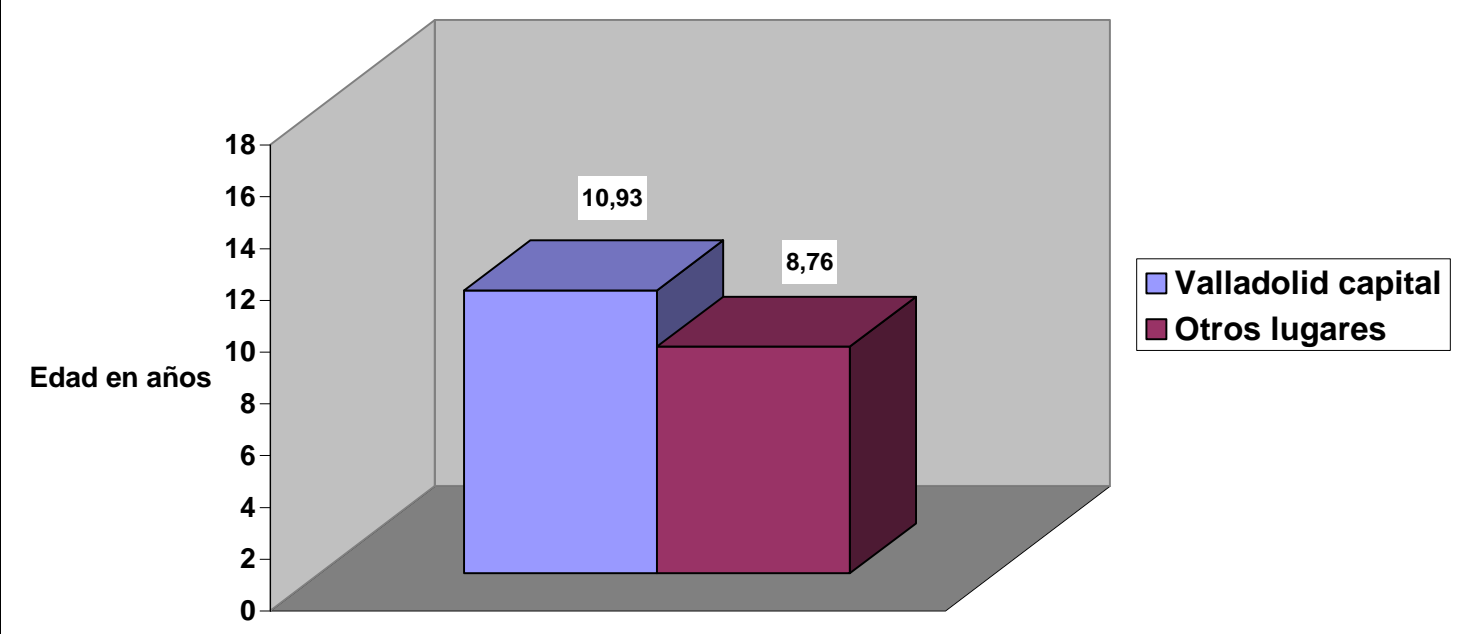

$p=0,0001$

En la tabla 52, se muestra la procedencia de los pacientes distribuidos por grupos de edad.

Tabla 52: Procedencia de los pacientes distribuidos según grupos de edad

\begin{tabular}{|c|cccccccc|}
\hline & $\begin{array}{c}\text { Valla } \\
\text { dolid }\end{array}$ & $\begin{array}{c}\text { Arroyo- } \\
\text { La } \\
\text { Flecha }\end{array}$ & $\begin{array}{c}\text { Laguna } \\
\text { de } \\
\text { Duero }\end{array}$ & $\begin{array}{c}\text { La } \\
\text { Cister } \\
\text { niga }\end{array}$ & $\begin{array}{c}\text { Tord } \\
\text { esilla } \\
\text { s }\end{array}$ & $\begin{array}{c}\text { Resto provincia } \\
\text { de Valladolid }\end{array}$ & $\begin{array}{c}\text { Comunidad de } \\
\text { Castilla y León }\end{array}$ & $\begin{array}{c}\text { Resto de } \\
\text { España }\end{array}$ \\
\hline $\begin{array}{c}\text { Menor o } \\
\text { igual 7 } \\
\text { años }\end{array}$ & 212 & 13 & 43 & 18 & 7 & 82 & 2 & 12 \\
$\begin{array}{c}8-13 \text { años } \\
\text { Mayor o } \\
\text { igual 14 } \\
\text { años }\end{array}$ & 44 & 6 & 4 & 2 & 2 & 13 & 4 & 3 \\
\hline Total & 634 & 22 & 32 & 11 & 4 & 63 & 7 & 10 \\
\hline
\end{tabular}


4.9. Relación entre la edad de los pacientes y la derivación previa de los pacientes al servicio de urgencias hospitalario por parte de otro servicio sanitario.

No se evidenciaron diferencias estadísticamente significativas entre la derivación previa de los pacientes al servicio de urgencias hospitalario por parte de otro servicio sanitario teniendo en cuenta su edad $(p=0,864)$. Tabla 53, figura 22.

Tabla 53: Relación entre la edad y la derivación del paciente al servicio de urgencias hospitalario.

\begin{tabular}{|c|ccccc|}
\hline \multirow{3}{*}{ Edad } & Derivado SI/NO & N & Porcentaje & Media & Desviación típica \\
\cline { 2 - 6 } & NO & 557 & $95,37 \%$ & 10,61 & 6,27 \\
& SI & 27 & $4,62 \%$ & 10,4 & 6,54 \\
\hline \multicolumn{5}{|c}{$\mathrm{p}=0,864$} \\
\hline
\end{tabular}

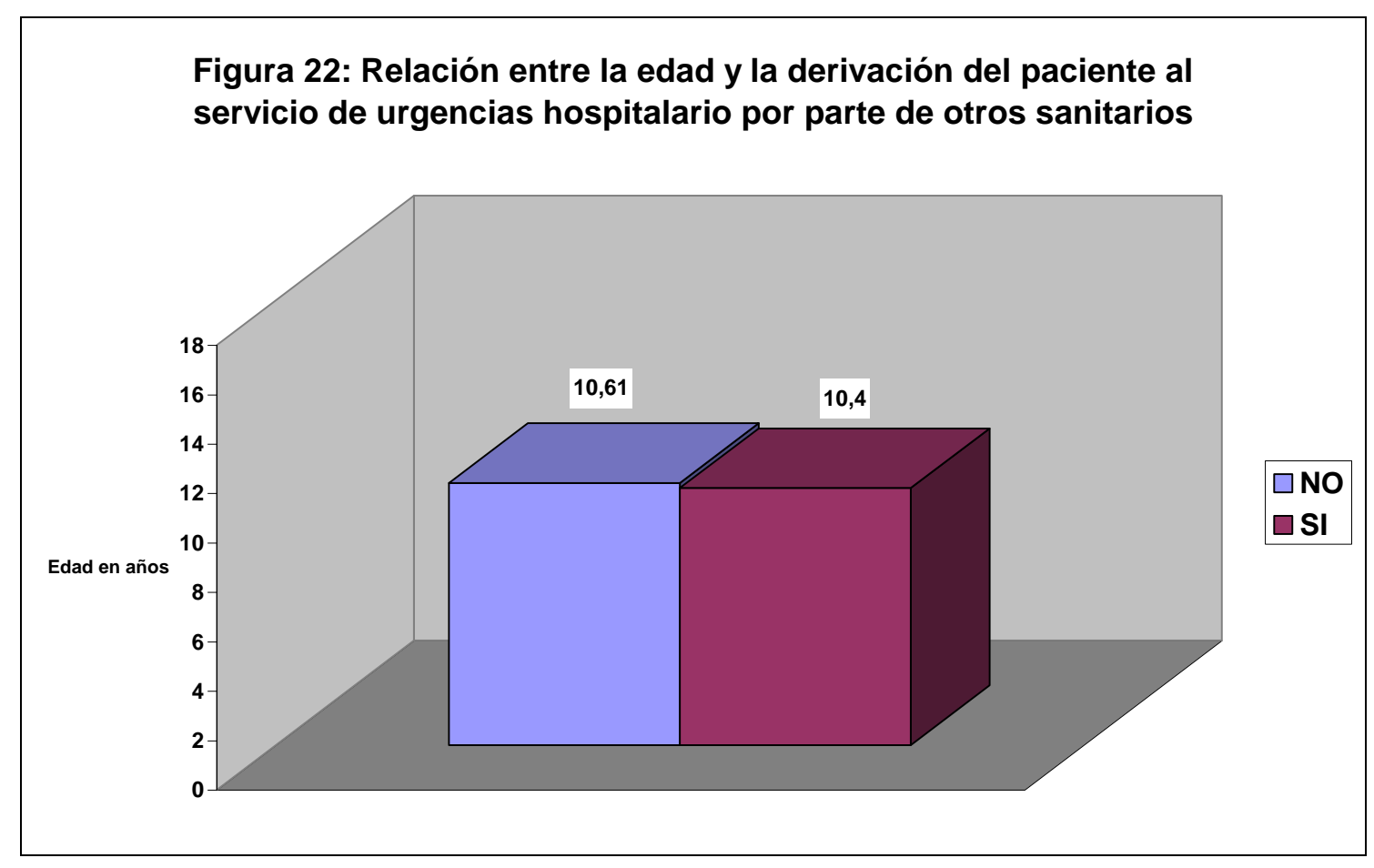

$p=0,864$ 
En la tabla 54, se divide a los pacientes por grupos de edades según la derivación al servicio de urgencias hospitalario por parte de otro servicio sanitario.

Tabla 54: Derivación al servicio de urgencias hospitalario por parte de otros servicios sanitarios distribuidos por grupos de edad

\begin{tabular}{|c|ccc|}
\hline & NO & Centro de Salud & Otro Hospital \\
\hline Menor o igual 7 años & 198 & 6 & 4 \\
8-13 años & 48 & 3 & 0 \\
Mayor o igual 14 años & 311 & 11 & 3 \\
\hline Total & 557 & 20 & 7 \\
\hline
\end{tabular}

\subsection{Acceso al servicio de urgencias hospitalario en vehículos de}

\section{Emergencias Sanitarias teniendo en cuenta la edad del paciente.}

Se evidenciaron diferencias estadísticamente significativas entre el acceso al servicio de urgencias hospitalario por parte de vehículos de Emergencias Sanitarias teniendo en cuenta su edad ( $p=0,0001)$ Tabla 55 , figura 23.

La edad media de los pacientes que acuden al servicio de urgencias hospitalario en vehículos de Emergencias Sanitarias es de 15,01 +/- 2,48 años y la de los que no lo necesitan es de 7,63 +/- 6,3 años.

Tabla 55: Relación entre la edad y la necesidad de transporte por vehículos de Emergencias Sanitarias

\begin{tabular}{|c|ccccc|}
\hline & Atendido por 112 & N & Porcentaje & Media & Desviación típica \\
\hline Edad & NO & 348 & $59,58 \%$ & 7,63 & 6,32 \\
& SI & 236 & $40,41 \%$ & 15,01 & 2,48 \\
\hline \multicolumn{5}{|c}{$p=0,0001$} \\
\hline
\end{tabular}


Figura 23: Relación entre la edad y el transporte por vehículos de Emergencias Sanitarias

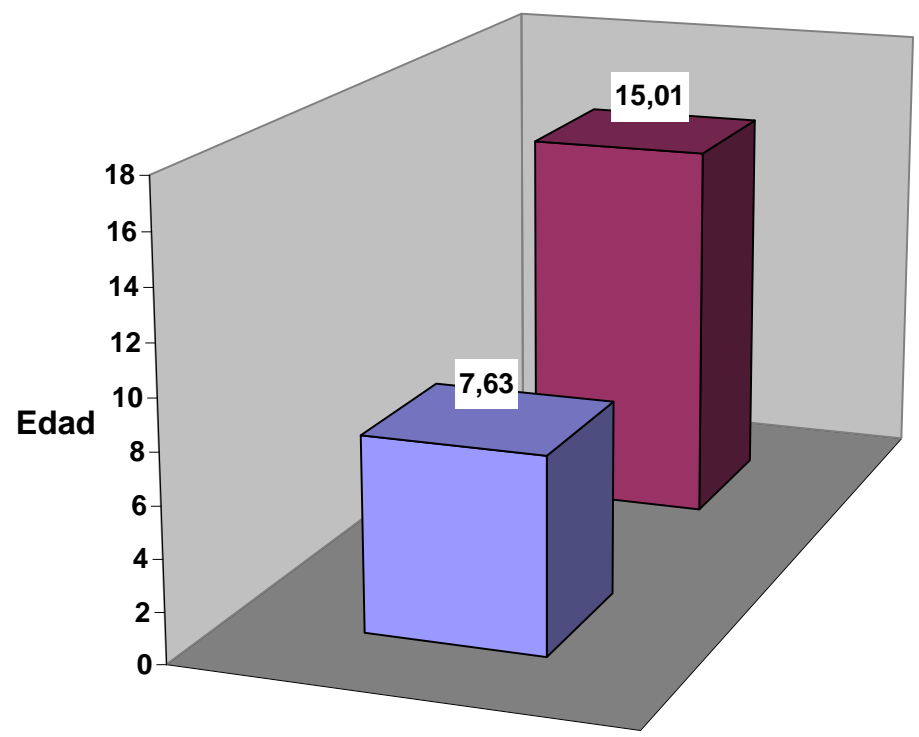

$\square$ NO

$\square \mathbf{S I}$

$p=0,0001$

En la tabla 56, se muestra la necesidad de transporte al servicio de urgencias hospitalario en vehículos de Emergencias Sanitarias, según los diferentes grupos de edad.

Tabla 56: Distribución por grupos de edades según la necesidad de acudir al servicio de urgencias hospitalario en vehículos de Emergencias Sanitarias

\begin{tabular}{|c|cccc|}
\hline & NO & $\%$ & Sí & $\%$ \\
\hline Menor o igual 7 años & 202 & $58,04 \%$ & 6 & $2,54 \%$ \\
8-13 años & 29 & $8,33 \%$ & 22 & $9,32 \%$ \\
Mayor o igual 14 años & 117 & $33,62 \%$ & 208 & $88,13 \%$ \\
\hline Total & 348 & $100 \%$ & 236 & $100 \%$ \\
\hline
\end{tabular}




\subsection{Relación entre la edad y la realización de analítica y otras pruebas}

complementarias diagnósticas en el servicio de urgencias hospitalario.

Se evidenciaron diferencias estadísticamente significativas entre la realización de analítica en el servicio de urgencias hospitalario teniendo en cuenta su edad ( $p=0,0001)$. Tabla 57 , figura 24 .

Tabla 57: Relación entre la edad y la realización de analítica en el servicio de urgencias hospitalario.

\begin{tabular}{|c|c|c|c|c|}
\hline & Realización de Analítica & $\mathrm{N}$ & Media & Desviación típica. \\
\hline \multirow[t]{3}{*}{ Edad } & NO & 266 & 7,24 & 6,34 \\
\hline & SI & 321 & 13,39 & 4,65 \\
\hline & \multicolumn{4}{|c|}{$p=0,0001$} \\
\hline
\end{tabular}

Figura 24: Relación entre la edad y la realización de analítica en el servicio de urgencias hospitalario

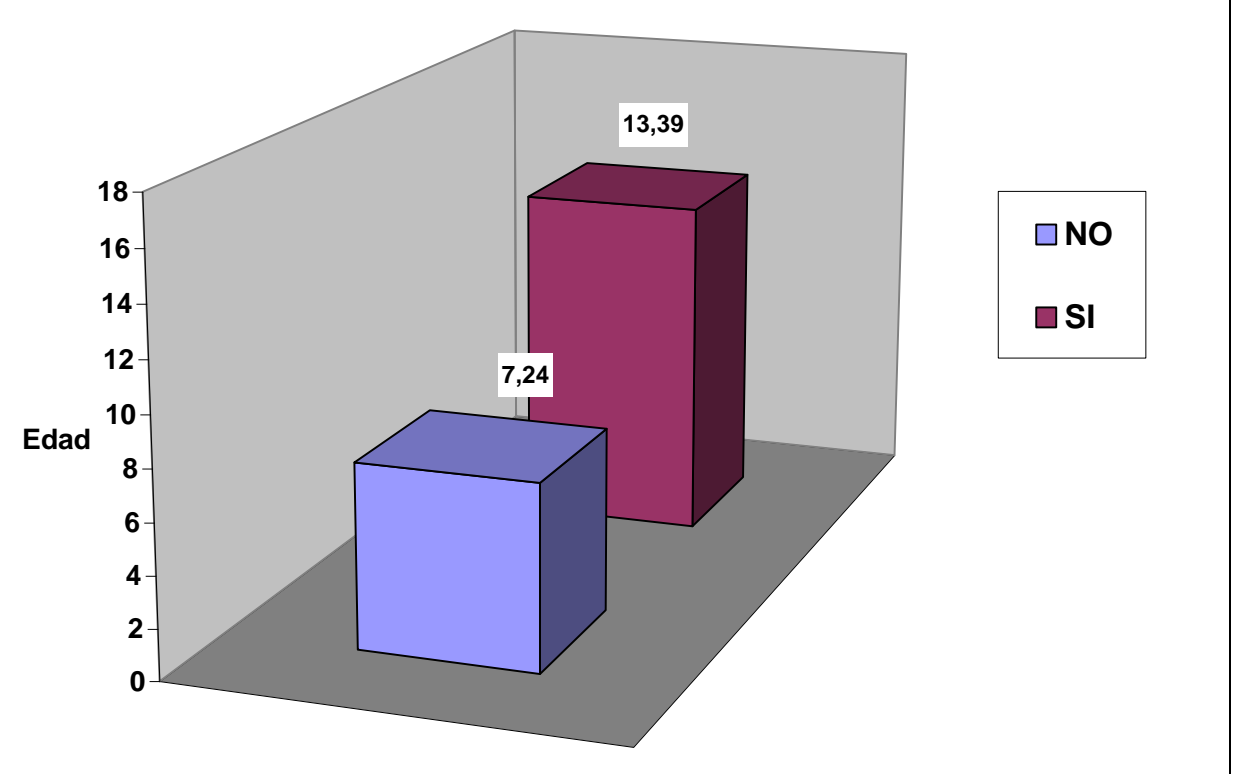

$p=0,0001$

Si se dividen los diferentes tipos de pruebas analíticas que se realizan en el servicio de urgencias hospitalario, encontramos los siguientes datos teniendo en cuenta su edad (tabla 58, figura 25): 
Tabla 58: Relación entre la edad y el tipo de pruebas realizadas en el servicio de urgencias hospitalario

\begin{tabular}{|cccc|}
\hline $\begin{array}{c}\text { Tipos de pruebas en el } \\
\text { servicio de urgencias } \\
\text { hospitalario }\end{array}$ & $\begin{array}{c}\text { Edad media NO } \\
\text { realización }\end{array}$ & $\begin{array}{c}\text { Edad media Sí } \\
\text { realización }\end{array}$ & $\begin{array}{c}\text { p- } \\
\text { valor }\end{array}$ \\
\hline Hemograma & $7,13+/-6,27$ & $14,13+/-3,82$ & 0,001 \\
\hline Bioquímica & $7,21+/-6,32$ & $13,95+/-4,07$ & 0,001 \\
\hline Hemostasia & $10,69+/-6,24$ & $9,53+/-6,75$ & 0,29 \\
Gasometría venosa & $10,22+/-6,38$ & $14,18+/-3,71$ & 0,001 \\
Gasometría arterial & $10,66+/-6,33$ & $10,3+/-5,04$ & 0,52 \\
Tóxicos en orina & $8,22+/-6,46$ & $15,02+/-2,29$ & 0,001 \\
ECG & $9,40+/-6,52$ & $14,45+/-3,23$ & 0,001 \\
Prueba imagen & $10,44+/-6,31$ & $13,85+/-4,61$ & 0,002 \\
\hline
\end{tabular}

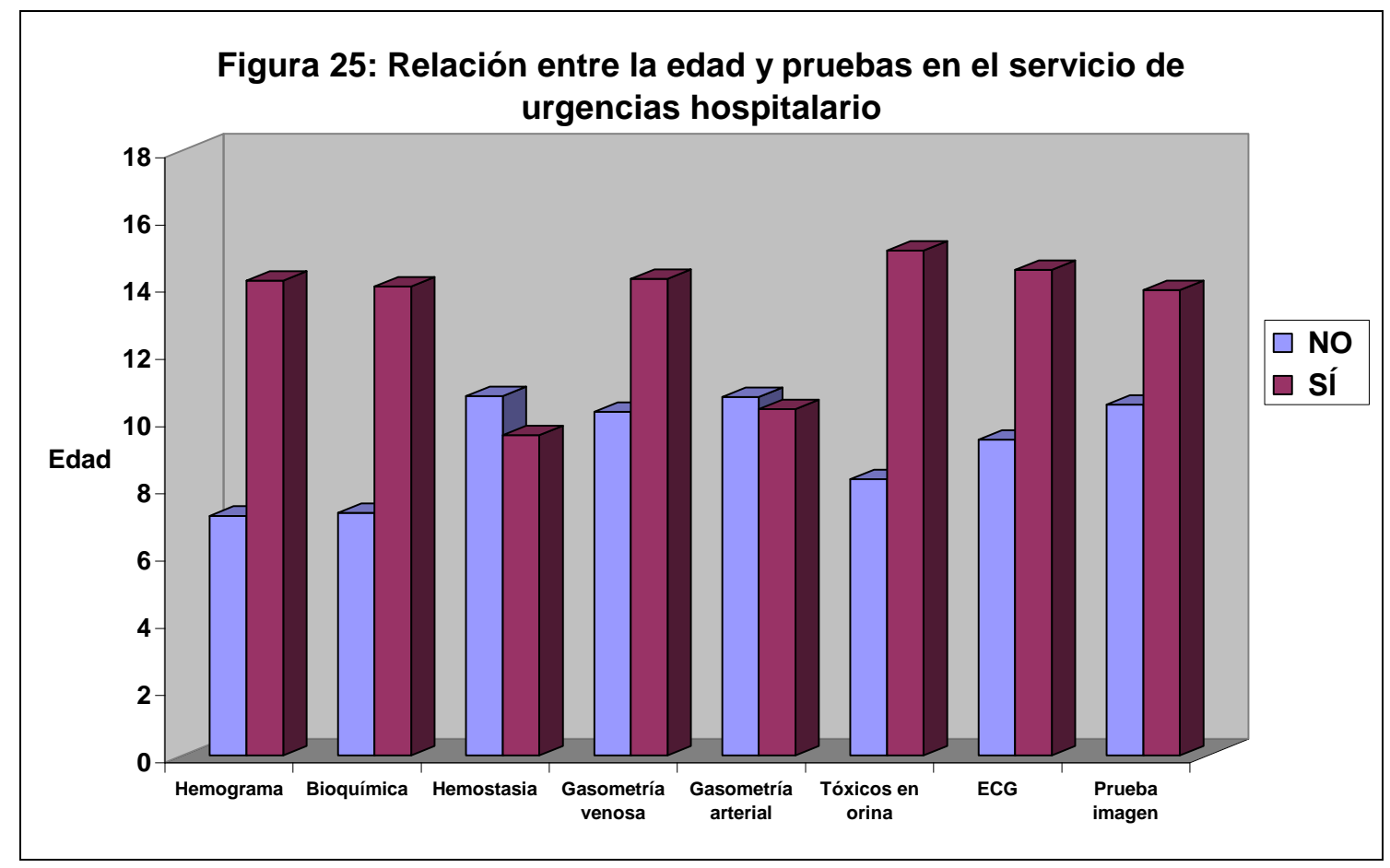

En la tabla 59 y figura 26, se muestra la realización de diferentes pruebas en el servicio de urgencias hospitalario distribuidas por grupos de edad. 
Tabla 59: Realización de pruebas en el servicio de urgencias hospitalario distribuidas por grupos de edad.

\begin{tabular}{|c|ccc|}
\hline & $\begin{array}{c}\text { Menores o iguales 7 } \\
\text { años }\end{array}$ & $\begin{array}{c}\text { Mayores o iguales 14 años } \\
\text { años }\end{array}$ \\
\hline Analítica & 45 & 30 & 246 \\
Sistemático & 23 & 29 & 238 \\
Bioquímica & 28 & 28 & 236 \\
Hemostasia & 15 & 2 & 17 \\
Gasometría venosa & 5 & 7 & 49 \\
Gasometría & 10 & & 11 \\
arterial & 10 & 15 & 187 \\
Tóxicos en orina & 5 & 16 & 119 \\
\hline ECG & 7 & 2 & 27 \\
\hline Pruebas de imagen & 4 & & \\
\hline
\end{tabular}

Figura 26: Realización de pruebas en el servicio de urgencias hospitalario distribuidas por grupos de edad

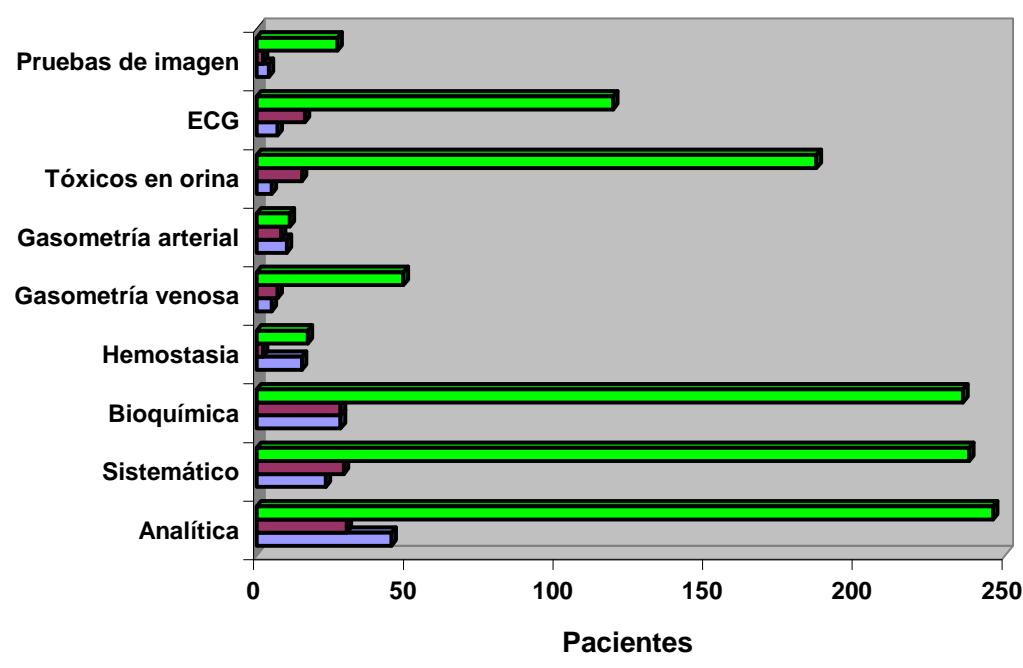

$\square$ Mayores o iguales 14 años प8-13 años

$\square$ Menores 0 iguales 7 años 


\subsection{Relación entre la edad y la administración de diferentes tratamientos}

en el servicio de urgencias hospitalario.

Se evidenciaron diferencias estadísticamente significativas entre la administración de tratamiento en el servicio de urgencias hospitalario teniendo en cuenta su edad ( $p=0,0001)$. Tabla 60 , figura 27.

Tabla 60: Relación entre la edad y el tratamiento en el servicio de urgencias hospitalario

\begin{tabular}{|c|c|c|c|c|}
\hline & Tratamiento & $\mathbf{N}$ & Media & Desviación típica \\
\hline Edad & NO & 240 & 8,38 & 6,52 \\
\hline & SI & 345 & 12,18 & 5,59 \\
\hline \multicolumn{5}{|c|}{$p=0,0001$} \\
\hline
\end{tabular}

Figura 27: Relación entre la edad y el tratamiento en el servicio de urgencias hospitalario

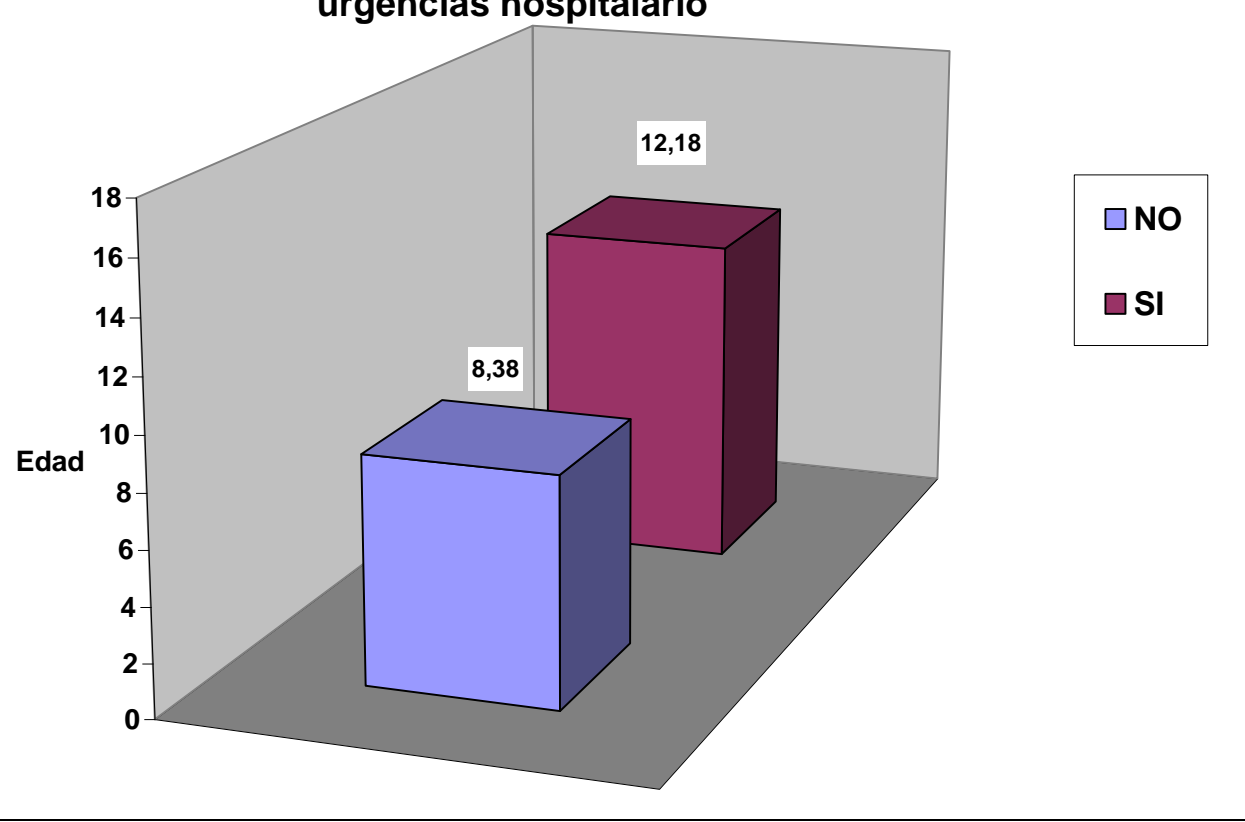

$p=0,0001$

En la tabla 61 y figura 28, se muestra la relación entre la edad de los pacientes y los diferentes tipos de tratamientos realizados en el servicio de urgencias hospitalario. 
Tabla 61: Relación entre edad y los diferentes tratamientos realizados en el servicio de urgencias hospitalario

\begin{tabular}{|c|ccc|}
\hline $\begin{array}{c}\text { Tipo de } \\
\text { tratamiento }\end{array}$ & $\begin{array}{c}\text { Edad media NO } \\
\text { tratamiento }\end{array}$ & $\begin{array}{c}\text { Edad media Sí } \\
\text { tratamiento }\end{array}$ & p valor \\
\hline Oxigenoterapia & $10,60+/-6,37$ & $11,07+/-4,94$ & 0,63 \\
Lavado gástrico & $10,91+/-6,17$ & $7,56+/-6,64$ & 0,001 \\
Carbón activado & $11,09+/-6,13$ & $6,30+/-6,05$ & 0,001 \\
Antídoto & $10,63+/-6,27$ & $9,71+/-7,27$ & 0,7 \\
Suero fisiológico & $8,68+/-6,5$ & $15,1+/-2,1$ & 0,001 \\
Suero glucosado & $10,29+/-6,38$ & $14,95+/-1,71$ & 0,001 \\
Antiemético & $9,58+/-6,45$ & $15,45+/-1,1$ & 0,001 \\
Analgésico & $10,48+/-6,31$ & $15,5+/-1,26$ & 0,001 \\
Protector gástrico & $10,29+/-6,35$ & $13,61+/-4,67$ & 0,001 \\
Tiamina & $10,62+/-6,27$ & $10,5+/-7,63$ & 0,95 \\
\hline
\end{tabular}

Figura 28: Relación entre la edad y diferentes tratamientos en el servicio de urgencias hospitalario

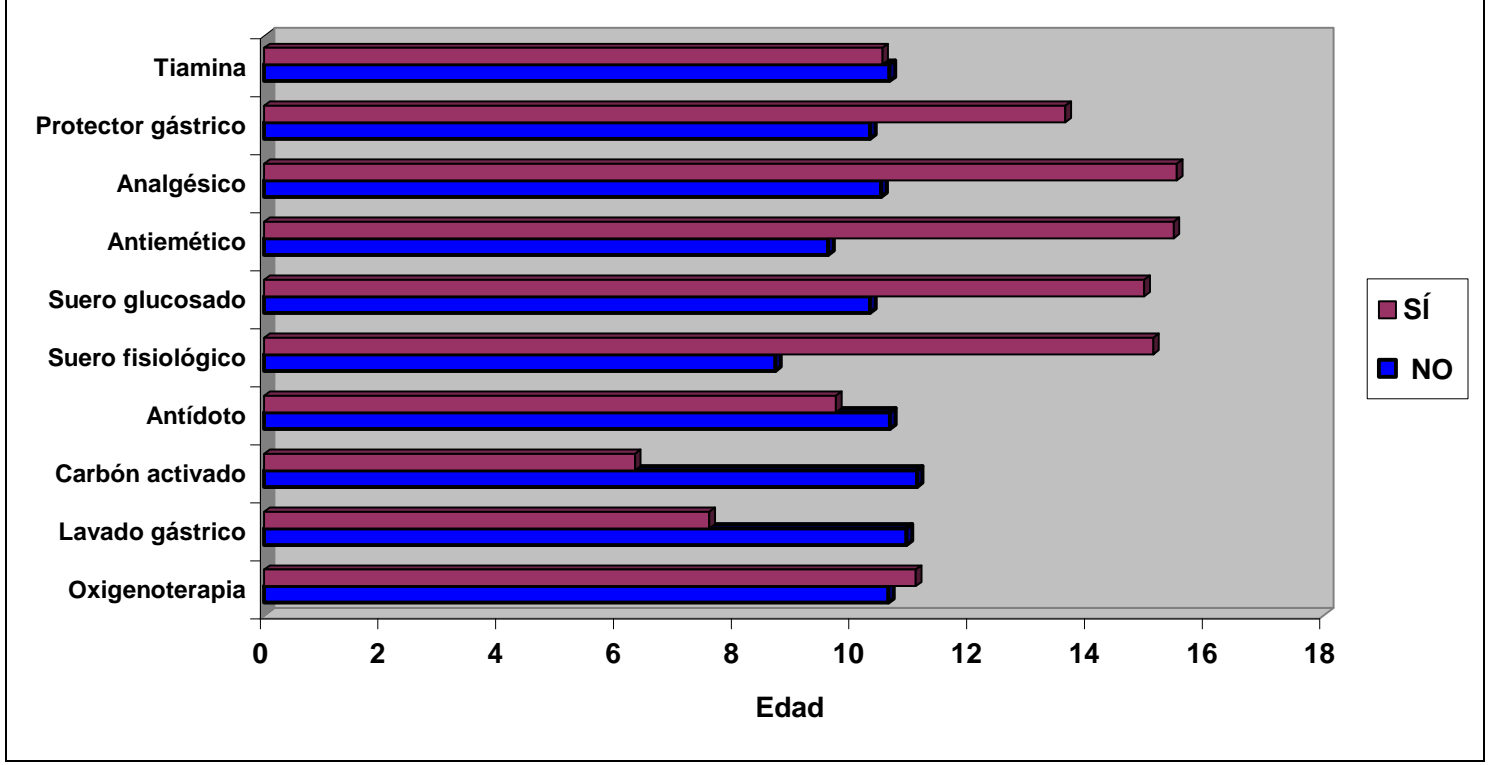

En la tabla 62 y la figura 29, se muestran los diferentes tratamientos que necesitan los pacientes en el servicio de urgencias hospitalario distribuidos por grupos de edad. 
Tabla 62: Tratamientos recibidos en el servicio de urgencias hospitalario distribuidos por grupos de edad

\begin{tabular}{|c|ccc|}
\hline Tratamiento & Menores o iguales 7 años & $8-13$ años & Igual o mayor a 14 años \\
\hline Oxigenoterapia & 13 & 9 & 21 \\
Lavado gástrico & 28 & 6 & 16 \\
Carbón activado & 38 & 5 & 14 \\
Antídotos & 3 & 0 & 4 \\
Suero fisiológico & 3 & 10 & 163 \\
Suero glucosado & 0 & 8 & 33 \\
Antieméticos & 0 & 4 & 101 \\
Analgésicos & 0 & 1 & 15 \\
Protector gástrico & 8 & 1 & 47 \\
Tiamina & 3 & 0 & 5 \\
Otros & 19 & 2 & 22 \\
\hline
\end{tabular}

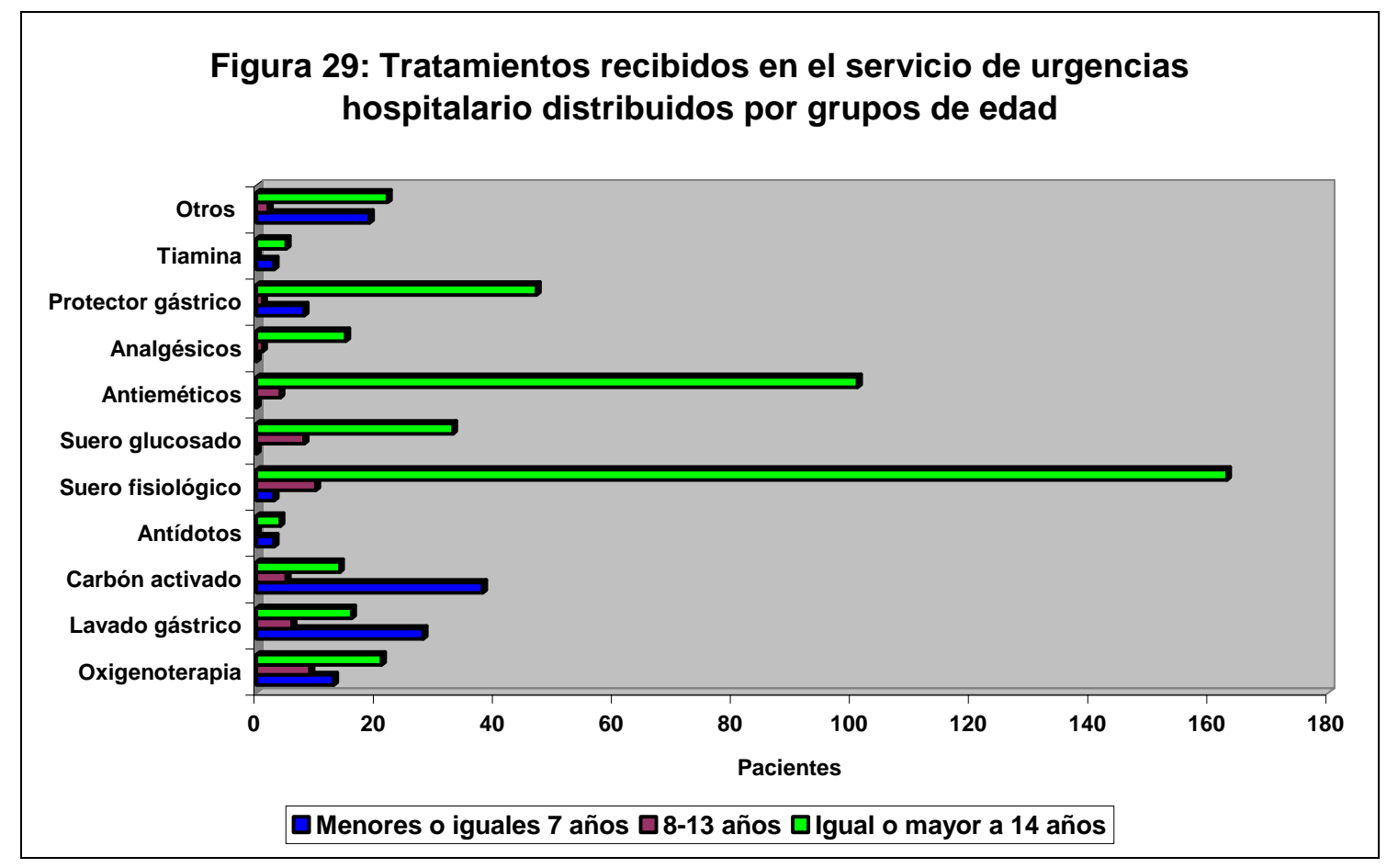


4.13. Relación entre la edad y el tiempo de permanencia en el servicio de urgencias hospitalario.

Se evidenciaron diferencias estadísticamente significativas entre el tiempo de observación en el servicio de urgencias hospitalario teniendo en cuenta su edad $(\mathbf{p}=\mathbf{0}, \mathbf{0 0 0 1})$. Tabla 63 , figura 30 .

Tabla 63: Relación entre la edad y la estancia en el servicio de urgencias hospitalarias

\begin{tabular}{|c|ccccc|}
\hline \multirow{2}{*}{ Edad } & $\begin{array}{c}\text { Estancia en urgencias } \\
\text { más de 2 horas }\end{array}$ & $\mathrm{N}$ & Porcentaje & Media & $\begin{array}{c}\text { Desviación } \\
\text { típica }\end{array}$ \\
& Menos de 2 horas & 412 & $41,44 \%$ & 6,63 años & 6,28 \\
& Mas de 2 horas & 582 & $58,55 \%$ & 12,63 años & 5,27 \\
\hline
\end{tabular}

Figura 30: Relación entre la edad y la permanencia en el servicio de urgencias hospitalario

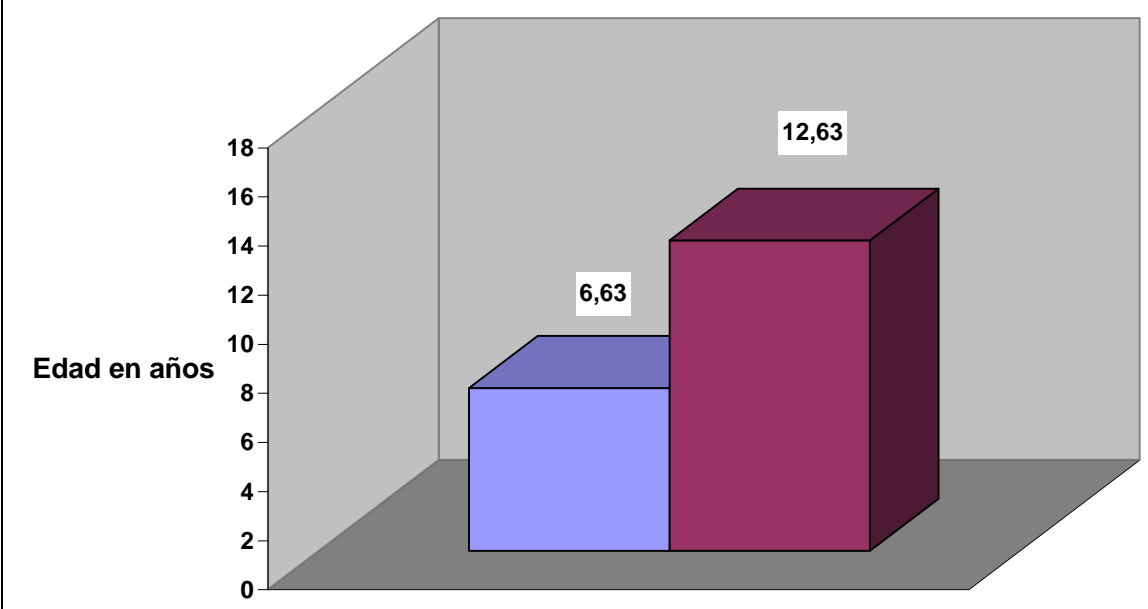


En la tabla 64 y figura 31 , se establece la relación entre la estancia en el servicio de urgencias hospitalario y los diferentes grupos de edad.

Tabla 64: Distribución por edades según la permanencia en el servicio de urgencias hospitalario

\begin{tabular}{|c|cccc|}
\hline & Menos de 2 horas & Porcentaje & Mas de 2 horas & Porcentaje \\
\hline Menor o igual 7 años & 274 & $66,50 \%$ & 115 & $19,75 \%$ \\
8-13 años & 20 & $4,85 \%$ & 58 & $9,96 \%$ \\
Mayor o igual 14 años & 118 & $28,64 \%$ & 409 & $70,27 \%$ \\
\hline Total & 412 & $100 \%$ & 582 & $100 \%$ \\
\hline
\end{tabular}

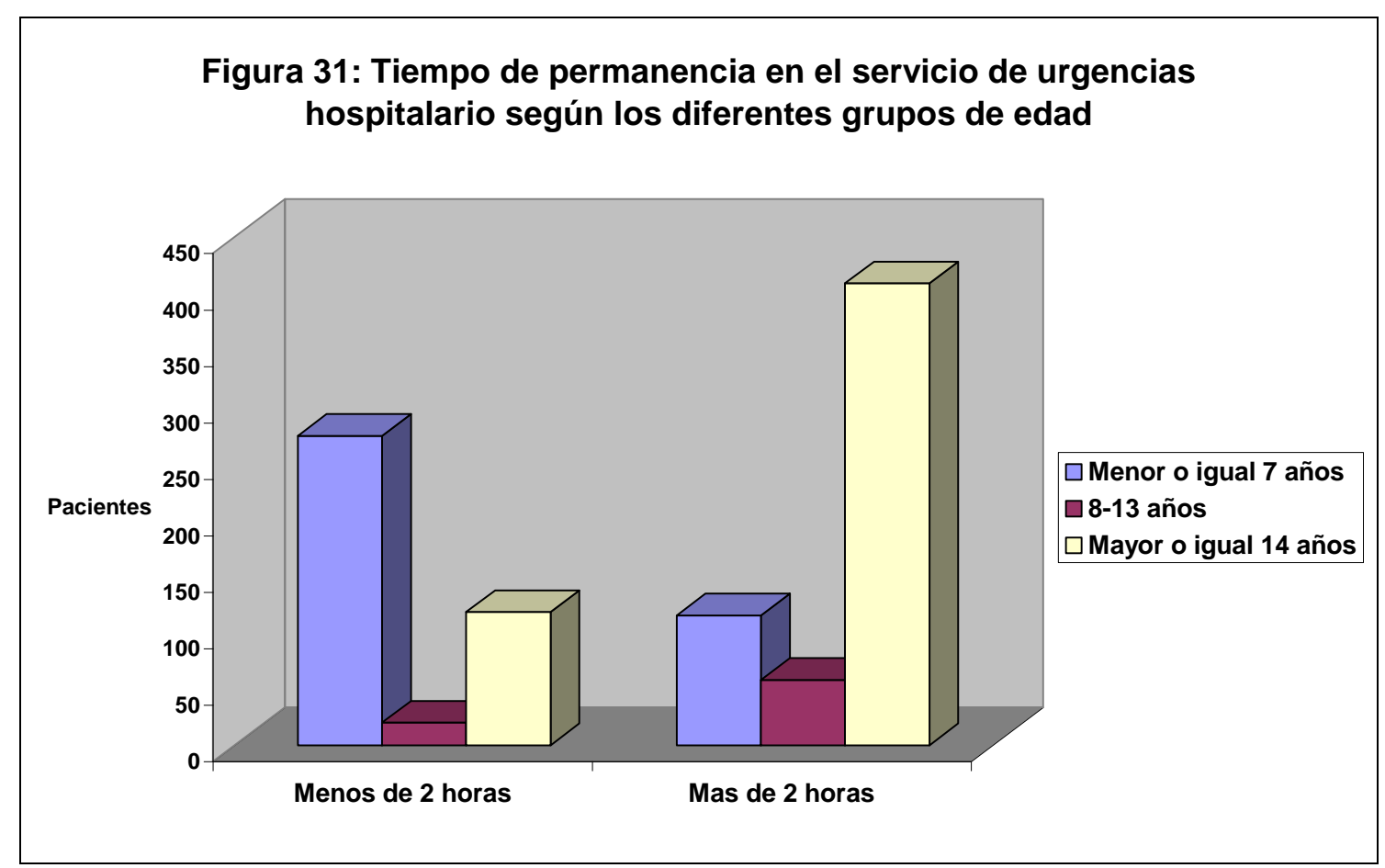

4.14. Relación entre la edad del paciente y el destino tras el paso por el servicio de urgencias hospitalario.

Se evidenciaron diferencias estadísticamente significativas entre el destino del paciente tras el paso por el servicio de urgencias hospitalario (dividido 
entre alta hospitalaria $u$ otras opciones) teniendo en cuenta su edad $(p=0,0001)$. Tabla 65, figura 32 .

Tabla 65: Relación entre la edad del paciente y su destino tras su estancia en el servicio de urgencias hospitalario

\begin{tabular}{|c|c|ccc|}
\hline \multicolumn{2}{|c|}{ Destino tras la urgencia } & $\mathrm{N}$ & Media & Desviación típ. \\
\hline \multirow{2}{*}{ Edad } & ALTA & 827 & 10,65 & 6,39 \\
& Otras opciones & 167 & 7,62 & 6,05 \\
& & & \\
\hline
\end{tabular}

Figura 32: Relación entre la edad y su destino tras la estancia en el servicio de urgencias hospitalario

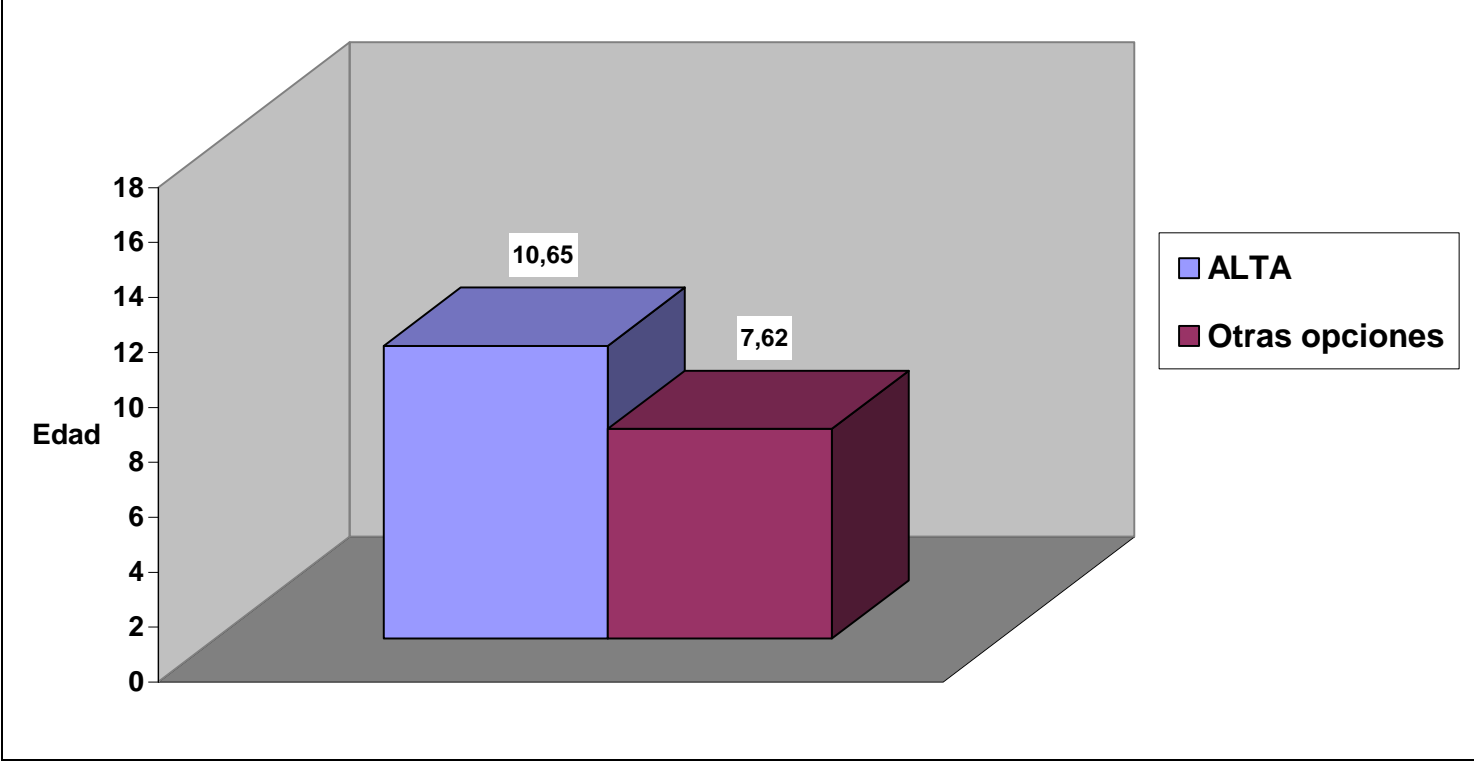

$p=0,0001$

En la tabla 66 y figura 33, se muestra la distribución por grupos de edad del destino del paciente tras la estancia en el servicio de urgencias hospitalario. 
Tabla 66: Distribución por grupos de edades de los pacientes según el destino tras la estancia en el servicio de urgencias hospitalario.

\begin{tabular}{|c|cccccccc|}
\hline & Alta & $\%$ & $\begin{array}{c}\text { Ingreso } \\
\text { Hospitalario }\end{array}$ & $\%$ & $\begin{array}{c}\text { Consulta } \\
\text { externa }\end{array}$ & $\begin{array}{c}\text { Deriva } \\
\text { ción }\end{array}$ & $\%$ \\
\hline $\begin{array}{c}\text { Menor o igual } \\
7 \text { años }\end{array}$ & 296 & $35,79 \%$ & 93 & $71,53 \%$ & 0 & $0 \%$ & 0 & $0 \%$ \\
$\begin{array}{c}\text { 8-13 años } \\
\begin{array}{c}\text { Mayor o igual } \\
14 \text { años }\end{array}\end{array}$ & 48 & $5,80 \%$ & 23 & $17,69 \%$ & 3 & $30 \%$ & 4 & $\begin{array}{c}14,81 \\
\%\end{array}$ \\
\hline Total & 883 & $58,40 \%$ & 14 & $10,76 \%$ & 7 & $70 \%$ & 23 & $\begin{array}{c}85,18 \\
\%\end{array}$ \\
\hline
\end{tabular}

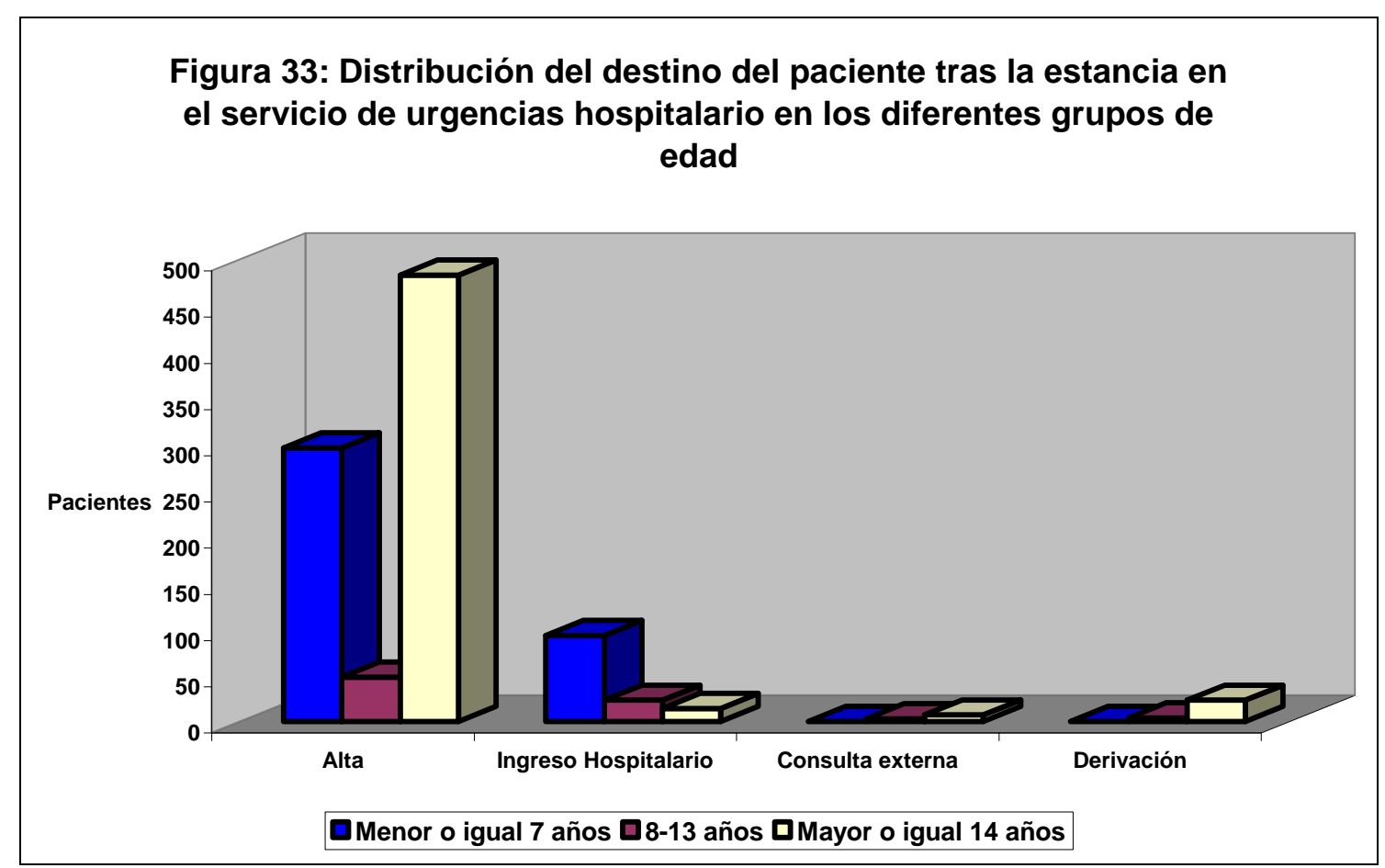

\subsection{Relación entre la edad y otros parámetros}

No se evidenciaron diferencias estadísticamente significativas entre la presencia de acompañamiento del paciente en el servicio de urgencias hospitalario teniendo en cuenta su edad $(p=0,15)$.

No se evidenciaron diferencias estadísticamente significativas entre el número de horas de ingreso en planta tras la intoxicación, teniendo en cuenta su 
edad divida en dos grupos menores o mayores de 14 años $(p=0,92)$ y menores o mayores de 7 años $(p=0,302)$

\subsection{Relación entre la edad y la necesidad de interconsulta a Psiquiatría} durante su estancia en el servicio de urgencias hospitalario.

Se evidenciaron diferencias estadísticamente significativas entre la necesidad de interconsulta con Psiquiatría durante su estancia en el servicio de urgencias hospitalario teniendo en cuenta su edad $(p=0,0001)$. Tabla 67 , figura 34.

Tabla 67: Relación entre la edad y la necesidad de interconsulta con el servicio de Psiquiatría durante su estancia en el servicio de urgencias hospitalario.

\begin{tabular}{|c|ccc|}
\hline Interconsulta con Psiquiatría & N & Media & Desviación típica \\
\hline NO & 377 & 10,54 & 6,38 \\
SI & 37 & 15,78 & 1,18 \\
& $\mathrm{p}=0,0001$ & & \\
\hline
\end{tabular}

Figura 34: Relación entre la edad y la necesidad de interconsulta con Psiquiatría durante su estancia en el servicio de urgencias hospitalario

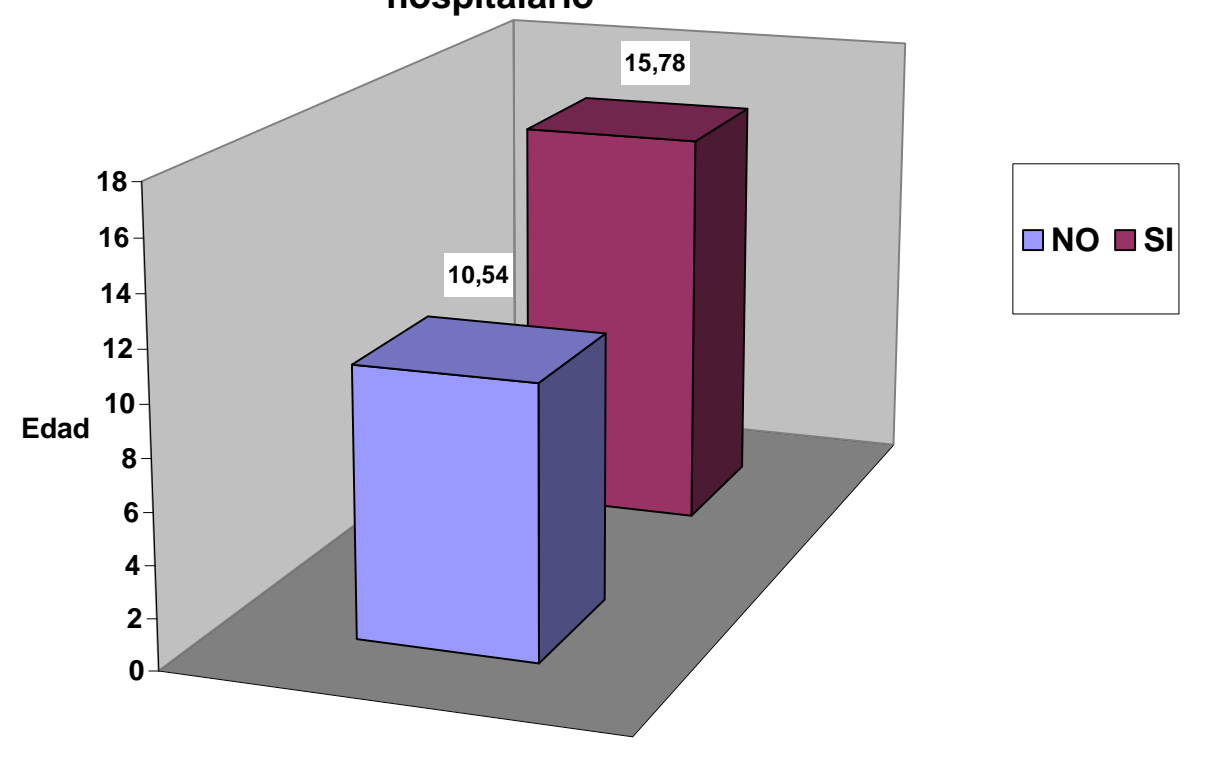

$p=0,0001$ 


\subsection{Relación entre la edad y la necesidad de consultar con Toxicología}

Se evidenciaron diferencias estadísticamente significativas entre la necesidad de consulta con Toxicología durante su estancia en el servicio de urgencias hospitalario teniendo en cuenta su edad $(p=0,0001)$. Tabla 68 , figura 35.

Tabla 68: Relación entre la edad y la necesidad de consulta con Toxicología durante la estancia en el servicio de urgencias hospitalario

\begin{tabular}{|c|ccc|}
\hline Toxicología & $\mathrm{N}$ & Media & Desviación típica \\
\hline NO & 338 & 11,82 & 5,89 \\
SI & 86 & 5,47 & 5,23 \\
& & $\mathrm{p}=0,0001$ & \\
\hline
\end{tabular}

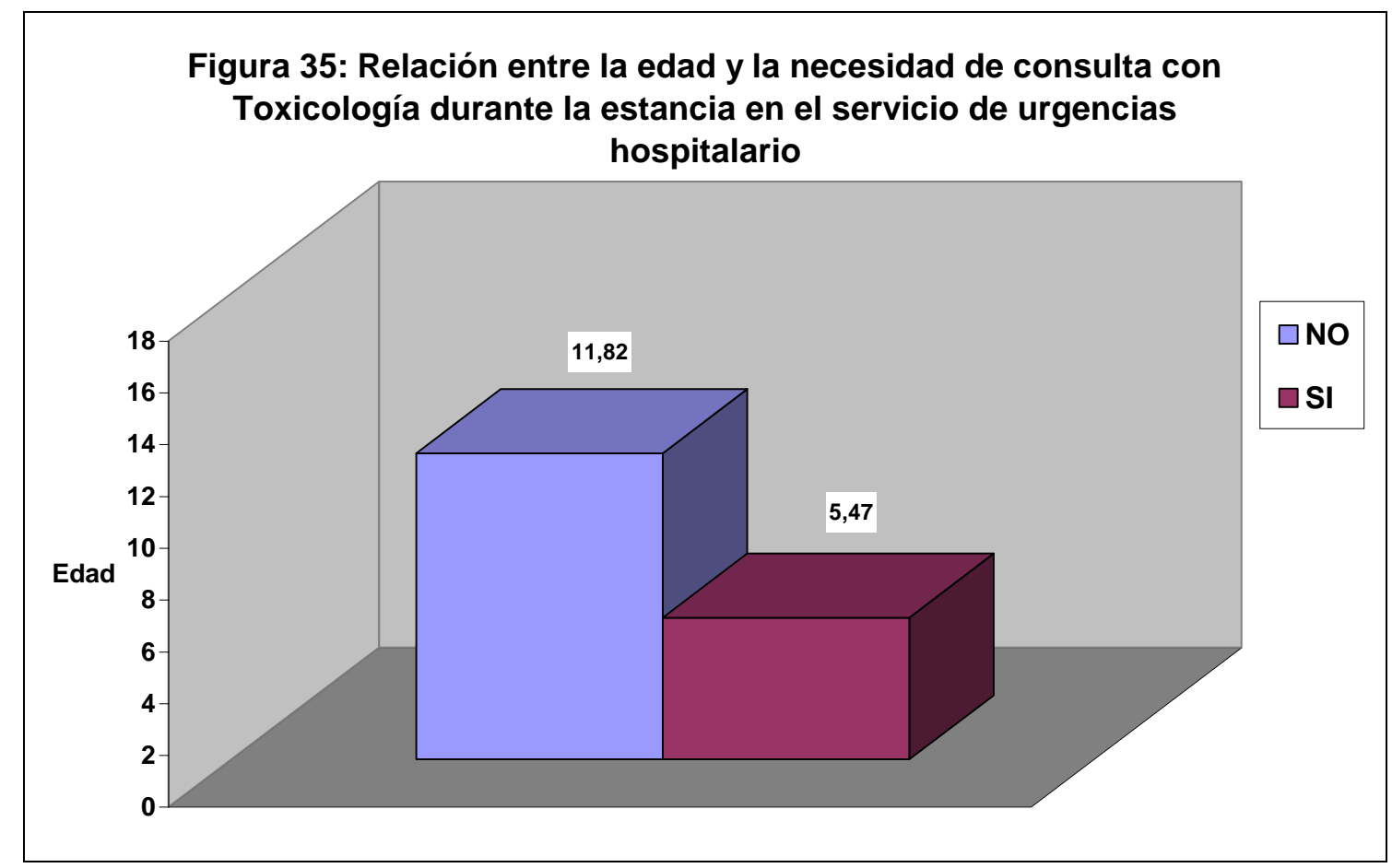

$p=0,0001$ 
En la tabla 69, se muestra la necesidad de consultar con Toxicología distribuida según grupos de edad.

Tabla 69: Interconsulta con Toxicología según grupos de edad

\begin{tabular}{|c|cccc|}
\hline & NO & $\%$ & SI & $\%$ \\
\hline Menor o igual 7 años & 88 & $26,03 \%$ & 60 & $69,76 \%$ \\
8-13 años & 27 & $7,98 \%$ & 17 & $19,76 \%$ \\
Mayor o igual 14 años & 223 & $65,97 \%$ & 9 & $10,46 \%$ \\
\hline & 338 & $100 \%$ & 86 & $100 \%$ \\
\hline
\end{tabular}

\subsection{Relación entre la edad y la propiedad de la medicación implicada en} las intoxicaciones medicamentosas.

Se evidenciaron diferencias estadísticamente significativas entre la propiedad de los fármacos en las intoxicaciones medicamentosas en el servicio de urgencias hospitalario teniendo en cuenta su edad $(p=0,025)$. Tabla 70 , figura 36.

Tabla 70: Relación entre la edad y la propiedad de los fármacos en las intoxicaciones medicamentosas

\begin{tabular}{|crcc|}
\hline Propietario de la Medicación & N & Media & Desviación típica \\
Paciente & 120 & 5,57 & 5,56 \\
Familiar & 84 & 4,01 & 4,33 \\
& $\mathrm{p}=0,025$ & & \\
\hline
\end{tabular}


Figura 36: Relación entre la edad y la propiedad de los fármacos en las intoxicaciones medicamentosas

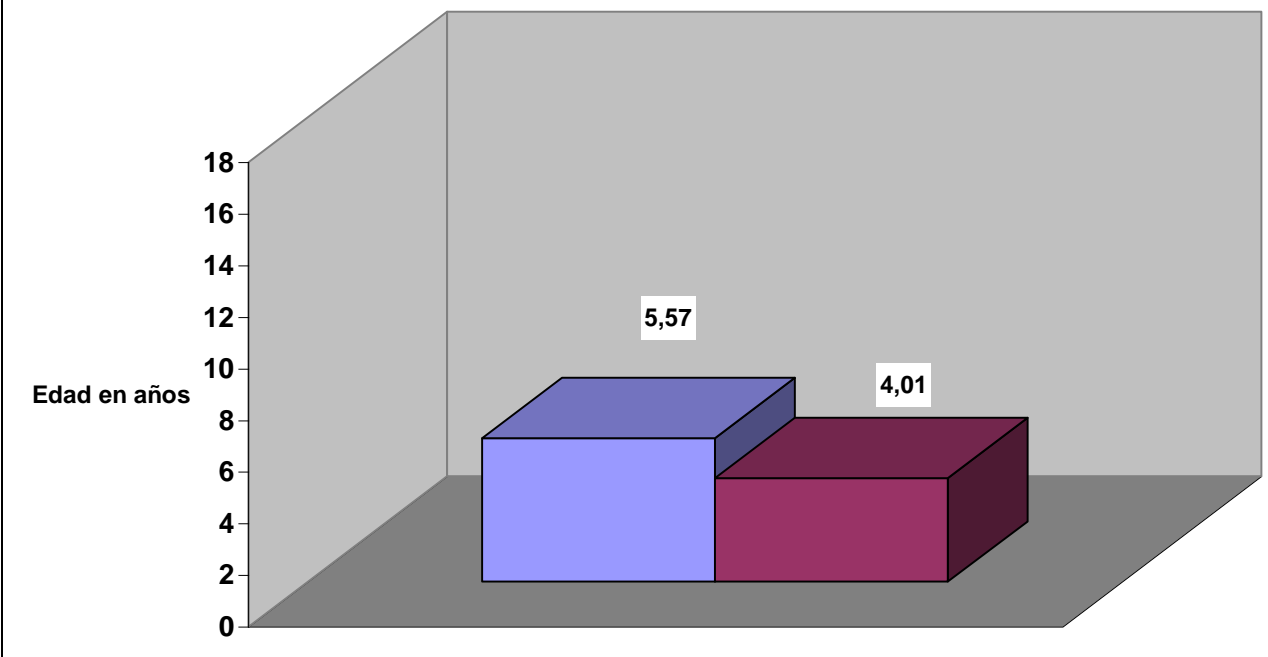

$p=0,025$

4.19. Relación entre la edad, el mecanismo de origen y los síntomas iniciales de la intoxicación por monóxido de carbono.

Si se divide a la población en mayores o iguales de 14 años, que son los pacientes atendidos habitualmente en los servicios de urgencias generales, y los menores de 14 años atendidos en Urgencias pediátricas; no se evidencian diferencias estadísticamente significativas entre el mecanismo de origen de las intoxicaciones por monóxido de carbono, teniendo en cuenta esta división de edad $(p=0,35)$; ni entre los síntomas iniciales de dicha intoxicación y esta división de edad $(p=0,20)$

Si la división en grupos de edad se establece en los 7 años, como en muchos trabajos de epidemiológicos españoles, no se evidencian diferencias estadísticamente significativas entre las el mecanismo de origen de las intoxicaciones por monóxido de carbono, teniendo en cuenta esta división de edad $(p=0,093)$; ni entre los síntomas iniciales de dicha intoxicación y la división de edad $(p=0,87)$ 
4.20. Relación entre la edad y el número de veces que acuden al servicio de urgencias hospitalario los pacientes en el mismo año en el que se produce la intoxicación.

Se evidenciaron diferencias estadísticamente significativas entre el número de veces que acuden al servicio de urgencias hospitalaria el mismo año que lo han hecho con el diagnóstico de intoxicación teniendo en cuenta su edad $(p=0,0001)$. Tabla 71 .

Tabla 71: Relación entre la existencia de consultas al servicio de urgencias hospitalarias el mismo año que lo han hecho por la intoxicación

\begin{tabular}{|crrc|}
\hline Consultas urgentes el mismo año & $\mathrm{N}$ & Media & Desviación típica \\
\hline$>=1$ & 250 & 8,1 & 6,84 \\
$<1$ & 744 & 10,83 & 6,14 \\
& $\mathrm{p}=0,0001$ & & \\
\hline
\end{tabular}

Si se divide a la población en mayores o iguales de 14 años, que son los pacientes atendidos habitualmente en los servicios de urgencias generales, y los menores de 14 años atendidos en Urgencias pediátricas; se evidencian diferencias estadísticamente significativas entre el número de veces que acuden al servicio de urgencias hospitalario en el mismo año que lo hacen por la intoxicación, teniendo en cuenta esta división de edad $(\mathbf{p}=\mathbf{0 , 0 0 0 1 )}$ Tabla 103 .Si la división en grupos de edad se establece en los 7 años, como en muchos trabajos de epidemiológicos españoles, se evidencian diferencias estadísticamente significativas entre las el número de veces que acuden al servicio de urgencias hospitalario el mismo año que lo hacen por la intoxicación, teniendo en cuenta esta división de edad ( $p=$ 0,0001) Tabla 72.

Tabla 72: Número de consultas al servicio de urgencias hospitalario el mismo año de la intoxicación distribuidos por grupos de edad

\begin{tabular}{|c|cccccccc|}
\hline & 0 & 1 & 2 & 3 & 4 & 5 & 6 & 7 \\
\hline Menor o igual 7 años & 253 & 71 & 28 & 13 & 10 & 8 & 5 & 1 \\
8-13 años & 68 & 6 & 2 & 0 & 1 & 0 & 0 & 1 \\
Mayor o igual 14 años & 423 & 74 & 20 & 7 & 1 & 0 & 2 & 0 \\
\hline & 744 & 151 & 50 & 20 & 12 & 8 & 7 & 2 \\
\hline & $\mathrm{p}=0,001$ \\
\end{tabular}


4.21. Relación entre la edad y el número de veces que acuden al servicio de urgencias hospitalario los pacientes en años previos al que se produce la intoxicación.

Se evidenciaron diferencias estadísticamente significativas entre el número de veces que acuden al servicio de urgencias hospitalaria en años previos al que lo han hecho con el diagnóstico de intoxicación teniendo en cuenta su edad $(p=0,027)$. Tabla 73 .

Tabla 73: Relación entre la presencia de consultas al servicio de urgencias hospitalario en los años previos a la intoxicación y la edad del paciente

\begin{tabular}{|cccc|}
\hline Consulta urgentes años previos & $\mathrm{N}$ & Media edad en años & Desviación típica \\
\hline$>=1$ & 661 & 9,83 & 6,48 \\
$<1$ & 333 & 10,78 & 6,29 \\
& $\mathrm{p}=0,027$ & & \\
\hline
\end{tabular}

4.22. Relación entre la edad y el número de veces que acuden al servicio de urgencias hospitalario los pacientes en años posteriores al que se produce la intoxicación.

Se evidenciaron diferencias estadísticamente significativas entre el número de veces que acuden al servicio de urgencias hospitalaria en años posteriores al que lo han hecho con el diagnóstico de intoxicación teniendo en cuenta su edad $(p=0,0001)$. Tabla 74 .

Tabla 74: Relación entre la existencia de consultas al servicio de urgencias hospitalarias en años posteriores al que lo han hecho por la intoxicación

\begin{tabular}{|cccc|}
\hline Consultas urgentes en años posteriores & $N$ & Media & Desviación típica \\
\hline$>=1$ & 492 & 9,09 & 6,55 \\
$<1$ & 478 & 11,26 & 6,07 \\
& $p=0,0001$ & & \\
\hline
\end{tabular}

Si se divide a la población en mayores o iguales de $\mathbf{1 4}$ años, que son los pacientes atendidos habitualmente en los servicios de urgencias generales, y los menores de 14 años atendidos en Urgencias pediátricas; se evidencian 
diferencias estadísticamente significativas entre el número de veces que acuden al servicio de urgencias hospitalario en años posteriores al que lo hacen por la intoxicación, teniendo en cuenta esta división de edad $(p=0,0001)$

Si la división en grupos de edad se establece en los 7 años, como en muchos trabajos de epidemiológicos españoles, se evidencian diferencias estadísticamente significativas entre las el número de veces que acuden al servicio de urgencias hospitalario en años posteriores al que lo hacen por la intoxicación, teniendo en cuenta esta división de edad $(p=\mathbf{0 , 0 0 0 1})$ Tabla 75.

Tabla 75: Número de consultas al servicio de urgencias hospitalarias en años posteriores a la intoxicación distribuidos por grupos de edad

\begin{tabular}{|c|c|c|c|c|c|c|c|c|c|c|c|c|c|c|c|c|c|c|c|c|c|c|c|c|}
\hline & 0 & 1 & 2 & 3 & 4 & 5 & 6 & 7 & 8 & 9 & $\begin{array}{l}1 \\
0\end{array}$ & 1 & $\begin{array}{l}1 \\
2\end{array}$ & 1 & 1 & 1 & 1 & $\begin{array}{l}1 \\
7\end{array}$ & 1 & 2 & 2 & $\begin{array}{l}2 \\
3\end{array}$ & 3 & 3 \\
\hline Menor o igual 7 & 14 & & 4 & 2 & 3 & 2 & 1 & 1 & & 1 & & & & & & & & & & & & & & \\
\hline años & 9 & 41 & 4 & 3 & 5 & 1 & 1 & 3 & 5 & 2 & 7 & 0 & 2 & 1 & 2 & 3 & 1 & 3 & 1 & 1 & 0 & 2 & 1 & 1 \\
\hline 8-13 años & 42 & 17 & 7 & 4 & 3 & 1 & 2 & 0 & 1 & 1 & 0 & 0 & 0 & 0 & 0 & 0 & 0 & 0 & 0 & 0 & 0 & 0 & 0 & 0 \\
\hline $\begin{array}{l}\text { Mayor o igual } \\
14 \text { años }\end{array}$ & $\begin{array}{c}28 \\
7\end{array}$ & 89 & $\begin{array}{l}4 \\
0\end{array}$ & $\begin{array}{l}2 \\
7\end{array}$ & $\begin{array}{l}1 \\
9\end{array}$ & $\begin{array}{l}1 \\
7\end{array}$ & 9 & 6 & 5 & 3 & 2 & 1 & 2 & 0 & 1 & 1 & 3 & 0 & 0 & 0 & 1 & 0 & 0 & 0 \\
\hline & $\begin{array}{c}47 \\
8\end{array}$ & $\begin{array}{c}14 \\
7\end{array}$ & $\begin{array}{l}9 \\
1\end{array}$ & $\begin{array}{l}5 \\
4\end{array}$ & $\begin{array}{l}5 \\
7\end{array}$ & $\begin{array}{l}3 \\
9\end{array}$ & $\begin{array}{l}2 \\
2\end{array}$ & $\begin{array}{l}1 \\
9\end{array}$ & $\begin{array}{l}1 \\
1\end{array}$ & $\begin{array}{l}1 \\
6\end{array}$ & 9 & 1 & 4 & 1 & 3 & 4 & 4 & 3 & 1 & 1 & 1 & 2 & 1 & 1 \\
\hline & & & & & & & & & & & & & & & & & & & & & & & & \\
\hline
\end{tabular}

\subsection{Relación entre la edad y el número de consultas ambulatorias.}

Si se divide a la población en mayores o iguales de 14 años, que son los pacientes atendidos habitualmente en los servicios de urgencias generales, y los menores de 14 años atendidos en Urgencias pediátricas; se evidencian diferencias estadísticamente significativas entre el número de consultas ambulatorias, teniendo en cuenta esta división de edad $(p=0,0001)$

Si la división en grupos de edad se establece en los 7 años, como en muchos trabajos de epidemiológicos españoles, se evidencian diferencias estadísticamente significativas entre las el número de consultas ambulatorias al que acuden, teniendo en cuenta esta división de edad ( $p=$ 0,0001) Tabla 76. 
Tabla 76: Relación entre la edad y el número de consultas ambulatorias

\begin{tabular}{|c|ccc|}
\hline Edad & $\mathrm{N}$ & Media & Desviación típica \\
\hline$>=14$ & 523 & 1,80 & 1,40 \\
$<14$ & 458 & 1,36 & 1,30 \\
& \multicolumn{2}{c}{$\mathrm{p}=0,0001$} \\
$>=7$ & 613 & 1,78 & 1,41 \\
$<7$ & 368 & 1,29 & 1,26 \\
\multicolumn{2}{r}{} & $\mathrm{p}=0,0001$ & \\
\hline
\end{tabular}

\section{Incidencia temporal.}

\subsection{Pacientes atendidos cada año en el servicio de urgencias}

\section{hospitalario.}

El año con un mayor número de intoxicaciones atendidas en urgencias fue el 2007 y el que menor el año 2004. (Tabla 77, Figura 37)

Tabla 77: Pacientes atendidos cada año

\begin{tabular}{|ccc|}
\hline Año de atención & Frecuencia & Porcentaje \\
\hline 2001 & 87 & 8,80 \\
$\mathbf{2 0 0 2}$ & 92 & 9,30 \\
2003 & 103 & 10,40 \\
$\mathbf{2 0 0 4}$ & 55 & 5,50 \\
2005 & 65 & 6,50 \\
$\mathbf{2 0 0 6}$ & 92 & 9,30 \\
$\mathbf{2 0 0 7}$ & 149 & 15,00 \\
$\mathbf{2 0 0 8}$ & 119 & 12,00 \\
\hline $\mathbf{2 0 0 9}$ & 119 & 12,00 \\
$\mathbf{2 0 1 0}$ & 113 & 11,40 \\
\hline
\end{tabular}




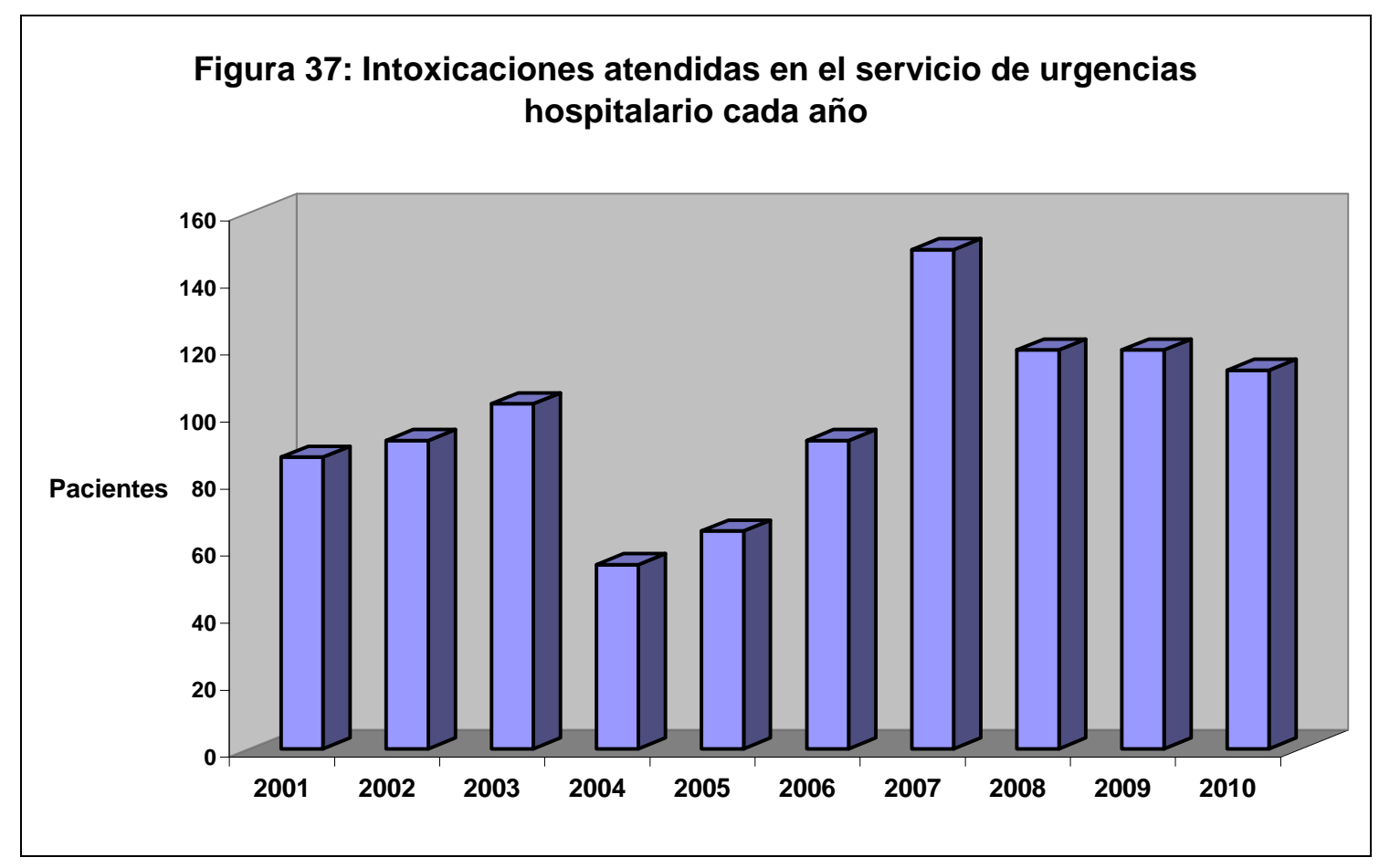

5.2 Pacientes atendidos cada mes en el servicio de urgencias hospitalario con diagnóstico de intoxicación.

Se analiza la incidencia en cada uno de los meses por separado, como se muestra en la Tabla 78 y figura 38.

Tabla 78: Pacientes intoxicados atendidos cada mes

\begin{tabular}{|ccc|}
\hline & Frecuencia & Porcentaje válido \\
Enero & 66 & 6,63 \\
Febrero & 82 & 8,24 \\
Marzo & 98 & 9,85 \\
\hline Abril & 83 & 8,35 \\
Mayo & 87 & 8,75 \\
Junio & 96 & 9,65 \\
Julio & 76 & 7,64 \\
Agosto & 69 & 6,94 \\
Septiembre & 82 & 8,24 \\
\hline Octubre & 81 & 8,14 \\
Noviembre & 81 & 8,14 \\
Diciembre & 93 & 9,35 \\
Total & 994 & 100 \\
\hline
\end{tabular}




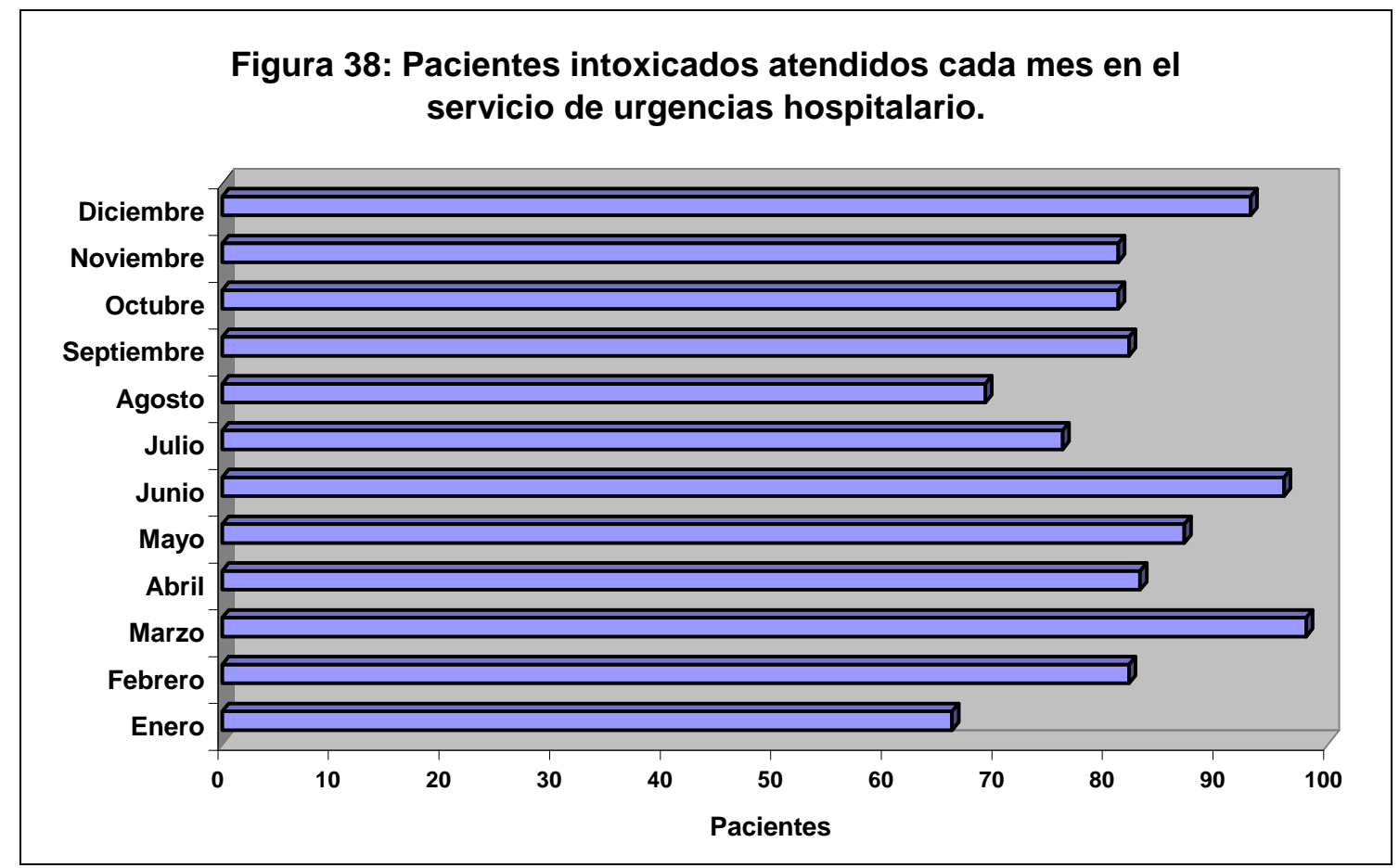

\subsection{Pacientes atendidos según la hora de admisión al servicio de} urgencias hospitalario.

Se registran los pacientes que son atendidos en el servicio de urgencias hospitalario con el diagnóstico de intoxicación según la hora de admisión al mismo, como se observa en la tabla 79, figura 39.

Tabla 79: Pacientes atendidos cada hora

\begin{tabular}{|ccc|}
\hline Hora de atención & Frecuencia & Porcentaje \\
\hline 0:00 - 0:59 & 66 & 6,60 \\
1:00 - 1:59 & 51 & 5,10 \\
2:00 - 2:59 & 23 & 2,30 \\
$3: 00-3: 59$ & 26 & 2,60 \\
$4: 00-4: 59$ & 16 & 1,60 \\
5:00 - 5:59 & 10 & 1,00 \\
$6: 00-6: 59$ & 10 & 1,00 \\
7:00 - 7:59 & 3 & 0,30 \\
$8: 00-8: 59$ & 4 & 0,40 \\
9:00-9:59 & 10 & 1,00 \\
$10: 00-10: 59$ & 13 & 1,30 \\
$11: 00-11: 59$ & 15 & 1,50 \\
& &
\end{tabular}




\begin{tabular}{|ccc|}
\hline $12: 00-12: 59$ & 32 & 3,20 \\
\hline $13: 00-13: 59$ & 33 & 3,30 \\
$14: 00-14: 59$ & 32 & 3,20 \\
$15: 00-15: 59$ & 43 & 4,30 \\
$16: 00-16: 59$ & 32 & 3,20 \\
$17: 00-17: 59$ & 36 & 3,60 \\
$18: 00-18: 59$ & 33 & 3,30 \\
$19: 00-19: 59$ & 43 & 4,30 \\
\hline $20: 00-20: 59$ & 78 & 7,80 \\
\hline $21: 00-21: 59$ & 117 & 11,80 \\
\hline $22: 00-22: 59$ & 149 & 15,00 \\
\hline $23: 00-23: 59$ & 119 & 12,00 \\
\hline
\end{tabular}

Figura 39: Intoxicaciones admitidas en el servicio de urgencias hospitalario cada hora

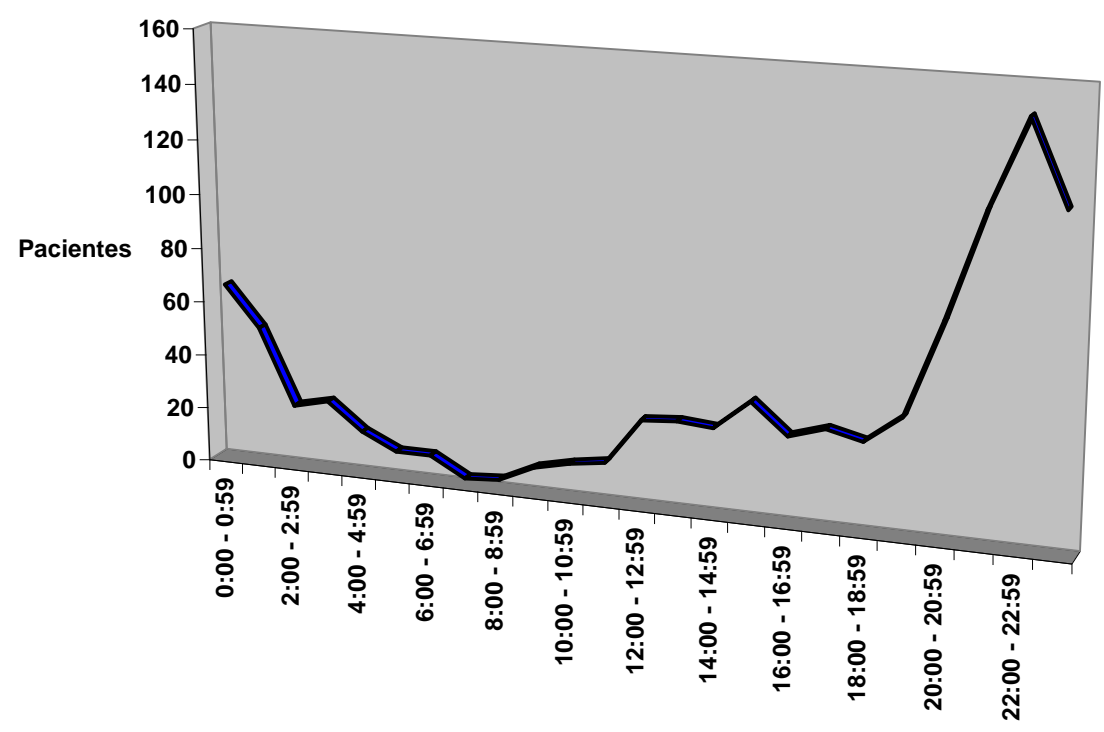




\section{Agente causal de la intoxicación.}

En este apartado vamos valorar las intoxicaciones más frecuentes.

\subsection{Intoxicación etílica.}

6.1.1. Distribución de la intoxicación etílica según el sexo.

La distribución por sexos en las intoxicaciones etílicas es 55,3\% en el caso de los hombres y $44,7 \%$ en las mujeres. Tabla 80 , figura 40 .

Tabla 80: Distribución por sexos en la intoxicación etílica

\begin{tabular}{|c|cc|}
\hline \multicolumn{2}{|c|}{ Frecuencia } & Porcentaje válido \\
\hline Hombre & 240 & 55,30 \\
Mujer & 194 & 44,70 \\
\hline Total & 434 & 100 \\
\hline \multicolumn{3}{|c|}{$\mathrm{p}=0,38$} \\
\hline
\end{tabular}

Figura 40: Distribución por sexos en la intoxicación etílica
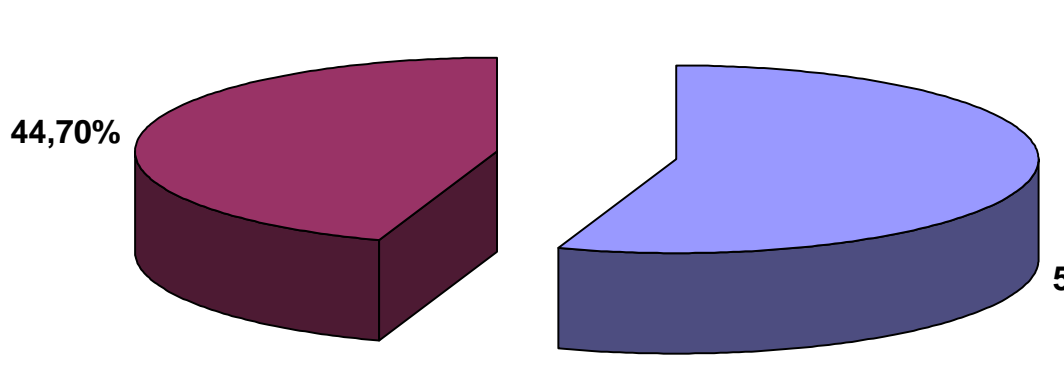

$p=0,38$ 
No se evidenciaron diferencias estadísticamente significativas en el sexo de los intoxicados teniendo en cuenta el diagnóstico de intoxicación etílica $(p=0,38)$

\subsubsection{Distribución por edades de la intoxicación etílica.}

La distribución por edades en las intoxicaciones etílicas es la que se muestra en la tabla 81 y figura 41.

Tabla 81: Distribución por edades de la intoxicación etílica

\begin{tabular}{|c|cc|}
\hline Edad & Frecuencia & Porcentaje \\
\hline 1 & 2 & 0,46 \\
2 & 1 & 0,23 \\
12 & 1 & 0,23 \\
13 & 19 & 4,37 \\
14 & 72 & 16,58 \\
15 & 116 & 26,72 \\
16 & 120 & 27,64 \\
17 & 103 & 23,73 \\
Total & 434 & 100 \\
\hline
\end{tabular}

Figura 41: Distribución por edades en la intoxicación etílica

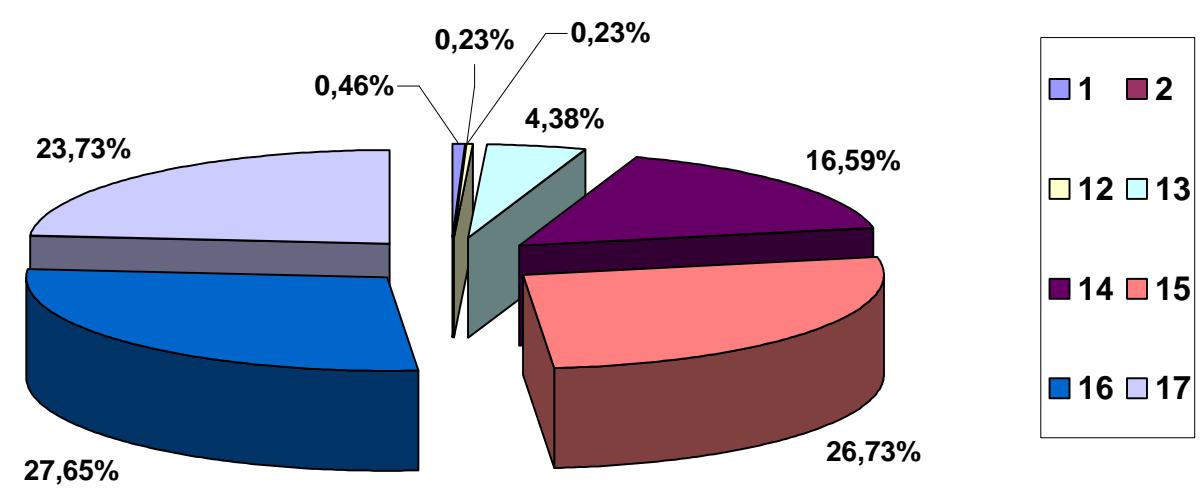


Se evidenciaron diferencias estadísticamente significativas en la edad de los intoxicados teniendo en cuenta el diagnóstico de intoxicación etílica $(p=$ 0,0001). Tabla 82, figura 42 .

Tabla 82: Relación entre la edad y el diagnóstico de intoxicación etílica

\begin{tabular}{|c|ccc|}
\hline Diagnóstico & $\mathrm{N}$ & Media & Desviación típica \\
\hline Intoxicación etílica & 434 & 15,39 & 1,65 \\
Resto de diagnósticos & 560 & 6,08 & 5,79 \\
& $\mathrm{p}=0,0001$ & \\
\hline
\end{tabular}

Figura 42: Relación entre la edad y el diagnóstico en el servicio de urgencias hospitalario de intoxicación etílica

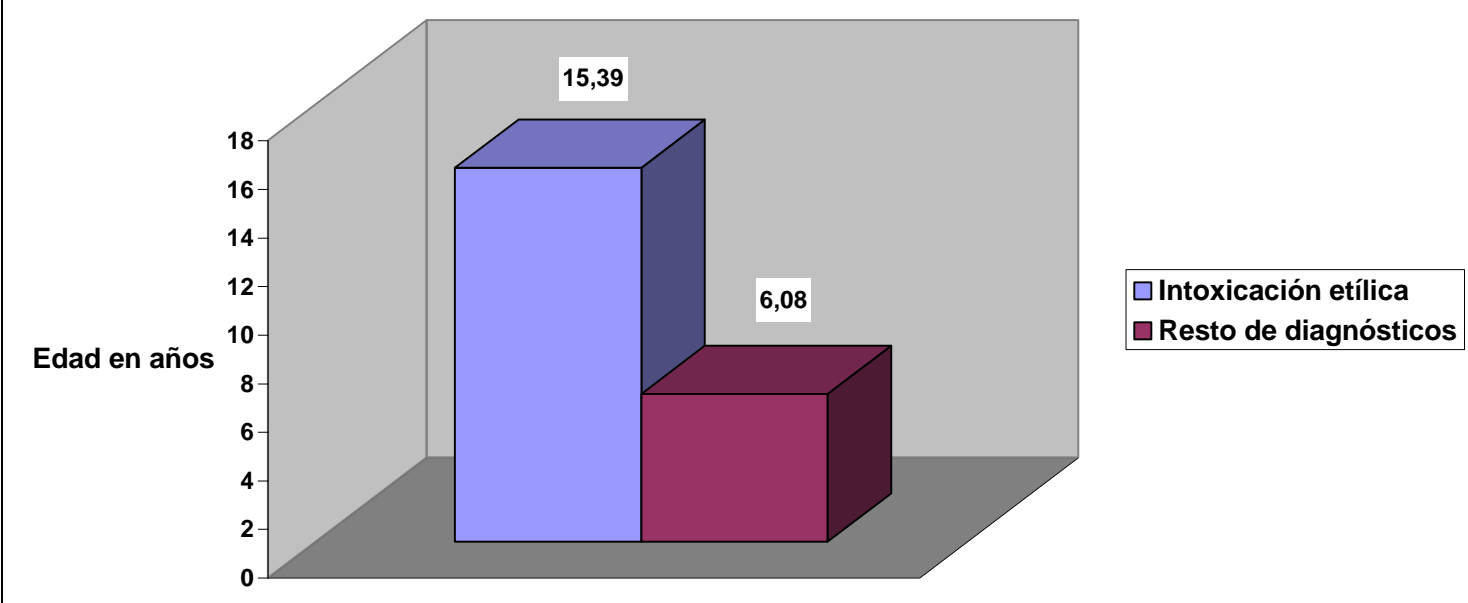

$p=0,0001$

6.1.3. Distribución del diagnóstico de intoxicación etílica en el servicio de urgencias hospitalario según los años de atención.

La distribución por años de la intoxicación etílica se muestra en la tabla 83 y figura 43. 
Tabla 83: Distribución por años en la intoxicación etílica

\begin{tabular}{|c|cc|}
\hline Año de atención & Frecuencia & Porcentaje válido \\
\hline 2001 & 39 & 8,98 \\
2002 & 25 & 5,76 \\
2003 & 31 & 7,14 \\
2004 & 21 & 4,83 \\
2005 & 30 & 6,91 \\
2006 & 38 & 8,75 \\
2007 & 70 & 16,12 \\
2008 & 65 & 14,97 \\
2009 & 56 & 12,90 \\
\hline 2010 & 59 & 13,59 \\
\hline Total & 434 & 100 \\
\hline
\end{tabular}

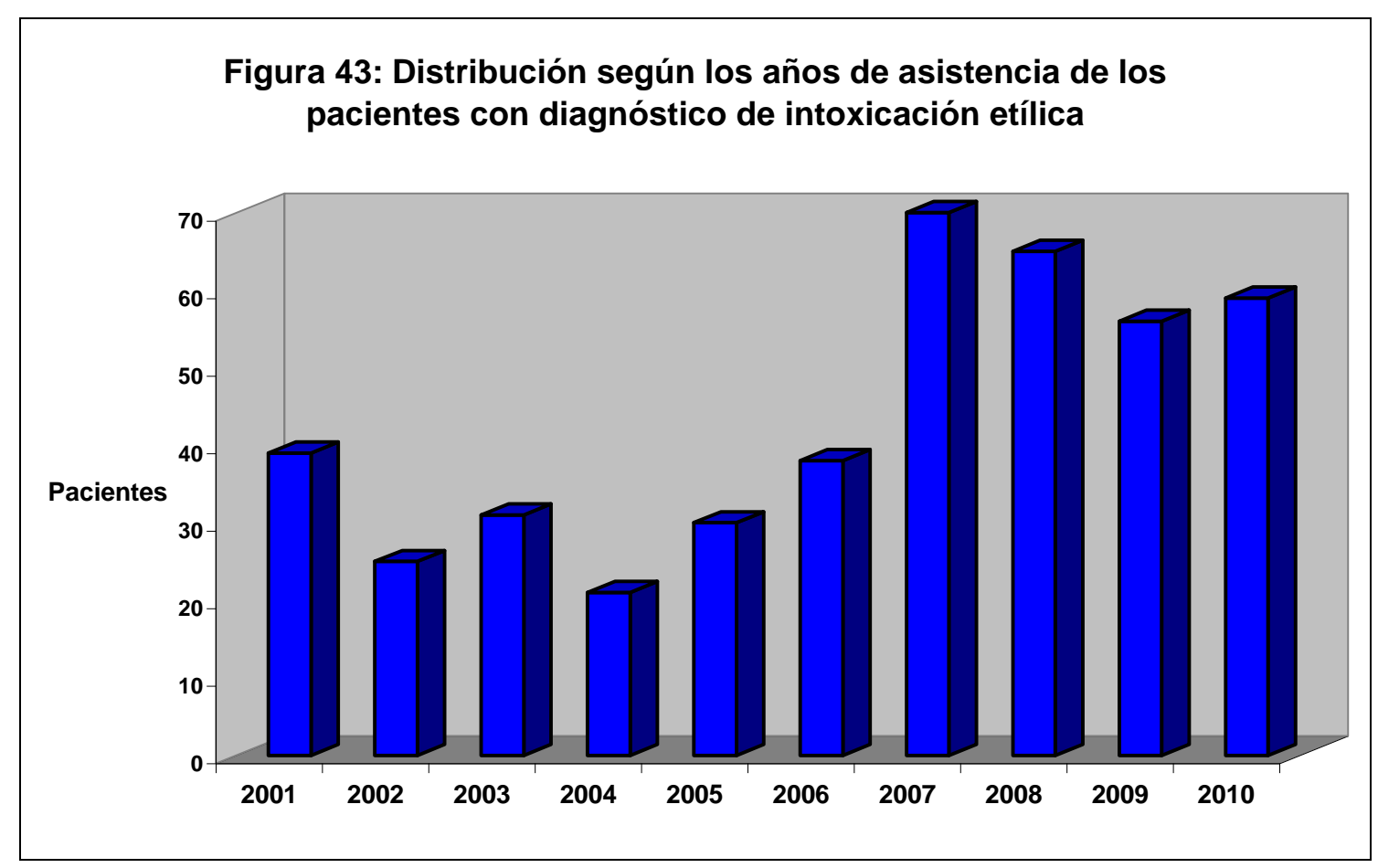

Se evidenciaron diferencias estadísticamente significativas en el año de la atención teniendo en cuenta el diagnóstico de intoxicación etílica ( $p=$ 0,001). Tabla 84, figura 44. 
Tabla 84: Relación entre el año de intoxicación y el diagnóstico de intoxicación etílica

\begin{tabular}{|c|c|c|c|c|c|c|c|c|c|c|}
\hline & 2001 & 2002 & 2003 & 2004 & 2005 & 2006 & 2007 & 2008 & 2009 & 2010 \\
\hline $\begin{array}{c}\text { Resto de } \\
\text { diagnósticos }\end{array}$ & 48 & 67 & 72 & 34 & 35 & 54 & 79 & 54 & 63 & 54 \\
\hline $\begin{array}{c}\text { Intoxicación } \\
\text { etílica }\end{array}$ & 39 & 25 & 31 & 21 & 30 & 38 & 70 & 65 & 56 & 59 \\
\hline Total & 87 & 92 & 103 & 55 & 65 & 92 & 149 & 119 & 119 & 113 \\
\hline \multicolumn{11}{|c|}{$p=0,001$} \\
\hline
\end{tabular}

Figura 44 : Relación entre el año de atención y el diagnóstico

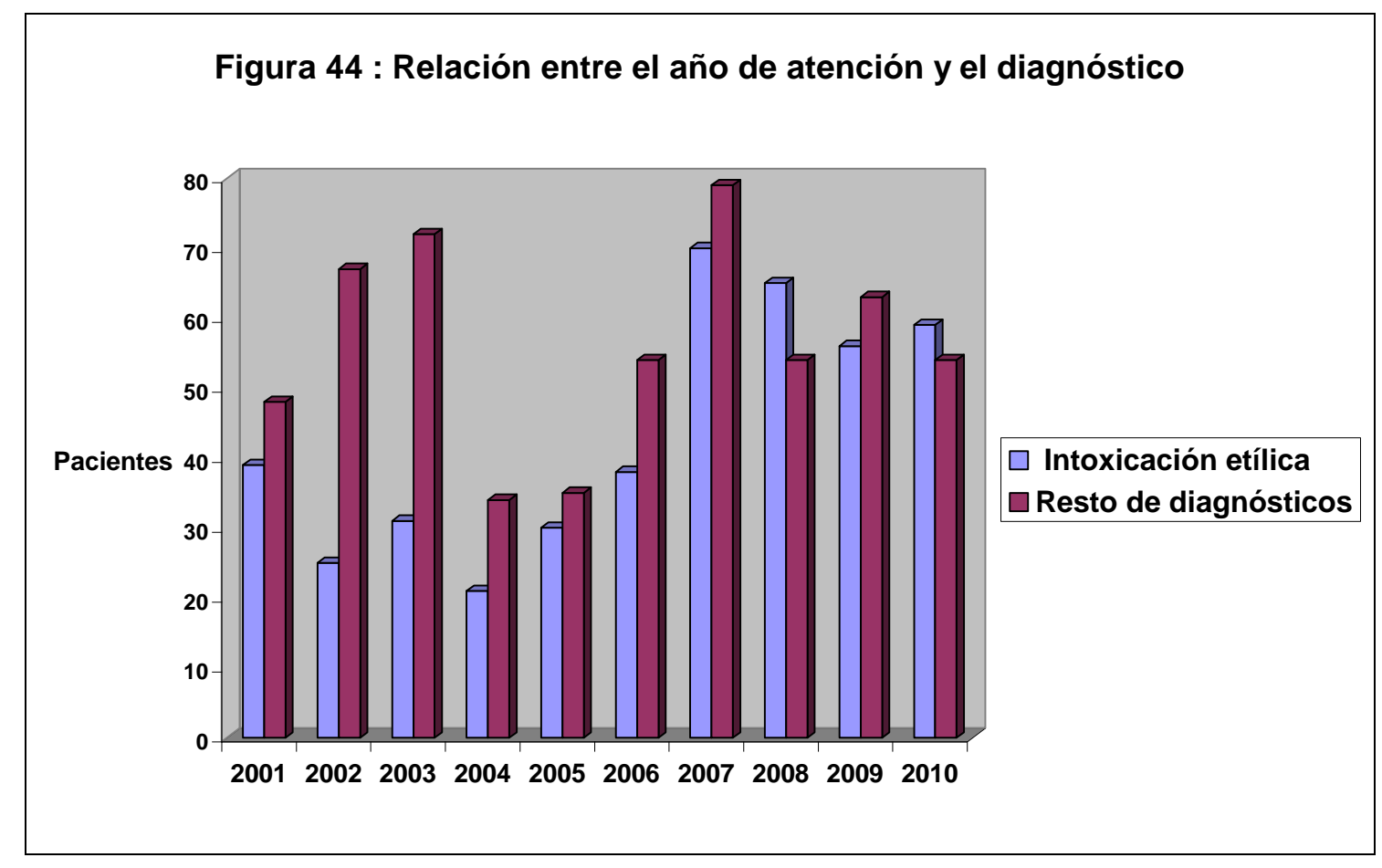

$p=0,001$ 
En la tabla 85, se muestra la edad media de atención en el servicio de urgencias hospitalario cada año con el diagnóstico de intoxicación etílica.

Tabla 85: Edad media de asistencia en el servicio de urgencias hospitalario cada año

\begin{tabular}{|cccc|}
\hline Año de atención & Edad media en años & $\mathbf{N}$ & Desviación típica \\
\hline 2001 & 15,44 & 39 & 0,912 \\
2002 & 15,28 & 25 & 3,14 \\
2003 & 15,77 & 31 & 1,17 \\
2004 & 15,29 & 21 & 1,14 \\
2005 & 15,67 & 30 & 1,18 \\
2006 & 15,66 & 38 & 0.99 \\
2007 & 15,66 & 70 & 1,10 \\
2008 & 15,43 & 65 & 1,19 \\
2009 & 15,11 & 56 & 2,16 \\
2010 & 14,88 & 59 & 2,27 \\
Total & 15,40 & 434 & 1,65 \\
\hline
\end{tabular}

\subsubsection{Distribución por meses en la intoxicación etílica.}

La distribución por meses en la intoxicación etílica es la siguiente. Tabla 86, figura 45.

Tabla 86: Distribución por meses en la intoxicación etílica

\begin{tabular}{|c|cc|}
\hline Mes de atención & Frecuencia & Porcentaje \\
\hline Enero & 26 & 5,99 \\
\hline Febrero & 28 & 6,45 \\
Marzo & 42 & 9,67 \\
Abril & 35 & 8,06 \\
Mayo & 43 & 9,9 \\
\hline Junio & 45 & 10,36 \\
Julio & 27 & 6,22 \\
Agosto & 36 & 8,29 \\
Septiembre & 51 & 11,75 \\
\hline Octubre & 29 & 6,68 \\
\hline Noviembre & 34 & 7,83 \\
\hline Diciembre & 38 & 8,75 \\
\hline Total & 434 & 100 \\
\hline
\end{tabular}




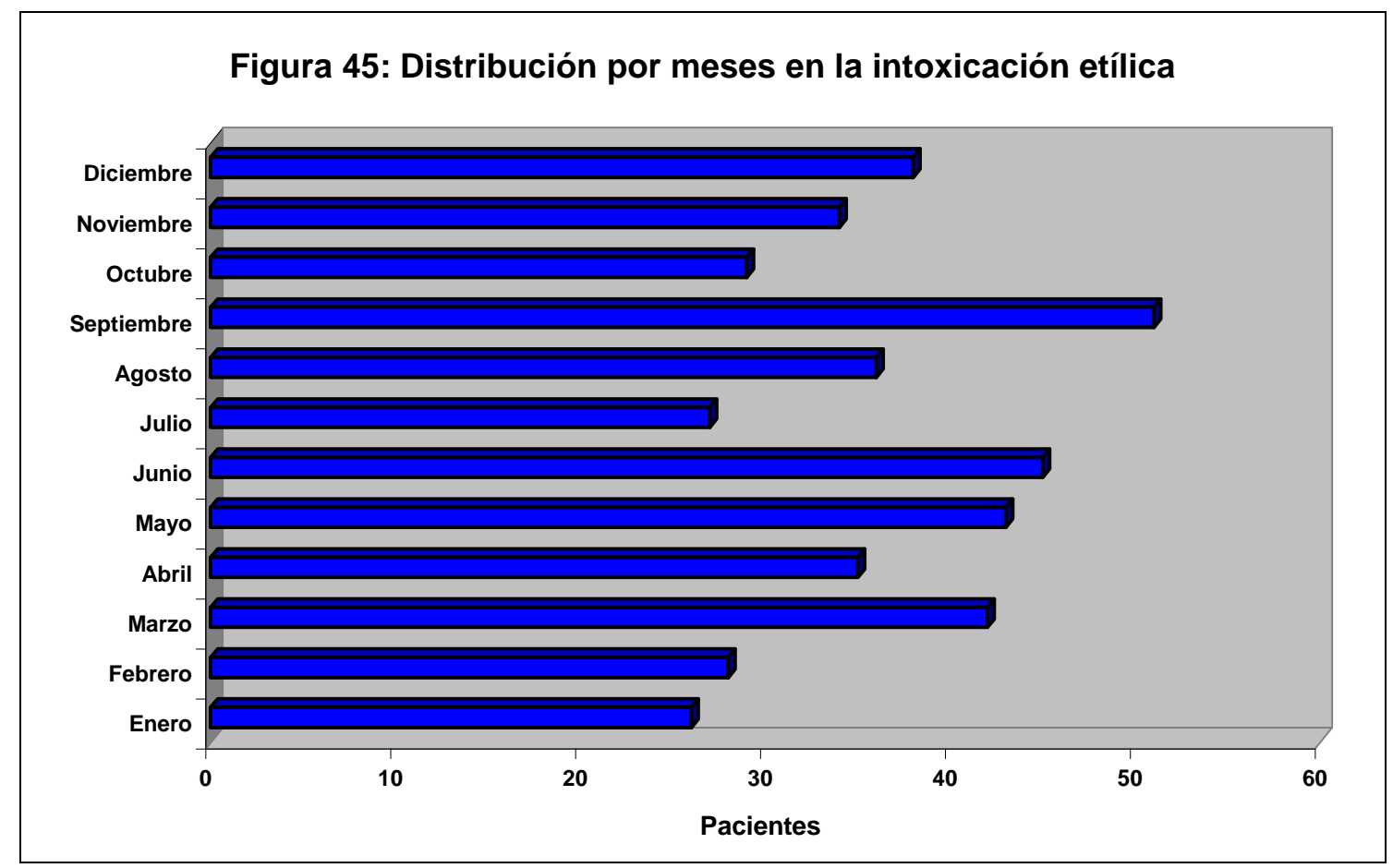

Se evidenciaron diferencias estadísticamente significativas en el mes de la atención teniendo en cuenta el diagnóstico de intoxicación etílica ( $p=$ 0,017). Tabla 87, figura 46.

Tabla 87: Distribución de los diagnósticos según los meses del año

\begin{tabular}{|c|c|c|c|c|c|c|c|c|c|c|c|c|}
\hline & $\begin{array}{c}\text { Ene } \\
\text { ro }\end{array}$ & $\begin{array}{l}\text { Febr } \\
\text { ero }\end{array}$ & $\begin{array}{c}\text { Mar } \\
\text { zo }\end{array}$ & $\begin{array}{l}\mathrm{Ab} \\
\mathrm{ril}\end{array}$ & $\begin{array}{l}\text { Ma } \\
\text { yo }\end{array}$ & $\begin{array}{c}\text { Jun } \\
\text { io }\end{array}$ & $\begin{array}{l}\text { Jul } \\
\text { io }\end{array}$ & $\begin{array}{l}\text { Agos } \\
\text { to }\end{array}$ & $\begin{array}{l}\text { Septie } \\
\text { mbre }\end{array}$ & $\begin{array}{c}\text { Octu } \\
\text { bre }\end{array}$ & $\begin{array}{c}\text { Noviem } \\
\text { bre }\end{array}$ & $\begin{array}{c}\text { Diciem } \\
\text { bre }\end{array}$ \\
\hline $\begin{array}{c}\text { Resto de } \\
\text { diagnósticos }\end{array}$ & 40 & 54 & 56 & 48 & 44 & 51 & 49 & 33 & 31 & 52 & 47 & 55 \\
\hline $\begin{array}{c}\text { Intoxicación } \\
\text { etílica }\end{array}$ & 26 & 28 & 42 & 35 & 43 & 45 & 27 & 36 & 51 & 29 & 34 & 38 \\
\hline Total & 66 & 82 & 98 & 83 & 87 & 96 & 76 & 69 & 82 & 81 & 81 & 93 \\
\hline \multicolumn{13}{|c|}{$p=0,017$} \\
\hline
\end{tabular}




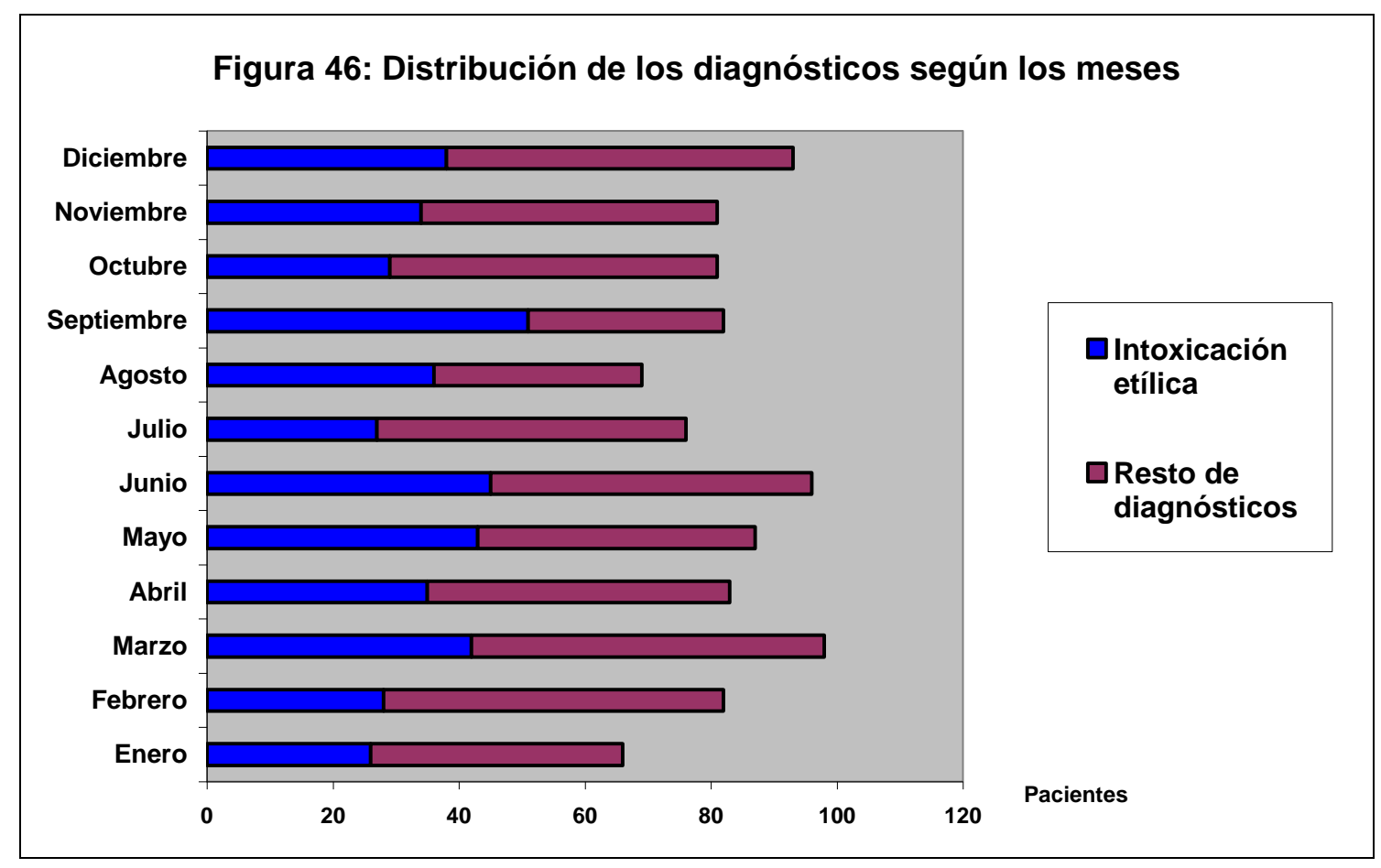

$p=0,017$ 


\subsubsection{Distribución por horas de la intoxicación etílica.}

La distribución de la intoxicación etílica según la hora de admisión en el servicio de urgencias hospitalarias es la que se muestra en la tabla 88 , figura 47.

Tabla 88: Distribución de la intoxicación etílica según la hora de admisión en el servicio de urgencias hospitalarias

\begin{tabular}{|c|cc|}
\hline & Frecuencia & Porcentaje \\
\hline $00: 00-00: 59$ & 40 & 9,21 \\
$01: 00-01: 59$ & 33 & 7,6 \\
\hline $02: 00-02: 59$ & 20 & 4,6 \\
$03: 00-03: 59$ & 16 & 3,68 \\
\hline $04: 00-04: 59$ & 11 & 2,53 \\
\hline $05: 00-05: 59$ & 8 & 1,84 \\
\hline $06: 00-06: 59$ & 6 & 1,38 \\
\hline $07: 00-07: 59$ & 1 & 0,23 \\
\hline $11: 00-11: 59$ & 1 & 0,23 \\
\hline $12: 00-12: 59$ & 1 & 0,23 \\
\hline $13: 00-13: 59$ & 2 & 0,46 \\
\hline $14: 00-14: 59$ & 1 & 0,23 \\
\hline $15: 00-15: 59$ & 2 & 0,46 \\
\hline $16: 00-16: 59$ & 2 & 0,46 \\
\hline $17: 00-17: 59$ & 2 & 0,46 \\
\hline $18: 00-18: 59$ & 4 & 0,92 \\
\hline $19: 00-19: 59$ & 6 & 1,38 \\
\hline $20: 00-20: 59$ & 30 & 6,91 \\
\hline $21: 00-21: 59$ & 77 & 17,74 \\
\hline $22: 00-22: 59$ & 96 & 22,11 \\
\hline $23: 00-23: 59$ & 75 & 17,28 \\
\hline Total & 434 & 100 \\
\hline & & \\
\hline
\end{tabular}




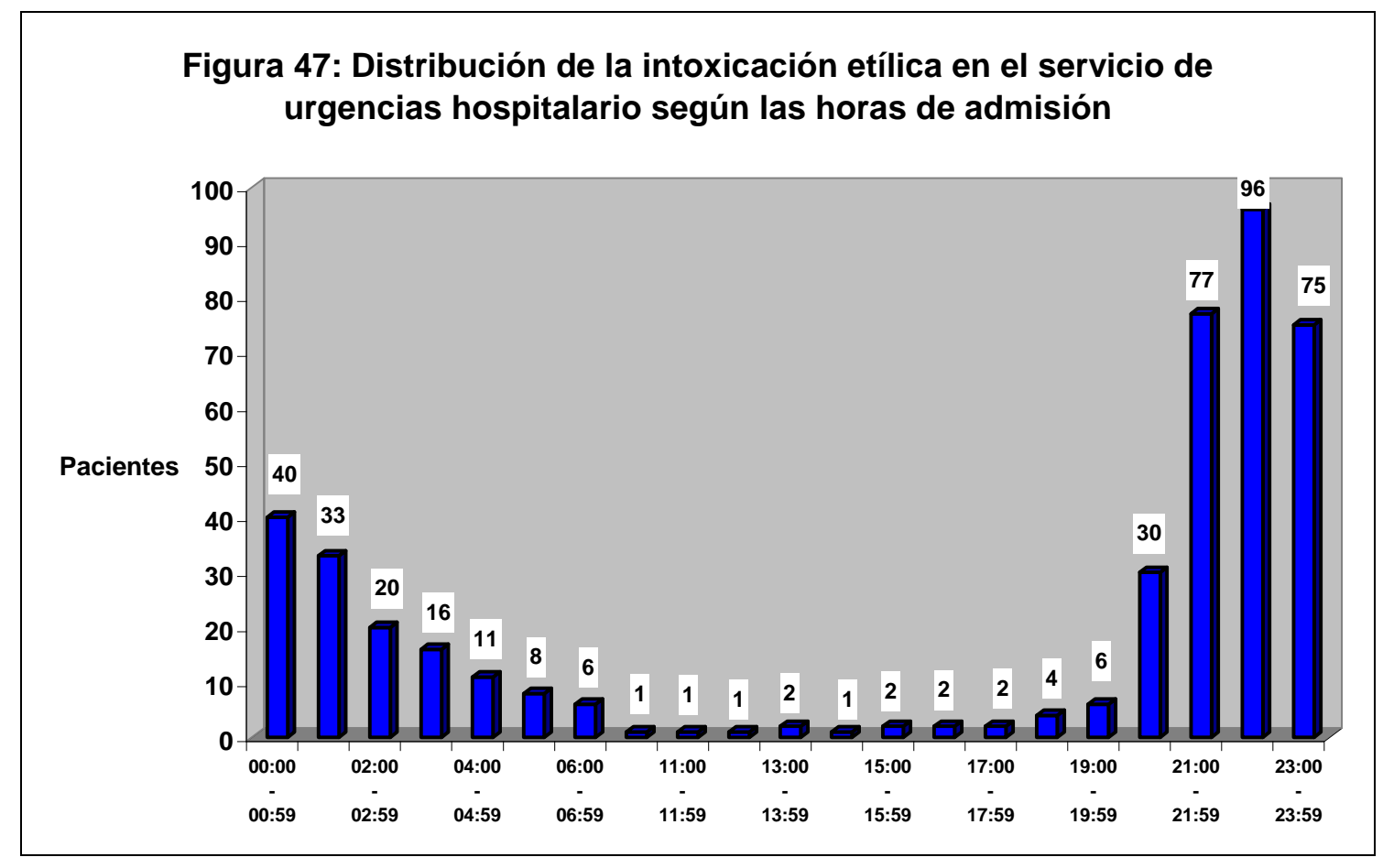

Se evidenciaron diferencias estadísticamente significativas en la hora de la admisión en el servicio de urgencias hospitalaria teniendo en cuenta el diagnóstico de intoxicación etílica $(p=\mathbf{0 , 0 0 0 1})$. Tabla 89, figura 48 .

Tabla 89: Relación entre el diagnóstico de intoxicación etílica y las horas de admisión en el servicio de urgencias hospitalario

\begin{tabular}{|c|c|c|c|c|c|c|c|c|c|c|c|c|}
\hline & $\begin{array}{c}00: 00 \\
- \\
00: 59\end{array}$ & $\begin{array}{c}01: 00 \\
- \\
01: 59\end{array}$ & $\begin{array}{c}02: 00 \\
- \\
02: 59\end{array}$ & $\begin{array}{c}03: 00 \\
- \\
03: 59\end{array}$ & $\begin{array}{c}04: 00 \\
- \\
04: 59\end{array}$ & $\begin{array}{c}05: 00 \\
- \\
05: 59\end{array}$ & $\begin{array}{c}06: 00 \\
- \\
06: 59\end{array}$ & $\begin{array}{c}07: 00 \\
- \\
07: 59\end{array}$ & $\begin{array}{c}08: 00 \\
- \\
08: 59\end{array}$ & $\begin{array}{c}09: 00 \\
- \\
09: 59\end{array}$ & $\begin{array}{c}10: 00 \\
- \\
10: 59\end{array}$ & $\begin{array}{c}11: 00 \\
- \\
11: 59\end{array}$ \\
\hline $\begin{array}{c}\text { Resto } \\
\text { diagnós } \\
\text { ticos }\end{array}$ & 26 & 18 & 3 & 10 & 5 & 2 & 4 & 2 & 4 & 10 & 13 & 14 \\
\hline $\begin{array}{c}\text { Intoxica } \\
\text { ción } \\
\text { etílica }\end{array}$ & 40 & 33 & 20 & 16 & 11 & 8 & 6 & 1 & 0 & 0 & 0 & 1 \\
\hline Total & 66 & 51 & 23 & 26 & 16 & 10 & 10 & 3 & 4 & 10 & 13 & 15 \\
\hline & $\begin{array}{c}12: 00 \\
- \\
-\end{array}$ & $\begin{array}{c}13: 00 \\
- \\
12.50\end{array}$ & $\begin{array}{c}14: 00 \\
-\end{array}$ & $\begin{array}{c}15: 00 \\
- \\
15.50\end{array}$ & $\begin{array}{c}16: 00 \\
- \\
-\end{array}$ & $\begin{array}{c}17: 00 \\
- \\
17.50\end{array}$ & $\begin{array}{c}18: 00 \\
- \\
10.50\end{array}$ & $\begin{array}{c}19: 00 \\
- \\
10.50\end{array}$ & $\begin{array}{c}20: 00 \\
-\end{array}$ & $\begin{array}{c}21: 00 \\
-\end{array}$ & $\begin{array}{c}22: 00 \\
-\end{array}$ & $\begin{array}{c}23: 00 \\
-\end{array}$ \\
\hline & \begin{tabular}{|c|}
$12: 59$ \\
31
\end{tabular} & $\frac{13: 59}{31}$ & $\begin{array}{c}14: 59 \\
31\end{array}$ & $\frac{15: 59}{41}$ & $\frac{16: 59}{30}$ & $\frac{17: 59}{34}$ & $\frac{18: 59}{29}$ & $\frac{19: 59}{37}$ & $\begin{array}{c}20: 59 \\
48\end{array}$ & $\begin{array}{c}21: 59 \\
40\end{array}$ & $\frac{22: 59}{53}$ & $\frac{23: 59}{44}$ \\
\hline & 1 & 2 & 1 & 2 & 2 & 2 & 4 & 6 & 30 & 77 & 96 & 75 \\
\hline & 32 & 33 & 32 & 43 & 32 & 36 & 33 & 43 & 78 & 117 & 149 & 119 \\
\hline
\end{tabular}


Figura 48: Relación entre la hora de admisión en el servicio de urgencias hospitalario y el diagnóstico de intoxicación etílica

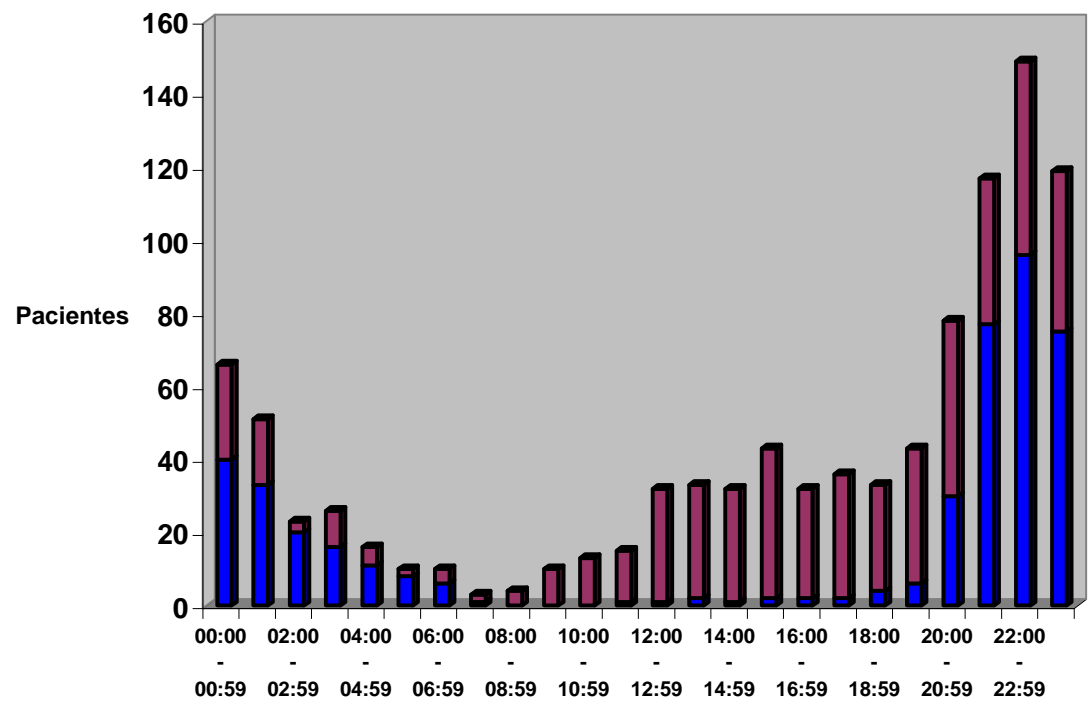

$\square$ Resto

diagnósticos

Intoxicación etílica

$p=0,0001$

\subsubsection{Distribución de la intoxicación etílica según la localidad de origen} de los pacientes.

Se distribuye a los pacientes con diagnóstico de intoxicación etílica, según la localidad de origen de los pacientes. Los resultados se muestran en la tabla 90 y figura 49.

Tabla 90: Distribución según la localidad de origen del paciente con intoxicación etílica

\begin{tabular}{|c|cc|}
\hline & Frecuencia & Porcentaje \\
\hline Valladolid & 328 & 75,57 \\
\hline Arroyo-La Flecha & 22 & 5,06 \\
Laguna de Duero & 18 & 4,14 \\
La Cisterniga & 7 & 1,61 \\
Tordesillas & 2 & 0,46 \\
Resto provincia de Valladolid & 44 & 10,13 \\
Comunidad de Castilla y León & 5 & 1,15 \\
Resto de España & 8 & 1,84 \\
\hline Total & 434 & 100 \\
\hline
\end{tabular}


Figura 49: Distribución de los pacientes con intoxicación etílica según su localidad de origen
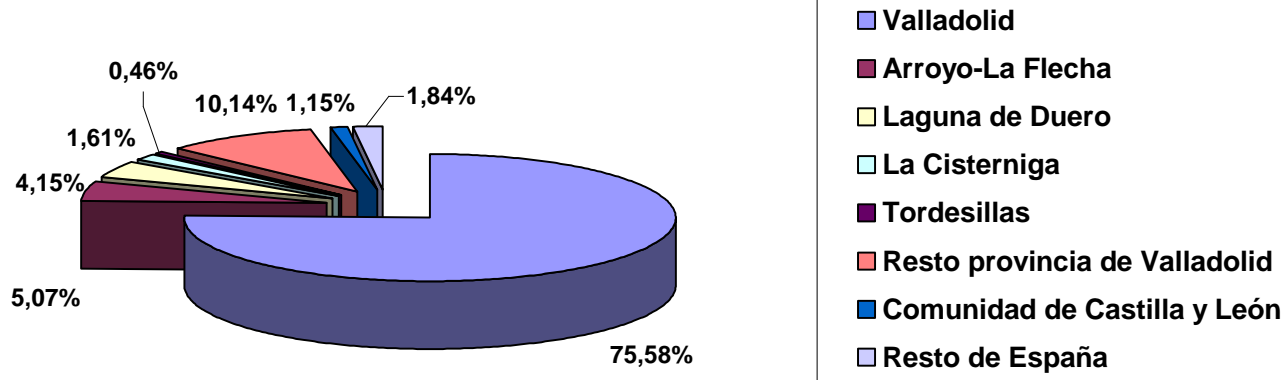

Se evidenciaron diferencias estadísticamente significativas en el origen de los pacientes teniendo en cuenta el diagnóstico de intoxicación etílica ( $p=$ 0,0001). Tabla 91, figura 50.

Tabla 91: Relación entre la localidad de origen de los pacientes y el diagnóstico de intoxicación etílica

\begin{tabular}{|c|cccccccc|}
\hline & $\begin{array}{c}\text { Valla } \\
\text { dolid }\end{array}$ & $\begin{array}{c}\text { Arroyo- } \\
\text { Llecha }\end{array}$ & $\begin{array}{c}\text { Laguna } \\
\text { de } \\
\text { Duero }\end{array}$ & $\begin{array}{c}\text { La } \\
\text { Cister } \\
\text { niga }\end{array}$ & $\begin{array}{c}\text { Tord } \\
\text { esilla } \\
\text { s }\end{array}$ & $\begin{array}{c}\text { Resto provincia } \\
\text { de Valladolid }\end{array}$ & $\begin{array}{c}\text { Comunidad de } \\
\text { Castilla y León }\end{array}$ & $\begin{array}{c}\text { Resto de } \\
\text { España }\end{array}$ \\
\hline $\begin{array}{c}\text { Resto } \\
\text { diagnósti } \\
\text { cos }\end{array}$ & 306 & 19 & 61 & 24 & 11 & 114 & 8 & 17 \\
$\begin{array}{c}\text { Intoxicaci } \\
\text { ón etílica }\end{array}$ & 328 & 22 & 18 & 7 & 2 & 44 & 5 & 8 \\
\hline Total & 634 & 41 & 79 & 31 & 13 & 158 & 13 & 25 \\
\hline \multicolumn{7}{c|}{$p=0,0001$} \\
\hline
\end{tabular}




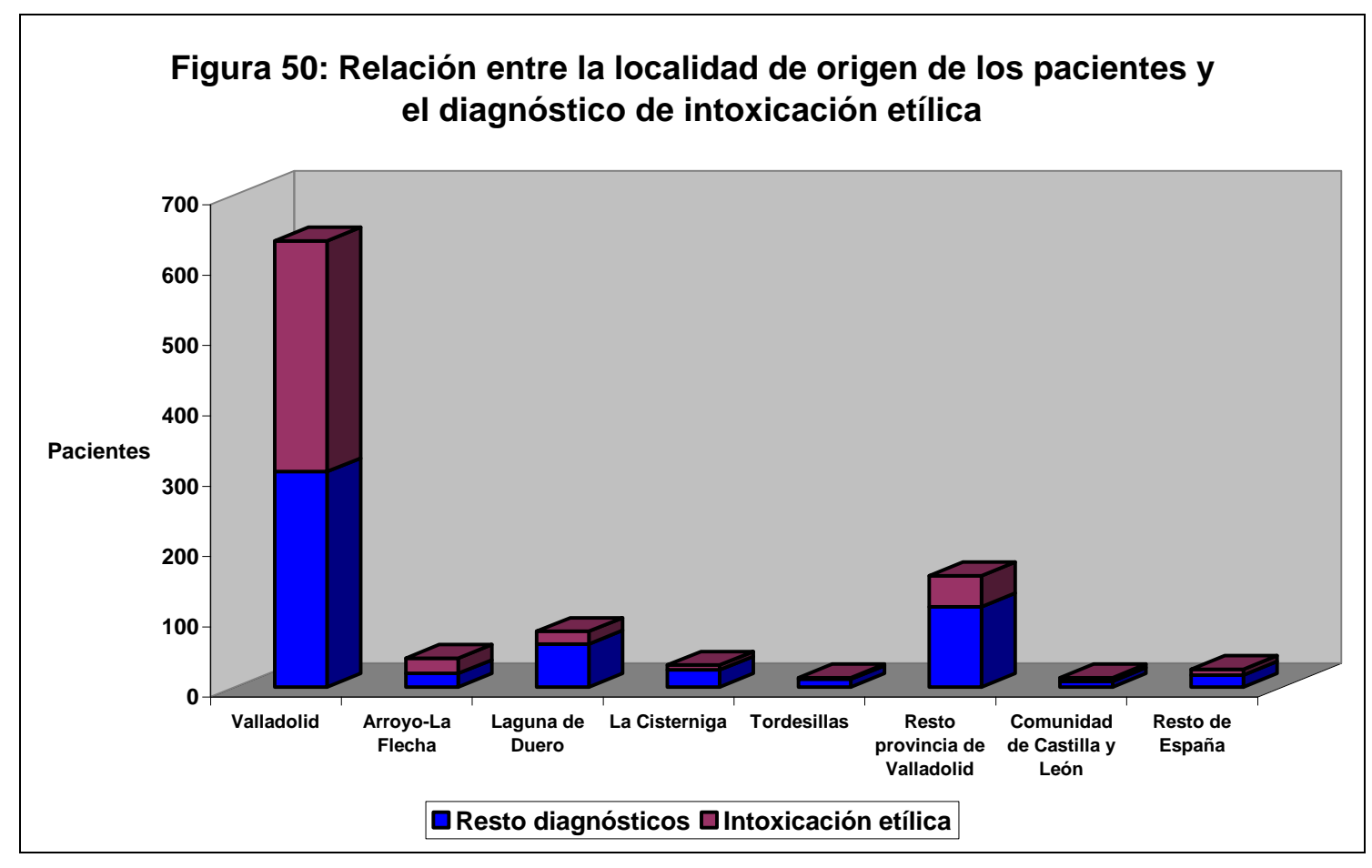

$p=0,0001$

\subsubsection{Antecedentes personales de los pacientes con intoxicación etílica}

Se distribuye a los pacientes con diagnóstico de intoxicación etílica, según la presencia o no de antecedentes personales. Los resultados se muestran en la tabla 92 y figura 51.

Tabla 92: Antecedentes personales en los pacientes con intoxicación etílica

\begin{tabular}{|c|cc|}
\hline & Frecuencia & Porcentaje \\
\hline NO & 278 & 64,06 \\
SI & 156 & 35,94 \\
\hline Total & 434 & 100 \\
\hline
\end{tabular}


Figura 51: Antecedentes personales en los intoxicados etílicos

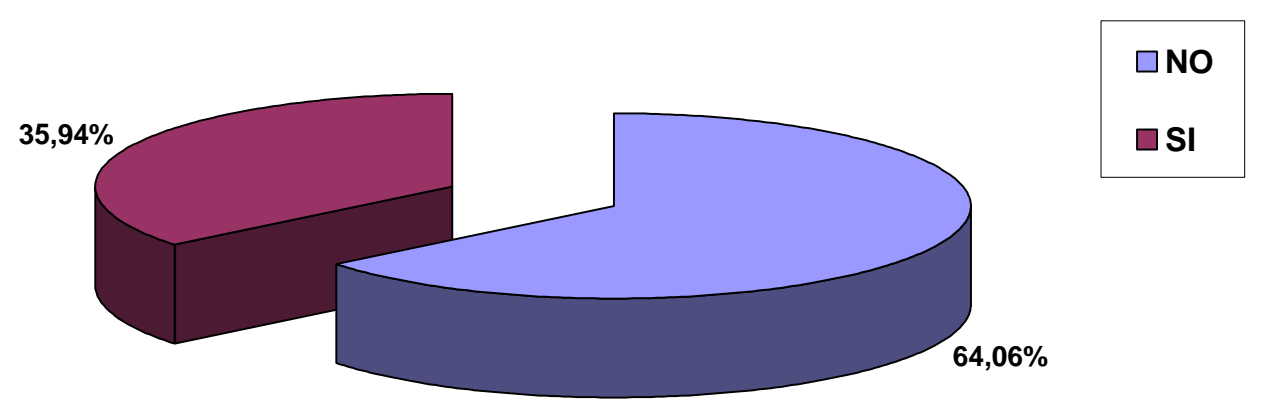

No se evidenciaron diferencias estadísticamente significativas en la presencia de antecedentes personales del paciente teniendo en cuenta el diagnóstico de intoxicación etílica. $(p=0,614)$. Tabla 93, figura 52.

Tabla 93: Relación entre la presencia de antecedentes personales y el diagnóstico de intoxicación etílica

\begin{tabular}{|c|cc|}
\hline & NO & SI \\
\hline Resto diagnósticos & 350 & 210 \\
Intoxicación etílica & 278 & 156 \\
\hline Total & 628 & 366 \\
\hline \multicolumn{2}{|c}{$\mathrm{p}=0,614$} & \\
\hline
\end{tabular}




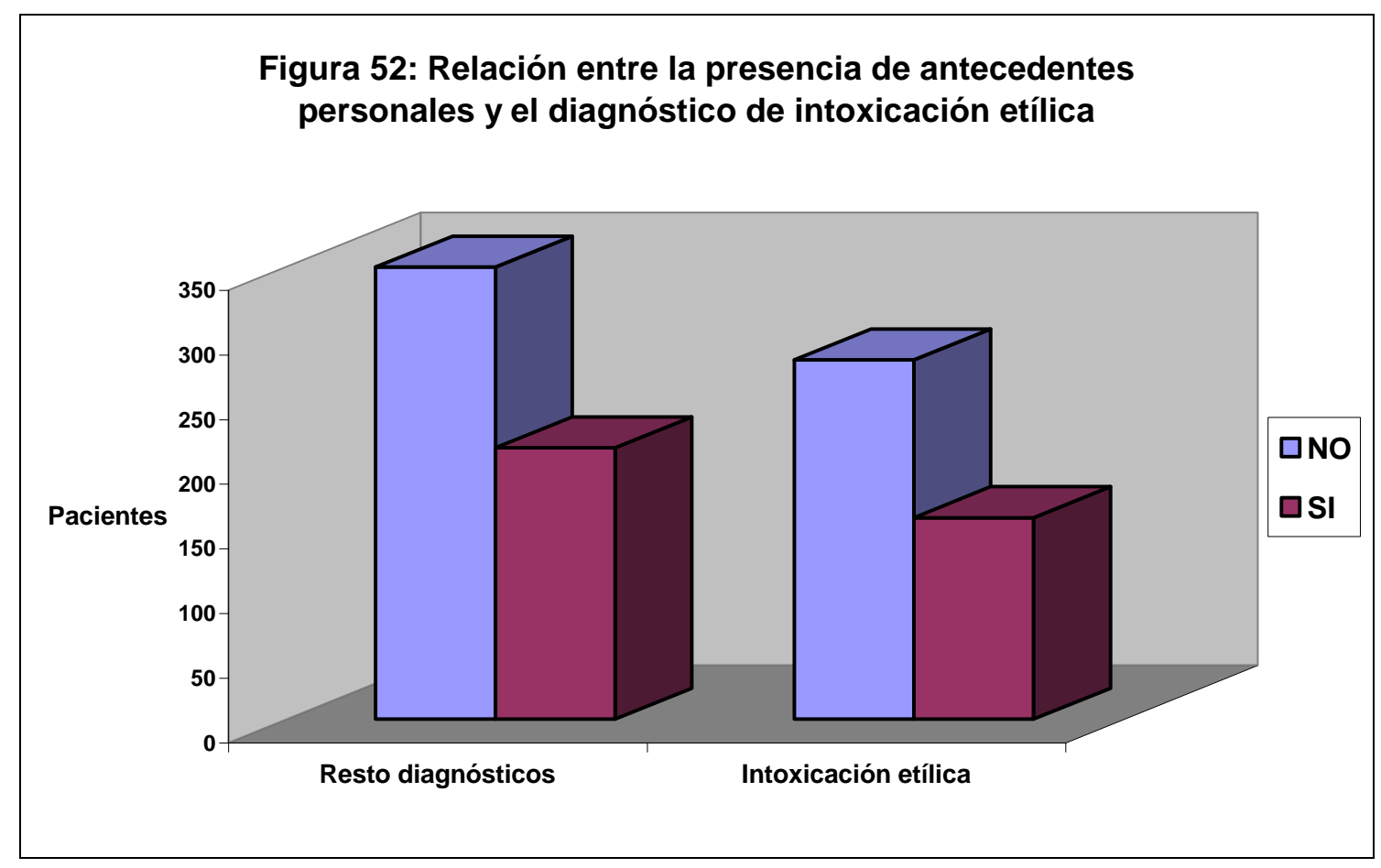

$p=0,614$

\subsubsection{Distribución de la intoxicación etílica según la derivación del} paciente al servicio de urgencias hospitalario por parte de personal sanitario.

Se distribuye a los pacientes con diagnóstico de intoxicación etílica, según la derivación al servicio de urgencias hospitalario por parte de personal sanitario. Los resultados se muestran en la tabla 94 y figura 53.

Tabla 94: Derivación al servicio de urgencias hospitalario por parte de personal sanitario de los pacientes con intoxicación etílica

\begin{tabular}{|c|cc|}
\hline & Frecuencia & Porcentaje \\
\hline NO & 271 & 96,78 \\
Centro de Salud & 9 & 3,21 \\
\hline Total & 280 & 100 \\
\hline
\end{tabular}


Figura 53: Derivación al servicio de urgencias hospitalario de los pacientes con diagnóstico de intoxicación etílica

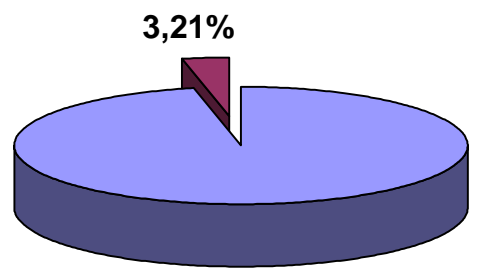

$\square$ Sin derivación

$\square$ Derivación desde el centro de Salud

$96,79 \%$

Se evidenciaron diferencias estadísticamente significativas en la derivación del paciente al servicio de urgencias hospitalario por parte de personal sanitario teniendo en cuenta el diagnóstico de intoxicación etílica $(p=0,036)$. Tabla 95, figura 54 .

Tabla 95: Relación entre la derivación del paciente al servicio de urgencias hospitalario por personal sanitario y el diagnóstico de intoxicación etílica

\begin{tabular}{|c|ccc|}
\hline & $\begin{array}{c}\text { Sin } \\
\text { derivación }\end{array}$ & $\begin{array}{c}\text { Derivación desde Centro de } \\
\text { Salud }\end{array}$ & $\begin{array}{c}\text { Derivación desde otro } \\
\text { hospital }\end{array}$ \\
\hline $\begin{array}{c}\text { Resto } \\
\text { diagnósticos }\end{array}$ & 286 & 11 & 7 \\
$\begin{array}{c}\text { Intoxicación } \\
\text { etílica }\end{array}$ & 271 & 9 & 0 \\
\hline Total & 557 & 20 & 7 \\
\hline \multicolumn{4}{|c}{} \\
\hline
\end{tabular}




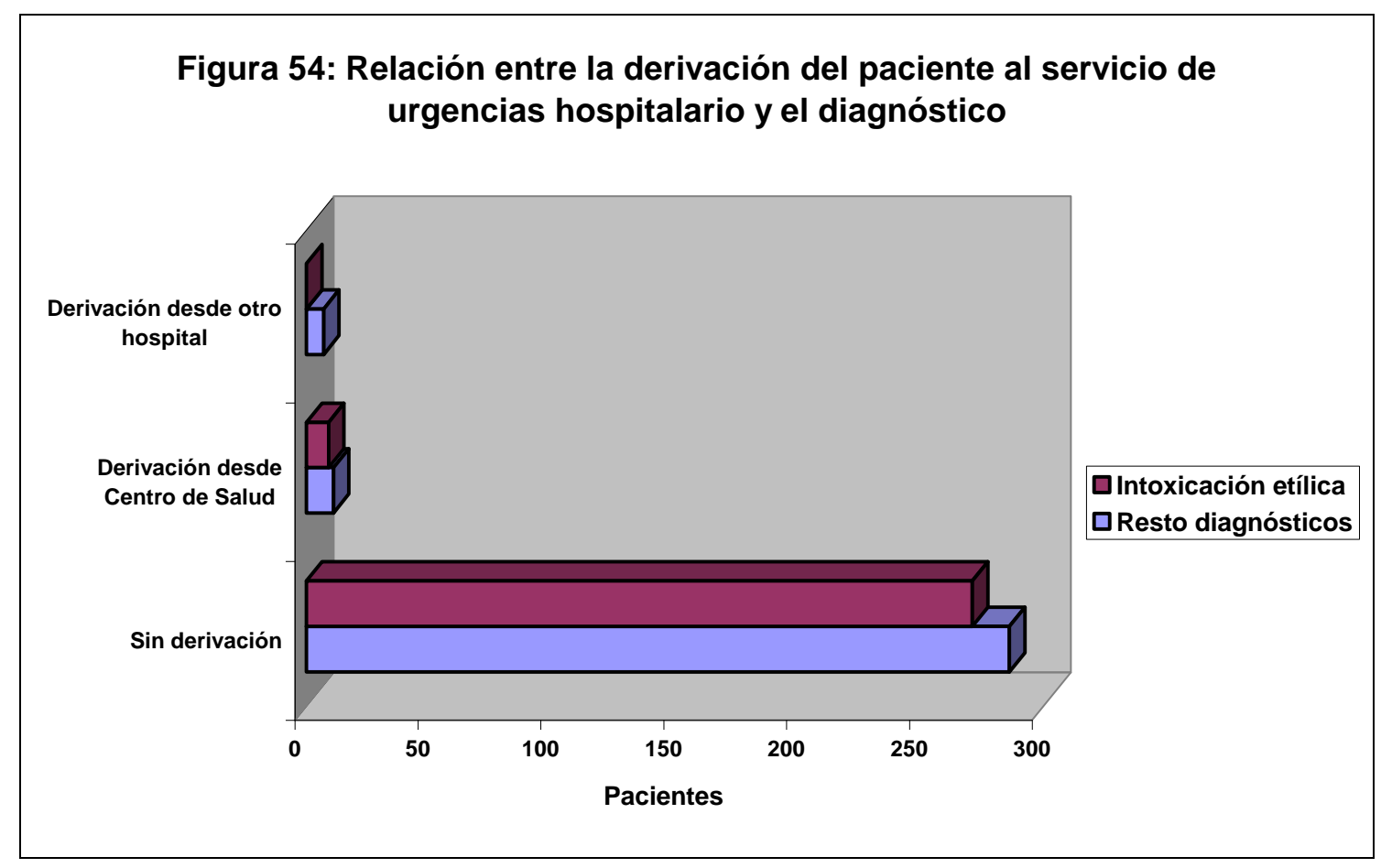

$p=0,036$

\subsubsection{Acompañamiento en el servicio de urgencias hospitalario de los pacientes con intoxicación etílica}

Se distribuye a los pacientes con diagnóstico de intoxicación etílica, según la presencia o no de acompañamiento durante su estancia en el servicio de urgencias hospitalario. Los resultados se muestran en la tabla 96 y figura 55.

Tabla 96: Acompañamiento durante la estancia en el servicio de urgencias hospitalario del paciente con intoxicación etílica

\begin{tabular}{|c|cc|}
\hline & Frecuencia & Porcentaje \\
\hline Solo & 5 & 8,62 \\
Familiar & 40 & 68,96 \\
Amigo & 12 & 20,68 \\
Representante legal & 1 & 1,72 \\
\hline Total & 58 & 100 \\
\hline
\end{tabular}


Figura 55: Acompañamiento en el servicio de urgencias

hospitalario del paciente con intoxicación etílica

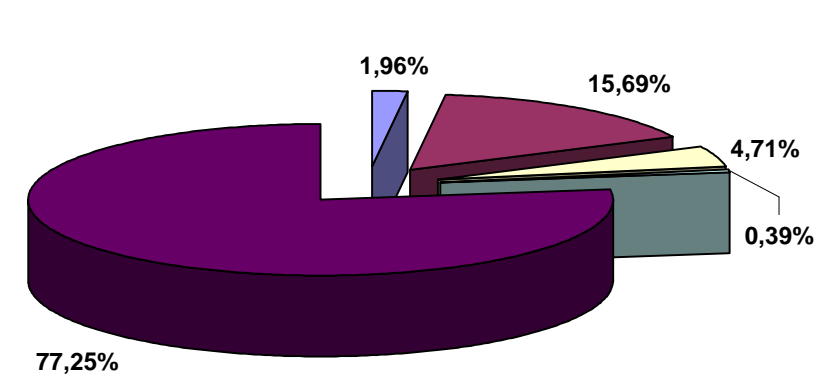

No se evidenciaron diferencias estadísticamente significativas en la presencia de acompañamiento del paciente durante su estancia en el servicio de urgencias hospitalario teniendo en cuenta el diagnóstico de intoxicación etílica. $(\mathbf{p}=\mathbf{0 , 0 1 4})$. Tabla 97 , figura 56.

Tabla 97: Relación entre la presencia de acompañante en el servicio de urgencias hospitalario y el diagnóstico de intoxicación etílica

\begin{tabular}{|c|cccc|}
\hline & Solo & Familiar & Amigo & Representante legal \\
\hline Resto diagnósticos & 1 & 42 & 1 & 1 \\
Intoxicación etílica & 5 & 40 & 12 & 1 \\
\hline Total & 6 & 82 & 13 & 2 \\
\hline \multicolumn{5}{|c}{$\mathrm{p}=0,014$} \\
\hline
\end{tabular}




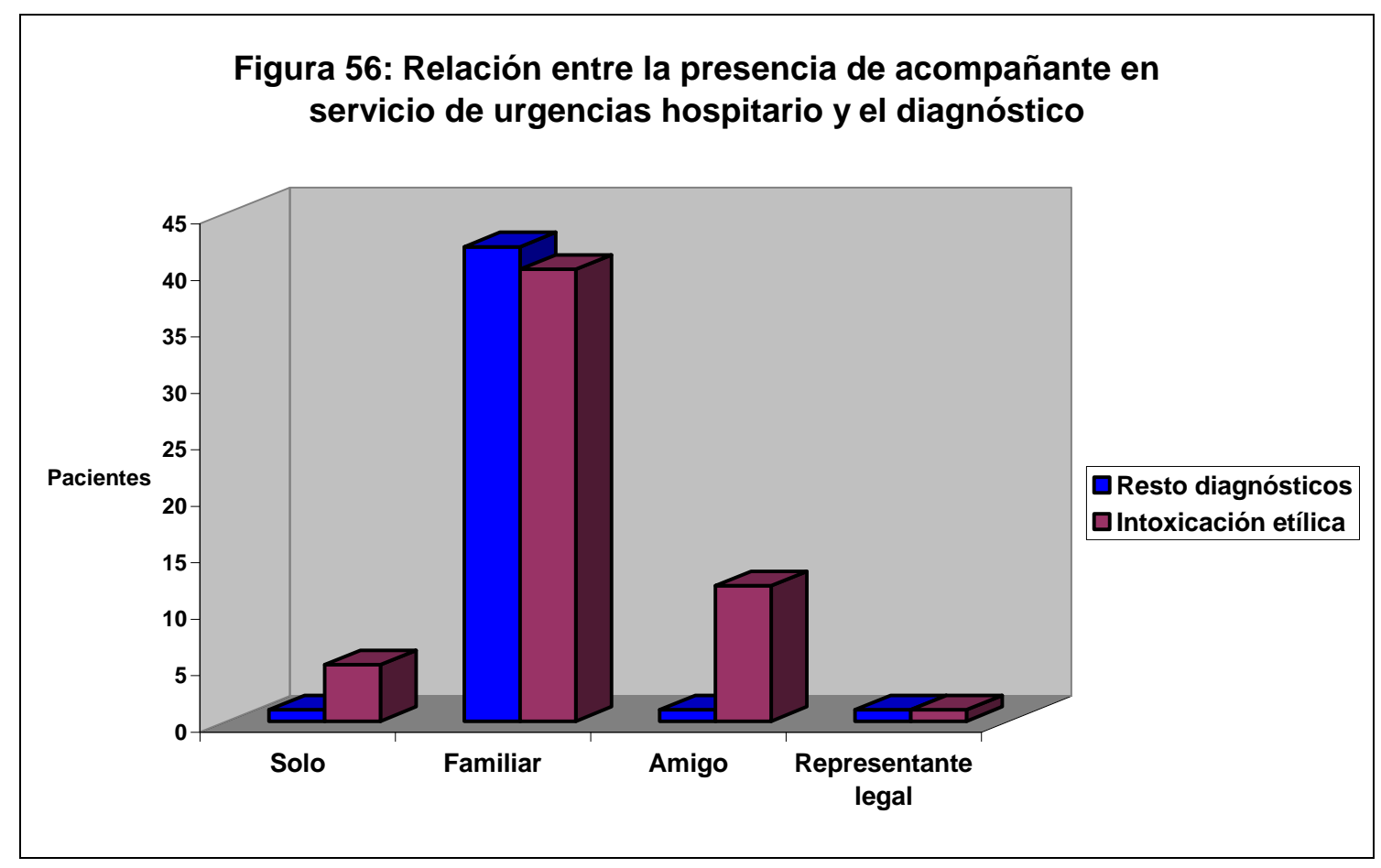

$p=0,014$

6.1.10. Distribución de la intoxicación etílica según la necesidad de transporte por vehículos de Emergencias Sanitarias.

Se distribuye a los pacientes con diagnóstico de intoxicación etílica, según la necesidad de acceder al Servicio de Urgencias Hospitalarias en vehículos de Emergencias Sanitarias. Los resultados se muestran en la tabla 98 y figura 57.

Tabla 98: Necesidad de transporte por vehículos de Emergencias Sanitarias en el caso de los pacientes con intoxicación etílica

\begin{tabular}{|c|cc|}
\hline & Frecuencia & Porcentaje \\
\hline NO & 81 & 28,92 \\
SI & 199 & 71,07 \\
\hline Total & 280 & 100 \\
\hline
\end{tabular}


Figura 57: Necesidad de transporte por vehículos de Emergencias Sanitarias en el caso de la intoxicación etílica

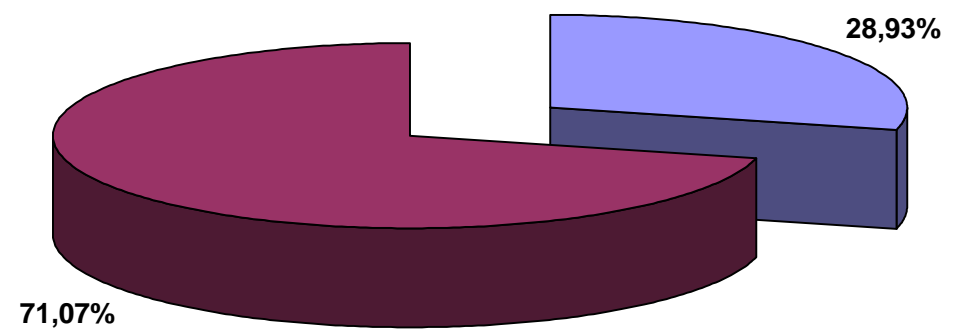

Se evidenciaron diferencias estadísticamente significativas en la necesidad de transporte del paciente intoxicado al servicio de urgencias hospitalario por medio de vehículos de Emergencias Sanitarias teniendo en cuenta el diagnóstico de intoxicación etílica $(p=0,0001)$. Tabla 99, figura 58 .

Tabla 99: Relación entre el diagnóstico de intoxicación etílica y la necesidad de transporte por vehículo de Emergencias Sanitarias

\begin{tabular}{|c|ccc|}
\hline & NO & SI & Total \\
\hline Resto diagnósticos & 267 & 37 & 304 \\
Intoxicación etílica & 81 & 199 & 280 \\
\hline Total & 348 & 236 & 584 \\
\hline \multicolumn{2}{l}{$\mathrm{p}=0,0001$} & & \\
\hline
\end{tabular}


Figura 58: Relación entre la necesidad de transporte a urgencias por vehículo de Emergencias Sanitarias y diagnóstico de intoxicación etílica

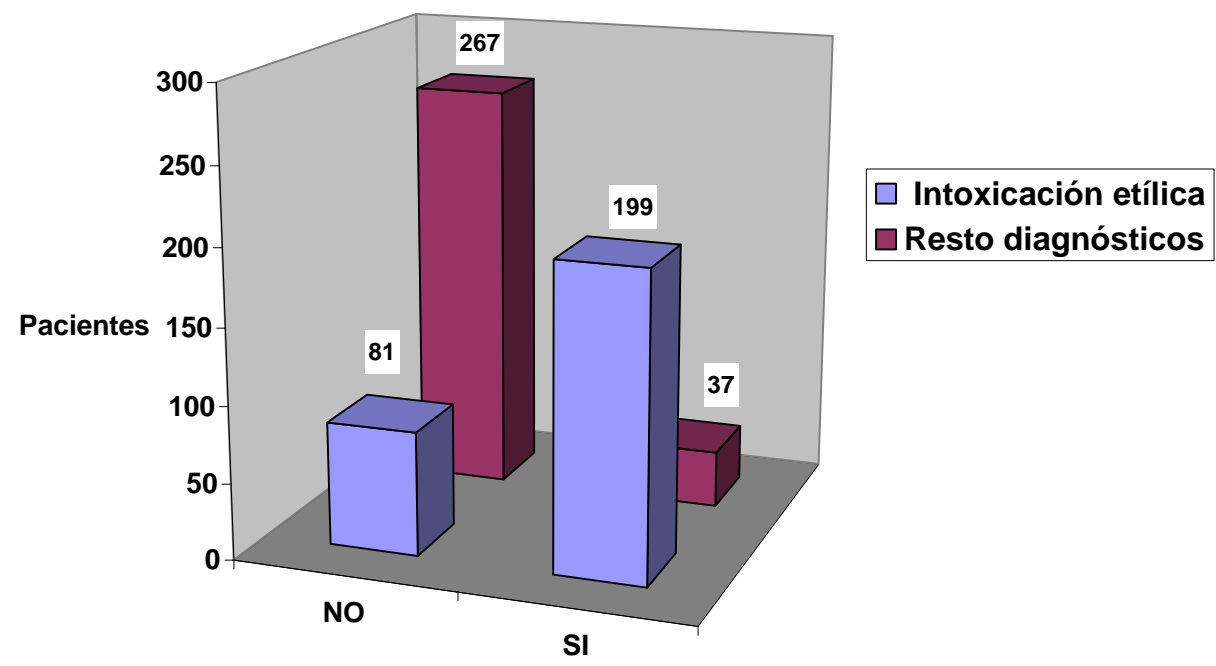

$p=0,0001$

\subsubsection{Distribución de la intoxicación etílica según la realización de} diferentes pruebas en el servicio de urgencias hospitalario.

Se distribuye a los pacientes con diagnóstico de intoxicación etílica, según la realización de pruebas diagnósticas en el servicio de urgencias hospitalario. Los resultados se muestran en la tabla 100 y figura 59.

Tabla 100: Realización de pruebas en el servicio de urgencias hospitalario en pacientes con intoxicación etílica

\begin{tabular}{|c|cccc|}
\hline Prueba & NO realización & Porcentaje & Sí realización & Porcentaje \\
\hline Analítica & 75 & 26,70 & 206 & 73,30 \\
\hline Sistemático sanguíneo & 81 & 28,90 & 199 & 71,10 \\
Bioquímica sanguínea & 84 & 29,90 & 197 & 70,10 \\
Hemostasia & 272 & 97,10 & 8 & 2,90 \\
Gasometría venosa & 236 & 84,30 & 44 & 15,70 \\
Gasometría arterial & 280 & 100 & 0 & 0 \\
Tóxicos en orina & 118 & 42,10 & 162 & 57,90 \\
ECG & 119 & 71,10 & 81 & 28,90 \\
\hline
\end{tabular}


Figura 59: Realización de pruebas diagnósticas en el servicio de urgencias hospitalarias en la intoxicación etílica

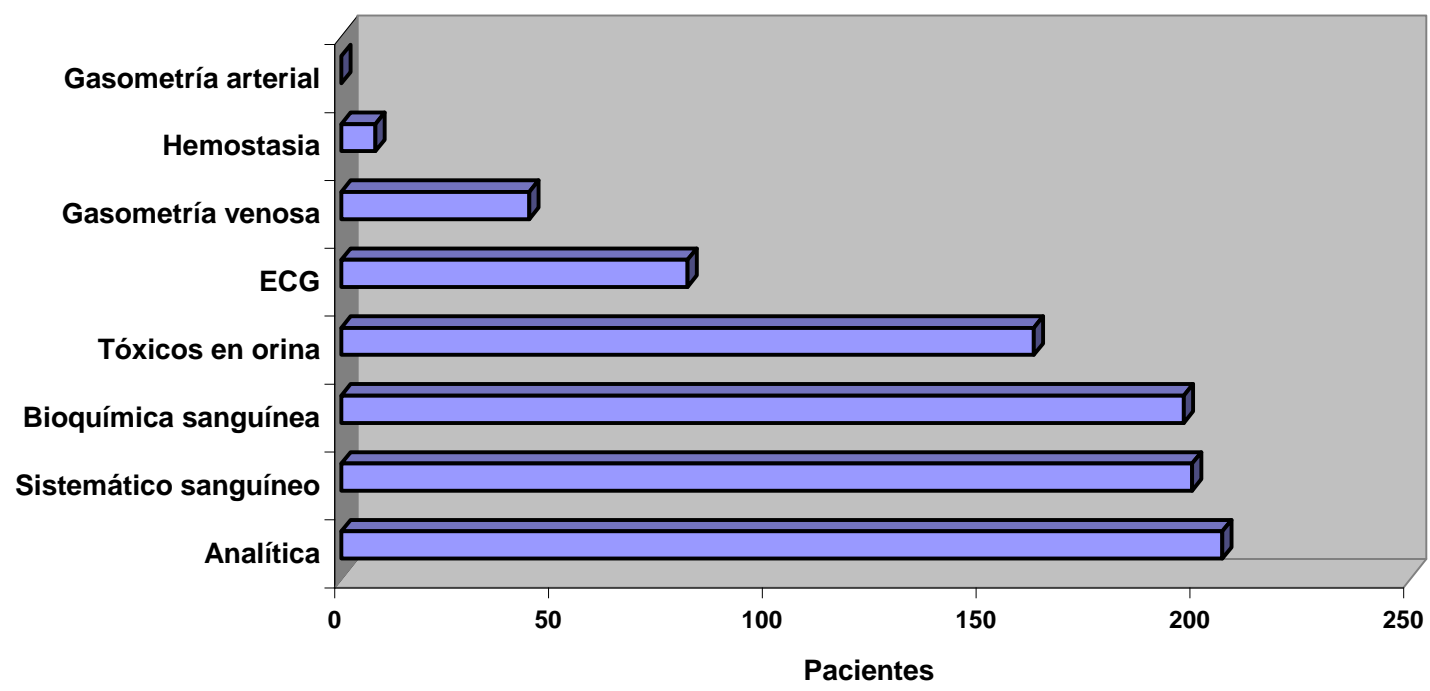

El caso de las pruebas de imagen realizadas durante su estancia en el servicio de urgencias hospitalario, se procede a mostrarlas en una tabla aparte. Tabla 101, figura 60.

Tabla 101: Realización de pruebas de imagen en los pacientes con intoxicación etílica

\begin{tabular}{|c|cc|}
\hline & Frecuencia & Porcentaje \\
\hline NO realización & 269 & 96,07 \\
Rx Torax & 5 & 1,78 \\
Rx Osea & 2 & 0,71 \\
Tomografia Axial Computerizada & 4 & 1,42 \\
Total & 280 & 100 \\
\hline
\end{tabular}


Figura 60: Realización de pruebas de imagen en los pacientes con intoxicación etílica

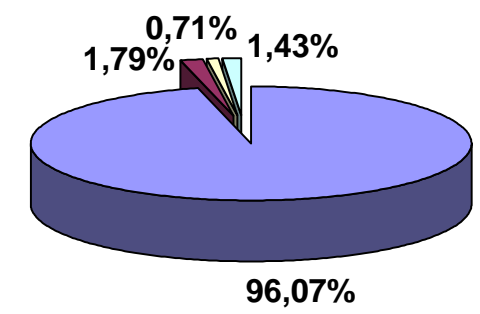

\subsubsection{Relación entre la realización de analítica en el servicio de} urgencias hospitalario y el diagnóstico de intoxicación etílica.

Se evidenciaron diferencias estadísticamente significativas en la realización de analítica en el servicio de urgencias hospitalario teniendo en cuenta el

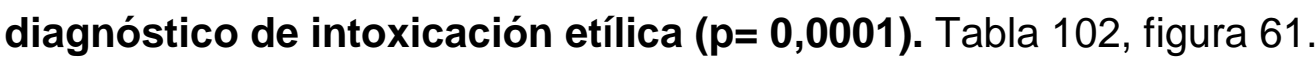

Tabla 102: Relación entre la realización de analítica y el diagnóstico de intoxicación etílica

\begin{tabular}{|c|cc|}
\hline Pruebas & Intoxicación etílica & Resto diagnósticos \\
\hline No analítica & 75 & 191 \\
Sí analítica & 206 & 115 \\
& $\mathrm{p}=0,0001$ & \\
\hline
\end{tabular}




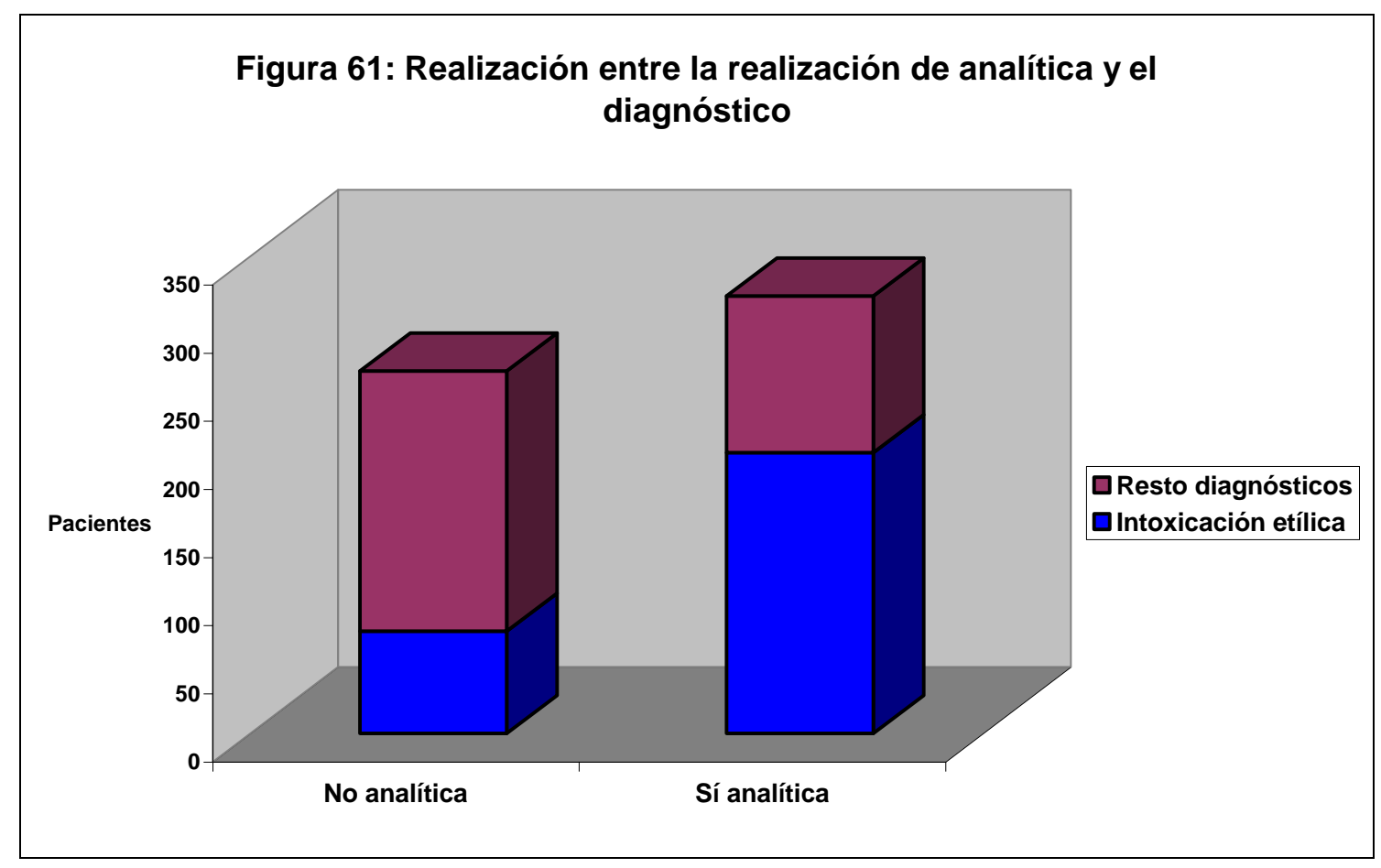

$p=0,0001$

\subsubsection{Relación entre la realización de sistemático de sangre en el} servicio de urgencias hospitalario y el diagnóstico de intoxicación etílica. Se evidenciaron diferencias estadísticamente significativas en la realización de sistemático sanguíneo en el servicio de urgencias hospitalario teniendo en cuenta el diagnóstico de intoxicación etílica $(\mathbf{p}=\mathbf{0 , 0 0 0 1})$. Tabla 103 , figura 62.

Tabla 103: Relación entre la realización de sistemático sanguíneo y el diagnóstico de intoxicación etílica

\begin{tabular}{|c|cc|}
\hline Pruebas & Intoxicación etílica & Resto diagnósticos \\
\hline No sistemático & 81 & 215 \\
Sí sistemático & 199 & 91 \\
& $\mathrm{p}=0,0001$ & \\
\hline
\end{tabular}




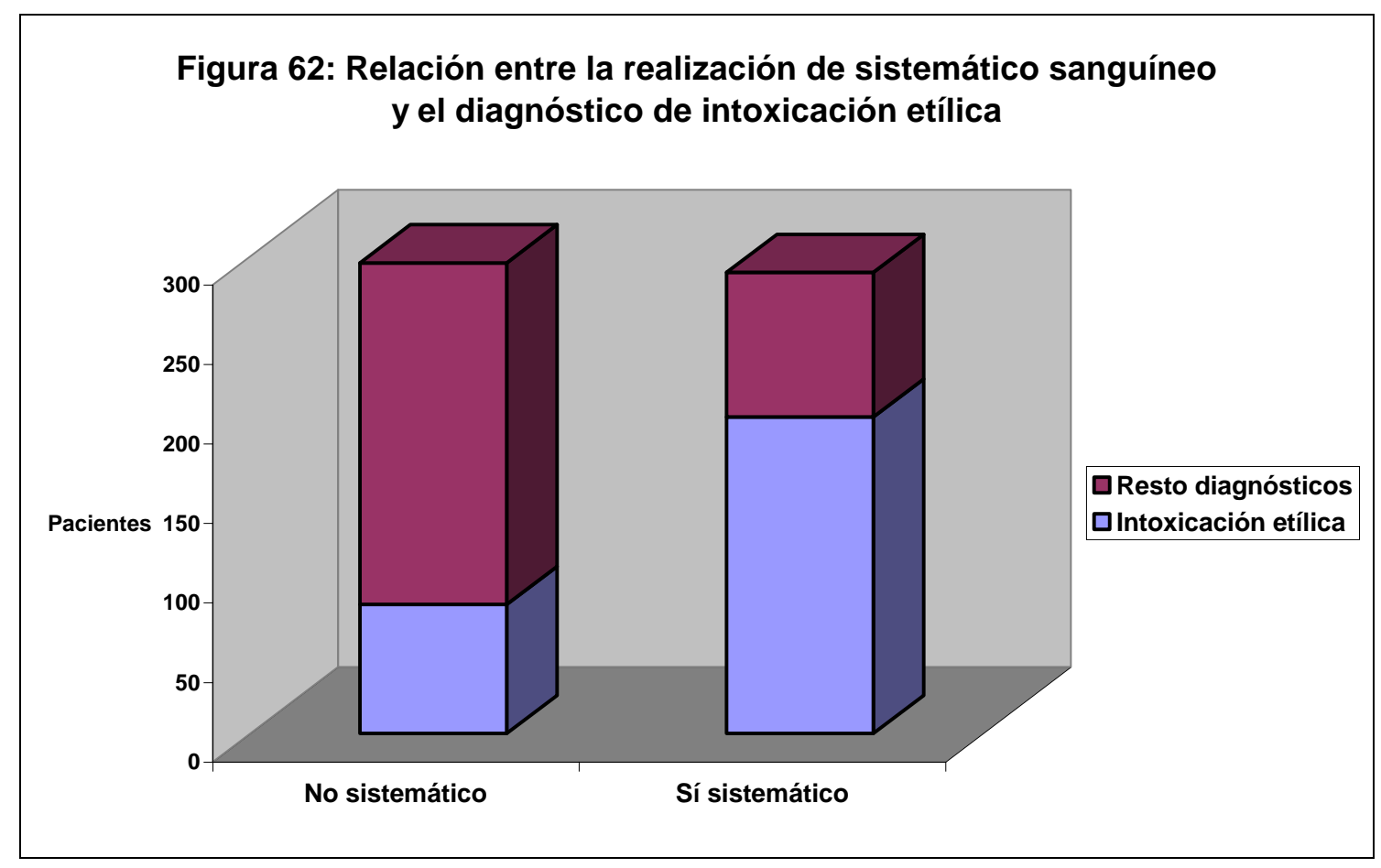

$p=0,0001$

\subsubsection{Relación entre la realización de bioquímica sanguínea en el} servicio de urgencias hospitalario y el diagnóstico de intoxicación etílica.

Se evidenciaron diferencias estadísticamente significativas en la realización de bioquímica sanguínea en el servicio de urgencias hospitalario teniendo en cuenta el diagnóstico de intoxicación etílica $(\mathbf{p}=\mathbf{0 , 0 0 0 1})$. Tabla 104 , figura 63.

Tabla 104: Relación entre la realización de bioquímica sanguínea y el diagnóstico de intoxicación etílica

\begin{tabular}{|c|cc|}
\hline Pruebas & Intoxicación etílica & Resto diagnósticos \\
\hline No bioquímica & 84 & 211 \\
Sí bioquímica & 197 & 95 \\
& $\mathrm{p}=0,0001$ & \\
\hline
\end{tabular}




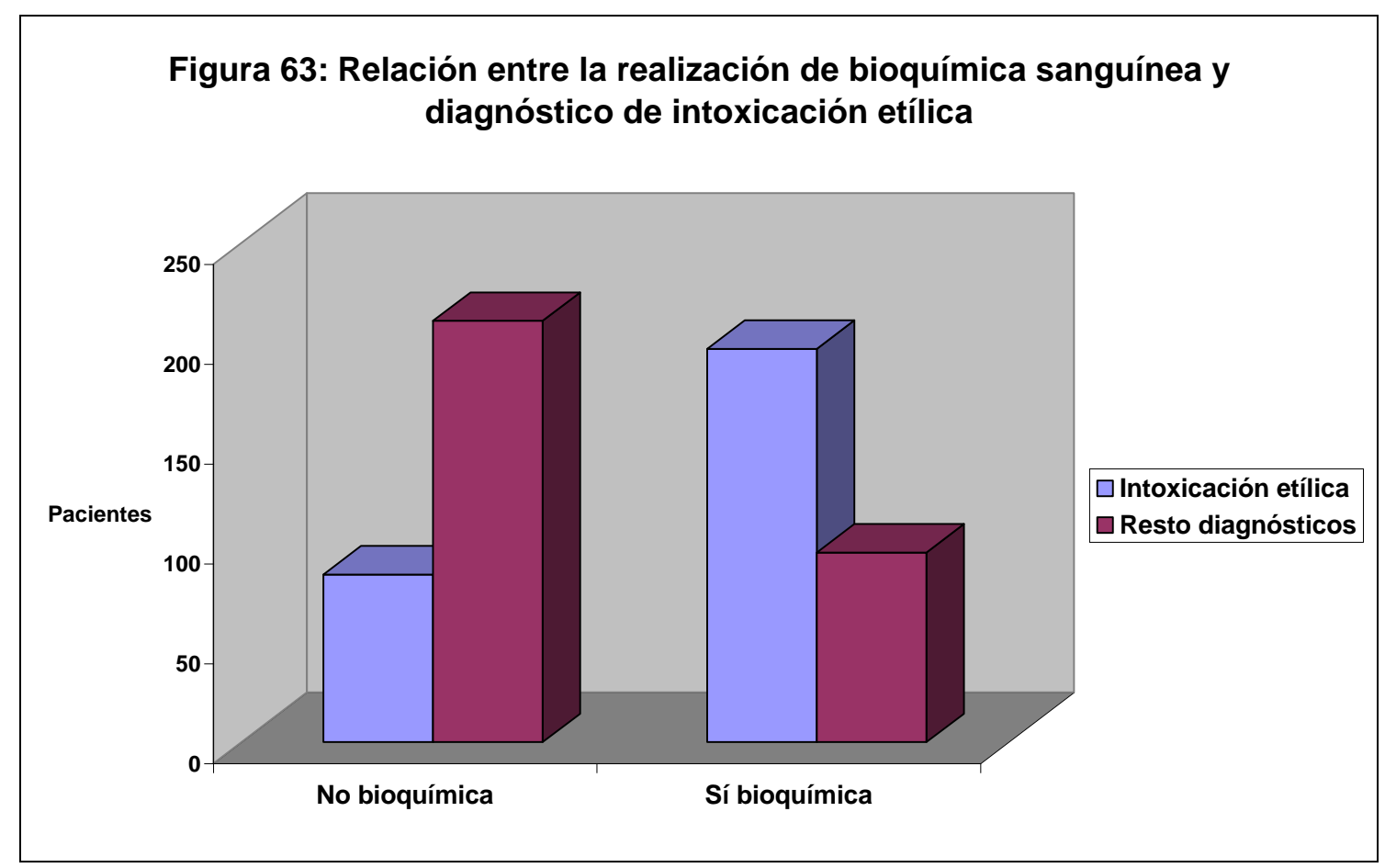

$p=0,0001$

\subsubsection{Relación entre la realización de hemostasia sanguínea en el} servicio de urgencias hospitalario y el diagnóstico de intoxicación etílica. Se evidenciaron diferencias estadísticamente significativas en la realización de hemostasia sanguínea en el servicio de urgencias hospitalario teniendo en cuenta el diagnóstico de intoxicación etílica $(p=\mathbf{0 , 0 0 3})$. Tabla 105 , figura 64.

Tabla 105: Relación entre la realización de hemostasia y el diagnóstico de intoxicación etílica

\begin{tabular}{|c|cc|}
\hline Pruebas & Intoxicación etílica & Resto diagnósticos \\
\hline No hemostasia & 272 & 279 \\
Sí hemostasia & 8 & 26 \\
& $\mathrm{p}=0,003$ & \\
\hline
\end{tabular}




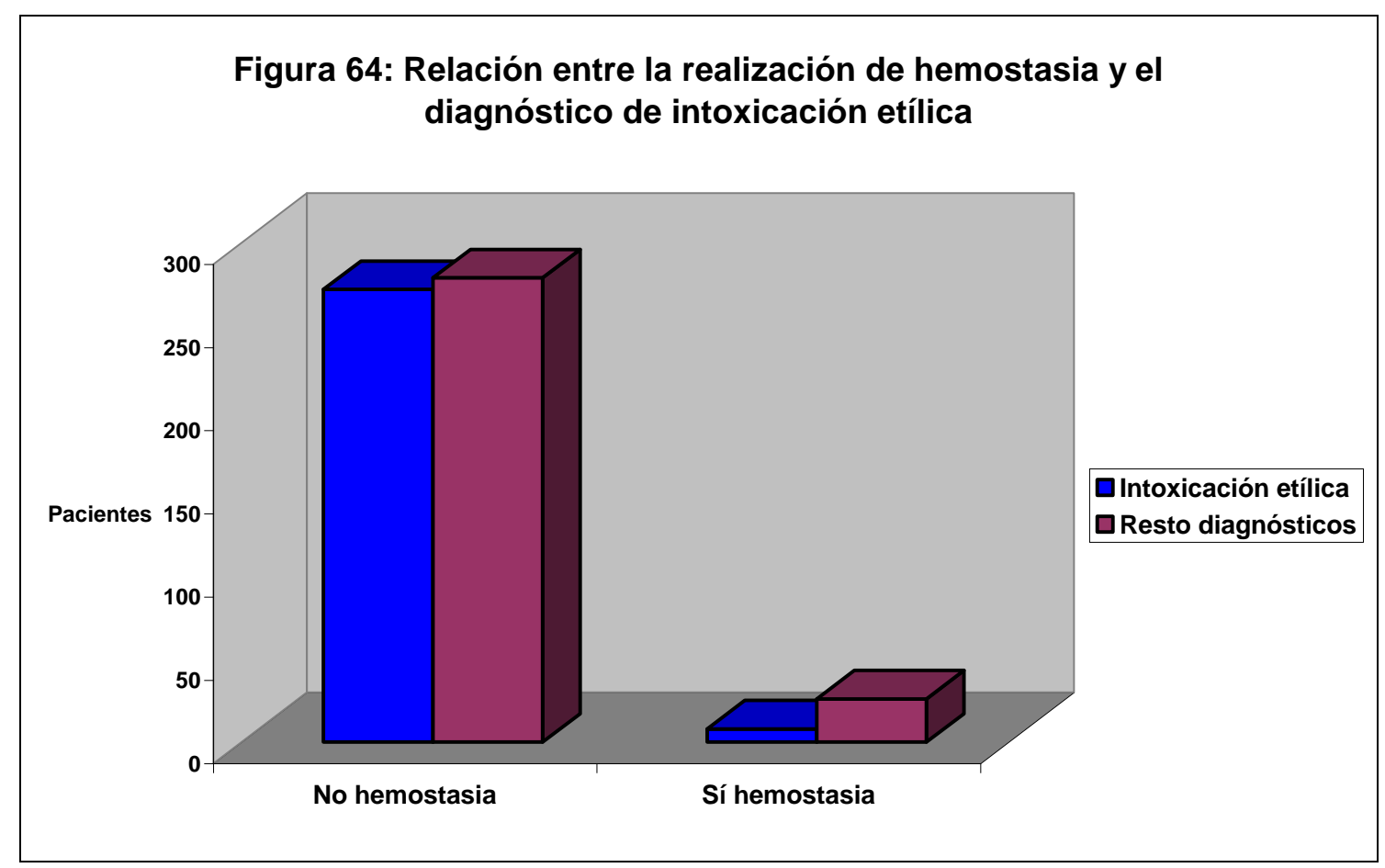

$p=0,003$

6.1.11.5. Relación entre la realización de gasometría venosa en el servicio de urgencias hospitalario y el diagnóstico de intoxicación etílica.

Se evidenciaron diferencias estadísticamente significativas en la realización de gasometría venosa en el servicio de urgencias hospitalario teniendo en cuenta el diagnóstico de intoxicación etílica $(\boldsymbol{p}=\mathbf{0 , 0 0 0 1})$. Tabla 106 , figura 65.

Tabla 106: Relación entre la realización de gasometría venosa y el diagnóstico de intoxicación etílica

\begin{tabular}{|c|cc|}
\hline Pruebas & Intoxicación etílica & Resto diagnósticos \\
\hline No gasometría venosa & 236 & 287 \\
Sí gasometría venosa & 44 & 17 \\
& $\mathrm{p}=0,0001$ & \\
\hline
\end{tabular}




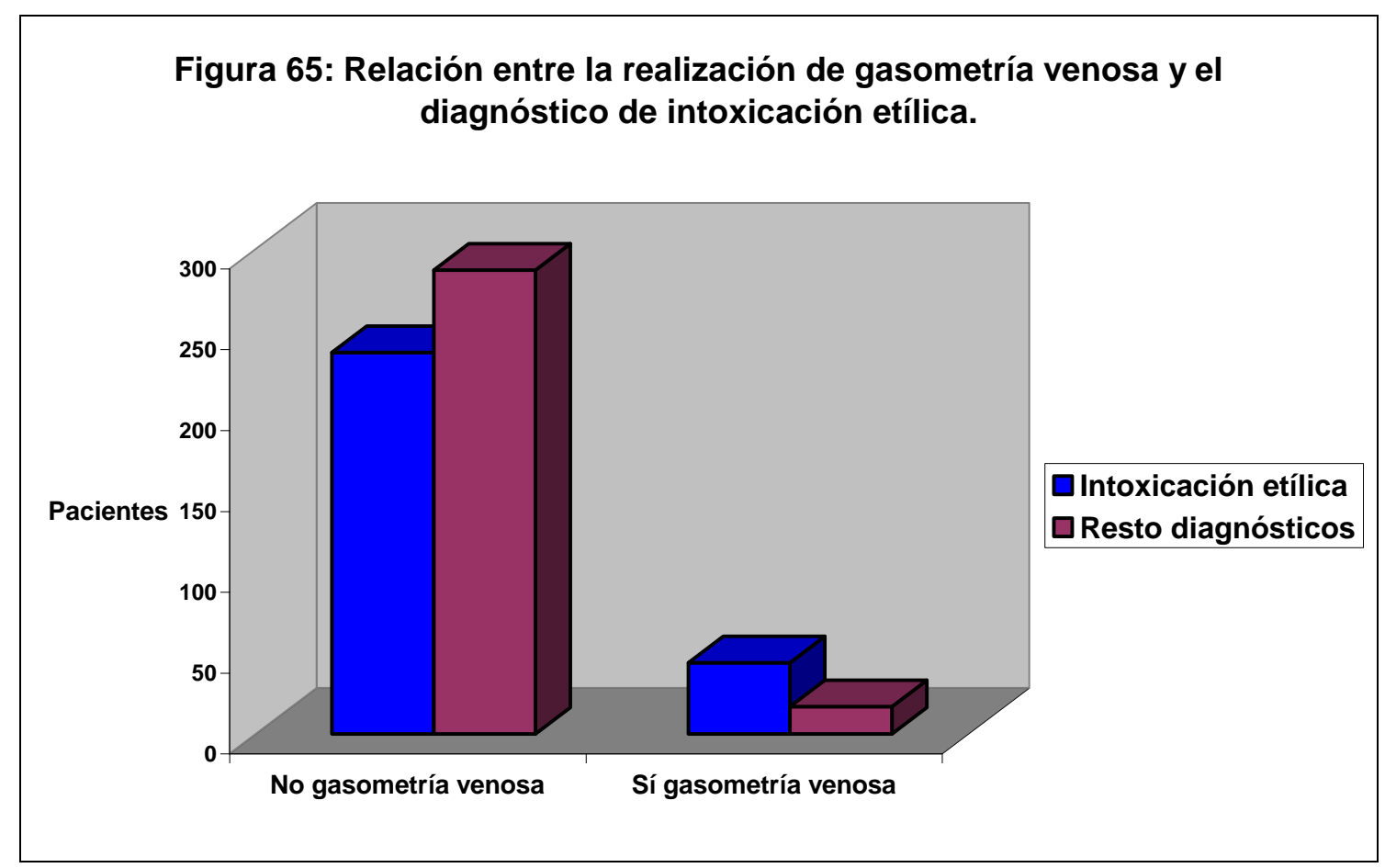

$p=0,0001$

\subsubsection{Relación entre la realización de gasometría arterial en el servicio} de urgencias hospitalario y el diagnóstico de intoxicación etílica.

Se evidenciaron diferencias estadísticamente significativas en la realización de gasometría arterial en el servicio de urgencias hospitalario teniendo en cuenta el diagnóstico de intoxicación etílica $(\mathbf{p}=\mathbf{0 , 0 0 0 1})$. Tabla 107 , figura 66.

Tabla 107: Relación entre la realización de gasometría arterial y el diagnóstico de intoxicación etílica

\begin{tabular}{|c|cc|}
\hline Pruebas & Intoxicación etílica & Resto diagnósticos \\
\hline No gasometría arterial & 280 & 275 \\
Sí gasometría arterial & 0 & 29 \\
& $\mathrm{p}=0,0001$ & \\
\hline
\end{tabular}




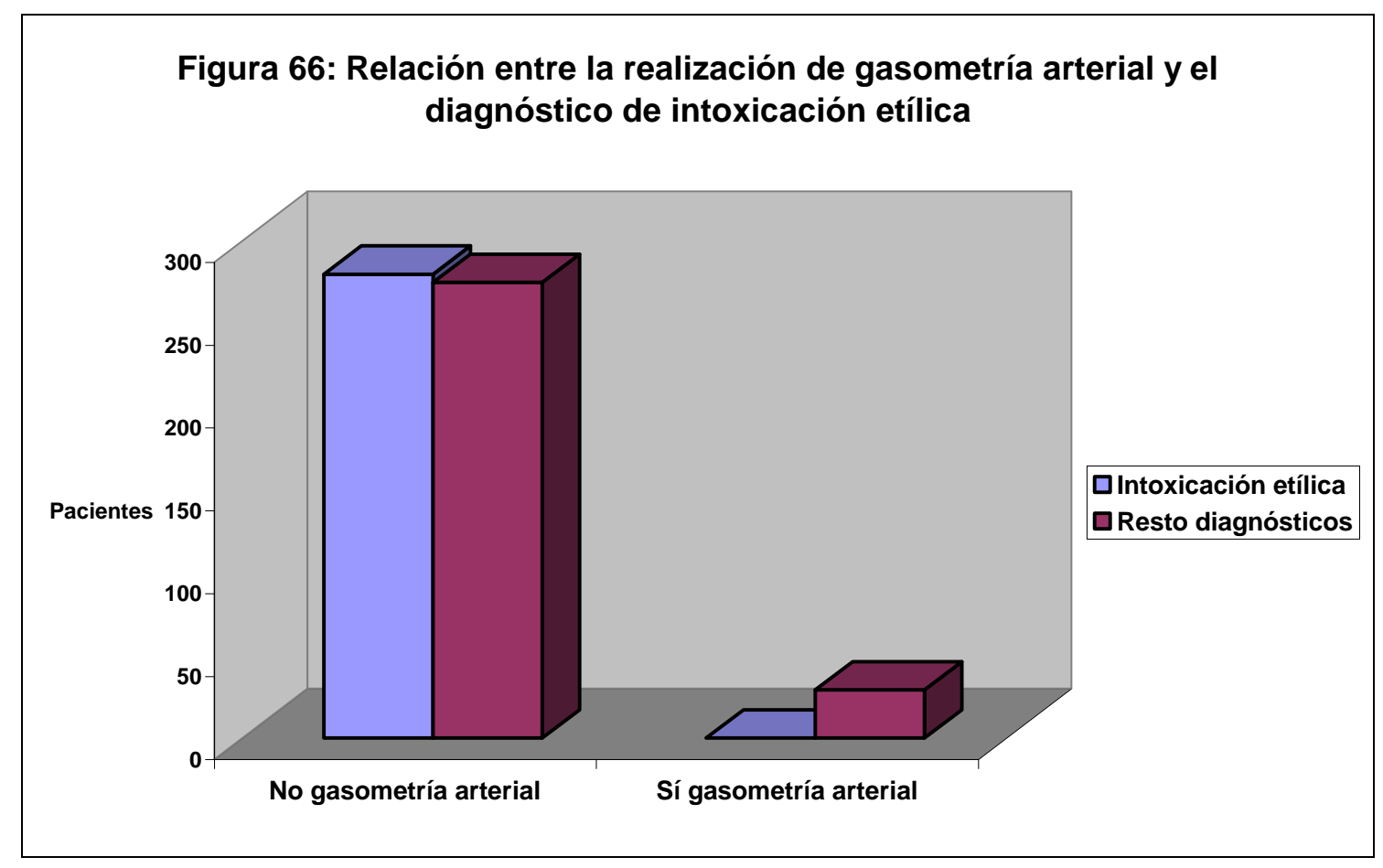

$p=0,0001$

6.1.11.7. Relación entre la realización de tóxicos en orina en el servicio de urgencias hospitalario y el diagnóstico de intoxicación etílica.

Se evidenciaron diferencias estadísticamente significativas en la realización de tóxicos en orina en el servicio de urgencias hospitalario teniendo en cuenta el diagnóstico de intoxicación etílica $(\mathbf{p = 0 , 0 0 0 1 )}$. Tabla 108, figura 67 .

Tabla 108: Relación entre la realización de tóxicos en orina y el diagnóstico de intoxicación etílica

\begin{tabular}{|c|cc|}
\hline Pruebas & Intoxicación etílica & Resto diagnósticos \\
\hline No tóxicos en orina & 118 & 259 \\
Sí tóxicos en orina & 162 & 45 \\
& $\mathrm{p}=0,0001$ & \\
\hline
\end{tabular}


Figura 67: Relación entre la realización de tóxicos en orina y el diagnóstico de intoxicación etílica

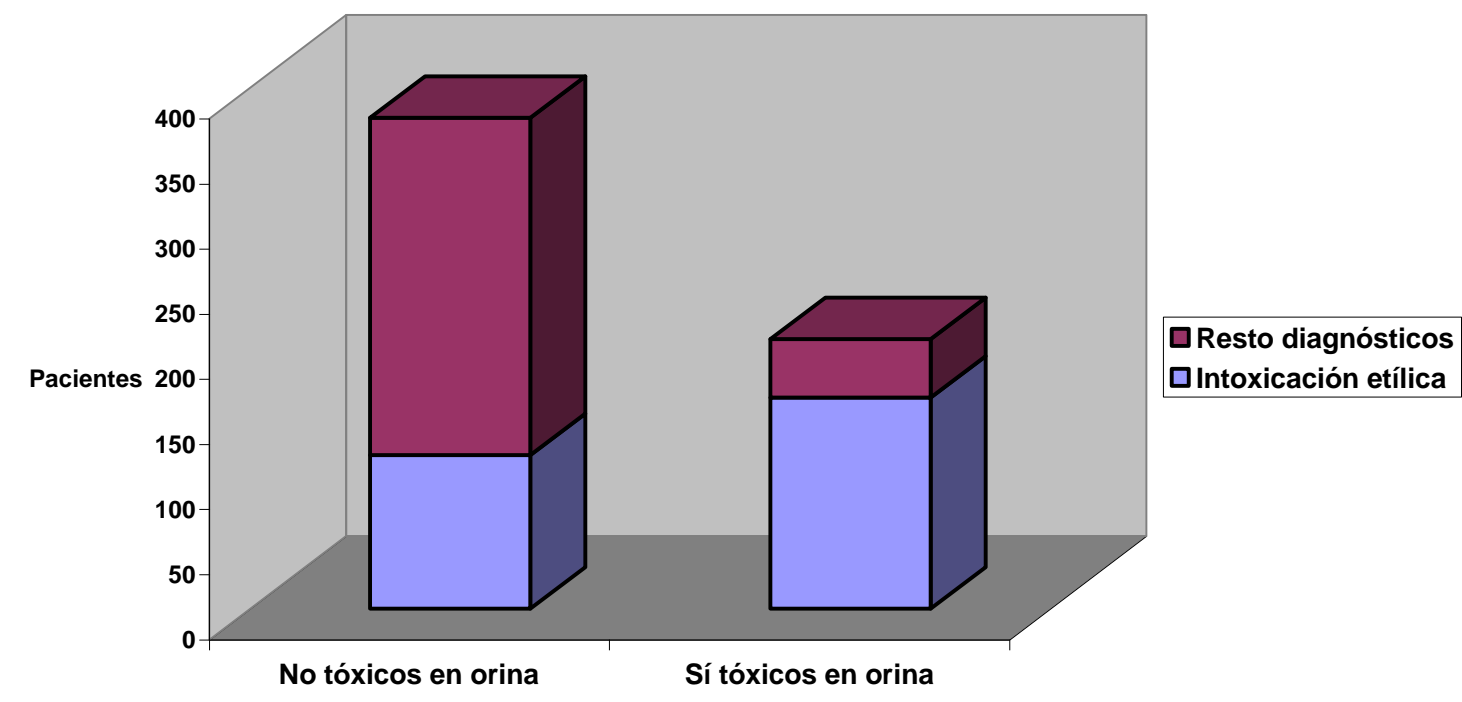

$p=0,0001$

6.1.11.8. Relación entre la realización de electrocardiografía en el servicio de urgencias hospitalario y el diagnóstico de intoxicación etílica.

Se evidenciaron diferencias estadísticamente significativas en la realización de electrocardiografía en el servicio de urgencias hospitalario teniendo en cuenta el diagnóstico de intoxicación etílica $(p=0,013)$. Tabla 109 , figura 68.

Tabla 109: Relación entre la realización de electrocardiografía y el diagnóstico de intoxicación etílica

\begin{tabular}{|c|c|c|}
\hline Pruebas & Intoxicación etílica & Resto diagnósticos \\
\hline No ECG & 199 & 243 \\
\hline Sí ECG & 81 & 61 \\
\hline \multicolumn{3}{|c|}{$p=0,013$} \\
\hline
\end{tabular}




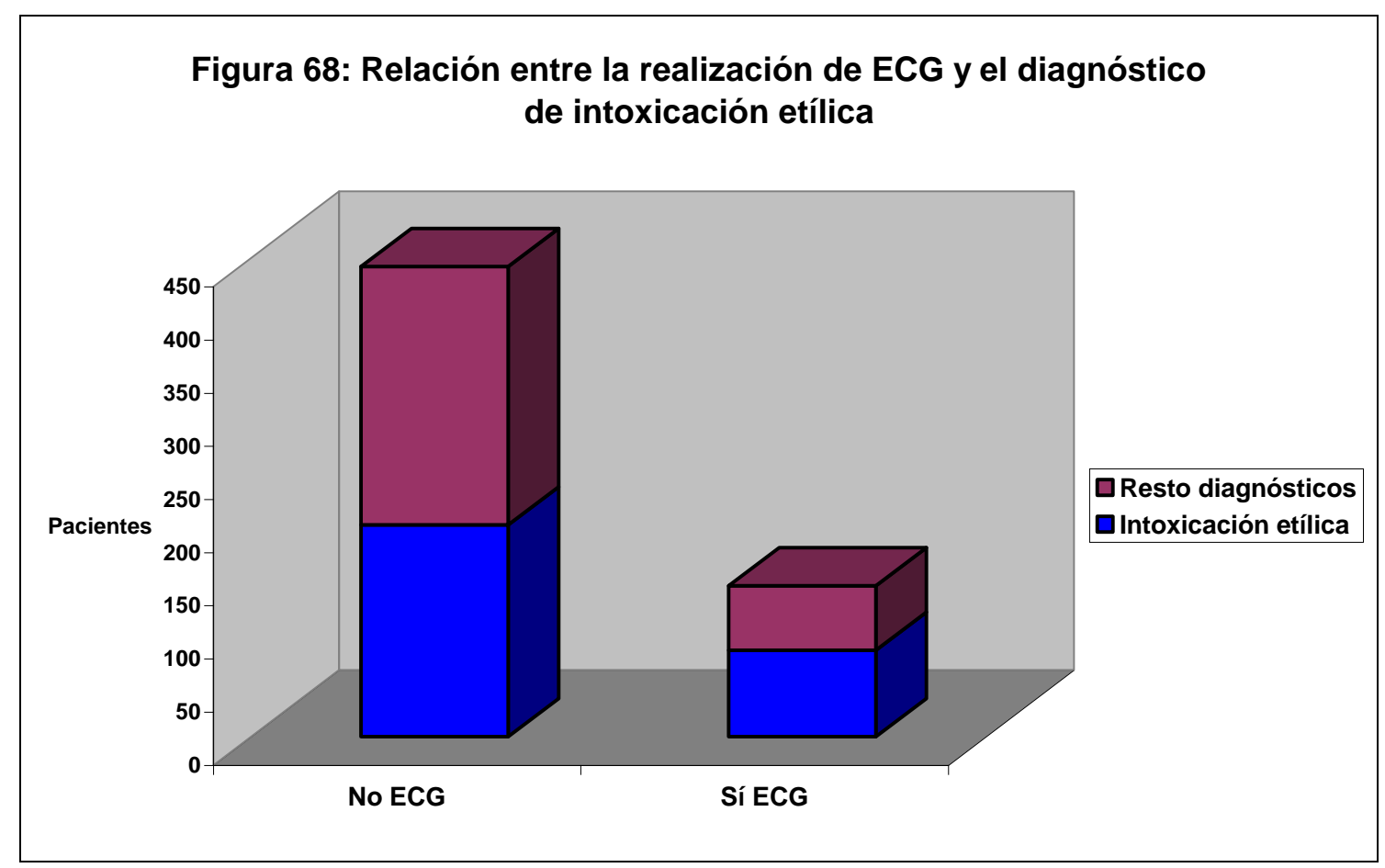

$p=0,013$

6.1.11.9. Relación entre la realización de pruebas de imagen en el servicio de urgencias hospitalario y el diagnóstico de intoxicación etílica.

No se evidenciaron diferencias estadísticamente significativas en la realización de pruebas de imagen en el servicio de urgencias hospitalario teniendo en cuenta el diagnóstico de intoxicación etílica $(p=0,084)$. Tabla 110 , figura 69 .

Tabla 110: Relación entre la realización de pruebas de imagen y el diagnóstico de intoxicación etílica

\begin{tabular}{|c|cc|}
\hline Pruebas & Intoxicación etílica & Resto diagnósticos \\
\hline No pruebas de imagen & 269 & 282 \\
Sí pruebas de imagen & 11 & 22 \\
& $\mathrm{p}=0,084$ & \\
\hline
\end{tabular}




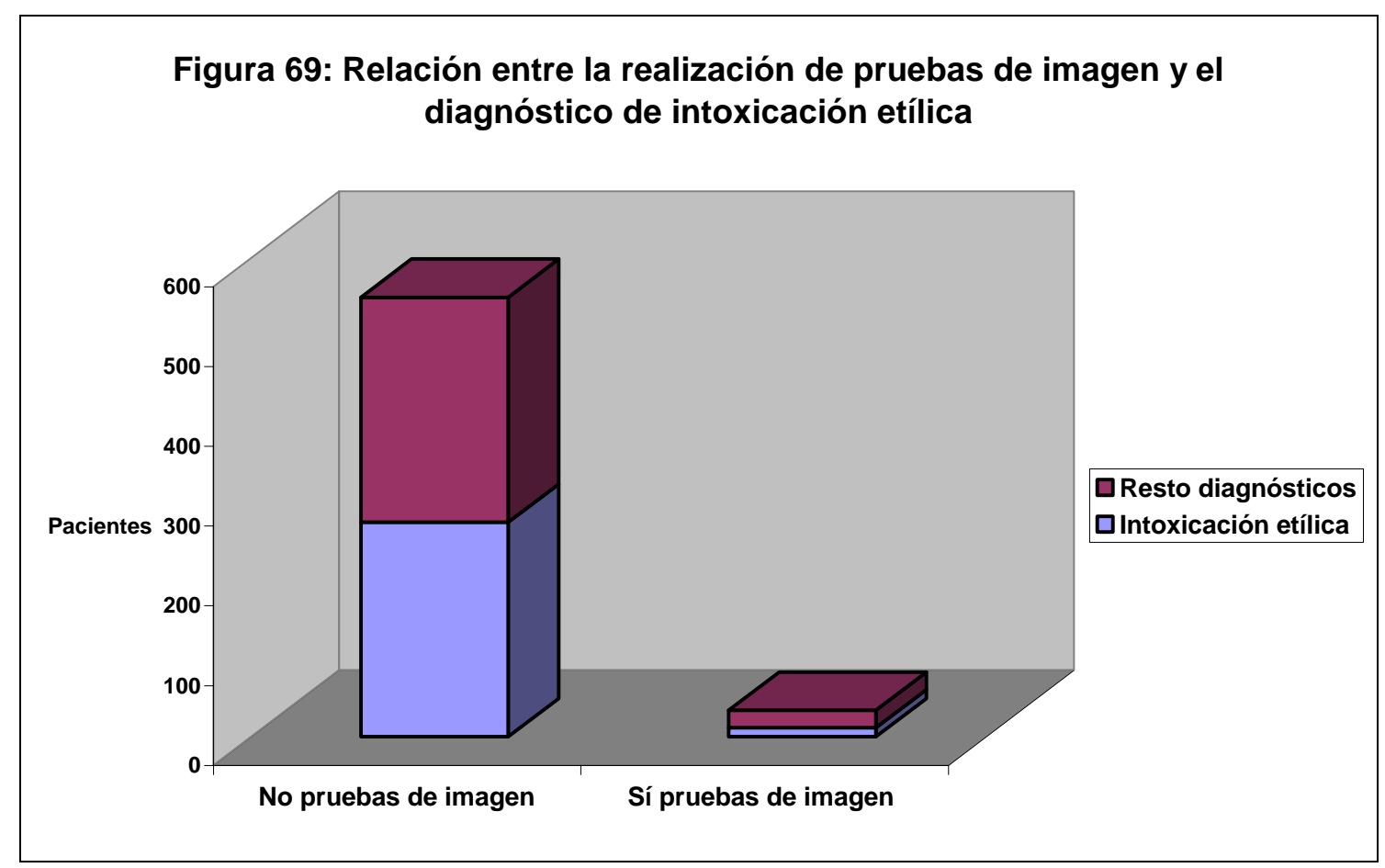

$p=0,084$

\subsubsection{Tratamiento recibido en los pacientes con intoxicación etílica}

Se distribuye a los pacientes con diagnóstico de intoxicación etílica, según el tratamiento recibido en el servicio de urgencias hospitalario. Los resultados se muestran en la tabla 111 y figura 70 .

Tabla 111: Tratamiento en el servicio de urgencias hospitalario en la intoxicación etílica

\begin{tabular}{|c|cc|}
\hline Tratamiento & Frecuencia & Porcentaje \\
\hline NO & 83 & 29,53 \\
SI & 198 & 70,46 \\
\hline Total & 281 & 100 \\
\hline
\end{tabular}


Figura 70: Tratamiento en las intoxicaciones etílicas

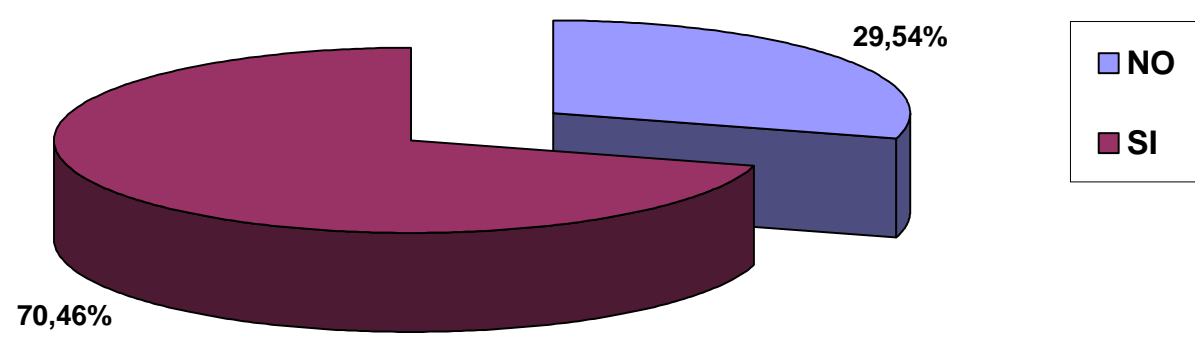

Se evidenciaron diferencias estadísticamente significativas en la necesidad de tratamiento en el servicio de urgencias hospitalario teniendo en cuenta el diagnóstico de intoxicación etílica $(p=\mathbf{0 , 0 0 0 1})$ Tabla 112, figura 71 .

Tabla 112: Relación entre la necesidad de tratamiento en el servicio de urgencias hospitalario y el diagnóstico de intoxicación etílica

\begin{tabular}{|c|ccc|}
\hline Diagnóstico & NO & SI & NO \\
\hline Resto diagnósticos & 157 & 147 & 304 \\
Intoxicación etílica & 83 & 198 & 281 \\
\hline Total & 240 & 345 & 585 \\
\hline \multicolumn{2}{|l}{$\mathrm{p}=0,0001$} & & \\
\hline
\end{tabular}




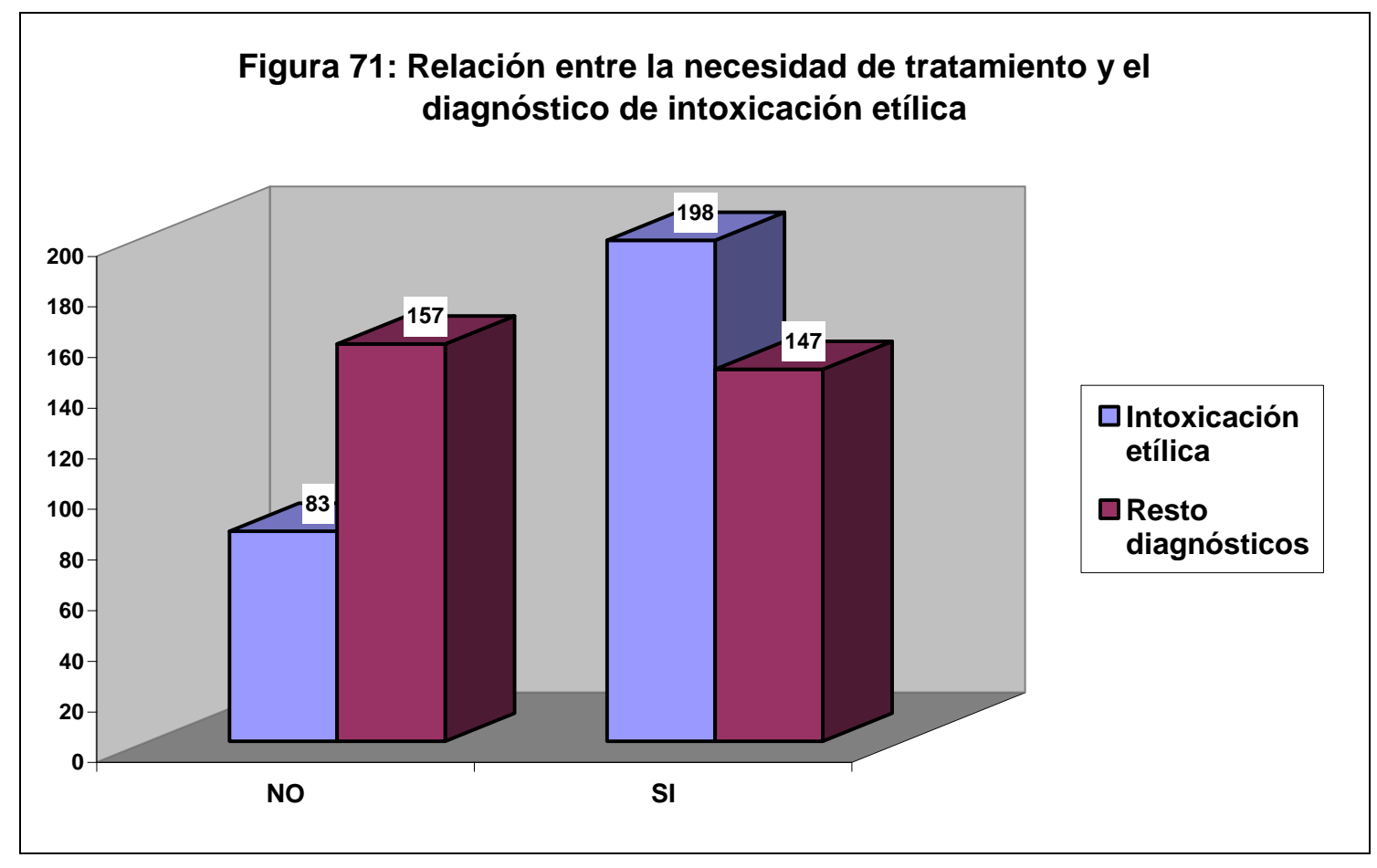

$p=0,0001$

\subsubsection{Tipos de tratamientos recibidos en los pacientes con}

\section{intoxicación etílica.}

Se distribuye a los pacientes con diagnóstico de intoxicación etílica, según el tipo de tratamiento recibido en el servicio de urgencias hospitalario. Los resultados se muestran en la tabla 113 y figura 72 .

Tabla 113: Tipo de tratamiento en el servicio de urgencias hospitalario en los pacientes con intoxicación etílica

\begin{tabular}{|c|cc|}
\hline Tipos de tratamiento & No & Sí \\
\hline Lavado gástrico & 280 & 0 \\
Carbón activado & 280 & 0 \\
Antídoto & 278 & 2 \\
\hline Suero fisiológico & 137 & 143 \\
Suero glucosado & 242 & 38 \\
Antiemético & 179 & 101 \\
Analgésico & 269 & 11 \\
Protector gástrico & 236 & 44 \\
Tiamina & 275 & 5 \\
Otros tratamientos & 269 & 12 \\
\hline
\end{tabular}




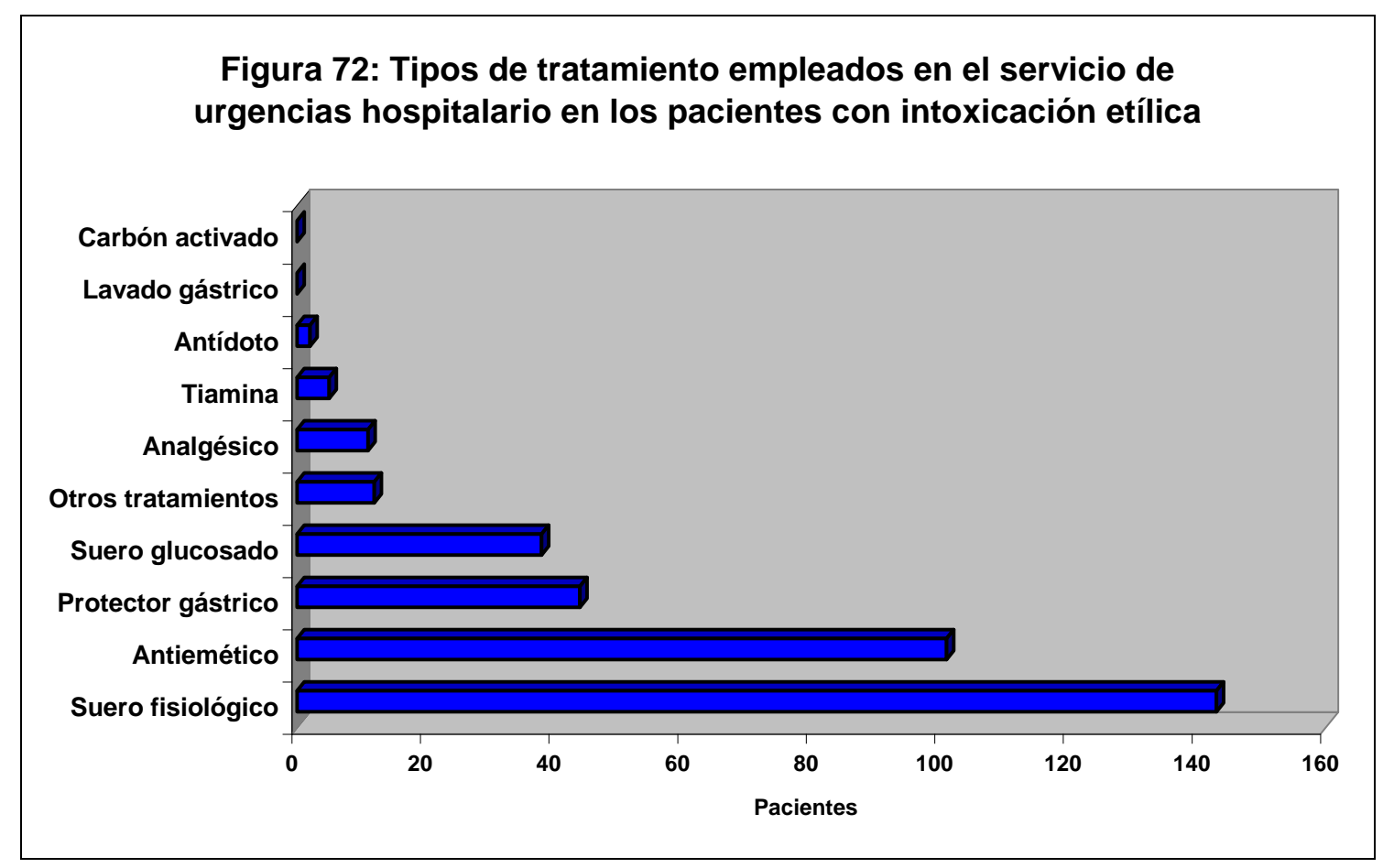

6.1.12.2. Relación entre el tratamiento con oxigenoterapia y el diagnóstico de intoxicación etílica.

Se evidenciaron diferencias estadísticamente significativas en la necesidad de tratamiento con oxigenoterapia en el servicio de urgencias hospitalario teniendo en cuenta el diagnóstico de intoxicación etílica $(p=0,0001)$ Tabla 114 , figura 73

Tabla 114: Relación entre el tratamiento con oxigenoterapia y la intoxicación etílica

\begin{tabular}{|c|cc|}
\hline Tratamiento & Intoxicación etílica & Resto diagnósticos \\
\hline Sí oxigenoterapia & 6 & 37 \\
No oxigenoterapia & 274 & 267 \\
& $p=0,0001$ & \\
\hline
\end{tabular}




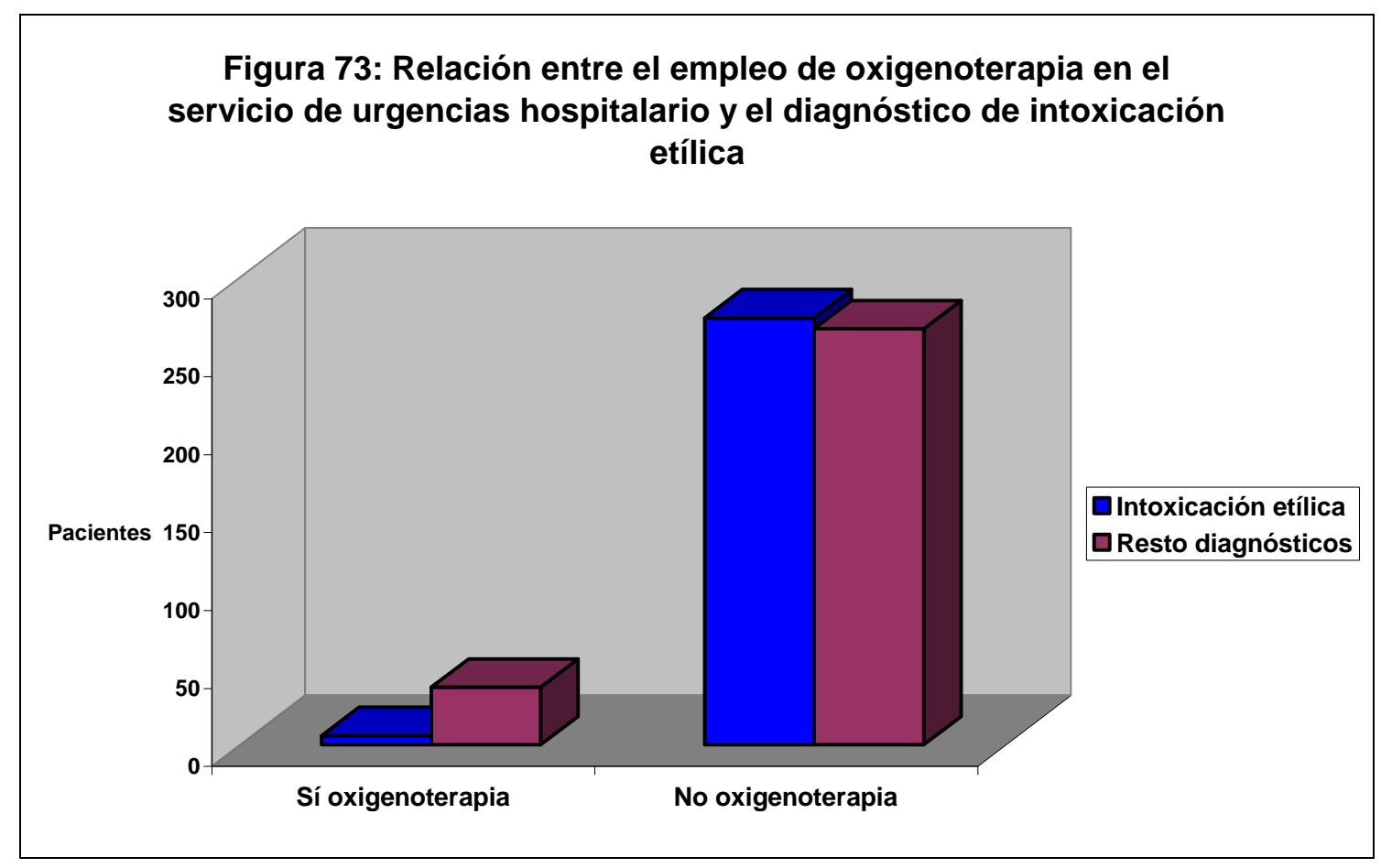

$p=0,0001$

\subsubsection{Relación entre la necesidad de lavado gástrico en el servicio de} urgencias hospitalario y el diagnóstico de intoxicación etílica.

Se evidenciaron diferencias estadísticamente significativas en la necesidad de realización de lavado gástrico en el servicio de urgencias hospitalario teniendo en cuenta el diagnóstico de intoxicación etílica $(\mathbf{p}=\mathbf{0 , 0 0 0 1 )}$ Tabla 115 , figura 74

Tabla 115: Relación entre la necesidad de lavado gástrico en el servicio de urgencias hospitalario y la intoxicación etílica

\begin{tabular}{|c|cc|}
\hline Tratamiento & Intoxicación etílica & Resto diagnósticos \\
\hline Lavado gástrico & 0 & 50 \\
No lavado gástrico & 280 & 253 \\
& $\mathrm{p}=0,0001$ & \\
\hline
\end{tabular}


Figura 74: Relación entre la realización de lavado gástrico en el servicio de urgencias hospitalario y el diagnóstico de intoxicación etílica

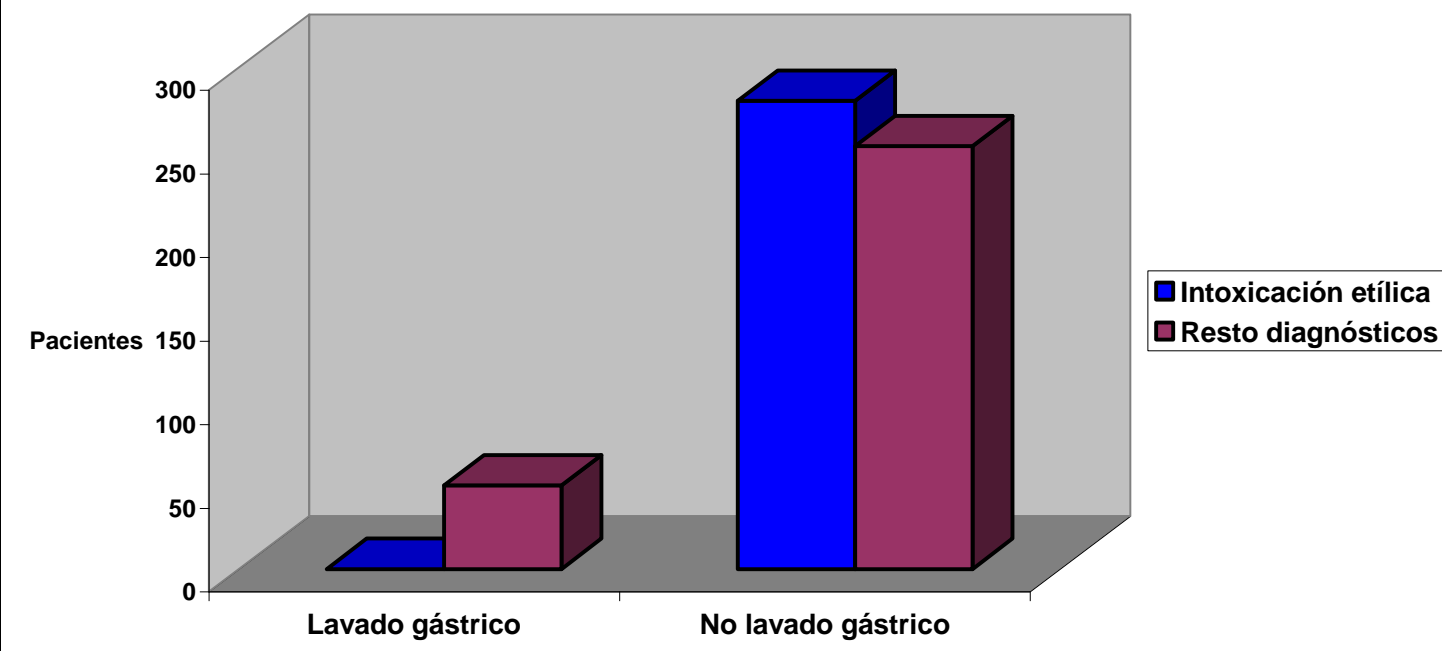

$p=0,0001$

\subsubsection{Relación entre la administración de carbón activado y el} diagnóstico de intoxicación etílica.

Se evidenciaron diferencias estadísticamente significativas en la necesidad de administración de carbón activado en el servicio de urgencias hospitalario teniendo en cuenta el diagnóstico de intoxicación etílica $(p=$ 0,0001) Tabla 116, figura 75.

Tabla 116: Relación entre la administración de carbón activado en el servicio de urgencias hospitalario y la intoxicación etílica

\begin{tabular}{|c|cc|}
\hline Tratamiento & Intoxicación etílica & Resto diagnósticos \\
\hline Carbón activado & 0 & 57 \\
No carbón activado & 280 & 245 \\
& $\mathrm{p}=0,0001$ & \\
\hline
\end{tabular}


Figura 75: Relación entre la administración de carbón activado en

el servicio de urgencias hospitalario y la intoxicación etílica

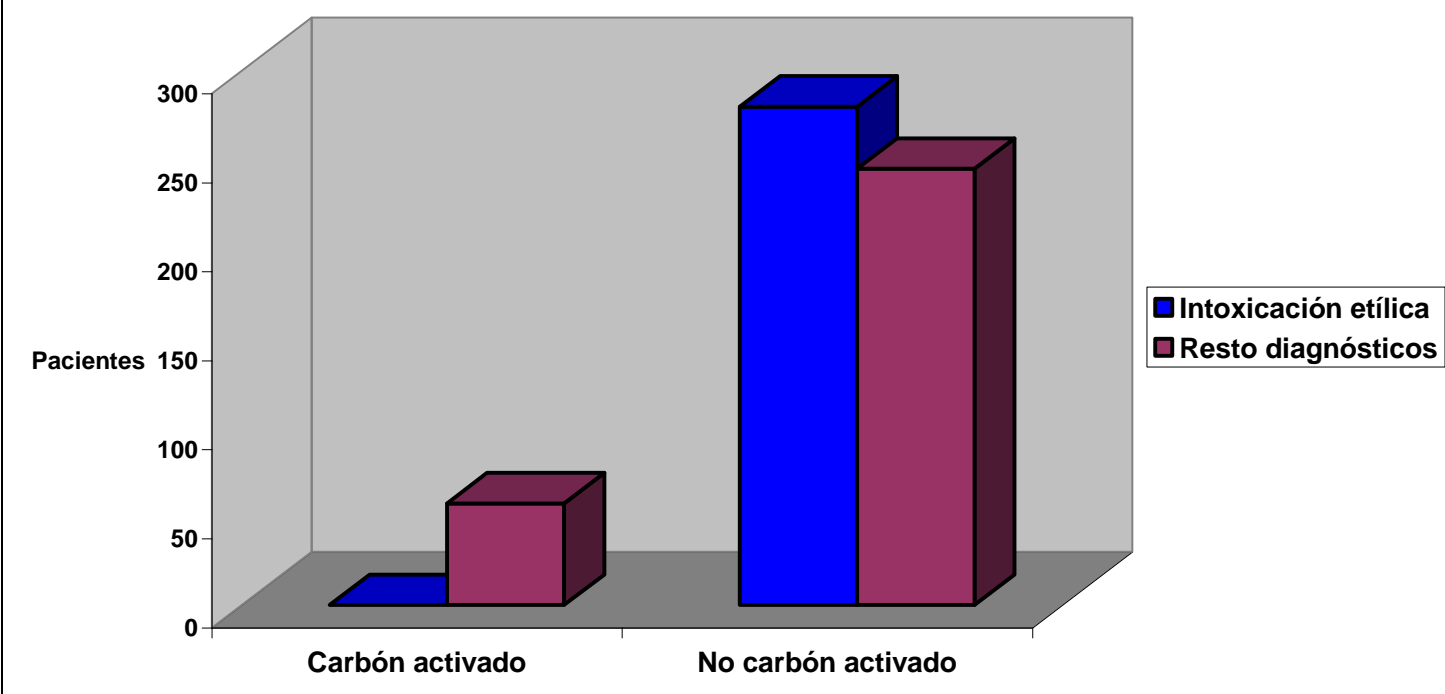

$p=0,0001$

\subsubsection{Relación entre la administración de suero fisiológico y el}

\section{diagnóstico de intoxicación etílica.}

Se evidenciaron diferencias estadísticamente significativas en la necesidad de administración de suero fisiológico en el servicio de urgencias hospitalario teniendo en cuenta el diagnóstico de intoxicación etílica $(p=$ 0,0001) Tabla 117, figura 76.

Tabla 117: Relación entre la administración de suero fisiológico en el servicio de urgencias hospitalario y la intoxicación etílica

\begin{tabular}{|c|cc|}
\hline Tratamiento & Intoxicación etílica & Resto diagnósticos \\
\hline Suero fisiológico & 143 & 33 \\
No suero fisiológico & 137 & 269 \\
& $\mathrm{p}=0,0001$ & \\
\hline
\end{tabular}




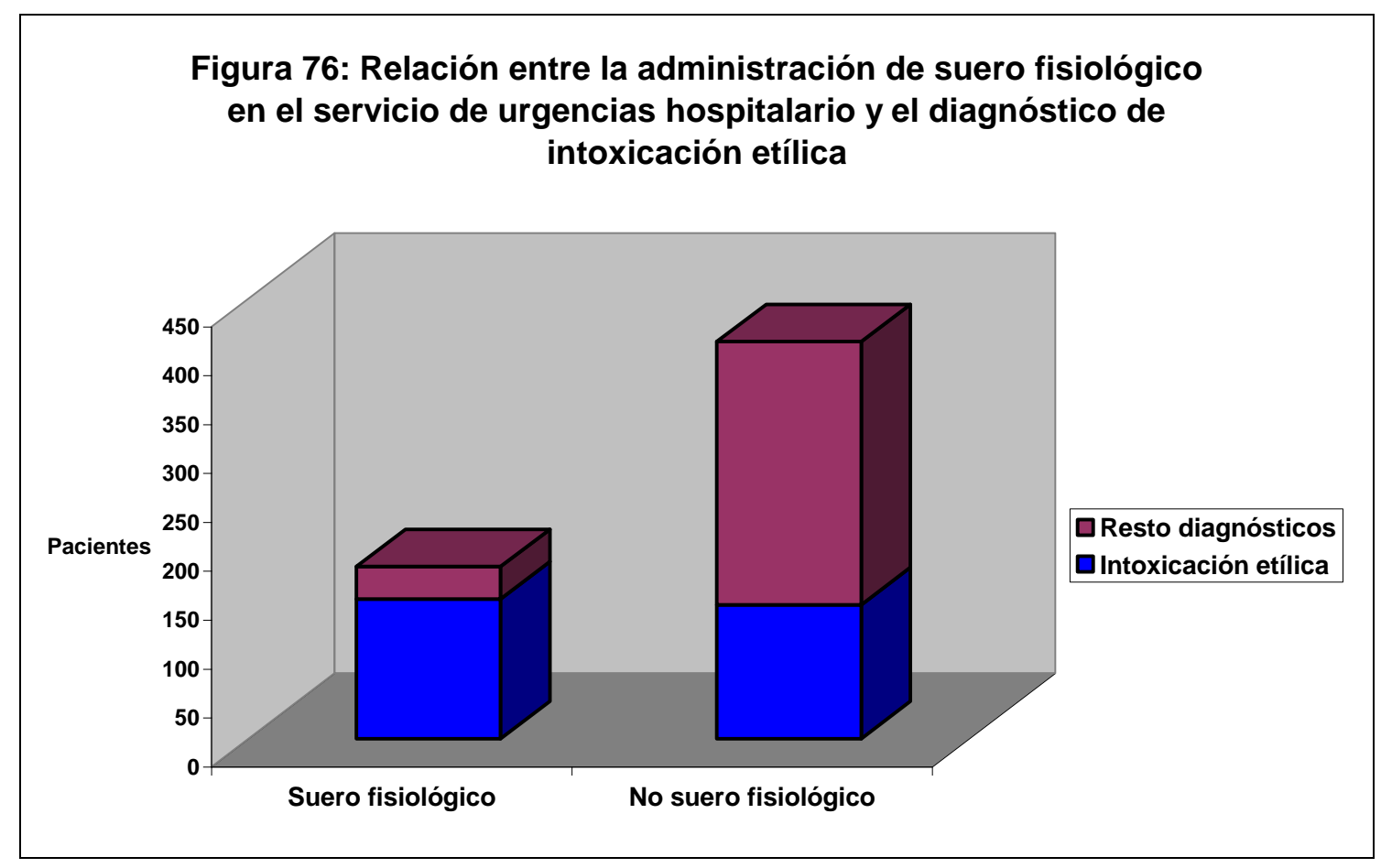

$p=0,0001$

\subsubsection{Relación entre la administración de suero glucosado y el}

\section{diagnóstico de intoxicación etílica.}

Se evidenciaron diferencias estadísticamente significativas en la necesidad de administración de suero glucosado en el servicio de urgencias hospitalario teniendo en cuenta el diagnóstico de intoxicación etílica ( $p=$ 0,0001) Tabla 118, figura 77.

Tabla 118: Relación entre la administración de suero glucosado en el servicio de urgencias hospitalario y la intoxicación etílica

\begin{tabular}{|c|cc|}
\hline Tratamiento & Intoxicación etílica & Resto diagnósticos \\
\hline Suero glucosado & 38 & 3 \\
No suero glucosado & 242 & 299 \\
& $\mathrm{p}=0,0001$ & \\
\hline
\end{tabular}




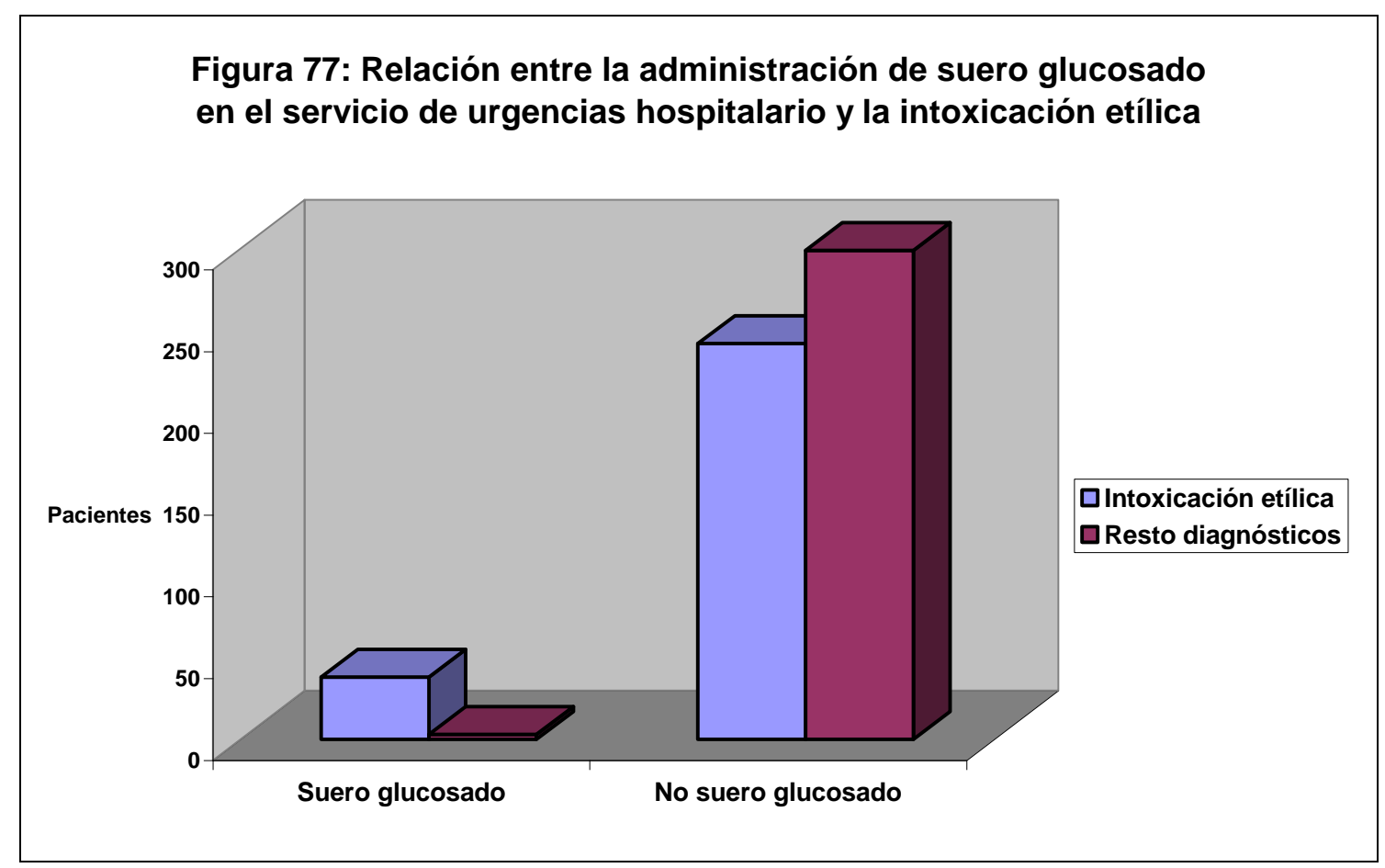

$p=0,0001$

\subsubsection{Relación entre la administración de antiemético y el diagnóstico} de la intoxicación etílica.

Se evidenciaron diferencias estadísticamente significativas en la necesidad de administración de antiemético en el servicio de urgencias hospitalario teniendo en cuenta el diagnóstico de intoxicación etílica $(p=0,0001)$ Tabla 119 , figura 78 .

Tabla 119: Relación entre la administración de antiemético en el servicio de urgencias hospitalario y la intoxicación etílica

\begin{tabular}{|c|cc|}
\hline Tratamiento & Intoxicación etílica & Resto diagnósticos \\
\hline Antiemético & 101 & 4 \\
No antiemético & 179 & 297 \\
& $\mathrm{p}=0,0001$ & \\
\hline
\end{tabular}




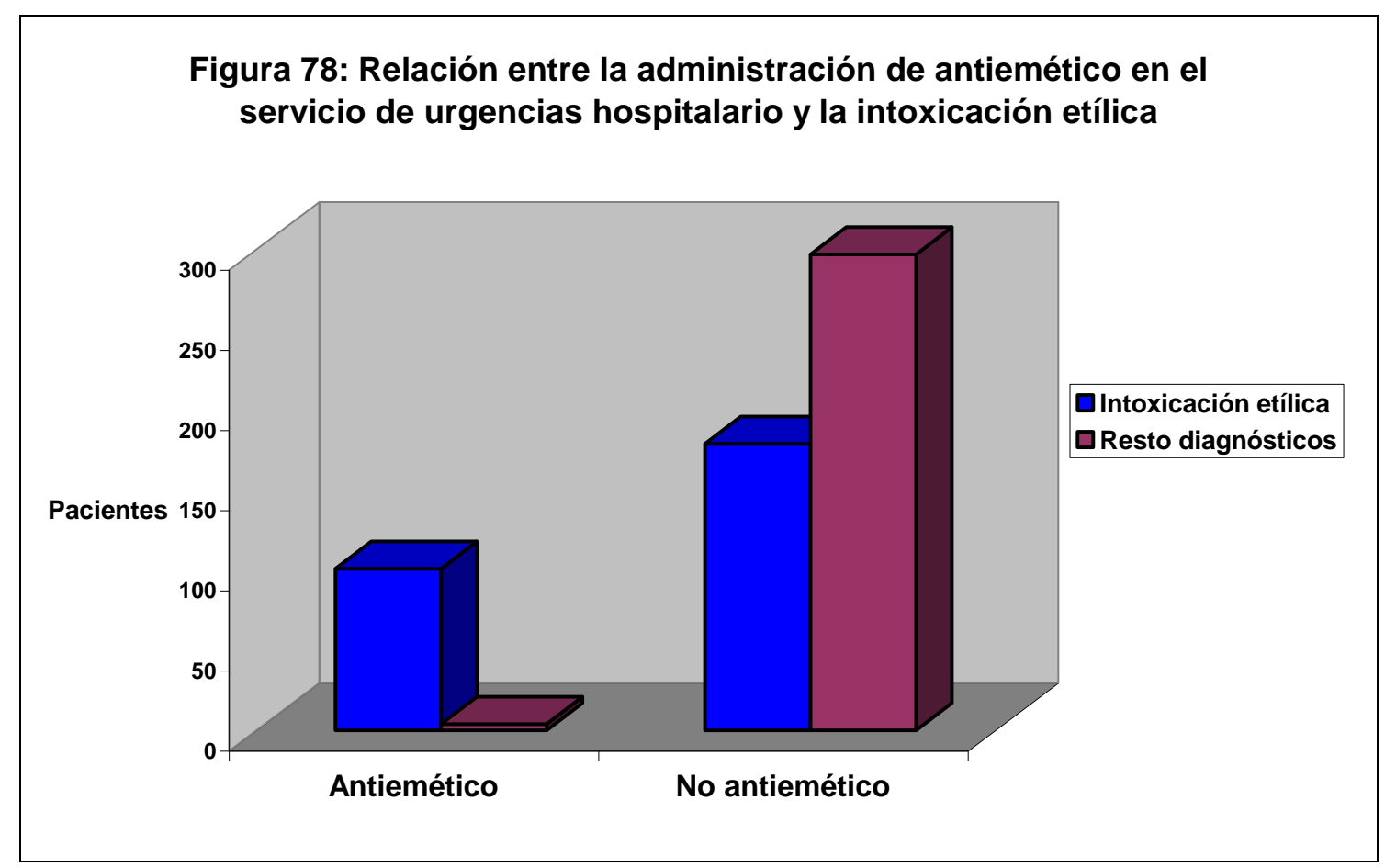

$p=0,0001$

\subsubsection{Relación entre la administración de protector gástrico y la} intoxicación etílica.

Se evidenciaron diferencias estadísticamente significativas en la necesidad de administración de protector gástrico en el servicio de urgencias hospitalario teniendo en cuenta el diagnóstico de intoxicación etílica ( $p=$ 0,0001) Tabla 120, figura 79

Tabla 120: Relación entre la administración de protector gástrico en el servicio de urgencias hospitalario y la intoxicación etílica

\begin{tabular}{|c|cc|}
\hline Tratamiento & Intoxicación etílica & Resto diagnósticos \\
\hline Protector gástrico & 44 & 12 \\
No protector gástrico & 236 & 289 \\
& $\mathrm{p}=0,0001$ & \\
\hline
\end{tabular}


Figura 79: Relación entre la administración de protector gástrico y el diagnóstico de intoxicación etílica

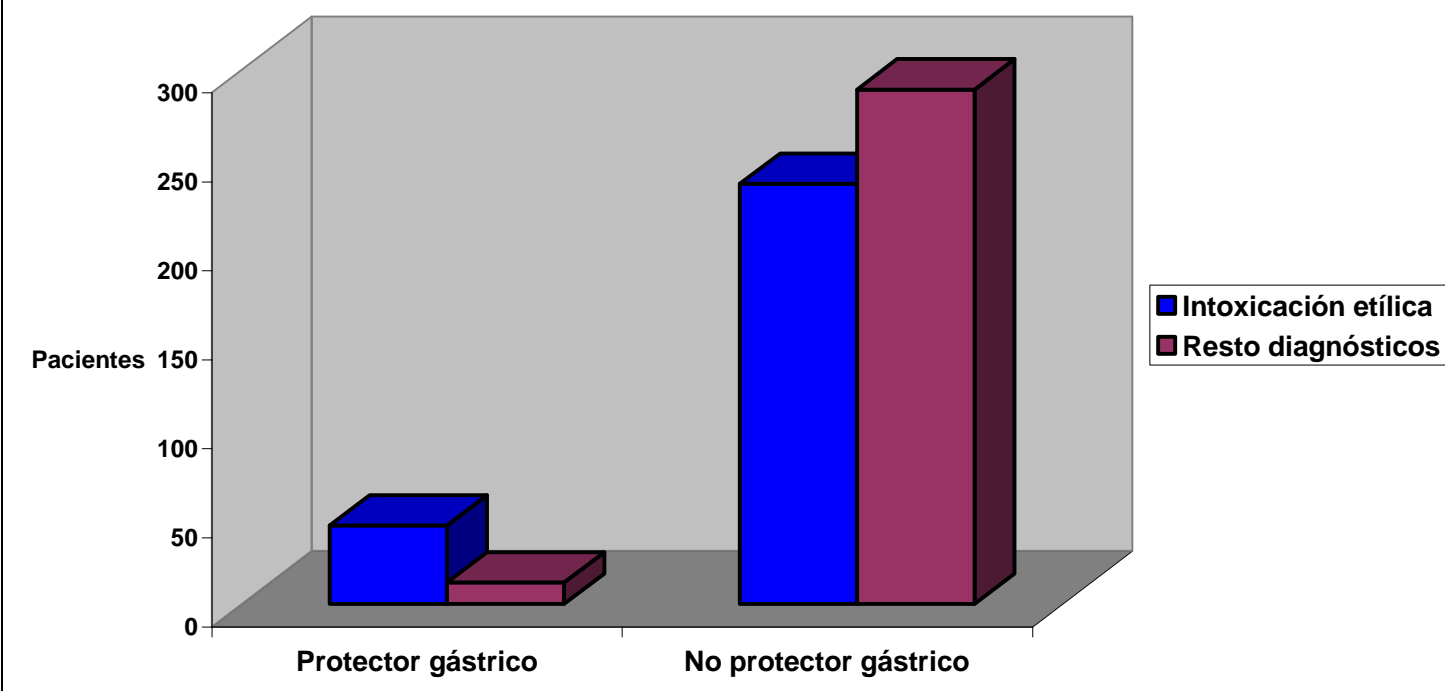

$p=0,0001$

\subsubsection{Relación entre la administración de otros tratamientos en el} servicio de urgencias hospitalario y la intoxicación etílica.

Se evidenciaron diferencias estadísticamente significativas en la necesidad de administración de otros tratamientos en el servicio de urgencias hospitalario teniendo en cuenta el diagnóstico de intoxicación etílica ( $p=$ 0,006) Tabla 121, figura 80.

Tabla 121: Relación entre la administración otros tratamientos en el servicio de urgencias hospitalario y la intoxicación etílica

\begin{tabular}{|c|cc|}
\hline Tratamiento & Intoxicación etílica & Resto diagnósticos \\
\hline Otros tratamientos & 12 & 31 \\
No otros tratamientos & 269 & 272 \\
& $p=0,006$ & \\
\hline
\end{tabular}




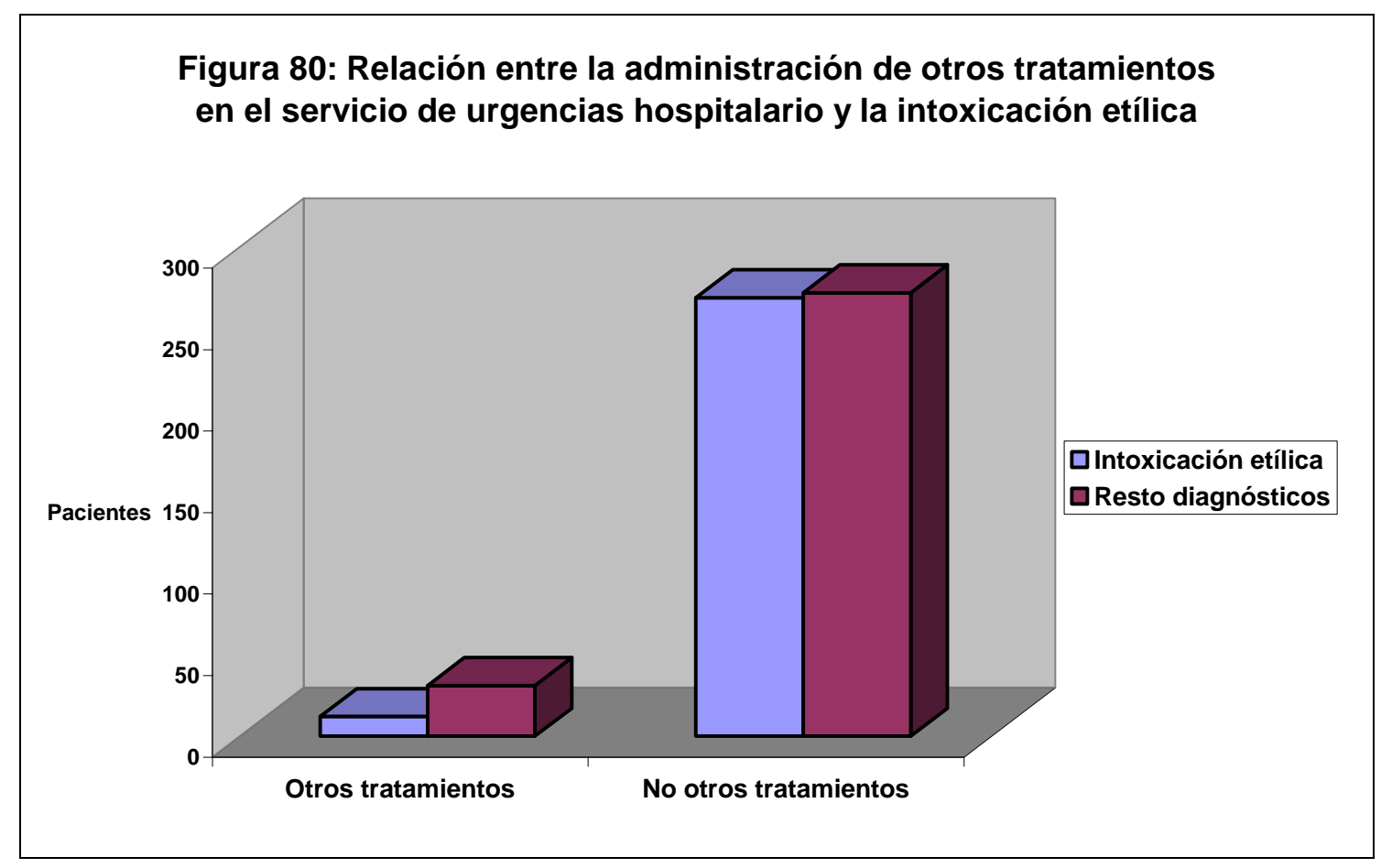

$p=0,006$

\subsubsection{Relación entre la administración de antídoto y el diagnóstico de} intoxicación etílica.

No se evidenciaron diferencias estadísticamente significativas en el tratamiento de con antídotos teniendo en cuenta el diagnóstico de intoxicación etílica $(p=0,298)$. Tabla 122 .

Tabla 122: Relación entre la administración de antídoto en el servicio de urgencias hospitalario y la intoxicación etílica

\begin{tabular}{|c|cc|}
\hline Tratamiento & Intoxicación etílica & Resto diagnósticos \\
\hline Antídoto & 2 & 5 \\
No antídoto & 278 & 297 \\
& $\mathrm{p}=0,298$ & \\
\hline
\end{tabular}




\subsubsection{Relación entre la administración de analgésicos y la intoxicación etílica.}

No se evidenciaron diferencias estadísticamente significativas en el tratamiento de con analgésicos teniendo en cuenta el diagnóstico de intoxicación etílica $(p=0,094)$. Tabla 123.

Tabla 123: Relación entre la administración de analgésicos en el servicio de urgencias hospitalario y la intoxicación etílica

\begin{tabular}{|c|cc|}
\hline Tratamiento & Intoxicación etílica & Resto diagnósticos \\
\hline Analgésicos & 11 & 5 \\
No analgésico & 269 & 297 \\
& $p=0,094$ & \\
\hline
\end{tabular}

\subsubsection{Relación entre la administración de tiamina en el servicio de urgencias hospitalario y la intoxicación etílica.}

No se evidenciaron diferencias estadísticamente significativas en el tratamiento de con tiamina teniendo en cuenta el diagnóstico de intoxicación etílica $(p=$ 0,412). Tabla 124.

Tabla 124: Relación entre la administración de tiamina en el servicio de urgencias hospitalario y la intoxicación etílica

\begin{tabular}{|c|cc|}
\hline Tratamiento & Intoxicación etílica & Resto diagnósticos \\
\hline Tiamina & 5 & 3 \\
No tiamina & 275 & 299 \\
& $p=0,412$ & \\
\hline
\end{tabular}

\subsubsection{Interconsulta con Psiquiatría durante la estancia en el} servicio de urgencias hospitalarias en los pacientes con intoxicación etílica

Se distribuye a los pacientes con diagnóstico de intoxicación etílica, según la necesidad de realizar interconsulta con Psiquiatría durante el tiempo de estancia en el servicio de urgencias hospitalarias. 
Se evidenciaron diferencias estadísticamente significativas en la necesidad de realizar interconsulta con Psiquiatría durante el tiempo de estancia en el servicio de urgencias hospitalarias teniendo en cuenta el diagnóstico de intoxicación etílica $(p=\mathbf{0}, 0001)$. Tabla 125 , figura 81 .

Tabla 125: Relación entre la necesidad de interconsulta con Psiquiatría y el diagnóstico de intoxicación etílica

\begin{tabular}{|c|ccc|}
\hline & No & Sí & Total \\
\hline Intoxicación etílica & 200 & 0 & 200 \\
Resto de diagnósticos & 177 & 37 & 214 \\
\hline Total & 377 & 37 & 414 \\
\hline$\quad \mathrm{p}=0,0001$ & & & \\
\hline
\end{tabular}

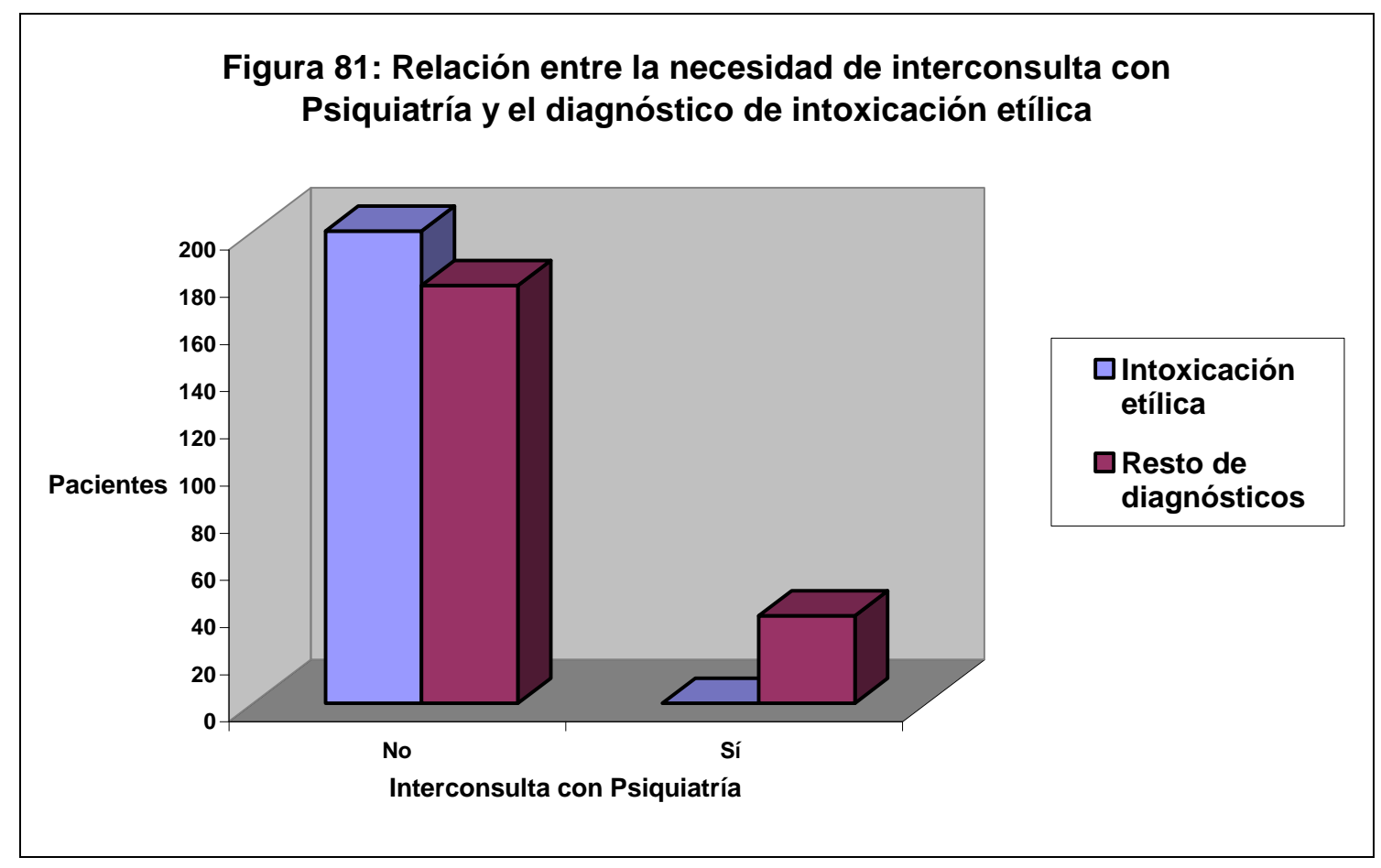

$p=0,0001$ 


\subsubsection{Interconsulta con Toxicología durante la estancia en el servicio de}

urgencias hospitalario en los pacientes con intoxicación etílica

Ninguno de los pacientes con intoxicación etílica precisaron realizar interconsulta con el servicio de Toxicología durante su estancia en el servicio de urgencias hospitalario.

Se evidenciaron diferencias estadísticamente significativas en la necesidad de realizar interconsulta con Toxicología durante el tiempo de estancia en el servicio de urgencias hospitalarias teniendo en cuenta el diagnóstico de intoxicación etílica $(p=\mathbf{0 , 0 0 0 1})$. Tabla 126, figura 82 .

Tabla 126: Relación entre la interconsulta con Toxicología y el diagnóstico de intoxicación etílica

\begin{tabular}{|c|ccc|}
\hline & NO & SI & Total \\
\hline Resto diagnósticos & 139 & 86 & 225 \\
Intoxicación etílica & 199 & 0 & 199 \\
\hline Total & 338 & 86 & 424 \\
\hline \multicolumn{2}{r}{$\mathrm{p}=0,0001$} & & \\
\hline
\end{tabular}




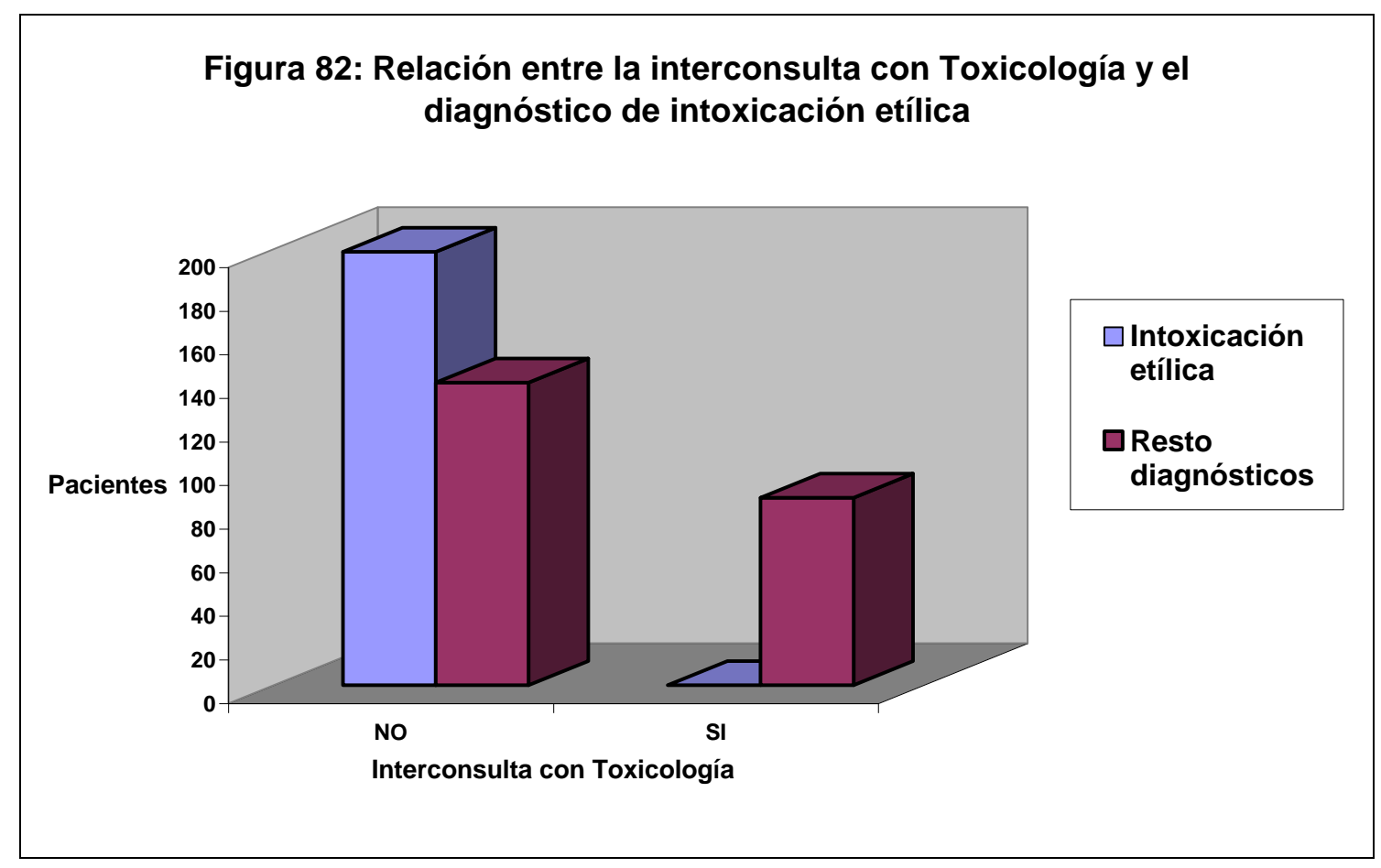

$p=0,0001$

\subsubsection{Distribución de la intoxicación etílica según el tiempo de} estancia en el servicio de urgencias hospitalario.

Se distribuye el tiempo de estancia en el servicio de urgencias hospitalario entre más o menos de 2 horas y los resultados se muestran en la tabla 127 y figura 83.

Tabla 127: Distribución del tiempo de estancia en el servicio de urgencias hospitalario en la intoxicación etílica

\begin{tabular}{|c|cc|}
\hline & Frecuencia & Porcentaje \\
\hline Menos de 2 horas & 105 & 24,19 \\
Mas de 2 horas & 329 & 75,8 \\
Total & 434 & 100 \\
\hline
\end{tabular}


Figura 83: Distribución del tiempo de permanencia en el servicio de urgencias de los pacientes con intoxicación etílica

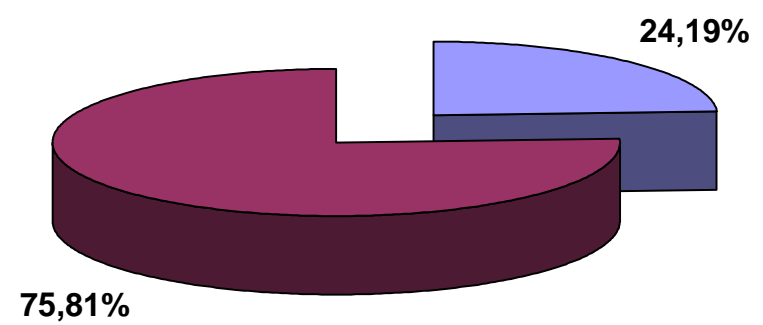

$75,81 \%$

Se evidenciaron diferencias estadísticamente significativas en el tiempo de permanencia en el servicio de urgencias hospitalario teniendo en cuenta el diagnóstico de intoxicación etílica $(p=0,0001)$. Tabla 128, figura 84

Tabla 128: Relación entre el tiempo de permanencia en el servicio de urgencias hospitalario y la intoxicación etílica

\begin{tabular}{|c|cc|c|}
\hline & Menos de 2 horas & Mas de 2 horas & Total \\
\hline Resto diagnósticos & 307 & 253 & 560 \\
Intoxicación etílica & 105 & 329 & 434 \\
\hline Total & 412 & 582 & 994 \\
\hline \multicolumn{2}{|l}{} \\
\hline
\end{tabular}




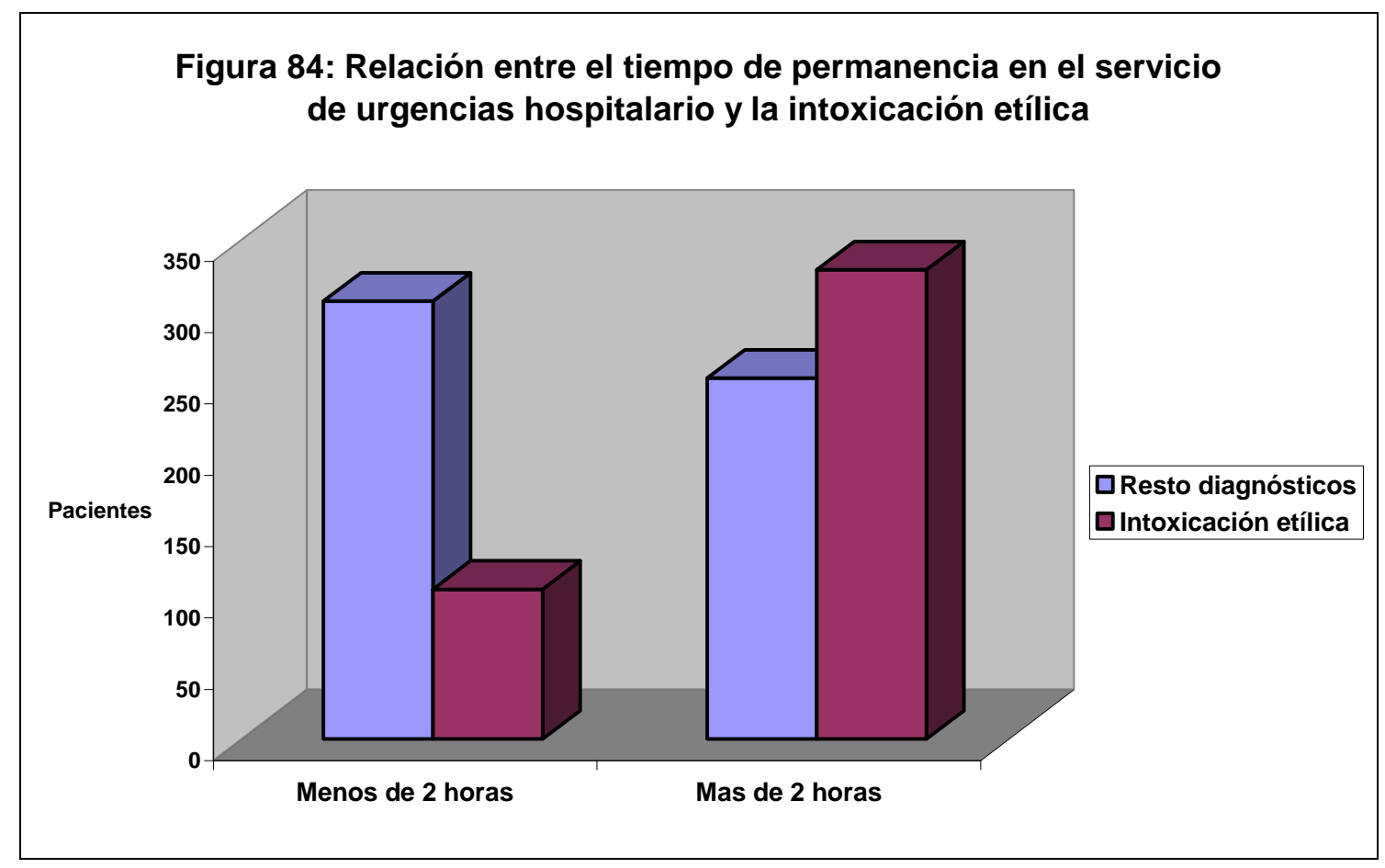

$p=0,0001$

\subsubsection{Distribución de la intoxicación etílica según el destino del} paciente tras su estancia en el servicio de urgencias hospitalario.

Se distribuye a los pacientes con diagnóstico de intoxicación etílica, según el destino del paciente tras su paso por el servicio de urgencias hospitalario. Los resultados se muestran en la tabla 129.

Tabla 129: Destino de los pacientes con intoxicación etílica tras su estancia en el servicio de urgencias hospitalario.

\begin{tabular}{|c|cc|}
\hline & Frecuencia & Porcentaje \\
\hline Alta & 429 & 98,84 \\
Ingreso Hospitalario & 5 & 1,15 \\
\hline Total & 434 & 100 \\
\hline
\end{tabular}

Se evidenciaron diferencias estadísticamente significativas en el destino tras su estancia en el servicio de urgencias hospitalario de los pacientes teniendo en cuenta el diagnóstico de intoxicación etílica $(p=0,0001)$. Tabla 130 , figura 85. 
Tabla 130: Relación entre el diagnóstico de intoxicación etílica y su destino tras la estancia en el servicio de urgencias hospitalario

\begin{tabular}{|c|ccccc|}
\hline & Alta & Ingreso Hospitalario & $\begin{array}{c}\text { Consulta } \\
\text { externa }\end{array}$ & Derivación & Total \\
\hline $\begin{array}{c}\text { Resto } \\
\text { diagnósticos } \\
\begin{array}{c}\text { Intoxicación } \\
\text { etílica }\end{array}\end{array}$ & 398 & 125 & 10 & 27 & 560 \\
\hline Total & 428 & 6 & 0 & 0 & 434 \\
\hline \multicolumn{7}{c}{$\mathrm{p}=0,0001$} \\
\hline
\end{tabular}

Figura 85: Relación entre el diagnóstico de intoxicación etílica y su destino tras la estancia en el servicio de urgencias hospitalario

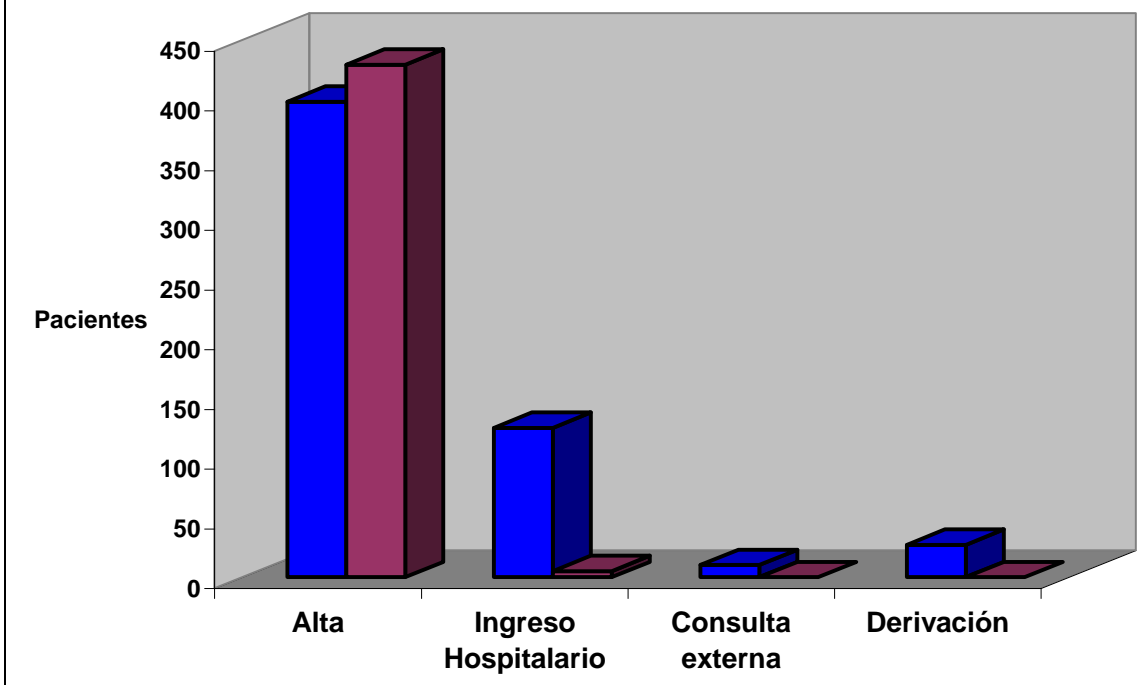

Resto diagnósticos Intoxicación etílica

$p=0,0001$

\subsubsection{Distribución de la intoxicación etílica según la planta donde} ingresa el paciente.

Se distribuye a los pacientes con diagnóstico de intoxicación etílica, según la planta donde se realiza el ingreso. Los resultados se muestran en la tabla 131. 
Tabla 131: Servicio a cargo del ingreso del paciente con intoxicación etílica

\begin{tabular}{|c|cc|}
\hline & Frecuencia & Porcentaje \\
\hline Pediatría & 1 & 16,66 \\
Medicina Interna & 1 & 16,66 \\
UCI & 2 & 33,33 \\
Otros & 2 & 33,33 \\
Total & 6 & 100 \\
\hline
\end{tabular}

Se evidenciaron diferencias estadísticamente significativas en el servicio a cargo del que se produce el ingreso teniendo en cuenta el diagnóstico de intoxicación etílica $(p=\mathbf{0 , 0 0 0 1})$. Tabla 132, figura 86 .

Tabla 132: Relación entre el servicio en el que se ingresa y el diagnóstico de intoxicación etílica

\begin{tabular}{|c|cccc|}
\hline & Pediatría & Medicina Interna & UCl & Otros \\
\hline Resto diagnósticos & 98 & 8 & 17 & 26 \\
Intoxicación etílica & 1 & 1 & 2 & 2 \\
\hline Total & 99 & 9 & 19 & 28 \\
\hline \multicolumn{7}{c}{$\mathrm{p}=0,0001$} \\
\hline
\end{tabular}

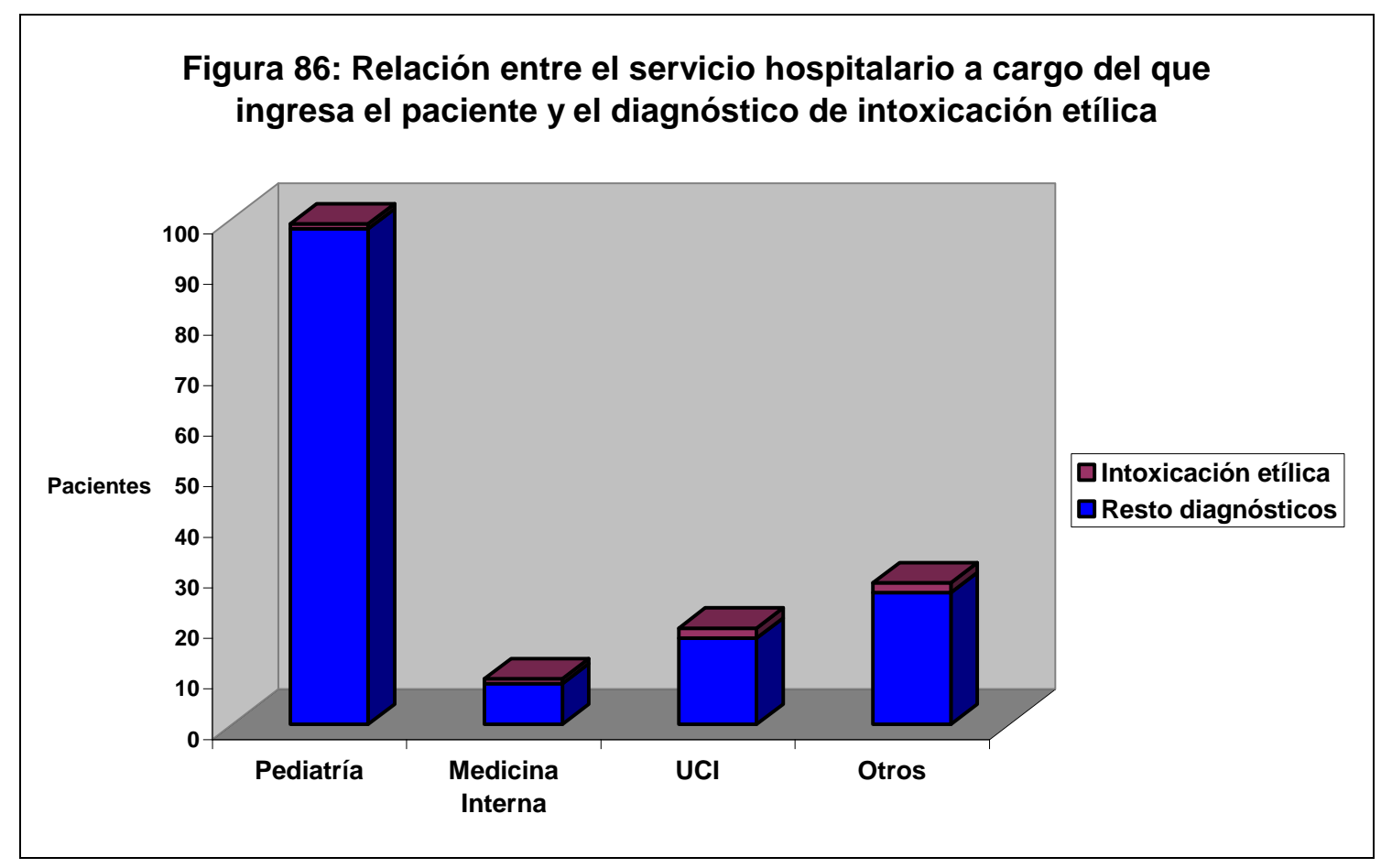

$p=0,0001$ 


\subsubsection{Tiempo de ingreso hospitalario en horas en los pacientes con}

\section{intoxicación etílica}

Sólo 5 pacientes con intoxicación etílica necesitan ingreso en planta. El tiempo de ingreso en horas se muestra en la tabla 133 y figura 87.

Tabla 133: Tiempo de ingreso en planta en los pacientes con intoxicación etílica

\begin{tabular}{|c|cc|}
\hline Tiempo en horas & Frecuencia & Porcentaje \\
\hline 24 & 3 & 60 \\
48 & 2 & 40 \\
\hline Total & 5 & 100 \\
\hline
\end{tabular}

Figura 87: Tiempo de ingreso en planta en las intoxicaciones etílicas

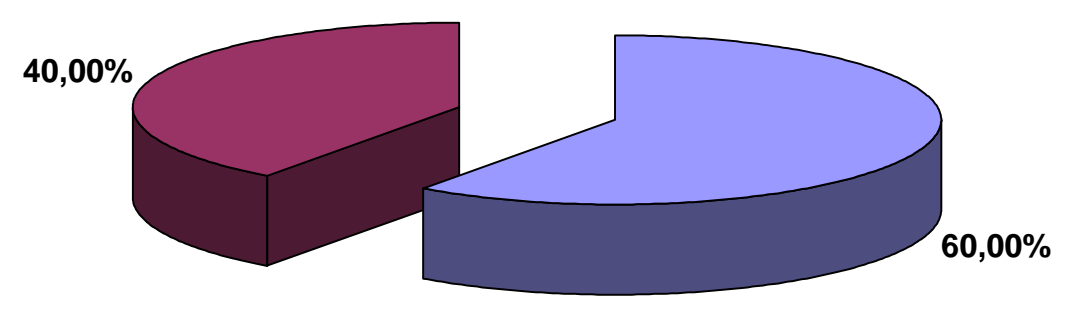

No se evidenciaron diferencias estadísticamente significativas en el tiempo de ingreso en planta medido en horas teniendo en cuenta el diagnóstico de intoxicación etílica $(p=0,564)$. Tabla 134 . 
Tabla 134: Relación entre el tiempo de ingreso y el diagnóstico de intoxicación etílica

\begin{tabular}{|c|ccc|}
\hline Diagnóstico & $\mathrm{N}$ & Media en horas & Desviación típica \\
\hline Resto diagnósticos & 123 & 27,06 & 25,06 \\
Intoxicación etílica & 5 & 33,6 & 13,14 \\
& & $\mathrm{p}=0,564$ & \\
\hline
\end{tabular}

\subsubsection{Cifras de alcoholemia encontradas en los pacientes con intoxicación etílica}

Se realiza la alcoholemia en sangre a 215 pacientes que acuden al servicio de Urgencias Hospitalario con el diagnóstico de intoxicación etílica. Tabla 135.

Tabla 135: Realización de alcoholemia en los pacientes con intoxicación etílica

\begin{tabular}{|ccc|}
\hline Realización de alcoholemia & Frecuencia & Porcentaje \\
\hline No & 219 & 50,5 \\
Sí & 215 & 49,5 \\
Total & 434 & 100 \\
\hline
\end{tabular}

No se evidenciaron diferencias estadísticamente significativas en la realización de alcoholemia teniendo en cuenta la edad del intoxicado $(p=0,37)$. Tabla 136.

Tabla 136: Edad media de los pacientes a los que se les realizó alcoholemia

\begin{tabular}{|ccc|}
\hline Realización alcoholemia & Edad media en años & Desviación típica \\
\hline No & 15,47 & 1,74 \\
Sí & 15,33 & 1,55 \\
& $\mathrm{p}=0,37$ & \\
\hline
\end{tabular}


No se evidenciaron diferencias estadísticamente significativas en la realización de alcoholemia teniendo en cuenta el sexo del intoxicado $(\mathbf{p = 0 , 7 0 )}$. Tabla 137.

Tabla 137: Relación entre el sexo y la realización de alcoholemia

\begin{tabular}{|c|c|c|c|}
\hline Realización de alcoholemia & Hombre & Mujer & Total \\
\hline No & 119 & 100 & 219 \\
\hline Sí & 121 & 94 & 215 \\
\hline Total & 240 & 194 & 434 \\
\hline \multicolumn{4}{|c|}{$p=0,70$} \\
\hline
\end{tabular}

Se evidenciaron diferencias estadísticamente significativas en la realización de alcoholemia teniendo en cuenta el tratamiento del intoxicado $(\mathbf{p}=\mathbf{0 , 0 0 0 1})$. Tabla 138.

Tabla 138: Relación entre la administración de tratamiento y la realización de alcoholemia

\begin{tabular}{|cccc|}
\hline Realización alcoholemia & No tratamiento & Sí tratamiento & Total \\
\hline No & 36 & 30 & 66 \\
Sí & 47 & 168 & 215 \\
Total & 83 & 198 & 281 \\
& $p=0,0001$ & & \\
\hline
\end{tabular}

No se evidenciaron diferencias estadísticamente significativas en la realización de alcoholemia teniendo en cuenta el destino del intoxicado tras el servicio de urgencias hospitalario $(p=0,50)$. Tabla 139

Tabla 139: Relación entre la realización de alcoholemia y el destino del paciente tras el servicio de urgencias hospitalario

\begin{tabular}{|ccc|}
\hline Realización alcoholemia & Alta & Ingreso hospitalario \\
\hline No & 216 & 3 \\
Sí & 213 & 2 \\
& $\mathrm{p}=0,50$ & \\
\hline
\end{tabular}




\subsubsection{Consultas al servicio de urgencias hospitalario el mismo año y en años posteriores que acuden por la intoxicación etílica}

Se distribuye a los pacientes con diagnóstico de intoxicación etílica, según el número de consultas al servicio de urgencias hospitalario en el mismo año en el que acuden por la intoxicación. Los resultados se muestran en la tabla 140141 y figura $88-89$

Tabla 140: Número de visitas al servicio de urgencias hospitalario el mismo año que acuden por la intoxicación etílica

\begin{tabular}{|c|cc|}
\hline Número de visitas a urgencias & Frecuencia & Porcentaje \\
\hline 0 & 360 & 82,94 \\
1 & 57 & 13,13 \\
2 & 12 & 2,76 \\
3 & 2 & 0,46 \\
6 & 2 & 0,46 \\
7 & 1 & 0,23 \\
\hline Total & 434 & 100 \\
\hline
\end{tabular}

Figura 88: Número de visitas al servicio de urgencias hospitalario el mismo año que acuden por la intoxicación etílica

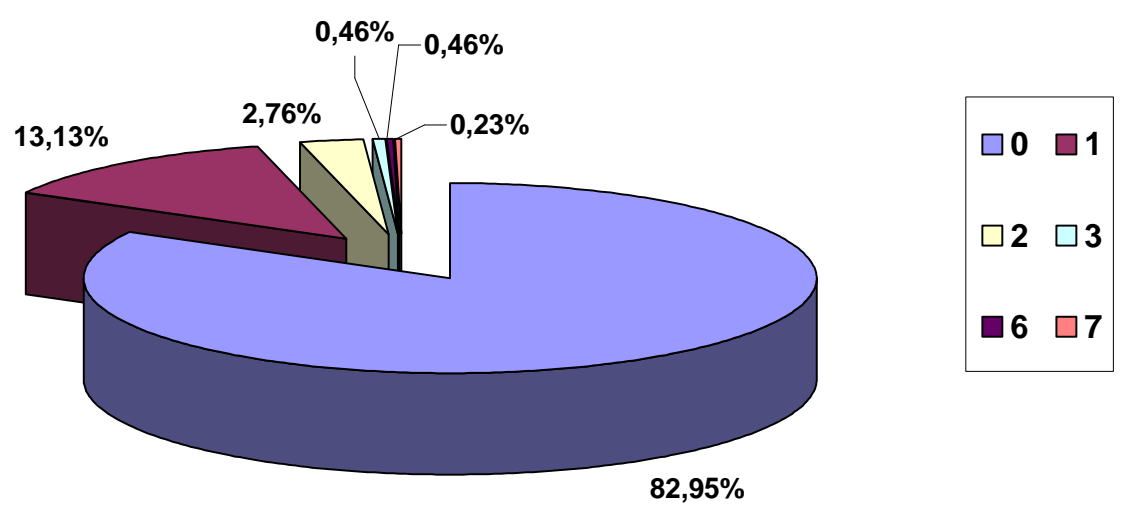


Tabla 141: Número de consultas al servicio de urgencias hospitalario en los años posteriores a la intoxicación etílica

\begin{tabular}{|ccc|}
\hline Número consultas & Frecuencia & Porcentaje \\
\hline 0 & 252 & 59,15 \\
1 & 72 & 16,90 \\
2 & 33 & 7,74 \\
3 & 16 & 3,75 \\
4 & 18 & 4,22 \\
5 & 11 & 2,58 \\
6 & 7 & 1,64 \\
7 & 5 & 1,17 \\
8 & 3 & 0,70 \\
9 & 1 & 0,23 \\
10 & 1 & 0,23 \\
11 & 1 & 0,23 \\
12 & 2 & 0,46 \\
14 & 1 & 0,23 \\
15 & 1 & 0,23 \\
16 & 1 & 0,23 \\
22 & 1 & 0,23 \\
\hline Total & 426 & 100 \\
\hline
\end{tabular}


Figura 89: Número de consultas en el servicio de urgencias hospitalario en los años posteriores a la intoxicación etílica

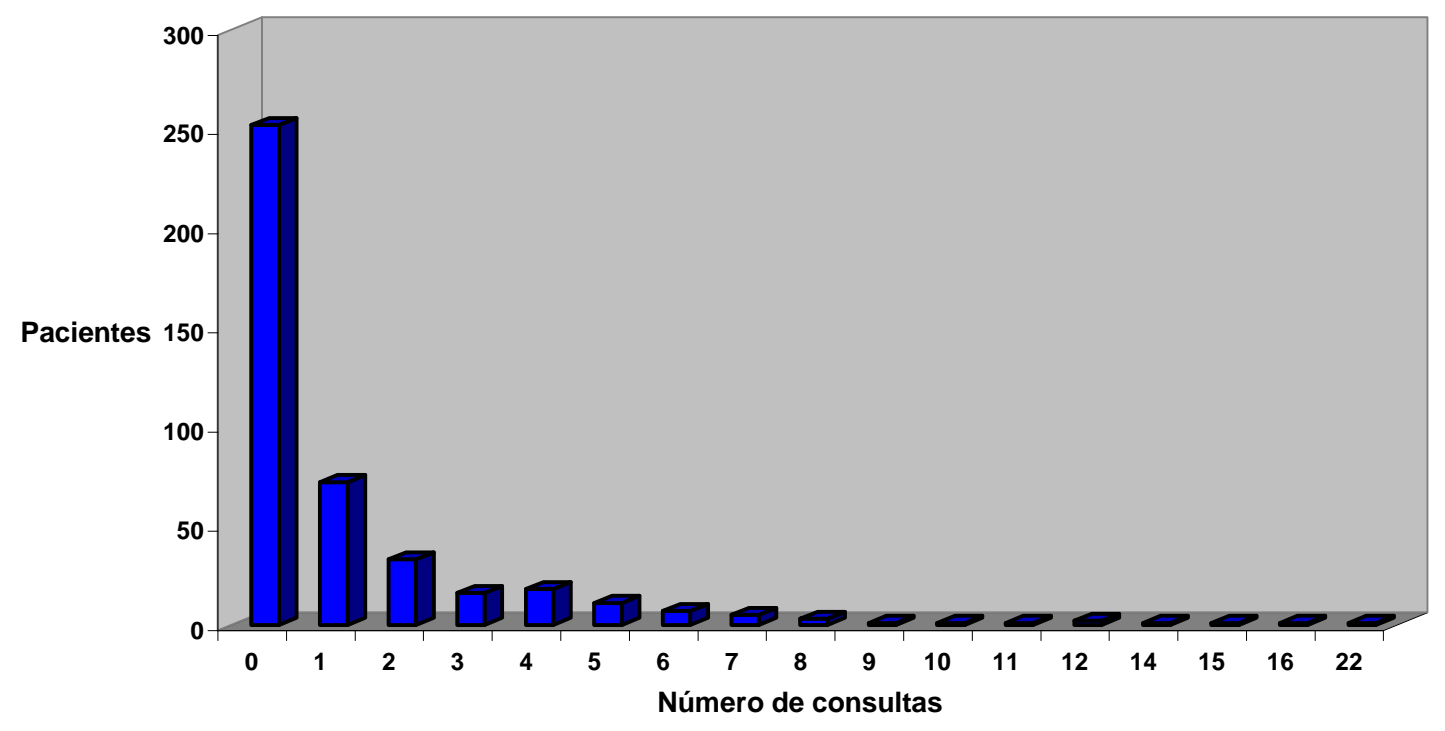

Se evidenciaron diferencias estadísticamente significativas en el número de consultas en el servicio de Urgencias Hospitalario el mismo año que acuden con la intoxicación y en años posteriores a la intoxicación teniendo en cuenta el diagnóstico de intoxicación etílica ( $\mathrm{p}=0,0001$ en ambos casos) Tabla 142 y 143, figura 90.

Tabla 142: Relación entre el número de consultas al servicio de urgencias hospitalario el mismo año de la intoxicación y el diagnóstico de intoxicación etílica

\begin{tabular}{|c|ccc|}
\hline Diagnostico & $\mathrm{N}$ & Media & Desviación típica \\
\hline Resto diagnósticos & 560 & 0,62 & 1,2 \\
Intoxicación etílica & 434 & 0,24 & 0,7 \\
& $\mathrm{p}=0,0001$ & \\
\hline
\end{tabular}

Tabla 143: Relación entre el número de consultas en el servicio de urgencias hospitalario en los años posteriores a la intoxicación y el diagnóstico de intoxicación etílica

\begin{tabular}{|c|ccc|}
\hline Diagnostico & $\mathrm{N}$ & Media & Desviación típica \\
\hline Resto diagnósticos & 544 & 2,62 & 4,09 \\
Intoxicación etílica & 426 & 1,25 & 2,51 \\
& $\mathrm{p}=0,0001$ & \\
\hline
\end{tabular}




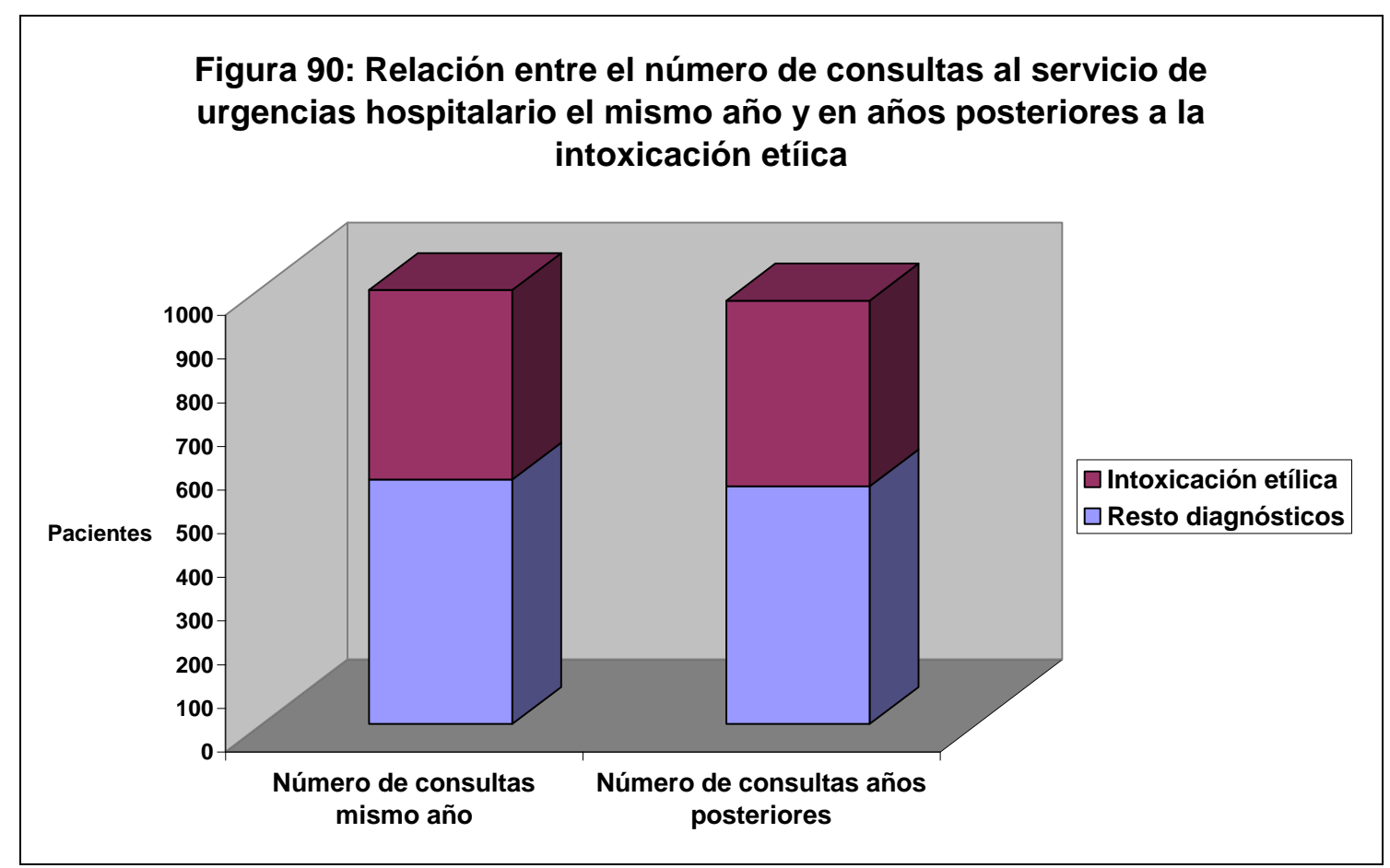

$p=0,0001$

6.1.21. Consultas al servicio de urgencias hospitalario en los años anteriores al que acuden por la intoxicación etílica

Se distribuye a los pacientes con diagnóstico de intoxicación etílica, según el número de consultas a urgencias en años anteriores al que acuden por la intoxicación. Los resultados se muestran en la tabla 144 y figura 91. 
Tabla 144: Número de consultas al servicio de urgencias hospitalario en los años previos a la intoxicación etílica

\begin{tabular}{|c|c|c|}
\hline Número de consultas a urgencias & Frecuencia & Porcentaje \\
\hline 0 & 161 & 37,09 \\
\hline 1 & 64 & 14,74 \\
\hline 2 & 48 & 11,05 \\
\hline 3 & 35 & 8,06 \\
\hline 4 & 27 & 6,22 \\
\hline 5 & 15 & 3,45 \\
\hline 6 & 21 & 4,83 \\
\hline 7 & 7 & 1,61 \\
\hline 8 & 5 & 1,15 \\
\hline 9 & 4 & 0,92 \\
\hline 10 & 6 & 1,38 \\
\hline 11 & 5 & 1,15 \\
\hline 12 & 8 & 1,84 \\
\hline 13 & 2 & 0,46 \\
\hline 14 & 3 & 0,69 \\
\hline 15 & 3 & 0,69 \\
\hline 16 & 4 & 0,92 \\
\hline 17 & 2 & 0,46 \\
\hline 19 & 2 & 0,46 \\
\hline 20 & 3 & 0,69 \\
\hline 21 & 2 & 0,46 \\
\hline 22 & 1 & 0,23 \\
\hline 25 & 1 & 0,23 \\
\hline 28 & 1 & 0,23 \\
\hline 36 & 2 & 0,46 \\
\hline 40 & 1 & 0,23 \\
\hline 42 & 1 & 0,23 \\
\hline
\end{tabular}




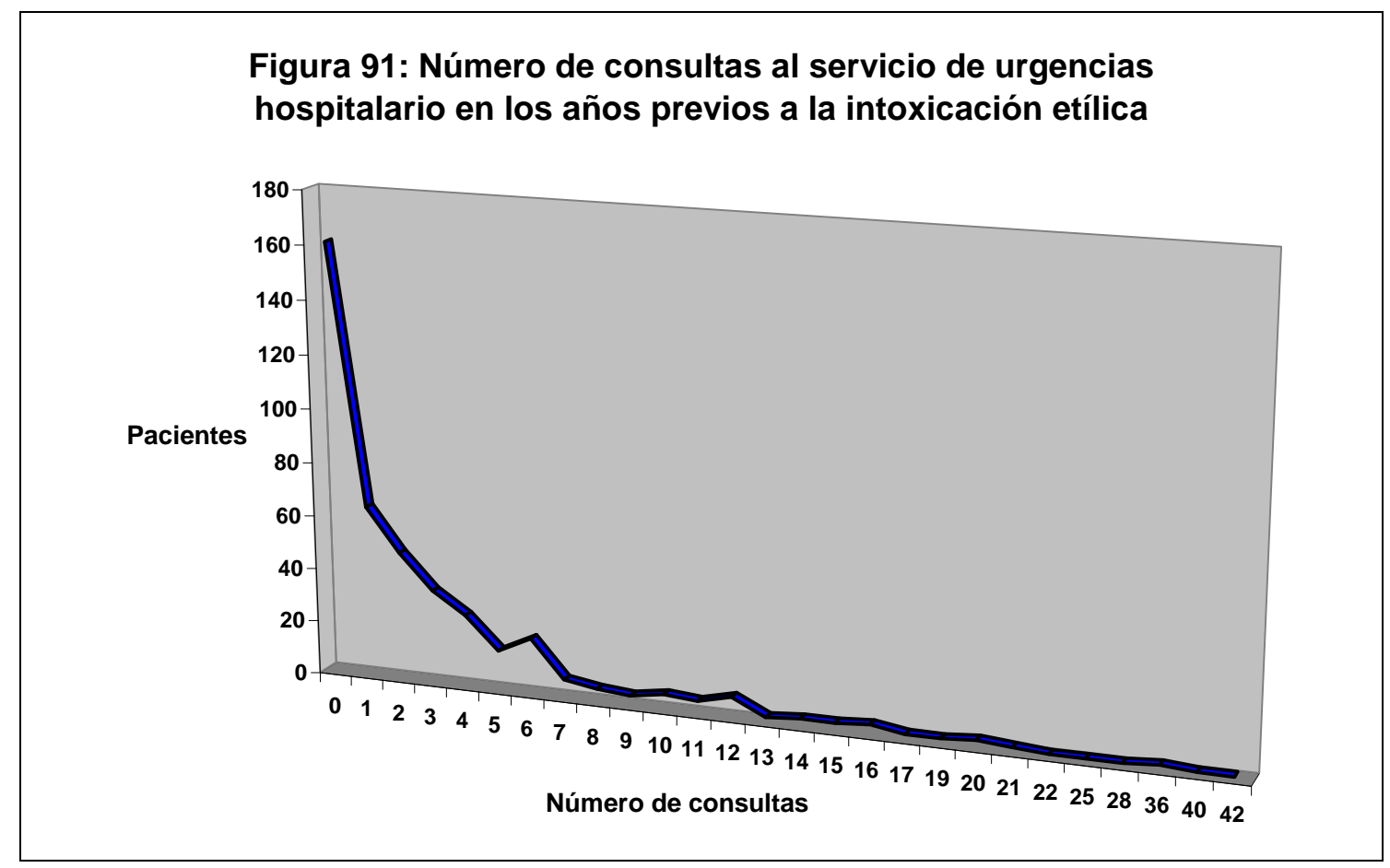

No se evidenciaron diferencias estadísticamente significativas en el número de consultas en Urgencias Hospitalarias en los años previos a la intoxicación teniendo en cuenta el diagnóstico de intoxicación etílica ( $p=$ 0,441) Tabla 145.

Tabla 145: Relación entre las consultas al servicio de urgencias hospitalario en los años previos a la intoxicación y el diagnóstico de intoxicación etílica

\begin{tabular}{|c|ccc|}
\hline Diagnóstico & $\mathrm{N}$ & Media & Desviación típica \\
\hline Resto diagnósticos & 560 & 3,69 & 4,81 \\
Intoxicación etílica & 434 & 3,43 & 5,73 \\
& $\mathrm{p}=0,441$ & \\
\hline
\end{tabular}




\subsubsection{Número de consultas ambulatorias solicitadas en los pacientes con intoxicación etílica}

Se evidenciaron diferencias estadísticamente significativas en el número de consultas ambulatorias teniendo en cuenta el diagnóstico de intoxicación etílica $(p=0,002)$ Tabla 146, figura 92.

Tabla 146: Relación entre el número de consultas ambulatorias y el diagnóstico de intoxicación etílica

\begin{tabular}{|c|ccc|}
\hline Diagnóstico & $\mathrm{N}$ & Media & Desviación típica \\
\hline Resto diagnósticos & 549 & 1,48 & 1,35 \\
Intoxicación etílica & 432 & 1,75 & 1,4 \\
& $\mathrm{p}=0,002$ & \\
\hline
\end{tabular}

Figura 92: Relación entre el número de consultas ambulatorias y la intoxicación etílica

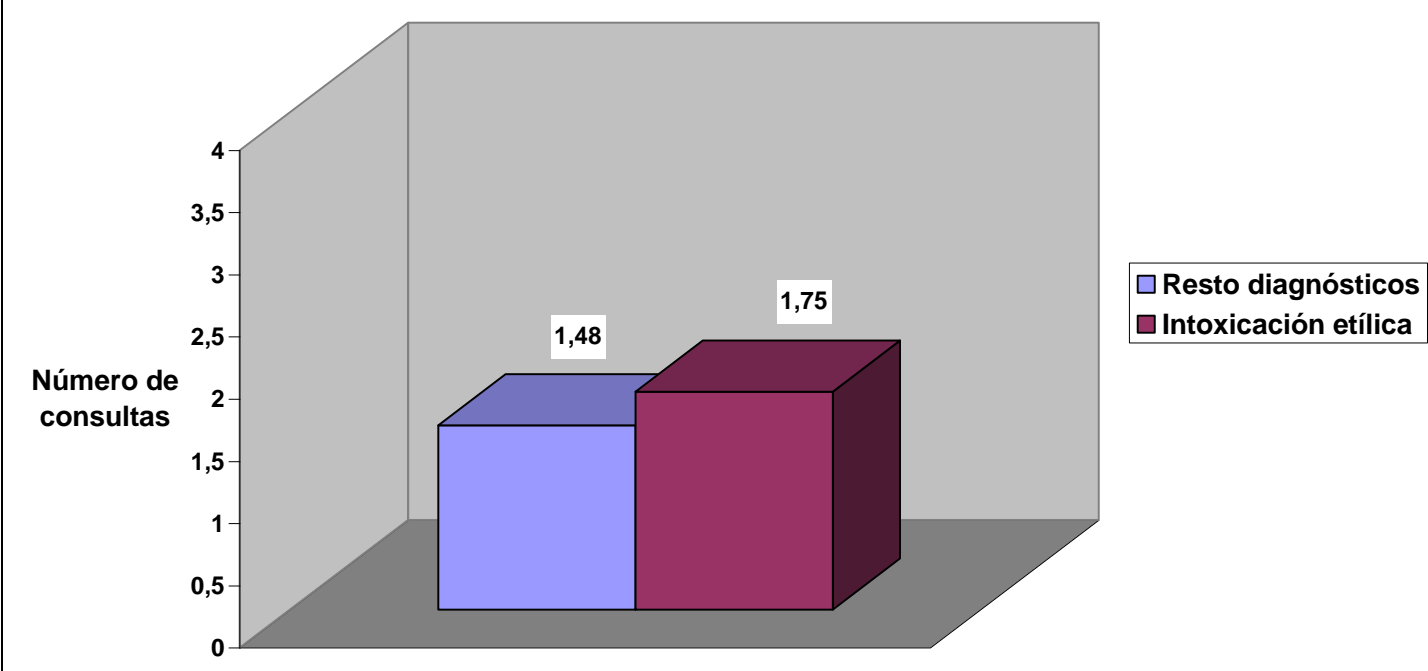

$p=0,002$ 


\subsection{Intoxicaciones medicamentosas.}

\subsubsection{Intoxicaciones medicamentosas y el sexo.}

Dentro de las intoxicaciones medicamentosas, el porcentaje de varones es de $46,81 \%$ y el de mujeres 53,18\%. Tabla 147, figura 93.

Se evidenciaron diferencias estadísticamente significativas en el sexo de los pacientes atendidos en el servicio de urgencias hospitalario teniendo en cuenta el diagnóstico de intoxicación medicamentosa $(p=0,008)$.

Tabla 147: Distribución por sexos en la intoxicación medicamentosa

\begin{tabular}{|c|c|c|c|c|}
\hline & & Resto intoxicaciones & Intoxicación medicamentosa & Total \\
\hline Hombre & $\mathrm{N}$ & 409 & 125 & 534 \\
& $\%$ & 56,30 & 46,80 & 53,70 \\
Mujer & $\mathrm{N}$ & 318 & 142 & 460 \\
& $\%$ & 43,70 & 53,20 & 46,30 \\
\hline
\end{tabular}




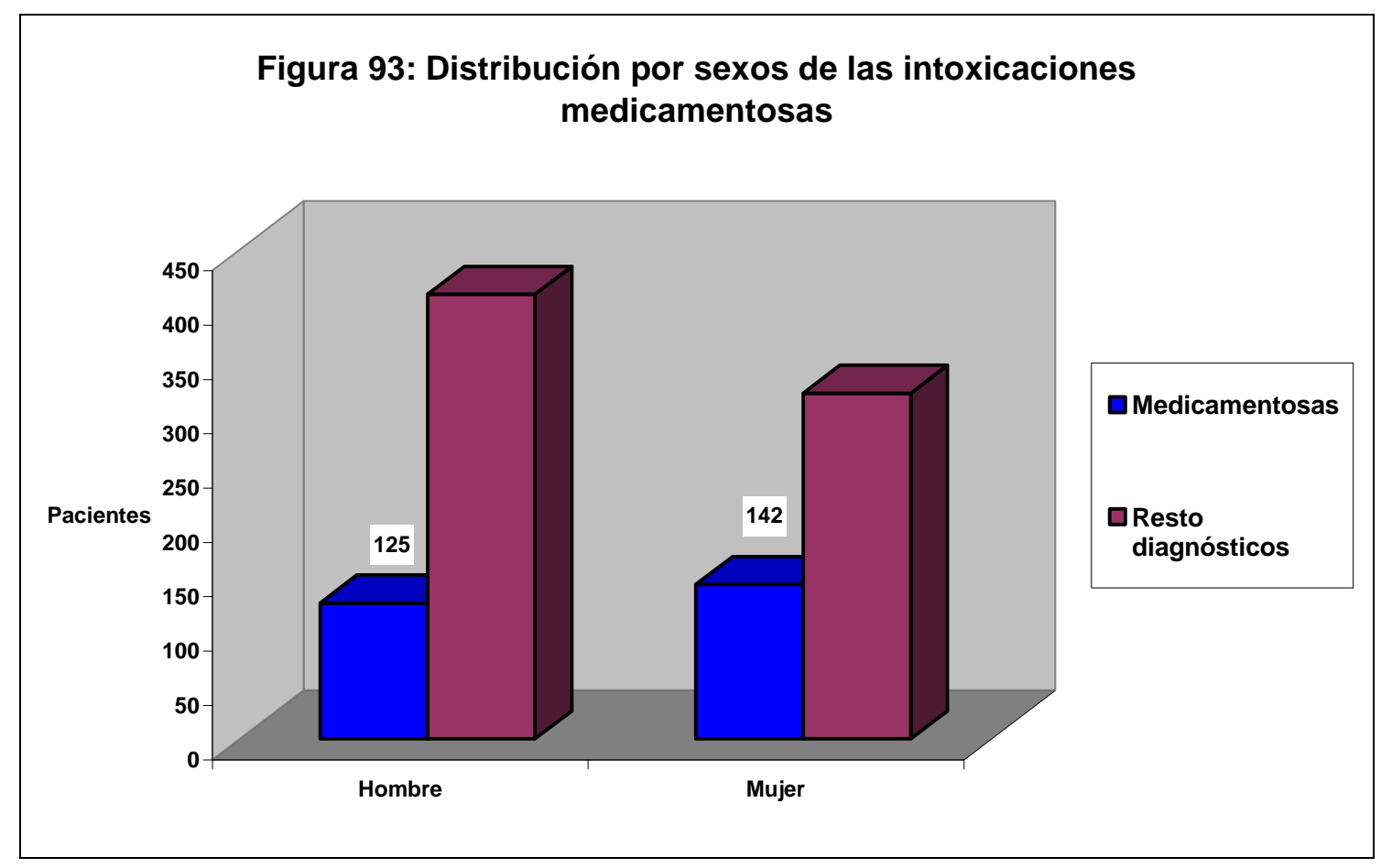

$p=0,008$

\subsubsection{Edad de los pacientes en las intoxicaciones medicamentosas.}

La edad media de los pacientes con diagnóstico de intoxicación medicamentosa es de 5,89+/- 5,74 años.

Se evidenciaron diferencias estadísticamente significativas en la edad de los pacientes atendidos en urgencias teniendo en cuenta el sexo en el diagnóstico de intoxicación medicamentosa $(p=0,0001)$. Tabla 148.

Tabla 148: Relación entre el sexo y la edad en los pacientes con intoxicación medicamentosa

\begin{tabular}{|ccc|}
\hline & Edad media & Desviación típica \\
\hline Hombre & 4,25 & 4,30 \\
Mujer & 7,33 & 6,43 \\
& & \\
\hline
\end{tabular}


Se evidenciaron diferencias estadísticamente significativas en la edad de los pacientes atendidos en urgencias teniendo en cuenta el diagnóstico de intoxicación medicamentosa $(p=\mathbf{0 , 0 0 0 1})$. Tabla 149 .

Tabla 149: Relación entre el diagnóstico de intoxicación medicamentosa y la edad del paciente

\begin{tabular}{|c|ccc|}
\hline & N & Media & Desviación típica \\
\hline Resto diagnósticos & 727 & 11,71 & 5,95 \\
Medicamentosas & 267 & 5,88 & 5,73 \\
& $\mathrm{p}=0,0001$ & \\
\hline
\end{tabular}

\subsubsection{Año de atención en urgencias de los pacientes con diagnóstico de intoxicación medicamentosa.}

Se evidenciaron diferencias estadísticamente significativas en el año de atención de los pacientes atendidos en el servicio de urgencias hospitalario teniendo en cuenta el diagnóstico de intoxicación medicamentosa ( $p=$ 0,031). Tabla 150, figura 94 .

Tabla 150: Distribución por años de los pacientes con intoxicación medicamentosa

\begin{tabular}{|c|cccccccccc|}
\hline & 2001 & 2002 & 2003 & 2004 & 2005 & 2006 & 2007 & 2008 & 2009 & 2010 \\
\hline Resto diagnósticos & 60 & 69 & 64 & 37 & 47 & 62 & 115 & 90 & 88 & 95 \\
Medicamentosas & 27 & 23 & 39 & 18 & 18 & 30 & 34 & 29 & 31 & 18 \\
\hline & 87 & 92 & 103 & 55 & 65 & 92 & 149 & 119 & 119 & 113 \\
\hline \multicolumn{10}{c}{$\mathrm{p}=0,031$} \\
\hline
\end{tabular}


Figura 94: Pacientes atendidos con diagnóstico de intoxicación medicamentosa cada año

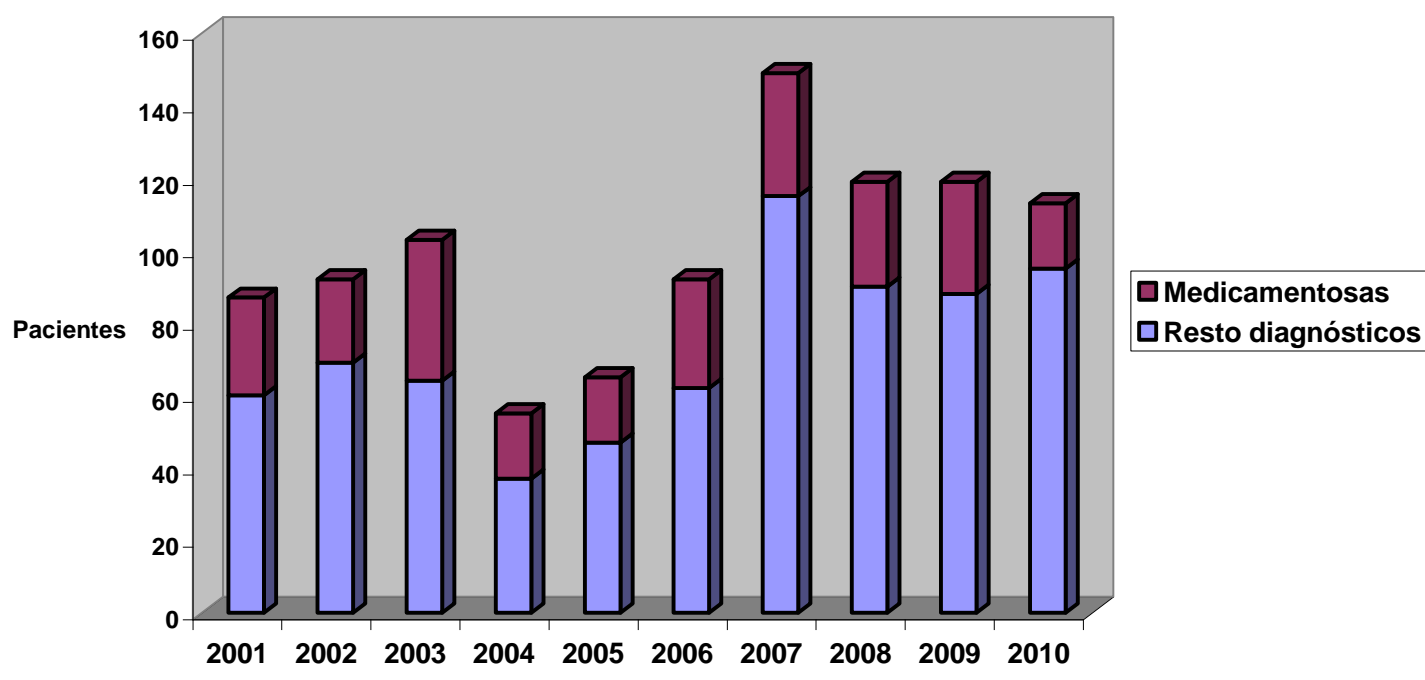

$p=0,031$

\subsubsection{Mes de atención en urgencias de los pacientes con diagnóstico de} intoxicación medicamentosa.

No se evidenciaron diferencias estadísticamente significativas en el mes de atención de los pacientes atendidos en el servicio de urgencias hospitalario teniendo en cuenta el diagnóstico de intoxicación medicamentosa $(p=$ 0,455). Tabla 151.

Tabla 151: Distribución de los pacientes con intoxicación medicamentosas según meses de asistencia al servicio de urgencias hospitalario

\begin{tabular}{|c|cccccc|}
\hline & Enero & Febrero & Marzo & Abril & Mayo & Junio \\
\hline Resto diagnósticos & 51 & 57 & 72 & 51 & 65 & 72 \\
\hline Medicamentosas & 15 & 25 & 26 & 32 & 22 & 24 \\
\hline & 66 & 82 & 98 & 83 & 87 & 96 \\
\cline { 2 - 8 } & \multicolumn{7}{c}{ Julio } & Agosto & Septiembre & Octubre & Noviembre & Diciembre \\
\cline { 2 - 8 } & 56 & 48 & 66 & 59 & 58 & 72 \\
\hline & 21 & 16 & 22 & 23 & 21 \\
\hline & 76 & 69 & 82 & 81 & 81 & 93 \\
\hline
\end{tabular}




\subsubsection{Distribución por horas de llegada al servicio de Urgencias}

Hospitalario de los pacientes con intoxicación medicamentosa.

Se evidenciaron diferencias estadísticamente significativas en la hora de atención de los pacientes atendidos en el servicio de urgencias hospitalario teniendo en cuenta el diagnóstico de intoxicación medicamentosa $(p=$ 0,0001). Tabla 152 y figura 95.

Tabla 152: Distribución por horas de llegada al servicio de Urgencias Hospitalario de los pacientes con intoxicación medicamentosa

\begin{tabular}{|c|cccccccccccc|}
\hline & $00: 00$ & $01: 00$ & $02: 00$ & $03: 00$ & $04: 00$ & $05: 00$ & $06: 00$ & $07: 00$ & $08: 00$ & $09: 00$ & $10: 00$ & $11: 00$ \\
& $00: 59$ & - & - & - & - & - & - & - & - & - & - & - \\
\hline $\begin{array}{c}\text { Resto } \\
\text { diagnóst } \\
\text { icos }\end{array}$ & 52 & 47 & 21 & 22 & 16 & 8 & 9 & 3 & 2 & 6 & 7 & 10 \\
$\begin{array}{c}\text { Medica } \\
\text { mentosa } \\
\text { s }\end{array}$ & 14 & 4 & 2 & 4 & 0 & 2 & 1 & 0 & 2 & 4 & 6 & 5 \\
\hline Total & 66 & 51 & 23 & 26 & 16 & 10 & 10 & 3 & 4 & 10 & 13 & 15 \\
\hline
\end{tabular}

\begin{tabular}{|ccccccccccccc|}
\hline $12: 00$ & $13: 00$ & $14: 00$ & $15: 00$ & $16: 00$ & $17: 00$ & $18: 00$ & $19: 00$ & $20: 00$ & $21: 00$ & $22: 00$ & $23: 00$ \\
- & - & - & - & - & - & - & - & - & - & - & - \\
$12: 59$ & $13: 59$ & $14: 59$ & $15: 59$ & $16: 59$ & $17: 59$ & $18: 59$ & $19: 59$ & $20: 59$ & $21: 59$ & $22: 59$ & $23: 59$ \\
\hline 17 & 16 & 11 & 23 & 16 & 23 & 21 & 28 & 54 & 100 & 123 & 92 \\
15 & 17 & 21 & 20 & 16 & 13 & 12 & 15 & 24 & 17 & 26 & 27 \\
\hline 32 & 33 & 32 & 43 & 32 & 36 & 33 & 43 & 78 & 117 & 149 & 119 \\
\hline
\end{tabular}


Figura 95: Relación entre la hora de admisión en el servicio de urgencias hospitalario y la intoxicación medicamentosa

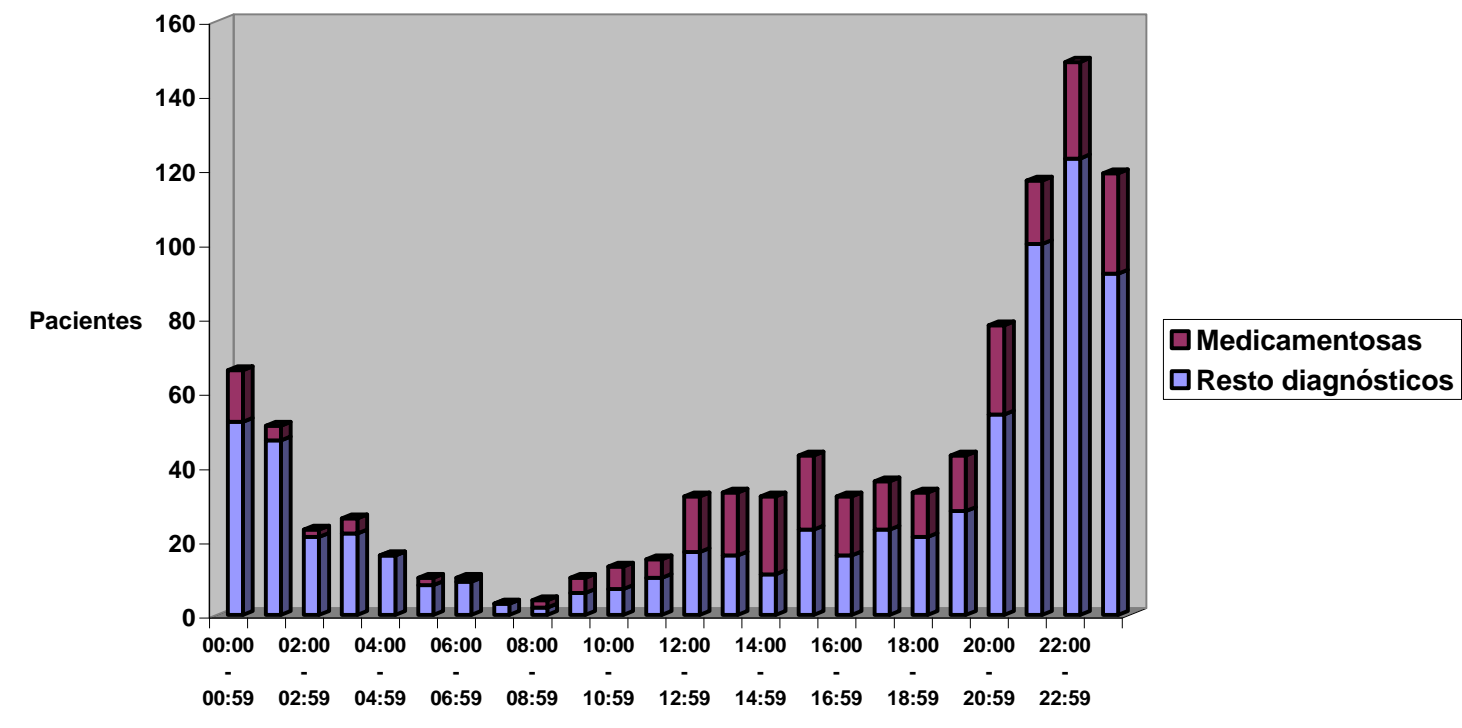

$p=0,0001$

\subsubsection{Localidad de origen de los pacientes con intoxicación} medicamentosas.

Se evidenciaron diferencias estadísticamente significativas en la localidad de origen de los pacientes teniendo en cuenta el diagnóstico de intoxicación medicamentosa $(p=0,001)$. Tabla 153 .

Tabla 153: Localidad de origen de los pacientes con intoxicación medicamentosa

\begin{tabular}{|c|cc|c|}
\hline & Valladolid capital & Otros lugares & Porcentajes \\
\hline Resto diagnósticos & 487 & 240 & $66,98 \% / 33.02 \%$ \\
Medicamentosas & 147 & 120 & $55,05 \% / 44.95 \%$ \\
\hline Total & 634 & 360 & \\
\hline \multicolumn{3}{|c|}{$p=0,001$} \\
\hline
\end{tabular}




\subsubsection{Necesidad de vehículos de Emergencias Sanitarias en los pacientes}

con intoxicación medicamentosa

Se evidenciaron diferencias estadísticamente significativas en la necesidad de vehículos de Emergencias Sanitarias para acceder al servicio de Urgencias Hospitalario de los pacientes teniendo en cuenta el

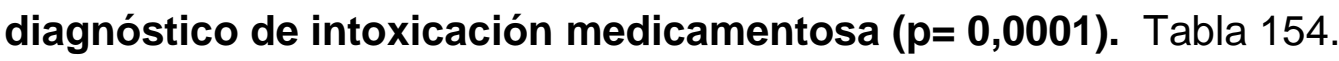

Tabla 154: Necesidad de vehículos de Emergencias Sanitarias para acceder al servicio de Urgencias Hospitalario en los pacientes con intoxicación medicamentosa

\begin{tabular}{|c|cc|c|}
\hline & NO & SI & Porcentajes \\
\hline Resto diagnósticos & 217 & 227 & $48,87 \% / 51,13 \%$ \\
Medicamentosas & 131 & 9 & $93,57 \% / 6,43 \%$ \\
\hline Total & 348 & 236 & 584 \\
\hline & $\mathrm{p}=0,0001$ \\
\hline
\end{tabular}

\subsubsection{Necesidad de pruebas durante la estancia en el servicio de} urgencias hospitalario de los intoxicados medicamentosos.

La necesidad de pruebas en el servicio de urgencias hospitalario del paciente con intoxicación medicamentosa se muestra en la tabla 155 y figura 96.

Tabla 155: Realización de pruebas diagnósticas en el servicio de urgencias hospitalario en las intoxicaciones medicamentosas

\begin{tabular}{|c|cc|cc|}
\hline & Sí & $\%$ & No & $\%$ \\
\cline { 2 - 5 } Analítica & 45 & 31,9 & 96 & 68,1 \\
Sistemático & 38 & 27 & 103 & 73 \\
Bioquímica & 42 & 29,8 & 99 & 70,2 \\
Hemostasia & 10 & 7,1 & 130 & 92,9 \\
Gasometría venosa & 8 & 5,7 & 132 & 94,3 \\
Gasometría arterial & 1 & 0,7 & 139 & 99,3 \\
Tóxicos en orina & 19 & 13,6 & 121 & 86,4 \\
ECG & 26 & 18,6 & 114 & 81,4 \\
Pruebas de imagen & 5 & 3,6 & 135 & 96,4 \\
\hline
\end{tabular}




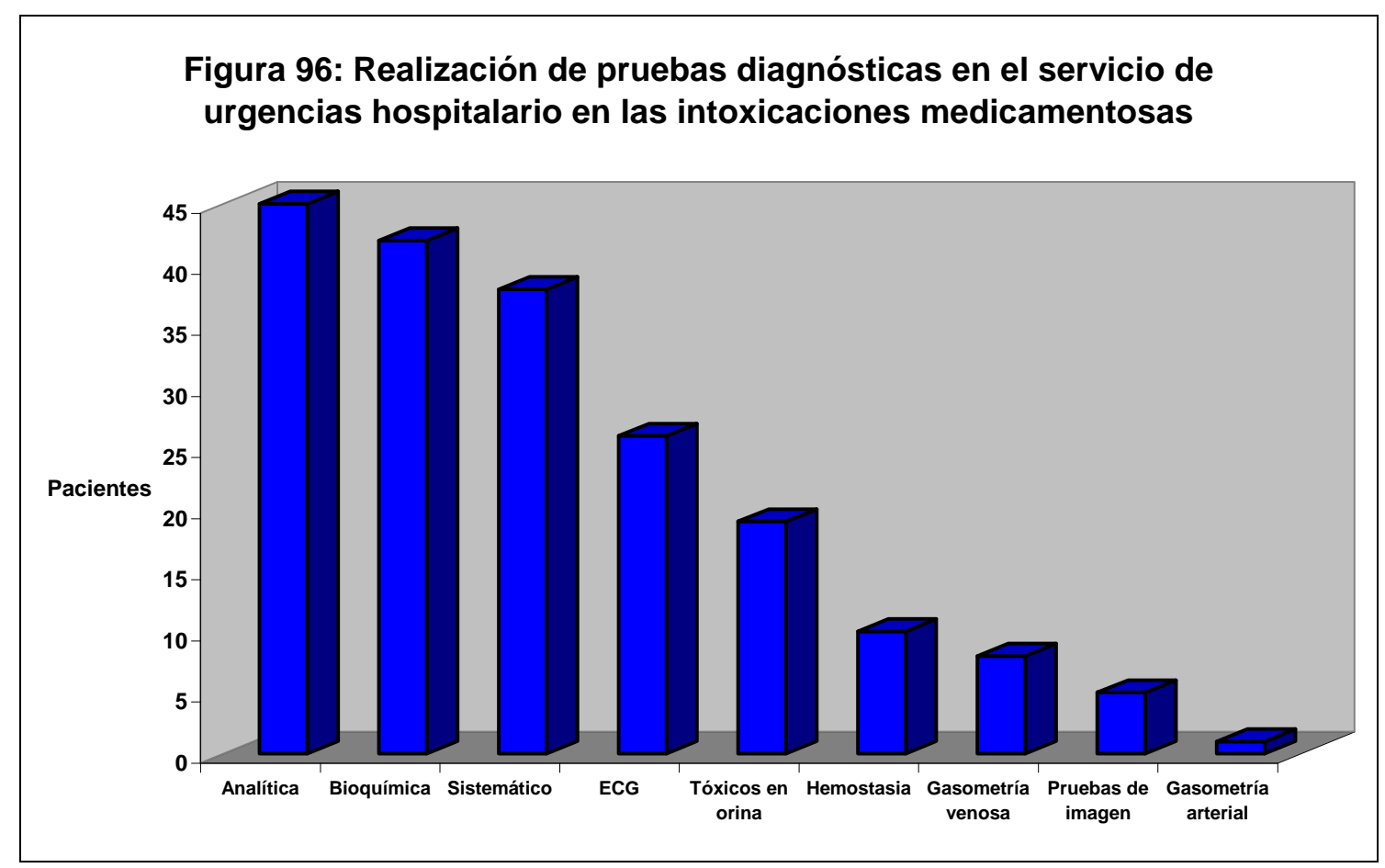

Se evidenciaron diferencias estadísticamente significativas en la realización de analítica en el servicio de Urgencias Hospitalario de los pacientes teniendo en cuenta el diagnóstico de intoxicación medicamentosa. Tabla 156, figura 97.

Tabla 156: Relación entre la realización de analítica en el servicio de urgencias hospitalario y la intoxicación medicamentosa

\begin{tabular}{|c|cc|}
\hline & Intoxicación medicamentosa & Resto intoxicaciones \\
\hline Sí analítica & 45 & 276 \\
No analítica & 96 & 170 \\
& $p=0,0001$ & \\
\hline
\end{tabular}


Figura 97: Relación entre la realización de analítica en el servicio de urgencias hospitalaria en las intoxicaciones medicamentosas

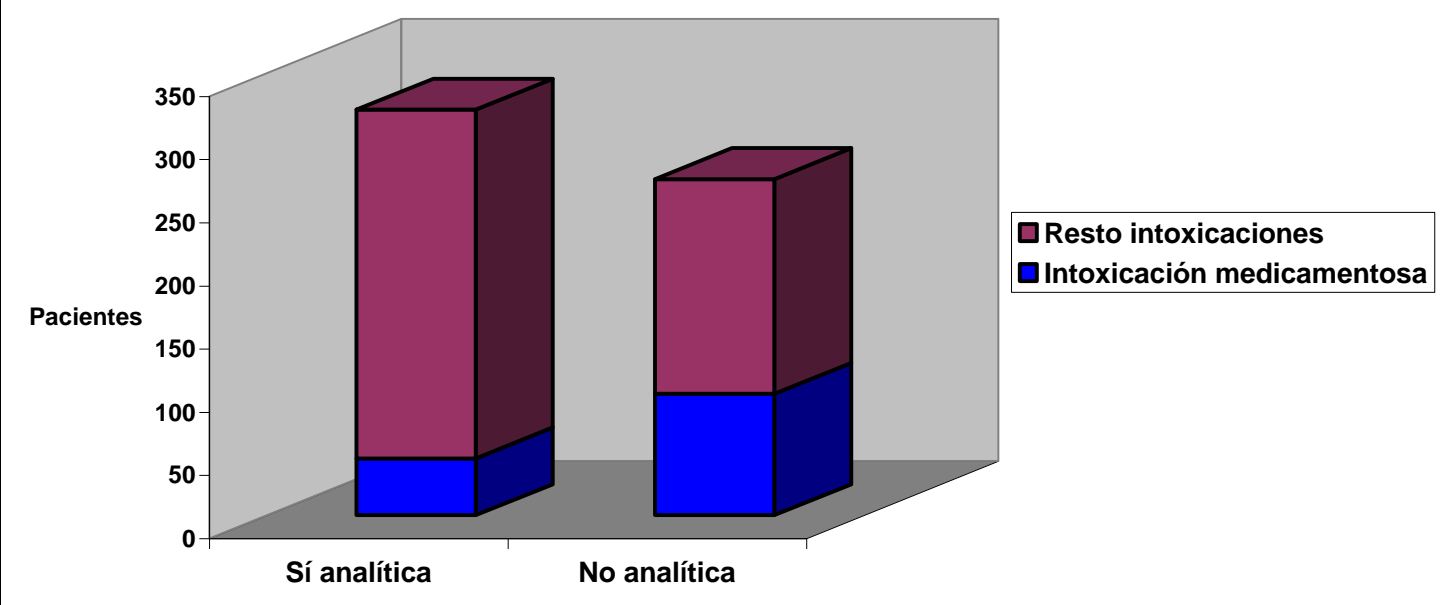

$p=0,0001$

Se evidenciaron diferencias estadísticamente significativas en la realización de sistemático de sangre en el servicio de Urgencias Hospitalario de los pacientes teniendo en cuenta el diagnóstico de intoxicación medicamentosa. Tabla 157, figura 98

Tabla 157: Relación entre la realización de sistemático sanguíneo y la intoxicación medicamentosa

\begin{tabular}{|c|cc|}
\hline & Intoxicación medicamentosa & Resto intoxicaciones \\
\hline Sí sistemático & 38 & 252 \\
No sistemático & 103 & 193 \\
& $\mathrm{p}=0,0001$ & \\
\hline
\end{tabular}


Figura 98: Relación entre la realización de sistemático sanguíneo en el servicio de urgencias hospitalario y la intoxicación medicamentosa

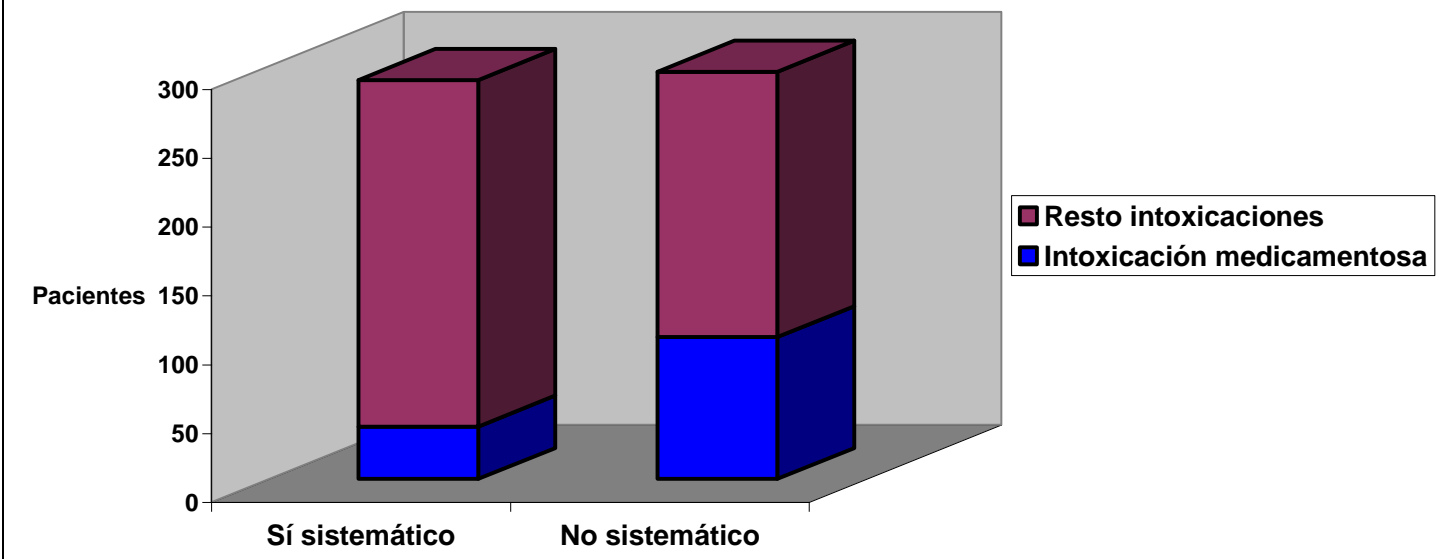

$p=0,0001$

Se evidenciaron diferencias estadísticamente significativas en la realización de bioquímica sanguínea en el servicio de Urgencias Hospitalario de los pacientes teniendo en cuenta el diagnóstico de intoxicación medicamentosa. Tabla 158, figura 99.

Tabla 158: Relación entre lla realización de bioquímica sanguínea en el servicio de urgencias hospitalario y la intoxicación medicamentosa

\begin{tabular}{|c|cc|}
\hline & Intoxicación medicamentosa & Resto intoxicaciones \\
\hline Sí bioquímica & 42 & 250 \\
No bioquímica & 99 & 196 \\
& $\mathrm{p}=0,0001$ & \\
\hline
\end{tabular}


Figura 99: Relación entre la realización de bioquímica sanguínea en el servicio de urgencias hospitalario y la intoxicación medicamentosa

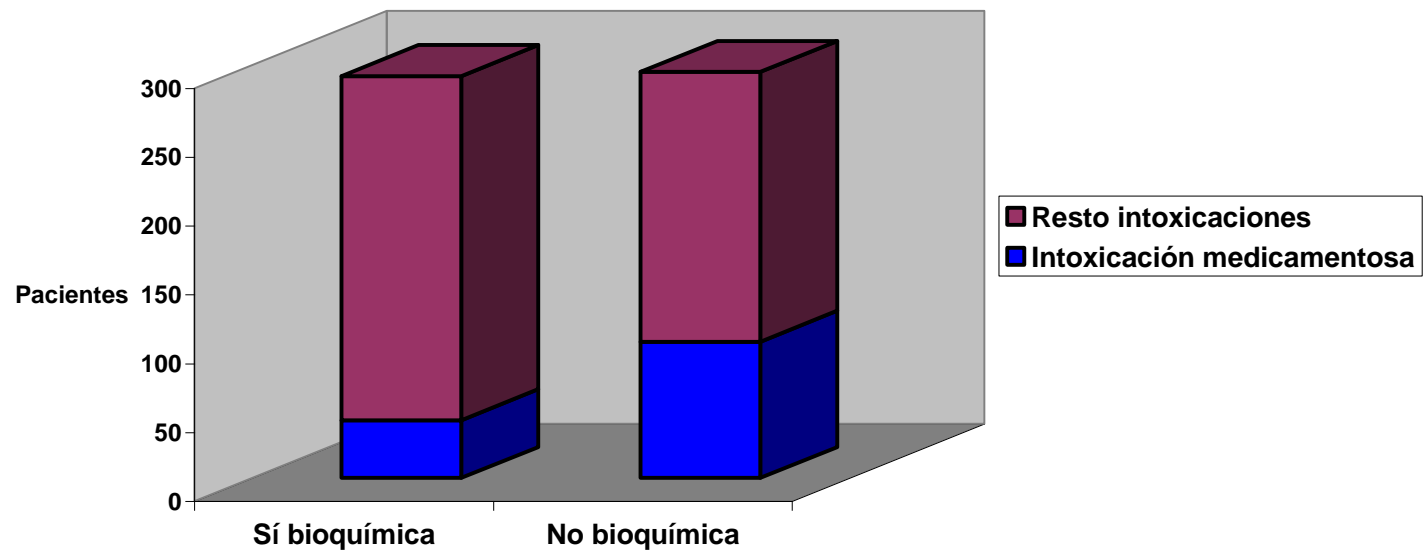

$p=0,0001$

No se evidenciaron diferencias estadísticamente significativas en la realización de hemostasia en el servicio de urgencias hospitalario de los pacientes teniendo en cuenta el diagnóstico de intoxicación medicamentosa. Tabla 159.

Tabla 159: Relación entre la realización de hemostasia en el servicio de urgencias hospitalario y la intoxicación medicamentosa

\begin{tabular}{|c|cc|}
\hline & Intoxicación medicamentosa & Resto intoxicaciones \\
\hline Sí hemostasia & 10 & 24 \\
No hemostasia & 130 & 421 \\
& $\mathrm{p}=0,44$ & \\
\hline
\end{tabular}

Se evidenciaron diferencias estadísticamente significativas en la realización de gasometría venosa en el servicio de urgencias hospitalario de los pacientes teniendo en cuenta el diagnóstico de intoxicación medicamentosa. Tabla 160, figura 100. 
Tabla 160: Relación entre la realización de gasometría venosa en el servicio de urgencias hospitalario y la intoxicación medicamentosa

\begin{tabular}{|c|cc|}
\hline & Intoxicación medicamentosa & Resto intoxicaciones \\
\hline Sí gasometría venosa & 8 & 53 \\
No gasometría venosa & 132 & 391 \\
& $\mathrm{p}=0,036$ & \\
\hline
\end{tabular}

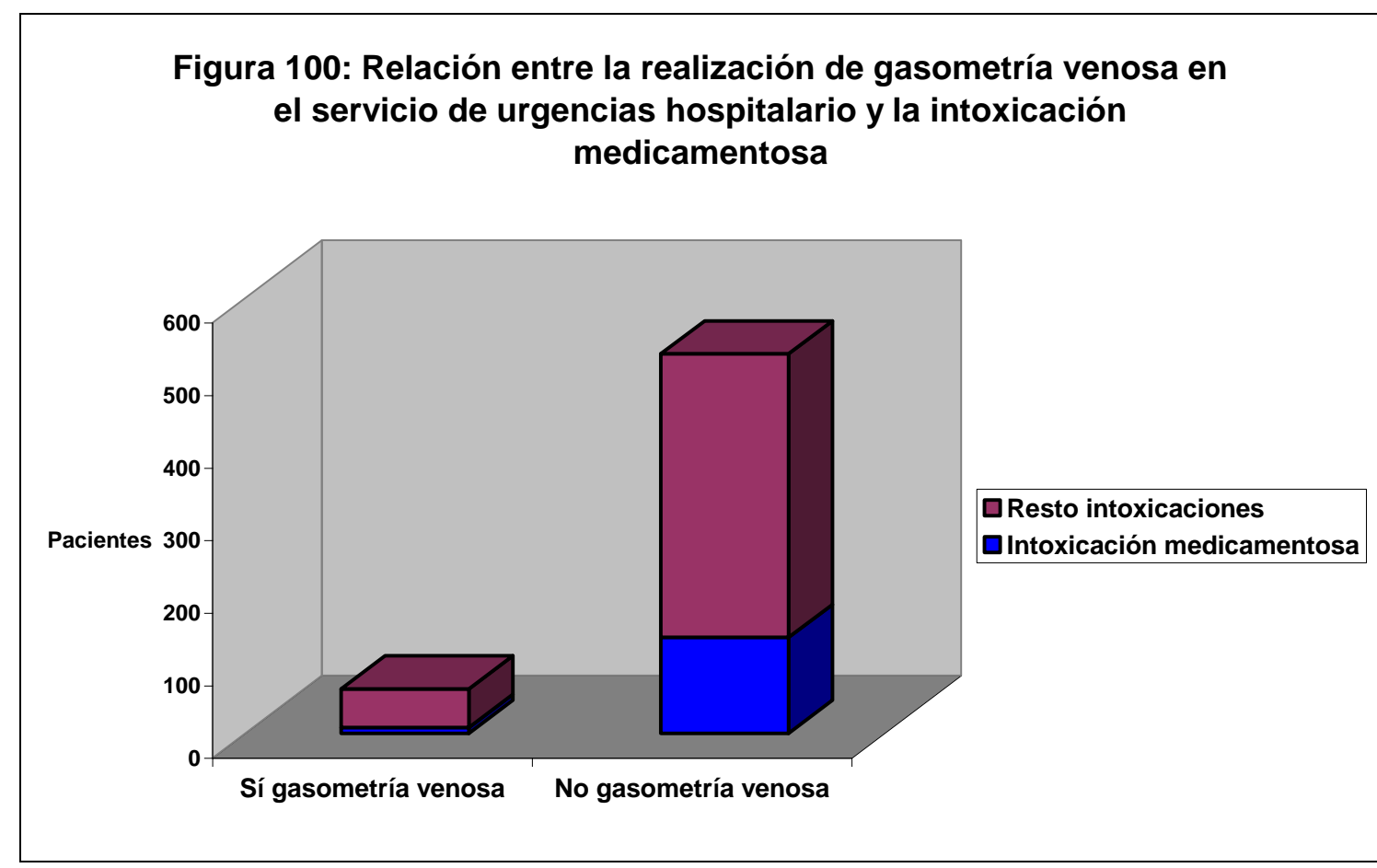

$p=0,036$

Se evidenciaron diferencias estadísticamente significativas en la realización de gasometría arterial en el servicio de urgencias hospitalario de los pacientes teniendo en cuenta el diagnóstico de intoxicación medicamentosa. Tabla 161, figura 101.

Tabla 161: Relación entre la realización de gasometría arterial en el servicio de urgencias hospitalario y la intoxicación medicamentosa

\begin{tabular}{|c|cc|}
\hline & Intoxicación medicamentosa & Resto intoxicaciones \\
\hline Sí gasometría arterial & 1 & 28 \\
No gasometría arterial & 139 & 416 \\
& $\mathrm{p}=0,008$ & \\
\hline
\end{tabular}


Figura 101: Relación entre la realización de gasometría arterial en el servicio de urgencias hospitalario y la intoxicación medicamentosa.

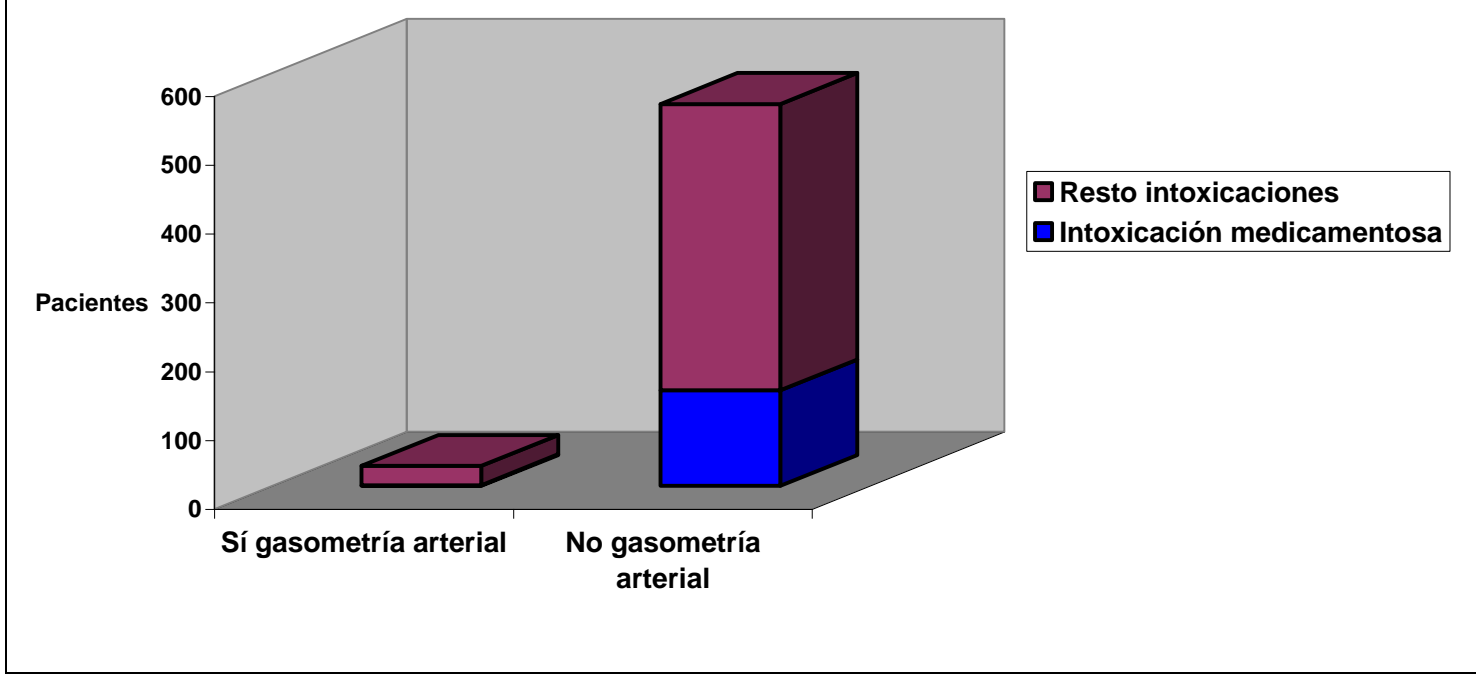

$p=0,008$

Se evidenciaron diferencias estadísticamente significativas en la realización de tóxicos en orina en el servicio de urgencias hospitalario de los pacientes teniendo en cuenta el diagnóstico de intoxicación medicamentosa. Tabla 162, figura 102.

Tabla 162: Relación entre la realización de tóxicos en orina en el servicio de urgencias hospitalario y la intoxicación medicamentosa

\begin{tabular}{|c|cc|}
\hline & Intoxicación medicamentosa & Resto intoxicaciones \\
\hline Sí tóxicos en orina & 19 & 188 \\
No tóxicos en orina & 121 & 256 \\
& $\mathrm{p}=0,0001$ & \\
\hline
\end{tabular}




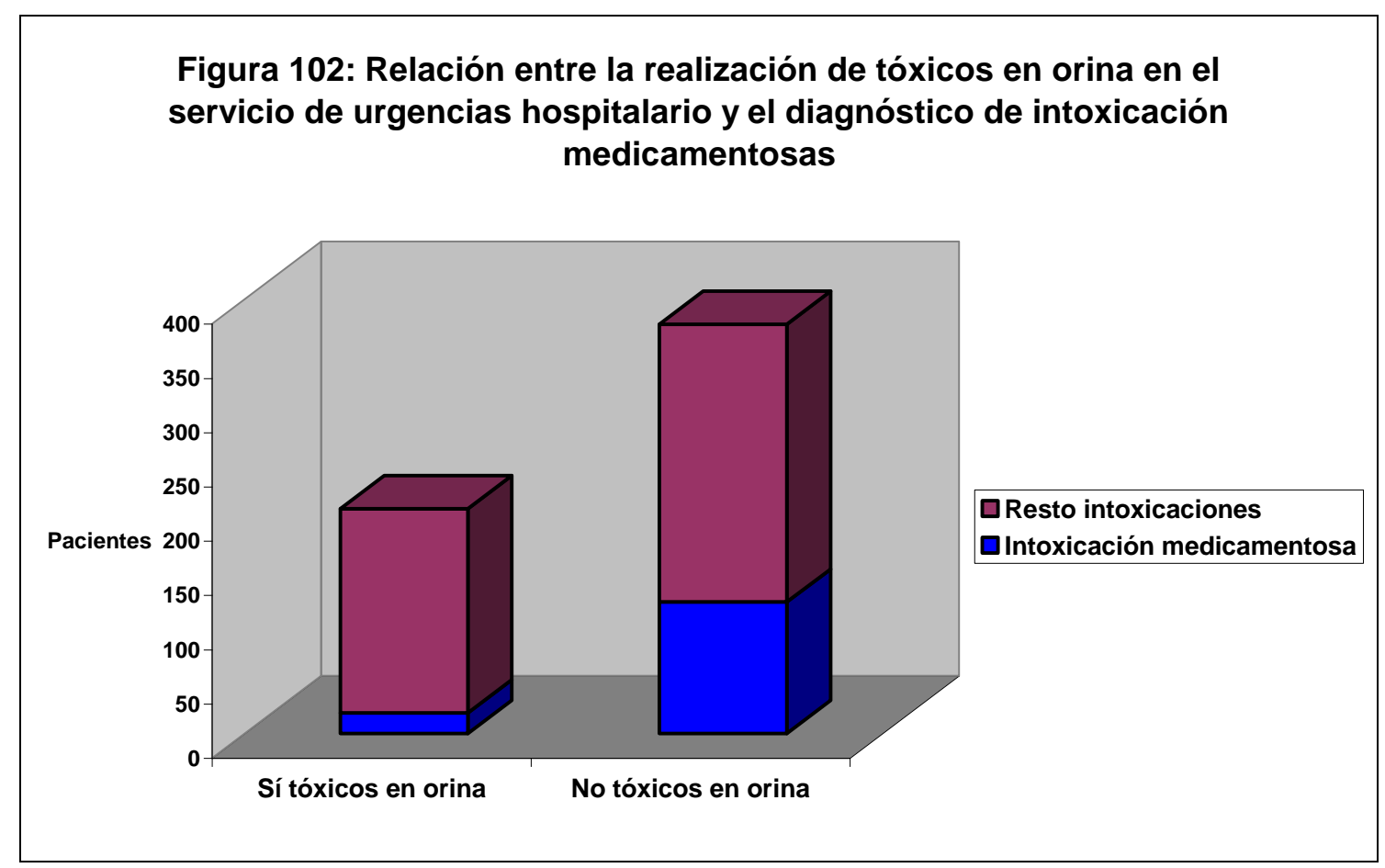

$p=0,0001$

No se evidenciaron diferencias estadísticamente significativas en la realización de electrocardiografía en el servicio de urgencias hospitalario de los pacientes teniendo en cuenta el diagnóstico de intoxicación medicamentosa. Tabla 163.

Tabla 163: Relación entre la realización de electrocardiografía en el servicio de urgencias hospitalario y la intoxicación medicamentosa

\begin{tabular}{|c|c|c|}
\hline & Intoxicación medicamentosa & Resto intoxicaciones \\
\hline Sí ECG & 26 & 116 \\
\hline No ECG & 114 & 328 \\
\hline \multicolumn{3}{|c|}{$p=0,069$} \\
\hline
\end{tabular}

No se evidenciaron diferencias estadísticamente significativas en la realización de pruebas de imagen en el servicio de urgencias hospitalario de los pacientes teniendo en cuenta el diagnóstico de intoxicación medicamentosa. Tabla 164. 
Tabla 164: Relación entre la realización de pruebas de imagen en el servicio de urgencias hospitalario y la intoxicación medicamentosa

\begin{tabular}{|c|cc|}
\hline & Intoxicación medicamentosa & Resto intoxicaciones \\
\hline Sí prueba imagen & 5 & 28 \\
No prueba de imagen & 135 & 416 \\
& $\mathrm{p}=0,222$ & \\
\hline
\end{tabular}

\subsubsection{Propietario de la medicación.}

Se recoge en las intoxicaciones medicamentosas, quién es el propietario de la misma, estableciéndose una división entre el propio paciente o el familiar. En todos los casos el propietario fue el mismo paciente o un familiar del mismo. Tabla 165.

Tabla 165: Propietario de la medicación en las intoxicaciones medicamentosas

\begin{tabular}{|ccc|}
\hline Propietario & Frecuencia & Porcentaje \\
\hline Paciente & 120 & 58,8 \\
Familiar & 84 & 41,2 \\
Total & 204 & 100 \\
\hline
\end{tabular}

\subsubsection{Necesidad de tratamiento durante la estancia en el servicio de urgencias hospitalario de los intoxicados medicamentosos.}

No se evidenciaron diferencias estadísticamente significativas en la administración de tratamiento en el servicio de urgencias hospitalario de los pacientes teniendo en cuenta el diagnóstico de intoxicación medicamentosa. Tabla 166.

Tabla 166: Relación entre los tratamientos realizados en el servicio de urgencias hospitalario teniendo en cuenta el diagnóstico de intoxicación medicamentosa

\begin{tabular}{|c|cc|}
\hline & Intoxicación medicamentosa & Resto intoxicaciones \\
\hline Sí tratamiento & 75 & 270 \\
No tratamiento & 65 & 175 \\
& $\mathrm{p}=0,136$ & \\
\hline
\end{tabular}




\subsubsection{Lavado gástrico en la intoxicación medicamentosa.}

Se evidenciaron diferencias estadísticamente significativas en la realización de lavado gástrico en el servicio de urgencias hospitalario de los pacientes teniendo en cuenta el diagnóstico de intoxicación medicamentosa. Tabla 167, figura 103.

Tabla 167: Relación entre la realización de lavado gástrico en el servicio de urgencias hospitalario teniendo en cuenta el diagnóstico de intoxicación medicamentosa

\begin{tabular}{|c|cc|}
\hline & Intoxicación medicamentosa & Resto intoxicaciones \\
\cline { 2 - 3 } Sí lavado gástrico & 43 & 7 \\
No lavado gástrico & 97 & 436 \\
& $\mathrm{p}=0,0001$ & \\
\hline
\end{tabular}

Figura 103: Relación entre la realización de lavado gástrico en el servicio de urgencias hospitalario y el diagnóstico de intoxicación medicamentosa

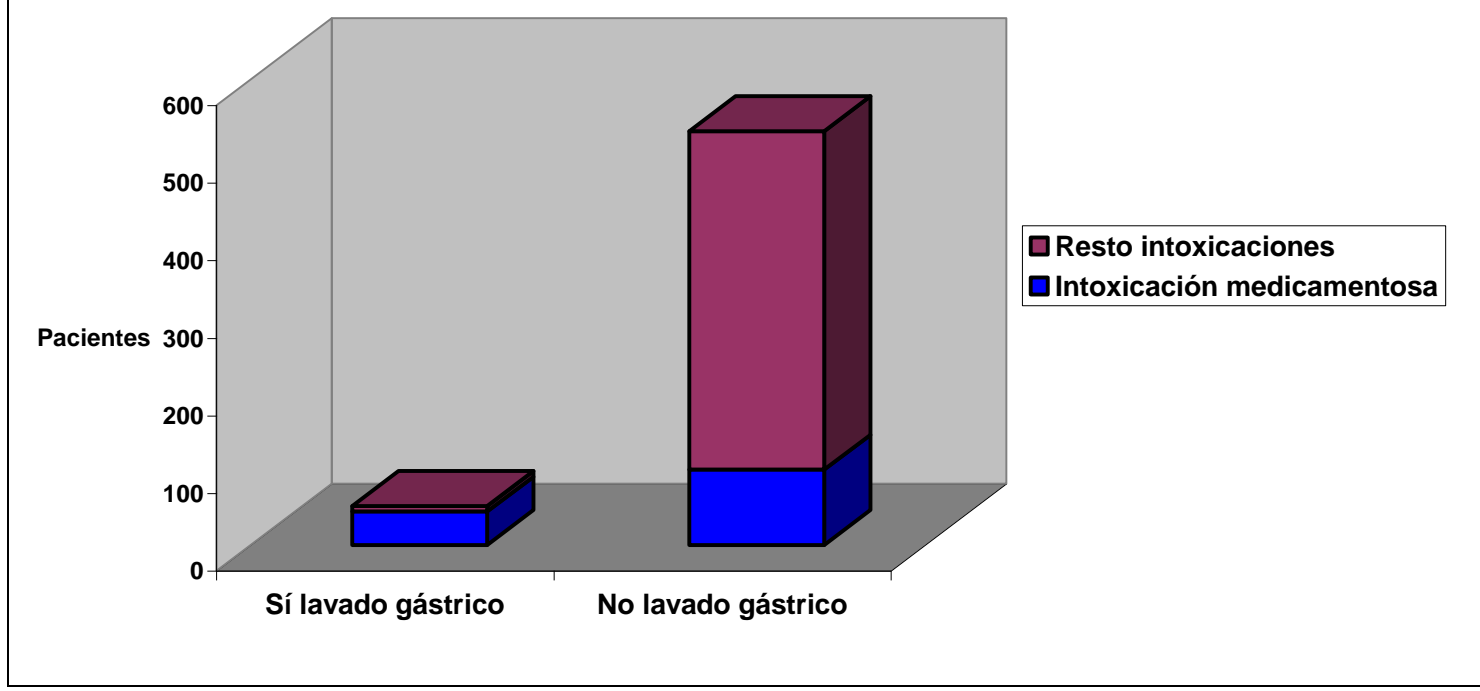

$p=0,0001$ 


\subsection{Relación entre el sexo del paciente con intoxicación}

medicamentosa y la realización de lavado gástrico en el servicio de urgencias hospitalario.

Se evidenciaron diferencias estadísticamente significativas en la realización de lavado gástrico en el servicio de urgencias hospitalario de los pacientes teniendo en cuenta el diagnóstico de intoxicación medicamentosa y el sexo del intoxicado. Tabla 168.

Tabla 168: Relación entre el lavado gástrico en el servicio de urgencias hospitalario y el sexo de las intoxicaciones medicamentosas

\begin{tabular}{|c|c|c|c|c|}
\hline & & No lavado gástrico & Sílavado gástrico & Total \\
\hline Hombre & $\mathrm{N}$ & 57 & 17 & 74 \\
& $\%$ & 58,80 & 39,50 & 52,90 \\
Mujer & $\mathrm{N}$ & 40 & 26 & 66 \\
& $\%$ & $41,20 \quad 00,50$ & 47,10 \\
\hline
\end{tabular}

\subsection{Relación entre la edad del paciente con intoxicación}

\section{medicamentosa y la realización de lavado gástrico en el servicio de} urgencias hospitalario.

Se evidenciaron diferencias estadísticamente significativas en la realización de lavado gástrico en el servicio de urgencias hospitalario de los pacientes teniendo en cuenta el diagnóstico de intoxicación medicamentosa y la edad del intoxicado. $(p=0,0001)$ Tabla 169.

Tabla 169: Relación entre el lavado gástrico en el servicio de urgencias hospitalario y la edad del paciente con la intoxicación medicamentosa

\begin{tabular}{|c|c|c|}
\hline & Edad media & Desviación típica \\
\hline No lavado gástrico & 5 & 5,1 \\
Sí lavado gástrico & 7,28 & 6,5 \\
& $p=0,0001$ & \\
\hline
\end{tabular}




\subsection{Relación entre la necesidad de trasporte en vehículos de}

Emergencias Sanitarias del paciente con intoxicación medicamentosa y la realización de lavado gástrico en el servicio de urgencias hospitalario.

Se evidenciaron diferencias estadísticamente significativas en la realización de lavado gástrico en el servicio de urgencias hospitalario de los pacientes teniendo en cuenta el diagnóstico de intoxicación medicamentosa y la necesidad de trasporte por vehículos de Emergencias Sanitarias. ( $p=$ 0,004) Tabla 170.

Tabla 170: Relación entre el lavado gástrico en el servicio de urgencias hospitalario y el trasporte en vehículos de Emergencias Sanitarias de las intoxicaciones medicamentosas

\begin{tabular}{|c|c|c|c|c|}
\hline & & $\begin{array}{c}\text { No trasportado por vehículo } \\
\text { Emergencias Sanitarias }\end{array}$ & $\begin{array}{c}\text { Sí vehículo de } \\
\text { Emergencias } \\
\text { Sanitarias }\end{array}$ & Total \\
\hline $\begin{array}{c}\text { No lavado } \\
\text { gástrico }\end{array}$ & $\mathrm{N}$ & 94 & 2 & 96 \\
$\begin{array}{c}\text { Sí lavado } \\
\text { gástrico }\end{array}$ & $\mathrm{N}$ & 72,30 & 22,20 & 69,10 \\
& $\%$ & 36 & 7 & 43 \\
& 27,70 & 77,80 & 30,90 \\
\hline
\end{tabular}

\subsection{Relación entre el número de sustancias que ocasionan la} intoxicación medicamentosa y la realización de lavado gástrico en el servicio de urgencias hospitalario.

Se evidenciaron diferencias estadísticamente significativas en la realización de lavado gástrico en el servicio de urgencias hospitalario de los pacientes teniendo en cuenta el diagnóstico de intoxicación medicamentosa y el número de sustancias que la ocasionan. $(p=0,011)$ Tabla 171. 
Tabla 171: Relación entre el lavado gástrico en el servicio de urgencias hospitalario y el número de sustancias que ocasiona la intoxicació medicamentosa

\begin{tabular}{|c|c|c|c|c|}
\hline & & Una sustancia & Más de una sustancia & Total \\
\hline No lavado gástrico & $\mathrm{N}$ & 95 & 2 & 97 \\
& $\%$ & 72 & 25 & 69,30 \\
Sí lavado gástrico & $\mathrm{N}$ & 37 & 6 & 43 \\
& $\%$ & 28 & 75 & 30,70 \\
\hline
\end{tabular}

\subsection{Relación entre el destino del paciente intoxicado}

medicamentoso tras su estancia en el servicio de urgencias hospitalario y la realización de lavado gástrico en el servicio de urgencias

\section{hospitalario.}

Se evidenciaron diferencias estadísticamente significativas en la realización de lavado gástrico en el servicio de urgencias hospitalario de los pacientes teniendo en cuenta el diagnóstico de intoxicación medicamentosa y el destino tras la estancia en el servicio de urgencias hospitalario. ( $p=$ 0,039) Tabla 172.

Tabla 172: Relación entre el lavado gástrico y el destino tras la estancia en el servicio de urgencias hospitalario en la intoxicación medicamentosa

\begin{tabular}{|c|c|c|c|c|}
\hline & & Alta & Otros destinos & Total \\
\hline No lavado gástrico & $\mathrm{N}$ & 76 & 21 & 97 \\
& $\%$ & 74,5 & 55,3 & 69,3 \\
Sí lavado gástrico & $\mathrm{N}$ & 26 & 17 & 43 \\
& $\%$ & 25,5 & 44,7 & 30,7 \\
& \multicolumn{3}{|c|}{$\mathrm{p}=0,039$} \\
\hline
\end{tabular}




\subsubsection{Administración de carbón activado en los pacientes con intoxicación medicamentosa.}

Se evidenciaron diferencias estadísticamente significativas en la administración de carbón activado en el servicio de urgencias hospitalario de los pacientes teniendo en cuenta el diagnóstico de intoxicación medicamentosa. $(p=0,039)$ Tabla 173, figura 104.

Tabla 173: Relación entre la administración de carbón activado en el servicio de urgencias hospitalarias teniendo en cuenta el diagnóstico de intoxicación medicamentosa

\begin{tabular}{|c|cc|}
\hline & Intoxicación medicamentosa & Resto intoxicaciones \\
\cline { 2 - 3 } Sí carbón activado & 51 & 6 \\
No carbón activado & 88 & 437 \\
& $\mathrm{p}=0,0001$ & \\
\hline
\end{tabular}

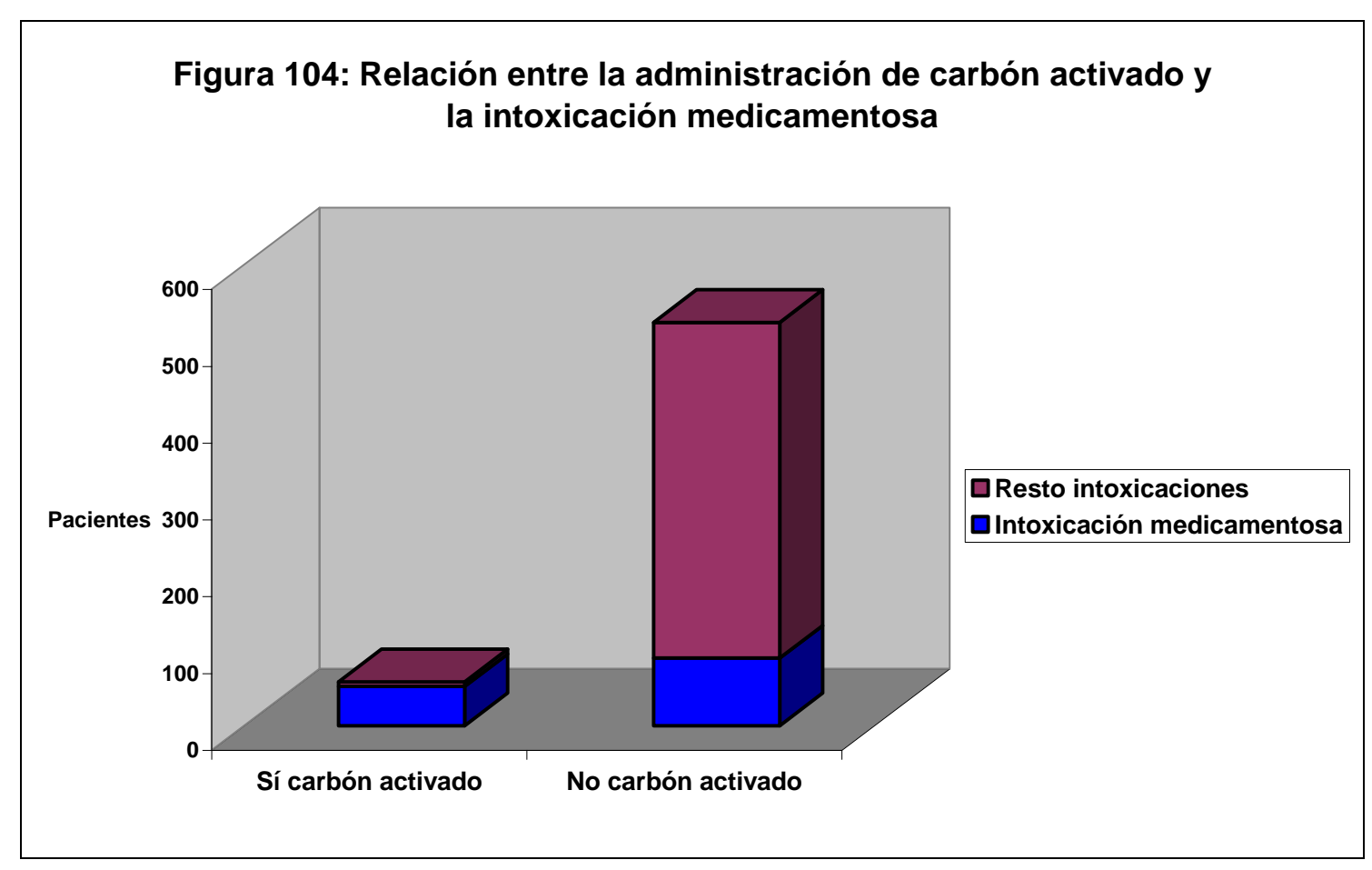

$p=0,0001$ 


\subsubsection{Administración de antídoto en los pacientes con intoxicación medicamentosa.}

No se evidenciaron diferencias estadísticamente significativas en la administración de antídoto en el servicio de urgencias hospitalario de los pacientes teniendo en cuenta el diagnóstico de intoxicación medicamentosa. $(p=0,77)$ Tabla 174.

Tabla 174: Relación entre la administración de antídoto teniendo en cuenta el diagnóstico de intoxicación medicamentosa

\begin{tabular}{|c|cc|}
\hline & Intoxicación medicamentosa & Resto intoxicaciones \\
\cline { 2 - 3 } Sí antídoto & 2 & 5 \\
No antídoto & 137 & 438 \\
& $\mathrm{p}=0,77$ & \\
\hline
\end{tabular}

\subsubsection{Administración de suero fisiológico en los pacientes con intoxicación medicamentosa.}

Se evidenciaron diferencias estadísticamente significativas en la administración de suero fisiológico en el servicio de urgencias hospitalario de los pacientes teniendo en cuenta el diagnóstico de intoxicación medicamentosa. ( $p=0,0001)$ Tabla 175, figura 104 .

Tabla 175: Relación entre la administración de suero fisiológico teniendo en cuenta el diagnóstico de intoxicación medicamentosa

\begin{tabular}{|c|cc|}
\hline & Intoxicación medicamentosa & Resto intoxicaciones \\
\cline { 2 - 3 } Sí suero fisiológico & 14 & 162 \\
No suero fisiológico & 125 & 281 \\
& $\mathrm{p}=0,0001$ & \\
\hline
\end{tabular}




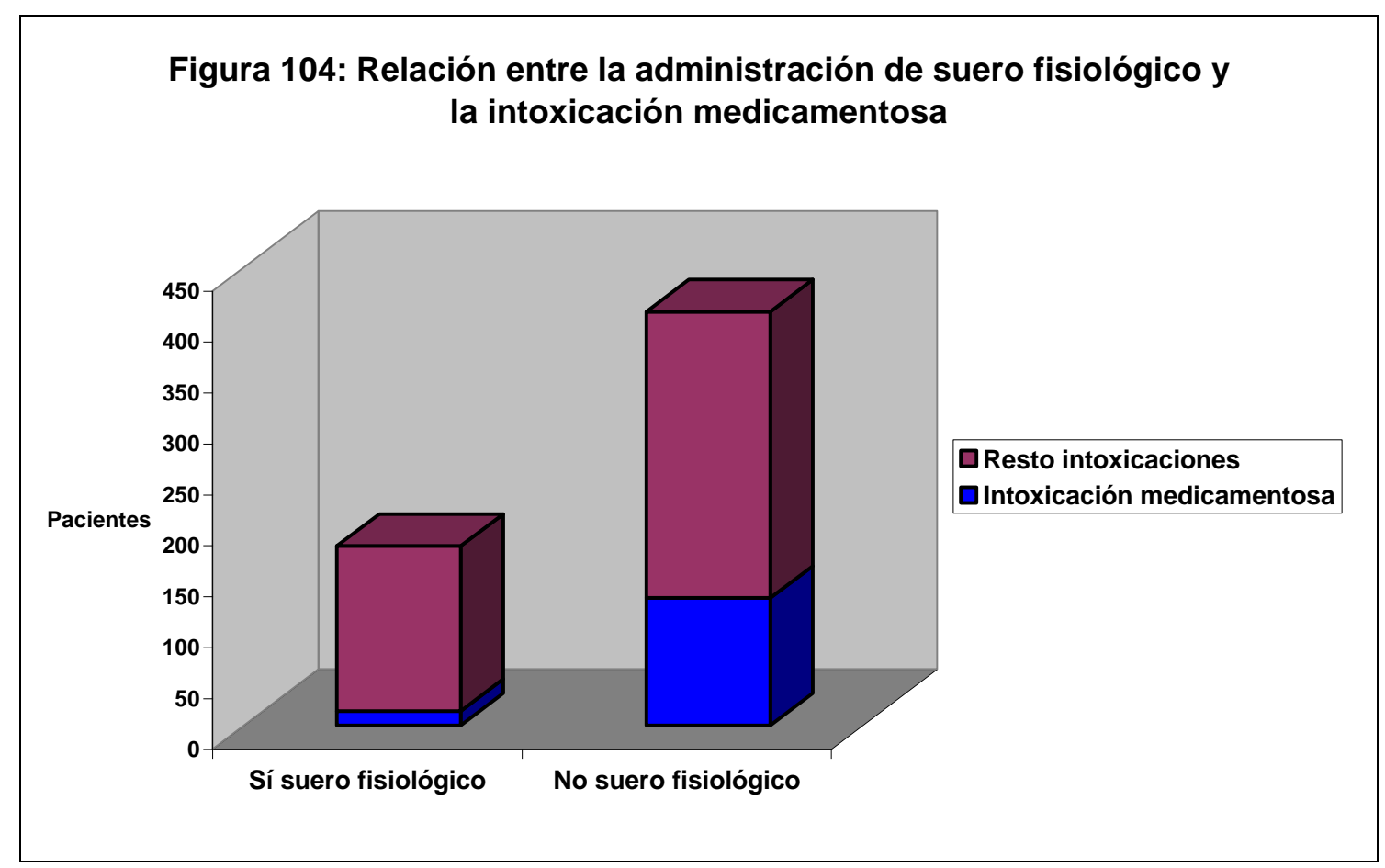

$p=0,0001$

\subsubsection{Administración de suero glucosado en los pacientes con}

\section{intoxicación medicamentosa.}

Se evidenciaron diferencias estadísticamente significativas en la administración de suero glucosado en el servicio de urgencias hospitalario de los pacientes teniendo en cuenta el diagnóstico de intoxicación medicamentosa. $(p=0,003)$ Tabla 176, figura 105.

Tabla 176: Relación entre la administración de suero glucosado teniendo en cuenta el diagnóstico de intoxicación medicamentosa

\begin{tabular}{|c|cc|}
\hline & Intoxicación medicamentosa & Resto intoxicaciones \\
\cline { 2 - 3 } Sí suero glucosado & 14 & 162 \\
No suero glucosado & 125 & 281 \\
& $\mathrm{p}=0,003$ & \\
\hline
\end{tabular}




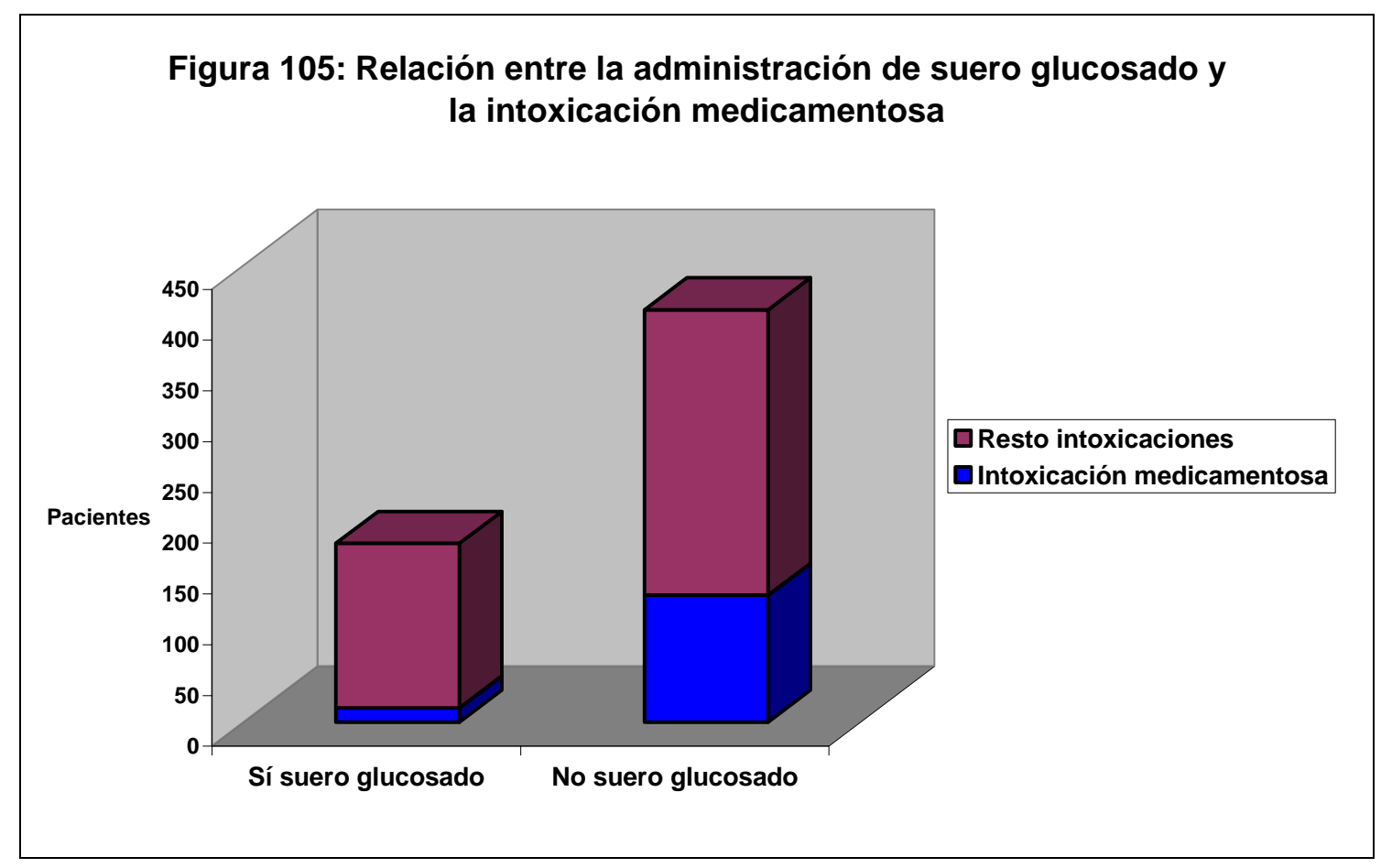

$p=0,003$

\subsubsection{Administración de antieméticos en los pacientes con} intoxicación medicamentosa.

Se evidenciaron diferencias estadísticamente significativas en la administración de antieméticos en el servicio de urgencias hospitalario de los pacientes teniendo en cuenta el diagnóstico de intoxicación medicamentosa. $(p=0,0001)$ Tabla 177, figura 106.

Tabla 177: Relación entre la administración de antieméticos teniendo en cuenta el diagnóstico de intoxicación medicamentosa

\begin{tabular}{|c|cc|}
\hline & Intoxicación medicamentosa & Resto intoxicaciones \\
\cline { 2 - 3 } Sí antiemético & 2 & 103 \\
No antiemético & 137 & 339 \\
& $\mathrm{p}=0,0001$ & \\
\hline
\end{tabular}




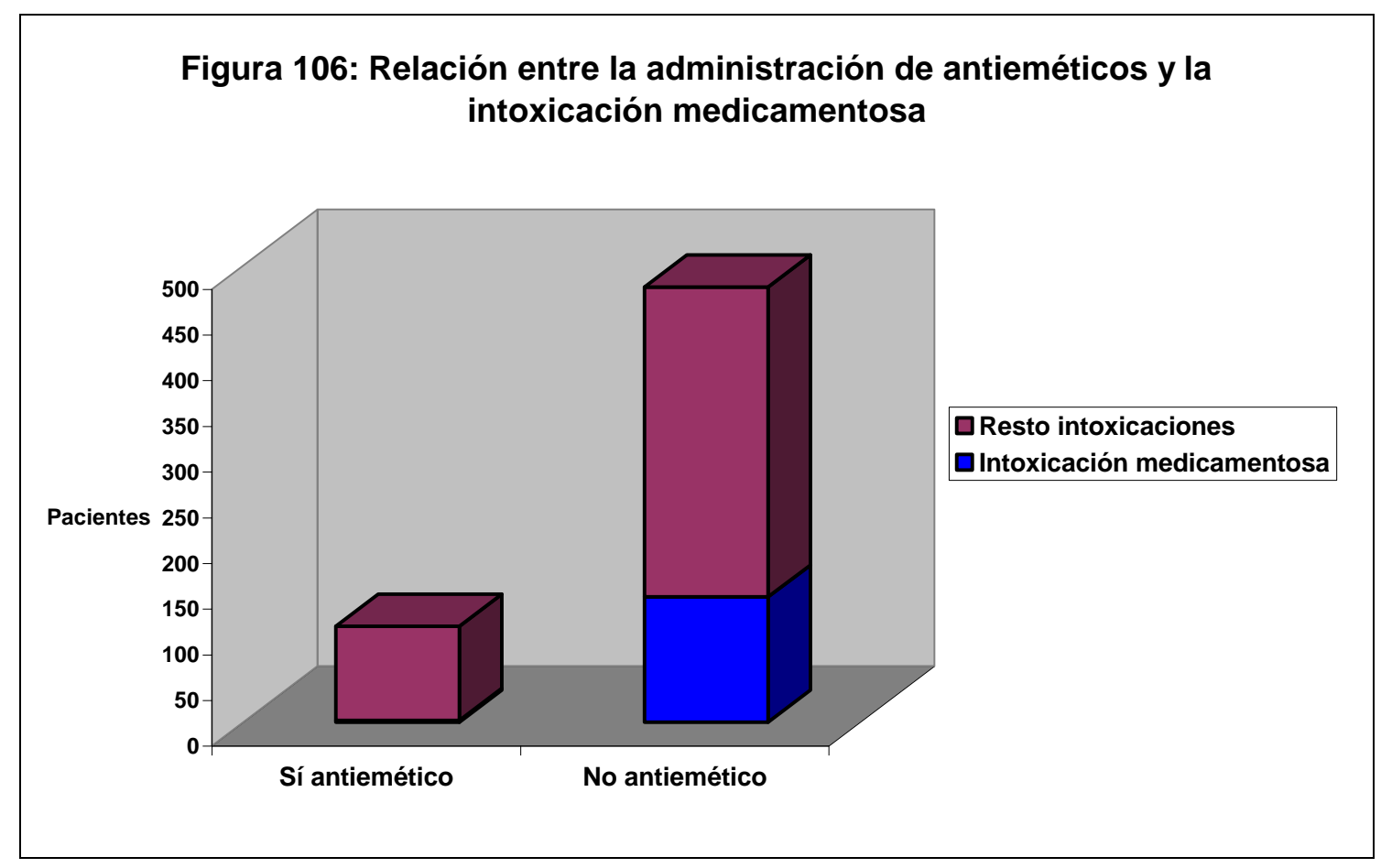

$\mathrm{p}=0,0001$

\subsubsection{Administración de analgésicos en los pacientes con intoxicación} medicamentosa.

Se evidenciaron diferencias estadísticamente significativas en la administración de analgésicos en el servicio de urgencias hospitalario de los pacientes teniendo en cuenta el diagnóstico de intoxicación medicamentosa. $(p=0,023)$ Tabla 178.

Tabla 178: Relación entre la administración de analgésicos teniendo en cuenta el diagnóstico de intoxicación medicamentosa

\begin{tabular}{|c|cc|}
\hline & Intoxicación medicamentosa & Resto intoxicaciones \\
\cline { 2 - 3 } Sí analgésico & 0 & 16 \\
No analgésicos & 139 & 427 \\
& $\mathrm{p}=0,023$ & \\
\hline
\end{tabular}




\subsubsection{Administración de protectores gástricos en los pacientes con intoxicación medicamentosa.}

Se evidenciaron diferencias estadísticamente significativas en la administración de protectores gástricos en el servicio de urgencias hospitalario de los pacientes teniendo en cuenta el diagnóstico de intoxicación medicamentosa. $(p=0,035)$ Tabla 179.

Tabla 179: Relación entre la administración de protectores gástricos teniendo en cuenta el diagnóstico de intoxicación medicamentosa

\begin{tabular}{|c|cc|}
\hline & Intoxicación medicamentosa & Resto intoxicaciones \\
\cline { 2 - 3 } Sí protector gástrico & 7 & 49 \\
No protectores gástricos & 132 & 393 \\
& $\mathrm{p}=0,035$ & \\
\hline
\end{tabular}

\subsubsection{Administración de tiamina en los pacientes con intoxicación medicamentosa.}

No se evidenciaron diferencias estadísticamente significativas en la administración de tiamina en el servicio de urgencias hospitalario de los pacientes teniendo en cuenta el diagnóstico de intoxicación medicamentosa. $(p=0,111)$ Tabla 180.

Tabla 180: Relación entre la administración de tiamina teniendo en cuenta el diagnóstico de intoxicación medicamentosa

\begin{tabular}{|c|cc|}
\hline & Intoxicación medicamentosa & Resto intoxicaciones \\
\cline { 2 - 3 } Sí tiamina & 0 & 8 \\
No tiamina & 139 & 435 \\
& $\mathrm{p}=0,111$ & \\
\hline
\end{tabular}

\subsubsection{Administración de oxigenoterapia en los pacientes con intoxicación medicamentosa.}

Se evidenciaron diferencias estadísticamente significativas en la administración de oxigenoterapia en el servicio de urgencias hospitalario de los pacientes 
teniendo en cuenta el diagnóstico de intoxicación medicamentosa. $(\mathbf{p}=\mathbf{0 , 1 1 1})$ Tabla 181.

Tabla 181: Relación entre la administración de oxigenoterapia teniendo en cuenta el diagnóstico de intoxicación medicamentosa

\begin{tabular}{|c|cc|}
\hline & Intoxicación medicamentosa & Resto intoxicaciones \\
\cline { 2 - 3 } Sí oxigenoterapia & 0 & 8 \\
No oxigenoterapia & 139 & 435 \\
& $\mathrm{p}=0,0001$ & \\
\hline
\end{tabular}

\subsubsection{Interconsulta a Psiquiatría en los intoxicados medicamentosos.}

Se evidenciaron diferencias estadísticamente significativas en la realización de interconsulta a Psiquiatría durante la estancia en el servicio de urgencias Hospitalario de los pacientes teniendo en cuenta el diagnóstico de intoxicación medicamentosa ( $\mathrm{p}=\mathbf{0 , 0 0 0 1 )}$. Tabla 182 y figura 107 .

Tabla 182: Relación entre la interconsulta con Psiquiatría y la intoxicación medicamentosa.

\begin{tabular}{|c|cc|c|c|}
\hline & NO & SI & $\%$ & Total \\
\hline Resto diagnósticos & 303 & 6 & $98,05 \% / 1,94 \%$ & 309 \\
Medicamentosas & 74 & 31 & $70,47 \% / 29,52 \%$ & 105 \\
\hline Total & 377 & 37 & 414 \\
\hline \multicolumn{7}{|c}{0,0001} \\
\hline
\end{tabular}




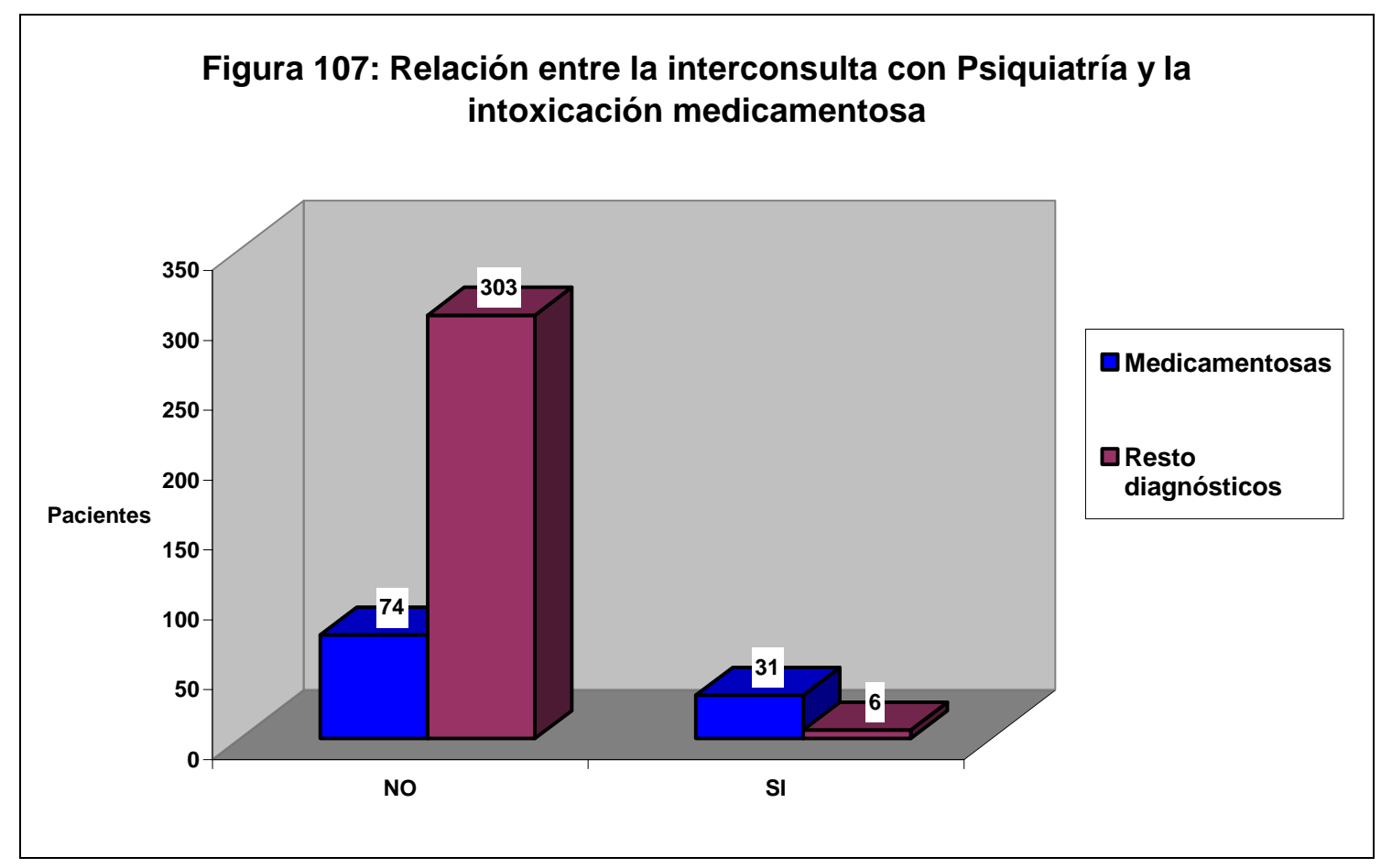

$p=0,0001$

\subsubsection{Relación entre la necesidad de consulta con Toxicología y la} intoxicación medicamentosa.

Se evidenciaron diferencias estadísticamente significativas en la realización de interconsulta a Toxicología durante la estancia en el servicio de Urgencias Hospitalarias de los pacientes teniendo en cuenta el diagnóstico de intoxicación medicamentosa $(p=0,0001)$. Tabla 183 y figura 108 .

Tabla 183: Relación entre la necesidad de consulta con Toxicología y la intoxicación medicamentosa.

\begin{tabular}{|c|ccc|c|}
\hline & NO & SI & $\%$ & Total \\
\hline Resto diagnósticos & 277 & 60 & $82,19 \% / 17,80 \%$ & 337 \\
Medicamentosas & 61 & 26 & $70,11 \% / 29,88 \%$ & 87 \\
\hline Total & 338 & 86 & 424 \\
\hline \multicolumn{5}{|c|}{0,0001} \\
\hline
\end{tabular}




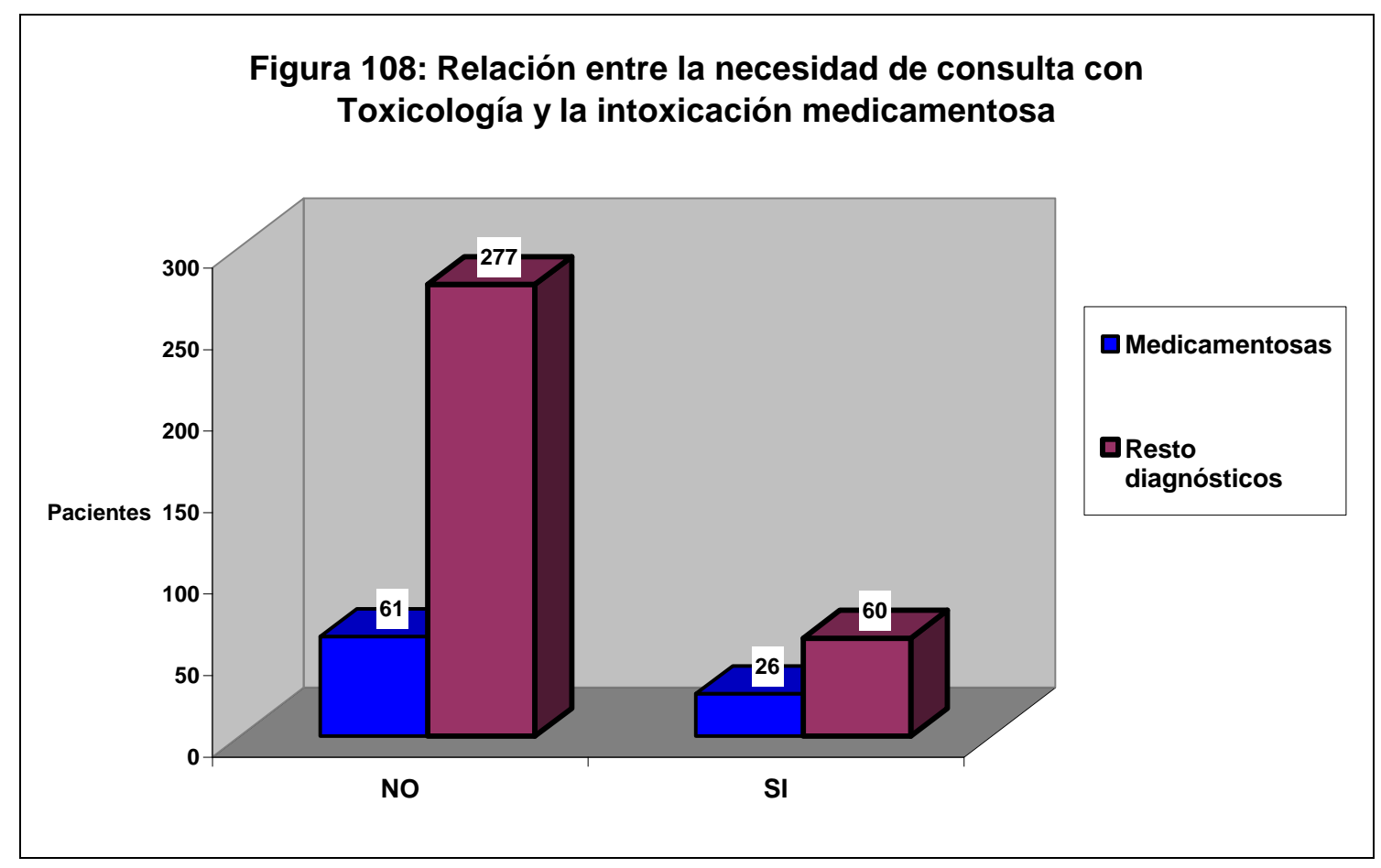

$p=0,0001$

\subsubsection{Estancia en el servicio de urgencias hospitalario de los pacientes} con intoxicación medicamentosa.

Se evidenciaron diferencias estadísticamente significativas en el tiempo de permanencia en el servicio de urgencias hospitalario teniendo en cuenta el diagnóstico de intoxicación medicamentosa $(p=0,0001)$. Tabla 184 y figura 109.

Tabla 184: Tiempo de permanencia en el servicio de urgencias hospitalario de los pacientes con intoxicación medicamentosa

\begin{tabular}{|c|cc|c|}
\hline & Menos de 2 horas & Mas de 2 horas & Porcentaje \\
\hline Resto diagnósticos & 271 & 456 & $37,27 \% / 62.73 \%$ \\
Medicamentosas & 141 & 126 & $52,80 \% / 47.2 \%$ \\
\hline Total & 412 & 582 & \\
\hline \multicolumn{2}{|c|}{$p=0,0001$} \\
\hline
\end{tabular}




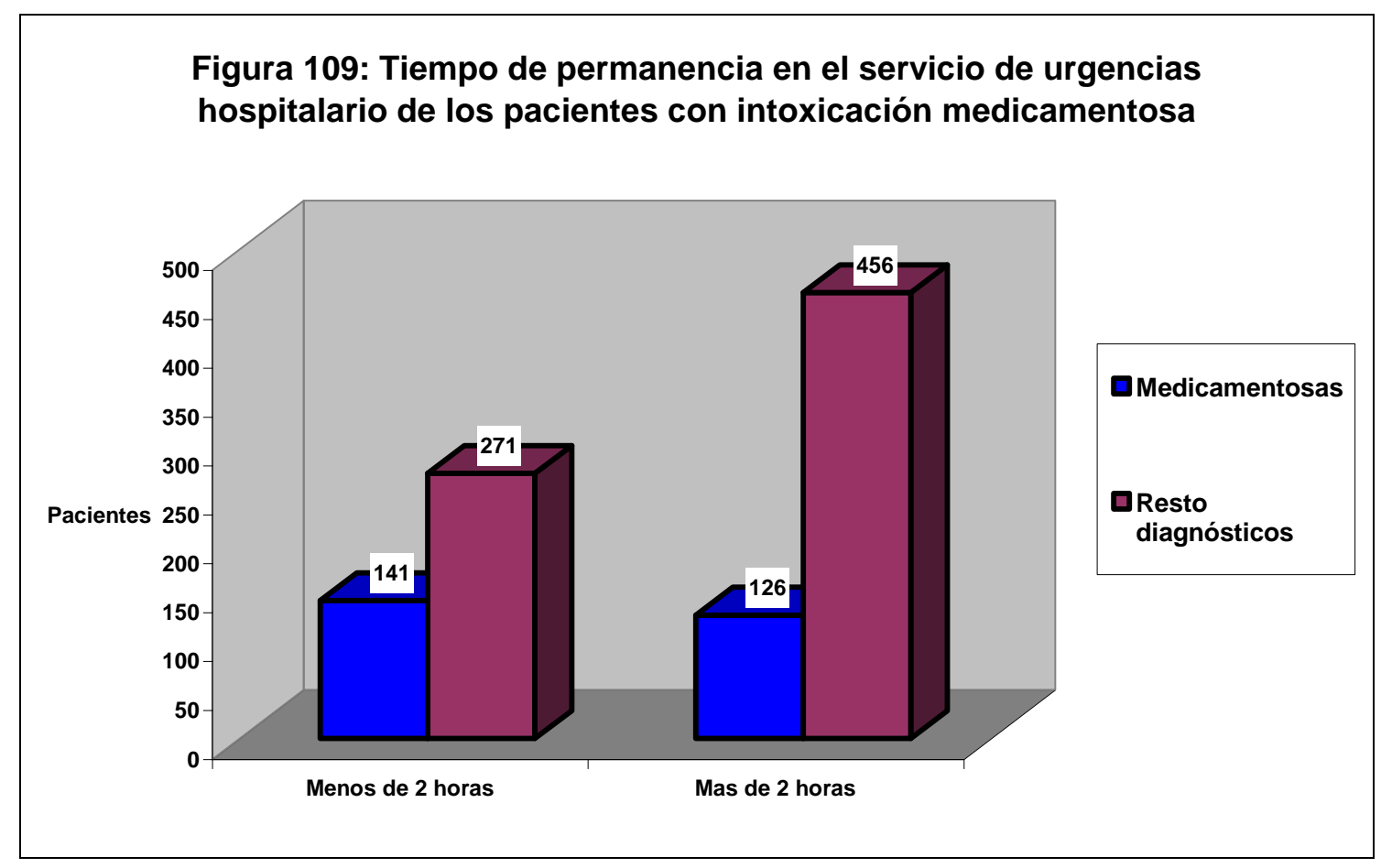

$p=0,0001$

6.2.14. Destino tras la estancia en el servicio de urgencias hospitalario de los pacientes con intoxicación medicamentosa.

El destino del paciente tras su estancia en el servicio de urgencias hospitalario se refleja en la tabla 185 y figura 110.

Tabla 185: Destino tras la estancia en el servicio de urgencias hospitalario de los pacientes con intoxicación medicamentosa.

\begin{tabular}{|c|cc|}
\hline & Frecuencia & Porcentaje \\
\hline Alta & 190 & 71,16 \\
\hline Ingreso Hospitalario & 57 & 21,34 \\
Derivación & 20 & 7,49 \\
\hline Total & 267 & 100 \\
\hline
\end{tabular}


Figura 110: Destino tras la estancia en el servicio de urgencias hospitalario
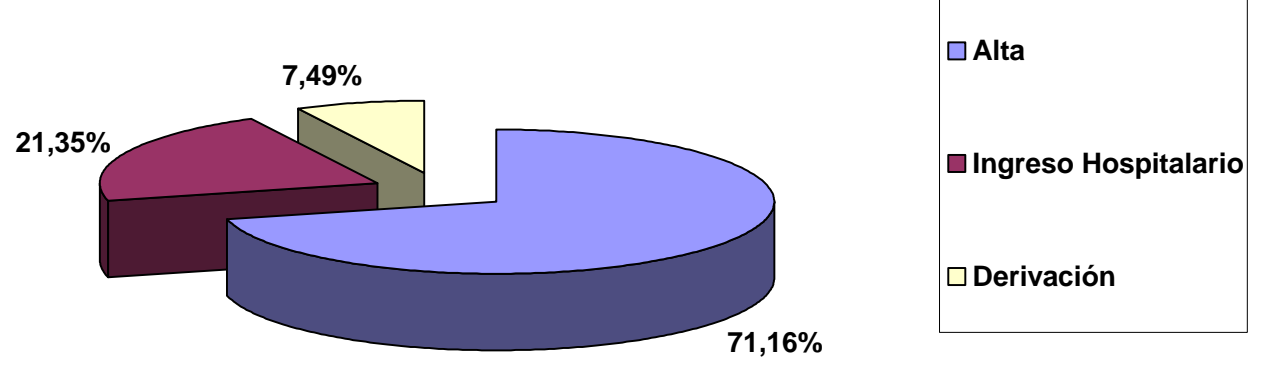

Se evidenciaron diferencias estadísticamente significativas en el destino tras la estancia en el servicio de Urgencias Hospitalario de los pacientes teniendo en cuenta el diagnóstico de intoxicación medicamentosa ( $p=$ 0,0001). Tabla 186.

Tabla 186: Destino de los pacientes con intoxicación medicamentosa

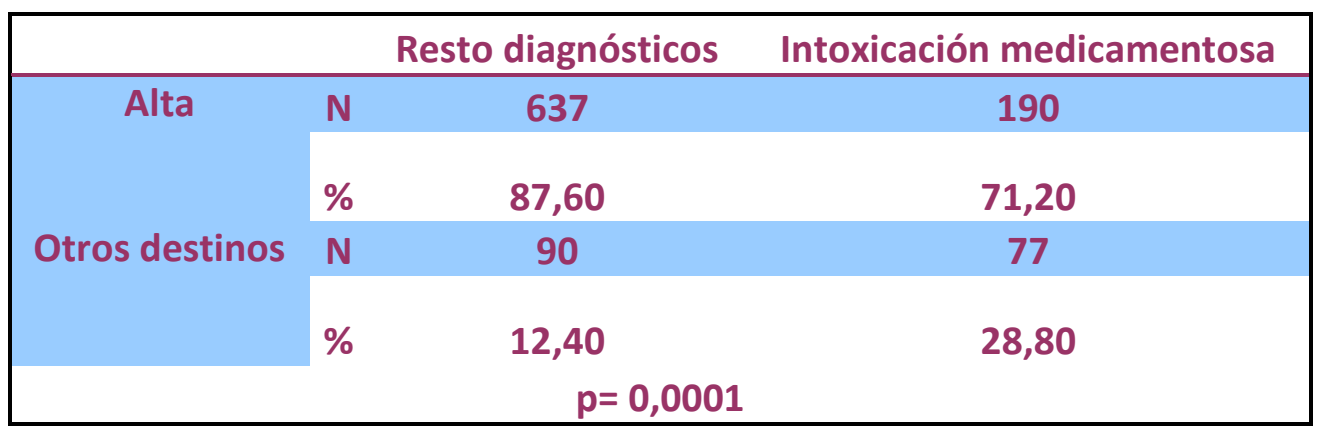

\subsubsection{Relación entre la intoxicación medicamentosa y diferentes} parámetros.

No se evidenciaron diferencias estadísticamente significativas en la derivación al servicio de Urgencias Hospitalario de los pacientes teniendo en cuenta el 
diagnóstico de intoxicación medicamentosa $(p=0,497)$; en el acompañamiento en su estancia en el servicio de urgencias hospitalarias $(p=0,572)$; Tampoco en la presencia de antecedentes personales del paciente teniendo en cuenta el diagnóstico de intoxicación medicamentosa $(p=$ 0,399); ni en el número de sustancias que ocasionan la intoxicación $(p=0,242)$; en quién es el propietario de la medicación que le ocasiona la intoxicación ( $p=0,402)$; ni en el número de consultas al servicio de urgencias hospitalarias en años previos al de la intoxicación ( $p=0,363)$; ni el número de consultas ambulatorias a las que es derivado el paciente $(p=0$, 833); ni al tiempo en horas de ingreso hospitalario necesario tras la

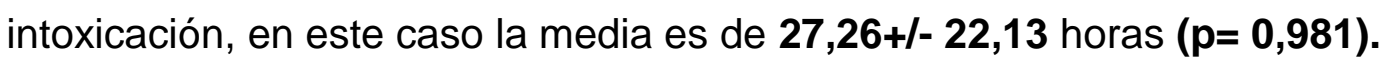

\subsubsection{Relación entre el número de consultas al servicio de Urgencias} Hospitalario en el mismo año y en los posteriores al que lo hacen con el diagnóstico de la intoxicación y el diagnóstico de intoxicación medicamentosa.

La media de consultas al servicio de Urgencias Hospitalario el mismo año de la intoxicación fue de 0,64+/- 1,22 consultas.

La media de las consultas al servicio de Urgencias Hospitalario en años posteriores al que lo hacen por la intoxicación fue de $2,79+/-4,73$ consultas.

Se evidenciaron diferencias estadísticamente significativas en el número de consultas al servicio de Urgencias Hospitalario el mismo año que acuden por la intoxicación teniendo en cuenta el diagnóstico de intoxicación medicamentosa $(p=0,002)$; por otra parte, también se evidenciaron diferencias estadísticamente significativas en el número de consultas al servicio de Urgencias Hospitalario en años posteriores al año que acuden por la intoxicación teniendo en cuenta el diagnóstico de intoxicación medicamentosa $(p=0,001)$. 


\subsection{Intoxicaciones por productos del hogar.}

\subsubsection{Sexo en las intoxicaciones por productos del hogar.}

No se evidenciaron diferencias estadísticamente significativas en el sexo de los pacientes teniendo en cuenta el diagnóstico de intoxicación por productos de limpieza. $(\mathbf{p}=\mathbf{0 , 2 9 7})$. Tabla 187.

Tabla 187: Relación entre el sexo y el diagnóstico de intoxicación por productos de limpieza

\begin{tabular}{|c|c|c|c|c|c|}
\hline & $\begin{array}{c}\text { Resto } \\
\text { diagnósticos }\end{array}$ & $\%$ & $\begin{array}{l}\text { Intoxicación por productos de } \\
\text { limpieza }\end{array}$ & $\%$ & Total \\
\hline Hombre & 486 & 53,23 & 48 & 59,25 & 534 \\
\hline Mujer & 427 & 46,76 & 33 & 40,74 & 460 \\
\hline & 913 & 100 & $p=0,297$ & 100 & 994 \\
\hline
\end{tabular}

\subsubsection{Edad en las intoxicaciones por productos del hogar.}

Se evidenciaron diferencias estadísticamente significativas en la edad de los pacientes teniendo en cuenta el diagnóstico de intoxicación por

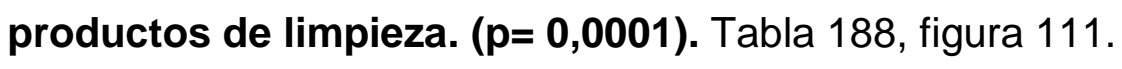

Tabla 188: Relación entre la edad y la intoxicación por productos de limpieza

\begin{tabular}{|c|c|c|}
\hline & Media & Desviación típica \\
\hline Intoxicación por productos de limpieza & 3,01 & 3,92 \\
\hline Resto diagnósticos & 10,78 & 6,23 \\
\hline \multicolumn{3}{|l|}{$p=0,0001$} \\
\hline
\end{tabular}




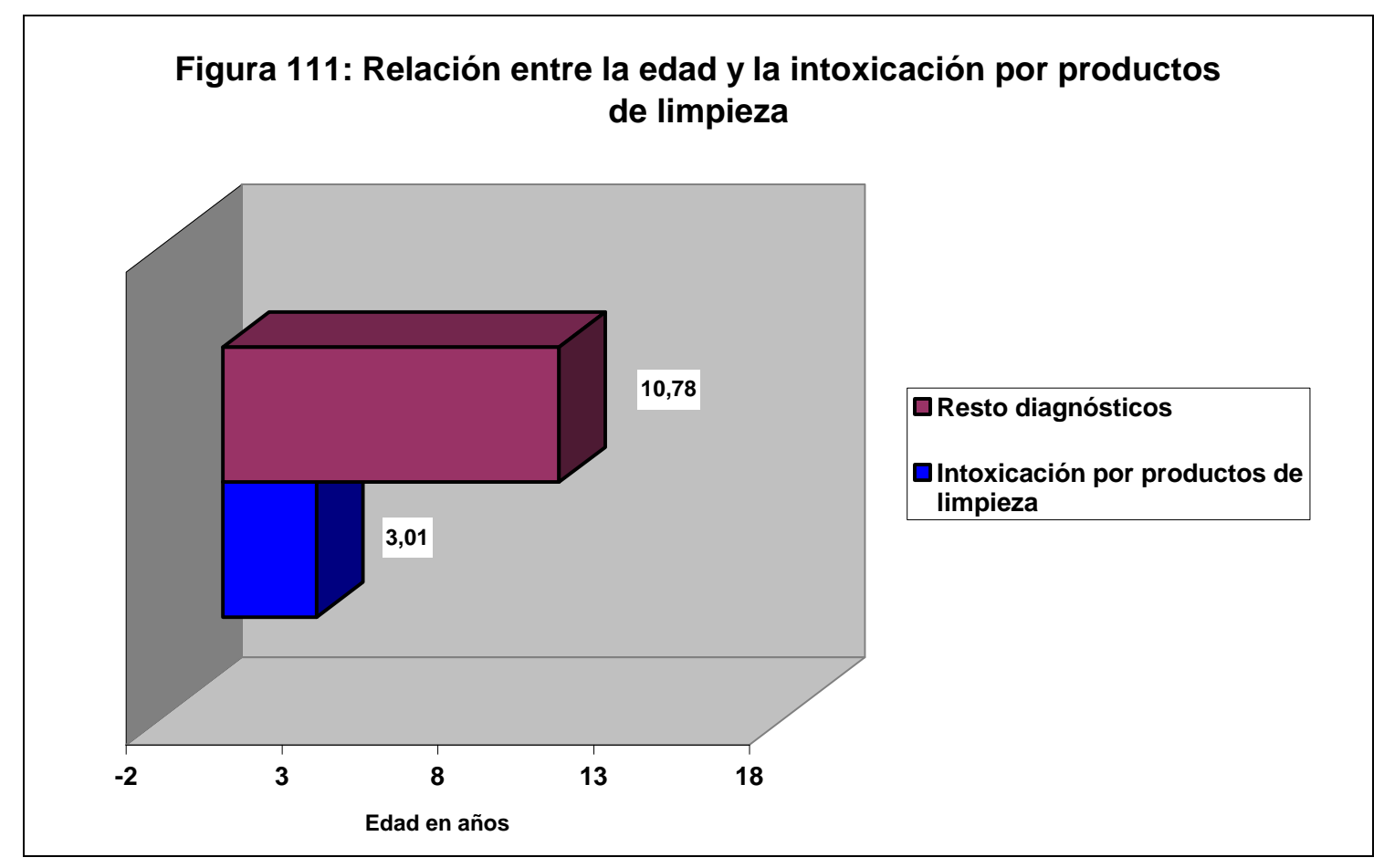

$p=0,0001$

\subsubsection{Año en el que ocurren las intoxicaciones por productos del hogar.}

No se evidenciaron diferencias estadísticamente significativas en el año en el que ocurre la intoxicación teniendo en cuenta el diagnóstico de intoxicación por productos de limpieza. $(\mathbf{p}=\mathbf{0 , 0 7 3})$. Tabla 189.

Figura 189: Relación entre el año y la intoxicación por productos de limpieza

\begin{tabular}{|c|cc|c|}
\hline $\begin{array}{c}\text { Año de } \\
\text { atención }\end{array}$ & $\begin{array}{c}\text { Frecuencia intoxicación } \\
\text { productos limpieza }\end{array}$ & $\%$ & $\begin{array}{c}\text { Frecuencia resto } \\
\text { diagnósticos }\end{array}$ \\
\hline 2001 & 5 & 6,40 & 82 \\
2002 & 12 & 15,40 & 79 \\
2003 & 9 & 11,50 & 93 \\
2004 & 4 & 5,10 & 51 \\
2005 & 4 & 5,10 & 60 \\
2006 & 4 & 5,10 & 88 \\
2007 & 19 & 24,40 & 130 \\
2008 & 4 & 5,10 & 115 \\
2009 & 10 & 12,80 & 109 \\
2010 & 7 & 9,00 & 106 \\
& & $p=0,073$ & \\
\hline
\end{tabular}




\section{4. Intoxicaciones por monóxido de carbono.}

\subsubsection{Relación entre sexo e intoxicaciones por monóxido de carbono.}

No se evidenciaron diferencias estadísticamente significativas en el sexo del paciente teniendo en cuenta el diagnóstico de intoxicación por monóxido de carbono. ( $p=\mathbf{0 , 0 6 2})$. Tabla 190.

Tabla 190: Relación entre el sexo y la intoxicación por monóxido de carbono

\begin{tabular}{|c|ccc|}
\hline & Resto diagnósticos & Intoxicación por CO & Total \\
\hline Hombre & 499 & 35 & 534 \\
Mujer & 415 & 45 & 460 \\
& 914 & 80 & 994 \\
& & & \\
& & & \\
\end{tabular}

\subsubsection{Relación entre la edad de los pacientes y la intoxicación por monóxido de carbono.}

No se evidenciaron diferencias estadísticamente significativas en la edad del paciente teniendo en cuenta el diagnóstico de intoxicación por monóxido de carbono. ( $p=\mathbf{0 , 3 2 9})$. Tabla 191.

Tabla 191: Relación entre la edad y el diagnóstico de intoxicación por monóxido de carbono

\begin{tabular}{|c|ccc|}
\hline & Frecuencia & Media edd en años & Desviación típica \\
\hline Resto diagnósticos & 914 & 10,19 & 6,54 \\
Intoxicación por CO & 80 & 9,6 & 5,09 \\
& \multicolumn{2}{|c}{$\mathrm{p}=0,329$} \\
\hline
\end{tabular}

\subsubsection{Relación entre el año de asistencia en el servicio de urgencias} hospitalario de los pacientes y la intoxicación por monóxido de carbono. La distribución temporal de la intoxicación por monóxido de carbono a lo largo de los años se muestra en la figura 112. 


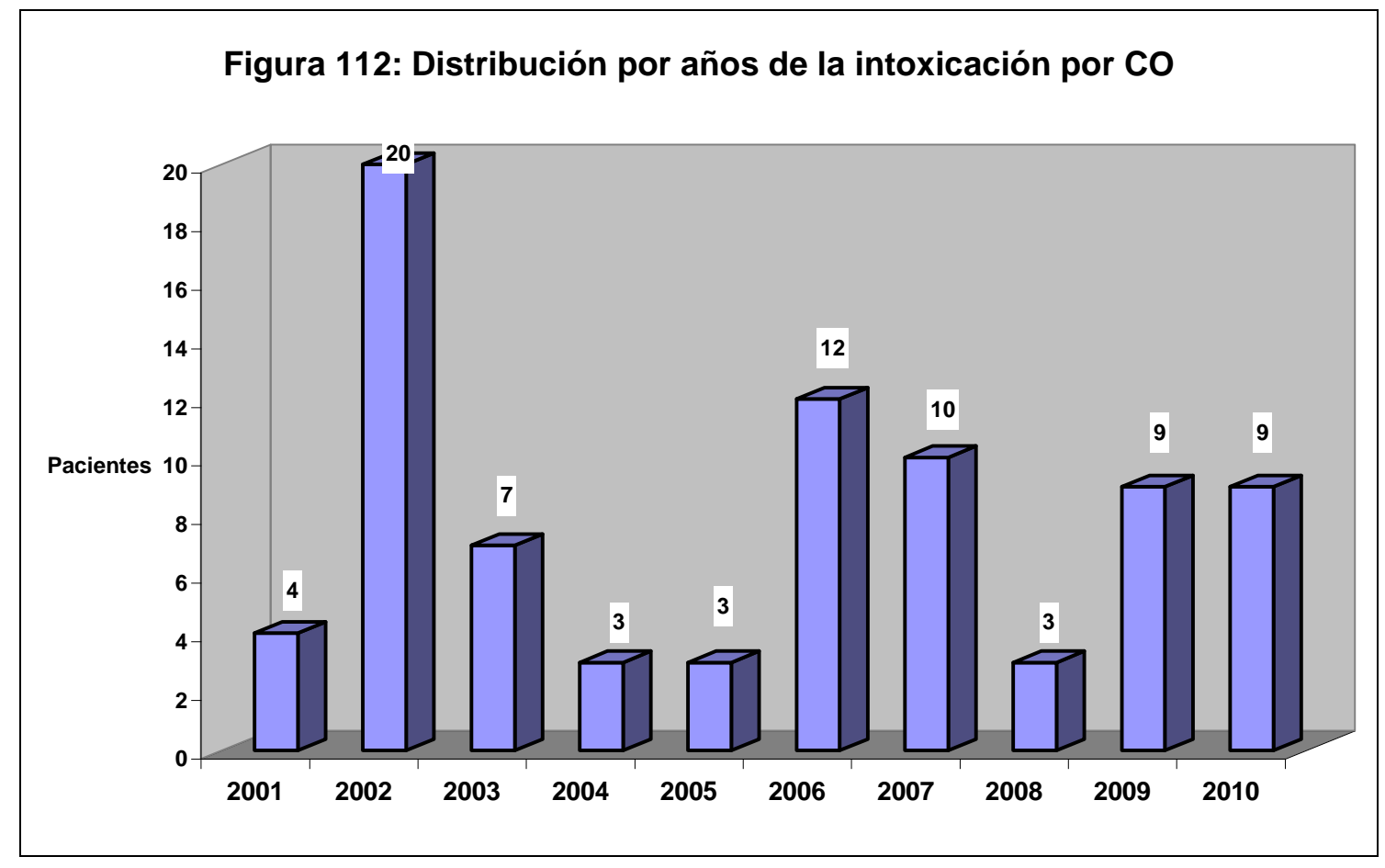

Se evidenciaron diferencias estadísticamente significativas en el año de asistencia en el servicio de urgencias hospitalario teniendo en cuenta el diagnóstico de intoxicación por monóxido de carbono. $(p=0,0001)$. Tabla 192 y figura 113.

Tabla 192: Relación entre el año de asistencia en el servicio de urgencias hospitalario y la intoxicación por monóxido de carbono.

\begin{tabular}{|c|c|cc|c|}
\hline & Resto diagnósticos & Intoxicación por CO & $\%$ & Total \\
\hline 2001 & 83 & 4 & 5,00 & 87 \\
2002 & 72 & 20 & 25,00 & 92 \\
2003 & 96 & 7 & 8,80 & 103 \\
2004 & 52 & 3 & 3,80 & 55 \\
2005 & 62 & 3 & 3,80 & 65 \\
2006 & 80 & 12 & 15,00 & 92 \\
2007 & 139 & 10 & 12,50 & 149 \\
2008 & 116 & 3 & 3,80 & 119 \\
2009 & 110 & 9 & 11,30 & 119 \\
2010 & 104 & 9 & 11,30 & 113 \\
\hline Total & 914 & 80 & 100 & 994 \\
\hline & \multicolumn{4}{|c}{} \\
\hline
\end{tabular}


Figura 113: Relación entre el año de asistencia en el servicio de urgencias hospitalario y la intoxicacion por monóxido de carbono

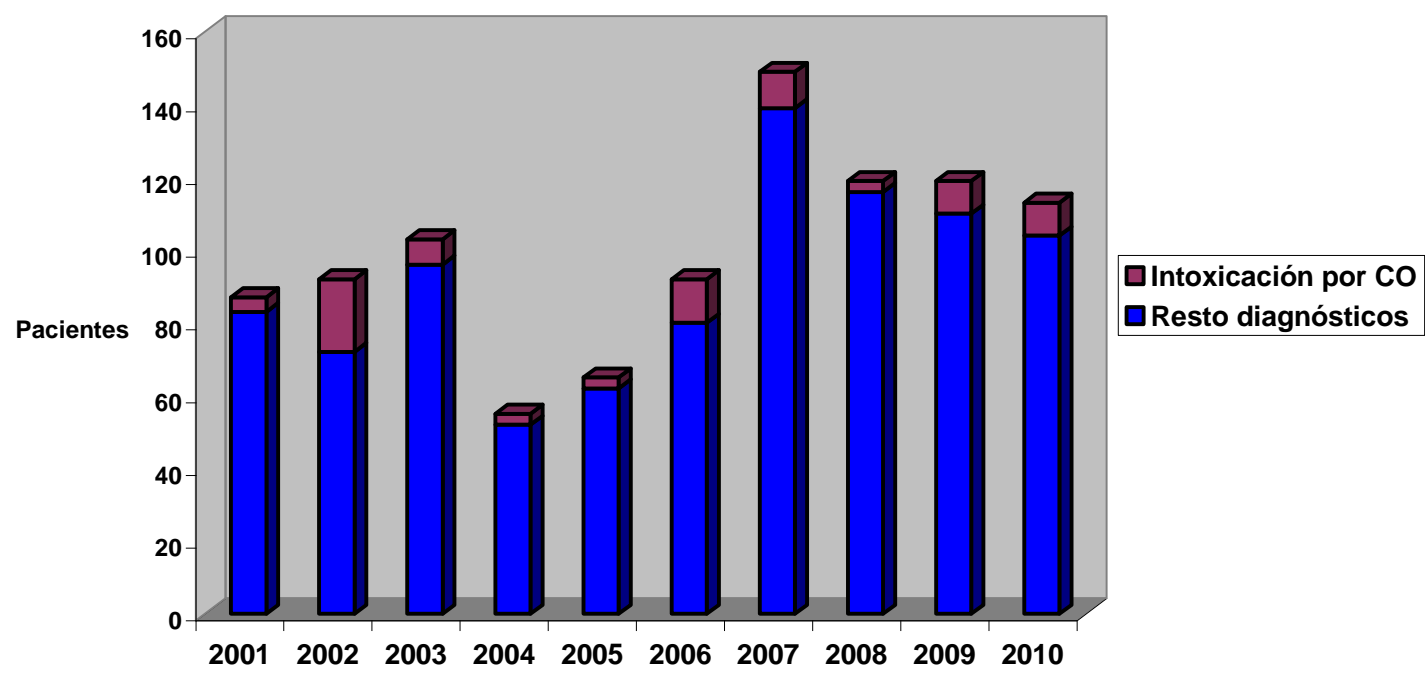

$p=0,0001$

\subsubsection{Mecanismo por el que se produce la intoxicación por monóxido de carbono}

En los 48 casos de intoxicación por monóxido de carbono registrados en el servicio de urgencias hospitalario del Hospital Universitario Río Hortega, durante estos diez años, se recoge el mecanismo por el que se produjo la intoxicación. Tabla 193, figura 114

Tabla 193: Mecanismo por el que se produce la intoxicación por monóxido de carbono

\begin{tabular}{|ccc|}
\hline Mecanismo & Frecuencia & Porcentaje \\
\hline Calderas... & 17 & 35,4 \\
Gas & 7 & 14,6 \\
Incendio & 4 & 8,3 \\
No recogido & 20 & 41,7 \\
\hline Total & 48 & 100 \\
\hline
\end{tabular}


Figura 114: Mecanismo por el que se produce la intoxicación por monóxido de carbono.

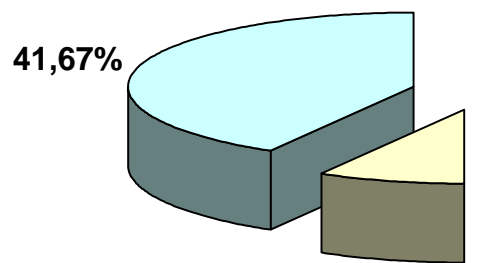

$8,33 \%$

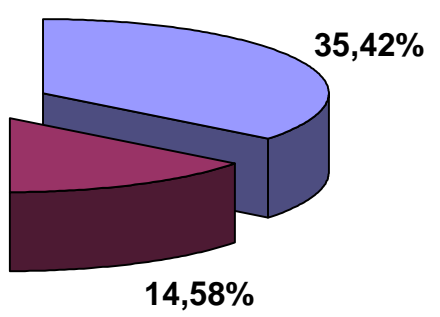

Calderas, estufas

$\square$ Gas

$\square$ Incendio

$\square$ No recogido

\subsubsection{Síntomas iniciales por los que demandan asistencia en los} pacientes intoxicados por monóxido de carbono.

En el caso de los 48 pacientes intoxicados por monóxido de carbono, se intenta recogen los primeros síntomas por los que solicitan asistencia sanitaria. Tabla 194, figura 115.

Tabla 194: Síntoma de debut de la intoxicación por CO

\begin{tabular}{|ccc|}
\hline Síntoma & Frecuencia & Porcentaje \\
\hline Digestivos & 16 & 33,30 \\
Neurológicos & 10 & 20,80 \\
Ninguno & 8 & 16,70 \\
Familiar & 5 & 10,40 \\
Síncope & 2 & 4,20 \\
Respiratorios & 2 & 4,20 \\
No recogidos & 5 & 10,40 \\
Total & 48 & 100 \\
\hline
\end{tabular}


Figura 115: Síntoma de debut de la intoxicación por monóxido de carbono

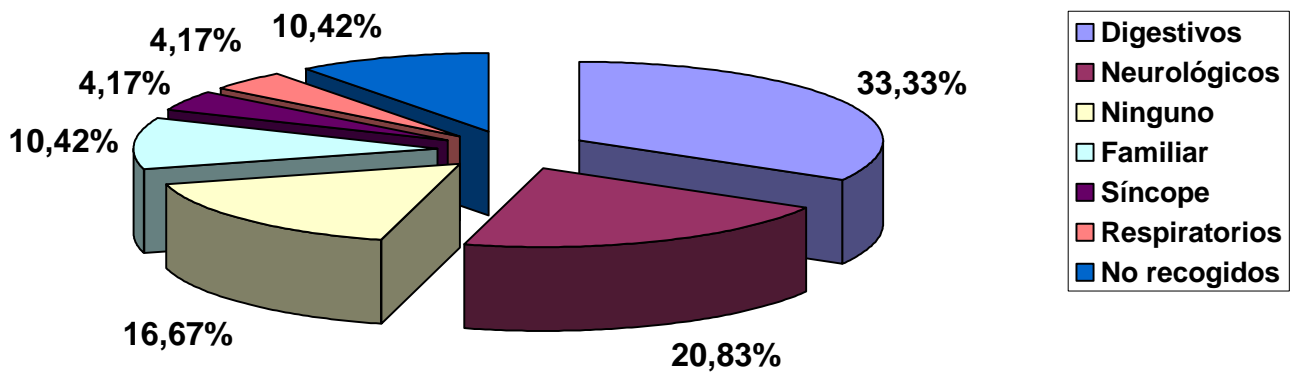

\subsubsection{Relación entre el tratamiento con oxigenoterapia en el servicio de} urgencias hospitalario en los pacientes con intoxicación por monóxido de carbono.

Se evidenciaron diferencias estadísticamente significativas en el tratamiento con oxigenoterapia en el servicio de urgencias hospitalario teniendo en cuenta el diagnóstico de intoxicación por monóxido de carbono. $(p=\mathbf{0 , 0 0 0 1})$. Tabla 195.

Tabla 195: Relación entre el tratamiento con oxigenoterapia en el servicio de urgencias y el diagnóstico de intoxicación por monóxido de carbono

\begin{tabular}{|c|ccc|}
\hline & Resto diagnósticos & Intoxicación por CO & Total \\
No oxigenoterapia & 532 & 9 & 541 \\
Sí oxigenoterapia & 8 & 35 & 43 \\
& 540 & 44 & 584 \\
& $p=0,0001$ & & \\
\hline
\end{tabular}




\subsubsection{Relación entre la necesidad de interconsulta con Toxicología durante la estancia en el servicio de urgencias hospitalario en los pacientes con intoxicación por monóxido de carbono.}

Se evidenciaron diferencias estadísticamente significativas en la necesidad de interconsulta con Toxicología durante su estancia en el servicio de urgencias hospitalario teniendo en cuenta el diagnóstico de intoxicación por monóxido de carbono. ( $p=\mathbf{0 , 0 0 0 1 )}$. Tabla 196.

Figura 196: Relación entre la necesidad de interconsulta con Toxicología durante la estancia en el servicio de urgencias hospitalario y la intoxicación por monóxido de carbono

\begin{tabular}{|c|ccc|}
\hline & Resto diagnósticos & Intoxicación por CO & Total \\
\hline No interconsulta Toxicología & 319 & 19 & 338 \\
Sí interconsulta Toxicología & 60 & 26 & 86 \\
& 379 & 45 & 424 \\
& $\mathrm{p}=0,0001$ & & \\
\hline
\end{tabular}

\subsubsection{Relación entre el destino del paciente tras su paso por el servicio de} urgencias hospitalario en los pacientes con intoxicación por monóxido de carbono.

Se evidenciaron diferencias estadísticamente significativas en el destino del paciente tras su paso por el servicio de urgencias hospitalario teniendo en cuenta el diagnóstico de intoxicación por monóxido de carbono. ( $p=$ 0,0001). Tabla 197 y figura 116.

Tabla 197: Relación entre el destino del paciente tras el paso por el servicio de urgencias hospitalario y la intoxicación por $\mathrm{CO}$

\begin{tabular}{|c|ccc|c|}
\hline & Resto diagnósticos & Intoxicación por CO & $\%$ & Total \\
\hline Alta & 786 & 41 & 51,25 & 827 \\
\hline Ingreso Hospitalario & 105 & 25 & 31,25 & 130 \\
Consulta externa & 2 & 8 & 10,00 & 10 \\
Derivación & 21 & 6 & 7,50 & 27 \\
& 914 & 80 & 994 & 994 \\
& \multicolumn{2}{r}{$\mathrm{p}=0,0001$} & & \\
\hline
\end{tabular}


Figura 116: Relación entre el destino del paciente tras el paso por el servicio de urgencias hospitalario y la intoxicación por $\mathrm{CO}$

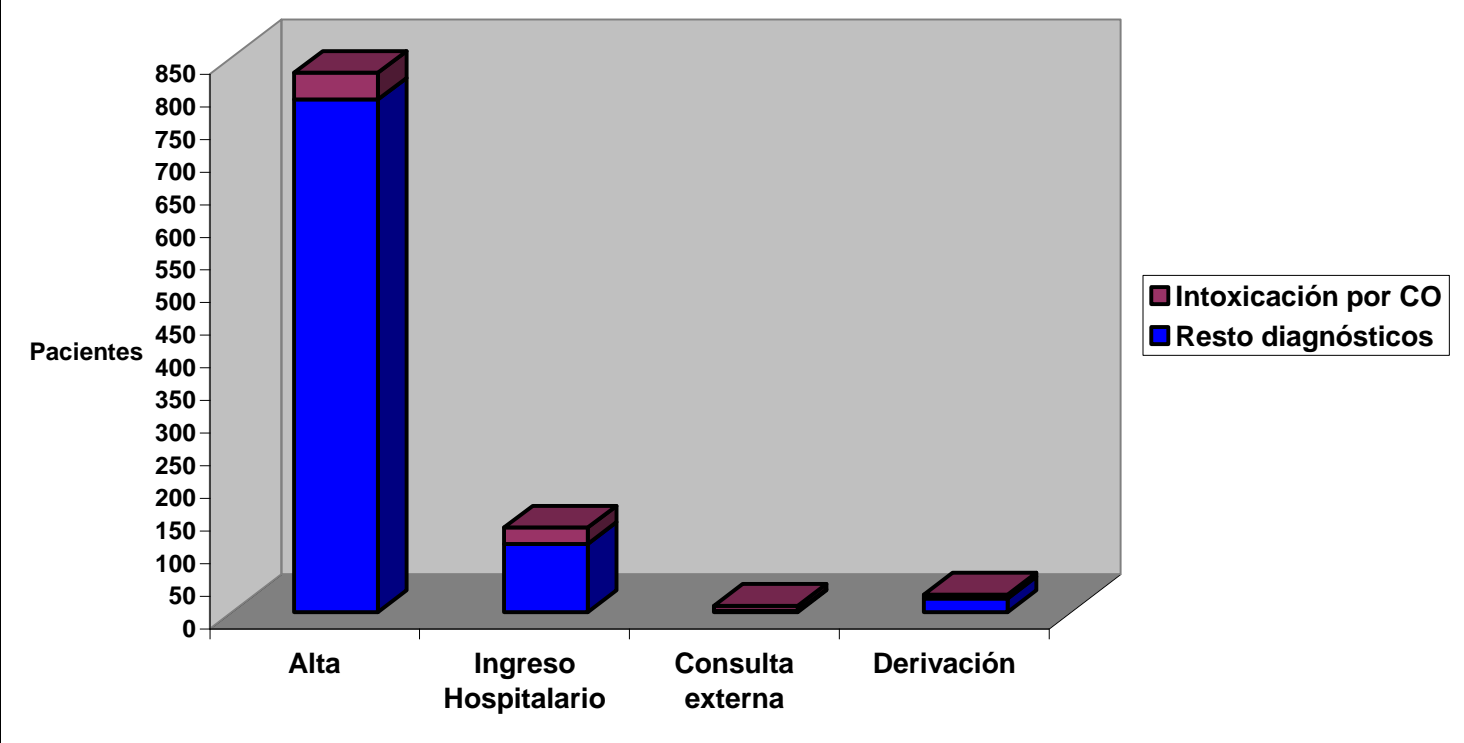

$p=0,0001$

\subsection{Intoxicación por productos industriales.}

\subsubsection{Relación entre el sexo y la intoxicación por productos industriales.}

No se evidenciaron diferencias estadísticamente significativas en el sexo del paciente teniendo en cuenta el diagnóstico de intoxicación por productos industriales. $(p=0,297)$. Tabla 198.

Tabla 198: Relación entre el sexo y la intoxicación por productos industriales

\begin{tabular}{|c|ccc|}
\hline & Resto intoxicaciones & Intoxicación por productos industriales & Total \\
\hline Hombre & 518 & 16 & 534 \\
Mujer & 451 & 9 & 460 \\
\hline & 969 & 25 & 994 \\
\hline \multicolumn{4}{r}{} \\
$\mathrm{n}=0,297$ & \\
\hline
\end{tabular}

\subsubsection{Relación entre la edad y la intoxicación por productos industriales.}

No se evidenciaron diferencias estadísticamente significativas en la edad del paciente teniendo en cuenta el diagnóstico de intoxicación por productos industriales. $(p=0,0001)$. Tabla 199. 
Tabla 199: Relación entre la edad del paciente y la intoxicación por productos industriales

\begin{tabular}{|c|c|c|}
\hline & Media & Desviación típica \\
\hline Resto intoxicaciones & 10,28 & 6,41 \\
\hline $\begin{array}{l}\text { Intoxicación por productos industriales } \\
\qquad p=0,0001\end{array}$ & 5,04 & 5,05 \\
\hline
\end{tabular}

\subsection{Intoxicación por productos cosméticos.}

\subsubsection{Sexo en la intoxicación por productos cosméticos.}

No se evidenciaron diferencias estadísticamente significativas en el año de la intoxicación teniendo en cuenta el diagnóstico de intoxicación por productos cosméticos. $(p=\mathbf{0 , 6 1 0})$. Tabla 200 .

Tabla 200: Relación entre el sexo y la intoxicación por productos cosméticos

\begin{tabular}{|c|cccc|}
\hline & Resto diagnósticos & Intoxicación productos cosméticos & Total \\
\hline Hombre & 521 & & 13 & 534 \\
Mujer & 451 & & 9 & 460 \\
& 972 & & 22 & 994 \\
& & $p=0,610$ & & \\
\hline
\end{tabular}

\subsubsection{Edad en la intoxicación por productos cosméticos.}

Se evidenciaron diferencias estadísticamente significativas en la edad de la intoxicación teniendo en cuenta el diagnóstico de intoxicación por productos cosméticos. $(p=0,0001)$. Tabla 201.

Tabla 201: Relación entre la edad del paciente y la intoxicación por productos cosméticos

\begin{tabular}{|c|ccc|}
\hline & $\mathrm{N}$ & Media & Desviación típica \\
\hline Resto diagnósticos & 972 & 10,34 & 6,37 \\
Intoxicación productos cosméticos & 22 & 1,72 & 1,42 \\
\hline \multicolumn{2}{|c|}{$\mathrm{p}=0,0001$} & & \\
\hline
\end{tabular}


6.6.3. Relación entre la interconsulta a Toxicología durante la estancia en el servicio de urgencias hospitalario y la intoxicación por productos cosméticos.

Se evidenciaron diferencias estadísticamente significativas en la necesidad de interconsulta a Toxicología durante su estancia en el servicio de urgencias hospitalario en cuenta el diagnóstico de intoxicación por productos cosméticos. ( $p=0,002)$. Tabla 202.

Tabla 202: Relación entre la interconsulta con Toxicología y la intoxicación por cosméticos

\begin{tabular}{|c|cc|c|}
\hline & $\begin{array}{c}\text { Resto } \\
\text { diagnósticos }\end{array}$ & $\begin{array}{c}\text { Intoxicación productos } \\
\text { cosméticos }\end{array}$ & Total \\
\hline $\begin{array}{c}\text { No interconsulta a } \\
\text { Toxicología }\end{array}$ & 334 & 4 & 338 \\
$\begin{array}{c}\text { Sí interconsulta a } \\
\text { Toxicología }\end{array}$ & 80 & 6 & 86 \\
& 414 & 10 & 424 \\
& $p=0,002$ & & \\
\hline
\end{tabular}

\subsubsection{Relación entre el destino tras la estancia en el servicio de urgencias} hospitalario y la intoxicación por productos cosméticos.

No se evidenciaron diferencias estadísticamente significativas en el destino tras la estancia en el servicio de urgencias hospitalario d la intoxicación teniendo en cuenta el diagnóstico de intoxicación por productos cosméticos. $(p=0,476)$. Tabla 203.

Tabla 203: Relación entre el destino tras su estancia en el servicio de urgencias hospitalario y la intoxicación por cosméticos

\begin{tabular}{|c|cc|c|}
\hline & Resto diagnósticos & $\begin{array}{c}\text { Intoxicación productos } \\
\text { cosméticos }\end{array}$ & Total \\
\hline Alta & 806 & 21 & 827 \\
Ingreso Hospitalario & 129 & 1 & 130 \\
Consulta externa & 10 & 0 & 10 \\
Derivación & 27 & 0 & 27 \\
& 972 & 22 & 994 \\
& & & \\
\hline
\end{tabular}




\subsection{Intoxicaciones por insecticidas.}

\subsubsection{Sexo en las intoxicaciones por insecticidas.}

No se evidenciaron diferencias estadísticamente significativas en el sexo del paciente teniendo en cuenta el diagnóstico de intoxicación por insecticidas. $(p=$ 0,229). Tabla 204.

Tabla 204: Relación entre el sexo del enfermo y la intoxicación por insecticidas

\begin{tabular}{|c|ccc|}
\hline & Resto diagnósticos & Intoxicación insecticidas & Total \\
\hline Hombre & 520 & 14 & 534 \\
Mujer & 453 & 7 & 460 \\
& 973 & 21 & 994 \\
& & & \\
\end{tabular}

\subsubsection{Edad en las intoxicaciones por insecticidas.}

Se evidenciaron diferencias estadísticamente significativas en la edad del paciente teniendo en cuenta el diagnóstico de intoxicación por insecticidas. $(p=0,0001)$. Tabla 205.

Tabla 205: Relación entre la edad y la intoxicación por insecticidas

\begin{tabular}{|c|cc|}
\hline & Media & Desviación típica \\
\hline Resto diagnósticos & 10,28 & 6,41 \\
Intoxicación insecticidas & 3,95 & 3,73 \\
& $\mathrm{p}=0,0001$ & \\
\hline
\end{tabular}

6.7.3. Relación entre la necesidad de interconsulta con Toxicología en las intoxicaciones por insecticidas.

Se evidenciaron diferencias estadísticamente significativas en la necesidad de interconsulta con Toxicología teniendo en cuenta el diagnóstico de intoxicación por insecticidas. $(p=0,033)$ Tabla 206. 
Tabla 206: Relación entre la necesidad de interconsulta con Toxicología en el servicio de urgencias hospitalario y la intoxicación por insecticidas

\begin{tabular}{|c|ccc|}
\hline \multirow{2}{*}{ No interconsulta Toxicología } & Resto diagnósticos & Intoxicación insecticidas & Total \\
Sí interconsulta Toxicología & 330 & 8 & 338 \\
& 80 & 6 & 86 \\
& 410 & 14 & 424 \\
& $\mathrm{p}=0,033$ & & \\
\hline
\end{tabular}

\subsubsection{Relación entre el destino tras la estancia en el servicio de urgencias hospitalario en las intoxicaciones por insecticidas.}

No se evidenciaron diferencias estadísticamente significativas entre el destino tras la estancia en el servicio de urgencias hospitalario teniendo en cuenta el

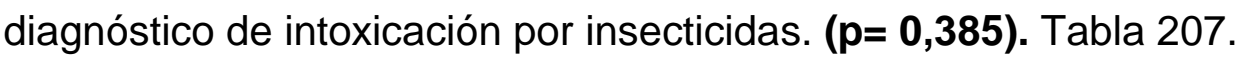

Tabla 207: Relación entre el destino tras el paso por el servicio de urgencias hospitalario y la intoxicación por insecticidas.

\begin{tabular}{|c|c|c|c|}
\hline & Resto diagnósticos & Intoxicación insecticidas & Total \\
\hline ALTA & 811 & 16 & 827 \\
\hline Otros destinos & 162 & 5 & 167 \\
\hline & $p=0$ & 21 & 994 \\
\hline
\end{tabular}

\subsection{Intoxicación por cannabis.}

\subsubsection{Sexo en las intoxicaciones por cannabis.}

No se evidenciaron diferencias estadísticamente significativas en el sexo del paciente teniendo en cuenta el diagnóstico de intoxicación por cannabis. $(p=$ 0,091). Tabla 208.

Tabla 208: Relación entre el sexo del enfermo y la intoxicación por cocaína

\begin{tabular}{|c|cccc|}
\hline & Resto diagnósticos & Intoxicación por cannabis & Total \\
\hline Hombre & 524 & 10 & 534 \\
Mujer & 457 & 3 & 460 \\
& 981 & 13 & 994 \\
& & & & \\
\hline
\end{tabular}




\subsubsection{Edad en las intoxicaciones por cannabis.}

Se evidenciaron diferencias estadísticamente significativas en la edad del paciente teniendo en cuenta el diagnóstico de intoxicación por cannabis. ( $p=$ 0,0001). Tabla 209.

Tabla 209: Relación entre la edad y la intoxicación por cannabis

\begin{tabular}{|c|cc|}
\hline & Media & Desviación típica \\
\hline Resto diagnósticos & 10,07 & 6,44 \\
Intoxicación por cannabis & 15,46 & 1,12 \\
$\mathrm{p}=0,0001$ & & \\
\hline
\end{tabular}

\subsubsection{Año de las intoxicaciones por cannabis.}

No se evidenciaron diferencias estadísticamente significativas en el año de atención en el servicio de urgencias hospitalario del paciente teniendo en cuenta el diagnóstico de intoxicación por cannabis. $(\mathbf{p}=\mathbf{0 , 5 6 5 )}$. Tabla 210. Figura 117.

Tabla 210: Relación entre el año de atención en el servicio de urgencias hospitalario y la intoxicación por cannabis

\begin{tabular}{|c|c|cc|c|}
\hline & Resto diagnósticos & Intoxicación por cannabis & $\%$ & Total \\
\hline 2001 & 86 & 1 & 7,69 & 87 \\
2002 & 92 & 0 & 0 & 92 \\
2003 & 102 & 1 & 7,69 & 103 \\
2004 & 55 & 0 & 0 & 55 \\
2005 & 65 & 0 & 0 & 65 \\
2006 & 91 & 1 & 7,69 & 92 \\
2007 & 146 & 3 & 23,07 & 149 \\
2008 & 115 & 4 & 30,76 & 119 \\
2009 & 117 & 2 & 15,38 & 119 \\
2010 & 112 & 1 & 7,69 & 113 \\
\hline & 981 & 13 & 100 & 994 \\
\hline & \multicolumn{4}{|l}{} \\
\hline
\end{tabular}


Figura 117: Relación entre el año de atención en el servicio de urgencias hospitalario y la intoxicación por cannabis.

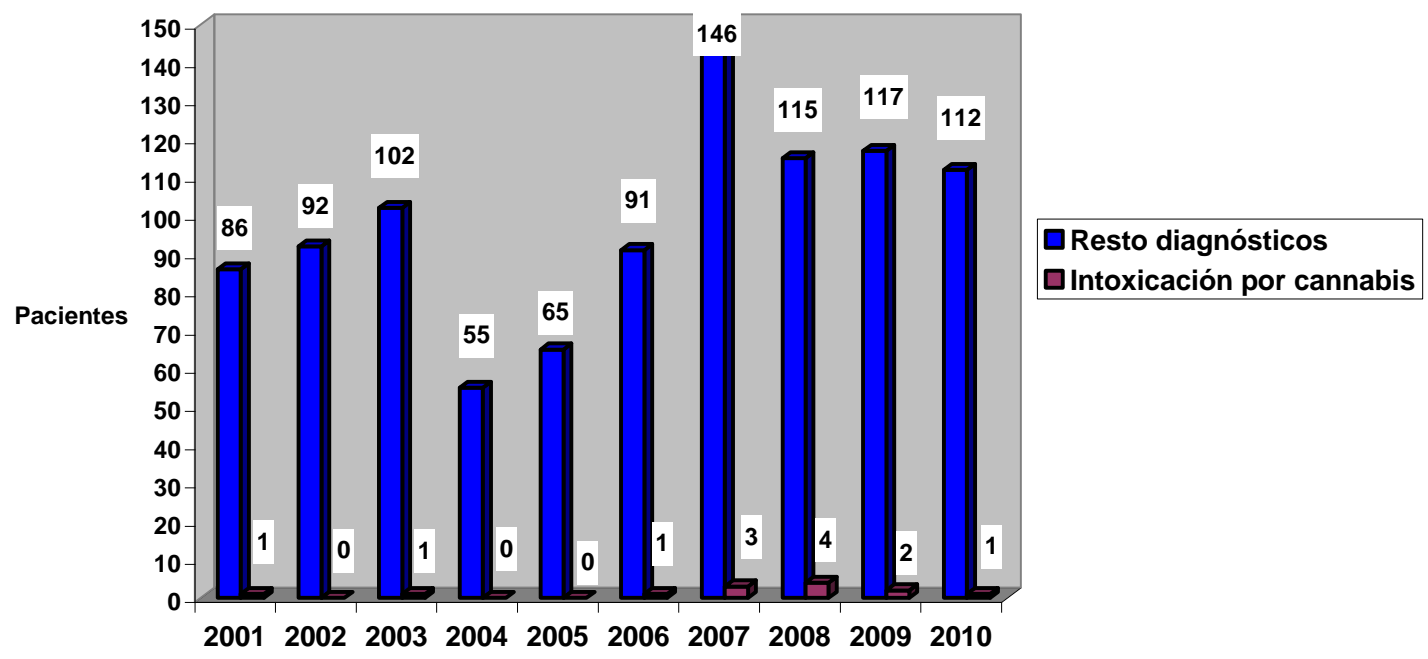

$p=0,565$

6.8.4. Hora de admisión en el servicio de urgencias hospitalario de las intoxicaciones por cannabis.

No se evidenciaron diferencias estadísticamente significativas en el año de atención en el servicio de urgencias hospitalario del paciente teniendo en cuenta el diagnóstico de intoxicación por cannabis. $(p=0,983)$. Tabla 211. Figura 118. 
Tabla 211: Relación entre la hora de admisión en el servicio de urgencias hospitalario y la intoxicación por cannabis

\begin{tabular}{|c|c|c|}
\hline & Resto diagnósticos & Intoxicación por cannabis \\
\hline $00: 00-00: 59$ & 65 & 1 \\
\hline 01:00- 01:59 & 50 & 1 \\
\hline 02:00 - 02:59 & 23 & 0 \\
\hline 03:00 - 03:59 & 25 & 1 \\
\hline 04:00 - 04:59 & 16 & 0 \\
\hline 05:00 - 05:59 & 10 & 0 \\
\hline $06: 00-06: 59$ & 10 & 0 \\
\hline 07:00 - 07:59 & 3 & 0 \\
\hline 08:00 - 08:59 & 4 & 0 \\
\hline 09:00 - 09:59 & 10 & 0 \\
\hline $10: 00-10: 59$ & 13 & 0 \\
\hline $11: 00-11: 59$ & 15 & 0 \\
\hline $12: 00-12: 59$ & 32 & 0 \\
\hline $13: 00-13: 59$ & 33 & 0 \\
\hline $14: 00-14: 59$ & 32 & 0 \\
\hline $15: 00-15: 59$ & 43 & 0 \\
\hline $16: 00-16: 59$ & 31 & 1 \\
\hline $17: 00-17: 59$ & 33 & 3 \\
\hline $18: 00-18: 59$ & 33 & 0 \\
\hline $19: 00-19: 59$ & 42 & 1 \\
\hline $20: 00-20: 59$ & 77 & 1 \\
\hline $21: 00-21: 59$ & 116 & 1 \\
\hline $22: 00-22: 59$ & 148 & 1 \\
\hline $23: 00-23: 59$ & 117 & 2 \\
\hline \multicolumn{3}{|c|}{$p=0,983$} \\
\hline
\end{tabular}


Distribución de la intoxicación por cannabis según la hora de admisión en el servicio de urgencias hospitalario

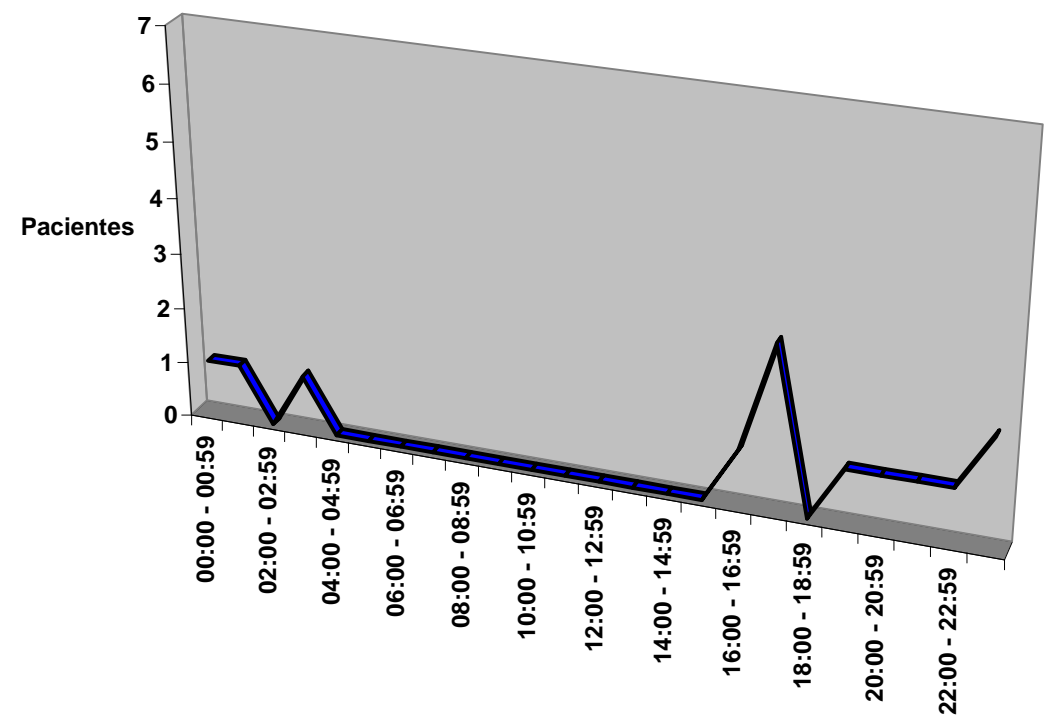

\subsubsection{Antecedentes personales de los pacientes con intoxicaciones por} cannabis.

No se evidenciaron diferencias estadísticamente significativas en la presencia de antecedentes personales del paciente teniendo en cuenta el diagnóstico de intoxicación por cannabis. ( $p=0,902)$. Tabla 212 .

Tabla 212: Relación entre la presencia de antecedentes personales en los pacientes y el diagnóstico de intoxicación por cannabis

\begin{tabular}{|c|ccc|c|}
\hline & $\begin{array}{c}\text { Resto } \\
\text { diagnósticos }\end{array}$ & $\begin{array}{c}\text { Intoxicación por } \\
\text { cannabis }\end{array}$ & $\%$ & Total \\
$\begin{array}{c}\text { No antecedentes } \\
\text { personales }\end{array}$ & 620 & 8 & 61,53 & 628 \\
Sí antecedentes personales & 361 & 5 & 38,46 & 366 \\
& 981 & 13 & 100 & 994 \\
& $p=0,902$ & & & \\
\hline
\end{tabular}

\subsubsection{Necesidad de trasporte de los pacientes en vehículos de}

\section{Emergencias Sanitarias en las intoxicaciones por cannabis.}

No se evidenciaron diferencias estadísticamente significativas en la necesidad de trasporte por vehículos de Emergencias Sanitarias teniendo en cuenta el diagnóstico de intoxicación por cannabis. $(p=\mathbf{0 , 7 3 1 ) . ~ T a b l a ~} 213$. 
Tabla 213: Relación entre la necesidad de trasporte por vehículos de Emergencias Sanitarias y la intoxicación por cannabis

\begin{tabular}{|c|c|cc|c|}
\hline & $\begin{array}{c}\text { Resto } \\
\text { diagnósticos }\end{array}$ & \multicolumn{2}{|l|}{$\begin{array}{l}\text { Intoxicación por } \\
\text { cannabis }\end{array}$} & $\begin{array}{c}\text { Tot } \\
\text { al }\end{array}$ \\
\hline $\begin{array}{c}\text { No vehículo de Emergencias } \\
\text { Sanitarias }\end{array}$ & 342 & 6 & 54,54 & 348 \\
$\begin{array}{c}\text { Sí vehículo de Emergencias } \\
\text { Sanitarias }\end{array}$ & 231 & 5 & 45,45 & 236 \\
& $\begin{array}{c}573 \\
\mathrm{p}=0,731\end{array}$ & 11 & 100 & 584 \\
\hline
\end{tabular}

\subsubsection{Relación entre el número de sustancias implicadas en la} intoxicación y el diagnóstico de intoxicación por cannabis.

No se evidenciaron diferencias estadísticamente significativas en el número de sustancias implicadas en la intoxicación y el diagnóstico de intoxicación por

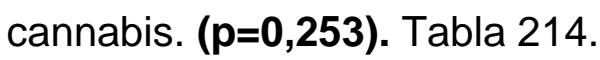

Tabla 214: Relación entre el número de sustancias que ocasionan la intoxicación y el diagnóstico de intoxicación por cannabis.

\begin{tabular}{|c|cc|}
\hline & Media del número de sustancias & Desviación típica \\
\hline Resto diagnósticos & 1,02 & 0,19 \\
Intoxicación por cannabis & 1,15 & 0,37 \\
& $\mathrm{p}=0,253$ & \\
\hline
\end{tabular}

\subsubsection{Destino de los pacientes en las intoxicaciones por cannabis tras el paso por el servicio de urgencias hospitalario}

No se evidenciaron diferencias estadísticamente significativas en el destino del paciente tras el paso por el servicio de urgencias hospitalario teniendo en cuenta el diagnóstico de intoxicación por cannabis. $(\mathbf{p}=\mathbf{0 , 1 0 3})$. Tabla 215. 
Tabla 215: Relación entre el destino del paciente tras la estancia en el servicio de urgencias hospitalario y la intoxicación por cannabis

\begin{tabular}{|c|cccc|}
\hline & Resto diagnósticos & Intoxicación por cannabis & Total \\
\hline ALTA & 814 & 13 & 827 \\
Otros & 167 & 0 & 167 \\
& 981 & 13 & 994 \\
& & & & \\
\hline
\end{tabular}

\subsection{Intoxicaciones por productos estimulantes.}

\subsubsection{Sexo de las intoxicaciones por productos estimulantes.}

No se evidenciaron diferencias estadísticamente significativas en el sexo de los pacientes teniendo en cuenta el diagnóstico de intoxicación por productos estimulantes. $(\mathbf{p}=\mathbf{0 , 1 4 6})$. Tabla 216 .

Tabla 216: Relación entre el sexo del paciente y la intoxicación por productos estimulantes

\begin{tabular}{|c|ccc|}
\hline & Resto intoxicaciones & Intoxicaciones por productos estimulantes & Total \\
\hline $\begin{array}{c}\text { Hombr } \\
\text { e }\end{array}$ & 527 & 7 & 534 \\
Mujer & 458 & 2 & 460 \\
& 985 & 9 & 994 \\
& & & \\
\end{tabular}

\subsubsection{Edad de las intoxicaciones por productos estimulantes.}

Se evidenciaron diferencias estadísticamente significativas en la edad de los pacientes teniendo en cuenta el diagnóstico de intoxicación por productos estimulantes. $(p=0,0001)$. Tabla 217 .

Tabla 217: Relación entre la edad y la intoxicación por productos estimulantes

\begin{tabular}{|c|cc|}
\hline & Media & Desviación típica \\
\hline Resto intoxicaciones & 10,1 & 6,44 \\
Intoxicaciones por productos estimulantes & 15 & 2,44 \\
$\mathrm{p}=0,0001$ & & \\
\hline
\end{tabular}




\subsubsection{Relación entre el destino tras el paso por el servicio de urgencias hospitalario y las intoxicaciones por productos estimulantes.}

No se evidenciaron diferencias estadísticamente significativas en el destino del paciente tras su estancia en el servicio de urgencias hospitalario teniendo en cuenta el diagnóstico de intoxicación por productos estimulantes. $(p=\mathbf{0 , 6 6 2})$. Tabla 218.

Tabla 218: Relación entre el destino del paciente tras su estancia en el servicio de urgencias hospitalario y la intoxicación por productos estimulantes

\begin{tabular}{|c|cc|c|}
\hline & Resto intoxicaciones & Intoxicaciones por productos estimulantes & Total \\
\hline $\begin{array}{c}\text { ALTA } \\
\text { Otro }\end{array}$ & 820 & 7 & 827 \\
$\mathrm{~s}$ & 165 & 2 & 167 \\
\hline & 985 & 9 & 994 \\
\hline \multicolumn{3}{|c|}{$\mathrm{p}=0,662$} \\
\hline
\end{tabular}

\subsection{Intoxicación por setas.}

\subsubsection{Sexo en la intoxicación por setas.}

No se evidenciaron diferencias estadísticamente significativas en el sexo del paciente teniendo en cuenta el diagnóstico de intoxicación por setas. $(\boldsymbol{p}=$ 0,524). Tabla 219.

Tabla 219: Relación entre el sexo del paciente y la intoxicación por setas

\begin{tabular}{|c|ccc|}
\hline & Resto intoxicaciones & Intoxicaciones por setas & Total \\
\hline Hombre & 530 & 4 & 534 \\
Mujer & 458 & 2 & 460 \\
& 988 & 6 & 994 \\
\hline
\end{tabular}




\subsubsection{Edad de las intoxicaciones por setas.}

No se evidenciaron diferencias estadísticamente significativas en la edad de los pacientes teniendo en cuenta el diagnóstico de intoxicación por setas. $(\mathbf{p}=$ 0,087). Tabla 220.

Tabla 220: Relación entre la edad y la intoxicación por setas

\begin{tabular}{|c|cc|}
\hline & Media & Desviación típica \\
\hline Resto intoxicaciones & 10,17 & 6,43 \\
Intoxicaciones por setas & 5,5 & 5,39 \\
\multicolumn{2}{|c|}{$\mathrm{p}=0,087$} & \\
\hline
\end{tabular}

\subsubsection{Destino tras la estancia en el servicio de urgencias hospitalario de} las intoxicaciones por setas.

No se evidenciaron diferencias estadísticamente significativas en el destino de los pacientes tras la estancia en el servicio de urgencias hospitalario los pacientes teniendo en cuenta el diagnóstico de intoxicación por setas. $(p=$ 0,277). Tabla 221.

Tabla 221: Relación entre el destino tras la estancia en el servicio de urgencias hospitalario y la intoxicación por setas

\begin{tabular}{|c|c|cc|c|}
\hline & Resto intoxicaciones & Intoxicaciones por setas & $\%$ & Total \\
\hline ALTA & $\mathbf{8 2 3}$ & 4 & 66,66 & 827 \\
Otros & 165 & 2 & 33,33 & 167 \\
\hline & 988 & 6 & 100 & 994 \\
\hline \multicolumn{4}{|c|}{$\mathrm{p}=\mathbf{0 , 2 7 7}$} \\
\hline
\end{tabular}

\subsection{Intoxicación por cocaína.}

\subsubsection{Sexo en la intoxicación por cocaína.}

No se evidenciaron diferencias estadísticamente significativas en el la sexo de los pacientes teniendo en cuenta el diagnóstico de intoxicación por cocaína. $(\mathrm{p}=0,353)$. Tabla 222. 
Tabla 222: Relación entre el sexo y la intoxicación por cocaína

\begin{tabular}{|c|cc|c|}
\hline & Resto diagnósticos & Intoxicación por cocaína & Total \\
\hline Hombre & 533 & 1 & 534 \\
Mujer & 460 & 0 & 460 \\
\hline & 993 & 1 & 994 \\
\hline \multicolumn{4}{|c|}{$\mathrm{p}=0,353$} \\
\hline
\end{tabular}

\subsubsection{Edad en la intoxicación por cocaína.}

No se evidenciaron diferencias estadísticamente significativas en la edad de los pacientes teniendo en cuenta el diagnóstico de intoxicación por cocaína. ( $p=$ 0,287). Tabla 223.

Tabla 223: Relación entre la edad del paciente y la intoxicación por cocaína

\begin{tabular}{|c|cc|}
\hline & Media & Desviación típica \\
\hline Resto diagnósticos & 10,14 & 6,43 \\
Intoxicación por cocaína & 17,00 & 0 \\
& $\mathrm{p}=0,287$ & \\
\hline
\end{tabular}

\subsubsection{Número de sustancias implicadas en la intoxicación por cocaína.}

Se evidenciaron diferencias estadísticamente significativas en el número de sustancias que ocasionan la intoxicación teniendo en cuenta el diagnóstico de intoxicación por cocaína. ( $p=0,0001)$. Tabla 224 .

Tabla 224: Relación entre el número de sustancias que ocasionan la intoxicación y el diagnóstico de intoxicación por cocaína

\begin{tabular}{|c|cc|c|}
\hline & Resto diagnósticos & Intoxicación por cocaína & Total \\
\hline 1 & 969 & 0 & 969 \\
\hline 2 & 20 & 0 & 20 \\
3 & 4 & 1 & 5 \\
\hline & 993 & 1 & 994 \\
\hline \multicolumn{3}{|c}{} \\
\hline
\end{tabular}




\subsubsection{Destino tras la estancia en el servicio de urgencias hospitalario y la intoxicación por cocaína.}

No se evidenciaron diferencias estadísticamente significativas en el destino de los pacientes tras la estancia en el servicio de urgencias hospitalario teniendo

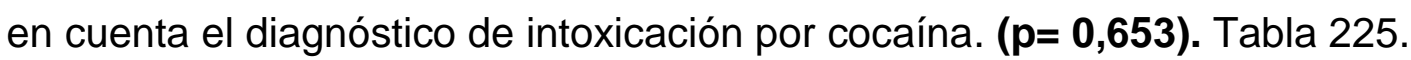

Tabla 225: Relación entre el destino del paciente tras la estancia en el servicio de urgencias hospitalario y la intoxicación por cocaína

\begin{tabular}{|c|cc|c|}
\hline & Resto diagnósticos & Intoxicación por cocaína & Total \\
\hline ALTA & 826 & 1 & 827 \\
Otros & 167 & 0 & 167 \\
\hline & 993 & 1 & 994 \\
\hline \multicolumn{4}{|r|}{} \\
\hline
\end{tabular}

\section{Localidad de origen de los pacientes}

Se establece una división entre los pacientes empadronados en Valladolid capital; en los principales pueblos del área de salud cuyo hospital de referencia es el Hospital Universitario Río Hortega, y los habitantes del resto de la provincia, comunidad o el resto de España. Tabla 226, figura 119.

Tabla 226: Localidad de origen

\begin{tabular}{|ccc|}
\hline Localidad & Frecuencia & Porcentaje \\
\hline Valladolid capital & 634 & 63,8 \\
Arroyo de la Encomienda & 41 & 4,10 \\
Laguna de Duero & 79 & 7,90 \\
La Cistérniga & 31 & 3,10 \\
Tordesillas & 13 & 1,30 \\
Resto provincia Valladolid & 158 & 15,90 \\
Resto Castilla y León & 13 & 1,30 \\
Resto de España & 25 & 2,50 \\
\hline
\end{tabular}


Figura 119: Localidad de origen de los pacientes intoxicados

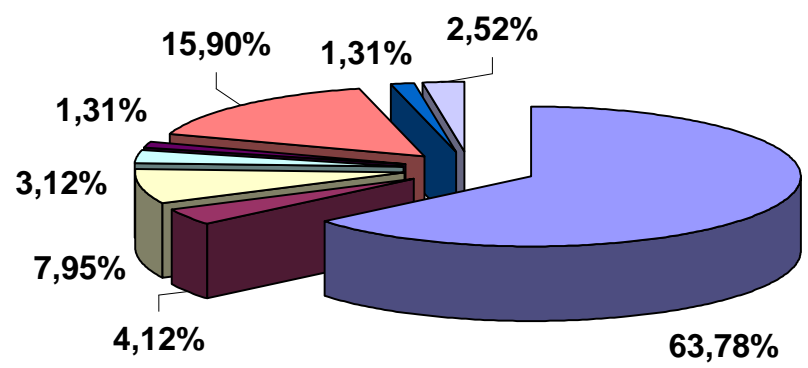

$\square$ Valladolid capital

$\square$ Arroyo de la Encomienda

$\square$ Laguna de Duero

$\square$ La Cistérniga

- Tordesillas

$\square$ Resto provincia Valladolid

$\square$ Resto Castilla y León

$\square$ Resto de España

Si se establece únicamente la división entre los pacientes de Valladolid capital y los pacientes de fuera de la localidad el resultado sería el siguiente, un $63,8 \%$ de los pacientes es de la capital, frente a un $36,2 \%$, es decir, 634 habitantes frente a 360. Tabla 227, figura 120.

Tabla 227: Procedencia de los intoxicados

\begin{tabular}{|ccc|}
\hline Localidad & Frecuencia & Porcentaje \\
\hline Valladolid capital & 634 & 63,80 \\
Resto & 360 & 36,20 \\
\hline
\end{tabular}


Figura 120: Procedencia de los pacientes

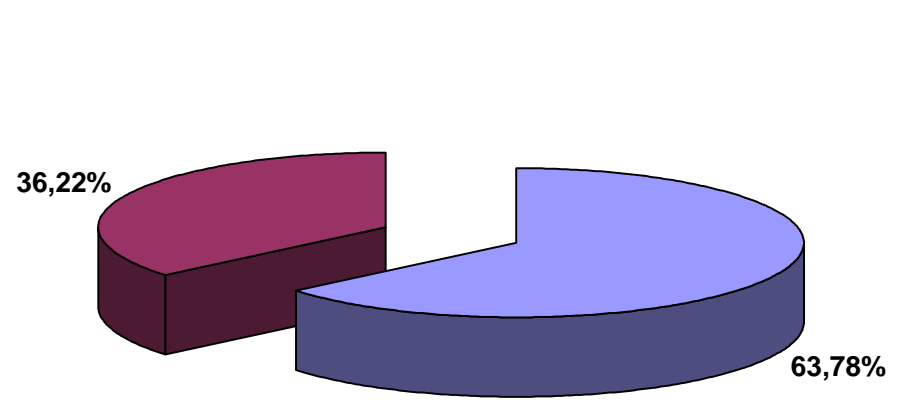

$\square$ Valladolid capital

$\square$ Resto

\section{Antecedentes personales de los pacientes intoxicados.}

Se registra la existencia o no de antecedentes personales de estos pacientes. Tabla 228, figura 121.

Tabla 228: Antecedentes personales de los intoxicados

\begin{tabular}{|ccc|}
\hline Antecedentes personales & Frecuencia & Porcentaje \\
\hline No & 628 & 63,2 \\
Sí & 366 & 36,8 \\
Total & 994 & 100 \\
\hline
\end{tabular}


Figura 121: Antecedentes personales de los intoxicados

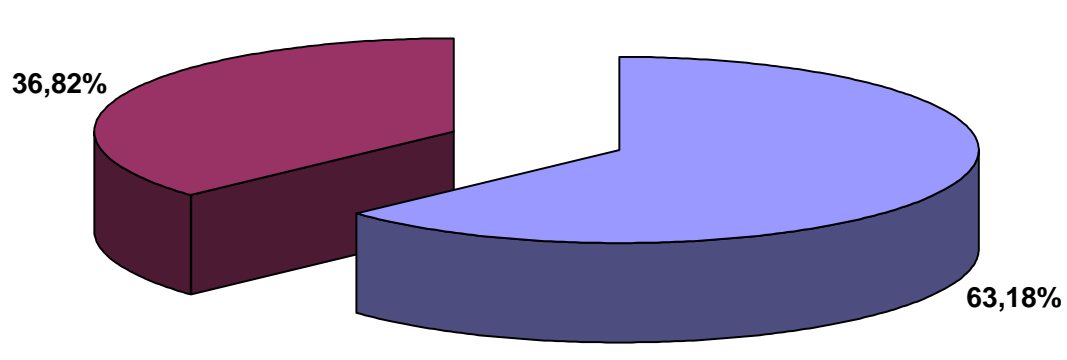

9. Derivación de los pacientes intoxicados al servicio de urgencias hospitalario por personal sanitario.

Se realiza una división entre los pacientes que acuden al servicio de urgencias hospitalario a petición propia, o los que son derivados desde un centro de salud o desde otro hospital. Tabla 229, figura 122.

En este caso, sólo se puede recoger dicha información de 584 historias.

Tabla 229: Derivación previa de los pacientes al servicio de urgencias hospitalario

\begin{tabular}{|ccc|}
\hline Derivación desde & Frecuencia & Porcentaje \\
\hline Sin derivación & 557 & 56,00 \\
Centro de salud & 20 & 2,00 \\
Otro hospital & 7 & 0,70 \\
Total & 584 & 58,8 \\
\hline
\end{tabular}


Figura 122: Derivación a urgencias de los pacientes intoxicados

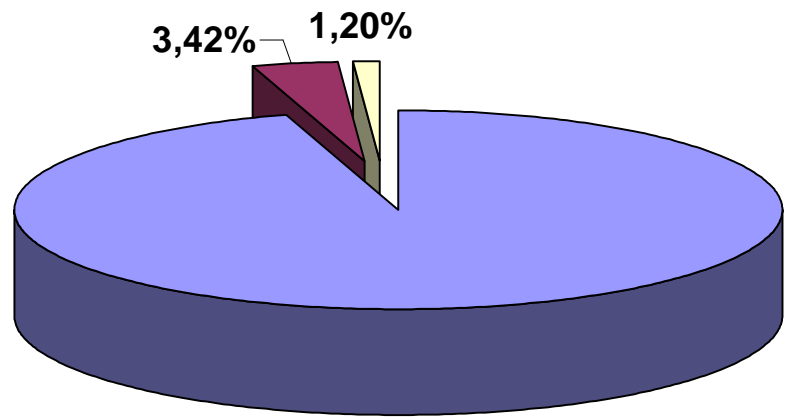

$\square$ Sin derivación

$\square$ Centro de salud

$\square$ Otro hospital

$95,38 \%$

\section{Acompañamiento del paciente en su estancia en el servicio de}

\section{urgencias hospitalario.}

Se registra en aquellas historias clínicas en las que es posible, si el paciente permanece en el servicio de urgencias hospitalario sólo 0 acompañado, y en este segundo caso, quién es la persona que se encuentra con él: familiar, amigos o representante legal. En 103 historias aparece reflejado si el menor está o no acompañado o por quién. Tabla 230, figura 123.

Tabla 230: Acompañamiento en la estancia en el servicio de urgencias hospitalario

\begin{tabular}{|ccc|}
\hline Acompañamiento & Frecuencia & Porcentaje \\
Familiar & 82 & 79,61 \\
Amigos & 13 & 12,62 \\
Sin acompañante & 6 & 5,83 \\
Representante legal & 2 & 1,94 \\
Total & 103 & 100 \\
\hline
\end{tabular}


Figura 123: Acompañamiento del paciente durante su estancia en el servicio de urgencias hospitalario.

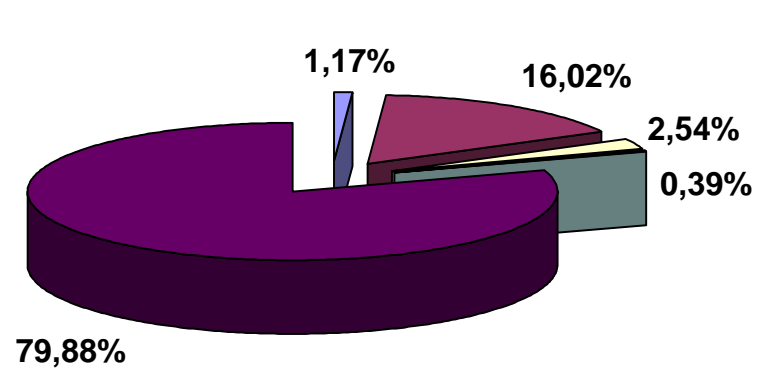

$\square$ Sin acompañante
$\square$ Familiar
$\square$ Amigos
$\square$ Representante legal
$\square$ No consta

$79,88 \%$

\section{Acceso al servicio de urgencias hospitalario en vehículos de}

\section{Emergencias Sanitarias}

En 584 pacientes se registró en la historia clínica si el paciente llegó o no en vehículo sanitario. En 236 casos, la forma de acudir al centro hospitalario fue gracias al transporte sanitario, sin especificar si este tipo de vehículo es un soporte vital básico o un soporte vital avanzado. Tabla 15, figura 7 . Esta cifra corresponde al 40,4 \% de las ocasiones.

En el 59,6\% de los casos, es decir, 348 pacientes, llegaron por otros medios. Tabla 231, figura 124.

Tabla 231: Acceso al servicio de urgencias hospitalario en vehículos de Emergencias Sanitarias

\begin{tabular}{|ccc|}
\hline Vehículo de Emergencias Sanitarias & Frecuencia & Porcentaje \\
\hline No & 348 & 59,6 \\
Si & 236 & 40,4 \\
Total & 584 & 100 \\
\hline
\end{tabular}




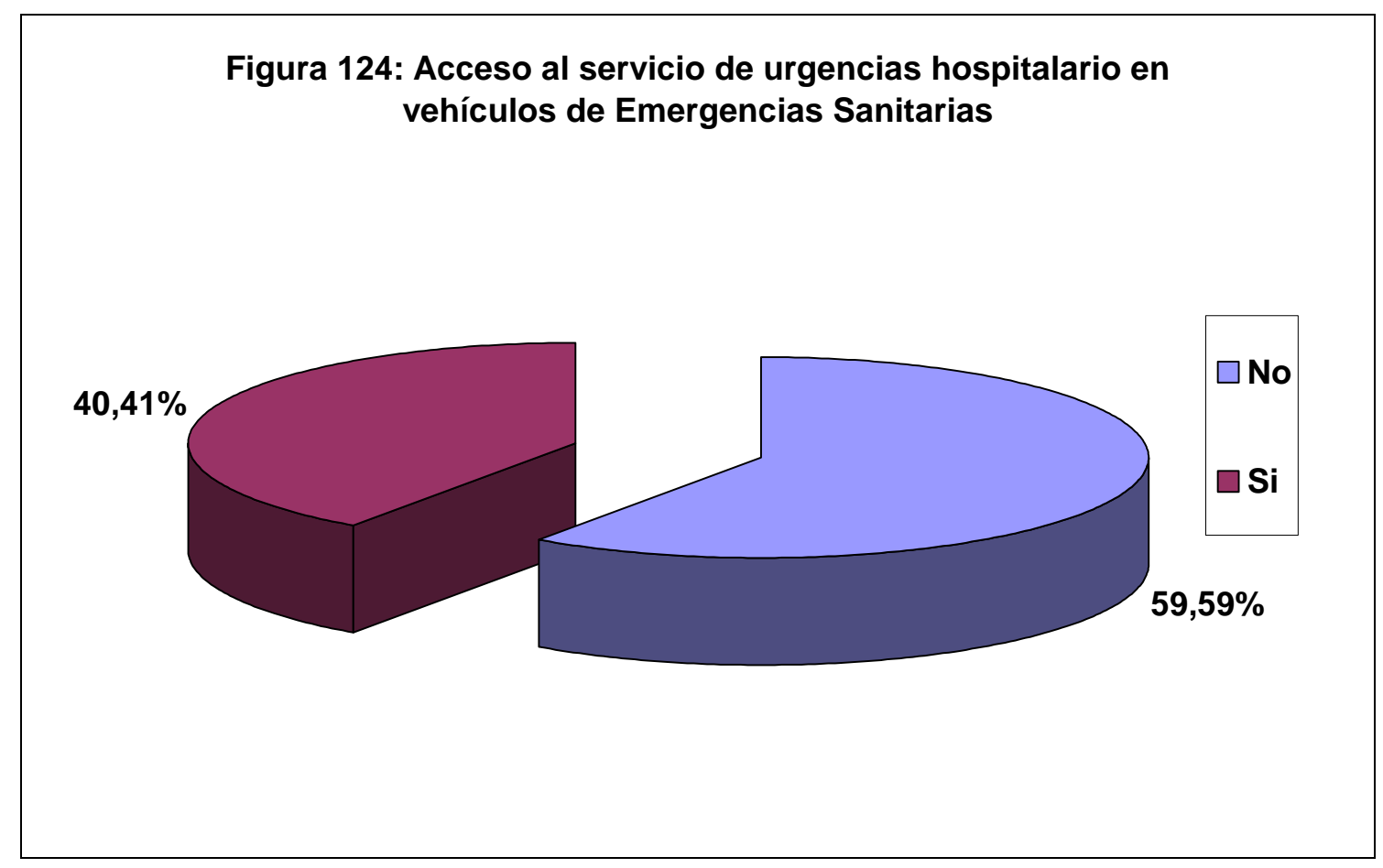

12. Realización de pruebas diagnósticas durante la estancia en el servicio de urgencias hospitalario.

12.1. Realización de analítica en el servicio de urgencias hospitalario.

Se registra la realización de una analítica de sangre u orina durante el periodo de estancia en el servicio de urgencias hospitalario. Tabla 232, figura 125.

Tabla 232: Realización de analítica

\begin{tabular}{|ccc|}
\hline Realización de analítica & Frecuencia & Porcentaje \\
\hline No & 266 & 45,30 \\
Sí & 321 & 45,70 \\
Total & 587 & 100
\end{tabular}



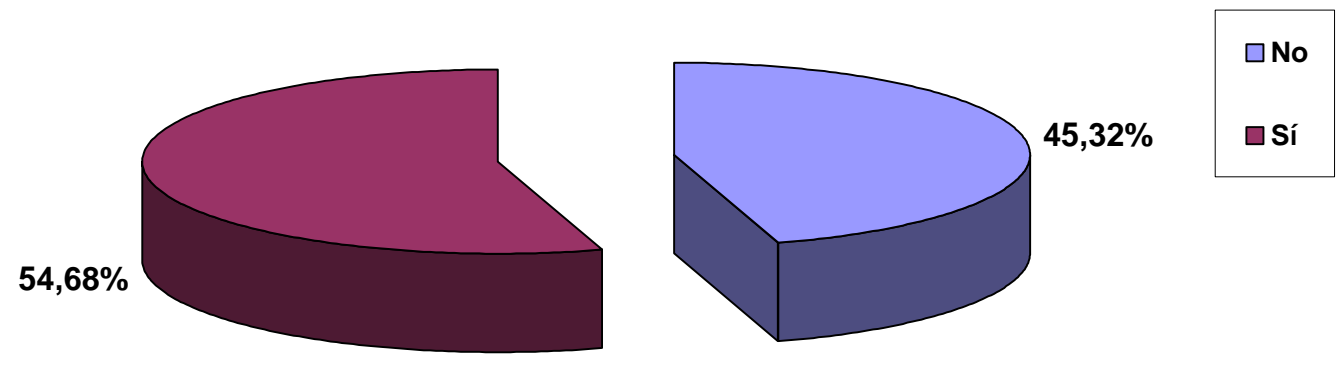

\subsection{Realización de diferentes tipos de analítica en el servicio de} urgencias hospitalario.

Se especifica dentro de aquellos pacientes sometidos en su estancia en el servicio de urgencias hospitalario qué tipo de pruebas analíticas se han llevado a cabo. Tablas 233-238, figura 126-127.

Tabla 233: Realización de sistemático de sangre

\begin{tabular}{|ccc|}
\hline Sistemático de sangre & Frecuencia & Porcentaje \\
\hline No & 296 & 50,50 \\
Sí & 290 & 49,50 \\
Total & 587 & 100 \\
\hline
\end{tabular}

Tabla 234: Realización de bioquímica sanguínea

\begin{tabular}{|ccc|}
\hline Bioquímica & Frecuencia & Porcentaje \\
\hline No & 295 & 50,30 \\
Sí & 292 & 49,70 \\
Total & 587 & 100 \\
\hline
\end{tabular}


Tabla 235: Realización de hemostasia sanguínea

\begin{tabular}{|ccc|}
\hline Bioquímica & Frecuencia & Porcentaje \\
\hline No & 551 & 94,20 \\
Sí & 34 & 5,80 \\
Total & 587 & 100 \\
\hline
\end{tabular}

Tabla 236: Realización de gasometría venosa

\begin{tabular}{|ccc|}
\hline Gasometría venosa & Frecuencia & Porcentaje \\
\hline No & 523 & 89,60 \\
Sí & 61 & 10,40 \\
Total & 586 & 100 \\
\hline
\end{tabular}

Tabla 237: Realización de gasometría arterial

\begin{tabular}{|ccc|}
\hline Gasometría arterial & Frecuencia & Porcentaje \\
\hline No & 555 & 95,00 \\
Sí & 29 & 5,00 \\
Total & 587 & 100 \\
\hline
\end{tabular}

Figura 126: Realización de diferentes tipos de analíticas en el servicio de urgencias hospitalario

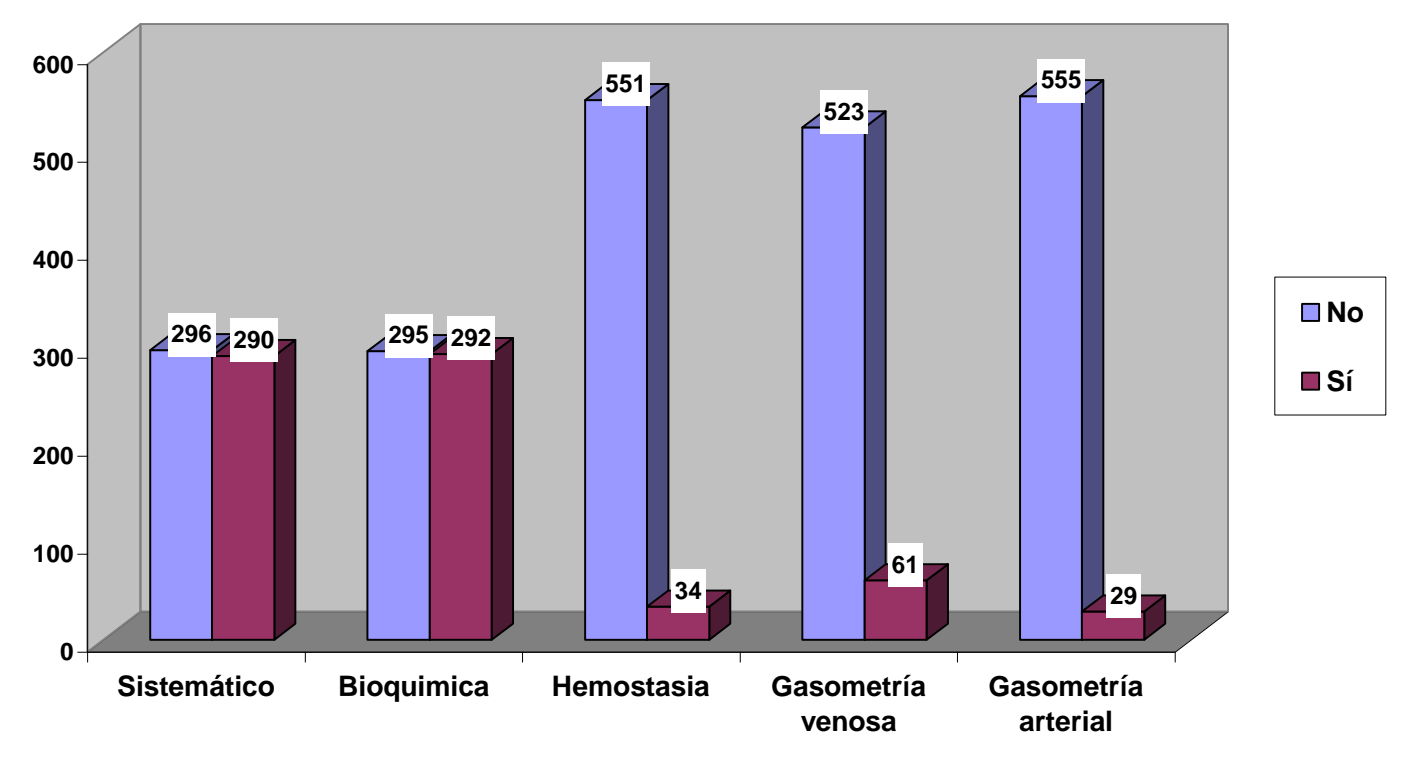


Tabla 238: Tóxicos en orina

\begin{tabular}{|ccc|}
\hline Tóxicos en orina & Frecuencia & Porcentaje \\
\hline No & 377 & 64,60 \\
Sí & 207 & 35,40 \\
Total & 584 & 100
\end{tabular}

Figura 127: Realización de prueba de tóxicos en orina en el servicio de urgencias hospitalario

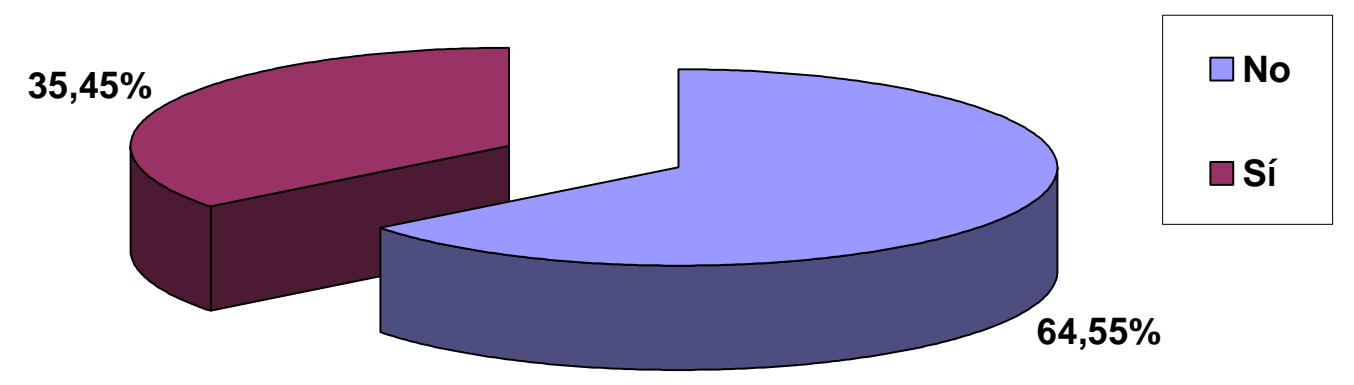

\subsection{Realización de electrocardiografía.}

Se registran aquellos pacientes sometidos a la realización de electrocardiografía durante su estancia en el servicio de urgencias hospitalario. Tabla 239, figura 128.

Tabla 239: Realización de electrocardiografía

\begin{tabular}{|ccc|}
\hline Electrocardiografía & Frecuencia & Porcentaje \\
\hline No & 442 & 75,70 \\
Sí & 142 & 24,30 \\
Total & 584 & 100
\end{tabular}


Figura 128: Realización electrocardiograma en el servicio de urgencias hospitalario

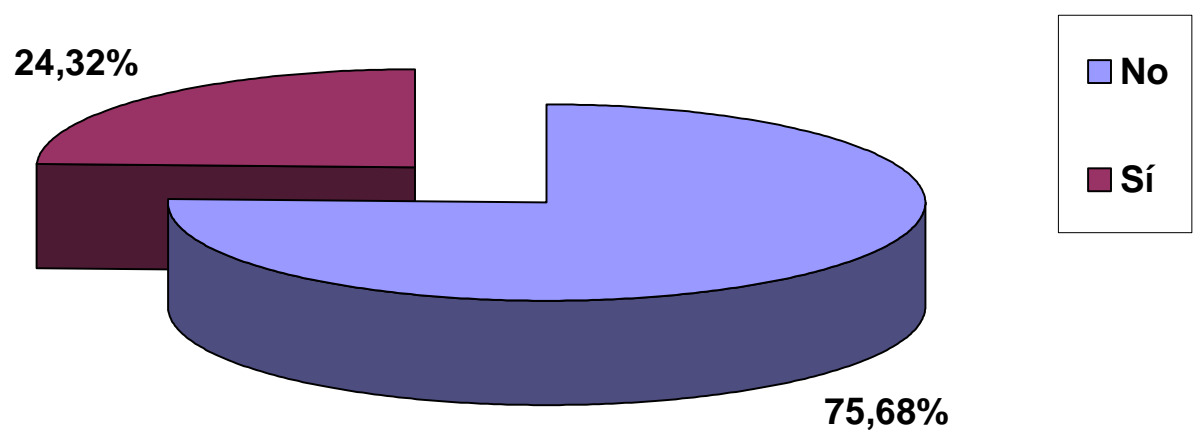

\subsection{Realización de pruebas de imagen.}

Se recoge la realización o no de pruebas de imagen en la estancia en el servicio de urgencias hospitalario de los pacientes intoxicados. Tabla 240, figura 129.

A su vez, dentro de aquellos que han sido sometidos a estas pruebas, se especifica el tipo necesario, estableciéndose diferentes categorías.

- Radiografía torácica

- Radiografía ósea

- Radiografía abdominal

- Ecografía

- Tomografía axial computarizada (TAC) 
Tabla 240: Realización de pruebas de imagen

\begin{tabular}{|ccc|}
\hline Prueba de imagen & Frecuencia & Porcentaje \\
\hline No & 551 & 94,30 \\
Radiografía tórax & 22 & 3,80 \\
Radiografía ósea & 3 & 0,50 \\
Radiografía abominal & 0 & 0 \\
Ecografía & 0 & 0 \\
TAC & 8 & 1,40 \\
Total & 584 & 100 \\
\hline
\end{tabular}

Figura 129: Realización de pruebas de imagen

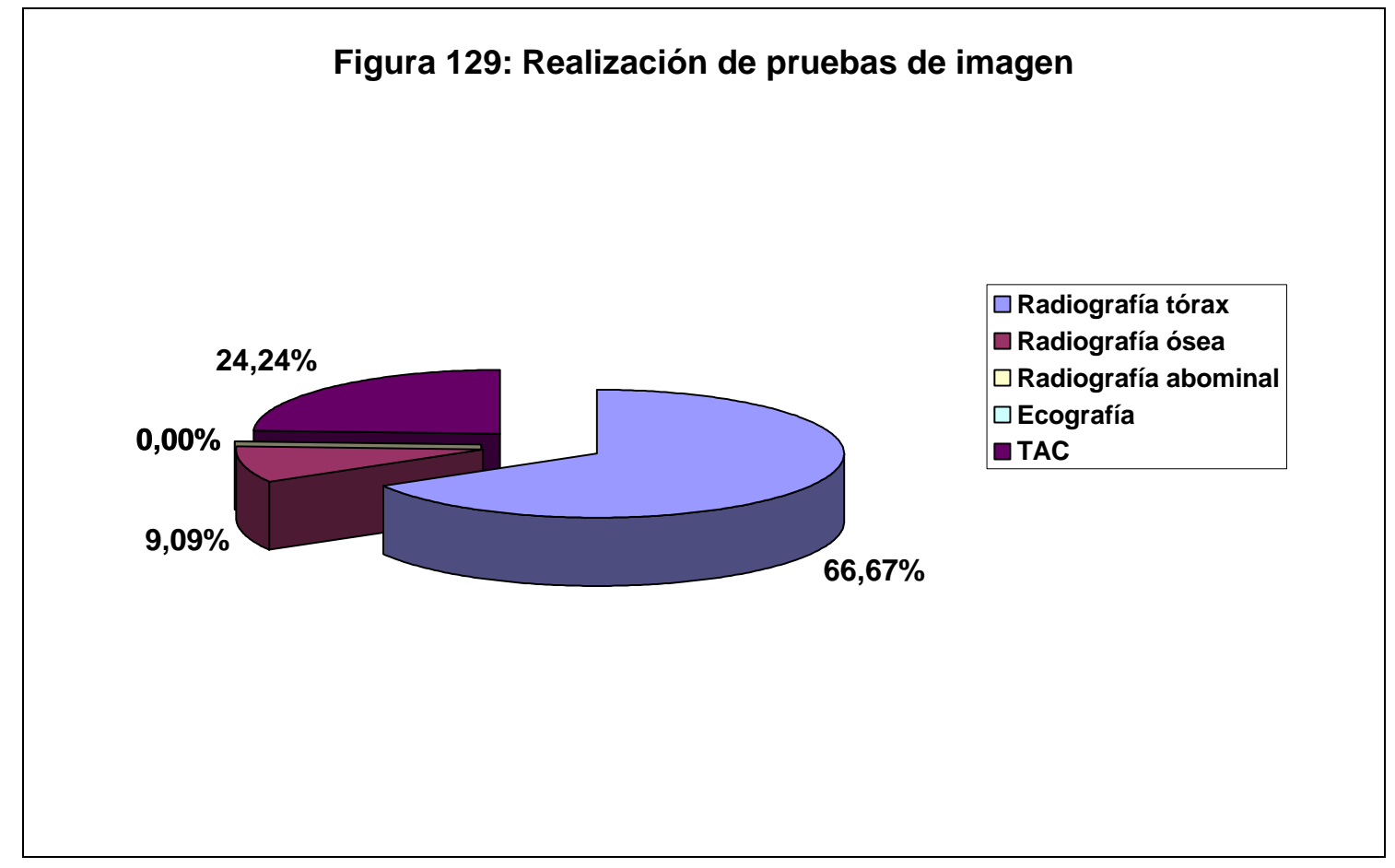

\section{Tratamiento durante la estancia en el servicio de urgencias} hospitalario.

13.1. Administración de oxigenoterapia durante la estancia en el servicio de Urgencias hospitalario.

Se especifica si durante la estancia en el Servicio de Urgencias Hospitalario el paciente intoxicado precisa la a 
dministración de oxigenoterapia por su patología. Tabla 241, figura 130.

Tabla 241: Administración de oxigenoterapia

\begin{tabular}{|ccc|}
\hline Oxigenoterapia & Frecuencia & Porcentaje \\
\hline No & 541 & 92,6 \\
Sí & 43 & 7,4 \\
Total & 584 & 100 \\
\hline
\end{tabular}

Figura 130: Administración de oxigenoterapia en el servicio de urgencias hospitalario

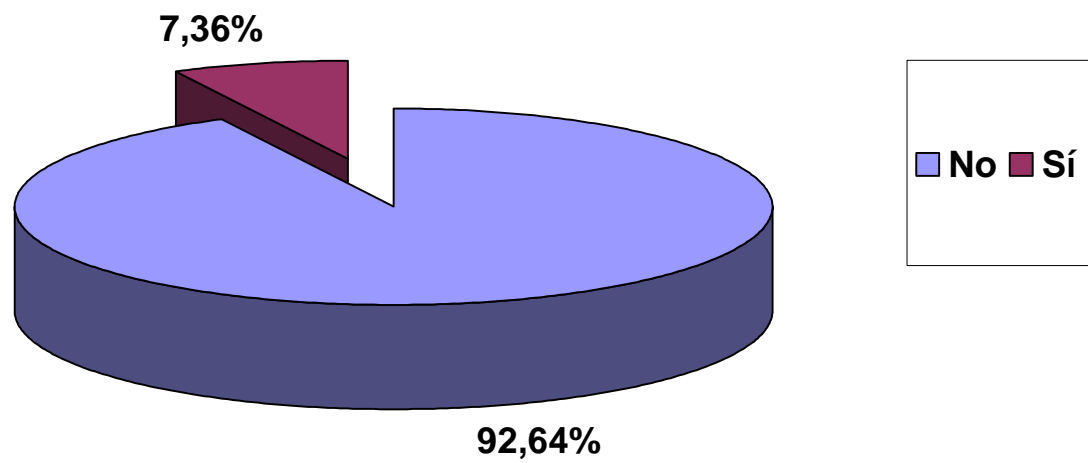

\subsection{Lavado gástrico.}

Dentro de los tratamientos realizados en urgencias podemos destacar la realización o no del lavado gástrico tabla 242, figura 131.

Tabla 242: Realización lavado gástrico en intoxicados

\begin{tabular}{|ccc|}
\hline Lavado gástrico & Frecuencia & Porcentaje \\
\hline No & 533 & 91,40 \\
Sí & 50 & 8,60 \\
Total & 583 & 100 \\
\hline
\end{tabular}


Figura 131: Lavado gástrico en el servicio de urgencias hospitalario

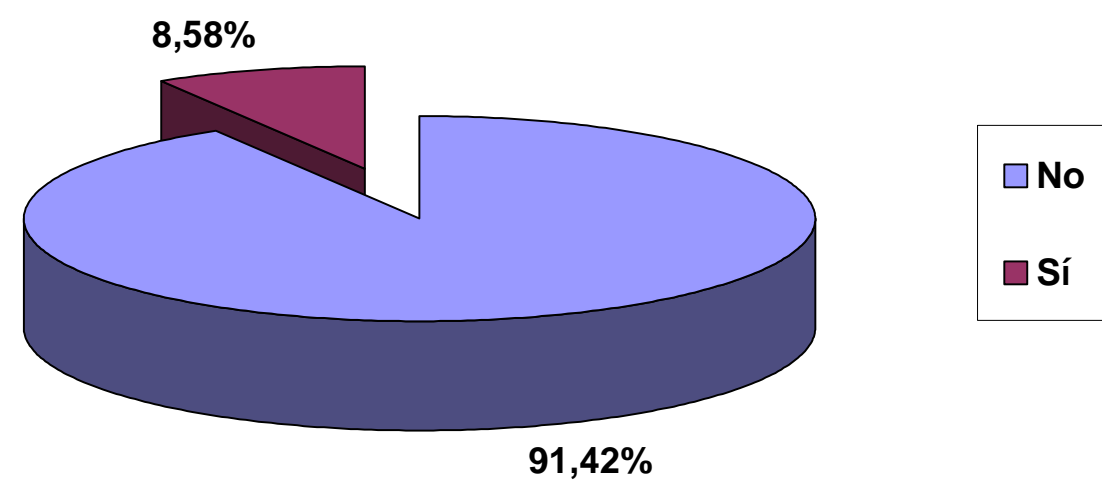

\subsection{Carbón activado.}

En la tabla 243 y figura 132 se muestra la administración o no de carbón activado en el servicio de urgencias hospitalario.

Tabla 243: Administración de carbón activado

\begin{tabular}{|ccc|}
\hline Carbón activado & Frecuencia & Porcentaje \\
\hline No & 525 & 90,20 \\
Sí & 57 & 9,80 \\
Total & 582 & 100 \\
\hline
\end{tabular}


Figura 132: Administración de carbón activado

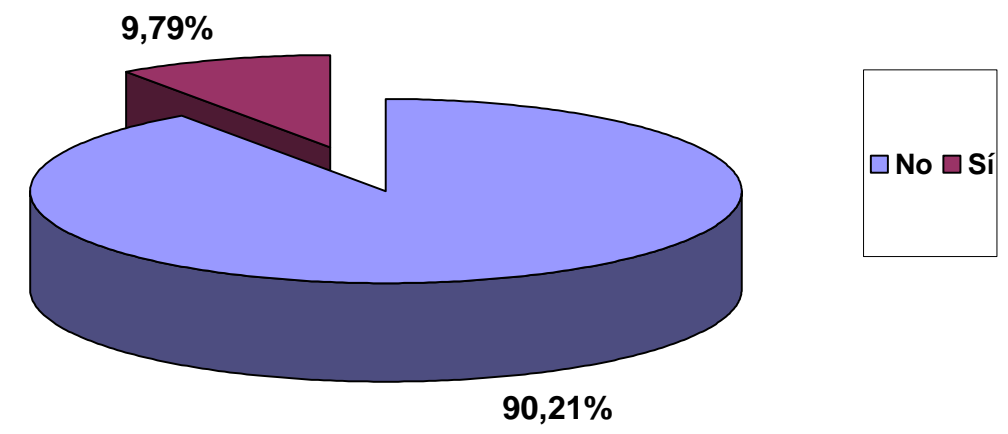

13.4. Administración de antídoto específico en el servicio de urgencias hospitalario.

Se recoge por otro lado, la administración de un antídoto específico que contrarreste los efectos del tóxico que ha sido recibido. Tabla 244.

Tabla 244: Administración de antídoto específico

\begin{tabular}{|ccc|}
\hline Antídoto & Frecuencia & Porcentaje \\
\hline No & 575 & 98,80 \\
Sí & 7 & 1,20 \\
Total & 582 & 100 \\
\hline
\end{tabular}

13.5. Administración de sueroterapia en el servicio de urgencias hospitalario.

Se registra la utilización de sueroterapia en su estancia en urgencias. Tabla 245, figura 133. 
Dentro de la misma se establece una diferencia entre el suero fisiológico y el suero glucosado.

Tabla 245: Administración de sueroterapia

\begin{tabular}{|ccc|}
\hline Sueroterapia & No & Sí \\
\hline Suero fisiológico & 406 & 176 \\
Suero glucosado & 541 & 41 \\
\hline
\end{tabular}

En este caso, hasta en un $30,2 \%$ de los pacientes que acuden a urgencias por intoxicación, se les pauta sueroterapia con suero fisiológico y un $7 \%$ con suero glucosado.

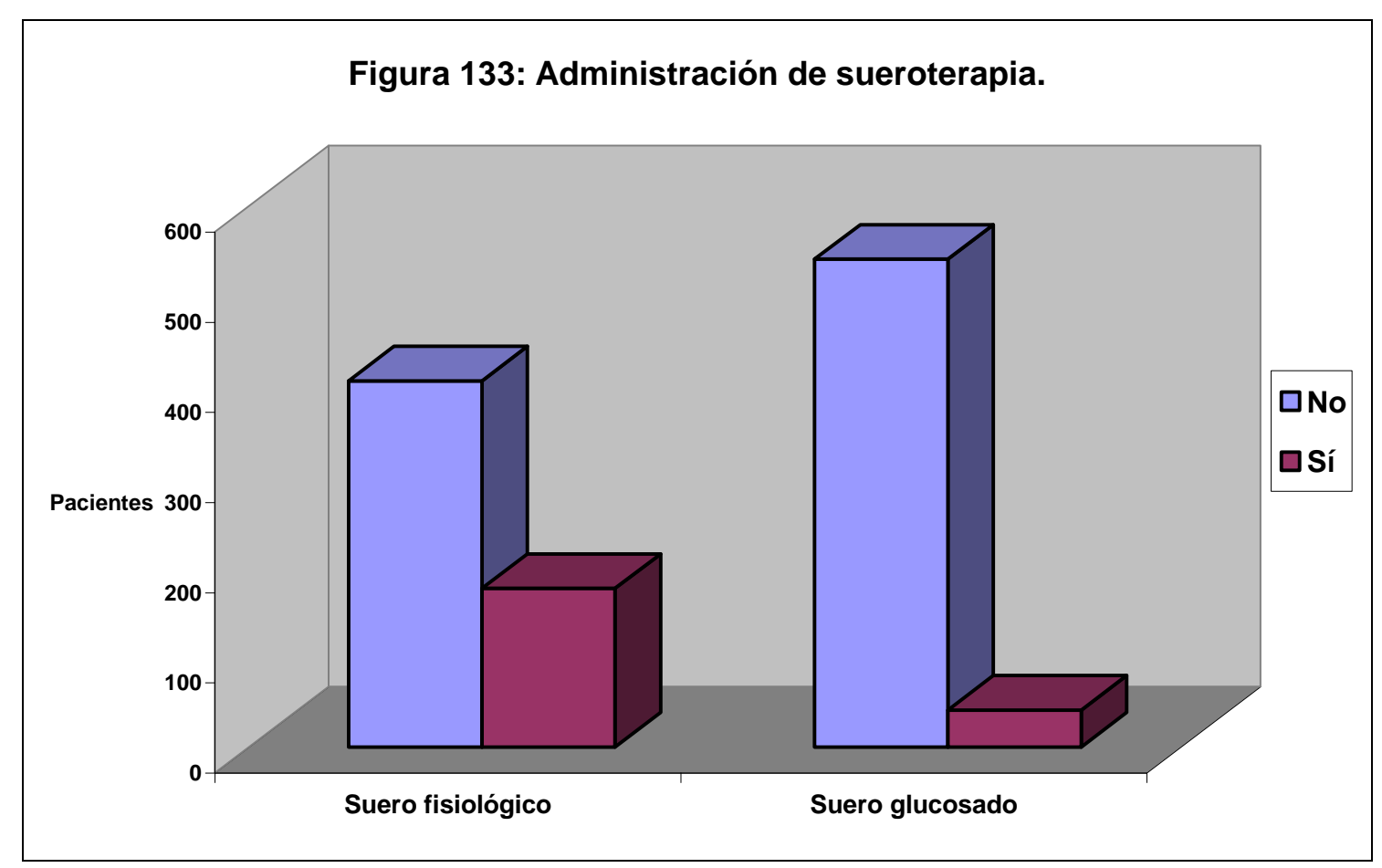

13.6. Administración de antieméticos.

La necesidad de tratamiento con antieméticos en urgencias es de hasta un $18,1 \%$ de los casos. Tabla 246, figura 134. 
Tabla 246: Administración de antiemético

\begin{tabular}{|ccc|}
\hline Antiemético & Frecuencia & Porcentaje \\
\hline No & 476 & 81,90 \\
SI & 105 & 18,10 \\
\hline Total & 582 & 100 \\
\hline
\end{tabular}

Figura 134: Administración de antieméticos

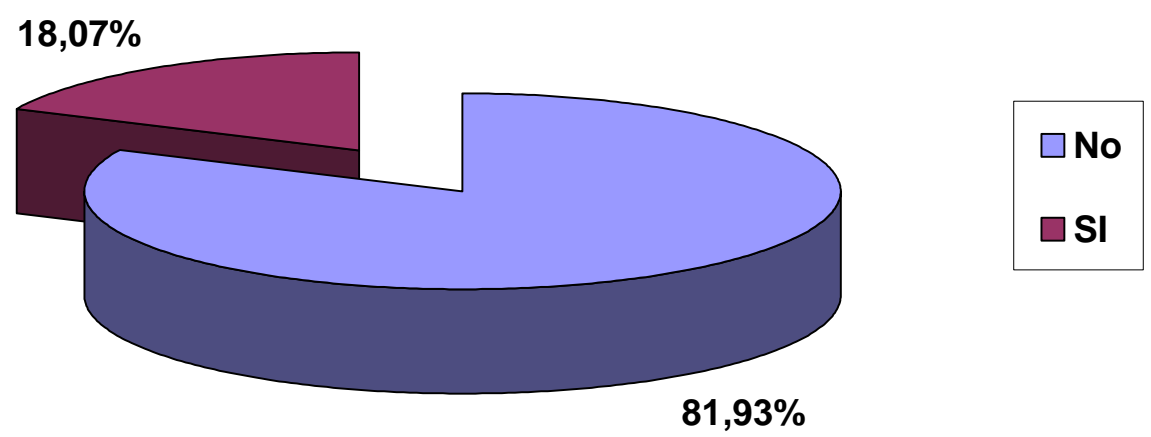

13.7. Administración de analgesia.

El empleo de analgesia en el servicio de urgencias hospitalario se muestra en la tabla 247 . En este caso, es necesaria en un $2,7 \%$ de los casos.

Tabla 247: Administración de analgesia

\begin{tabular}{|ccc|}
\hline Analgesia & Frecuencia & Porcentaje \\
\hline No & 566 & 97,30 \\
SI & 16 & 2,70 \\
\hline Total & 582 & 100 \\
\hline
\end{tabular}




\subsection{Administración de protectores gástricos}

En el caso de los protectores gástricos, el tanto por ciento de utilización en el servicio de urgencias hospitalario es un poco mayor, registrándose que se pauta en un 9,6\% de los pacientes intoxicados. Tabla 248, figura 135.

Tabla 248: Administración de protector gástrico

\begin{tabular}{|ccc|}
\hline Protector gástrico & Frecuencia & Porcentaje \\
\hline No & 525 & 90,40 \\
SI & 56 & 9,60 \\
\hline Total & 581 & 100 \\
\hline
\end{tabular}

Figura 135: Administración de protectores gástricos

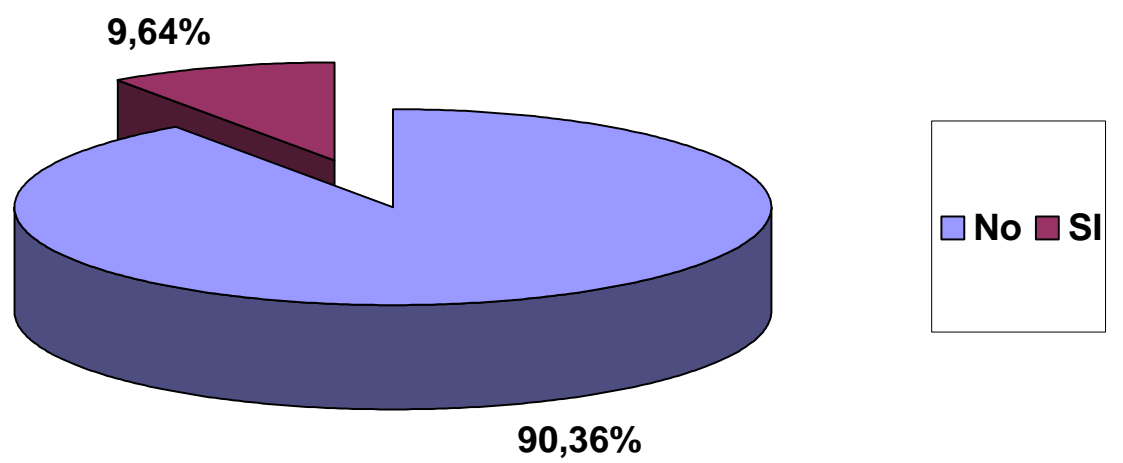

\subsection{Administración de tiamina.}

Se establece una valoración del empleo de Tiamina $300 \mathrm{mg}$ en el servicio de urgencias hospitalario. Tabla 249.

Tabla 249: Administración de tiamina

\begin{tabular}{|ccc|}
\hline Tiamina & Frecuencia & Porcentaje \\
\hline No & 574 & 98,60 \\
SI & 8 & 1,40 \\
\hline Total & 582 & 100 \\
\hline
\end{tabular}




\subsection{Administración de otro tipo de tratamiento.}

Además de los anteriores tratamientos, el paciente intoxicado puede haber recibido otro tipo de fármaco que por representar un tanto por ciento muy pequeño, no se especifica. Tabla 250.

Tabla 250: Administración de otros tratamientos no especificados

\begin{tabular}{|ccc|}
\hline Otro tratamiento & Frecuencia & Porcentaje \\
\hline No & 541 & 92,60 \\
SI & 43 & 7,40 \\
\hline Total & 584 & 100 \\
\hline
\end{tabular}

\section{Realización de interconsulta con el Servicio de Psiquiatría.}

Se establece la necesidad de consultar con el psiquiatra de guardia la valoración del paciente en un $8,9 \%$ de los casos de los intoxicados. Tabla 251 , figura 136.

Tabla 251: Interconsulta con Servicio de Psiquiatría

\begin{tabular}{|ccc|}
\hline Interconsulta con Psiquiatría & Frecuencia & Porcentaje \\
\hline No & 377 & 91,10 \\
SI & 37 & 8,90 \\
\hline Total & 414 & 100 \\
\hline
\end{tabular}




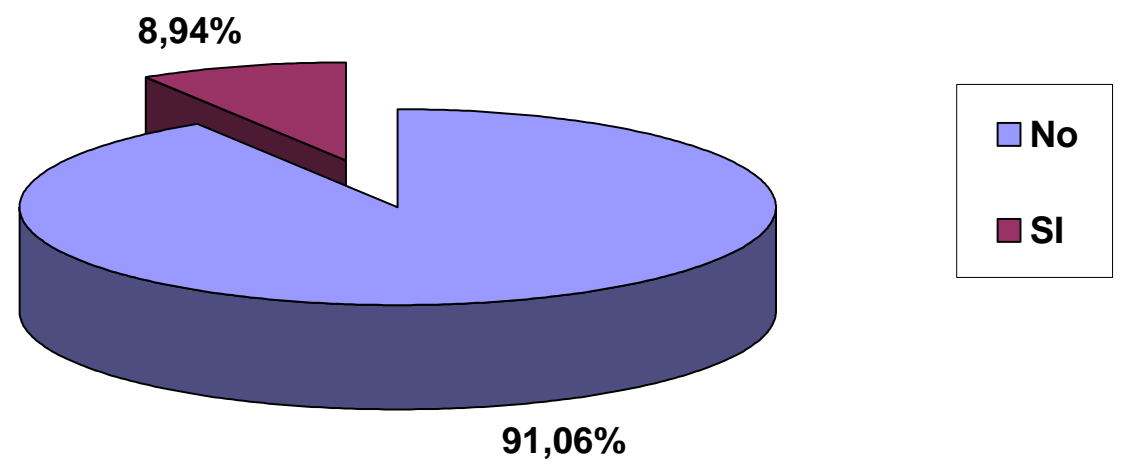

\section{Realización de interconsulta con Toxicología.}

Se registra la necesidad de consultar sobre la intoxicación recibida en el servicio de urgencias hospitalario, con el servicio de Toxicología, ya sea el hospitalario, o telefónicamente con el Instituto Nacional de Toxicología (en el número de teléfono disponible las 24 horas del día 914112676). Tabla 252, figura 137.

Tabla 252: Interconsulta con Toxicología

\begin{tabular}{|ccc|}
\hline Interconsulta con Toxicología & Frecuencia & Porcentaje \\
\hline No & 338 & 79,70 \\
SI & 86 & 20,30 \\
\hline Total & 424 & 100 \\
\hline
\end{tabular}




\section{Figura 137: Interconsulta con Toxicología}

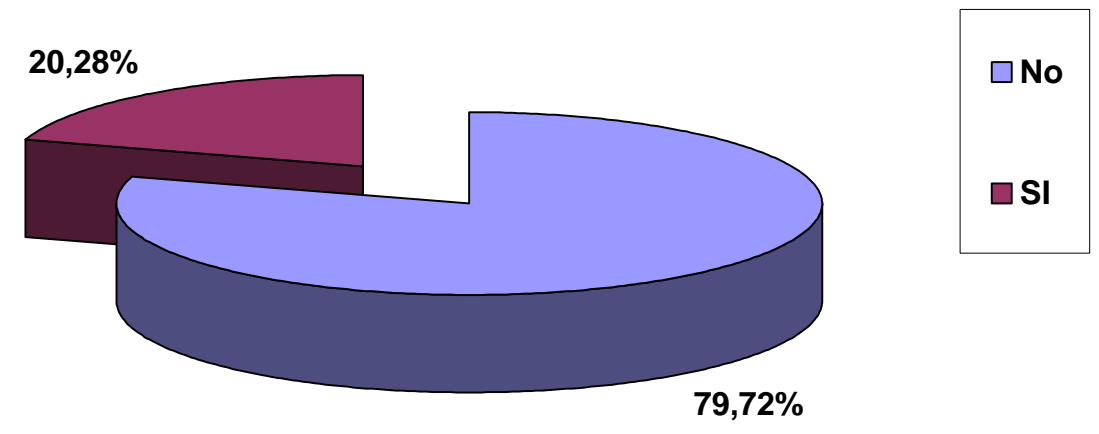

\section{Tiempo de permanencia en el servicio de urgencias hospitalario.}

La media en minutos de estancia en urgencias corresponde a 215,62 +/- 196, 23 minutos.

La mediana en minutos de permanencia es de 153.

Para un manejo más sencillo de los datos obtenidos, podemos distribuir la población en dos grupos diferenciados: aquellos que permanecen más de 2 horas en el servicio de urgencias hospitalario o los que están menos de 120 minutos. Se muestra en la tabla 253, figura 138.

Tabla 253: Permanencia en el servicio de urgencias hospitalario de los pacientes

\begin{tabular}{|ccc|}
\hline & Frecuencia & Porcentaje \\
\hline Menos de 2 horas & 412 & 41,44 \\
Mas de 2 horas & 582 & 58,55 \\
Total & 994 & 100 \\
\hline
\end{tabular}


Figura 138: Estancia en el servicio de urgencias hospitalario de los pacientes intoxicados

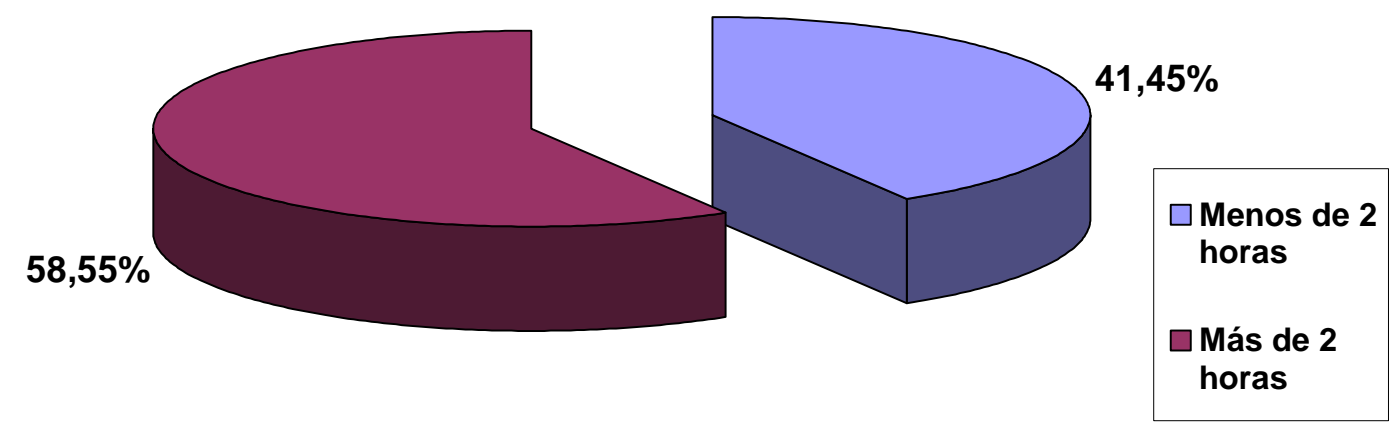

\section{Destino tras la estancia en el servicio de urgencias hospitalario.}

En el $84,8 \%$ de las ocasiones, los pacientes atendidos en el servicio de urgencias hospitalario con el diagnóstico de intoxicación, son dados de alta sin necesidad de ingreso hospitalario. El 15,2\% restante, precisa de ingreso hospitalario. Tabla 254, figura 139.

Tabla 254: Destino tras la estancia en el servicio de urgencias hospitalario

\begin{tabular}{|ccc|}
\hline Destino & Frecuencia & Porcentaje \\
\hline Alta & 839 & 84,40 \\
Ingreso en Pediatría & 99 & 10,00 \\
Ingreso en Medicina Interna & 9 & 0,90 \\
Ingreso en Unidad Cuidados Intensivos & 19 & 1,90 \\
Ingreso en otros servicios & 28 & 2,80 \\
\hline
\end{tabular}


Figura 139: Destino de los pacientes intoxicados tras su estancia en el servicio de urgencias hospitalario

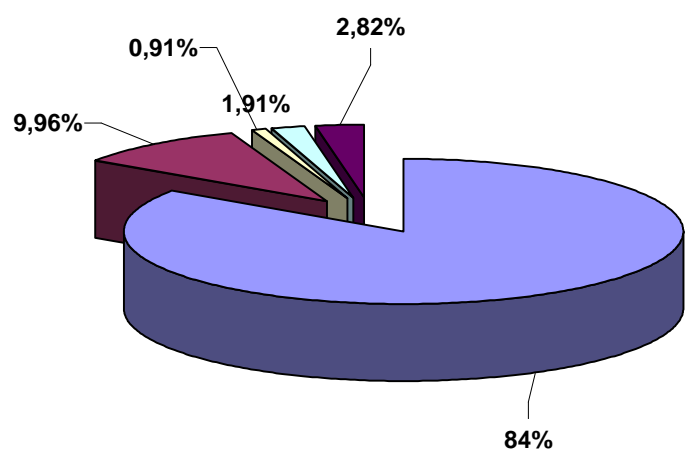

$\square$ Alta

u Ingreso en Pediatría

$\square$ Ingreso en Medicina Interna

$\square$ Ingreso en Unidad Cuidados Intensivos

$\square$ Ingreso en otros servicios

\section{Tiempo de ingreso hospitalario tras su estancia en el servicio de}

\section{urgencias hospitalario.}

En aquellos pacientes cuyo destino tras la estancia en el servicio de urgencias hospitalario es el ingreso hospitalario, se recoge la estancia en horas que permanecen ingresados. Tabla 255, figura 140.

La media del ingreso es de 27,32 +/- 24,71 horas.

El paciente que más tiempo permanece ingresado es de 168 horas, el equivalente a 7 días. 
Tabla 255: Horas de ingreso hospitalario tras el alta de urgencias

\begin{tabular}{|cc|}
\hline Tiempo ingresados en horas & Frecuencia \\
\hline 1 & 3 \\
2 & 1 \\
3 & 1 \\
4 & 1 \\
5 & 7 \\
8 & 11 \\
10 & 1 \\
11 & 1 \\
12 & 6 \\
14 & 1 \\
15 & 2 \\
16 & 1 \\
17 & 1 \\
\hline 24 & 69 \\
48 & 13 \\
72 & 3 \\
96 & 3 \\
120 & 2 \\
168 & 1 \\
\hline
\end{tabular}


Figura 140: Horas de ingreso hospitalario de los intoxicados.

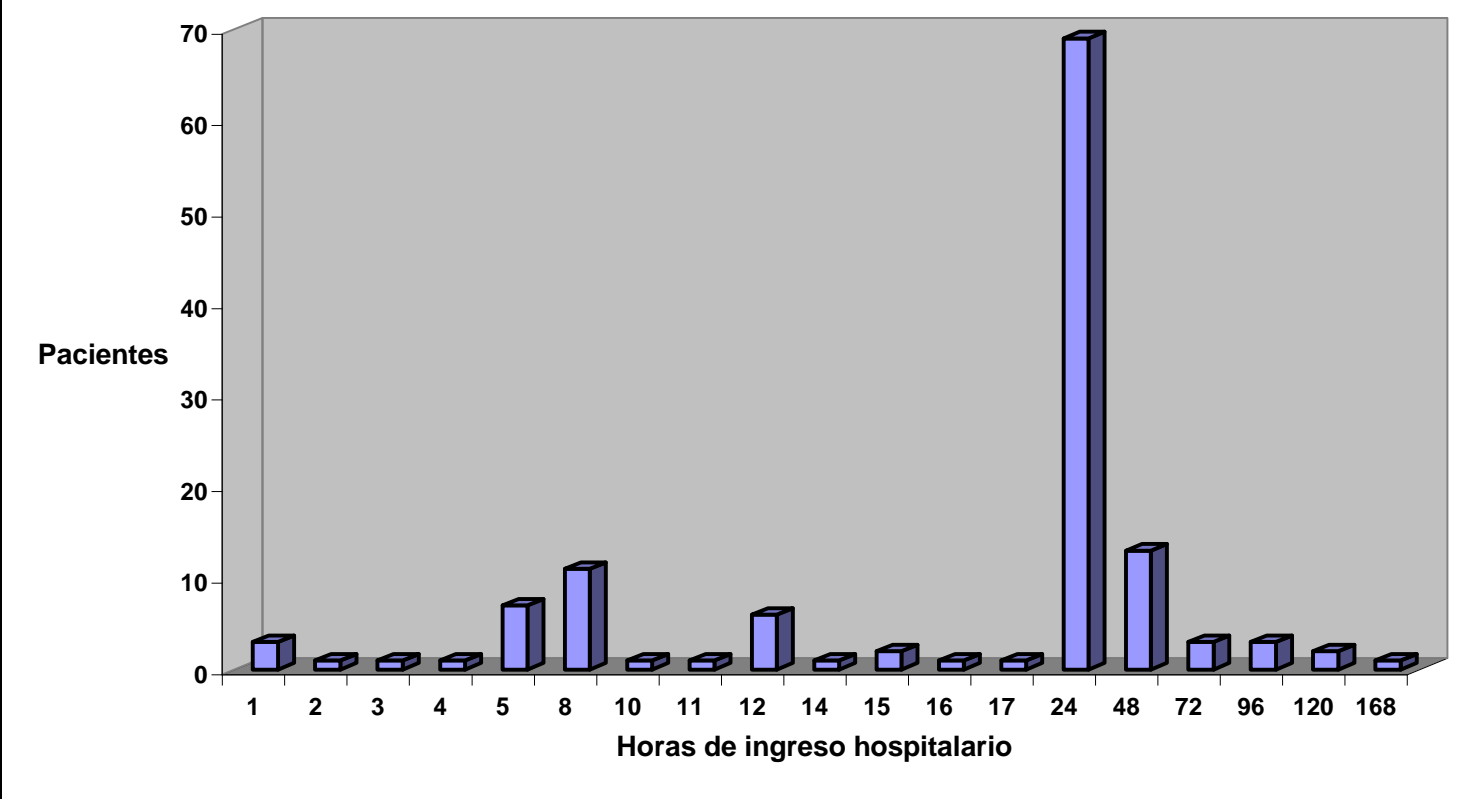

19. Número de veces que acuden estos pacientes al servicio de urgencias hospitalario en el mismo año en el que se produce la asistencia por intoxicación.

Se recogen el número de veces que estos pacientes intoxicados acuden al servicio de urgencias hospitalario por el mismo o diferente motivo, a lo largo del año en el que se produce esta asistencia por intoxicación. Tabla 256, figura 141.

Tabla 256: Número de veces que acuden al servicio de urgencias hospitalario el mismo año de la intoxicación

\begin{tabular}{|ccc|}
\hline Consultas mismo año & Frecuencia & Porcentaje \\
\hline 0 & 744 & 74,80 \\
1 & 151 & 15,20 \\
2 & 50 & 5,00 \\
3 & 20 & 2,00 \\
4 & 12 & 1,20 \\
5 & 8 & 0,80 \\
6 & 7 & 0,70 \\
7 & 2 & 0,20 \\
Total & 994 & 100 \\
\hline
\end{tabular}


Figura 141: Consultas al servicio de urgencias hospitalario el mismo año en el que lo hace por la intoxicación
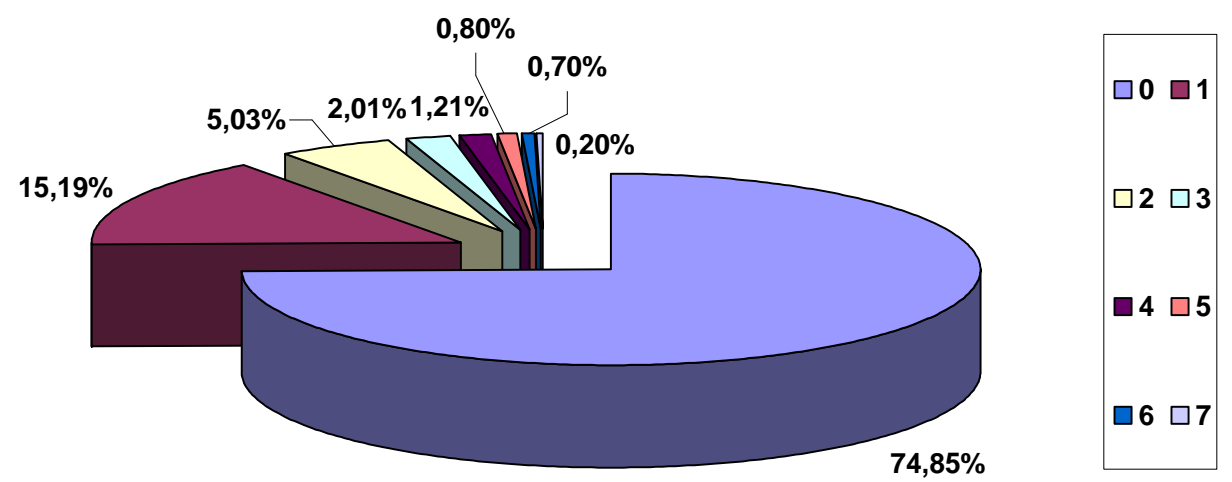

Si se establece el motivo por el que realizan las siguientes visitas al servicio de urgencias hospitalario en el mismo año en el que lo hacen por la intoxicación. (Tabla 257, figura 142):

Tabla 257:Tipo de urgencias por las que acuden al servicio de urgencias hospitalario el mismo año de la intoxicación

\begin{tabular}{|ccc|}
\hline Tipo de urgencia & Frecuencia & Porcentaje \\
\hline General & 152 & 60,32 \\
\hline Traumatológicas & 51 & 20,24 \\
\hline Intoxicación & 28 & 11,11 \\
\hline Otorrinolaringológicas & 7 & 2,78 \\
Oftalmológicas & 4 & 1,59 \\
Psiquiátricas & 3 & 1,19 \\
Otras & 3 & 1,19 \\
Ginecológicas & 2 & 0,79 \\
Precisan ingreso & 2 & 0,79 \\
\hline Total & 252 & 100 \\
\hline
\end{tabular}


Figura 142: Tipo de urgencias por las que acuden al servicio de urgencias hospitalario el mismo año en el que acuden por intoxicación

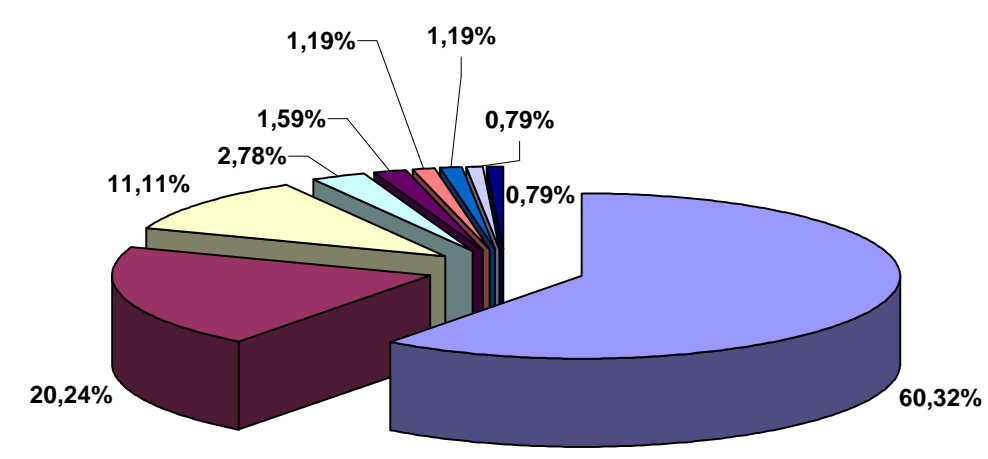

$\square$ General
$\square$ Traumatológicas
$\square$ Intoxicación
$\square$ Otorrinolaringológicas
$\square$ Oftalmológicas
$\square$ Psiquiátricas
$\square$ Otras
$\square$ Ginecológicas
$\square$ Precisan ingreso

\section{Visitas al servicio de urgencias hospitalario en los años anteriores al}

\section{registrado.}

Se recogen el número de veces que estos pacientes han necesitado asistencia sanitaria por parte del servicio de Urgencias Hospitalario Río Hortega. Tabla 258, figura 143.

La media de consultas es de 3,58 +/- 5,23 visitas a urgencias y la mediana 2.

Tabla 258: Número de consultas en el servicio de urgencias hospitalario en los años previos a la intoxicación

\begin{tabular}{|ccc|}
\hline Consultas & Frecuencia & Porcentaje \\
0 & 333 & 33,50 \\
1 & 143 & 14,38 \\
2 & 111 & 11,16 \\
3 & 80 & 8,04 \\
4 & 61 & 6,13 \\
5 & 57 & 5,73 \\
6 & 37 & 3,72 \\
7 & 25 & 2,51 \\
8 & 16 & 1,60
\end{tabular}




\begin{tabular}{|lcc|}
\hline 9 & 17 & 1,71 \\
\hline 10 & 20 & 2,01 \\
\hline 11 & 11 & 1,10 \\
\hline 12 & 24 & 2,41 \\
\hline 13 & 5 & 0,50 \\
\hline 14 & 5 & 0,50 \\
\hline 15 & 10 & 1,00 \\
\hline 16 & 8 & 0,80 \\
\hline 17 & 5 & 0,50 \\
18 & 2 & 0,20 \\
\hline 19 & 3 & 0,30 \\
\hline 21 & 5 & 0,50 \\
\hline 22 & 4 & 0,40 \\
\hline 23 & 1 & 0,10 \\
\hline 25 & 2 & 0,20 \\
\hline 26 & 2 & 0,20 \\
\hline 35 & 1 & 0,10 \\
\hline 36 & 1 & 0,10 \\
\hline 40 & 1 & 0,10 \\
\hline 42 & 2 & 0,20 \\
\hline Total & 1 & 0,10 \\
\hline & 1 & 0,10 \\
\hline & 994 & 100 \\
\hline
\end{tabular}

Figura 143: Consultas al servicio de urgencias hospitalario en los años previos a la intoxicación

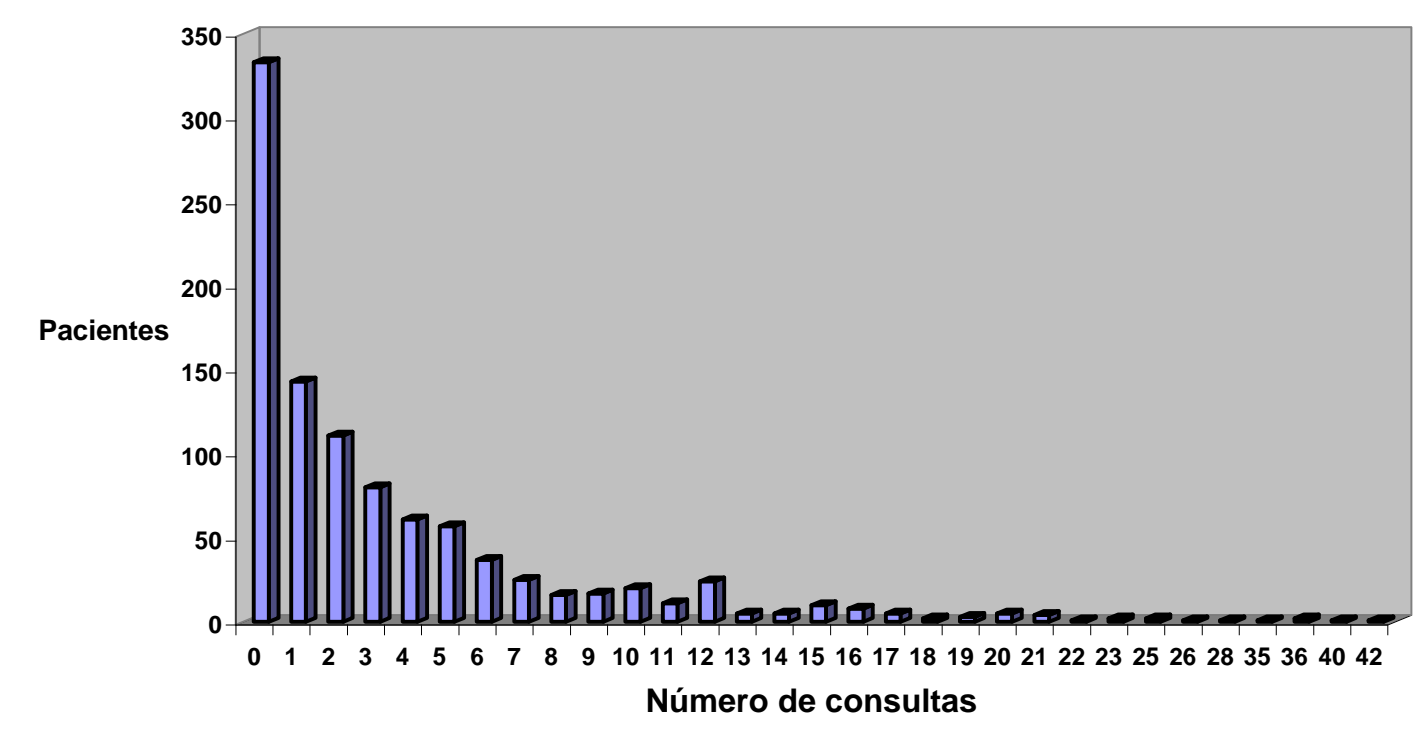




\section{Consultas en el servicio de urgencias hospitalario en los años}

\section{posteriores a la intoxicación.}

Se registra el número de veces que estos pacientes intoxicados acuden al servicio de urgencias hospitalario en los años posteriores a su atención por la intoxicación. Tabla 259, figura 144.

La media de consultas de los pacientes es de 2,02 +/- 3,55 visitas y la mediana de 1.

Tabla 259: Número de consultas al servicio de urgencias hospitalario en los años posteriores a la intoxicación

\begin{tabular}{|c|c|c|}
\hline Consultas & Frecuencia & Porcentaje válido \\
\hline 0 & 478 & 49,27 \\
\hline 1 & 147 & 15,15 \\
\hline 2 & 91 & 9,38 \\
\hline 3 & 54 & 5,56 \\
\hline 4 & 57 & 5,87 \\
\hline 5 & 39 & 4,02 \\
\hline 6 & 22 & 2,26 \\
\hline 7 & 19 & 1,95 \\
\hline 8 & 11 & 1,13 \\
\hline 9 & 16 & 1,64 \\
\hline 10 & 9 & 0,92 \\
\hline 11 & 1 & 0,10 \\
\hline 12 & 4 & 0,41 \\
\hline 13 & 1 & 0,10 \\
\hline 14 & 3 & 0,30 \\
\hline 15 & 4 & 0,41 \\
\hline 16 & 4 & 0,41 \\
\hline 17 & 3 & 0,30 \\
\hline 19 & 1 & 0,10 \\
\hline 20 & 1 & 0,10 \\
\hline 22 & 1 & 0,10 \\
\hline 23 & 2 & 0,20 \\
\hline 30 & 1 & 0,10 \\
\hline 35 & 1 & 0,10 \\
\hline Total & 970 & 100 \\
\hline
\end{tabular}


Gráfico 144: Consultas en el servicio de urgencias hospitalario en los años posteriores a su visita por intoxicación

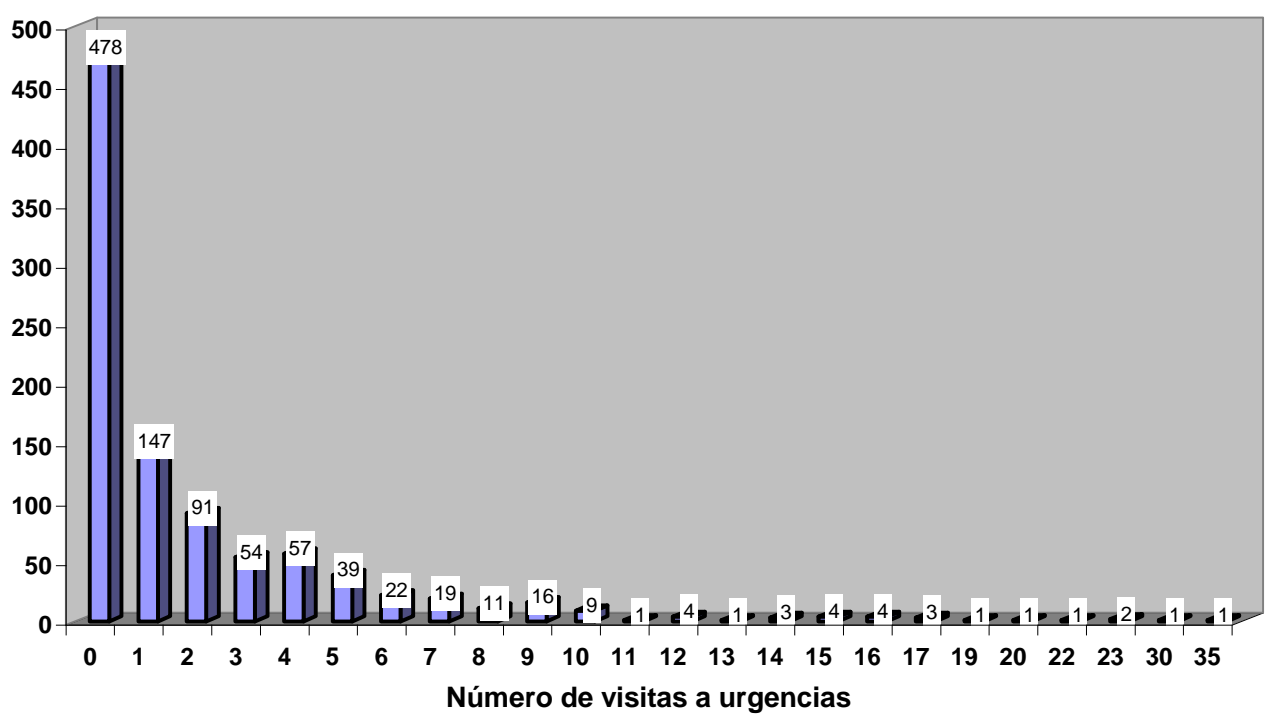

\section{Consultas externas hospitalarias de los pacientes intoxicados.}

Se recoge el número de veces que acuden a consultas externas los pacientes que han sido atendidos por intoxicaciones en el servicio de urgencias hospitalario. Tabla 260, figura 145.

Se recoge hasta un máximo de cuatro especialidades, siendo la media de 1,6 $+/-1,37$ consultas y la mediana de 1 consulta.

Tabla 260: Número de consultas externas hospitalarias en pacientes intoxicados

\begin{tabular}{|ccc|}
\hline Consultas & Frecuencia & Porcentaje válido \\
\hline 0 & 288 & 29,40 \\
1 & 212 & 21,61 \\
2 & 212 & 21,61 \\
3 & 140 & 14,27 \\
4 & 129 & 13,14 \\
Total & 981 & 100 \\
\hline
\end{tabular}




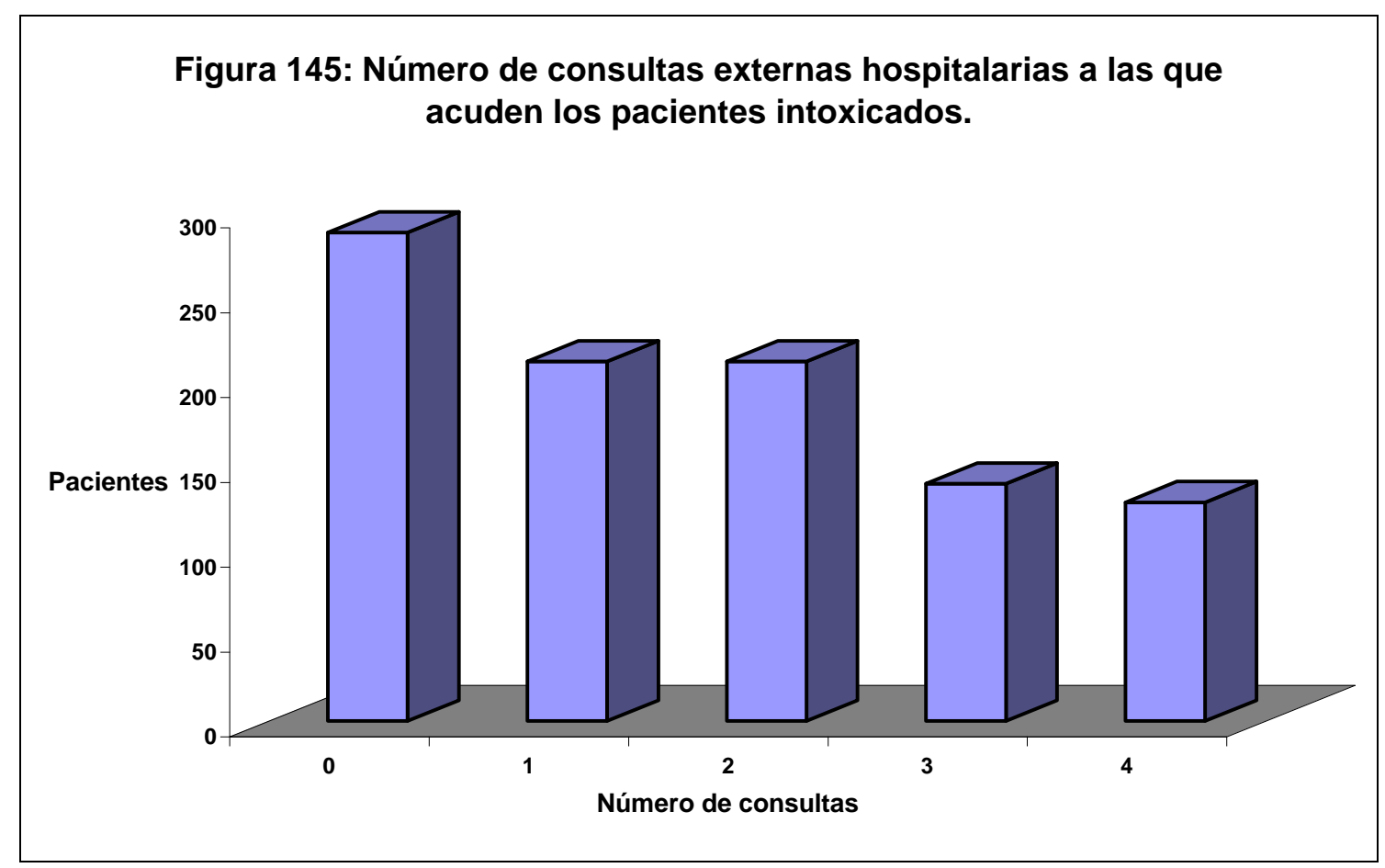

Dentro de las especialidades a las que acuden los pacientes que han sido atendidos por una intoxicación podemos valorar las más demandadas. Tabla 261, figura 146.

Tabla 261: Especialidades ambulatorias a las que acuden los pacientes intoxicados

\begin{tabular}{|ccc|}
\hline Visita & No & Si \\
\hline Dermatología & 771 & 210 \\
Oftalmología & 678 & 303 \\
ORL & 784 & 197 \\
Traumatología & 701 & 280 \\
Psiquiatría & 974 & 7 \\
Endocrinología & 923 & 58 \\
Alergología & 855 & 126 \\
Medicina Interna & 979 & 1 \\
Ginecología & 914 & 67 \\
Maxilofacial & 916 & 65 \\
\hline
\end{tabular}


Figura 146: Especialidades ambulatorias a las que acuden los pacientes intoxicados.

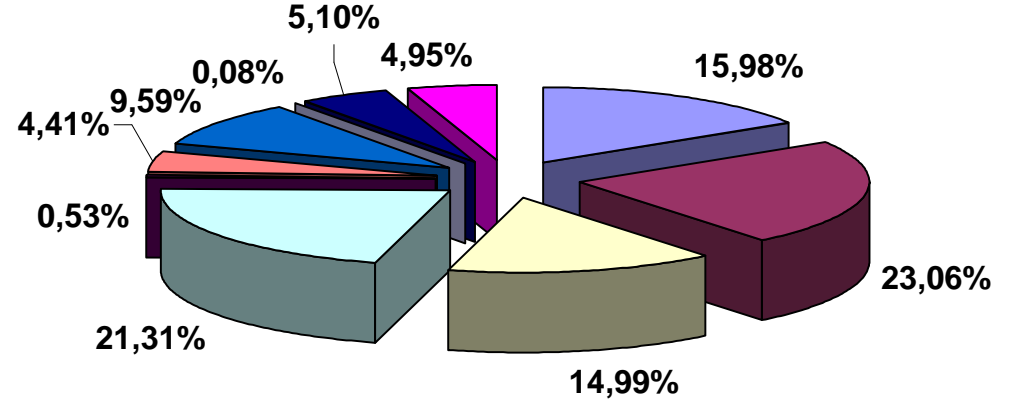

$\square$ Dermatología

口Oftalmología

$\square$ ORL

$\square$ Traumatología

- Psiquiatría

$\square$ Endocrinología

$\square$ Alergología

$\square$ Medicina Interna

- Ginecología

$\square$ Maxilofacial 


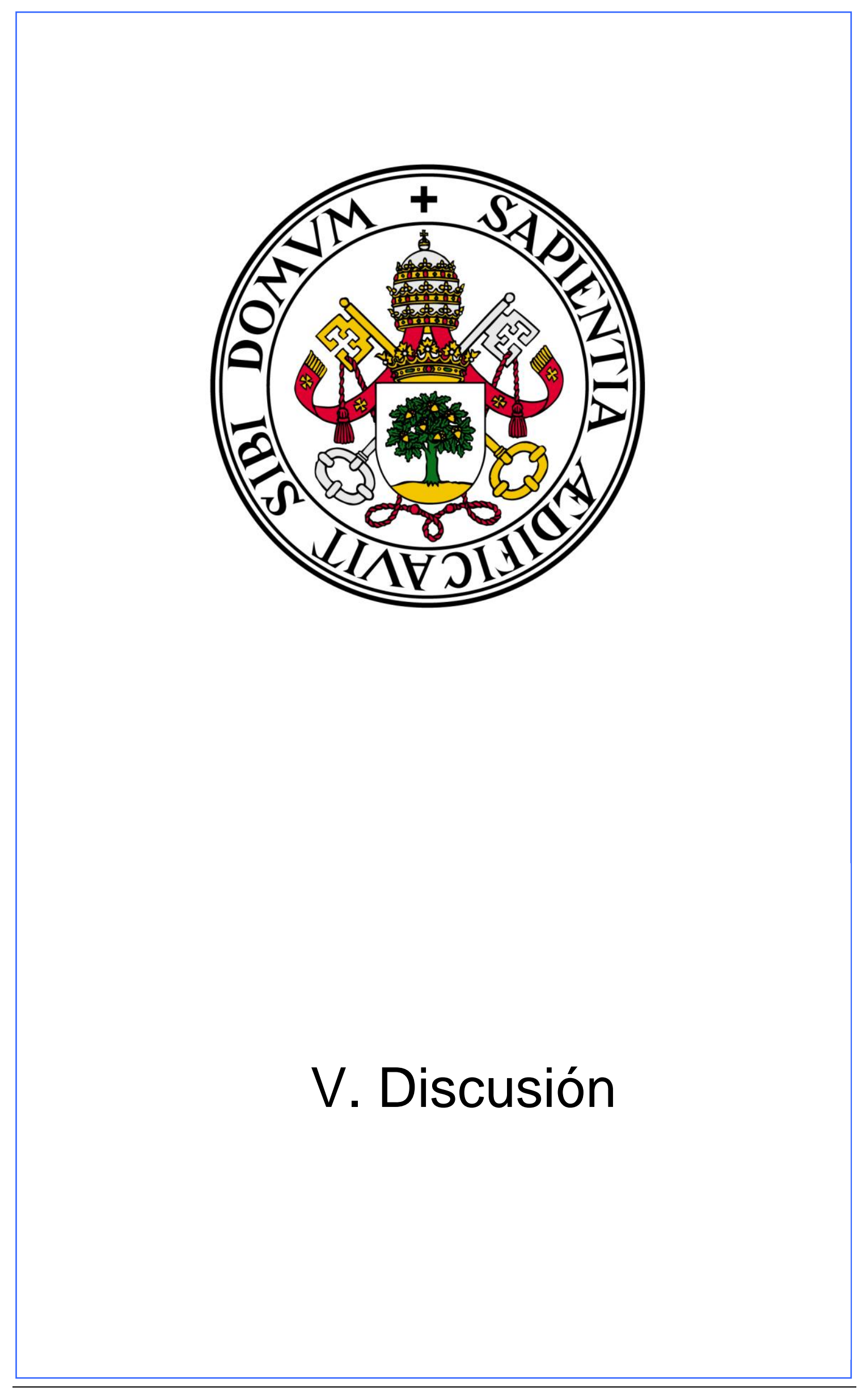





\section{Discusión}

Dadas las características del ámbito de estudio, un hospital de tercer nivel, que el estudio se realiza a lo largo de diez años completos (2001 a 2010) e incluye a pacientes desde recién nacidos hasta los 18 años, y todos los tipos de intoxicaciones, podemos inferir que los resultados obtenidos, nos acercan a la situación epidemiológica actual de las intoxicaciones agudas en menores de edad en nuestro entorno.

El Hospital Universitario Río Hortega de Valladolid es centro de referencia de consultas a la Unidad de Toxicología Clínica para la Comunidad Autónoma de Castilla y León, sin embargo estas no se reciben a través del Servicio de Urgencias, por lo que estas consultas no son recogidas en el estudio.

Dicho hospital se califica como "Hospitox", es decir, Hospital de tercer nivel que aglutina determinaciones analíticas que precisan técnicas complejas, que requieren personal especializado o que son de utilización muy esporádica, y que debería contemplarse al menos uno en cada Comunidad Autónoma

Las historias clínicas del servicio de urgencias del Hospital Universitario Río Hortega sólo se encuentran informatizadas completamente desde el año 2006, lo que ha supuesto un problema a la hora de acceso a la información de los parámetros estudiados. La obligada revisión manual de las historias clínicas, evidenció que muchos de los parámetros estudiados no se encontraban recogidos o resultaban ilegibles, por lo que no pudieron ser registrados, circunstancia que sucedió en las historias clínicas de los años 2001 a $2005 .{ }^{50}$

Por tanto, de las 994 historias clínicas de las que se pueden obtener datos, sólo en 584 se pueden recogen todos los parámetros estudiados.

Por otra parte, conviene reseñar que el servicio de urgencias del Hospital Universitario Río Hortega, los pediatras atienden en la zona de urgencias pediátricas a niños menores de 14 años, mientras que en la zona de urgencias generales se atiende a todas las personas con edad superior a 14 años; es 
decir, los niños de 14 a 18 años son atendidos en la zona de urgencias generales.

Dentro de los estudios realizados a nivel español respecto a las intoxicaciones en menores de edad, podemos considerar que nuestro trabajo es el primero que engloba a todos los jóvenes menores de 18 años durante un periodo de tiempo tan extenso como diez años.

Por otro lado, también es el primer trabajo que realiza una estimación de la incidencia de intoxicaciones en menores de edad, teniendo como referencia el número de pacientes menores de 18 años inscritos en la Seguridad Social por tarjeta sanitaria en dicho Área de Salud: 0,27\%.

Conviene recalcar que nuestra incidencia de intoxicaciones en menores de edad respecto a las urgencias atendidas en el Servicio de Urgencias Hospitalario Río Hortega en los menores de 18 años es de 0,41\%. Si bien este dato está dentro del rango objetivado en otros estudios, no es comparable, ya que la incidencia que calculan es respecto únicamente la atención en el Servicio de Urgencias Pediátricas y oscilan entre 0,76\% (Mintegui) ${ }^{42}, 0,30 \%$ (Azkunaga) ${ }^{37}$ y (Mintegui) ${ }^{45}$

Existen otros trabajos similares, pero que no alcanzan una muestra tan extensa, como son el de Rey ${ }^{51}$, que recoge los pacientes con intoxicaciones por drogas de abuso mayores de 14 años durante 10 años; o el de Palomar ${ }^{52}$ (intoxicaciones durante 9 años, pero también sólo mayores de 14 años); sólo de pacientes en edad pediátrica, no se encuentran muestras recogidas durante un periodo de tiempo tan extenso.

\section{Sexo.}

La distribución por sexos en el conjunto de las intoxicaciones recogidas, muestra una ligera tendencia al sexo masculino, 53,7\% frente a $46,3 \%$ de mujeres.

Este hecho se superpone en la mayoría de los trabajos elaborados en nuestro medio. Así el estudio de Mintegui de $1990{ }^{42}$ muestra una distribución similar $57,7 \%$ para los varones; en estudios con una menor muestra, como es el de 
Boscá $2003^{9}$, la distribución por sexos es casi del $50 \%$; en el estudio de Mintegi de $2006^{33}$ existe paridad en cuanto al sexo. En los más recientes como el de Azkunaga en menores de 7 años del $2013^{35}$ el porcentaje es de $53,7 \%$ en el caso de los hombres, y en los últimos datos del Boletín de Intoxicaciones de la Sociedad Española de Urgencias Pediátricas ${ }^{86}$ la división por géneros es de 328 varones frente a 314 mujeres.

La mayor proporción de varones, se relaciona con un mayor consumo de alcohol y drogas ilegales por parte de estos. (Tablas 14, 22, 23 y 25)

El hecho argumental de que el aumento de intoxicaciones en varones respecto a mujeres es debido al alcohol y las drogas ilegales, se expone también en otras dos tesis doctorales, Sola ${ }^{87}$ y Carmona ${ }^{88}$, aunque en ellas se estudia la población mayor de 14 años, por tanto no son del todo comparables sus datos con los recogidos en el presente estudio.

Esta distribución por sexos en el caso de las drogas de abuso, se reproduce en los diferentes estudios, como el caso del artículo publicado en Adicciones por De Miguel Bouza ${ }^{29}$ en el que se observa que un $76,7 \%$ de estas intoxicaciones están sufridas por varones.

En la encuesta ESTUDES ${ }^{49}$ del 2010, , se observa que los chicos consumen en mayor proporción que las chicas todas las drogas ilegales, mientras que con el tabaco y los tranquilizantes o hipnóticos sucede lo contrario. No obstante, la prevalencia de consumo intensivo de cualquier droga de comercio ilegal es mayor en los varones que en las mujeres

En el caso del consumo de cannabis, sólo o en combinación con otras sustancias, el estudio de Velasco ${ }^{38}$ nos muestra que el $60,6 \%$ de los sujetos eran varones.

En nuestro estudio, se establece una relación significativa en el destino del paciente tras su estancia en urgencias teniendo en cuenta su sexo. Un porcentaje mayor de varones son los que necesitan ingreso hospitalario o son dados de alta sin precisar ser derivados a otro servicio. Por el contrario, las jóvenes son las que con más frecuencia reciben el alta con derivación a una consulta externa o bien son derivadas a otro centro hospitalario. En el caso del 
centro hospitalario, en muchas ocasiones se refiere a un centro de salud mental. Esto se produjo porque hasta el año 2009 el Hospital Universitario Río Hortega carecía de Unidad de Psiquiatría, estando ésta en un hospital al que había que derivar a los pacientes que precisaban valoración psiquiátrica. En el resto de ocasiones fueron derivaciones por necesidad de tratamiento en una cámara hiperbárica en otro hospital; o bien, por traslado al pertenecer al otro Área de Salud de Valladolid.

Este resultado por tanto, iría asociado a la relación significativa que se produce entre la necesidad de interconsulta con el servicio de Psiquiatría teniendo en cuenta el sexo del paciente; ya que el número de interconsultas es mayor en el caso de las mujeres (81,08\% frente a 18,91\%). Este dato se podría explicar por la mayor prevalencia de algunas enfermedades psiquiátricas en mujeres, tal como los trastornos alimentarios. Cabe destacar 3 artículos al respecto en el que se observan los siguientes resultados: Díaz de Neira $2013^{89}$, en el que de 267 casos registrados, el 64\% son féminas; González- Juárez, $2007^{90}$, , en el que se encuentra un riesgo de esta enfermedad en jóvenes de 12 a 18 años de $11,8 \%$ en el caso de las mujeres y de $4,6 \%$ en el caso de los hombres; o bien el artículo de Muro-Sanz de $2007^{91}$, en el que se detectan nuevos casos de la enfermedad en el $2,31 \%$ de mujeres frente a $0,17 \%$ de varones.

En el Boletín del Grupo de Intoxicaciones de la Sociedad Española de Urgencias de Pediatría de febrero de 2013, se muestra una comunicación enviada a la $62^{\circ}$ congreso de la Asociación Española de Pediatría en el que se muestra la actualidad de los intentos de suicidio mediante intoxicaciones voluntarias. En este caso, destaca que el 89,3\% de los casos, es llevado a cabo por mujeres, y en todos ellos, siempre mayores de 11 años ${ }^{92}$; igual que en Azkunaga $2011^{37}$, intentos autolíticos son llevados a cabo por mujeres en un $92,3 \%$.

Estos resultados también son similares en el artículo de Duce Tello de 1998, ${ }^{93}$ en el que entre los 14 y los 18 años, los pacientes con gestos autolíticos, correspondían en un 85\% al género femenino. 
En el informe del 2009, sobre la salud mental de niños y adolescentes en España ${ }^{94}$, se muestra que la prevalencia de bulimia nerviosa en mujeres es del $1-3 \%$, y en los hombres 10 veces menor.

\section{Edad.}

La media de edad corresponde a 10,15+/-6,43 años y la mediana a 14 años, observándose dos picos de edades claros (figura 11), niños pequeños hasta los 3 años y otro pico correspondiente a jóvenes de 14 a 18 años.

Estos datos son similares en otros estudios como el de Azkunaga de $2011,{ }^{37} \mathrm{O}$ Herranz en el $2003^{95}$ en el que se observan los dos grandes grupos de edad, el de menores de 5 años, que representa el $91,70 \%$ de los casos y el de mayores de 12 años, 32,2\%.

O bien los datos resumen del Observatorio de Toxicología Infantil correspondientes a los 5 años de 2008 a 2013, en el que también realzan dos grandes picos, menores de 5 años y mayores de 13 años.

Los datos aportados por la Memoria SIT del 2011 del Ministerio de Justicia, ${ }^{96}$ difieren un poco de estos resultados; ya que aquí lo que contabilizan son las llamadas al Servicio de Toxicología, por lo que el pico de adolescentes que aparece en otros estudios, en este caso no resulta llamativo, puesto que estos jóvenes no consultan telefónicamente. Sin embargo, si que se muestra un aumento importante del número de llamadas correspondientes a niños de 19 a 36 meses (edades en las que empiezan las intoxicaciones involuntarias).

Cabe destacar en nuestros resultados, que la media de edad de los pacientes atendidos cada año va aumentando según pasan los años, siendo mayor la edad de los pacientes atendidos desde que el Hospital Universitario Río Hortega se encuentra en su actual ubicación. Podríamos intentar explicar este dato porque muchos jóvenes con intoxicaciones etílicas acceden a este hospital por medio de vehículos de Emergencias Sanitarias, mientras que anteriormente no se utilizaba tanto este medio de trasporte, y al encontrarse 
los dos hospitales muy cercanos, en la misma zona urbana, se distribuía la atención a dichas intoxicaciones entre ambos.

La media de edad de los pacientes atendidos cada mes es mayor en los meses en los que la ciudad o los pueblos de la zona tienen festividades importantes, es decir, Agosto, Septiembre y Diciembre. Esto coincide también con un mayor número de intoxicaciones etílicas registradas en las urgencias hospitalarias, junto con los meses de mayo y junio (fechas de finales de exámenes.)

En el artículo de Fernández, P.(2002) ${ }^{97}$ sobre intoxicaciones en Santiago de Compostela, no se muestra una relación estadísticamente significativa en cuanto al mes de atención en urgencias de pediatría.

La media de edad de los pacientes según la hora de admisión de los pacientes en el servicio de urgencias muestra claras diferencias (figuras 18 y 19). Las horas de la noche y madrugada corresponden a pacientes de mayor edad (lo que se relaciona con las intoxicaciones etílicas) y los picos de salida del colegio o guardería, así como salida del trabajo de los padres o cuidadores, con niños más pequeños.

\section{Año y mes de atención en el servicio de urgencias hospitalario.}

El año de mayor número de intoxicaciones registradas fue el 2007 y el que menor el 2004. Como se muestra en la tabla 77 y figura 37 , el número de intoxicaciones ha ido aumentando a lo largo de los años.

Este hecho, podría explicarse por varios motivos. Quizá por el aumento importante de intoxicaciones etílicas en los últimos años (Mintegui 2012 ${ }^{45}$ ) y secundariamente de drogas de abuso; por la menor edad de inicio de consumo de las mismas (encuesta ESTUDES y Encuesta de consumo problemático de cannabis en jóvenes españoles ${ }^{49,98}$ ) y por el aumento de traslados de estas intoxicaciones, sobre todo de pacientes encontrados en las calles, en vehículos de Emergencias Sanitarias al hospital. Otro motivo del aumento de esta 
demanda es que muchos pacientes acuden directamente al servicio de urgencias hospitalarias sin ser derivados por otro sanitario, o bien, que desde el centro de salud se deriva al servicio de urgencias hospitalario, sobre todo a petición de los padres (Ruiz, 2007 ${ }^{99}$ )

Si comparamos estos datos con los del Observatorio Toxicológico Infantil, de diciembre $2013^{86}$, en el que se muestran los datos recogidos en los cinco años de historia del Observatorio, se observa que el año con una mayor prevalencia de intoxicaciones en niños fue el 2010 y el de menor el 2008.

En cuanto a los meses con mayor número de intoxicaciones en nuestro estudio son en orden Marzo, Junio y Diciembre, lo que coincidiría con final de exámenes y fiestas de Navidad y los de menor en orden creciente, Enero, Agosto y Julio.

Las intoxicaciones por consumo de alcohol y por drogas de abuso se relacionan con el tiempo de ocio, haciéndolas más frecuentes en meses de carácter festivo y en horario nocturno. Estos datos son similares a otros estudios como los de Sola ${ }^{87}$, Carmona ${ }^{88}$, Bugarín ${ }^{100}$, Hermida ${ }^{101}$, Martín ${ }^{102}$.

En el estudio de Fernández de $2002^{97}$, se observa una mayor incidencia de intoxicaciones en Febrero y en Julio, aunque sin existir una relación estadísticamente significativa.

\section{Diagnóstico y tipo de sustancias implicadas en las intoxicaciones.}

En nuestro trabajo, el tipo de diagnóstico por el que acuden al servicio de urgencias, así como las sustancias que lo ocasionan es la intoxicación por ingesta de alcohol (43,70\%), seguido de su medicación habitual (15,10\%), el monóxido de carbono $(8,00 \%)$ y finalmente los productos de limpieza.

Si comparamos nuestros datos con estudios del 2006 como el de Mintegi ${ }^{33}$, los datos son bastante diferentes pues en su caso la primera causa con las 
intoxicaciones son medicamentosas, seguidas por las debidas a productos de limpieza, etanol y monóxido de carbono.

En estudios más actuales como el del Boletín del Observatorio de intoxicaciones en pediatría de junio $2012^{109}$, el $49,3 \%$ de los casos de intoxicaciones son por fármacos, seguida de los productos del hogar en el $19,8 \%$, etanol en el $10,3 \%$, sin especificar en el $6 \%$, cosméticos en el $4,5 \%$ y monóxido de carbono en el $3 \%$

Esta disparidad de datos respecto a los nuestros se puede explicar porque en algunos centros recogen datos sólo hasta los 14 años, en otros hasta los 15, y en otros hasta los 18 inclusive o no. No existen por tanto, datos fiables de esa franja de edad, que nosotros sí presentamos.

Por otra parte, en nuestro estudio las intoxicaciones por monóxido de carbono suponen la tercera causa de intoxicaciones en menores de edad y este hecho no ocurre en el resto de España. Esto es debido a las especiales condiciones climatológicas de nuestra provincia y a la utilización de sistemas de calefacción calderas o estufas de cisco que en otras partes de España no se usan tanto.

En las consultas atendidas por el Servicio Toxicológico, recogidas en $2011^{66}$, el $53,82 \%$ son por intoxicaciones medicamentosas, el 17,8\% por productos de limpieza, por cosméticos en el $5,5 \%$ y por plaguicidas en el $6,7 \%$. En este tipo de consultas no aparecen las drogas de abuso ni el etanol, ya que no se realizan llamadas telefónicas para preguntar por sus posibles efectos secundarios, estas drogas son ingeridas por los jóvenes con fines recreacionales, con la idea de que casi no existe peligro para su salud física y psíquica.

En las recogidas por el Servicio de Toxicología irlandés del año $2010^{84}$ el orden de las intoxicaciones en menores de 19 años es: en primer lugar las producidas por medicamentos y drogas de abuso (no las separan), en segundo lugar las producidas por productos industriales, en tercero lugar las producidas por productos de limpieza, en cuarto lugar las producidas plaguicidas y en quinta posición las inducidas por los cosméticos. 
En nuestro trabajo, si analizamos sólo los menores de 7 años el principal diagnóstico es la intoxicación medicamentosa en un 49,6\%; en segundo lugar los productos de limpieza en el 18,5\%; en tercer lugar intoxicación por monóxido de carbono en el $8 \%$; en cuarto lugar los cosméticos con el 5,7\%; en quinto lugar los productos industriales con el 5,1\%. Los datos recogidos en EEUU por el American Association of Poison Control en 201067 en menores de 5 años difieren bastante de los encontrados en nuestro estudio. El 13,18\% de las intoxicaciones son por producidas por productos cosméticos, el $9,35 \%$ por analgésicos, el 9,24\% por productos de limpieza, y el 3,22\% por pesticidas. Comparados con los datos en menores de 7 años del $2013^{35}$ de Azkunaga en España son también distintos a los de EEUU, el porcentaje más alto de intoxicaciones es por medicamentos con el $59 \%$, por productos del hogar en el $24,4 \%$ y por cosméticos en el $5,8 \%$.

Nuestro trabajo tiene unos datos concordantes con los del resto de España, salvo en que el tercer puesto lo ocupan las intoxicaciones por monóxido de carbono por las peculiares condiciones climatológicas y sociales de nuestro entorno. Sin embargo, son bastante diferentes a las estadounidenses, donde la primera causa de intoxicación son los productos cosméticos frente a las medicamentosas que se presentan en los estudios españoles. Esto podría explicarse por la política sanitaria y farmacológica existente en ese país: mientras en EEUU sólo acceden al número de comprimidos, pastillas, sobres necesarios para el tiempo que le ha sido prescrito por el facultativo (salvo el caso de los analgésicos o antipiréticos que son comprados sin receta médica), en España, aún seguimos con la costumbre de almacenar fármacos en el domicilio, y muchas veces al alcance de los más pequeños del hogar.

\subsection{Intoxicaciones etílicas.}

Actualmente, el alcohol es el agente que más intoxicaciones produce en España en la edad pediátrica. Azkunaga $2012^{36}$

En nuestro estudio, el alcohol es el tóxico implicado en una mayor cantidad de intoxicaciones, en concreto en 434 de las 994. Hasta la fecha en España, no 
ha sido publicada una muestra tan grande de menores de edad con intoxicación etílica.

La incidencia de las intoxicaciones etílicas ha ido aumentando a lo largo de los años. (Figura 43). En España, las consultas en urgencias por este motivo, se han duplicado por 2,5 en los últimos 10 años (Mintegi, 2012) ${ }^{45}$

Este hecho es recogido también en otros estudios realizados con intoxicaciones etílicas en menores de edad, como el de Azkunaga $2012^{36}$, en las que el alcohol se erige como el principal causante de intoxicaciones en niños entre los años 2008 a 2010, desbancando a los fármacos.

Por otra parte, también desciende la edad media de las intoxicaciones etílicas registradas. La edad media de los pacientes con dicha intoxicación es de 15,4+/- 1,45 años, pero si analizamos año por año, comprobamos que la edad media de las intoxicaciones también va disminuyendo. Tabla 85.

Esto va a favor de los datos encontrados en la encuesta ESTUDES ${ }^{98}$ en los que la edad media del consumo semanal de alcohol va disminuyendo según pasan los años siendo en el 2002 de 14,95 años y en el 2010 de 14,75 años. En la encuesta ESTUDES del 2012-2013 ${ }^{128}$, la edad media de inicio del consumo de alcohol es de 13,9 años.

En el estudio de Sanz de $2009^{121}$, la edad media de los pacientes con intoxicación etílica corresponde a 16,2+/- 1,2 años. Este estudio se llevó a cabo durante el 2007, por lo tanto, los intoxicados en nuestro medio son más jóvenes.

En Europa, según el informe de Alcohol del 2007 del Plan Nacional sobre drogas $^{129}$, casi todos los estudiantes de 15-16 años (>90\%) han bebido alcohol alguna vez en la vida. La edad media de inicio se sitúa en los 12,5 años y la primera embriaguez, en los 14 años.

Si se valoran estudios a nivel europeo como el alemán Schöberl $2008^{130}$, que analiza 173 niños con diagnóstico de intoxicación etílica atendidos durante los años 1998 a 2994 en un hospital alemán, nos muestra que la edad media es de 14,5 años. 
Si por el contrario, se comparan nuestros resultados con otros estudios canadienses, Weinberg $2006^{131}$,la media de edad de los jóvenes analizados con intoxicación etílica continúa siendo similar, 14,5 años siendo la distribución hombres/mujeres del $50 \%$.

La distribución por sexos en el caso de nuestro estudio es de 55,29\% hombres frente al $44,7 \%$ mujeres. En el estudio de Sanz ${ }^{121}$, la distribución por sexos es justo el $50 \%$

En el Informe Reitox de España de $2011^{132}$, nos muestra una distribución cercana al $50 \%$ en cuanto al sexo en el consumo de etanol en jóvenes españoles entre los 14 y los 18 años.

El mes con mayor número de casos registrados en nuestro estudio es Septiembre, lo que coincide con las fiestas locales, seguido de Junio y Mayo (fechas de finales de exámenes y curso escolar.) Datos similares que asocien las intoxicaciones etílicas con estas fechas se encuentran en las tesis doctorales de Sola ${ }^{87}$, Carmona ${ }^{88}$ y Martín ${ }^{102}$.

Las horas de máxima afluencia de intoxicaciones etílicas en el servicio de urgencias hospitalario fueron las nocturnas, desde las 20 horas a las 2 horas de la madrugada, lo que coincide con las horas de salida con fin recreacional de los jóvenes. Estos datos coinciden los encontrados en el trabajo de Sanz de $2009^{121}$

Se realizan en estos pacientes, bastante más determinaciones sanguíneas que en pacientes con otro tipo de intoxicaciones. En muchas de ellas, se solicitan los tóxicos en orina, para valorar si junto al alcohol hay otro tipo de tóxico acompañante. Se realizó analítica de sangre con niveles de alcoholemia en 215 casos y en 36 de esos casos la alcoholemia superaba los $200 \mathrm{mg} / \mathrm{dl}$. , con una edad media de 15,11 años. A partir de estas cifras superiores de 200 $\mathrm{mg} / \mathrm{dl}$. el paciente presenta una sintomatología muy florida. (Roldán, 2003) ${ }^{133}$

La cifra media de alcoholemia registrada corresponde a 21,75 +/- 67,96 mg/dL. En el estudio de Sanz ${ }^{121}$, la cifra media de alcoholemia es bastante mayor, $186+/-56,2 \mathrm{mg} / \mathrm{dL}$. El nivel de etanol en sangre, sería necesario pedirlo siempre en lactantes y niños pequeños tras la exposición al etanol; mientras 
que en el caso de adolescentes sólo estaría indicado en aquellos con síntomas moderados-graves de intoxicación y no en síntomas leves. Mintegui ${ }^{45}$. Podríamos concluir, que la petición de alcoholemia en el servicio de urgencias de nuestro hospital no está del todo justificada, ya que se solicitan muchas alcoholemias a jóvenes con intoxicaciones leves.

En cuanto a la forma de acceder al servicio de urgencias hospitalario, un $71,07 \%$ son transportados por vehículos de emergencias sanitarias, un porcentaje mucho mayor que en el caso de otras intoxicaciones.

De todos los pacientes con intoxicación etílica, sólo 5 ingresaron, aunque 2 lo hicieron en el servicio de Cuidados Intensivos.

Por tanto, basándonos en la levedad del cuadro, el elevado número de altas hospitalarias tras la atención urgente y los resultados de las alcoholemias realizadas, se podría concluir que se trasladan en ambulancia un número excesivo de pacientes, que podrían ser trasladados, si lo precisaran, por otros medios, o que incluso no deberían de llegar a un servicio de urgencias hospitalario. El uso inapropiado del sistema de urgencias y emergencias como consecuencia de intoxicaciones etílicas leves, puede repercutir en que un recurso importante para la atención sanitaria urgente, como son las ambulancias, no esté disponible cuando una emergencia lo necesita. Ya hay estudios, como el de Martín $2012^{134}$ en el que se analiza el coste beneficio del empleo de tanto medio de transporte público, y se preguntan si todas las ambulancias son necesarias.

Las cifras de jóvenes a los que se les administra tratamiento en el servicio de urgencias hospitalario $70,46 \%$, son bastante más altas que la de estudios como el de Sanz, en las que sólo el 34\% recibe tratamiento.

Además, estos pacientes permanecen en los servicios de urgencias hospitalarios más tiempo que el resto de intoxicaciones; con los gastos que ello genera (Lee, 2011) ${ }^{135}$. No existen estudios sobre datos económicos que generen en nuestro país las intoxicaciones etílicas, pero ante la cantidad de recursos que consumen, deben de ser elevados (se utilizan más ambulancias, se administran más tratamientos...) 
Es por tanto el consumo de alcohol en jóvenes un problema que abarca diferentes estamentos de la sociedad: es un problema social, económico, laboral, sanitario, educacional. Desde hace mucho tiempo, se están llevando a cabo numerosos intentos de prevenir y disminuir el consumo de alcohol en gente joven ${ }^{136-144}$, aunque por el momento parece que no resultan muy efectivos a la luz del presente estudio.

\subsection{Intoxicaciones medicamentosas}

La edad media de las intoxicaciones medicamentosas es de 5,88 años, dentro de ellas la edad media varía según el tipo de intoxicación, así la edad por intoxicación debida a benzodiacepinas o antidepresivos es mayor que la de paracetamol que es 3,09 años. Esto puede ser debido a que el paracetamol es un fármaco habitual en todas las viviendas españolas, y que los padres no tienen tanto cuidado al guardar esta medicación como lo pueden tener con otras. Además, otra de las formas de producirse la intoxicación por paracetamol es la debida a un error en el cálculo de la dosificación.

En los protocolos sobre intoxicaciones agudas de Nogué de $2010^{54}$, destacan dentro de los fármacos las intoxicaciones por paracetamol, seguido de benzodiacepinas y antidepresivos.

En el estudio sobre intoxicaciones por antitérmicos de Conejo de $2002^{39}$ el $73,2 \%$ de las intoxicaciones por antitérmicos son en niños de 1 a 3 años; y en el artículo de Wang de $2013^{125}$ la edad media de las intoxicaciones por paracetamol fue de 2,6 años.

En el artículo de Li de $2013^{145}$ indica que la mayoría de las intoxicaciones por paracetamol en EEUU durante 1993 a 2007 han sido por menores de 5 años; disminuyendo la incidencia en adultos; y en el artículo de Budnitz de $2011^{146}$ refiere una incidencia en EEUU de ingestión no supervisada de paracetamol en menores de 6 años que corresponde al 13,4\% de todas las intoxicaciones por paracetamol en el país durante los años 2006 a 2007.

En el Boletín del grupo de intoxicaciones de junio 2012, los fármacos más comunes en las intoxicaciones son 19,6\% antitérmicos, 15,9\% benzodiacepinas, 9,8\% antiinflamatorios, $22,4 \%$ resto de fármacos. En nuestro 
estudio, dentro de las intoxicaciones medicamentosas, los fármacos que las causan son en primer lugar los fármacos habituales tomados por el paciente, en segundo lugar paracetamol, en tercer lugar benzodiacepinas, los AINES en cuarto lugar y por último los antidepresivos. Son por tanto, resultados similares.

En el artículo de Burghardt de $2013^{147}$, muestra un aumento de las intoxicaciones en niños año tras año; y lo atribuye al aumento de prescripciones de los adultos; y en el artículo del mismo año de Spiller ${ }^{148}$ se refleja un aumento del número de intoxicaciones medicamentosas en menores de 6 años, hasta ser el 33\% de todas ellas. Nuestros resultados no avalarían este supuesto, ya que en las intoxicaciones medicamentosas en las que aparece el propietario de la medicación, en el 58,8\% son intoxicaciones causadas por fármacos prescritos para el propio intoxicado.

En nuestro estudio, las intoxicaciones medicamentosas no aumentan con el paso de los años.

En el estudio del 2011 de Azkunaga $^{37}$ que evalúa la evolución a través de 5 años, no existen variaciones respecto a las intoxicaciones medicamentosas en general, aunque sí que muestran un descenso de las intoxicaciones por paracetamol, por los nuevos envases seguros que se comercializan en España.

\subsection{Intoxicaciones por monóxido de carbono.}

La intoxicación por monóxido de carbono es una de las principales causas de muerte de origen tóxico en países desarrollado. ${ }^{47}$

En las muestras del Observatorio Toxicológico de $2012^{109}$, las intoxicaciones por monóxido de carbono corresponden al 3\% de todas las intoxicaciones.

El número de intoxicaciones por monóxido de carbono producidas cada años en España podría ser alrededor de 2500 por año, de las que un centenar serían mortales. ${ }^{155}$ En un estudio realizado en el 2001, durante 18 meses en los dos hospitales de Valladolid, se diagnosticaron 158 intoxicaciones por monóxido de carbono (Dueñas Laita) ${ }^{48}$ 
En nuestro estudio, las intoxicaciones por monóxido de carbono, constituyen la tercera causa de intoxicaciones en menores de edad. La edad media de dicha intoxicación es de 9,6 +/- 5 años.

En este tipo de intoxicaciones, muchas veces los pacientes acuden a los centros sanitarios asintomáticos; por ello, la coximetría debería ser solicitada a los familiares que hayan estado expuestos a la fuente de $\mathrm{CO}$, cuando se confirma el diagnóstico en alguno de los miembros de la familia. ${ }^{156}$ (recogido en Docohumo 2010, Dueñas Laita) De hecho, si observamos los síntomas iniciales que presentan los pacientes en nuestro estudio, un $16,6 \%$ de los intoxicados acuden asintomáticos al servicio de urgencias hospitalario, y un $10,4 \%$ son menores de edad trasladados por haber encontrado una intoxicación por monóxido de carbono en los familiares que les acompañaban.

No obstante, en algunos estudios como el de Macías Robles de $2009^{18}$, se detecta un descenso de las intoxicaciones por monóxido de carbono de manera global, en adultos y niños. Aunque en el informe FETOC del 2010 y en los previos, nos habla de que el monóxido de carbono es el responsable, en un $37 \%$, de las intoxicaciones por productos químicos en España.

En este tipo de pacientes, es importante realizar una determinación en sangre de carboxihemoglobina. En nuestros pacientes se realizan únicamente 28 determinaciones en los 48 pacientes que acuden a urgencias. De ellos, 15 presentan valores de $\mathrm{COHb}$ por encima del $5 \%$, lo que sería diagnóstico de intoxicación por $\mathrm{CO}$ en jóvenes no fumadores.

\subsection{Intoxicaciones por productos del hogar.}

En nuestro estudio, 72 intoxicaciones fueron en menores de 7 años (88\%); 4 de 8-13 años (4,93\%) y 5 en mayores de 14 años $(6,17 \%)$.

Son la segunda causa de intoxicaciones en menores en España. ${ }^{45}$ suponiendo un $19 \%$ de todas las intoxicaciones. La mayoría de ellas se producen entre los 1 y 4 años de forma accidental.

En el artículo de Franklin de $2008^{149}$ relata que el $13,2 \%$ de las intoxicaciones en niños en EEUU en dicho año correspondían a intoxicaciones por productos de limpieza. 
En el artículo de McKenzie de $2010^{150}$ nos muestra la evolución en EEUU de las intoxicaciones en menores de 1990 a 2006. Nos muestra como los casos han ido disminuyendo año tras año. El $72 \%$ de los casos corresponden a niños de 1 a 3 años y el 45,9\% a menores de un año. En nuestro estudio, el número de intoxicaciones por productos de limpieza oscila poco a lo largo de los años.

En los últimos años, está apareciendo una nueva intoxicación por detergentes en menores que antes no existía, las intoxicaciones por las pastillas de lavavajillas y lavadoras (Bramuzzo, 2013; Bonney, 2013; Williams, 2012 y 2014) ${ }^{151-154}$ Son de colores y parecen gominotas, por lo que son atrayentes para la población infantil. Como consecuencia de este auge de intoxicaciones, muchas compañías están realizando cambios en sus envases.

\subsection{Intoxicaciones por drogas.}

El aumento de la incidencia del consumo de alcohol y drogas ilegales en jóvenes, es un tema que preocupa mucho a nivel socio, político, económico, sanitario. En España, el Plan Nacional sobre Drogas está en vigor desde el año 1985, y publica periódicamente estudios sobre la epidemiología, manejo, prevención y tratamiento de esta problemática. ${ }^{157} \mathrm{~A}$ su vez existen planes autonómicos y locales o municipales.

A nivel europeo existe el European Monitoring Centre for Drugs and Drugs Addiction. Este organismo, publica anualmente las memorias de la actividad ${ }^{158-}$ 166

Según la encuesta EDADES del $2009^{167}$, realizadas desde 1995 al 2009, las drogas de comercio ilegal más extendidas en España consumidas por personas entre 15 y 65 años fueron en orden decreciente el cannabis, la cocaína en polvo y el éxtasis. La edad media del primer consumo, difiere de los datos de otras encuestas, ya que en el caso del cannabis es 18,6 años, la cocaína en polvo 20,9 años y el éxtasis 20,46 años.

Estos datos, son diferentes a los encontrados en la Encuesta ESTUDES realizada en 2010 a estudiantes entre 14 y 18 años $^{49}$. Los jóvenes aquí bajan la edad media de inicio de consumo de sustancias: etanol e inhalables volátiles entre los 13 y 14 años; cocaína, éxtasis, alucinógenos y anfetaminas entre los 
14,9 años y los 15,5 años. En la encuesta ESTUDES del 2012-2013 ${ }^{128}$ la edad de inicio de consumo de sustancias sitúa el etanol en los 13,9 años, los inhalantes volátiles en los 13,4 años, el cannabis en los 14,9 años, la cocaína, las anfetaminas y el éxtasis en los 15,5 años.

El Proyecto Europeo de encuestas de estudiantes sobre el alcohol y otras drogas (ESPAD) ${ }^{168}$, recoge datos sobre el consumo de sustancias tóxicas en estudiantes europeos entre 15 y 16 años. España no pertenece a este proyecto, pero sí hay un acuerdo por el que se comparan los datos de la encuesta ESTUDES con la ESPAD. En el 2011, el 17\% de los encuestados habían probado el cannabis, el $3 \%$ éxtasis y el $3 \%$ anfetaminas.

El estudio del 2014 de Vázquez ${ }^{169}$, muestra los datos de una encuesta similar a la ESTUDES pero sólo en la provincia de Valladolid. Los datos apuntaban que los jóvenes vallisoletanos habían consumido alguna vez en la vida en un $77,2 \%$ bebidas alcohólicas, un $36,7 \%$ tabaco, un $17 \%$ cannabis, un $2,5 \%$ tranquilizantes con receta, un 1,9\% Speed o anfetaminas; un $1,3 \%$ tranquilizantes sin receta, un $1,1 \%$ éxtasis, un $1 \%$ cocaína y un $0,7 \%$ inhalantes volátiles. En los últimos 30 días habían probado alcohol el 64\%, tabaco el $20,3 \%$ y el resto de drogas el $6,26 \%$

En nuestro estudio, la edad media de las intoxicaciones etílicas recogidas es 15,4+/-1,65 años, cannabis $15,46+/-1,12$ años y cocaína 17 años. Se recogen 434 intoxicaciones etílicas, 13 intoxicaciones por cannabis, 6 intoxicaciones por anfetaminas y tan sólo 1 intoxicación por cocaína.

Si comparamos los datos de nuestro estudio con los obtenidos en otras series como en el servicio de urgencias pediátricas del Hospital Sant Joan de Déu en el periodo comprendido entre octubre 2007 y marzo $2008^{54}$, podemos comprobar que los datos son parecidos. Se atienden 227 intoxicaciones, de ellas, el 24,6\% fueron en jóvenes con consumo recreacional. El tóxico que ocasiona la visita a urgencias fue en 57 de ellas etanol, cannabis en 13, disolventes de pintura para inhalar en 3 , inhalación de pegamento en un caso 
y otro caso intoxicación por anfetaminas. Ocho jóvenes estaban intoxicados por más de un tóxico.

Otro estudio con el que podemos comparar nuestros resultados es el de Arias Constatí de $2010^{21}$. En él se atienden durante 30 meses 321 pacientes de los cuales, 272 eran intoxicaciones etílicas y 110 otras sustancias. La edad media fue de 16,1+/- 1,2 años. La distribución por sexos era mujeres 50,5\%. El tóxico empleado fue el alcohol en 272 casos, 84 cannabis, 11 cocaína, 10 anfetaminas, 8 benzodiacepinas, 7 inhalación disolventes, 5 opiáceos.

Los resultados por tanto son similares salvo en el número de casos recogidos, puesto que nuestro estudio abarca 10 años y los otros dos trabajos implican ámbito es bastante menor.

Podemos atribuir estas diferencias a un mayor consumo de algunas sustancias en determinadas zonas, como el consumo de cannabis en jóvenes en la zona de Levante que es superior a otras zonas del interior de la península. De hecho en la encuesta ESTUDES ${ }^{49}$, la prevalencia de consumo de cannabis en los últimos 30 días en Cataluña es de $21,3 \%$ en los jóvenes de la comunidad, frente a un $15,1 \%$ en Castilla y León.

Esta distribución irregular en España, también ha sido registrada en otras tesis doctorales como la de Rey en $2009^{51}$, en la que al comparar la incidencia de intoxicaciones por drogas de abuso en el Área Sanitaria de Santiago de Compostela con las intoxicaciones atendidas en el Hospital Clinic de Barcelona, se observa que en el centro catalán la incidencia dobla la media nacional.

En España por tanto, el cannabis es la droga ilegal más extendida, y la que antes comienza a consumirse ${ }^{170}$.

En nuestro estudio se recogen únicamente 13 intoxicaciones, lo que corresponde a un $1,3 \%$ de todas las intoxicaciones agudas en menores de edad. En otras 12 intoxicaciones además de otra sustancia está implicado el cannabis. Por tanto, entre el grupo de drogas ilegales, es la primera causa. La edad media de los afectados es de 15,46+/- 1,12 años y la distribución por sexos es claramente a favor de los hombres 10:3. La afluencia a urgencias ha 
ido aumentando a lo largo de los años, resultado extrapolable al aumento del consumo en España como se ha explicado anteriormente.

La hora de menor afluencia a urgencias son las horas de instituto por la mañana, pero la distribución es homogénea entre la tarde y primeras horas de la noche. Todos los pacientes fueron dados de alta domiciliaria.

En el caso del uso del cannabis como segunda sustancia implicada en la intoxicación, el diagnóstico principal corresponde en 8 casos a intoxicación etílica y en 4 a intoxicación por productos estimulantes (bebidas energéticas).

Se está reflejando en estudios como el de Briere de $2011^{171}$, que el consumo simultáneo de alcohol y cannabis, aumenta los riesgos de ambos y además, la probabilidad de convertirse en adictos.

En los estudios que encontramos en España sobre las intoxicaciones por cannabis, como el caso de Velasco $2010^{38}$, se recogen únicamente en un año 72 casos, con una edad media de 16,2+/-1,2 años y una distribución por sexos de $60,6 \%$ varones. En este estudio, sólo son dados de alta el 58,3\% frente al $100 \%$ de nuestros pacientes. Se les realizó seguimiento ambulatorio en el $13,9 \%$ de los casos, frente a sólo uno de nuestros jóvenes. Por tanto, deberíamos preguntarnos si ante el diagnóstico de intoxicación por cannabis en nuestras urgencias, no deberíamos de ponernos en contacto con servicios sociales o psiquiatría ambulatoria para un seguimiento y posible desintoxicación de los jóvenes. Esta misma cuestión, ya ha sido planteada en otros servicios de urgencias como es se refleja en el artículo de Matalí de $2009^{172}$.

Otro de los temas a tener en cuenta en relación con el consumo de esta sustancia, es la sensación de no estar ante una droga que tienen gran cantidad de jóvenes. Se olvidan de la problemática asociada; en el estudio de Schubart de $2011^{173}$ nos muestra cómo hasta en un $10 \%$ de los intoxicados por cannabis en menores de 12 años sufren experiencias psicóticas. La relación entre el consumo de cannabis y diferentes enfermedades mentales quedan reflejados en algunos artículos actuales: (Morales-Muñoz) ${ }^{174}$, (Coppola) ${ }^{175}$, (Philipps) $)^{176}$, $(\text { Bally })^{177}$, (Gutiérrez-Rojas) ${ }^{178}$ 
Respecto a las intoxicaciones por anfetaminas corresponden en nuestro estudio al $0,6 \%$, y acompañan como segunda sustancia al resto de intoxicaciones en el 7,69\% de los casos de poliintoxicaciones ( 2 casos: una intoxicación por cocaína y otra por cannabis). La edad media de estos pacientes es de 14,67+/-2,87 años.

En la escala ESTUDES del $2010^{49}$, la edad media de inicio de consumo es de 15,5 años. Por tanto, nuestros consumidores de anfetaminas son más jóvenes.

En 2009 entre las drogas ilegales de más extendidas en España fueron el cannabis y la cocaína con prevalencias en los últimos 12 meses del 2,6\%.

La evolución de la prevalencia de la cocaína alguna vez en la vida en la población entre los 15 y los 64 años en España de 1995 al 2009, ha ido aumentando de forma muy significativa. ${ }^{179}$. Esto supone además de un alto consumo en jóvenes, una mayor exposición pasiva en los hijos de consumidores. (García-Algar) ${ }^{180,181}$

En nuestro estudio sólo hay una persona con intoxicación por cocaína, un joven varón de 17 años.

Podemos concluir que la distribución geográfica varía el consumo de sustancias y eso afecta a los resultados encontrados en el presente estudio. En el estudio de Salazar de $2014^{34}$, se observa por ejemplo que las intoxicaciones con fines recreacionales son más habituales en País Vasco, Navarra y Zaragoza que en el resto de las comunidades autónomas españolas.

\subsection{Intoxicaciones por cosméticos.}

En nuestro estudio existen 22 casos de intoxicaciones por productos cosméticos. La edad media de esta intoxicación es de 1,73+/- 1,42 años. Esta cifras son comparables con las recogidas por el Boletín del grupo de intoxicaciones de la sociedad española de urgencias de pediatría de Diciembre $2013^{86}$.

En el estudio de Azkunaga de $2013^{35}$, las intoxicaciones por cosméticos representan el $5,8 \%$ de los casos, siendo el tercer tóxico que más 
intoxicaciones genera. Si en nuestros pacientes sólo analizamos los menores de 7 años, las intoxicaciones por cosméticos suponen el 5,7\% de todas las intoxicaciones, produciéndose en el $59,1 \%$ de los casos en varones.

Los datos de la memoria $\mathrm{SIT}^{66}$ nos hablan de que el 5,5\% de las llamadas recibidas en la consulta de toxicología son por cosméticos.

De nuestros 22 casos, en 10 de ellos fue necesario realizar consulta con el servicio de toxicología, por tanto, estos datos serían comparables con los anteriores.

En 12 casos el tratamiento recibido por los pacientes de nuestro estudio fue el lavado gástrico con el carbón activado.

\subsection{Intoxicación por productos industriales.}

En nuestro estudio se recogen 25 casos con una edad media de 5,04+/-5,05 años, con una mayor presencia en el sexo masculino $64 \%$.

Sin embargo, en nuestro estudio, el tipo de productos industriales con el que se intoxican los jóvenes no son muy peligrosos, ya que el $84 \%$ de ellos reciben el alta y sólo el $46,2 \%$ reciben tratamiento. Si el contacto hubiese sido con productos con una alta toxicidad, se les procedería a realizar tratamiento a todos ellos (Rodríguez) ${ }^{182}$, (Wattigney) ${ }^{183}$

\subsection{Intoxicaciones por pesticidas}

En nuestro estudio se producen 21 intoxicaciones por pesticidas, con una edad media de 3,95+/-3,73 años y una mayor frecuencia de varones (66,7\%). Estos resultados son comparables a los de Perry, 2014 de Inglaterra ${ }^{184}$ en que recoge los datos de las intoxicaciones por pesticidas en un periodo de 9 años, de los que se desprende que la mayoría ocurren en niños menores de 4 años. 


\subsection{Intoxicaciones por setas.}

Sólo encontramos en nuestro estudio 6 pacientes, con una edad media de $3,8+/-4,82$ años. Ninguno de los casos resulta grave, ya que no requieren ingreso en cuidados intensivos.

En España se han recogido desde hace 6 años 122 casos de intoxicaciones por setas, que afectaron a 356 pacientes.(Piqueras) ${ }^{185}$

Según los datos de la memoria SIT del 2011, la ingesta de setas es responsable únicamente del $0,2 \%$ de las llamadas.

\section{Procedencia de los pacientes intoxicados.}

La mayoría de los pacientes que acuden al servicio de urgencias hospitalario son de Valladolid capital $(63,80 \%)$. Dentro de los pueblos de la provincia, la incidencia más alta de intoxicaciones en menores de edad está en aquellos municipios con una mayor natalidad, como Laguna de Duero, Arroyo de la Encomienda o La Cistérniga.

La media de edad de los pacientes de Valladolid capital es de 10,93 años y la del resto de 8,76 años. Estos resultados podrían ser explicados porque los pacientes más pequeños son trasladados en vehículos particulares por la familia, cuando consideran estos que sufren intoxicaciones peligrosas, mientras que en la ciudad los jóvenes tienen un acceso más fácil al hospital, sin que sea preciso el concurso de la familia; además, a mayor edad, más consumo recreacional en la propia ciudad, con el consiguiente acceso al servicio de urgencias hospitalario.

\section{Acceso al servicio de urgencias en vehículos de emergencias sanitarias.}

En el 59,6\% de los casos, es decir, 348 pacientes, no precisaron este servicio; mientras que 236 menores, 40,4\% sí que lo utilizaron. En nuestro estudio sólo 
uno de los jóvenes acudió al servicio de urgencias hospitalario en soporte vital avanzado.

Cabe destacar, que el Servicio de Emergencias Sanitarias de Castilla y León en funcionamiento desde el año 2000 ha ido aumentando paulatinamente el número de su flota de ambulancias y el personal que trabaja para ellos.

En el Boletín del grupo de intoxicaciones de urgencias pediátricas de Abril $2014^{104}$, muestran una comunicación sobre actuaciones prehospitalarias que refleja que se utilizó una ambulancia en el $25 \%$ de las intoxicaciones, y dentro este tanto por ciento, medicalizada en el 33\%. Y en el Boletín de Febrero 2013 nos muestra que en las intoxicaciones con fines suicidas, el $35.7 \%$ acuden en transporte sanitario.

Esta diferencia en cuanto a la necesidad de trasporte por una unidad médica de emergencias de nuestro estudio al del Boletín de intoxicaciones de urgencias pediátricas de Abril 2014, puede deberse a varias causas: que nuestros menores presenten intoxicaciones menos graves, de hecho en nuestro estudio no existe ningún exitus; o bien, que la cercanía al hospital haga que no sea necesario activar los soportes vitales avanzados, ya que las necesidades de atención del paciente se cubran mejor en el hospital que en el ámbito de la extrahospitalaria, tal como el lavado gástrico o la administración de carbón activado. Por otra parte, la población de Valladolid ciudad y provincia está muy envejecida con una amplia morbimortalidad, y en el momento actual existen en la ciudad 3 soportes vitales avanzados durante el día y dos por la noche; y en la provincia únicamente el de Medina del Campo. Estas ambulancias habitualmente se encuentran ocupadas durante prácticamente todo el día en patologías de los adultos, por lo que sólo atienden patologías infantiles de carácter grave, que en el caso de las intoxicaciones registradas en nuestro trabajo son escasas.

En nuestro estudio se evidenciaron diferencias estadísticamente significativas entre el acceso a urgencias por parte de vehículos de Emergencias Sanitarias teniendo en cuenta su edad. 
Esto se explica porque la mayoría de los niños son transportados en vehículos familiares, y los adolescentes que necesitan vehículos de emergencia presentan intoxicaciones por drogas de abuso o son víctimas de intentos autolíticos.

En el estudio de Azkunaga de $2013^{35}$, el transporte se realizó en vehículo familiar en el $86 \%$ y $7,6 \%$ en vehículos de emergencias. Si comparamos estos datos, es decir en menores de 7 años con los nuestros, en la muestra el tanto por ciento de utilización de transporte sanitario es menor, el 2,5\%.

En el trabajo de Fernández Egido ${ }^{105}$, se muestra claramente que la mayoría de las intoxicaciones que precisan utilizar vehículos de Emergencias Sanitarias se dan en adultos de 26 a 45 años. En el caso de los menores de edad, son divididos en dos grupos: de recién nacidos a 15 años y de 16 a los 25 años. Los menores o iguales a 15 años precisan vehículos de emergencias sanitarias en un $1,1 \%$ por intentos autolíticos, y en segundo lugar por intoxicación etílica en $0,6 \%$, seguido de intoxicación por benzodiacepinas, antidepresivos, analgésicos y salicilatos por este orden. En los jóvenes de 16 a 25 años: 4,6\% por intoxicación etílica, seguida por los intentos autolíticos en el $4,5 \%$, intoxicaciones por benzodiacepinas en el 2,5\%, cocaína en el 1,2\% y el resto de intoxicaciones en un porcentaje muy bajo.

En otro estudio de Shah, M. de $2009^{106}$ refiere que de todos los transportes realizados por vehículos de emergencia a niños en EEUU durante el periodo de 1997 al 2000, el 62\% fue trasladado a consecuencia de traumatismos 0 intoxicaciones. No establece diferencias entre estas dos categorías. La diferencia de estos datos estadounidenses con los españoles se debe sobre todo a las características geográficas, demográficas y económicas que diferencian los EEUU de España.

\section{Realización de pruebas durante su estancia en el servicio de urgencias.}

No hay reglas fijas respecto a las pruebas que se deben realizar de forma rutinaria. El sentido común del médico y el juicio clínico es el que prima en la

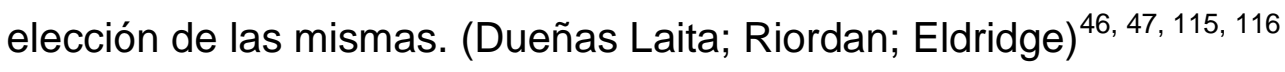


La edad media de los pacientes a los que se les realizan pruebas diagnósticas en el servicio de urgencias hospitalario es mayor que la edad media de los pacientes a los que no se les realiza, excepto para la hemostasia y la gasometría arterial. (Tablas 57 y 58)

Esto podríamos explicarlo porque en el caso de los más pequeños se tiende a no someterles a exploraciones cruentas si no son estrictamente necesarias, y además las intoxicaciones que presentan no se benefician de pruebas analíticas, ni se pueden determinar los niveles de sustancia en sangre y/o en orina.

En el caso de la hemostasia y la gasometría arterial, son pruebas bastante específicas que se necesitan en las intoxicaciones por raticidas o por monóxido de carbono, y que no pueden dejar de realizarse pese a la corta edad del afectado.

Los tóxicos en orina y sangre detectan sustancias que son consumidas por jóvenes de mayor edad. Además, pueden resultar útiles en las ingestas voluntarias de tóxicos desconocidos. Olgun ${ }^{117}$, Martin ${ }^{118}$, Shannon ${ }^{119}$; por tanto, es lógico que en nuestro estudio la población sometida a esta analítica sea de mayor edad.

En el año 2010, Arias Constantini, V. ${ }^{120}$, publicó un artículo en el que se preguntaba por la necesidad de realizar analítica a todos los pacientes con intoxicación etílica en urgencias. Llegaban a la conclusión de que ésta sólo sería necesaria si es un caso que atente a la seguridad del tráfico o cuando no haya certeza de la sustancia consumida, en pacientes con alteración de la conciencia, y cuando ninguno de los acompañantes sean capaces de dar una información verídica.(Sanz) ${ }^{121}$ (Charalambous) ${ }^{122}$.

Sólo habría que solicitar aquellas en las que la medición de sustancias modificaran en algo el diagnóstico o tratamiento del paciente: coma de origen desconocido, convulsiones en el contexto de una poliintoxicación.

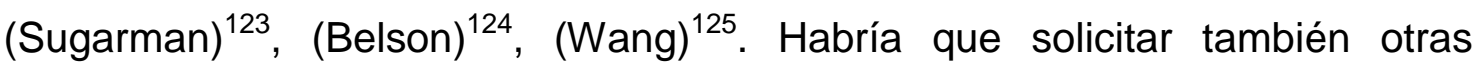
pruebas como es el caso de ECG en pacientes con dolor torácico con diagnóstico de intoxicación por monóxido de carbono (Dueñas Laita) ${ }^{126}$, o por 
cocaína $(\text { Lahoz })^{127}$, ya que en ambos casos pueden darse síndromes coronarios en el contexto de la sobredosis.

En nuestro estudio podríamos concluir que se solicitan demasiadas pruebas ya que en casi la mitad de los pacientes intoxicados menores de edad se les somete a la extracción en el servicio de urgencias hospitalario de una analítica con hemograma y bioquímica, y en el caso de los tóxicos en orina en el $35,40 \%$. En el caso de las intoxicaciones etílicas este porcentaje es aún mayor: el $73,30 \%$ de los pacientes son sometidos a analíticas. Estos datos reflejan una medicina defensiva, con petición de pruebas innecesarias. Este hecho ocurre también en otros estudios como el Sanz ${ }^{121}$, en el que se realiza petición de tóxicos en orina en el $62,8 \%$ de los jóvenes con intoxicación etílica.

\section{Tratamiento de las intoxicaciones.}

En nuestro estudio han recibido tratamiento el 58,9\% de los pacientes con intoxicación. Esto va a favor de lo ya expuesto anteriormente que muchos de los pacientes que acuden con diagnósticos de intoxicaciones son leves o están producidas por agentes que en la dosis a la que se han expuesto no resultan dañinos.

La indicación de descontaminación gastrointestinal y la técnica a realizar ha sido y es un tema ampliamente discutido. La Academia Americana de Toxicología y la Asociación Europea de Toxicólogos Clínicos redactaron en 1997 unas primeras recomendaciones, revisadas con posterioridad, y que constituyen un consenso internacional basado en la evidencia científica. En ellas se aconseja que sólo se emplee el lavado gástrico en cuatro supuestos: cuando la ingestión es de gran cantidad de tóxico, si el tóxico ingerido es realmente peligroso, si se realiza el procedimiento en la primera hora tras la ingesta y siempre que no existan contraindicaciones a la técnica.

En España era anteriormente un método ampliamente utilizado. En el estudio de Munné $2005^{186}$, concluía que los criterios de consenso sobre el desuso del 
lavado gástrico en las intoxicaciones agudas no habían sido aún adoptados por la mayoría de los hospitales españoles. La utilización del carbón activado en España ha ido aumentando progresivamente, pasando de un $25 \%$ de empleo en 1995 a un 46\% en el 2006 (Burillo Putze) ${ }^{187}$. De hecho, hasta el artículo de Burillo Putze en el $2008^{14}$, no se había recogido en ningún artículo español que el carbón activado fuera el método de descontaminación digestiva más empleado en el país y que mejorara las asistencias en el servicio de urgencias. En nuestro estudio sólo se realiza lavado gástrico en un $9 \%$ de la población que acude a urgencias con el diagnóstico de intoxicación y un $10 \%$ reciben como tratamiento carbón activado. Estos datos dejan ver que el empleo del lavado gástrico aún es ampliamente empleado en el servicio de urgencias de nuestro hospital. Si comparamos nuestro estudio con el trabajo de Velasco $2014^{188}$ en el que analizan el avance en el tratamiento de las intoxicaciones en pediatría estudiándolo en 3 periodos 2001-2002; 2008-2009; 2009-2011; se abre una puerta a la esperanza pues con el paso de los años los servicios de urgencias hospitalarios han ido aceptando y llevando a cabo las recomendaciones que indican las guías clínicas.

Si analizamos los pacientes de nuestro estudio a los que se le realiza lavado gástrico (50 pacientes), es cierto que la distribución por años indica un porcentaje del $4 \%$ en el 2006 , del $58 \%$ en el periodo $2007-2008$ y del $38 \%$ en el periodo 2009-2010 (no disponiendo de datos de los años anteriores al no estar informatizadas las historias anteriores al 2006). Por tanto, el porcentaje de los pacientes que se someten a lavado gástrico va disminuyendo también a lo largo de los años.

El diagnóstico por el que acuden los pacientes que se someten a lavado gástrico es en un $86 \%$ la intoxicación medicamentosa, seguida por intoxicación por insecticidas, productos de limpieza, cosméticos y setas.

Fueron dados de alta el $62 \%$ de los pacientes que se sometieron a lavado gástrico. Esto habla a favor de la gravedad de la intoxicación, con lo cual se cumpliría uno de los criterios que abogan por la realización del lavado gástrico.

Cuando se realizo lavado gástrico en el tratamiento de la intoxicación, se realizó interconsulta con Toxicología en un 30,6\% de las ocasiones. 
En nuestro estudio el carbón activado se emplea en un $10 \%$ de las intoxicaciones registradas, frente al $95,35 \%$ de las intoxicaciones registradas por Velasco $2014^{188}$. El empleo del carbón activado, en dosis única o en dosis repetidas, está indicado en la ingesta por vía oral de casi todos los tóxicos menos los alcoholes, metales, litio, cáusticos y potasio ${ }^{46}$. Es útil en las primeras 2 horas de la ingesta y se repite a las 4 horas si son fármacos con circulación enterohepática.

La edad media de los pacientes a los que se les administró carbón activado fue de 6,3+/-6 años. Su empleo ha sido constante a lo largo de los años. En el $89,5 \%$ de los casos se utilizó en pacientes con intoxicaciones medicamentosas, seguido de un $3,5 \%$ setas y raticidas y un 1,8\% intoxicación por cosméticos. El $68 \%$ de estos pacientes son dados de alta, pero el $53,84 \%$ de los pacientes que ingresan lo hacen en la Unidad de cuidados intensivos, por tanto, la gravedad de las intoxicaciones de los pacientes en los que se emplea el carbón activado, como en el caso de los que se someten a lavado gástrico, es mayor.

Cuando se administró carbón activado se interconsulta con Toxicología en un $40 \%$ de los casos.

Podríamos atribuir la baja cantidad de pacientes a los que se administra carbón activado, al miedo a las reacciones adversas que puedan presentarse. Sin embargo, se ha visto en diversos estudios, que la mayoría de los pacientes que sufren reacciones adversas al carbón son leves. (Amigó ${ }^{189}$ )

La administración de antídotos en el servicio de urgencias en nuestro estudio se realiza en 7 pacientes con diagnóstico de intoxicación por paracetamol, insecticidas y productos de limpieza. En estos casos, sólo se precisó la interconsulta con Toxicología en el 33,3\% de los pacientes.

La cuestión que subyace tras esta poca cantidad de antídotos empleados es el motivo por el que no se ha empleado en el resto de intoxicaciones por paracetamol (55). De estas 55 intoxicaciones ninguna fue transportada por unidades de emergencia sanitaria por pensar que el antídoto le haya sido administrado allí, y sólo dos casos fueron valorados por atención primaria. De 
estos jóvenes ingresan 15 pacientes (en ellos se supone una mayor gravedad de la ingestión).

En el caso de las intoxicaciones por paracetamol, está aprobada la utilización de forma empírica de antídoto cuando no se conoce severidad, concentraciones plasmáticas o tiempo transcurrido desde su ingesta. Se ha valorado en diferentes estudios el riesgo beneficio de su empleo y todos concluyen el beneficio de su pronta utilización. (Hedeland) ${ }^{190}$, (Heard $)^{191}$ (Freeman) $^{192}$ (Whyte) $^{193}$ (Betten) $^{194}$

Se podría mejorar el mejor empleo de los antídotos en las intoxicaciones en menores, como se comenta en el estudio de Martínez Sánchez ${ }^{195}$, donde se indica que la administración de antídotos en los servicios de urgencias pediátricas es muy infrecuente. La solución podría pasar por un reciclaje del personal médico que tratan dichas intoxicaciones.

\section{Interconsulta con la unidad de Salud Mental.}

En nuestro estudio, se precisa interconsulta con el servicio de Psiquiatría en un $8,9 \%$ de las intoxicaciones.

Los pacientes son todos de edad superior a 13 años, con una edad media de 15,78+/- 1,18 años. El $81,1 \%$ de los pacientes son mujeres y el $43,2 \%$ de los pacientes tienen antecedentes psiquiátricos. La mitad de los intoxicados son sometidos a lavado gástrico y administración de carbón activado.

La relación a favor de las mujeres se observa también el en estudio de Hermida $^{196}$, sobre pacientes de 15 a 19 años con intoxicación con fin autolítico, donde el predominio está claramente a favor de las mujeres.

En el Boletín del Grupo de trabajo de intoxicaciones de la Sociedad Española de Urgencias de Pediatría de Febrero de 2013, se aborda el tema de las intoxicaciones en niños con fines suicidas. El 5,6\% de los casos de intoxicaciones producidas entre 2008 y 2012 fueron con fines autolíticos, 
produciéndose en jóvenes mayores de 11 años y siendo responsables casi siempre los fármacos (psicofármacos y paracetamol). En nuestro estudio, $83,3 \%$ son intoxicaciones medicamentosas, seguido de $8,1 \%$ sustancias sin especificar, $5,4 \%$ intoxicaciones por productos industriales y $2,7 \%$ intoxicaciones por cannabis. En un 18,9\% de las ocasiones hay más de una sustancia causante de la intoxicación. La sustancia responsable de la intoxicación es en un 27\% la medicación habitual del joven, en el 24,3\% benzodiacepinas; en el 21,6\% AINES, en el 13,5\% no está especificado en la historia y el resto de tóxicos más o menos en la misma proporción corresponden a hachís, antidepresivos, paracetamol, productos de cosmética y productos industriales

La mitad de estos pacientes fueron traídos al hospital en vehículos de Emergencias Sanitarias frente al $40 \%$ del global del estudio.

Si comparamos estos datos con los de pacientes ingresados ya en unidades de salud mental por intentos autolíticos (Bella) ${ }^{197}$, la media de edad es mucho menor que en nuestro estudio: 12,84 años, y la distribución por sexos también va a favor del femenino $56,10 \%$. En el $90 \%$ de los casos, los pacientes habían sido ingresados por intoxicación medicamentosa, sobre todo psicofármacos, y los fármacos prescritos para ellos o sus familiares. Por tanto, estos datos son similares a los encontrados en nuestra investigación.

En otro estudio anterior, esta vez realizado en España (Venceslá) ${ }^{198}$, la prevalencia de intentos autolíticos es mayor en mujeres y en un $84,8 \%$ de los casos es debida a intoxicación medicamentosa.

Otra de las causas de interconsulta con el Servicio de Psiquiatría en los menores afectados por una intoxicación sería la agitación en pacientes consumidores de drogas y alcohol y los síndromes de de abstinencia de opiáceos, hipnóticos y sedantes. Realmente ésta es la causa más frecuente de agitación psicomotriz en los adultos en urgencias, y actualmente debería de ser considerada en múltiples ocasiones en el caso de los menores de edad. ${ }^{199}$ 


\section{Interconsulta con Toxicología}

En nuestro estudio se realizan 86 consultas con Toxicología. La edad media de los pacientes afectados es de 5,48+/-5,23 años. La distribución por sexos es ligeramente superior en el sexo masculino $54,7 \%$.

Los diagnósticos fundamentales por los que se produce esta consulta con Toxicología son las intoxicaciones por monóxido de carbono y las intoxicaciones medicamentosas, seguidas por las intoxicaciones por productos de limpieza, productos industriales, intoxicaciones por cosméticos y por insecticidas, y en último lugar para las intoxicaciones por setas. Dentro de las intoxicaciones medicamentosas, los fármacos que originan más consultas a toxicología son los prescritos para el propio paciente o la familia, seguido de las benzodiacepinas y los AINES y en último lugar, la intoxicación por paracetamol.

Si tenemos en cuenta los datos de la Memoria SIT del $2011^{66}$, los datos son similares, siendo las causas de consulta más frecuentes las intoxicaciones medicamentosas, seguido de las intoxicaciones por productos de limpieza, plaguicidas y cosméticos.

En nuestro estudio difieren los datos en lo que respecta a las intoxicaciones por monóxido de carbono. Como se ha visto la incidencia en nuestro medio es mayor que en el resto de España, esto es debido a las condiciones climáticas, a la costumbre de uso de braseros y estufas que no se tiene en otras comunidades autónomas. Esta diferencia también se observa en las memorias anuales Irlandesas ${ }^{75}$, en las que en jóvenes menores de 19 años, la causa principal de consulta son las intoxicaciones medicamentosas, seguidas de las industriales, productos de limpieza y cosmética.

En cuanto a los meses del año en los que se producen las consultas al servicio de Toxicología, en nuestro caso, la distribución es de mayor en diciembre $(17,4 \%)$, seguido de mayo $(16,3 \%)$, octubre $(11,6 \%)$ y enero $(10,5 \%)$. En la Memoria SIT el mes con mayor demanda es mayo (9\%), seguido de julio, marzo y abril. Estos datos podrían tener una explicación, ya que como el 
monóxido de carbono es la primera causa junto con los medicamentos de consulta con Toxicología, los meses de frío generan una mayor demanda de consultas a toxicología, cosa que no sucede en el caso del Instituto Nacional de Toxicología y Ciencias Forenses.

\section{Tiempo de permanencia en el servicio de urgencias.}

La media en minutos de estancia en el servicio de urgencias hospitalario corresponde a 215,62 +/- 196, 23 minutos, con una mediana de 153 minutos de permanencia en el servicio de urgencias.

No se encuentran muchos estudios con los que comparar este dato. El estudio de Goicochea ${ }^{103}$ muestra tiempo de estancia que fue de 94 minutos, pero sólo cuenta con los menores de 14 años y engloba no sólo las intoxicaciones sino también las lesiones.

En nuestro caso, se evidenciaron diferencias estadísticamente significativas entre el tiempo de observación en urgencias teniendo en cuenta la edad. Tabla 63 y figura 30. Permanecen mayor número de minutos en el servicio de urgencias los pacientes de mayor edad.

Esto es debido a que los niños que acuden al servicio de urgencias hospitalario por una intoxicación aguda, a veces ni siquiera han llegado a ingerir la sustancia en dosis tóxica, por lo que se envían rápidamente al domicilio; y en las intoxicaciones agudas verdaderas, los pediatras prefieren ingresarlos en la planta o en la unidad de cuidados intensivos, para realizar la observación clínica y mantenerles monitorizados.

En el caso de los adolescentes, la mayoría son sometidos a pruebas diagnósticas en el servicio de urgencias hospitalario, ya que en muchas ocasiones no se sabe exactamente cuál es el tóxico que ha provocado la intoxicación; se asocia el consumo de drogas y alcohol con lesiones traumáticas que precisan pruebas de imagen; o bien, al ser intentos autolíticos o drogadicción, se solicita interconsulta con el servicio de psiquiatría, lo que 
demora el tiempo de estancia en el servicio de urgencias hospitalario. La mayoría de las intoxicaciones en estos jóvenes no son graves, por lo que se les deja más tiempo de observación en el servicio de urgencias hospitalario, pero se evita un ingreso en planta innecesario.

\section{Destino tras el paso por la urgencia hospitalaria.}

Sólo el $15,2 \%$ de las intoxicaciones precisa de un ingreso hospitalario, frente al $84,8 \%$ de las ocasiones que son dados de alta con o sin derivación a consulta externa.

Esto puede ser explicado porque un gran porcentaje de las urgencias por intoxicaciones agudas no revisten gravedad, o no son dosis tóxicas las empleadas. (Mateu Sancho; Mc Guian) ${ }^{46,47,54}$. ${ }^{107,} 108$

Este tanto por ciento de ingresos es mayor en otros servicios de urgencias, quizá porque muchas veces la observación se hace directamente en la planta. ${ }^{109}$ En este artículo de Azkunaga junio 2012, el 48,6\% de los intoxicados no son dados de alta; y en estudios posteriores ${ }^{86}$, se indica que ingresan el $50,46 \%$ de los intoxicados. Aunque en la UCl sólo lo hacen el 1,09\%, frente al $1,9 \%$ que lo hacen en nuestro estudio. Podría concluirse por tanto, que los criterios para ingreso en la unidad de cuidados intensivos en nuestro estudio son similares a los del resto de España.

Si comparamos nuestros datos sobre los pacientes que ingresan en cuidados intensivos y los EEUU (Even) ${ }^{110}$, en nuestro estudio se recogen 19 casos, con una edad media de 5,47+/-5,51 años, 52,6\% mujeres, y el diagnóstico por el que ingresan en la Unidad de Cuidados Intensivos es en un $68,4 \%$ intoxicación medicamentosa, $10,5 \%$ etílica, y en un tanto por ciento igual de 5,3\% intoxicaciones por monóxido de carbono, insecticidas, productos de limpieza e industriales. En el caso de Even, respecto a la edad los dividen en dos grandes grupos, menores de 3 años y mayores de 13 años; siendo la causa principal de la intoxicación, igual que en nuestro estudio, la medicamentosa. 
La edad media de los pacientes dados de alta es un poco mayor que la de los niños que ingresan o son derivados a otro hospital $(10,65$ años frente a 7,62 .)

En el estudio de Azkunaga de $2013^{35}$ que sólo incluye a los menores de 7 años, el $45,4 \%$ de los pacientes ingresan, y 3 lo hacen en la unidad de cuidados intensivos. En nuestro estudio, de los menores de 7 años el $71,5 \%$ de los pacientes ingresan, esto puede deberse a la no presencia en nuestro hospital de una unidad de corta estancia para observación; por tanto, muchos ingresan únicamente para observación.

En el año 2004, Domingo A. ${ }^{111}$ y anteriormente, Casanova ${ }^{112}$, Trapero ${ }^{113}$, Gómez ${ }^{114}$, repasaban los criterios de ingreso pediátricos y afirmaban que se procede a ingresar hospitalariamente a niños que podrían beneficiarse de estar ambulatoriamente. Este beneficio, podría plantearse ante los futuros ingresos de los menores en nuestro hospital.

\section{Consultas en el servicio de urgencias hospitalario en el mismo año de la intoxicación, previos y posteriores.}

Las consultas realizadas en las urgencias pediátricas han experimentado un notable aumento, pese al descenso de la natalidad. Esto ocurre no sólo en nuestro país sino en el resto de países desarrollados (Yamamoto) ${ }^{200}$ (Mintegi) $^{201}$. Lo que supone un gasto sanitario enorme (Ruger) ${ }^{202}$. El problema es que no todos los pacientes que acuden a urgencias hospitalarias lo necesitan, y la mayoría podrían solucionar sus problemas en el centro de salud. (Aranaz) ${ }^{203}$

En nuestro estudio, se han separado las consultas en urgencias hospitalarias el mismo año de la intoxicación, en los previos y en los posteriores para sopesar si estos jóvenes que acuden por intoxicaciones tienen más tendencia a ser hiperfrecuentadores. Para ello además se observa si los pacientes que han acudido por intoxicaciones presentan antecedentes personales en el 
momento de la intoxicación, que expliquen la necesidad de recurrir a los servicios de urgencias hospitalarios con tan corta edad.

El $63,3 \%$ de los jóvenes no presentan ningún antecedente personal reflejado en las historias clínicas; $18,6 \%$ antecedentes no catalogados (en este punto se engloba una gran cantidad de entidades, haciendo una especial mención a la patología endocrinológica, sobre todo diabetes); 4,8\% pulmonares (en su mayoría asma); 4,3\% traumatológicos; 4,2\% psiquiátricos; $1,5 \%$ urológicos; $1,1 \%$ cardiacos, $0,5 \%$ digestivos; $0,4 \%$ ginecológicos; $0,2 \%$ sociales. Por tanto, la mayoría de nuestros jóvenes no tienen patología crónica que explique la demanda asistencial.

Los episodios de consulta urgente hospitalaria el mismo año de la intoxicación tienen una cifra media de $0,46+/-1,03$ consultas; en años anteriores 3,58+/5,23 consultas y en los posteriores $2,02+/-3,55$ consultas. Se observa por tanto, que los pacientes utilizan de manera inapropiada el servicio de urgencias.

Si valoramos el motivo por el que acuden al servicio de urgencias hospitalario el mismo año, los años anteriores y los años posteriores, cabe destacar que la primera causa es patología general, la segunda corresponde a causa traumatológica y la tercera una nueva intoxicación. Por tanto, deberíamos valorar si no sería útil establecer nuevas guías de prevención de accidentes infantiles, o bien, investigar en una posible adicción en el joven que requiera un seguimiento extrahospitalario.

En el estudio de Riba de $2004^{204}$, se observa que una gran cantidad de los pacientes que acuden de forma frecuente a urgencias hospitalarias, lo constituyen los pacientes pediátricos, sobre todo, menores de 2 años. Lo que achacan a la inexperiencia de los padres y concluyen que debería propiciar cambios en la organización y coordinación entre niveles sanitarios, ya que muchas de las cuestiones por las que acuden se solucionarían en los centros de salud. 
Respecto al número de consultas ambulatorias hospitalarias que a las que acuden los pacientes intoxicados, la media está en 1,6+/- 1,37 consultas. En este punto, se puede discutir sobre la capacidad que tienen los médicos de atención primaria, tanto pediatras como los de población general, para solicitar determinadas pruebas que les ayuden con el diagnóstico, la demanda de los pacientes, la confianza que radica en ellos... (Pueyo, 2011) ${ }^{205}$ 


\section{Limitaciones de nuestro estudio.}

El presente estudio puede presentar ciertas limitaciones que hay que tener en cuenta a la hora de ser valorado.

En primer lugar, como ya se expuso anteriormente, sólo se encuentran informatizadas completamente las historias clínicas del Hospital Universitario Río Hortega desde el año 2006. Esto ha supuesto un problema a la hora de acceso a la información de los parámetros estudiados. Por tanto, de las 994 historias clínicas de las que se pueden obtener datos, sólo en 584 se pueden recogen todos los parámetros estudiados.

En segundo lugar, hay que tener en cuenta la peculiar organización de la atención pediátrica en nuestro servicio de urgencias, donde los menores de 14 años son atendidos en las urgencias pediátricas y los mayores de esta edad en las urgencias de adultos. La disparidad de criterios de inclusión existente en los estudios encontrados hasta la fecha, que se adaptan a la diferente organización de los servicios de urgencia hospitalarios de las distintas comunidades españolas hacen complicado la comparación de los resultados. Dependiendo de las características del hospital la atención de los menores de edad se realiza siempre en las urgencias generales, o bien en las urgencias pediátricas hasta una edad y posteriormente en las de adultos; muchos centros no disponen de servicios de psiquiatría de guardia; el acceso a los diferentes kits diagnósticos no es el mismo; la distribución geográfica de los pacientes es bastante diferente según la provincia en la que habite el sujeto.

Por otra parte, no existen protocolos de actuación unificados en España a la hora de atender a los pacientes intoxicados.

En tercer lugar, el Hospital Río Hortega, no disponía de Unidad de Agudos de Psiquiatria ubicada dentro del propio hospital en los primeros años de recogida de datos. Por ello, podrían haberse perdido parte de los pacientes con intoxicaciones medicamentosas leves y algunos de los pacientes psiquiátricos crónicos politoxicómanos que acudieran directamente a la unidad de referencia 
de psiquiatría del Hospital Villacián. No nos es posible asegurar, que en el $100 \%$ de los pacientes que eran dados de alta del servicio de urgencias y derivados al Hospital Villacian para interconsulta por psiquiatría, acudieran a dicho servicio. 


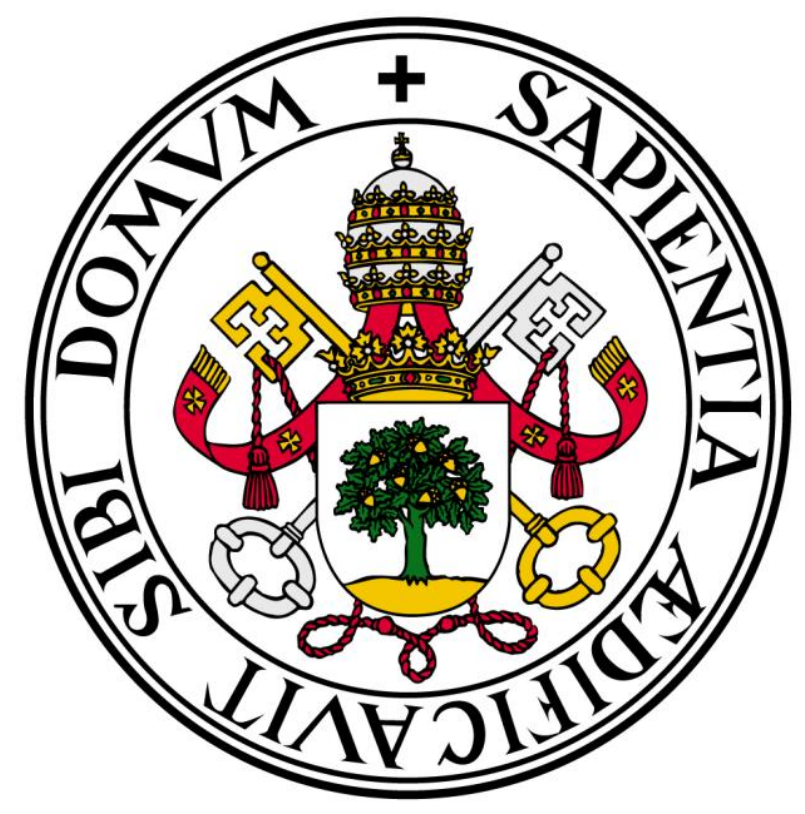

VI. Conclusiones 


\section{Conclusiones.}

1. La incidencia de las intoxicaciones agudas en la población menor de 18 años, atendidas en el Hospital Universitario Río Hortega de Valladolid, en la década de 2001-2010 fue de 0,41\%.

2. Se objetivan dos claros perfiles de pacientes intoxicados. El primero sería el de un joven de 15,4 años de edad media atendido por intoxicación etílica, sin diferencia en cuanto a la incidencia por sexo, que acude en horario nocturno, procedente de Valladolid capital, transportado por un vehículo de Emergencias Sanitarias. El segundo sería el de una niña de 5,92 años de edad por intoxicación medicamentosa, que acude en horario de tarde o noche, procedente de Valladolid capital y es transportada por un vehículo familiar.

3. El número de intoxicaciones atendidas en menores de edad ha ido aumentando, al igual que la edad media de las intoxicaciones, con el paso de los años.

4. El número de intoxicaciones etílicas en menores de edad ha aumentado notablemente a lo largo de los años, disminuyendo la edad media de los consumidores.

5. A los menores que presentan intoxicación etílica se les realizan pruebas complementarias y se les administra tratamiento, siendo dados de alta desde el servicio de urgencias sin complicaciones.

6. Los menores que presentan intoxicaciones medicamentosas no suelen precisar pruebas diagnósticas ni tratamiento hospitalario, siendo dados de alta desde el servicio de urgencias sin complicaciones.

7. La asistencia recibida por los menores de edad que acuden al servicio de urgencias es similar a los datos registrados en otros hospitales de tercer nivel nacionales. 
8. Se evidencia la realización de excesivas pruebas diagnósticas innecesarias a los menores intoxicados.

9. El número de tratamientos innecesarios realizados a los menores intoxicados ha disminuido sustancialmente a lo largo del periodo de estudio. 


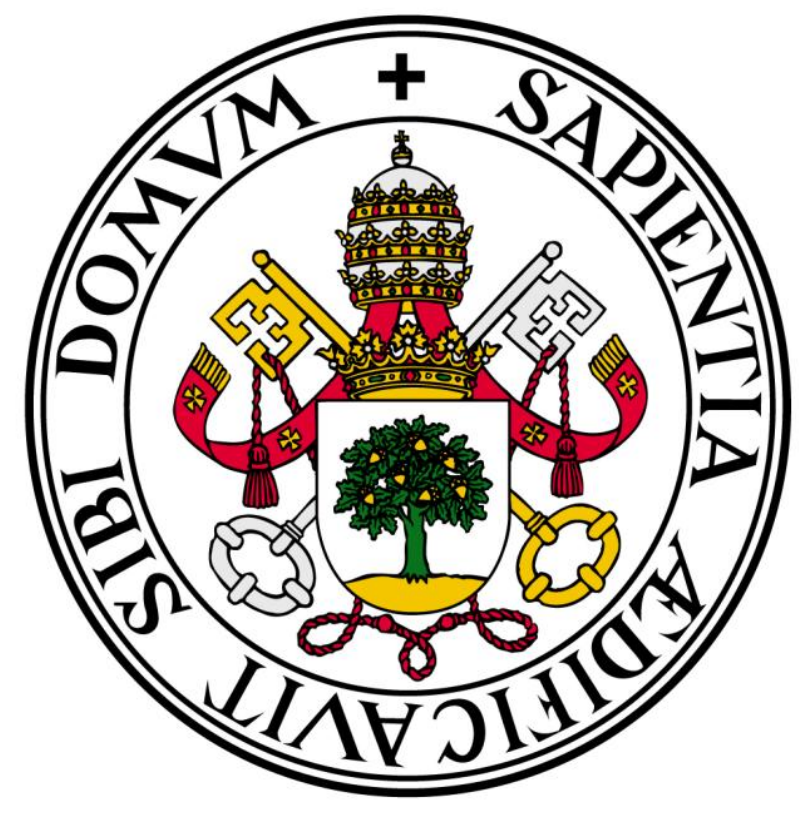

VII. Anexo I. 



\section{ANEXO I}

Tesis españolas relacionadas con las intoxicaciones

1. Título: Intervención del servicio de Farmacología Clínica en el tratamiento de las intoxicaciones agudas ingresadas en una unidad de cuidados intensivos.

Autor: Frati Pereira M. Elisa.

Año: 1982

Universidad: Autónoma de Barcelona.

Resumen: Se describe el perfil epidemiológico del paciente intoxicado agudo que requiere ingresar en la UCIG. Se estudian las características asistenciales. Otro objetivo es la racionalización y actualización de los tratamientos sociológicos.

2. Titulo: Intoxicación medicamentosa aguda grave. Patología respiratoria asociada.

Autor: Nogué Xarau, Santiago.

Año 1986.

Universidad: Barcelona.

Resumen: Se analiza la patología respiratoria que ha presentado una serie de intoxicaciones graves que han provocado ingreso en la unidad de cuidados intensivos, comparándola con la que se ha presentado en otra serie paralela de intoxicaciones no medicamentosas y estableciendo unos factores pronósticos de su presentación. 
3. Título: Accidentes domésticos por intoxicaciones infantiles en la zona sur de Madrid.

Autor: Andarías Moríñigo, Rosa M.

Universidad Complutense de Madrid.

Año: 1989

Resumen: Recogida de datos de 963 historias clínicas de niños atendidos en 4 centros hospitalarios de la zona en el año 1987 debidos a intoxicaciones. Se recogen los siguientes parámetros: sexo, edad, tipo de intoxicación, tipo de tóxico, lugar del accidente, data, clase social, antecedentes, tiempo hasta la asistencia médica hospitalaria, estancia, ingreso, evolución, diagnóstico y tratamiento.

4. Título: Insecticidas organofosforados: intoxicaciones agudas. Intoxicaciones crónicas.

Autor: Yelamos Rodríguez, Fernando.

Universidad: Granada

Fecha: 1990.

Resumen: Se recogen 187 casos de intoxicaciones agudas por insecticidas organofosforados, atendidas por el doctorando en el servicio de Urgencias del Hospital Torrecardenas de Almería, durante 6 años (1981 a 1986.)

Todos estos pacientes siguen un mismo tratamiento protocolizado. Se analizan los resultados encontrados según el tóxico, la vía de absorción, motivo de la intoxicación y factores pronósticos añadidos.

5. Título: Evolución de las urgencias toxicológicas en un hospital general.

Autor: Monteis Catot, Jaume. 
Año: 1990.

Universidad autónoma de Barcelona.

Resumen: Se ha valorado la evolución de las intoxicaciones atendidas en el Hospital del Mar desde 1983 a 1988, poniendo especial hincapié en los cambios en el patrón toxicológico de las intoxicaciones medicamentosas.

6. Título: Epidemiología de las intoxicaciones infantiles.

Autor: Bedoya Pérez, Rafael

Año: 1991.

Universidad de Sevilla.

Resumen: Se realizó un estudio prospectivo de todos los niños atendidos por intoxicación aguda en el Hospital Infantil Universitario Virgen del Rocío, desde Febrero 1990 al 31 Enero de 1991. El objeto del estudio era conocer la magnitud del problema de las intoxicaciones infantiles en dicho medio, los rasgos epidemiológicos más llamativos y establecer medidas preventivas.

7. Título: Intoxicaciones agudas. Estudio epidemiológico. Repercusión hospitalaria en un área sanitaria.

Autor: Rodríguez Getino, José Ángel.

Universidad de Oviedo.

Año 1991.

Resumen: Estudio retrospectivo de la demanda asistencial por intoxicaciones agudas en el área de urgencias del hospital de referencia durante 4 años (desde 1985 a 1989.)

8. Título: Evolución del patrón clínico asistencial en las intoxicaciones agudas graves. 
Autor: Palomar Martínez, Mercedes.

Año: 1992.

Universidad Autónoma de Barcelona.

Resumen: Se analizaron los episodios de intoxicación aguda graves ingresados en la unidad de cuidados intensivos del Hospital General Vall D'Hebron desde el 1 de Enero de 1981 al 31 de Diciembre de 1990. Se revisaron factores clínicos, epidemiológicos y terapéuticos.

9. Título: Estudio epidemiológico de las intoxicaciones agudas atendidas en el Hospital Clínico Universitario de Zaragoza en el período 1990-1994.

Autor: Sola García, José Luis.

Año: 1995.

Universidad de Zaragoza.

Resumen: Evalúa la bondad del sistema de datos de recogida de intoxicaciones por parte del Servicio de Toxicología y estudia las principales características de las intoxicaciones agudas atendidas en el Hospital Clínico Universitario de Zaragoza en el periodo 1990-1994.

10. Título: Estudio epidemiológico y características psicosociales de las intoxicaciones en el ámbito hospitalario.

Autor: Hermida Lazcano, Ignacio.

Universidad Santiago de Compostela.

Año 1998.

Resumen: Se estudian 999 casos de intoxicaciones atendidos en un hospital de tercer nivel durante el año 1995. Se recogen las características psicológicas y sociales de los 96 pacientes cuya intoxicación fue voluntaria. 
11 Título: Intoxicaciones adultas y pediátricas atendidas en el Hospital General de Galicia durante el periodo 1993-96. Aspectos epidemiológicos, clínicos, terapéuticos y analíticos.

Nombre: Ortega Beneitez, Magdalena

Universidad: Santiago de Compostela

Fecha de lectura: 22/07/1998

Resumen: Estudio de 1385 intoxicaciones agudas, correspondientes a 1192 adultos y 193 niños, atendidos durante el período de 4 años en Hospital General de Galicia de Santiago de Compostela. Se estudió el número y tipo de intoxicaciones, su distribución respecto al sexo, edad, procedencia, cronología, tóxico, antecedentes, clínica, evolución y tratamientos.

12. Título: Variaciones epidemiológicas en las intoxicaciones agudas y voluntarias atendidas en el Hospital 12 de Octubre de Madrid de 1979 a 1994.

Autor: Dorado Pombu, Soledad

Universidad Complutense de Madrid.

Año: 1999

Resumen: se han revisado las características de los pacientes atendidos por intoxicación aguda voluntaria en el Servicio de Urgencias del Hospital universitario "12 de Octubre" de Madrid en los años 1979, 1985, 1990 y 1994. Se consideraron la edad (siempre mayores de 14 años), sexo, tóxico responsable, día de la semana, mes, antecedentes, destino y motivo que determinó la intoxicación. Según el motivo se agruparon en intoxicaciones con fines suicidas, por alcohol, drogas ilícitas y sobredosificación por automedicación. Según los tóxicos en aquellos que tienen uso terapéutico y los que no lo tienen. Se consideró además un grupo formado por los pacientes graves que precisaron ingreso en la Unidad de Cuidados Intensivos. 
13. Toxicología aguda en los hospitales públicos de Cataluña.

Autor: Pere Munné Mas

Universidad: Universitat de Barcelona.

Año: 1999

Resumen: Estudio descriptivo sobre las intoxicaciones en 24 hospitales públicos de Cataluña en el periodo de 1995.

14 Título: Epidemiología de las urgencias toxicológicas por drogas de abuso en el área sanitaria de Santiago de Compostela. Período 1997-2007.

Autor: Rey Barbosa, María Cristina

Universidad Santiago de Compostela.

Año 2009.

Resumen: Se realiza estudio observacional de carácter descriptivo longitudinal y retrospectivo sobre las intoxicaciones por drogas de abuso que llegan a los servicios de urgencias del área sanitaria de Santiago de Compostela entre los años 1997 a 2007. Se analiza la evolución temporal de características epidemiológicas, clínicas, analíticas y terapéuticas.

15 Título: Urgencias hospitalarias por intoxicaciones agudas por intoxicaciones agudas: estudio multicéntrico nacional.

Autor: Burillo Putze, Guillermo.

Universidad de La Laguna.

Año: 2010.

Resumen: Se realiza un estudio prospectivo multicéntrico nacional transversal de las intoxicaciones agudas en 24 horas atendidas en urgencias hospitalarias de 24 hospitales españoles en el período de un año comprendido entre septiembre de 2005 y agosto 2006. 
Se analizaron las características demográficas, la asistencia, intencionalidad, síntomas, diagnóstico, tratamiento y destino paciente.

16 Titulo: Estudio sociológico, epidemiológico, clínico y terapéutico de las intoxicaciones agudas asistidas en el Consorcio Hospital General Universitario de Valencia en el periodo de un año.

Autor: Herrera de Pablo, Pere.

Universidad de Valencia

Año: 2010

Resumen: Análisis de forma prospectiva desde el punto de vista sociológico, epidemiológico, clínico y terapéutico, las intoxicaciones agudas en mayores de 14 años, asistidas durante un año natural en el servicio de urgencias de un hospital de tercer nivel de un área predominantemente urbana.

17. Título: Estudio multicéntrico de las intoxicaciones agudas en Zaragoza.

Autor: Juan Carmona.

Universidad de Zaragoza

Año: 2010

Resumen: Análisis de forma prospectiva de las intoxicaciones atendidas en los servicios de urgencia de los hospitales de Zaragoza durante el periodo comprendido entre 1 de enero de 2000 y 31 diciembre 2000.

18 Título: Epidemiología y calidad asistencial en el paciente intoxicado en un hospital de tercer nivel.

Autora: Beatriz Martín Pérez

Universidad de Valladolid 
Año: 2012

Resumen: Conocimiento de la calidad asistencial de los paciente intoxicados que acuden al Servicio de Urgencias del Hospital Universitario Río Hortega; Determinando los indicadores del CALITOX, estudiarlos y mejorarlos si fuera posible; Conocer la epidemiología de las intoxicaciones agudas en nuestro ámbito.

19 Titulo: Aportación analítica al proceso de atención al paciente intoxicado. Experiencia en la Unidad de Toxicología Clínica del Hospital Universitario Son Dureta.

\section{Autor: Bartomeu Castanyer Puig}

Universidad de Barcelona

Año: 2012.

Resumen: El estudio de los datos del registro ha conseguido conocer la realidad de la epidemiologia de la intoxicación en nuestro entorno geográfico. Asimismo, las encuestas realizadas han permitido valorar objetivamente el grado de conocimiento que poseen los clínicos que atienden a enfermos intoxicados sobre diferentes aspectos relativos a la toxicología analítica, y se han obtenido orientaciones que han facilitado conocer con una base objetiva lo que se espera del laboratorio, y cómo valora el clínico solicitante al laboratorio y los resultados analíticos suministrados.

En relación a la intoxicación por Paracetamol, la estimación de la semivida del PCT en los casos en que no es posible aplicar el nomograma de RumackMattew, posibilita obtener de manera rápida y fácil una información objetiva útil que permite valorar la adecuación o no de instaurar el tratamiento específico, y que aporta un dato complementario útil en la valoración pronostica de la intoxicación por PCT.

La determinación de PCT en orina ha mostrado ser una herramienta útil para descartar una ingesta de PCT en las 24 horas previas, por lo que resulta adecuada su inclusión en el algoritmo de actuación del paciente pediátrico con sospecha de intoxicación aguda por PCT. 
20. Título: Identificación de factores de riesgo en las intoxicaciones pediátricas. Autor: Beatriz Azkunaga Santibañez.

Año: 2013.

Universidad del País Vasco.

Resumen: Un análisis de las circunstancias que rodean las intoxicaciones agudas pediátricas puede facilitar la identificación de las situaciones de riesgo más habituales en nuestro entorno. Esto requiere investigar los mecanismos últimos responsables de la intoxicación, siendo un primer paso necesario para poder diseñar estrategias preventivas que puedan se eficaces a nuestros niños y adolescentes. 


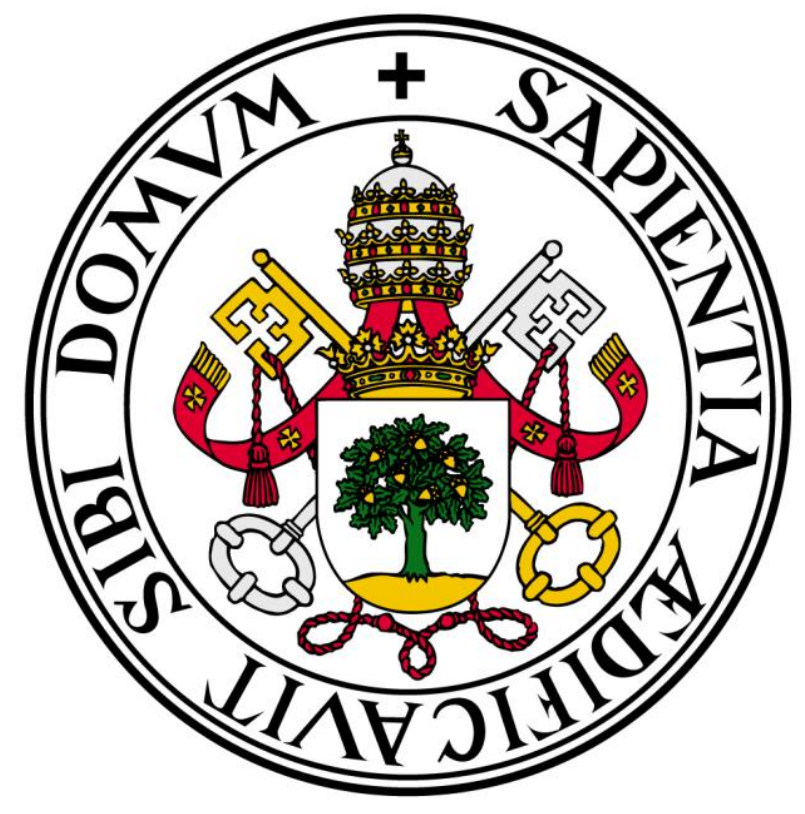

VIII. Bibliografía 


\section{Bibliografía}

1. Dueñas Laita A. Intoxicaciones agudas en medicina de urgencia y cuidados críticos. Barcelona. Elsevier-Masson 1999;3-6.

2. Vallverdu J. La evolución de la toxicología: de los venenos a la evaluación de riesgos. Revista de toxicología 2005;22:153-161.

3. Nolla Panadés R. Estudio estadístico de los intoxicados ingresados en el Hospital Clínico y Provincial de Barcelona durante el quinquenio 1951-1955. Arch Españ Med 1956;(2):283-288.

4. Milla J, Camp J, Borras A, Munne P, Anguita A. Epidemiology of the acute intoxication in Barcelona. Acta Pharmacol Toxicol (Copenh) 1977;41 Suppl 2:562-569.

5. Carpintero JM, Ochoa FJ, Ruiz JI, Bragado L, Palacios G, Ramalle-Gómara E. Prevalencia de las intoxicaciones agudas en Urgencias de La Rioja. Emergencias 2000;(12):92-97.

6. Riquelme RA, Burillo-Putze G, Jimenez SA, Hardisson DLT. Epidemiología global de la intoxicación aguda en un área de salud. Atención Primaria 2001;28(7):506.

7. Espinosa G, Miró O. Intoxicación por éxtasis líquido:estudio de 22 casos. Med Clin 2001;(117):56-58.

8. Mintegui S, Benito F, Vázquez MA, Fernández A, Gortazar P, Grou G. Intoxicaciones en urgencias: cambios epidemiológicos en los últimos 10 años. An Esp Pediatr 2002; $56(1): 23-29$

9. Bosca SB, Marco Garbayo JL, Robles PE, San Martin Ciges MA. Intoxicaciones accidentales en niños en el Hospital General de Requena 1997-2002. Atención Primaria 2003;32(10):603-604.

10. Burillo G, Munné $P$, Dueñas $A$. National multicenter study of acute intoxication in emergency departaments of Spain. European Journal of Emergency Medicine 2003;(10):101-104.

11. Sanjurjo E, Nogué $S$, Miró O, Munné P. Análisis de las consultas generadas por el consumo de éxtasis en un servicio de urgencias. Medicina Clinica 2004;(123):90-92.

12. Sanjurjo $E$, Montori $E$, Nogué $S$, Sánchez $M$, Munné $P$. Urgencias por cocaína: un problema emergente. Med Clin 2006;(126):616-619.

13. Pastó Cardona L, Martorell Puigserver C, Mercadal Orfila G, Machí Ribes JJ, Jordar Massanès. Intoxicaciones agudas en el servicio de urgencias de un hospital universitario de nivel III: cambios producidos en los últimos diez años. Revista Toxicología 2007;(24):36-41.

14. Burillo $G$, Munné $P$, Dueñas $A$ et al. Intoxicaciones agudas: perfíl epidemiológico y clínico, y análisis de las técnicas de descontaminación digestiva utilizadas en los 
servicios de urgencias españoles en el año 2000 - Estudio HISPATOX. Emergencias 2008;(20):15-26.

15. Galicia M, Nogué S, Sanjurjo E, Miró O. Evolución de las consultas urgentes relacionadas con el consumo de cocaína durante el período 2002-2007. Emergencias 2008;(20):385-390.

16. Hernández SH., Hoffman RS. Perspectivas en el consumo de cocaína. Emergencias 2008;(20):371-373.

17. Piñana López A. Grupo de urgencias SEMERGEN. Intoxicación etílica aguda en adolescentes. Siete Días Médicos (revista en internet) 2009.

18. Macías Robles MD, Fernández Carreira JM, García Suárez I, Fernández Diéguez O, Redondo Torres $\mathrm{G}$. Evolución epidemiológica de las intoxicaciones agudas por gases tóxicos atendidas durante el periodo de 2004 a 2007 en urgencias de un hospital comarcal. Emergencias 2009;21(5):350-353.

19. Clemente Rodríguez C, Aguirre Tejedo A, Echarte Pazos J, Puente Palacios I, Iglesias Lepine ML, Supervía Caparrós A. Diferencias entre hombres y mujeres en las características de las intoxicaciones. Emergencias 2010;(22):435-440.

20. Sanchez SA, Redondo MS, Garcia Vicario MI, Velazquez MA. Episodios de urgencia hospitalarios relacionados con el consumo de alcohol en personas de entre 10 y 30 años de edad en Castilla y León durante el periodo de 2003-2010. Rev Esp Salud Publica 2012;86(4):409-417.

21. Arias Constantiní V, Sanz Marcos N, Trenchs Sainz de La Maza V, Curcoy Barcenilla Al, Matalí Costa J, Luaces Cubells C. Uso de drogas psicoactivas por parte de adolescentes y consultas en servicio de urgencias. Med Clin 2010;134(13):583-586.

22. Pita-Fernández $\mathrm{S}$, Lombardía-Cortiña $\mathrm{M}$, Orozco-Veltran D, Gil-Guillén V. Manifestaciones clínicas de la intoxicación por digital en pacientes ancianos en los servicios de urgencia. Arch Gerontol Geriatr 2011;53(2):106-110.

23. Croche Santander B, lonso Salas MT, Loscertales Abril M. Intoxicación accidental por cannabis: presentación de cuatro casos pediátricos en un hospital terciario del sur de España. Arch Argent Pediatr 2011;109(1):4-7.

24. Alonso CL, Ruiz HJ, Villalobos RJ, Gaitero TJ, Perez RT, Canedo VE. Intoxicación por Vitamina $D$ en recién nacidos inmigrantes Latinoamericanos. Serie de 3 casos. An Pediatr (Barc ) 2011;74(6):409-412.

25. Galicia $\mathrm{M}$, Nogué $\mathrm{S}$, Miró $\mathrm{O}$. Intoxicación por éxtasis: 505 casos consecutivos atendidos en urgencias. Emerg Med J 2011;28(6):462-466.

26. Clemente Rodríguez C, Echarte Pazos JL, Aguirre Tejedo A, Puente Palacios I, Iglesias Lepine ML, Supervía Caparrós A. Diferencias en las intoxicaciones de los españoles y los extranjeros atendidas en urgencias. Emergencias 2011;23:271-275.

27. Badia $M$, Justes $M$, Serviá $L$ et al. Clasificación de las enfermedades mentales en una Unidad de Cuidados Intensivos. Med Intensiva 2011;35(9):539-545. 
28. López J, Pérez X, Labad J, Esteve F, Manez R, Javierre C. Higher requirements of dialysis in severe lithium intoxication. Hemodial Int 2012;16(3):407-413.

29. de Miguel-Bouzas JC, Castro-Tubio E, Bermejo-Barrera AM, Fernandez-Gomez P, Estevez-Nunez JC, Tabernero-Duque MJ. Estudio epidemiológico de las intoxicaciones agudas tratadas en un hospital gallego entre 2005 y 2008. Adicciones 2012;24(3):239246.

30. Muñoz R, Borobia A, Quintana M, Martínez-Virto AM, Frías J, Carcas AJ. Desarrollo y validación de un programa de toxicovigilancia con detección automatizada de casos en un hospital terciario (SAT-HULP). Emergencias 2013;(25):423-429.

31. Estévez Rueda MJ, Guisado Vasco P, Alba Muñoz L, Carpena Zafrilla M, Gallego Rodríguez $\mathrm{P}$, Trueba Vicente A. Revisión de 862 pacientes portadores de drogas intraabdominales (body packers) ingresados en una unidad de vigilancia de urgencias. Protocolo de manejo. Emergencias 2013;(25):451-458.

32. Moran Chorro I, Baldirá Martínez de Irujo J, Marruecos-Sant L, Nogué S. Toxicología clínica. Madrid, Grupo difusión, 2011.

33. Mintegi S, Fernandez A, Alustiza J et al. Emergency visits for childhood poisoning: a 2year prospective multicenter survey in Spain. Pediatr Emerg Care 2006;22(5):334-338.

34. Salazar J, Zubiaur O, Azkunaga B, Molina JC, Mintegi S. Diferencias territoriales en las intoxicaciones agudas en menores de 14 años en España. An Pediatr (Barc) 2014;80(3):1-5.

35. Azkunaga B, Mintegi S, Salmon N, Acedo Y, Del AL. [Poisoning in children under age 7 in Spain. Areas of improvement in the prevention and treatment]. An Pediatr (Barc) 2013;78(6):355-360.

36. Azkunaga B, Mintegui S, Del Arco L, Bizcarra I. Cambios epidemiológicos en las intoxicaciones atendidas en los servicios de urgencias pediátricos españoles entre 2001 y 2010: incremento de las intoxicaciones etílicas. Emergencias 2012;24:376-379.

37. Azkunaga B, Mintegi S, Bizkarra I, Fernandez J. Toxicology surveillance system of the Spanish Society of Paediatric Emergencies: first-year analysis. Eur J Emerg Med 2011;18(5):285-287.

38. Velasco AE, Trenchs Sainz de la Maza, Curcoy Barcenilla Al, Velasco RJ, Matali Costa JL, Luaces CC. ¿Quién da positivo por cannabis en las urgencias pediátricas? An Pediatr (Barc ) 2010;72(6):385-390.

39. Conejo Menor JL, Lallana Dupla MT. Intoxicación por antitérmicos. Anales de Pediatría 2002;56(4):318-323.

40. Vazquez P, Lopez-Herce J, Galaron P, Merello GC. Niveles de carboxihemoglobina y factores de riesgo de las intoxicaciones por monóxido de carbono en niños. Med Clin (Barc ) 1997;108(1):1-3.

41. Repetto MR. Pediatric poisonings due to cleansing agents reported in 1994 to the Toxicological Information Service of Seville, Spain. Vet Hum Toxicol 1996;38(6):435437. 
42. Mintegui RS, Velasco Vicente JV, Villate CA et al. Evaluación de los aspectos epidemiológicos de los casos de intoxicaciones en un servicio de urgencias pediátrico. An Esp Pediatr 1990;33(6):528-530.

43. Armas $\mathrm{H}$, Baselga $\mathrm{C}$, Calvo l et al. Aspectos epidemiológicos de las intoxicaciones accidentales en niños en Aragón. An Esp Pediatr 1980;13(9):761-770.

44. Gonzalez D, Tauler E, Llorens J. Intoxicaicones por mordeduras de víboras en niños: revisión de seis casos. An Esp Pediatr 1980;13(2):151-156.

45. Mintegi Satc. Manual de intoxicaciones en pediatría. 3a edición. Grupo de trabajo de intoxicaciones de la Sociedad Española de Urgencias de Pediatría 2012.

46. Dueñas Laita A. (2012) iTox. Urgencias por intoxicación en el adulto y en el niño. (Versión 1.0.1.) (Mobile application software). Disponible en http://itunes.apple.com

47. Dueñas Laita A. iTox. Urgencias por intoxicaciones en el adulto y niño. 1a ed. Adalia, Madrid, 2014.

48. Duenas-Laita A, Ruiz-Mambrilla M, Gandia F et al. Epidemiology of acute carbon monoxide poisoning in a Spanish region. J Toxicol Clin Toxicol 2001;39(1):53-57.

49. Encuesta estatal sobre el uso de drogas en estudiantes de enseñanzas secundarias 1994-2010. Ministerio de Sanidad y Consumo. Gobierno de España. 2010

50. Ayala MA, Jiménez MA, Rodríguez MC, Burillo Putze G. Sobre los informes de asistencia en urgencias. Med Clin (Barc) 2004;122-139.

51. Rey MC. Epidemiología de las urgencias toxicológicas por drogas de abuso en el área sanitaria de Santiago de Compostela. Período 1997-2007. (Tesis Doctoral). Universidad Santiago de Compostela.; 2009.

52. Palomar M. Evolución del patrón clínico asistencial en las intoxicaciones agudas graves. (Tesis Doctoral). Universidad Autónoma de Barcelona.; 1992.

53. Mintegi S, at col. Manual de intoxicaciones en pediatría. 2a edición. Madrid, Ergón, 2008.

54. Nogué S. Intoxicaciones agudas. Bases para el tratamiento en un servicio de urgencias. 2010.

55. Ferrer A, Nogué Saet. Estudio de vigilancia epidemiológica de intoxicaciones causadas por productos químicos. 2001

56. Ferrer A, Nogué $S$, Dueñas Laita A, at col. Estudio de vigilancia epidemiológica de intoxicaciones causadas por productos químicos. 2002

57. Ferrer A, Nogué Satc. Estudio de vigilancia epidemiológica en los servicios de urgencias de intoxicaciones causadas por productos químicos. 2003

58. Ferrer A, Nogué S, et.col. Estudio de vigilancia epidemiológica en los servicios de urgencias, de las intoxicaciones causadas por productos químicos. 2004 
59. Ferrer A, Nogué $S$, at col. Estudio de vigilancia epidemiológica en los servicios de urgencias, de las intoxicaciones causadas por productos químicos. 2005

60. Ferrer A, Nogué $S$, at col. Estudio de vigilancia epidemiológica en los servicios de urgencias, de las intoxicaciones causadas por productos químicos. 2006

61. Ferrer A, Nogué S. Estudio de vigilancia epidemiológica en los servicios de urgencias, de las intoxicaciones causadas por productos químicos. 2007

62. Ferrer A, Nogué S. Estudio de vigilancia epidemiológica en los servicios de urgencias, de las intoxicaciones causadas por productos químicos. 2008

63. Ferrer $A$, Nogué $S$, at col. Estudio de vigilancia epidemiológica en los servicios de urgencias, de las intoxicaciones causadas por productos químicos. 2009

64. Ferrer A, Nogué S, at.col. Estudio de vigilancia epidemiológica en los servicios de urgencias, de las intoxicaciones causadas por productos químicos. 2010

65. LoVecchio F, Curry SC, Waszolek Kea. Poison control centers decrease emergency healthcare utilization costs. J Med Toxicology 2008;4:221-224.

66. Vallejo de Torres G. Memoria SIT 2011. Instituto Nacional de Toxicología y Ciencias Forenses 2011.

67. Bronstein AC, Spyker DA, Cantinela Jr LR, Green J, Rumack BH, Dart RC. 2010 Annual Report of the American Association of Poison Control Center's National Poison Data System (NPDS): 28th Annual Report. Clinical Toxicology 2011;49(10):910-941.

68. Bronstein AC, Spyker DA, Cantinela Jr LR, Green J, Rumack BH, Giffin SL. 2009 Annual Report of the American Association of Poison Control Centers' National Poison Data System (NPDS): 27th Annual Report. Clinical Toxicology 2010;48:979-1178.

69. Bronstein AC, Spyker DA, Cantinela Jr LR, Green J, Rumack BH, Giffin SL. 2008 Annual Report of the American Association of Poison Control Centers' National Poison Data System (NPDS): 26th Annual Report. Clinical Toxicology 2009;47:911-1084.

70. Bronstein AC, Spyker DA, Cantinela Jr LR, Green J, Rumack BH, Heard SE. 2006 Annual Report of the American Association of Poison Control Centers' National Poison Data System. Clinical Toxicology 2006;45:815-917.

71. Lai MW, Klein- Schwartz W, Rodgers Jr G et al. 2005 Annual Report of the American Association of Poison Control Centers' National Poisoning and Exposure Database. 2005

72. Watson W, Litoviz T, Rodgers Jr G et al. 2004 Annual Report of the American Association of Poison Control Centers Toxic Exposure Surveillance System. 2004

73. Watson W, Litoviz T, Rodgers Jr G et al. 2003 Annual Report of the American Association of Poison Control Centers Toxic Exposure Surveillance System. 2003

74. Watson W, Litovitz T, Rodgers Jr G et al. 2002 Annual Report of the American Association of Poison Control Centers Toxic Exposure Surveillance System. 2002 
75. Jackson G, Good AM. National Poisons information services. Annual report 2009/2010 and five years review. 2010

76. Beaumont Hospital. National Posions Information Centre. Annual report 2002. 2002

77. Beaumont Hospital. National Poisons Information Centre. Annual Report 2003. 2003

78. Beaumont Hospital. National Poisons Information Centre. Annual Report 2004. 2004

79. Beaumont Hospital. National Poisons Information Centre. Annual report 2005. 2005

80. Beaumont Hospital. National Poisons information centre. Annual report 2006. 2006

81. Tracey J, Casey P, Cooke A et al. Poisons information centre of Ireland. Annual report 2007. 2007

82. Tracey J, Casey P, Cooke A et al. Poisons Information Centre of Ireland. Annual report 2008. 2008

83. Tracey J, Casey P, Cooke A et al. Poisons information centre of Ireland. Annual report 2009. 2009

84. Tracey J, Duggan E, Casey P et al. Poisons information center Ireland. Anual report 2010. 2010

85. Villa A, Cochet A, Tan Guyodo G. Les intoxications signalées aux centres antipoison français en 2006. 2006

86. Azkunaga B, Martínez L, Mintegi S, Pociello N. Boletín. Grupo de trabajo de intoxicaciones de la sociedad española de urgencias de pediatría. Diciembre 2013. 2013;15:1-4

87. Sola JL. Estudio epidemiológico de las intoxicaciones agudas atendidas en el Hospital Clínico Universitario de Zaragoza en el período 1990-1994. (Tesis Doctoral). Universidad de Zaragoza.; 1995.

88. Carmona J. Estudio multicéntrico de las intoxicaciones agudas en Zaragoza.(Tesis Doctoral). Universidad de Zaragoza.; 2010.

89. Díaz de Neira $\mathrm{M}$, et al. Prevalencia y funciones de los pensamientos y conductas autoagresivas en una muestra de adolescentes evaluados en consulta externa de salud mental. Revista de Psiquiatría y Salud Mental (Barcelona) 2013;6(4)

90. González-Juárez C, Pérez - Pérez E, Martín Cabrera B, Mitja Pau I, Roy de Pablo R, Vázquez de la Torre $P$. Detección de adolescentes con riesgo de sufrir desordenes alimentarios. Atención Primaria 2007;39(4):189-194.

91. Muro-Sanz P, Amador - Campos JA. Prevalence of eating disorders in a Spanish community adolescent sample. Eating and Weight disorder 2007;12(1):1-6.

92. Azkunaga B, Martínez L, Mintegi S, Pociello N. Boletín. Grupo de trabajo de intoxicaciones de la sociedad española de urgencias de pediatría. Febrero 2013. 2013;13:1-4. 
93. Duce $\mathrm{S}$, López $\mathrm{E}, \mathrm{Navas} \mathrm{V}$ et al. Intoxicaciones medicamentosas voluntarias atendidas en un servicio de urgencias. Emergencias 1998;10(4):225-233.

94. Castro J, Escandell I, Escudero C et al. Informe sobre la salud mental de niños y adolescentes. Cuadernos técnicos. 2009.

95. Herranz M, Clerigué N. Intoxicación en niños: Metahemoglobinemia. Anales del Sistema Sanitario de Navarra 2003;26:209-223.

96. Vallejo de Torres G. Memoria 2011 del Instituto Nacional de Toxicología y Ciencias Forenses. Instituto Nacional de Toxicología y Ciencias Forenses 2011.

97. Fernández P. Intoxicaciones pediátricas atendidas en el Hospital de Santiago de Compostela (1993-1996). Revista de toxicología 2002; 20(3):216-220.

98. Consumo problemático de cannabis en estudiantes españoles de 14-18 años: validación de escalas. Informes, estudios e investigación en drogodependencias Ministerio de Sanidad y Política Social 2009.

99. Ruiz C, Escorihuela R. Causas de consulta especializada en pacientes mayores de 7 años procedentes de atención primaria. Anales de Pediatria 2007;66(3):313-328.

100. Bugarin R, Galego P, Gude F, García A, Galbán C. Estudio de las intoxicaciones etílicas agudas en un servicio de urgencias. An Medicina Interna (Madrid) 2000;17(11):588591.

101. Hermida I. Estudio epidemiológico y características psicosociales de las intoxicaciones en el ámbito hospitalario. (Tesis Doctoral). Universidad Santiago de Compostela.; 1998.

102. Martín B. Epidemiología y calidad asistencial en el paciente intoxicado en un hospital de tercer nivel. (Tesis Doctoral). Universidad de Valladolid.; 2012.

103. Goicochea JA, Nieto MA, Laguna A, Larrocha D, Canto VD, Murillo F. Características de las urgencias hospitalarias del Servicio Andaluz de Salud durante 2012 a partir del Conjunto Mínimo Básico de Datos de Urgencias. Revista Española Salud Pública 2013;87(6):587-600.

104. Azkunaga B, Martínez L, Mintegi S, Pociello N. Boletín. Grupo de trabajo de intoxicaciones de la sociedad española de urgencias de pediatría. Abril 2014. 2014;16(1):4

105. Fernández Egido C, García Herrero G, Romero García R, Marquina Santos AJ. Intoxicaciones agudas en las urgencias extrahospitalarias. Emergencias 2008;20(5):328-331.

106. Shah M, Cushman JT, O Davis C, Bazarian JJ, Auinger P, Friedman B. Epidemiología del uso de los servicios de emergencias médicas por parte de la población pediátrica: un análisis del estudio National Hospital Ambulatory Medical Care Survey. Prehospitalary Emergency Care (edición española) 2009;2(2).

107. Mateu Sancho J. El niño intoxicado. Barcelona: MC ediciones; 1995. 
108. McGuigan MA. Guideline Consensus Panel. Guideline for the out-of-hospital management of human exposures to minimally toxic substance. J Toxicol Clin Toxicol 2003;41:907-917.

109. Azkunaga B, Martínez L, Mintegi S. Boletín. Grupo de trabajo de intoxicaciones de la sociedad española de urgencias de pediatría. Junio 2012. 2012;11:1-4.

110. Even KM, Armsby CC, Bateman ST. Poisonings requiring admission to the pediatric intensive care unit: A 5-year review. Clin Toxicol (Phila) 2014;52(5):519-524.

111. Domingo A, García JJ, Luaces C, Gelabert G, Pou J. Criterios de adecuación de ingresos en urgencias pediátricas. An Pediatr (Barc ) 2004;61(5):442-454.

112. Casanova C, Gascón P, Calvo F, Tomás M, Paricio JM, Blasco L. Uso inapropiado de la hospitalización en Pediatría. Validación de la versión española del Pediatric Appropriateness Evaluation Protocol. Gaceta Sanitaria 1999;13:301-303.

113. Trapero B, Lorenzo S, Alonso B, Pastor V. Evaluación de la adecuación del ingreso y la estancia hospitalaria mediante el Pediatric Appropriateness Evaluation Protocol.

Calidad Asistencial 2002;17:624-631.

114. Gómez JL, Bonillo A, González-Ripoll M, Jiménez R, Aguirre FJ, López J. Utilidad del Pediatric Appropriateness Evaluation Protocol para detectar deficiencias de circuitos asistenciales hospitalarios. An Pediatr (Barc) 2004;60:228-235.

115. Riordan M, Rylance G. Poisoning in Children 1: General management. Arch Dis Child 2002;87:392-396.

116. Eldridge DL, Van Eyk J, Kornegay C. Pediatric toxicology. Emerg Med Clin North Am 2007; 25:283-308.

117. Olgun H, Yildirim ZK, Karacan M, Ceviz N. Clinical, electrocardiographic, and laboratory findings in children with amitriptyline intoxication. Pediatr Emerg Care 2009;25:170-173.

118. Martin G, Belson MD. The utility of toxicologic analisis in children with suspected ingestions. Pediatr Emerg Care 1999;15:383-387.

119. Shannon M. Ingestion of toxic substances by children. New England Journal 2002;342:186-191.

120. Arias Constantini V, Trenchs Sainz de La Maza V, Sanz N, Curcoy Al, Luaces C. Valoración de la necesidad de analítica sanguínea a los adolescentes con intoxicación etílica aguda en un servicio de urgencias. An Pediatr 2010;73:288-290.

121. Sanz N, Arias Constantini V, Trenchs Sainz de La Maza V, Curcoy Al, Matalí J, Luaces C. Consultas por intoxicación etílica aguda en un servicio de urgencias pediátricas. An Pediatr 2009;70(2):132-136.

122. Charalambous MP. Alcohol and the accident and emergency department: a current review. Alcohol Alcohol 2007;37:307-312. 
123. Sugarman JM, Rodgers GB, Paul RL. Utility of toxicology screening in a pediatric emergency department. Pediatr Emerg Care 1997;13(3):194-197.

124. Belson MG, Simon HK, Sullivan K, Geller RJ. The utility of toxicologic analysis in children with suspected ingestions. Pediatr Emerg Care 1999;15(6):383-387.

125. Wang GS, Deakyne S, Bajaj L, Yin S, Heard K, Roosevelt G. The limited utility of screening laboratory tests and electrocardiograms in the management of unintentional asymptomatic pediatric ingestions. The Journal of Emergency Medicine 2013;45(1):34-38.

126. Dueñas Laita A, Nogué-Xarau S, Burillo-Putze G, Ruiz-Mambrilla M. Infarto de miocardio asociado a una intoxicación por monóxido de carbono. Med Clin 2006;126(8).

127. Lahoz R, Jordán I, Jiménez L, Hermogenes F. Infarto agudo de miocardio por cocaína. An Pediatr 2009;70(4):397-398.

128. Ministerio de Sanidad. Encuesta estatal sobre uso de drogas en enseñanzas secundarias (ESTUDES) España 2012/2013. 2013

129. Moyá C, Sánchez A, Flores J et al. Alcohol. Informes de la comisión clínica. Centro de publicaciones del Ministerio de Sanidad y Consumo Gobierno de España 2007;2.

130. Schoberl S, Nickel P, Schmutzer G, Siekmeyer W, Kiess W. [Acute ethanol intoxication among children and adolescents. A retrospective analysis of 173 patients admitted to a university children hospital]. Klin Padiatr 2008;220(4):253-258.

131. Weinberg L, Wyatt JP. Children presenting to hospital with acute alcohol intoxication. Emerg Med J 2006;23(10):774-776.

132. Reitox National Focal Point. 2011 National Report ( 2010 data) to the EMCDDA Spain. 2011

133. Roldán J, Frauca C, Dueñas Laita A. Intoxicación por alcoholes. Anales del Sistema Sanitario de Navarra 2003;26(1):129-139.

134. Marin N, Newbury-Birch D, Duckett J et al. A retrospective analysis of the nature, extent and cost of alcohol-related emergency calls to the ambulance service in an English region. Alcohol and alcoholism (Oxford) 2012;47(2):191-197.

135. Lee GA, Forsythe M. Is alcohol more dangerous than heroin? The physical, social and financial costs of alcohol. Int Emerg Nurs 2011;19(3):141-145.

136. Foxcroft D, Ireland D, Lowe G, Breen R. WITHDRAWN: Primary prevention for alcohol misuse in young people. Cochrane Database Syst Rev 2011;(9):CD003024.

137. Foxcroft DR, Ireland D, Lister-Sharp DJ, Lowe G, Breen R. Primary prevention for alcohol misuse in young people. Cochrane Database Syst Rev 2002;(3):CD003024.

138. Foxcroft DR, Tsertsvadze A. Universal school-based prevention programs for alcohol misuse in young people. Cochrane Database Syst Rev 2011;(5):CD009113. 
139. Hellandsjo Bu ET, Watten RG, Foxcroft DR, Ingebrigtsen JE, Relling G. Teenage alcohol and intoxication debut: the impact of family socialization factors, living area and participation in organized sports. Alcohol Alcohol 2002;37(1):74-80.

140. Kuntsche E, Muller S. Why do young people start drinking? Motives for first-time alcohol consumption and links to risky drinking in early adolescence. Eur Addict Res 2012;18(1):34-39.

141. Perez MA, Redondo OM, Mesa G, I, Jimenez P, I, Martinez Fernandez ML, Perez MR. Motivación del consumo de alcohol por adolescentes de un instituto urbano. Aten Primaria 2010;42(12):604-611.

142. Rehm J, Patra J, Popova S. Alcohol-attributable mortality and potential years of life lost in Canada 2001: implications for prevention and policy. Addiction 2006;101(3):373384.

143. Rothwell $\mathrm{H}$, Segrott J. Preventing alcohol misuse in young people aged 9-11 years through promoting family communication: an exploratory evaluation of the Kids, Adults Together (KAT) Programme. BMC Public Health 2011;11:810.

144. Stolle M, Sack PM, Thomasius R. Binge drinking in childhood and adolescence: epidemiology, consequences, and interventions. Dtsch Arztebl Int 2009;106(19):323328.

145. Li C, Martin BC. Trends in emergency department visits attributable to acetaminophen overdoses in the United States: 1993-2007. Pharmacoepidemiology and drugs safety 2011;20(8):810-818.

146. Budnitz DS, Lovegrove MC, Crosby AE. Emergency department visits for overdoses of acetaminophen-containing products. American Journal of Preventive Medicine 2011;40(6):585-592.

147. Burghardt LC, Ayers JW, Brownstein JS, Bronstein AC, Ewald MB, Bourgeoi FT. Adult prescription drug use and pediatric medication exposures and poisonings. Pediatrics 2013;132(1):1-12.

148. Spiller HA, Beuhler MC, Ryan ML, Borys DJ, Aleguas A, Bosse GM. Evaluation of changes in poisoning in young children: 2000 to 2010. Pediatric Emerg Care 2013;29(5):635-640.

149. Franklin RL, Rodgers GB. Unintentional child poisonings treated in United States hospital emergency departments: national estimates of incident cases, populationbased poisoning rates, and product involvement. Pediatrics 2008;122(6):1244-1251.

150. McKenzie LB, Ahir N, Stolz U, Nelson NG. Household cleaning product-related injuries treated in US emergency departments in 1990-2006. Pediatrics 2010;126(3):509-516.

151. Bramuzzo M, Amaddeo A, Facchina G, Neri E, Martelossi S, Barbi E. Liquid detergent capsule ingestion: a new pediatric epidemic? Pediatr Emerg Care 2013;29(3):410-411.

152. Bonney A, Mazor S, Goldman RD. Laundry detergent capsules and pediatric poisoning. Canadian Family Physician 2013;12:1295-1296. 
153. Williams H, Baterman DN, Thomas SH, Thompson JP, Scott RA, Vale JA. Exposure to liquid detergent capsules: a study undertaken by the UK National Poisons Information Service. Clinical Toxicology (Philadelphia) 2012;50(8):776-780.

154. Williams $\mathrm{H}$, Jones $\mathrm{S}$, Wood $\mathrm{K}$ et al. Reported toxicity in 1486 liquid detergent capsule exposures to the UK National Poisons Information Service 2009-2012, including their ophthalmic and CNS effects. Clin Toxicol (Phila) 2014;52(2):136-140.

155. Nogué S, Dueñas Laita A. Monóxido de carbono: un homicidio invisible y silencioso. Med Clin 2005;124(8):300-301.

156. Dueñas-Laita A, Burillo Putze $G$, Alonso JR et al. Bases del manejo clínico de la intoxicación por humo de incendios. Docohumo Madrid 2010. Medicina Intensiva 2010;34(9):609-619.

157. Martínez IM, Nieto MA, Del Pueyo B. Medios de comunicación y dependencia. Plan Nacional sobre Drogas. 2000

158. Observatorio Europeo de las Drogas y las Toxicomanías. 2002: informe anual sobre el problema de la drogodependencia en la Unión Europea y en Noruega. 2002

159. Observatorio Europeo de las Drogas y las Toxicomanías. Informe anual 2003: el problema de la drogodependencia en la Unión Europea y en Noruega. 2003

160. Observatorio Europeo de las Drogas y las Toxicomanías. Informe anual 2004: el problema de la drogodependencia en la Unión Europea y en Noruega. 2004

161. Observatorio Europeo de las Drogas y las Toxicomanías. Informe anual 2005: el problema de la drogodependencia en Europa. 2005

162. Observatorio Europeo de las Drogas y las Toxicomanías. Infome anual 2006: el problema de la drogodependencia en Europa. 2006

163. Observatorio Europeo de las Drogas y las Toxicomanías. Informe anual 2007: el problema de la drogodependencia en Europa. 2007

164. Observatorio Europeo de las Drogas y las Toxicomanías. Informe anual 2008: el problema de la drogodependencia en Europa. 2008

165. Observatorio Europeo de las Drogas y las Toxicomanías. Informe anual 2009: el problema de la drogodependencia en Europa. 2009

166. Observatorio Europeo de las Drogas y las Toxicomanías. Informe anual 2010: el problema de la drogodependencia en Europa. 2010

167. EDADES: Encuesta domiciliaria sobre alcohol y drogas en España. Plan Nacional sobre Drogas Gobierno de España 2009.

168. Hibell B, Guttorssom U, Ahlstrom S et al. The 2007 ESPAD Report. Substance Use Among Students in 35 European Countries. ESPAD: The European School Survey Project on Alcohol and Other Drugs 2007. 
169. Vázquez MA, Muñoz MF, Fierro A, Alfaro M, Rodríguez M, Rodríguez L. Consumo de sustancias adictivas en los adolescentes de 13 a 18 años y otras conductas de riesgo relacionadas. Rev Ped Atención Primaria 2014;16:125-134.

170. Moyá C, Álvarez C, Sánchez C et al. Cannabis II. Informes de la comisión clínica. Centro de publicaciones del Ministerio de Sanidad y Política Social 2009;2.

171. Briere FN, Fallu JS, Descheneaux A, Janosz M. Predictors and consequences of simultaneous alcohol and cannabis use in adolescents. Addict Behav 2011;36(7):785788.

172. Matalí JL, Pardo M, Trenchs Sainz de La Maza V, Serrano E, Gabaldon S, Luaces C. Consumo de drogas en adolescentes. Dilema ético en el abordaje diagnósticoterapéutico. An Pediatr 2009;70(4):386-390.

173. Schubart CD, van Gastel WA, Breetvelt EJ et al. Cannabis use at a young age is associated with psychotic experiences. Phisologycal Medicine 2011;41(6):1301-1310.

174. Morales-Muñoz I, Jurado-Barba R, Ponce $G$ et al. Characterizing cannabis-induced psychosis: A study with prepulse inhibition of the startle reflex. Psiquiatry Research 2014;220 (1)535-540.

175. Coppola M, Mondola R. Cannabis consumption systemic adverse effects. International Journal of High Risk Behavoirs and Addiction 2014;28(3).

176. Phillips N, Milne B, Silsbury C et al. Addressing adolescent substance use in a paediatric health-care setting. Journal of Paediatric and Chil Health 2014;50(9):726-731.

177. Bally N, Zullino D, Aubry JM. Cannabis use and first manic episode. Journal of Affective Disorders 2014;165:103-108.

178. Gutiérrez-Rojas L, De Irala J, Martínez-González MA. Efectos del cannabis sobre la salud mental en jóvenes consumidores. Rev Med Univ Navarra 2006;50(1):3-10.

179. Álvarez E, Brime B, González J et al. Observatorio Español de las Drogas y las Toxicomanías. Informe 2011. Ministerio de Sanidad, Política Social e Igualdad 2011.

180. Garcia-Algar O, Lopez N, Bonet M, Pellegrini M, Marchei E, Pichini S. 3,4methylenedioxymethamphetamine (MDMA) intoxication in an infant chronically exposed to cocaine. Ther Drug Monit 2005;27(4):409-411.

181. Garcia-Algar O, Papaseit E, Velasco M et al. Consultas en urgencias de pediatría por intoxicación aguda por drogas de abuso. An Pediatr (Barc ) 2011;74(6):413-419.

182. Rodríguez L, Martínez L, Quintillá JM, Trenchs Sainz de La Maza V, Vila V, Luaces C. Ingesta de cáusticos: situación actual y puesta al día de las recomendaciones. An Pediatr (Barc ) 2011;65(5):334-340.

183. Wattigney WA, Kaye WE, Orr MF. Acute hazardous substance releases resulting in adverse health consequences in children: Hazardous Substances Emergency Events Surveillance system, 1996-2003. Journal of Environmental Health 2007;70(4):17-24. 
184. Perry L, Adams RD, Bennett AR et al. National toxicovigilance for pesticide exposures resulting in health care contact - An example from the UK's National Poisons Information Service. Clin Toxicol (Phila) 2014;52(5):549-555.

185. Piqueras J. Intoxicaciones por setas, una actualización. Revista Española de Medicina Legal 2014;40(1):19.

186. Munné P. Variabilidad de la descontaminación digestiva en nuestro medio. Revista de Tóxicología 2005;22:83.

187. Burillo Putze G, Munné Mas P. Carbón activado en las intoxicaciones agudas:¿está todo dicho? Medicina Clinica 2010;135(6):260-262.

188. Velasco R, et al. Impact of a working group of a grastrointestinal descontamination in Spanish emergency departament. European Journal of Emergency of Medicine 2014;21(3):195-198.

189. Amigó $\mathrm{M}$, Nogué $\mathrm{S}$, Miró $\mathrm{O}$. Carbón activado en 575 casos de intoxicaciones agudas. Seguridad y factores asociados a las reacciones adversas. Med Clin (Barc) 2010;135:243-249.

190. Hedeland RL, Andersen J, Askob N, Iskandar A, Jorgensen MH. Early predictors of severe acetaminophen-induced hepatotoxicity in a paediatric population referred to a tertiary paediatric department. Acta Paediatr 2014;103 (11)1179-1186.

191. Heard K, Rumack BH, Green JL et al. A single-arm clinical trial of a 48-hour intravenous $\mathrm{N}$-acetylcysteine protocol for treatment of acetaminophen poisoning. Clin Toxicol (Phila) 2014;52(5):512-518.

192. Freeman R. MHRA recommendations on the use of intravenous acetylcysteine in paracetamol overdose. Arch Dis Child Educ Pract Ed 2014;99(1):37-40.

193. Whyte IM, Francis B, Dawson AH. Safety and efficacy of intravenous N-acetylcysteine for acetaminophen overdose: analysis of the Hunter Area Toxicology Service (HATS) database. Curr Med Res Opin 2007;23(10):2359-2368.

194. Betten DP, Cantrell FL, Thomas SC, William SR, Clark RF. A prospective evaluation of shortened course oral $\mathrm{N}$-acetylcysteine for the treatment of acute acetaminophen poisoning. Ann Emerg Med 2007;50(3):272-279.

195. Martínez L, Imario AF, Escuredo L, Macao P, Trenchs Sainz de La Maza V, Luaces C. Uso de antídotos en un servicio de urgencias pediátrico. Anales de Pediatría 2014;81(4)220-225.

196. Hermida I, Fernández P, Ferrer A, Bermejo AM, Tabernero MJ. Perfil psicosocial de pacientes ingresados por intoxicación aguda voluntaria. Rev Toxicol 2003;20:33-37.

197. Bella M, Fernández R, Willington J. Intento de suicidio en niños y adolescentes: depresión y trastorno de conducta disocial como patologías más frecuentes. Arch Argen Pediatr 2010;108(2):124-129. 
198. Venceslá JF, Moriana JA. Conducta autolítica y parasuicida. Características sociodemográficas en población infantojuvenil de ámbito rural. Rev Asoc Esp Neuropsiq 2002;21(84):49-64.

199. Jiménez MT, Aragó J, Nuño A, Loño J, Ochando G. Atención al paciente agitado, violento o psicótico en urgencias: un protocolo pendiente para una patología en aumento. Anales de Pediatría 2005;63(6):526-536.

200. Yamamoto LG, Zimmermann KR, Butts RJ et al. Characteristics of frequent pediatric emergency department users. Pediatr Emerg Care 1995;11(6):340-346.

201. Mintegi S, Benito J, García S, Corrales A, Bartolomé MJ, Trebolazaba N. Demanda y asistencia en un servicio de urgencias hospitalario. An Pediatr (Barc ) 2004;61(2):156161.

202. Ruger JP, Ritcher CJ, Spitznagel EL, Lewis LM. Analysis of costs, length of stay, and utilization of emergency department services by frequent users: implications for health policy. Acad Emerg Med 2004;11(12):1311-1317.

203. Aranaz JM, Martínez R, Gea MT, Rodrigo V, Antón P, Gómez F. ¿Por qué los pacientes utilizan los servicios de urgencias hospitalarios por iniciativa propia? Gaceta Sanitaria 2006;20(4).

204. Riba D, Rodríguez-Rosich A, Gázquez M, Buti S. Pacientes hiperfrecuentadores en los servicios de urgencias. Estudio descriptivo en un hospital de segundo nivel. Emergencias 2004;16:178-183.

205. Pueyo MJ, Baranda L, Valderas J, Starfield B, Rajmil L. Papel del pediatra de atención primaria y coordinación con atención especializada. An Pediatr (Barc) 2011;75(4):247-252. 
